\title{
Systematics of the Onychoteuthidae Gray, 1847 (Cephalopoda: Oegopsida)
}

K.S. Bolstad

\author{
A thesis submitted to the \\ Earth \& Oceanic Sciences Research Institute \\ Auckland University of Technology \\ in fulfilment of the requirements for the degree of \\ Doctor of Philosophy \\ supervised by Dr Steve O'Shea \\ 2008
}




\section{TABLE OF CONTENTS}

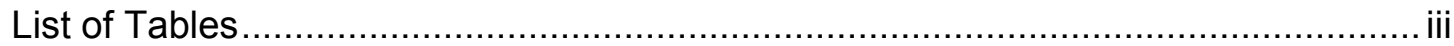

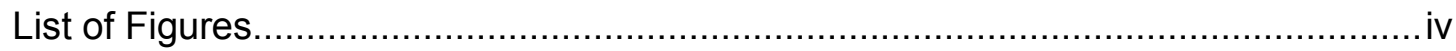

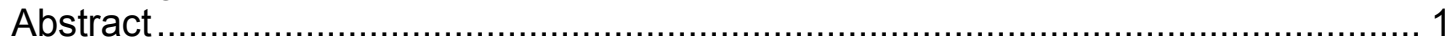

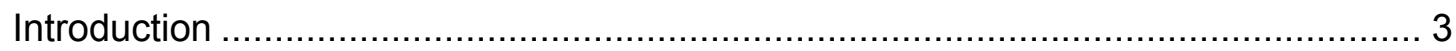

Foreword - Generic and Familial Nomenclature ............................................... 3

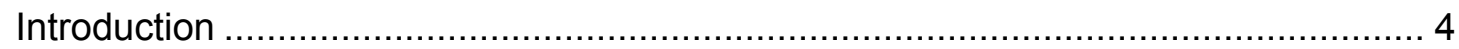

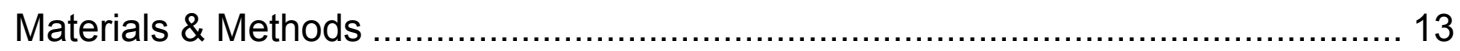

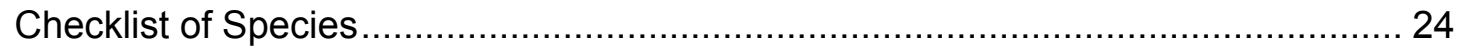

Quick Visual Reference to the Onychoteuthidae ................................................. 25

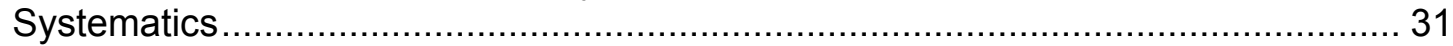

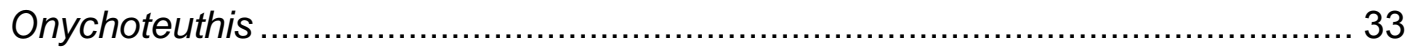

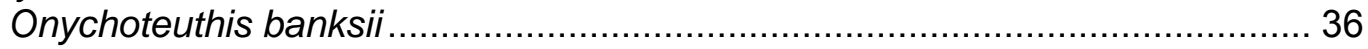

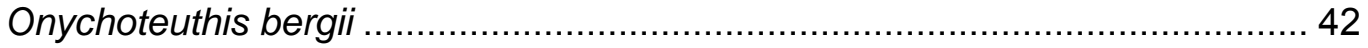

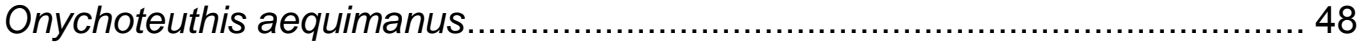

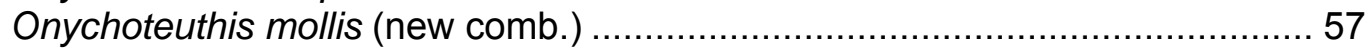

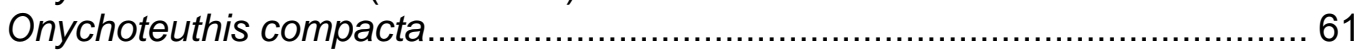

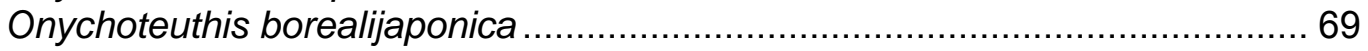

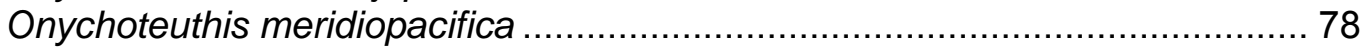

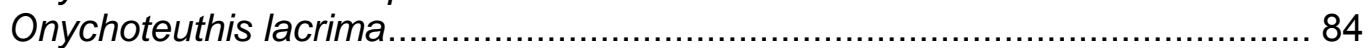

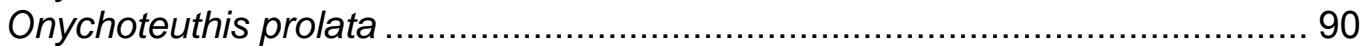

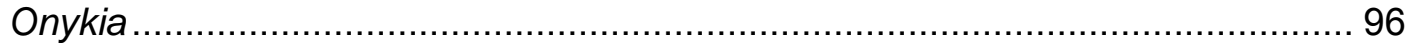

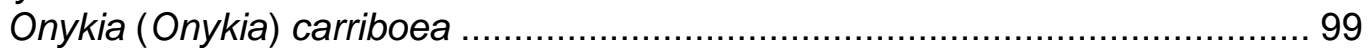

Onykia (Onykia) robusta (new comb.)..................................................... 106

Onykia (Onykia) loennbergii (new comb.) ................................................ 118

Onykia (Onykia) aequatorialis (new comb.) …........................................ 125

Onykia (Onykia) robsoni (new comb.) ........................................................ 131

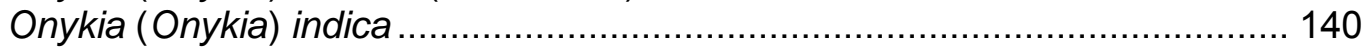

Onykia (Moroteuthopsis) ingens (new comb.) ......................................... 145

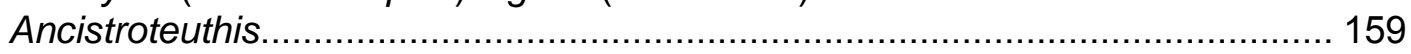

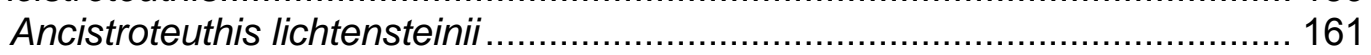

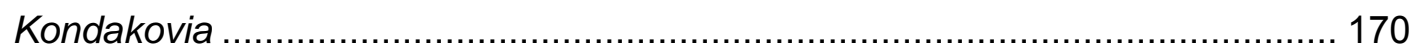

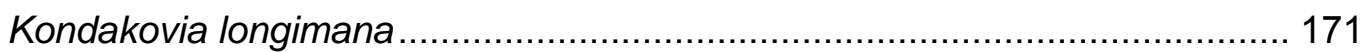

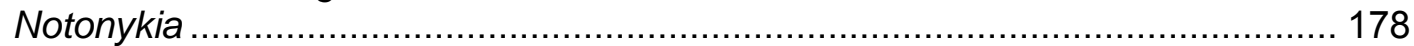

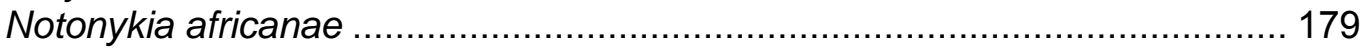

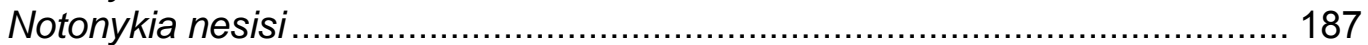

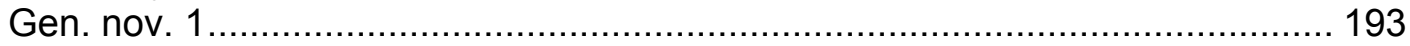

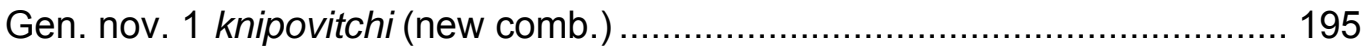

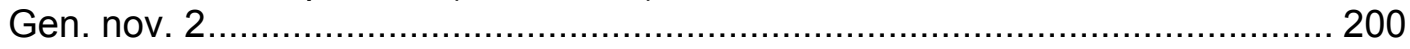

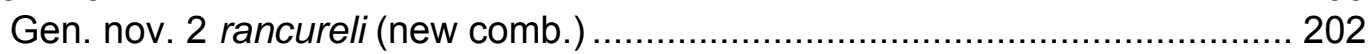

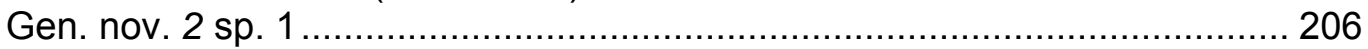

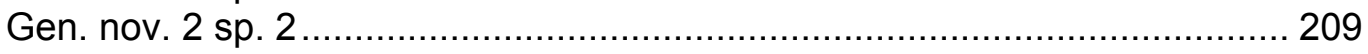

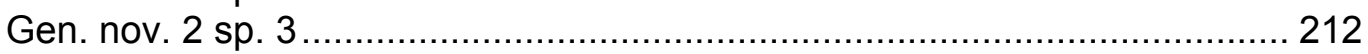

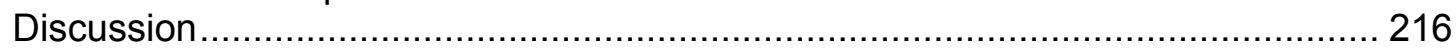

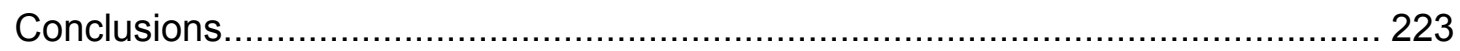

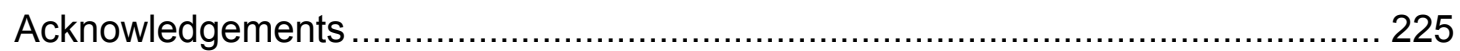

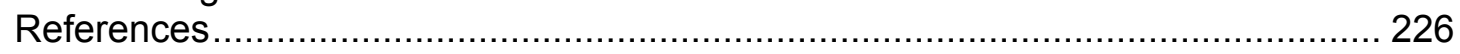

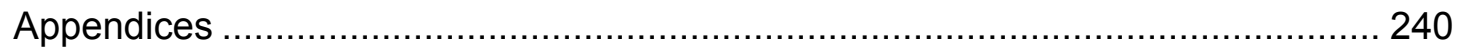

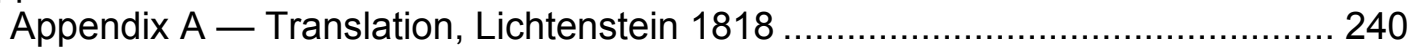

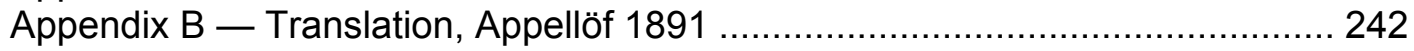

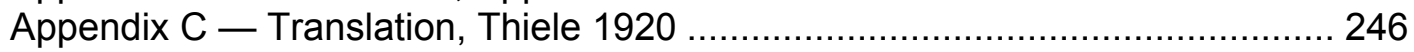

Appendix D — Translation, Adam 1962 ................................................... 249

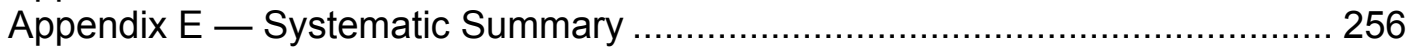




\section{ATTESTATION OF AUTHORSHIP}

I hereby declare that this submission is my own work and that, to the best of my knowledge and belief, it contains no material previously published or written by another person (except where explicitly defined in the acknowledgements), nor material that, to a substantial extent, has been submitted for the award of any other degree or diploma from a university or institute of higher learning.

Kathrin S. Bolstad 


\section{LIST OF TABLES}

1. Comparative table of onychoteuthid genera

2. Comparative table of species in the genus Onychoteuthis

3. Measurements and counts of Onychoteuthis banksii

4. Measurements and counts of Onychoteuthis bergii

5. Measurements and counts of Onychoteuthis aequimanus

6. Measurements and counts of Onychoteuthis compacta

7. Measurements and counts of Onychoteuthis borealijaponica

8. Measurements and counts of Onychoteuthis meridiopacifica

9. Measurements and counts of Onychoteuthis lacrima

10. Measurements and counts of Onychoteuthis prolata

11. Comparative table of species in the genus Onykia

12. Measurements and counts of Onykia (Onykia) carriboea

13. Measurements and counts of Onykia (Onykia) robusta

14. Measurements and counts of Onykia (Onykia) loennbergii

15. Measurements and counts of Onykia (Onykia) aequatorialis

16. Measurements and counts of Onykia (Onykia) robsoni

17. Measurements and counts of Onykia (Onykia) indica

18. Measurements and counts of Onykia (Moroteuthopsis) ingens

19. Sexually dimorphic characters in the lower beaks of Onykia (Moroteuthopsis) ingens

20. Measurements and counts of Ancistroteuthis lichtensteinii

21. Measurements and counts of Kondakovia longimana

22. Measurements and counts of Notonykia africanae

23. Measurements and counts of Notonykia nesisi

24. Measurements and counts of Gen. nov. 1 knipovitchi

25. Measurements and counts of Gen. nov. 2 rancureli and Gen. nov. 2 spp. 1-3 


\section{LIST OF FIGURES}

1. Distribution of material examined in this study

2. Measures and counts used in text

3. Onychoteuthid hook morphology and terminology

4. Paralarval sucker morphology and terminology

5. Radular morphology and terminology

6, 7. Onychoteuthis banksii

8, 9. Onychoteuthis bergii

10-13. Onychoteuthis aequimanus

14. Onychoteuthis mollis

15, 16. Onychoteuthis compacta

17-19. Onychoteuthis borealijaponica

20,21 . Onychoteuthis meridiopacifica

22,23 . Onychoteuthis lacrima

24,25 . Onychoteuthis prolata

26-28. Onykia (Onykia) carriboea

29-33. Onykia (Onykia) robusta

34-36. Onykia (Onykia) loennbergii

37, 38. Onykia (Onykia) aequatorialis

39-41. Onykia (Onykia) robsoni

42, 43. Onykia (Onykia) indica

44-48. Onykia (Moroteuthopsis) ingens

49-52. Ancistroteuthis lichtensteinii

53-55. Kondakovia longimana

56-58. Notonykia africanae

59,60 . Notonykia nesisi

61, 62. Gen. nov. 1 knipovitchi

63. Walvisteuthis virilis

64-67. Gen. nov. 2 rancureli and Gen. nov. 2 spp. 1-3 


\section{ABSTRACT}

Squids in the family Onychoteuthidae Gray, 1847 have been reported from every ocean but the Arctic, are taken frequently in deep-sea fisheries bycatch, and are ecologically important in the diets of many marine predators including cetaceans, pinnipeds, sharks, and seabirds. However, the diversity and systematics of the family have remained poorly understood. Of the $60+$ nominal species, 12-14 have generally been accepted in recent studies. Challenges to clarity include insufficient species descriptions, original descriptions published in eight languages and often based solely on early life stages, non-designation or subsequent loss of type material, and the existence of several unresolved species complexes.

In light of the general systematic disarray of the Onychoteuthidae, a global revision of the family follows, based on $\sim 1500$ specimens examined from 19 repositories. Type material has been examined wherever possible; for some species, photographs of type specimens, original illustrations, and/or the original descriptions have provided the only information available. It has not been possible to fully disambiguate taxa in some cases (e.g. Gen. nov. 2), given the limited material and information available, but for all species treated in this revision ( 25 out of 26 species; no material was available for Kondakovia nigmatullini), descriptions and illustrations are provided to a consistent standard that will enable their reidentification. External and internal morphological characters and states are described for subadult to adult stages of most species, with external characters reported through ontogeny as permitted by available material. Historically important characters are treated (general external morphology, body proportions, tentacle clubs, photophores, gladius, lower beak, radula), augmented by several more recently recognised characters (palatine teeth, detailed morphology of the tentacular hooks in adults, tentacular suckers in paralarvae, chromatophore patterns). The systematic value of both historical and new morphological characters at the generic and species levels are discussed; at all ontogenetic stages, tentacular club and hook morphology are considered the most valuable characters, although body proportions and gladius also prove useful.

Partial disambiguation of the Onychoteuthis banksii complex has been possible in the Pacific and Atlantic Oceans, resulting in the resurrection of Onychoteuthis bergii Lichtenstein, 1818 and Onychoteuthis aequimanus Gabb, 1868, the description of two new species, Onychoteuthis lacrima and Onychoteuthis prolata (in press), and the expansion of one species' recognised distribution (Onychoteuthis compacta) to include the Atlantic Ocean. 
The genus Moroteuthis Verrill, 1881 is considered a junior synonym of Onykia Lesueur, 1821, in accordance with the findings of several earlier authors. However, morphological differences in the species 'Moroteuthis' ingens necessitate the resurrection of the subgenus Moroteuthopsis Pfeffer, 1908b, with all other Onykia species placed into a new subgenus, Onykia (Onykia). Sexual dimorphism is reported in the beaks of Onykia (Moroteuthopsis) ingens (new comb.), and revised sex-specific equations are given for estimating this species' biomass based on LRL.

Morphological and historical genetic data suggest a more distant relationship between Onykia and the species 'Moroteuthis' knipovitchi Filippova, 1972 than was suggested by earlier classifications. This species is therefore considered to represent an undescribed genus, herein referred to as Gen. nov. 1, which cannot be more fully diagnosed and described at present due to limited material.

The generic position of 'Onykia' rancureli (Okutani, 1981) is also uncertain; it may be allied to Walvisteuthis virilis Nesis \& Nikitina, 1986 (family Walvisteuthidae Nesis \& Nikitina, 1986), but confirmation is impossible without examining type material of $W$. virilis. A second new genus, Gen. nov. 2, is therefore described for 'Onykia' rancureli and several morphological variants reported from the Pacific and Atlantic Oceans.

Given that the majority of available onychoteuthid material was collected after 1950, resulting in the descriptions of over half of the generally accepted genera and species since 1960, ongoing collection programmes are necessary to further resolve onychoteuthid systematics. 
Generic affiliations of many species have been revised subsequent to their original descriptions; in references to literature where this is the case, species names are given with the author's originally attributed genus in quotes, followed by the species' current systematic designation, e.g. 'Onychoteuthis' lichtensteinii (= Ancistroteuthis lichtensteinii). Where the original genus has been placed into synonymy with a senior name, the junior name, given in quotes, is followed by the senior name in parentheses, with an equal sign, e.g. 'Moroteuthis' (= Onykia). The onychoteuthid genera recognised in this thesis are Onychoteuthis Lichtenstein, 1818 (abbreviated Os.); Onykia Lesueur, 1821 (abbreviated Ok.); Ancistroteuthis Gray, 1849; Kondakovia Filippova, 1972; and Notonykia Nesis, Roeleveld \& Nikitina, 1998. The species 'Moroteuthis' knipovitchi Filippova, 1972 is considered referable to a new genus not yet described or named due to lack of available material, and so referred to herein as 'Gen. nov. 1.' The species 'Onykia' rancureli (Okutani, 1981), and several allied morphological forms, are likewise considered referable to a new genus, referred to as 'Gen. nov. 2.' The genus Onykia is subdivided into two subgenera, Onykia Lesueur, 1821 - indicated e.g. Ok. (Ok.) robsoni - and Moroteuthopsis Pfeffer, 1908b, indicated e.g. Ok. (M.) ingens.

The family name 'Onychoteuthidae' is technically incorrect according to the rules for forming family-group names (Article 29.3, International Code of Zoological Nomenclature (ICZN)). Since the stem of the type genus, Onychoteuthis, is Onychoteuthid-, the grammatically correct family name would be Onychoteuthididae. However, ICZN Article 29.3.1.1 states that the spelling in prevailing usage is to be maintained; thus the original spelling, Onychoteuthidae (prevalent since its description in 1847) is retained herein. 


\section{INTRODUCTION}

Squids in the family Onychoteuthidae Gray, 1847 ('hooked' squids) have been known to science for nearly 200 years. They have been recorded from every ocean except the Arctic, are taken frequently in deep-sea fisheries bycatch (Jackson \& Mladenov 1994, Anderson et al. 1998), and are ecologically important in the diets of many marine predators including cetaceans (e.g. Clarke et al. 1976, 1993, 1994; Clarke 1980; Clarke \& MacLeod 1980, 1982b; Gómez-Villota 2007), pinnipeds (e.g. Clarke \& Macleod 1982a, c), sharks (Cherel \& Duhamel 2004), and seabirds (e.g. Imber \& Russ 1975, Imber 1976, Moore et al. 1998, Piatkowski et al. 2001); indeed, some species, such as Kondakovia longimana, Gen. nov. 2 rancureli (new comb.), and Onychoteuthis meridiopacifica are represented almost exclusively in collections by ex-gut-content specimens. Despite their 'abundance' and frequency of capture in fisheries trawls (Anderson et al. 1998), they are relatively poorly represented in collections, with only $\sim 1500$ specimens available from 19 repositories, most of which have been collected only within the past 50 years (Fig. 1).

Onychoteuthids are most readily identifiable by the double row of hooks on each tentacle club (visible in most species by $M L \sim 15 \mathrm{~mm}$ ) and the lack of dentition on the adults' arm suckers. Additional familial characters include the simple, straight mantlefunnel locking apparatus; the DDVV attachment of the buccal connectives; and the 'step' in the jaw angle of the lower beak. Photophores and secondary (dorso-lateral) occipital folds are also present in some genera (Onychoteuthis, Ancistroteuthis and Notonykia (secondary occipital folds, no photophores)).

Although some genera (Onykia, Kondakovia) have tissues with high concentrations of ammonium ions (Nesis 2000), rendering them unpalatable to humans, several species from the non-ammoniacal groups (e.g. Onychoteuthis borealijaponica and Ancistroteuthis lichtensteinii) are of minor commercial value in the areas where they occur (Zuev \& Nesis 1971, Okutani \& Murata 1983, Nesis 1987, Gillespie 1997). Several species attain giant sizes; Onykia (Onykia) robusta reaches ML 1.97m (pers. obs.) and is elsewhere reported to attain ML 2.3m (Nesis 1987), and Kondakovia longimana regularly attains $\mathrm{ML}>800 \mathrm{~mm}$; while others remain small (Onychoteuthis meridiopacifica matures at ML 60-90mm).

Since the first two species were described in 1817 and 1818 (Onychoteuthis banksii and Os. bergii), 12 genera and more than 60 species have been attributed to the 
Onychoteuthidae (Sweeney \& Young 2003). Studies investigating the group's systematics (e.g. Pfeffer 1912, Tsuchiya \& Okutani 1992, Young \& Harman 1987, Kubodera et al. 1998) have identified a number of dubious taxa, junior synonyms, and unresolved species complexes, and suggest the presence of additional undescribed taxa.

Recent accounts place the number of accepted genera and species at six and 12 (Nesis 1987), five and 13 (Kubodera et al. 1998), and six and 14 (Vecchione et al. 2003). Challenges to clarifying systematics within the family have included insufficient species descriptions (the most meagre, that of Os. banksii, comprises two lines of Latin and makes no mention of the characteristic tentacular hooks), original descriptions and relevant literature published in eight languages and often based on early life stages (not yet displaying the most important systematic characters, resulting in two frequently cited genera, Moroteuthis and Chaunoteuthis, representing life stages of other genera), and lack of type material (not extant, never designated, or lost for over half of the named species).

The first species of onychoteuthid was described over 200 years ago, and with it began the systematic snarls that would intrigue teuthologists for more than two centuries. 'Loligo' cardioptera Lesueur \& Petit in Peron, 1807 was described from one paralarva ( $\mathrm{ML} \sim 10 \mathrm{~mm}$ ) and appears to have been a small specimen of Onykia; however, the species was illustrated but not formally described, and although the specimen was located by Lu et al. (1995), it could not be found for examination at MNHN in 2007, so its status remains unresolved.

This auspicious beginning was followed a decade later by the establishment of 'Loligo' banksii Leach, 1817 (= Onychoteuthis banksii), reported from the Gulf of Guinea. The description (in its entirety 'L. pedibus ordinariis antliis globosis, pinnis rhombum efformantibus,' simply identifying 'ordinary tentacles with globular suckers' and 'fins forming a rhombus') contained no salient morphological details. As a result, Os. 'banksii' became a catch-all name for many squid species in many subsequently recognised genera for most of the next two centuries, despite subsequent attributions of 27 additional species to the genus Onychoteuthis - beginning in the following year. Lichtenstein (1818) received two specimens, found on board a ship (one in the crow's nest, $10 \mathrm{~m}$ above sea level) from the Cape of Good Hope, which he named Os. bergii, providing a description of its tentacular morphology. For this new species he created the genus Onychoteuthis, to which he also attributed Os. 'bellonii' (as he named the 
species described by Belon (1553, p. 340)), 'Onychoteuthis' fabricii (= Gonatus fabricii), and Os. 'molinae' (as he renamed Sepia unguiculata Molina, 1782, though Lichtenstein later (1820) recognised unguiculata as the senior name). A translation of the original description of Os. bergii and the genus Onychoteuthis can be found in Appendix A. However, the name Os. bergii was largely ignored, and although Férussac \& d'Orbigny (1834-1848) illustrated two specimens, both they and Gray (1849) considered Os. bergii a synonym of Os. banksii.

Within several years, a second related genus was recognised. Lesueur (1821) described Onykia, attributing two new species to it, 'Onykia' angulatus and Onykia carriboea (= Ok. (Ok.) carriboea). (Lesueur's 'Loligo' bartlingii, now considered a junior synonym of Os. banksii (fide d'Orbigny 1845), was described in the same publication, but the specimen lacked tentacle clubs so Lesueur did not realise its affinity with the other two species.) Ok. (Ok.) carriboea was described from a small specimen (75mm total length), and was said to inhabit floating fuci in the Gulf of Mexico and Gulf Stream; its distinguishing features were given as two series of hooks alternating with suckers at their bases, and 'a blue to a purple, or yellow, \&c.' colouration. These character states, as well as the small size of all known specimens attributed to $O k$. (Ok.) carriboea, are indicative of early ontogenetic stages; to date, no adult of OK. (Ok.) carriboea has been positively identified. (The status of Ok. angulata remains uncertain; the holotype was found by Lu et al. (1995) but was not located for examination during collection visits for this study.) Recent authors (Tsuchiya \& Okutani 1992, Wakabayashi et al. 2007, discussed below) have concluded that commonly accepted species in the younger genus 'Moroteuthis' Verrill, 1881 (known largely from subadult, adult and spent specimens) probably represent the later ontogenetic stages of historically enigmatic Onykia species.

Between 1820 and 1840, four more species were described: Loligo felina Blainville, 1823; Loligo uncinata Quoy \& Gaimard, 1825; Onychoteuthis fleuryi Reynaud in Lesson, 1831; and Loligo laticeps Owen, 1836. Unfortunately the descriptions of these specimens are insufficient to attribute them with confidence to any recognised species (although in most cases it is apparent that the specimens in question were, in fact, onychoteuthids), and the type specimens are not extant (the former three were originally MNHN specimens and were neither found by Lu et al. (1995) nor during a collection visit made for this study in 2007; and the type repository of $L$. laticeps has never been established). 
Férussac \& d'Orbigny (1834-1848) were the next to contribute significantly to knowledge of the onychoteuthid taxa. They described Os. banksii with an extensive synonymy, Os. cardioptera with a similarly long synonymy that included the senior name Ok. (Ok.) carriboea; and three new species, 'Onychoteuthis' lichtensteinii (= Ancistroteuthis lichtensteinii), 'Onychoteuthis dussumieri,' and Os. platyptera. These were illustrated in detail, as were a number of named species they considered synonyms of older names, including Os. lessonii Férussac in Lesson 1831, and Os. lesueurii d'Orbigny in Férussac \& d'Orbigny 1835, both listed under Os. banksii in the latter text, and Os. peratoptera (listed in their text under the new name Os. platyptera). Illustrations were also given based on available material for some species not previously illustrated (Os. banksii and Os. bergii), and reproduced or reinterpreted from earlier authors (Os. angulata, Os. bartlingii and Ok. (Ok.) carriboea from Lesueur (1821), and Os. fleuryi from Reynaud in Lesson 1831). Férussac \& d'Orbigny's work (1834-1848) represented the first synopsis of known onychoteuthid taxa.

Gray $(1847,1849)$ believed that the hooked squids should be grouped together into a single family and accordingly described the Onychoteuthidae (first mentioned in 1847, defined in 1849), to which he attributed nine genera. These he separated into three groups: those bearing hooks on the tentacles only (the true onychoteuthid genera, Onychoteuthis, Onykia, and a new genus, Ancistroteuthis Gray, 1849, differentiated from Onychoteuthis by the lack of free rachis on the gladius); those bearing hooks on both the arms and tentacles (Enoploteuthis d'Orbigny in Rüppell, 1844; Ancistrocheirus Gray, 1849; Abralia Gray, 1849; 'Octopodoteuthis' (= Octopoteuthis) Rüppell 1844; and the fossil Acanthoteuthis, all since moved into other families); and one, Ommastrephes d'Orbigny, 1834, entirely without hooks (likewise subsequently moved to its own family). Because of the diverse armature of these genera, the family diagnosis specified only the presence of a terminal pad of suckers on the club, rhomboidal fins terminally situated on the mantle, a lanceolate gladius, 'naked' eyes (lacking a corneal membrane) with a deep anterior sinus, and the presence of primary (ventro-lateral) occipital folds. However, subsequent authors (Pfeffer 1900, 1908a, 1908b, 1912; Naef 1921/1923; Thiele 1935 - see below) progressively revised the family diagnosis to eventually include the characters now considered most diagnostic, namely, the presence of three primary occipital folds on the head, arms with two rows of suckers, tentacle clubs with two rows of hooks on the manus, and buccal connective formula DDVV. 
Between 1840 and 1880 several new taxa were described by various authors, including: Os. krohnii Vérany, 1847; 'Onychoteuthis' kamtschatica Middendorff, 1849 (= Gonatus kamtschaticus, fide Nesis 1987); Os. brevimanus and Os. rutilus Gould, 1852; Loligo plagioptera Souleyet, 1852; 'Onychoteuthis hamatus' (= Ancistroteuthis lichtensteinii Férussac, 1835, fide Adam 1942) and 'Onychoteuthis perlatus' Risso, 1854 (= Chiroteuthis veranyi Férussac, 1834, fide Adam 1942); Os. fusiformis Gabb, 1862; Os. aequimanus Gabb, 1868; Onychoteuthis lobipennis Dall, 1871; and 'Ommastrephes robustus' Verrill, 1876 (= Ok. (Ok.) robusta). It is not possible to accurately determine the identity of most of these nominal taxa, due to: insufficient morphological detail in the original description and/or illustrations; type specimens not being designated, being incomplete/poorly conserved or no longer extant; and/or no type locality having been designated. The exceptions are Os. aequimanus and $O K$. (Ok.) robusta - the syntypes of both are extant, and retain systematically valuable characters/states.

Ok. (Ok.) robusta was the first onychoteuthid species recognised to attain giant size, although there was some confusion as to its identity and generic affiliation. Dall (1873), who first reported the specimens that would later become the syntypes, provisionally attributed them to Os. bergii. Verrill (1876) recognised them as a new species, which he described and named 'Ommastrephes' robustus (according to Dall's suggestion). Steenstrup (1882) placed the species in Lestoteuthis (= Gonatus), and Verrill (1881) placed it into a new genus, Moroteuthis, characterised by a narrow gladius with a solid, laminate, cartilaginous rostrum; simple, ovate mantle-funnel-locking apparatus; three primary occipital folds; large sagittate fins; and smooth-ringed suckers. 'Moroteuthis' (= Onykia) robusta was the type species, by monotypy. However, Smith (1881) concurrently described a new species from southern Patagonia, 'Onychoteuthis' ingens, that would later be recognised as a second 'Moroteuthis' species. Verrill (1881) also proposed a new generic name, Teleoteuthis, as a replacement for 'Onychia' (an emended spelling of Onykia proposed by Latreille (1885), but preoccupied by a genus of Lepidoptera); however, the original spelling of Onykia is the oldest taxonomically valid name for the genus. Verrill (1882) attributed a new species to 'Teleoteuthis' (= Onykia), 'T. agilis' (= Ok. (Ok.) carriboea).

Near the end of the $19^{\text {th }}$ century, Pfeffer began to publish the first of several extensive works on cephalopod systematics, eventually setting a new standard for detailed descriptions and illustrations, and discussing the systematic importance of various morphological characters. In 1884 he described a new genus, Steenstrupiola (= 
Onykia, prior to development of the tentacular hooks, which he recognised in 1912), and five new species: S. chilensis and S. atlantica; 'Onychia' binotata, which he later (1912) synonymised with Ok. (Ok.) carriboea, and 'Onychia' curta; and Os.

brachyptera. These descriptions include the first mention of teeth around the inner margin of the paralarval tentacular suckers, an important systematic character (Nixon \& Dilly 1977, Harman \& Young 1985, Salcedo-Vargas 1995, Bolstad 2007). In 1900 Pfeffer provided the first keys for identifying known onychoteuthid species, as well as describing the genus Teleonychoteuthis (= Onychoteuthis), and diagnosing (but not describing or illustrating) two new species, 'Teleoteuthis' (= Onykia) appellöfi and 'Teleoteuthis' verrilli. (Hoyle (1904) also included A. 'lichtensteini' and Os. 'banksi' in his key of dibranchiate cephalopods.) In 1908(a) Pfeffer published a description and detailed illustrations of Os. 'banksii' (which appears, based on its proportions, to depict Os. compacta Berry, 1913), as well as a description and synonymy of Ok. (Ok.) robusta, with figures redrawn from Verrill (1876). In his 1912 tome on the Cephalopoda of the Plankton Expedition, he dedicated nearly 60 pages (in the English translation) and 14 plates to the Onychoteuthidae, giving several diagnostic keys based on different morphological characters; full discussions of histories, synonymies, descriptions, illustrations, and distributions for each recognised species; and several tables of specimen measurements. In spite of the occasional oversight or inconsistency in the text and illustrations (e.g. the illustration of 'Tetronychoteuthis dussumierii' showing secondary nuchal folds, a probable composite of the head/brachial crown and mantle of two separate specimens), this monumental and thorough treatment of the family is an excellent body of information on most of the species treated therein. Pfeffer's illustrations and discussion of the gladius and tentacular morphology are of particular value.

During Pfeffer's ongoing investigations, several additional species were described by other authors: 'Chaunoteuthis' (= Onychoteuthis) mollis Appellöf, 1891 (containing the first report of spermatophore implantation within the genus Onychoteuthis, and within the Onychoteuthidae - see Appendix B), and 'Teleoteuthis' (= Onykia) caroli and ' $T$.' jattai Joubin, 1900. As these taxa were published, Pfeffer incorporated them into his works, redescribing and illustrating or synonymising them as he deemed appropriate.

With improved availability of increasingly detailed descriptions and illustrations of existing species, 'new' species descriptions became likewise more detailed and thorough, with a few exceptions. The four onychoteuthid species described between 1900 and 1930 - 'Teleoteuthis' compacta Berry, 1913 (= Os. compacta); 'Moroteuthis' 
loennbergii Ishikawa \& Wakiya, 1914 (= Ok. (Ok.) loennbergii); 'Moroteuthis' aequatorialis Thiele, 1920 (= Ok. (Ok.) aequatorialis) (original description translated in Appendix C); and Os. borealijaponica Okada, 1927 (simply diagnosed based on the elliptical intestinal photophores) - are all considered taxonomically valid herein. During this time, several detailed accounts of cephalopod fauna from specific geographic regions also contained improved descriptions and illustrations of existing taxa. Naef (1921/1923), describing the Gulf of Naples fauna, included descriptions and illustrations of Os. 'banksi' (including reports of early ontogenetic stages), $A$. lichtensteinii and 'Chaunoteuthis' (= Onychoteuthis) mollis, as well as a thorough discussion of paralarval morphology and ontogenetic changes of these species. Sasaki (1929) similarly redescribed and illustrated 'Moroteuthis' (= Onykia) robusta, 'M.' loennbergii, and Os. borealijaponica, the latter however considered a junior synonym of Os. banksii therein.

Adam (1942) next began to investigate onychoteuthid systematics, and recognised Risso's (1854) two 'Onychoteuthis' species as belonging to existing taxa (see above). In his 1952 report on cephalopods from the South Atlantic, off the coast of Africa, he included descriptions and illustrations of 'Onychoteuthis banksii' (which appear to have been a mix of Os. banksii (sensu stricto) and Os. bergii) along with a table of measurements and an extensive synonymy. In 1962, he gave a lengthy description and discussion of an A. lichtensteinii specimen from off Angola (with somewhat different body proportions from previously reported Mediterranean specimens), and described a new species, 'Moroteuthis' robsoni (= Ok. (Ok.) robsoni). Both descriptions are translated in Appendix D.

New species and genera continued to appear through the following decades, including Ok. 'japonica' Taki, 1964 (= Ok. (Ok.) robusta Verrill, 1876, fide Tsuchiya \& Okutani 1992); 'Moroteuthis' knipovitchi (= Gen. nov. 1 knipovitchi (new comb.)) and Kondakovia longimana Filippova, 1972 (placed into its own new genus due to full series of marginal suckers retained on the tentacle clubs through maturity); 'Onykia' rancureli (= Gen. nov. 2 rancureli (new comb.)) and Ok. (Ok.) indica Okutani, 1981; 'Moroteuthis pacifica' (= Ok. (Ok.) robusta) Okutani, 1983; Os. meridiopacifica Rancurel \& Okutani, 1990 (originally reported as 'Onychoteuthis sp.' by Rancurel (1970)); and Notonykia africanae Nesis, Roeleveld \& Nikitina, 1998, placed into its own genus on the basis of unique tentacular club morphology, with a low number of hooks and high number of suckers on the terminal pad. 
During the latter half of the $20^{\text {th }}$ century, additional information on specific morphological characters became available for some onychoteuthid species, through a number of studies comparing features across a wide range of taxa. These characters include lower beaks (Clarke 1962, 1966, 1980, 1986), gladius (Toll 1982, Bizikov 1996), spermatophores (Hess 1987), and tentacular hooks (Engeser \& Clarke 1988, Young \& Harman 1998). Several papers addressing individual species also appeared, in some cases reporting isolated stranding or capture events (e.g. Torchio 1967, Nesis 1970, and Okutani \& Ida 1986, for 'Chaunoteuthis' (= Onychoteuthis) mollis; Lu \& Williams 1994 and Vacchi et al. 1994 for K. longimana); in some cases investigating specific aspects of a species' biology, ecology or morphology (for Os. borealijaponica: Murata \& Ishii 1977, Murata et al. 1982, Okutani \& Murata 1983; for Ok. (M.) ingens: Jackson 1993, 1995, 1997, 2001; Jackson \& Jackson 2004; Jackson \& Mladenov 1994; Jackson et al. 1997, 1998; Cherel \& Duhamel 2003); and occasionally investigating systematics of particular species or groups of species (e.g. Tsuchiya \& Okutani 1992 for Ok. (Ok.) robusta; Young \& Harman 1987 for paralarval Onychoteuthis species in Hawai'ian waters). Additionally, onychoteuthids have been included in (or the focus of) several recent genetic studies, including Bonnaud et al. (1998), Carlini \& Graves (1999), Lindgren et al. (2004), and Wakabayashi et al. (2007).

Recent zoogeographical works and identification guides (both worldwide and for more localised regions) have included variously detailed descriptions and illustrations of onychoteuthids, including Young 1972; Roper et al. 1984, 1985; Nesis 1987; Guerra Sierra 1992; and Okutani 2005. Several authors have also reviewed and summarised known information about the Onychoteuthidae; Kubodera et al. (1998) gave diagnoses, brief descriptions and illustrations (reproduced from other relevant publications) for each of the 13 species they accepted at the time - Os. banksii, compacta, borealijaponica, meridiopacifica; A. lichtensteinii including four distinct morphological forms, two of which have since been identified as the species Notonykia africanae and N. nesisi; Ok. (Ok.) carriboea and 'Onykia' rancureli (= Gen. nov. 2 rancureli); 'Moroteuthis' robusta, loennbergii, robsoni (= Ok. (Ok.) robusta, loennbergii, robsoni) and 'Moroteuthis' ingens (= Ok. (M.) ingens); 'Moroteuthis' knipovitchi (= Gen. nov. 1 knipovitchi); and $K$. longimana - along with discussions of some of the systematic problems in the family, such as the Os. banksii complex and the status of the genera Onykia, 'Moroteuthis' and 'Chaunoteuthis.'

Nesis (2000) discussed known distribution patterns, various morphological character states and their evolutionary polarity (primitive or advanced), and likely interfamilial 
relationships. He concluded that the family consisted of two groups, the ammoniacal, neutrally buoyant genera ('Moroteuthis' and Kondakovia) and the dense, muscular, negatively buoyant genera (Onychoteuthis, Onykia, Ancistroteuthis and Notonykia), and that Kondakovia and Onychoteuthis were the most derived groups.

An online reference project, the Tree of Life, has also published a summary of known information about the Onychoteuthidae (www.tolweb.org/Onychoteuthidae), including species diagnoses, photographs, illustrations, SEMs, and a list of all nominal taxa within the family (similar to that in Appendix E). Although the project is young and has not been peer reviewed to date, the pages have been collaboratively created by several authors who have previously published on the Onychoteuthidae and are respected experts in their fields.

The present familial revision was begun in 2003 and has resulted in four relevant recent publications on aspects of onychoteuthid systematics. Bolstad (2006) reported sexual dimorphism in the beaks of Ok. (M.) ingens, and Bolstad (2007) redescribed the onychoteuthid fauna of the New Zealand region - Os. 'banksii' (= Os. aequimanus), Ok. (M.) ingens and Ok. (Ok.) robsoni, and Notonykia africanae and the new species N. nesisi. A third publication (Bolstad in press) partially resolves the Os. 'banksii' complex from the Pacific Ocean, and describes two new species, Os. lacrima and Os. prolata. In the interest of presenting a complete family revision, the information contained in these publications is reproduced in the Systematics section below. A fourth publication, Laptikhovsky et al. (2008), describes a second species of Kondakovia, $K$. nigmatullini, from the sub-Antarctic; however, this material was not received in sufficient time for inclusion in this revision. 
MATERIALS \& METHODS

Material examined

Original descriptions and, where possible, type material of all previously described nominal onychoteuthid species have been examined. Preserved and limited fresh specimens were loaned from, or examined at, the following institutions, between March 2003 and December 2008:

ANSP — Philadelphia Academy of Natural Sciences, Philadelphia, Pennsylvania, USA BMNH - The Natural History Museum, London, UK

CASIZ - California Academy of Natural Sciences, San Francisco, California, USA IASOS - Institute of Antarctic and Southern Ocean Studies, University of Tasmania, Hobart, Tasmania, Australia

JAMSTEC - Japan Agency for Marine-earth Science and Technology MNHN - Muséum National d'Histoire Naturelle, Paris, France NIWA - National Institute of Water \& Atmospheric Research Ltd, Wellington, New Zealand

NMFS - National Marine Fisheries Service, Honolulu, Hawai'i, USA

NMNZ - Museum of New Zealand Te Papa Tongarewa, Wellington, New Zealand NMV - National Museum of Victoria, Melbourne, Australia

NSMT - National Museum of Nature and Science, Tokyo, Japan

SAM - South African Museum, Cape Town, South Africa

SBMNH - Santa Barbara Museum of Natural History, Santa Barbara, California, USA

UMML - Rosenstiel School of Marine and Atmospheric Science, Miami, Florida, USA (formerly University of Miami Marine Lab)

USNM - National Museum of Natural History, Smithsonian Institution, Washington, DC, USA

ZMMGU — Zoological Museum, Moscow State University, Moscow, Russia

ZMB - Zoologisches Museum, Museum für Naturkunde der Humboldt-Universität, Berlin, Germany

ZMBN — Zoological Museum, Universitetet i Bergen, Norway

ZMH - Zoologisches Museum der Universität Hamburg, Hamburg, Germany

Material collected on the 'Dana' Expedition (Carlsbergfondets oceanografiske Ekspedition omkring Jorden 1928-1930 under Ledelse af Professor Johannes 
Schmidt) was examined at (but not part of the collections belonging to) UMML; these specimens are indicated by registration numbers beginning with 'D,' e.g. D4075 XI. Specimens examined in collaboration with R. Young at the University of Hawai'i (Honolulu) have not been formally accessioned into a museum collection and have been given temporary reference numbers beginning with KSB (e.g. KSB-001). Type specimens selected from this material and several additional specimens will be accessioned into SBMNH and USNM collections; their temporary KSB-numbers are followed by [SBMNH] or [USNM] to indicate their future location.

Collection data for many of these Hawai'ian specimens and many older specimens from other collections are incomplete or unavailable; these data have been presented as completely as possible. All collection dates are given as $\mathrm{dd} / \mathrm{mm} / \mathrm{yyyy}$. Specimens examined are listed in order of decreasing latitude and secondarily by dorsal mantle length (in multiple specimen lots, by ML of the largest specimen). Sexes are provided for the majority of specimens, except in cases where dissection was unjustified, or where the specimen was too juvenile to determine its sex, indicated 'sex indet.' Specimens in lots of more than three individuals were generally not sexed.

Additional collection acronyms used in text are:

AUT - Auckland University of Technology

BT — bottom temperature

BTT - bottom trawl

CMT - Cobb midwater trawl

D (e.g. D4075 XI) — Dana Expedition (material housed but not accessioned at UMML) EMWT — Engels midwater trawl

FMMWT — fine-meshed midwater trawl

FRV — fisheries research vessel

FTS - fisheries training ship

$\mathrm{HN}$ - hand net

IKMWT — Isaacs-Kidd midwater trawl

IYGPT — international Young gadoid pelagic trawl

JAMARC - Japan Marine Fishery Resources Research Center

MBC - Mesopelagic Boundary Community Cruises (conducted by the University of Hawai'i)

MFish — New Zealand Ministry of Fisheries 
MIDOC - midwater opening and closing net

MT - motorised trawler

$\mathrm{MV}$ - motorised vessel

MWT — midwater trawl

NFD - no further data

NMFS — United States National Marine Fisheries Service

$\mathrm{NN}$ - neuston net

NORFANZ - New Zealand and Australia Norfolk Ridge-Lord Howe Rise Biodiversity Voyage

RFRL — Tokai Regional Fisheries Research Laboratory, Tokyo

RMT — rectangular midwater trawl

RV - research vessel

SOP — New Zealand MFish Scientific Observer Programme

ShT - shrimp trawl

SrT - Sergia trawl

ST - surface temperature $\left({ }^{\circ} \mathrm{C}\right)$

TMT — Tucker midwater trawl

TC - Townsend Cromwell Cruises (conducted by the University of Hawai'i)

TOTO - Tongue of the Ocean (Bermuda)

USNS — United States Naval Ship

$[\mathrm{x}]$ — data missing/illegible (e.g. station numbers)

Distribution maps are based solely on the specimens examined herein. Material was available from most major ocean currents (Fig. 1), with the following currents best represented:

Pacific - North Pacific, North Equatorial, Kuroshio and Kuroshio Extension, California, South Equatorial, and East Australian, and the North and South Equatorial countercurrents

Atlantic - Gulf Stream, North Atlantic Drift, Canary, North Equatorial, Equatorial Counter, and South Equatorial

Material from Antarctic and sub-Antarctic waters, and from the Indian Ocean, was relatively scarce, and very little or no material was available from the large southeastern gyre formed by the South Pacific, Mentor and Peru currents; the Brazil Current; the Benguelen Current; and the South Atlantic and South Indian currents. 
A number of specimens, in particular older material and paralarval or juvenile specimens, could not be identified with confidence to species level due to their size, lack of intermediate stages to facilitate identification, damage or poor conservation. These specimens are intentionally excluded from systematic treatment.

Specimens photographed using environmental scanning electron microscopy (ESEM) were critical-point dried, sputter-coated in gold-palladium, and photographed at the University of Auckland.

The synonymy for each species is limited to citations providing substantial morphological accounts, including species and specific character descriptions and/or
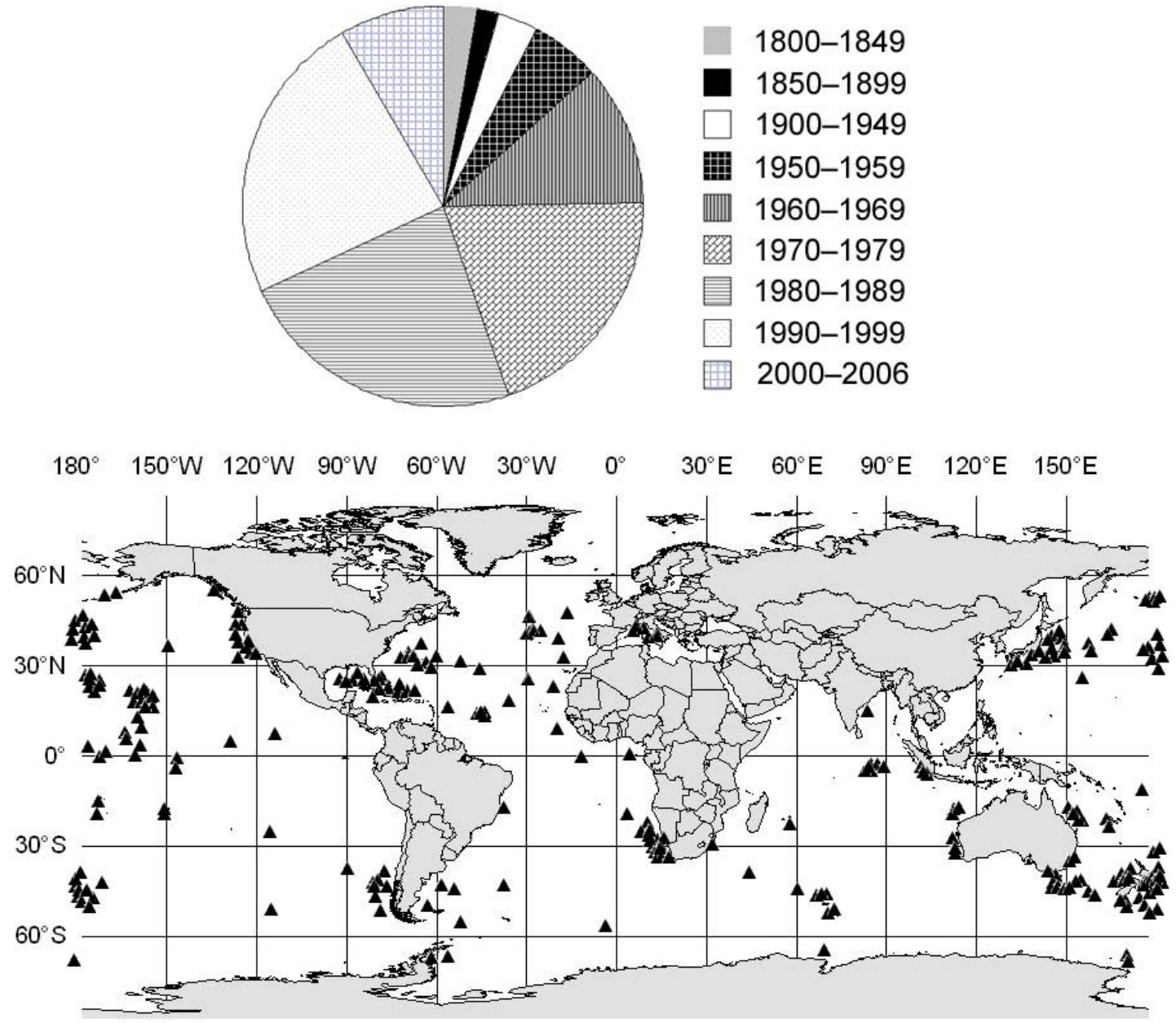

Fig. 1 - temporal (by collection year) and geographic distribution of onychoteuthid material examined in this study. 
illustrations, and references to type material. Citations not detailed enough to permit confident identification of a taxon, where the material was not available for reexamination, are omitted. Specifically, many references to Os. 'banksii' and OK. 'carriboea' in the literature have been excluded, since the characters differentiating species at one time attributed to the Os. 'banksii' complex (all Onychoteuthis species apart from Os. meridiopacifica), and small Onykia specimens, are relatively cryptic and were not recognised until recently. Taxa included in genetic studies (e.g. Bonnaud et al. 1998, Wakabayashi et al. 2007) have been included only where species identity is unambiguous or could be confirmed through re-examination of material.

Morphological terminology, measures, indices and counts

Species descriptions, and specimen measurements, indices and counts generally follow the guidelines given by Roper \& Voss (1983). Major measurements are also illustrated in Fig. 2; their 'index' (e.g. mantle width index, MWI) is calculated as a percentage of the mantle length (see definitions below). Measurements of features symmetrical across the midline (e.g. arms, tentacles) were taken on the more complete side of the specimen and are indicated ' $R$ ' or ' $L$ ' in the tables. Ranges of indices are given in text as $X-Y-Z$, where $X$ is the lowest observed value, $Y$ is the mean index, and $Z$ is the highest observed value; in the case of the arms, the range is given as $X-Y_{I}-Y_{I I-I V}-Z$, where $Y_{I}$ is the mean index of Arms I and $Y_{I I-I V}$ is the mean index of Arms II-IV; where the observed range was less than $5 \% \mathrm{ML}$, only the mean is given.

Morphological measurements used in text and tables include:

$\mathrm{ML}$ - mantle length (dorsal)

$\mathrm{MW} / \mathrm{MWI}$ - mantle width / index, as \% ML

$\mathrm{HL} / \mathrm{HLI}$ - head length / index (measured along dorsal midline, from anterior tip of dorsal nuchal cartilage to separation of Arms I)

$\mathrm{HW} / \mathrm{HWI}$ - head width / index

FL / FLI — fin length / index

FW / FWI - fin width / index

$\mathrm{TnL} / \mathrm{TnLI}$ - tentacle length / index

$\mathrm{CL} / \mathrm{CLI}$ - tentacle club length (including carpus) / index 

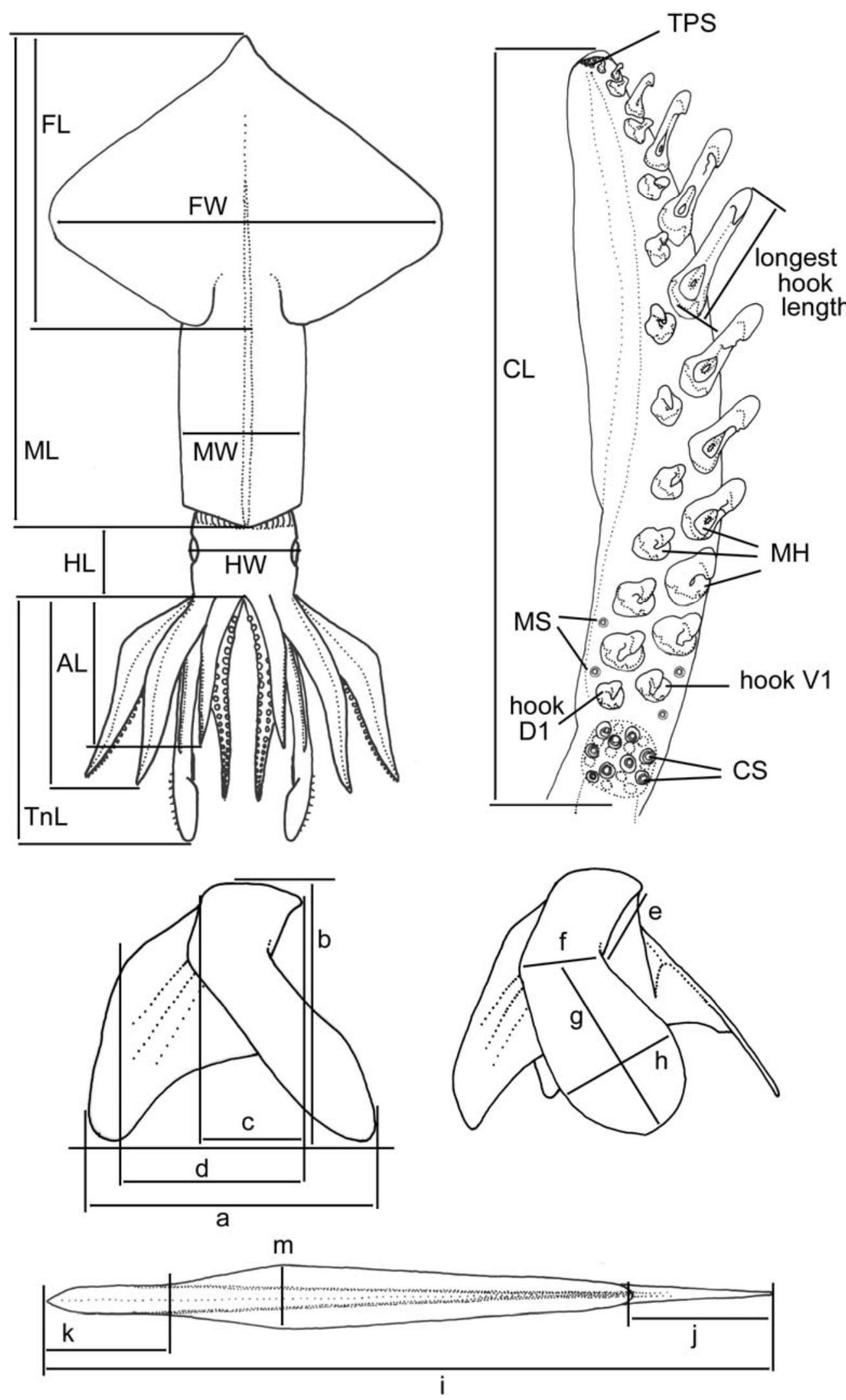

Fig. 2 - Onychoteuthid measures and counts. Acronyms in upper two figures as defined in text; A-H) lower beak: (A) length, (B) depth, (C) length of hood, (D) length of hood and crest combined, (E) lower rostral length (LRL), (F) wing width level with jaw angle, $(G)$ wing length, $(H)$ greatest wing width; $J-L)$ gladius: $(J)$ length of rostrum, $(K)$ length of free rachis, $(L)$ gladius length $(G L),(M)$ greatest width. 

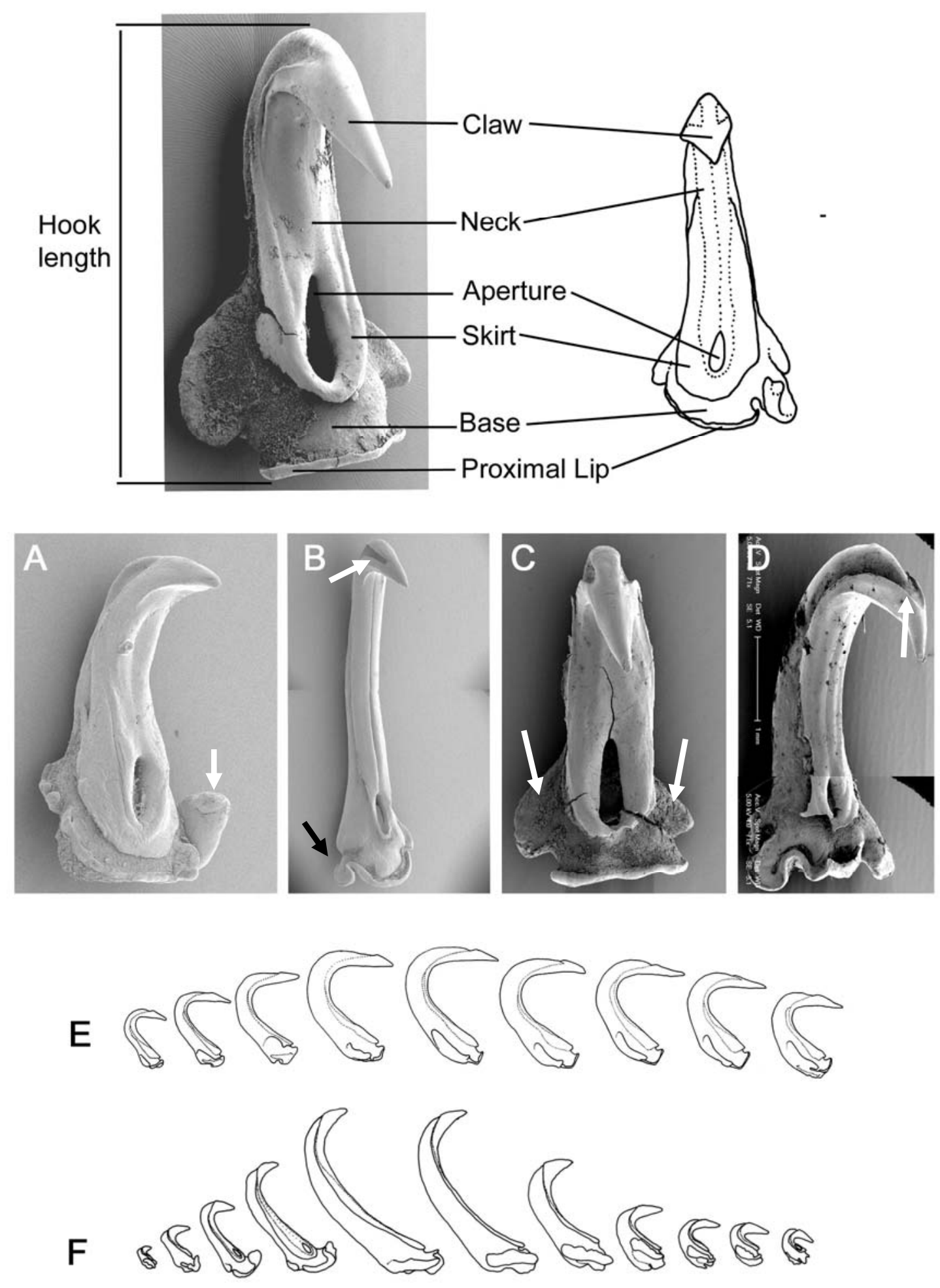

Fig. 3 - Terminology and morphology of onychoteuthid tentacular hooks. A) Os. compacta hook V10; B) A. lichtensteinii hook V6; C) Ok. (Ok.) robsoni hook V13; D) N. africanae hook $V 5$; E) $K$. longimana, partial ventral hook series, with strongly recurved hooks ( $<90^{\circ}$ in lateral profile); F) Os. aequimanus, ventral hook series, with shallowly recurved hooks $\left(>90^{\circ}\right)$. Arrows indicate: A) asymmetrical base produced into spike; B) deep lateral grooves on claw (white), asymmetrical base not produced into spike (black); C) symmetrical base; D) lateral blades and grooves joining across distal extremity of claw. 
CS - count of carpal suckers on the tentacle club

$\mathrm{MH}-$ count of manus hooks on the tentacle club

MS - count of marginal suckers (on manus of the tentacle club)

TPS - count of terminal pad suckers on the tentacle club

$\mathrm{GL}$ - gladius length

$\mathrm{LRL}$ - lower rostral length

The breadths of keels on the arms and tentacles are reported as a percentage of the breadth of the corresponding arm or tentacle at the same point along its length, e.g. an aboral keel that is widest midway along the arm and attains a breadth equal to that of the arm at the same point is described as 'attaining $100 \%$ arm width at $50 \%$ arm
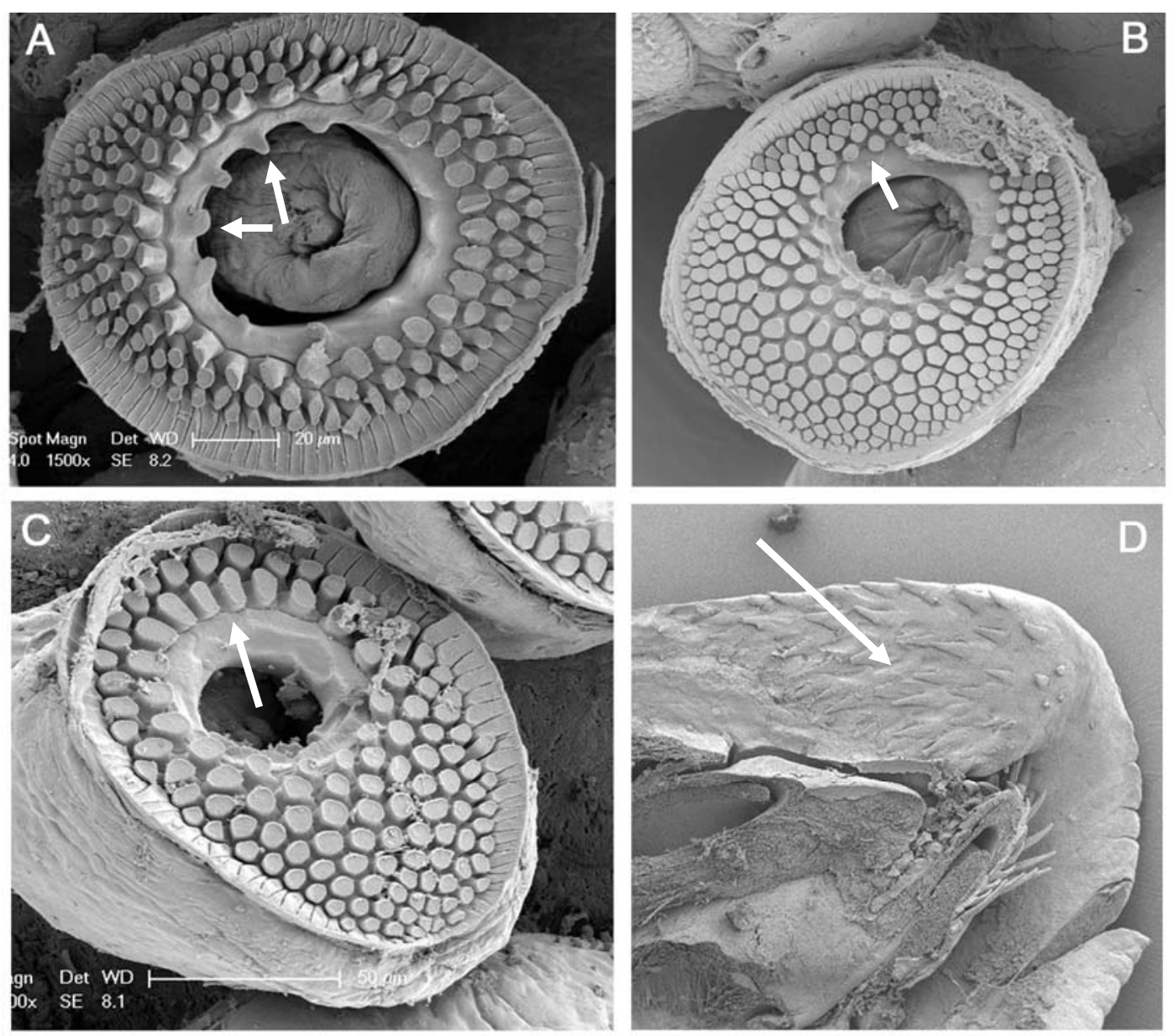

Fig. 4 - Morphology of onychoteuthid paralarval tentacular suckers and adult buccal mass. A) Ok. (M.) ingens (circular type); B) N. africanae (distally square type); C, D) Os. aequimanus: C) ovoid type sucker, D) radular apparatus. Arrows indicate: A) teeth around inner margin of sucker rings; B) circular-faced pegs on oral face of sucker; C) polygonal-faced pegs on oral face of sucker; D) palatine palp. 
length.' The swimming keel and lateral membrane breadths on the arms are given as a percentage of the arm dimension visible when the keel is in profile (for Arms I and IV, arm width; for Arms II and III, arm depth).

Lower beak terminology follows Clarke (1980) (see Fig. 2); palatine teeth are observed as in O'Shea (1999), situated medially on the lateral buccal palps (fide Messenger \& Young 1999), as illustrated in Fig. 4D for Onychoteuthis aequimanus.

Hook terminology follows Young \& Harman (1998), with additional terminology defined in Fig. 3; individual hooks are identified according to dorsal/ventral row and numbered from proximal-most to distal-most (e.g. first ventral hook, next to carpus $=\mathrm{V} 1$; first dorsal = D1) and hook length is measured from proximal lip to distal extremity of claw (excluding recurved portion of claw).

\section{Onychoteuthid characters and character states}

The following characters, in combination, have proven useful in diagnosing genera and species within the family Onychoteuthidae.

1. Mantle. Structure of the epidermis, which may be smooth, rugose, or longitudinally ridged; posterior shape, e.g. tapering or remaining broad ventral to the fins; visibility of the gladius through the dorsal midline and of the rostrum ventrally through the posterior tissue of the mantle; distribution of chromatophores in the ventral posterior region, e.g. present over the entire ventral surface or absent from a posterior patch.

2. Fins. Overall shape may be ovate, paddle-shaped, heart-shaped, sagittate or rhombic; whether or not the posterior fin margin is produced beyond the tip of the mantle; fin length and width relative to $\mathrm{ML}$.

3. Head. Width of the head relative to the mantle width; presence/absence and number of secondary (dorsal to dorso-lateral) occipital folds and/or occipital crest; shape of the funnel groove, which may be broadly U-shaped or trapezoidal, V-shaped, or broadly $\mathrm{V}$-shaped posteriorly, becoming very acute to nearly parallel-sided anteriorly; presence/absence of a Y-shaped ridge inside the funnel groove; relative lengths of the funnel-mantle components of the locking apparatus to each other and ML. 
4. Arms. Lengths relative to each other and ML; number of suckers; presence/absence and extent of development of the keels on Arms I-III (located on the aboral surface, directed away from the arm, and generally of uniform width along the arm or widest medially), and of lateral membranes on Arms IV (located at the aboro-lateral edge of the arm, in the same plane as the aboral surface, and widest proximally or of uniform width along the arm).

5. Tentacles. Length relative to $\mathrm{ML}$; length of the tentacle club relative to $\mathrm{ML}$; number of carpal suckers, hooks on the manus (with 'manus' herein applied solely to the hook-bearing part of the club), terminal pad suckers, and marginal suckers if present; position and length of the largest ventral hook (excluding recurved portion of claw) relative to the paired dorsal hook and to club length; symmetry/asymmetry in the bases of the larger ventral hooks (see Fig. $3 A-C$ ); presence/absence of an enlarged ventral spike on the more distal ventral hooks (Fig. 3A); sculpture of the hook claw, which may have deep lateral grooves (Fig. 3B) or broad lateral 'blades' meeting at the midline of the claw (Fig. 3D); general shape and obtuse/acute recurvature of the hooks in lateral profile. Additionally, in paralarvae and juveniles, shape of the tentacular suckers; number, shape and arrangement of the concentric rows of pegs on the oral face of the sucker rings; presence/absence and number of teeth around the inner sucker ring margin; diameter of the marginal suckers (where present) relative to hook length; ML at which the first hooks begin to differentiate, at which all hooks are differentiated and all fully developed; and $M L$ (if any) at which all marginal suckers are lost.

6. Lower beak (see Fig. 2B-H). In lateral profile: length vs depth; lengths of the hood and of the whole crest (hood and visible crest combined) relative to the baseline; position of the posterior hood margin (lying close to vs well above the crest); presence/absence of a shoulder ridge or wing fold obscuring the jaw angle. In general: relative length of the LRL to the wings; greatest width of the wings relative to their width at the jaw angle; presence and size of any cartilaginous inserts on the wings or in the jaw angle region; length of the 'step'/ angle ridge on the inner surface of the lateral wall; whether or not the lateral walls are indented below the crest; strength and span of the lateral wall fold.

7. Radula (see Fig. 5). (All teeth observed near the bending plane.) Height of each tooth in a transverse row relative to that of the rachidian; tricuspid vs unicuspid rachidian and length/orientation of the lateral cusps (where present) 
relative to the mesocone; unicuspid vs bicuspid first lateral tooth and length/orientation of the outer cusp (where present) relative to the inner cusp.

8. Palatine palp. Number and distribution of the palatine teeth over the surface of the palp and their length relative to the rachidian height.

9. Gladius (see Fig. 2J-M). Overall shape; length of the free rachis (where present) and rostrum relative to total GL; greatest width relative to $G L$ and position (as \% GL, measured from anterior end) at which greatest width is attained; depth of the dorsal keel, where present; shape of the rostrum in dorsal view, lateral profile and cross-section.

10. Photophores. Presence/absence, shape and relative size of intestinal photophores; presence/absence, size (as \% eye circumference) and shape of photophores on ventral periphery of eyeball.
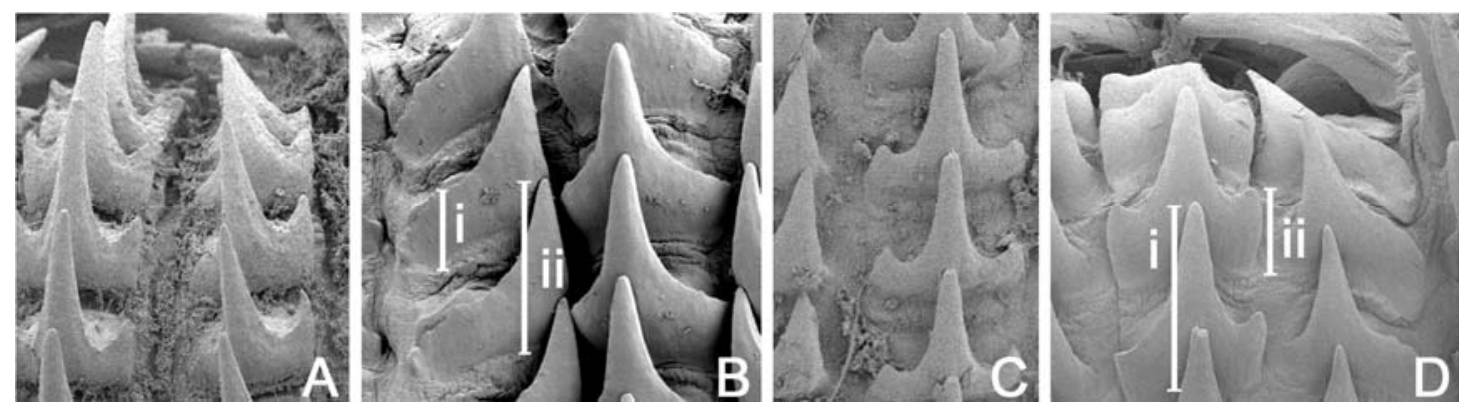

Fig. 5 - types and measurements of rachidian and first marginal radular teeth: A) strongly tricuspid rachidian and strongly bicuspid first lateral; B) unicuspid rachidian (apparent asymmetry due to angle of photography) and first lateral (i, base height, ii, overall first marginal height); (C) strongly tricuspid rachidian with 'hooked' lateral cusps; D) weakly tricuspid rachidian and unicuspid first lateral (i, rachidian height, ii, lateral cusp height). 


\section{CHECKLIST OF SPECIES}

Family Onychoteuthidae Gray, 1847

Genus Onychoteuthis Lichtenstein, 1818

Onychoteuthis banksii (Leach, 1817)

Onychoteuthis bergii Lichtenstein, 1818

Onychoteuthis aequimanus Gabb, 1868

Onychoteuthis mollis (Appellöf, 1891) (new comb.)

Onychoteuthis compacta (Berry, 1913)

Onychoteuthis borealijaponica Okada, 1927

Onychoteuthis meridiopacifica Rancurel \& Okutani, 1990

Onychoteuthis lacrima (in press)

Onychoteuthis prolata (in press)

Genus Onykia Lesueur, 1821

Onykia (Onykia) carriboea Lesueur, 1821

Onykia (Onykia) robusta (Verrill, 1876) (new comb.)

Onykia (Onykia) loennbergii (Ishikawa \& Wakiya, 1914) (new comb.)

Onykia (Onykia) aequatorialis (Thiele, 1921) (new comb.)

Onykia (Onykia) robsoni (Adam, 1962) (new comb.)

Onykia (Onykia) indica Okutani, 1981

Onykia (Moroteuthopsis) ingens (Smith, 1881) (new comb.)

Genus Ancistroteuthis Gray, 1849

Ancistroteuthis lichtensteinii (Férussac, 1835)

Genus Kondakovia Filippova, 1972

Kondakovia longimana Filippova, 1972

Kondakovia nigmatullini Laptikhovsky, Arkhipkin \& Bolstad, 2008

Genus Notonykia Nesis, Roeleveld and Nikitina, 1998

Notonykia africanae Nesis, Roeleveld \& Nikitina, 1998

Notonykia nesisi Bolstad, 2007

Gen. nov. 1

Gen. nov. 1 knipovitchi (Filippova, 1972) (new comb.)

Gen. nov. 2

Gen. nov. 2 rancureli (Okutani, 1981) (new comb.)

Gen. nov. 2 sp. 1

Gen. nov. 2 sp. 2

Gen. nov. 2 sp. 3 

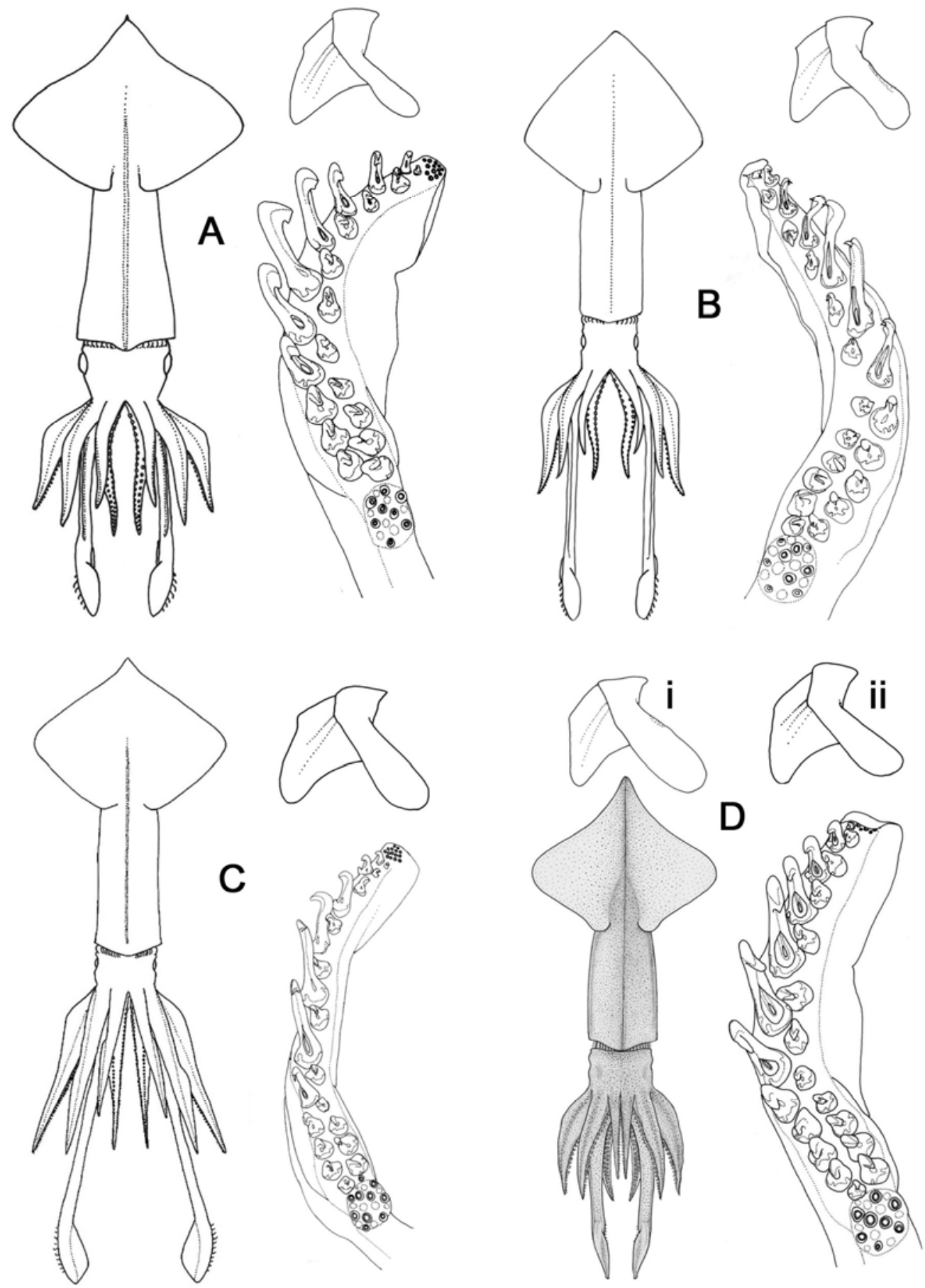

A) Onychoteuthis banksii, pp. 36-41; B) Onychoteuthis bergii, pp. 42-47; C) Onychoteuthis aequimanus, pp. 48-56; C) Onychoteuthis compacta, pp. 61-68: (i) 'humped' beak form, (ii) 'normal' beak form. 

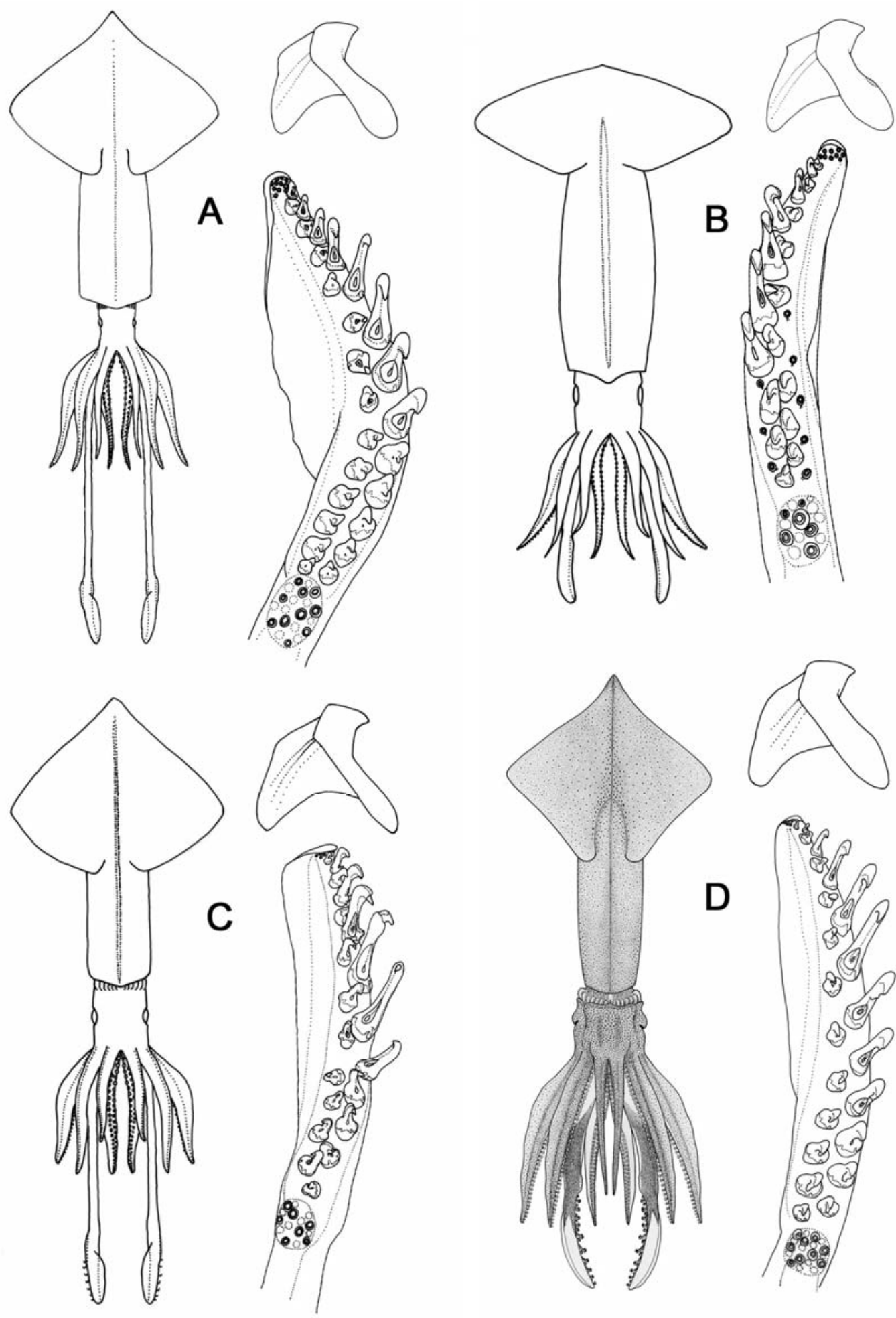

D

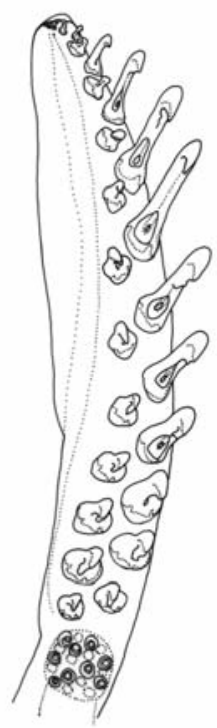

A) Onychoteuthis borealijaponica, pp. 69-77; B) Onychoteuthis meridiopacifica, pp. 78-83; C) Onychoteuthis lacrima, pp. 84-89; C) Onychoteuthis prolata, pp. 90-95. 


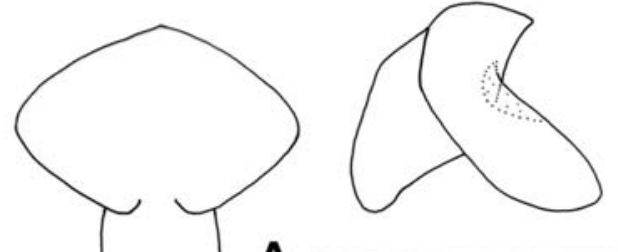

A
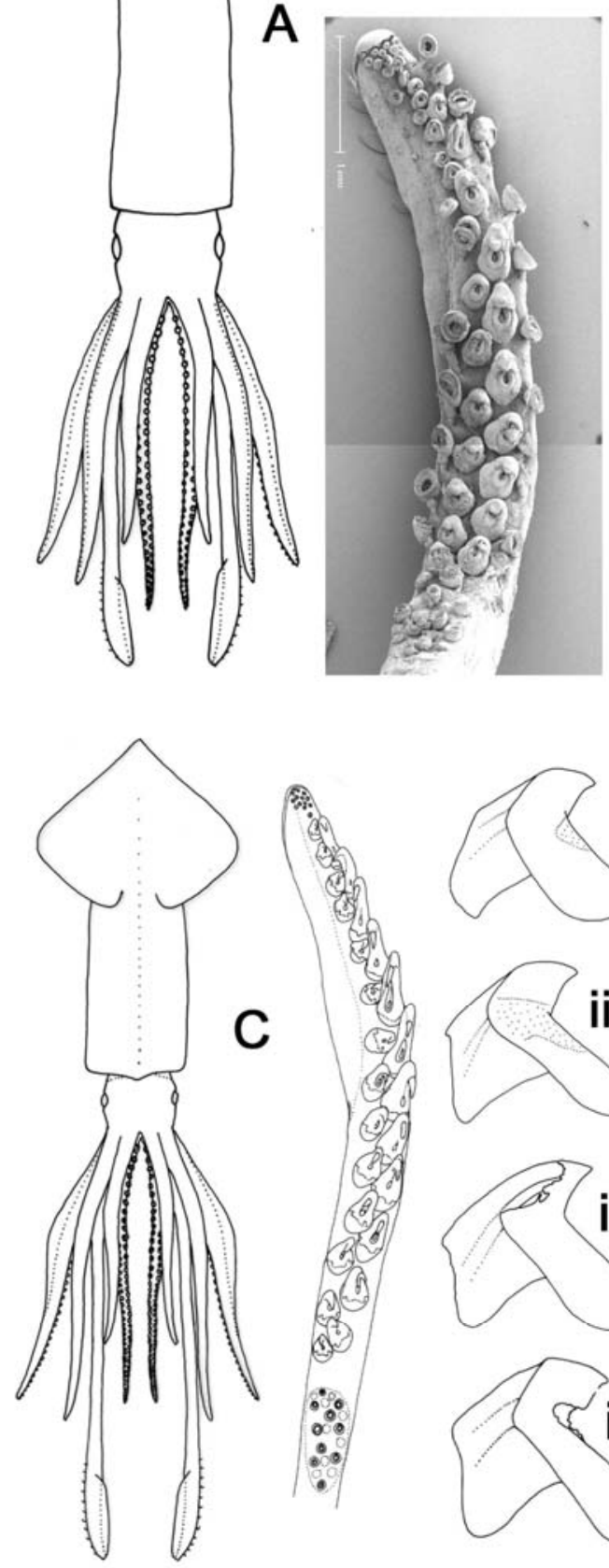
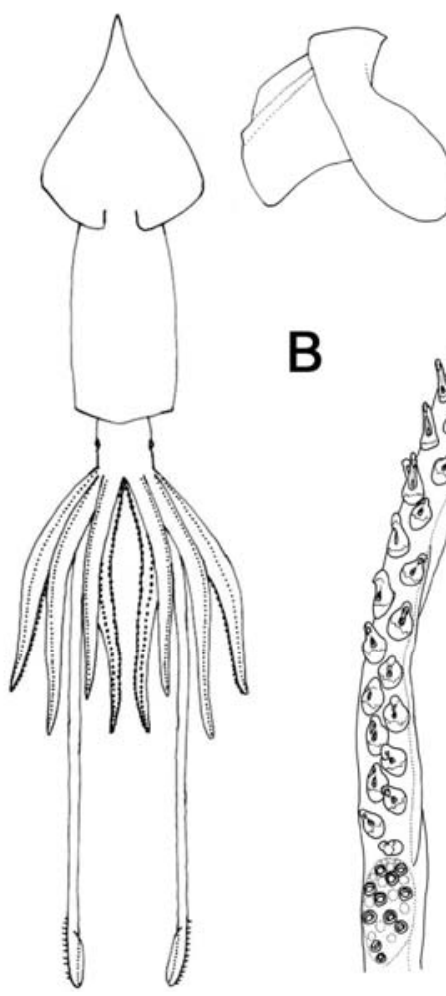

B
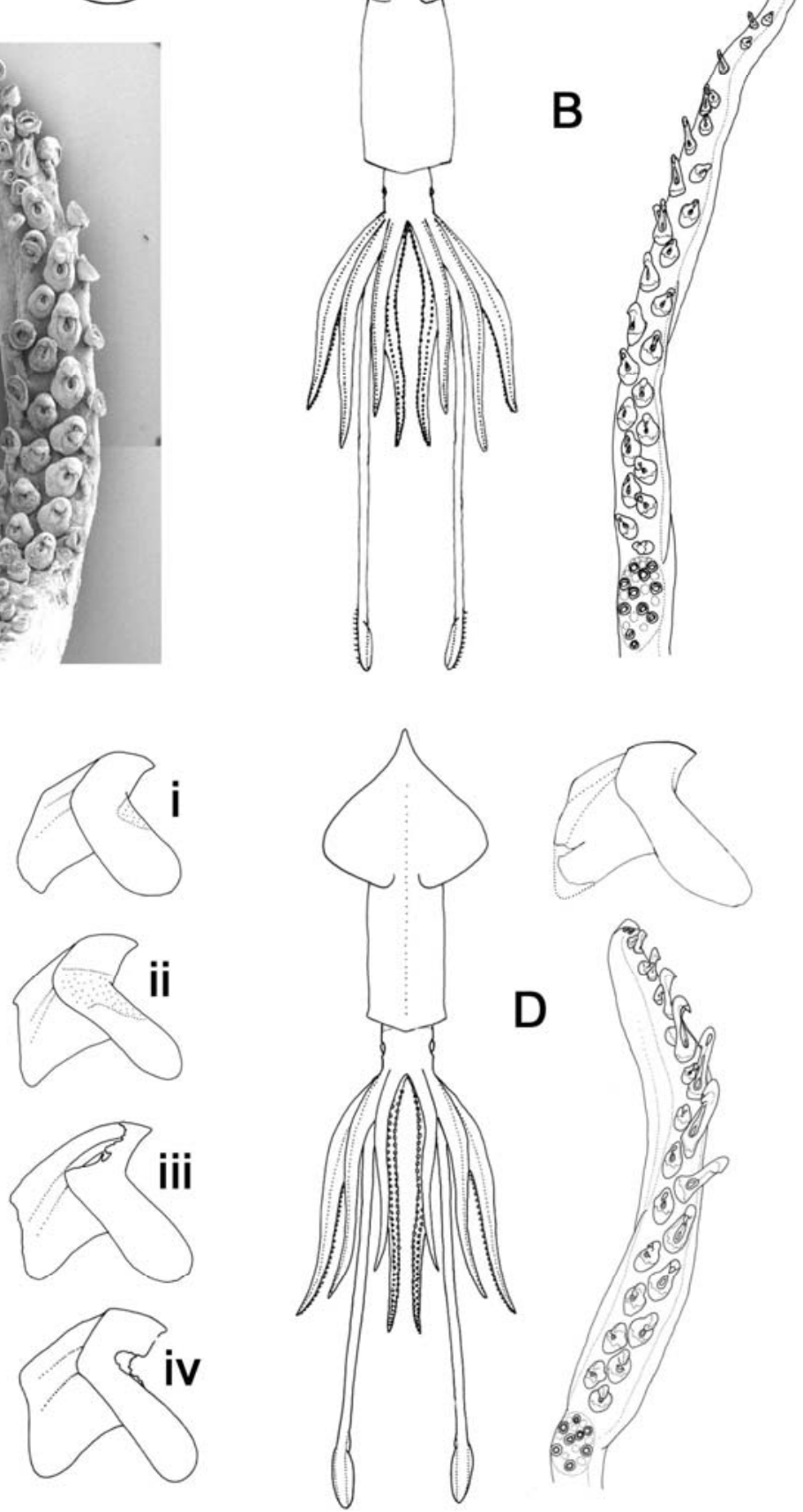

A) Onykia (Onykia) carriboea, pp. 99-105; B) Onykia (Onykia) robusta, pp. 106-117;

C) Onykia (Moroteuthopsis) ingens, pp. 145-158: (i) subadult $q$, (ii) subadult $\hat{\sigma}$, (iii) adult + , (iv) adult ${ }_{3}$; D) Onykia (Onykia) loennbergii, pp. 118-124. 

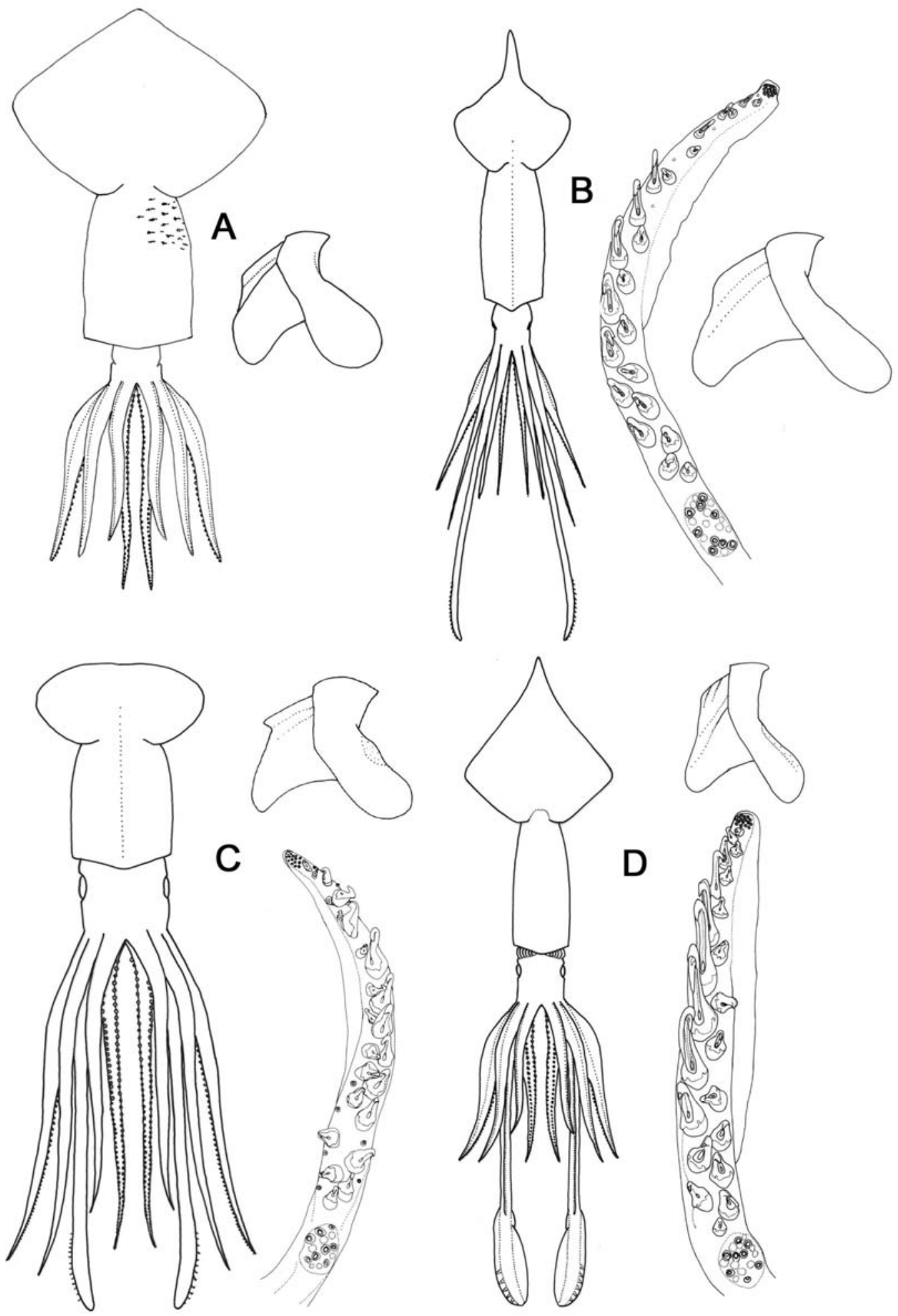

A) Onykia (Onykia) aequatorialis, pp. 125-129; B) Onykia (Onykia) robsoni, pp. 131139; C) Onykia (Onykia) indica, pp. 140-144; D) Ancistroteuthis lichtensteinii, pp. 161169. 

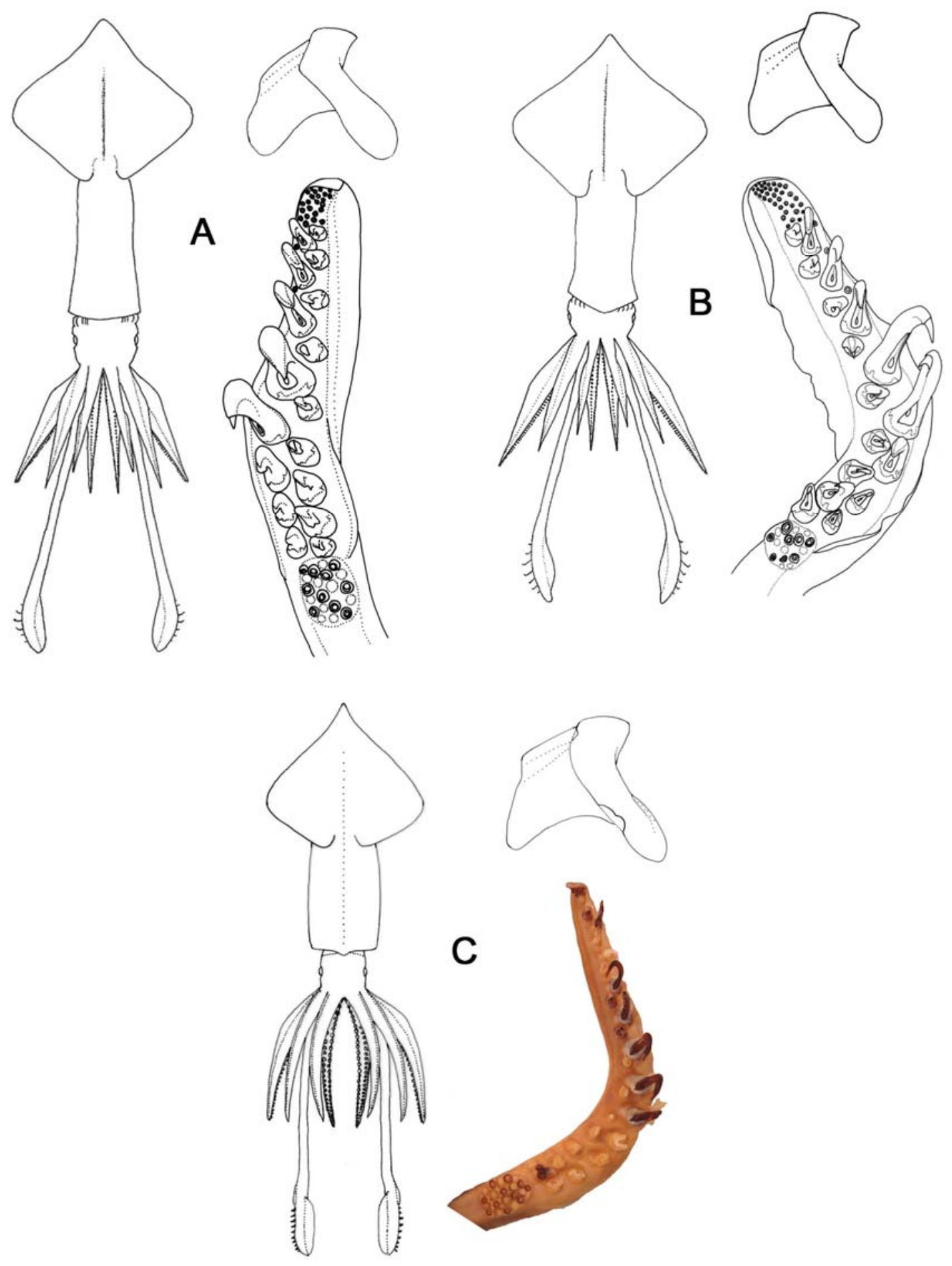

A) Notonykia africanae, pp. 179-186; B) Notonykia nesisi, pp. 187-193; C) Gen. nov. 1 knipovitchi, pp. 195-199. 

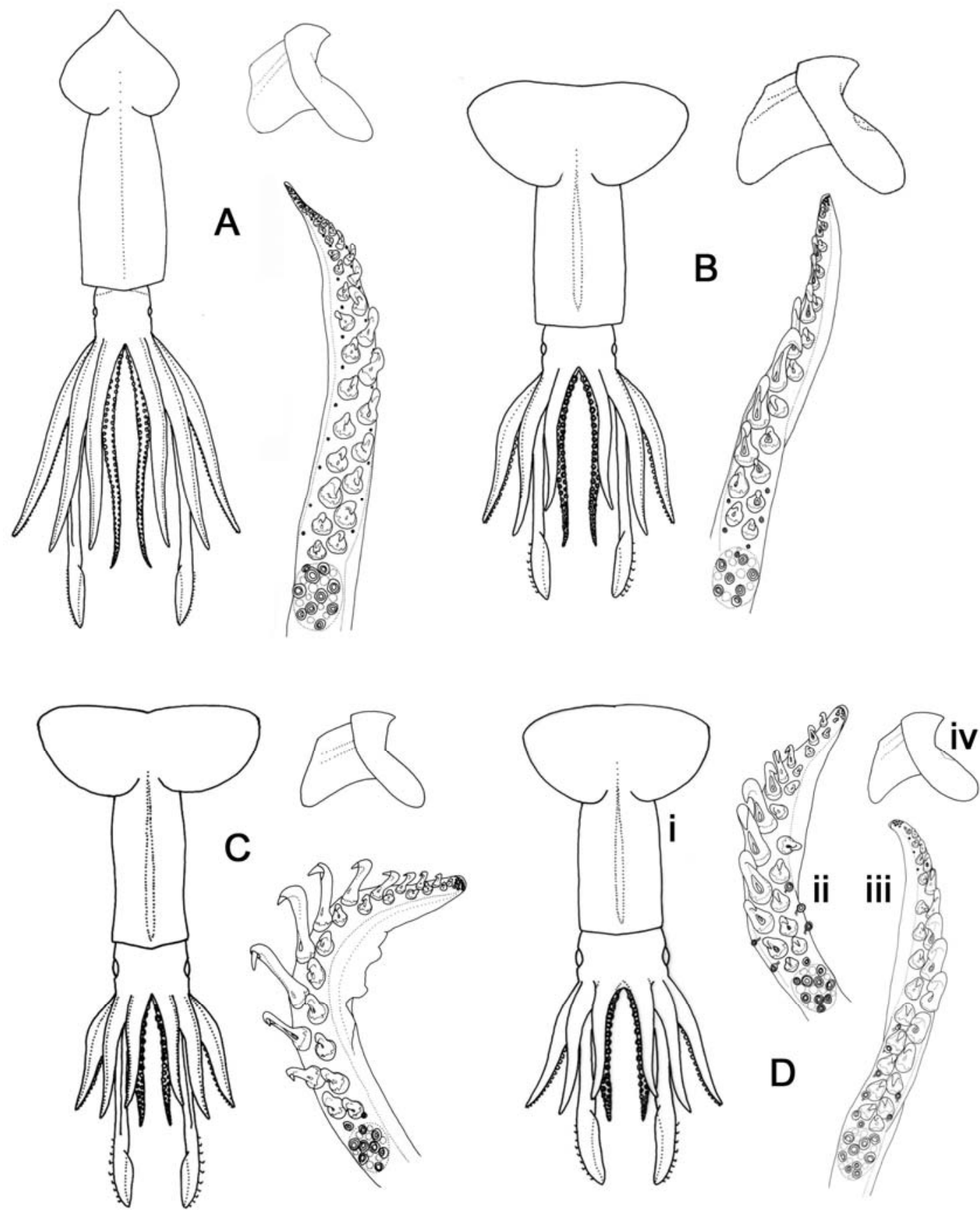

A) Kondakovia longimana, pp. 171-177; B) Gen. nov. 2 rancureli, pp. 202-206; C) Gen. nov. 2 sp. 1, pp. 206-209; D) Gen. nov. 2 sp. 2 (pp. 209-211) and 3 (pp. 212214): (i) Gen. nov. 2 sp. 3 (Gen. nov. 2 sp 2 nearly identical, but lacking inter-arm membranes), (ii) Gen. nov. 2 sp. 3 tentacle club, (iii) Gen. nov. 2 sp. 2 tentacle club, (iv) Gen. nov. 2 sp. 3 beak. 


\section{SYSTEMATICS}

\section{Family Onychoteuthidae Gray, 1849}

Diagnosis - Small- to large-bodied squids (ML 60mm-2m at maturity) with simple, straight mantle-funnel locking apparatus, and buccal connective formula DDVV; head with three primary (ventro-lateral) occipital folds and variably with secondary (dorsolateral) occipital folds; photophores variably present on ventral surface of intestine and surface of eye; arms with double row of suckers, adentate in post-paralarval stages; manus of tentacle clubs with double row of hooks, variably with small marginal suckers along dorsal and ventral manus margins; lower beak with pronounced 'step' in jaw angle, forming angle ridge along interior surface of lateral wall; males without hectocotylus; gladius with primary conus and rostrum. 


\begin{tabular}{|c|c|c|c|c|c|c|c|c|c|c|c|}
\hline 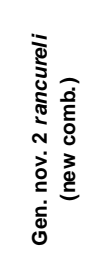 & $\begin{array}{l}\text { 志 } \\
\text { 岁 }\end{array}$ & $\begin{array}{l}\text { 志 } \\
\text { 产 }\end{array}$ & 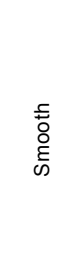 & 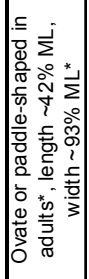 & 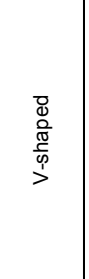 & 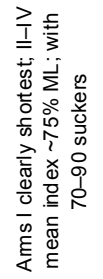 & 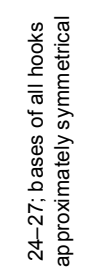 & 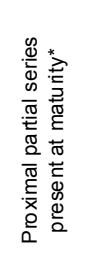 & $\underset{\infty}{\bar{D}}$ & 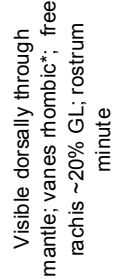 & 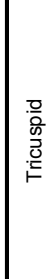 \\
\hline 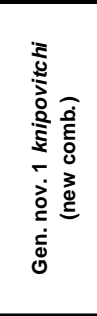 & 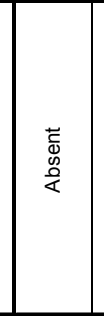 & 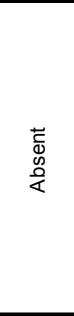 & 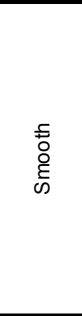 & 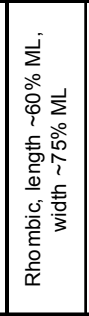 & 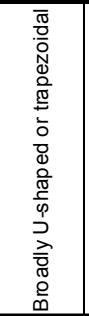 & 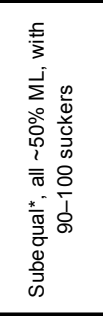 & 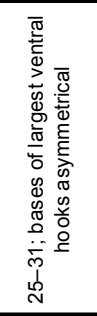 & 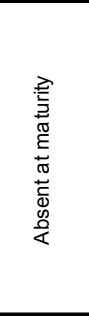 & స్ & 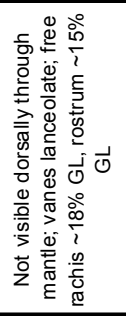 & \begin{tabular}{|l} 
\\
\\
$\frac{2}{0}$ \\
0 \\
$\frac{0}{5}$ \\
$\frac{3}{5}$ \\
\end{tabular} \\
\hline 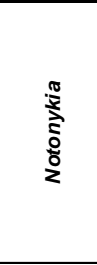 & 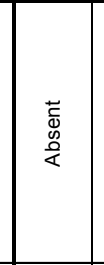 & $\begin{array}{l}\text { 总 } \\
\text { 岕 }\end{array}$ & 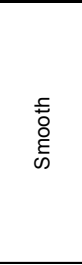 & 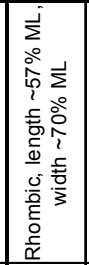 & $\begin{array}{l}\frac{\sigma}{0} \\
\frac{0}{\sigma} \\
\frac{\sigma}{p} \\
>\end{array}$ & 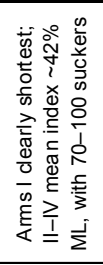 & 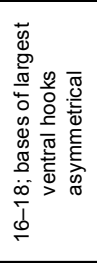 & 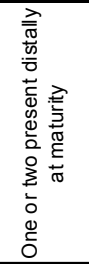 & $\frac{9}{1}$ & 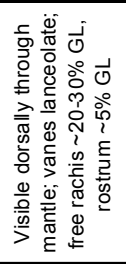 & \begin{tabular}{|l} 
\\
$\frac{0}{0}$ \\
$\frac{0}{0}$ \\
$\frac{0}{5}$ \\
5
\end{tabular} \\
\hline 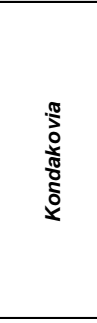 & 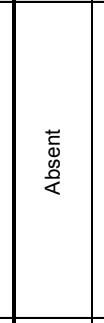 & 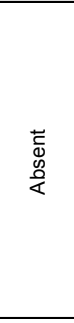 & 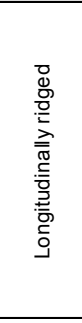 & 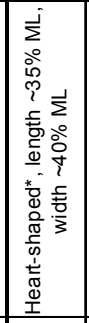 & 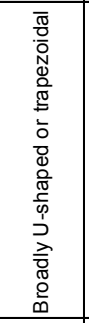 & 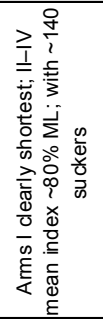 & 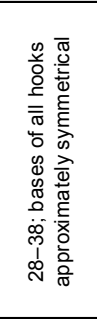 & 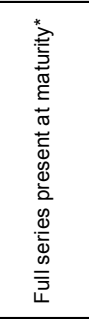 & 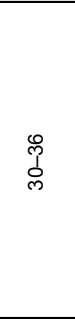 & 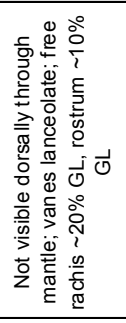 & 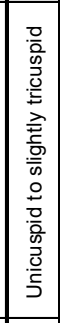 \\
\hline 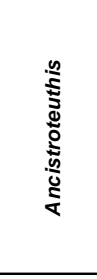 & 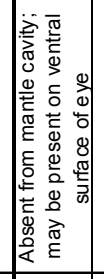 & 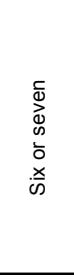 & 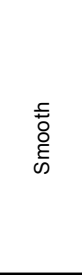 & 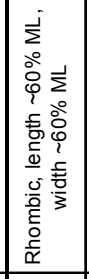 & $\begin{array}{l}\frac{\sigma}{0} \\
\frac{0}{\sigma} \\
\frac{\sigma}{p} \\
>\end{array}$ & 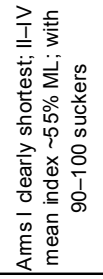 & 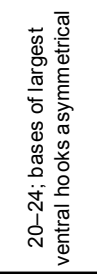 & 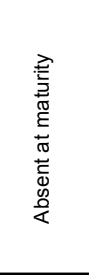 & 号 & 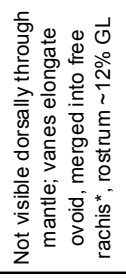 & 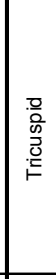 \\
\hline 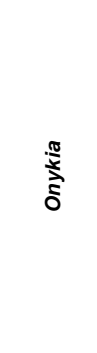 & $\begin{array}{l}\text { 志 } \\
\text { 晏 }\end{array}$ & 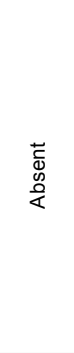 & 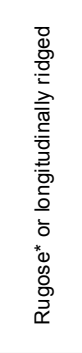 & 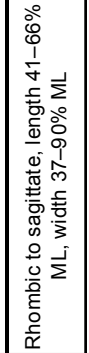 & 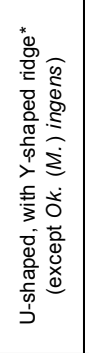 & 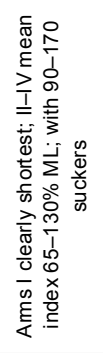 & 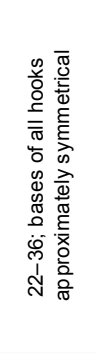 & 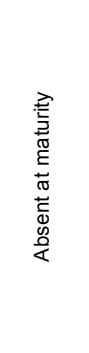 & $\frac{\infty}{1}$ & 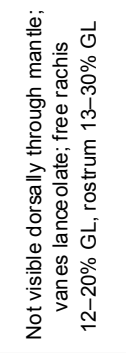 & 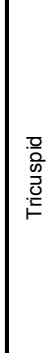 \\
\hline 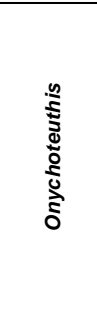 & 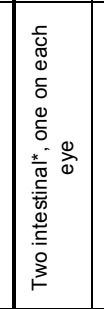 & $\begin{array}{l}\frac{\bar{g}}{\underline{g}} \\
\frac{\mathrm{o}}{\mathrm{x}} \\
\text { is }\end{array}$ & 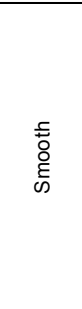 & 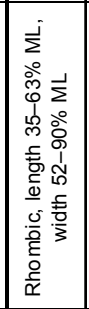 & 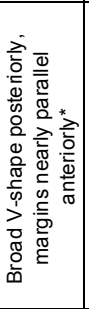 & 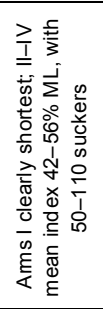 & 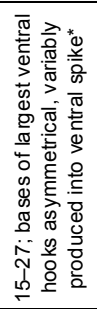 & 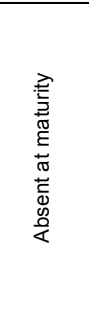 & $\frac{\infty}{1}$ & 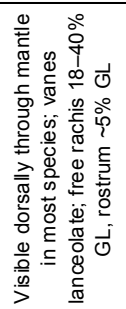 & 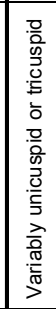 \\
\hline & 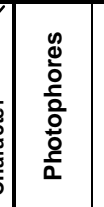 & 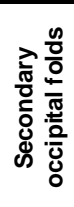 & 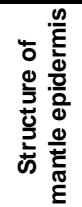 & $\stackrel{n}{\frac{n}{4}}$ & 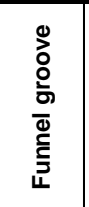 & $\stackrel{n}{\sum_{x}^{2}}$ & 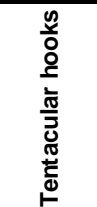 & 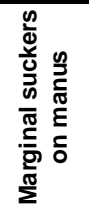 & 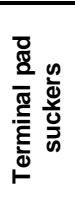 & 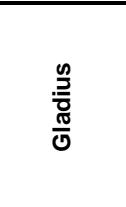 & 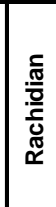 \\
\hline
\end{tabular}




\section{Onychoteuthis Lichtenstein, 1818}

Chaunoteuthis Appellöf, 1891: 1-29, Pls 1-4. Type species Chaunoteuthis mollis Appellöf, 1891, by monotypy.

Teleonychoteuthis Pfeffer, 1900: 158. Type species Teleonychoteuthis krohnii Vérany, 1847, by monotypy.

Type species — Onychoteuthis bergii Lichtenstein, 1818.

Diagnosis - Intestine with two photophores, one over posterior extremity of ink-sac, one near anus; ventral periphery of each eyeball with one distinct photophore; occipital region with six to ten secondary folds; gladius clearly visible through dorsal mantle musculature in most species; funnel groove broadly $\mathrm{V}$-shaped posteriorly, with nearly parallel margins anteriorly; arms 21-66\% ML; manus with 15-27 hooks, with bases of largest ventral hooks asymmetrical, variably produced into spike; length of longest ventral hook $16-25 \% \mathrm{CL}$; gladius with posteriorly directed rostrum, $\sim 5 \% \mathrm{GL}$.

Remarks - The systematic instability of the cosmopolitan tropical/temperate genus Onychoteuthis has been long recognised (Young 1972, Kubodera et al. 1998, Vecchione et al. 2003). Os. banksii had become a catch-all name for many morphologically similar taxa, including the type species, Os. bergii, two of the additional three species generally recognised by recent authors (Os. compacta and Os. borealijaponica), and about twenty additional nominal species (see e.g. Pfeffer 1912, Adam 1952). The name 'Os. banksii' has typically been applied to any narrowbodied specimen with 20-23 hooks on each tentacular club, two round photophores on the ventral surface of the intestine, distinct ocular photophores, and rhombic fins of length $\sim 55 \% \mathrm{ML}$. However, these characters have been widely believed to characterise several to many undescribed species (Young 1972, Nesis 1987, Young \& Harman 1987, Kubodera et al. 1998, Vecchione et al. 2003), so new characters and states are necessary to differentiate them.

The confusion surrounding Onychoteuthis taxa stems from a number of separate systematic problems. The type specimens of the four aforementioned species are, respectively: juvenile and damaged (holotype, Os. banksii, Fig. 6B), small juvenile and extensively damaged (holotype, Os. compacta, Fig. 15B), not extant (Os. borealijaponica, never designated), and partially digested (holotype and paratypes, Os. meridiopacifica). The original descriptions are, respectively: two lines long and based on a single juvenile specimen, rather more detailed and well illustrated but based on a 
single small juvenile; restricted to a description of the intestinal photophores only, and relatively more comprehensive but based on partially digested material.

While Os. borealijaponica and Os. meridiopacifica are morphologically distinct, the remaining seven Onychoteuthis species treated herein are very similar, requiring the identification of new characters and their states for reliable differentiation. Identification of existing, and recognition and reliable differentiation of new taxa have been aided by the earlier efforts of several colleagues who had independently investigated important characters for diagnosing species within this genus, namely Seki (2001), Vecchione and Young (see Vecchione et al. 2003). These colleagues are accordingly named as co-authors on the relevant new species as follows: Os. lacrima Bolstad \& Seki and Os. prolata Bolstad, Vecchione \& Young (both in press).

Key character states of all species of Onychoteuthis are given in Table 2. 


\begin{tabular}{|c|c|c|c|c|c|c|c|c|c|}
\hline $\begin{array}{l}\frac{\mathscr{g}}{\bar{z}} \\
\frac{\mathrm{c}}{0}\end{array}$ & $\begin{array}{l}\text { 离 } \\
\text { 站 }\end{array}$ & 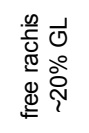 & 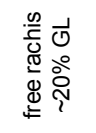 & 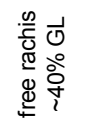 & 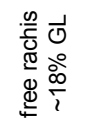 & 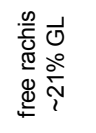 & 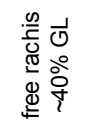 & 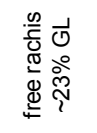 & 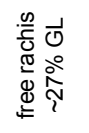 \\
\hline 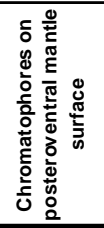 & 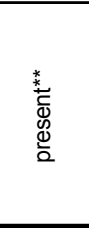 & $\begin{array}{l}\text { है } \\
\text { o } \\
\text { 亲 }\end{array}$ & 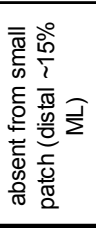 & $\begin{array}{l}\text { है } \\
\text { o } \\
\text { 亲 }\end{array}$ & 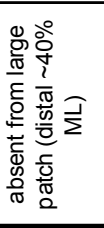 & 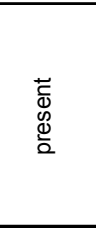 & 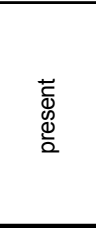 & 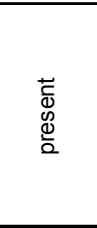 & 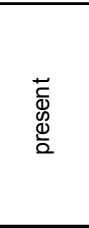 \\
\hline 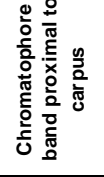 & 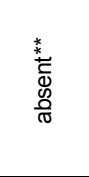 & 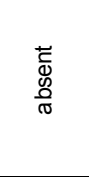 & 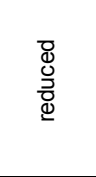 & $\begin{array}{l}\text { ज्ञ } \\
0 \\
\text { 竎 } \\
\text { J }\end{array}$ & 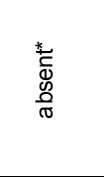 & 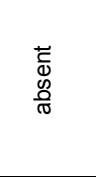 & 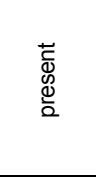 & 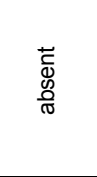 & 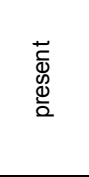 \\
\hline 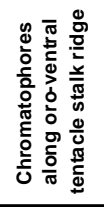 & $\begin{array}{l}\text { 毒 } \\
\text { 兽 }\end{array}$ & 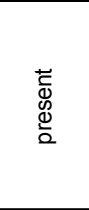 & $\begin{array}{l}\text { 蒿 } \\
\text { 边 }\end{array}$ & 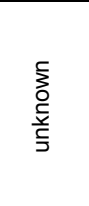 & 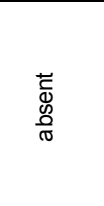 & 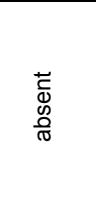 & 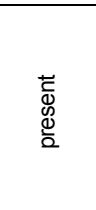 & 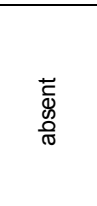 & 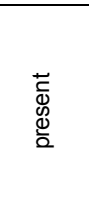 \\
\hline 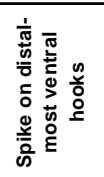 & 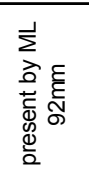 & 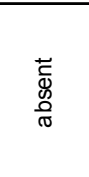 & 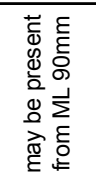 & $\begin{array}{l}\text { 离 } \\
\text { 亲 } \\
\text { s. }\end{array}$ & 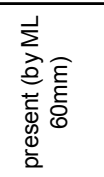 & 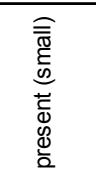 & 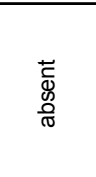 & 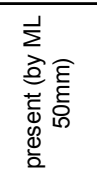 & 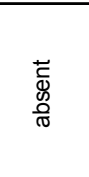 \\
\hline 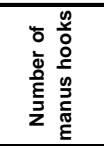 & 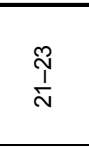 & 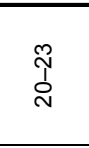 & $\begin{array}{l}\text { స్} \\
\stackrel{\sigma}{\sigma}\end{array}$ & 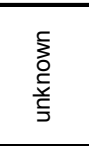 & $\begin{array}{l}\stackrel{N}{N} \\
\stackrel{N}{N}\end{array}$ & స్ స్ & $\begin{array}{l}\stackrel{9}{T} \\
\stackrel{1}{L}\end{array}$ & 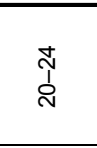 & ণิ స్ \\
\hline 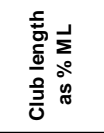 & 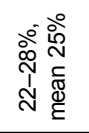 & 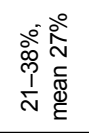 & 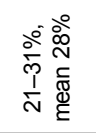 & 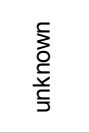 & 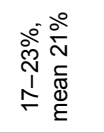 & 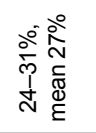 & 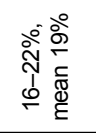 & 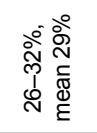 & 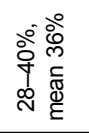 \\
\hline 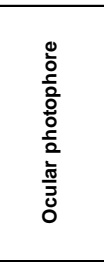 & 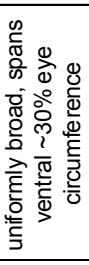 & 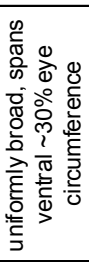 & 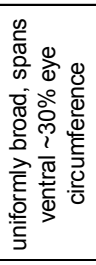 & 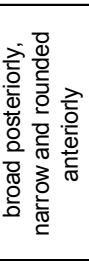 & 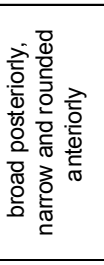 & 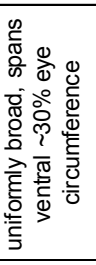 & 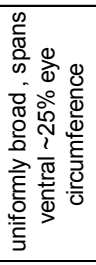 & 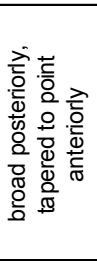 & 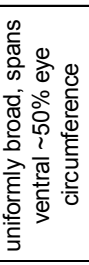 \\
\hline 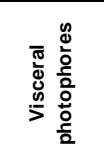 & 0 & & & & 0 & $\begin{array}{l}0 \\
\bigcap\end{array}$ & $\begin{array}{l}0 \\
0\end{array}$ & 0 & \\
\hline j] & 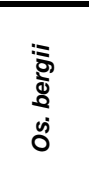 & 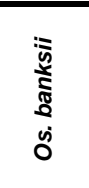 & טit & 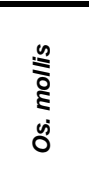 & 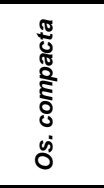 & 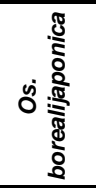 & 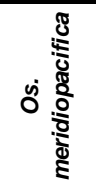 & 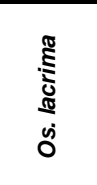 & $\begin{array}{l}\frac{\pi}{\pi} \\
\frac{\pi}{0} \\
\frac{0}{2} \\
\dot{d j} \\
0\end{array}$ \\
\hline
\end{tabular}


Onychoteuthis banksii (Leach, 1817) (Figs 6, 7, Tables 2, 3)

Loligo banksii Leach, 1817: 141.

Onychoteuthis banksii (Leach, 1817) - Toll (1982): 51-55, PI. 5A, (1998): Fig. 31; Sweeney \&

Roper (1998): 572; Lipinski et al. (2000): 106; Bolstad (in press).

Onychoteuthis banksi [sic] (Leach, 1817) — Clarke (1986): 72-74, Fig. 32a.

Type material examined - BMNH 1986266, holotype, ML $31 \mathrm{~mm}$, Gulf of Guinea, NFD.

Additional material examined (59 specimens) - UMML 31.2965, ${ }^{\AA}, \mathrm{ML} 110 \mathrm{~mm}, 41^{\circ} 37^{\prime} \mathrm{N}$, $27^{\circ} 00^{\prime} \mathrm{W}, 500 \mathrm{~m}, 21 / 06 / 1931,0045 \mathrm{~h}$, Dana $4193 \mathrm{VI}$; BMNH 20070558, §̊, ML 85mm, $41^{\circ} 10.5^{\prime} \mathrm{N}, 14^{\circ} 38^{\prime} \mathrm{W}$, surface, 28/05/1955, 'Discovery' Investigations Stn 3245, HN; D4075 XI, + , ML 81mm, 38 $29^{\prime} \mathrm{N}, 2^{\circ} 08^{\prime} \mathrm{E}, 600 \mathrm{~m}, 22 / 05 / 1930,0230 \mathrm{~h}$, Dana Expedition Stn $4075 \mathrm{XI}$; UMML 31.1302, sex indet., ML 45mm, 31 ${ }^{\circ} 05^{\prime} \mathrm{N}, 62^{\circ} 35^{\prime} \mathrm{W}, 04 / 04 / 1957$, MV Delaware; USNM 726945, $q$, ML 59mm, $31^{\circ} \mathrm{N}, 64^{\circ} \mathrm{W}$, Bermuda, 800-0m, 03/09/1968, RV Trident Acre 4-1-N; USNM 575945, 2 sex indet., ML 32, $24 \mathrm{~mm}, 29^{\circ} 00^{\prime} \mathrm{N}, 88^{\circ} 02^{\prime} \mathrm{W}$, East of Mississippi River Delta, 247m, 27/04/1961, RV Oregon 3254, 20m MWT; UMML 31.1307, 2 q, ML 73, 71mm, $28^{\circ} 28^{\prime} \mathrm{N}, 43^{\circ} 10^{\prime} \mathrm{W}$, surface, 18/07/1964, 0330h, RV

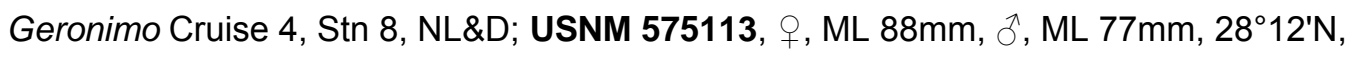
$87^{\circ} 49^{\prime} \mathrm{W}$, south of Mobile, Alabama, Gulf of Mexico, surface, -/-/1952, RV Oregon Stn 1008, dipnet; USNM 727512, , $\mathrm{ML} 49 \mathrm{~mm}, 25^{\circ} 35^{\prime} \mathrm{N}, 79^{\circ} 25^{\prime} \mathrm{W}$, Florida, North Atlantic, surface, 22/08/1962, RV Gerda, dipnet and light; USNM 575114, sex indet., ML 48mm, $25^{\circ} 30^{\prime} \mathrm{N}, 92^{\circ} 00^{\prime} \mathrm{W}$, S of Marsh Island, Louisiana, 3162m [sic], 10/03/1954, RV Oregon, drift net; UMML 31.984, 24 sex indet., ML $87-59 \mathrm{~mm}, 25^{\circ} 21^{\prime} \mathrm{N}, 79^{\circ} 41^{\prime} \mathrm{W}$, surface, 27/09/1962, RV Gerda Stn 75; UMML 31.986, 4 sex indet., ML 68-46mm, 2520'N, $79^{\circ} 42^{\prime} \mathrm{W}$, surface, 22/06/1962, RV Gerda Stn 40; UMML 31.577, ๆ, ML 73mm, ð, ML $70 \mathrm{~mm}$, sex indet., $\mathrm{ML} 59 \mathrm{~mm}, 2^{\circ} 20-24^{\circ} 17.6^{\prime} \mathrm{N}, 78^{\circ} 02-01.5^{\prime} \mathrm{W}, 1 \mathrm{~m}$ over $1071 \mathrm{~m}$,

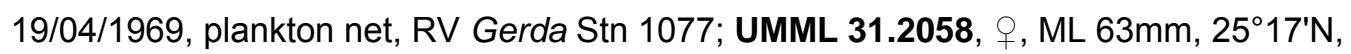
$77^{\circ} 55^{\prime} \mathrm{W}, 17 / 08 / 1966$, RV Tursiops Stn 324; UMML 31.983, ð, ML 58mm, $24^{\circ} 35^{\prime} \mathrm{N}$, $80^{\circ} 01^{\prime} \mathrm{W}$, surface, 13/06/1968, RV Gerda Stn 1006; UMML 31.39, 2 \%, ML 70, 66mm, $23^{\circ} 59^{\prime} \mathrm{N}, 81^{\circ} 48.5^{\prime} \mathrm{W}$, between Havana and Key West, -/12/1951, RV Yara (Cuban Navy); UMML 31.701, 2 sex indet., ML 34, 31mm, $7^{\circ} 16.2-15.3^{\prime} \mathrm{N}, 79^{\circ} 50.8-52.9^{\prime} \mathrm{W}$, 03/05/1967, RV Pillsbury; USNM 885307, sex indet., ML 53mm, $10^{\circ} 52^{\prime} \mathrm{N}, 22^{\circ} 09^{\prime} \mathrm{W}$, 608-592m, 15/04/1971, 1952-2027h, RV Walther Herwig Stn 490-II-71, 1600-mesh EMWT; D4000, + , ML 69mm, $0^{\circ} 31^{\prime} \mathrm{S}, 11^{\circ} 02^{\prime} \mathrm{W}$, surface, 04/03/1930, 0235h, Dana Expedition Stn 4000; CASIZ 030487, sex indet., ML 45mm, $10^{\circ} 01.5^{\prime} \mathrm{S}, 80^{\circ} 05^{\prime} \mathrm{W}$, 04/03/1935, Stn N-40; CASIZ 030502, §ิ, ML 84mm, 2 sex indet, ML 76, 62mm, $25^{\circ} 00^{\prime} \mathrm{S}, 22^{\circ} 20^{\prime} \mathrm{W}, 04 / 01 / 1935$, Stn N22-23, coll. T. Crocker; CASIZ 030513, วิ, ML $83 \mathrm{~mm}$, sex indet., $\mathrm{ML} 67 \mathrm{~mm}, 33^{\circ} 09^{\prime} \mathrm{S}, 74^{\circ} 12^{\prime} \mathrm{W}$, off Chile, 02/02/1935, Yacht Zaca Stn N29, coll. T. Crocker.

Unlocalised material examined (3 specimens) - MNHN 632, ㅇ, ML 121mm, coll. d'Orbigny, NFD; MNHN 626, $\uparrow$, ML 120mm, Atlantic Ocean, 1834, coll. d'Orbigny, NFD; MNHN 633, ㅇ, ML $91 \mathrm{~mm}$, Atlantic Ocean, 1830, coll. Damon, NFD. 
Distribution (Fig. 6A) - Central and North Atlantic (including Gulf of Mexico); $800-0 \mathrm{~m}$.

Diagnosis — Intestinal photophores circular, well defined, with diameter of anterior $\sim 75 \%$ that of posterior; ocular photophore uniformly broad, spanning ventral $\sim 30 \%$ eye circumference; chromatophores absent from tentacle stalk proximal to carpus; chromatophores present along oro-ventral stalk ridge; tentacle club length $\sim 27 \% \mathrm{ML}$, with 20-23 long, slender, strongly recurved hooks; distal-most ventral hook bases not produced into spike.

Description (Figs 6B-7I) - Mantle muscular, width 19-23-27\% ML, conical overall, tapering nearly uniformly; gladius visible dorsally as prominent ridge along proximal $80 \% \mathrm{ML}$; rostrum visible ventrally through posterior $10 \% \mathrm{ML}$ and may pierce mantle posteriorly through at least $\mathrm{ML} 49 \mathrm{~mm}$; small, firm, oblong ventral keel, $\sim 10 \% \mathrm{ML}$, present anterior to visible portion of rostrum. Intestinal photophores circular, with diameter of anterior $\sim 75 \%$ that of posterior. Fins rhombic, length $\sim 57 \% \mathrm{ML}$, width 52 73-89\% ML; posterior margins concave posteriorly, becoming slightly convex anteriorly; anterior margins straight to slightly convex.

Head rounded, length 18-21-29\% ML, width 14-21-29\% ML, depth 19\% ML, anteriorly constricted prior to brachial crown; occipital region typically with six secondary folds. Ocular photophore uniformly broad, with rounded ends, spanning ventral $\sim 30 \%$ eye diameter. Funnel groove broad, well-defined $V$-shape posteriorly, constricted anteriorly into narrow $\mathrm{V}$; funnel aperture opens directly below posterior eye margin. Funnel component of locking apparatus 15\% ML; mantle component $\sim 33 \%$ ML.

Arms $28-32_{I}-43_{I I-I V}-48 \% \mathrm{ML}$, formula II=III=IV>I, each with 70-90 suckers in two rows (ML 50-90mm), bordered on either side by low trabeculate membrane; sucker diameter 40-50\% arm width. Aboral keels present on Arms I-III: that on Arms I 10\% arm width over distal 60-70\% arm length; that on Arms II 50\% arm depth over entire arm length; that on Arms III widening to $\sim 100 \%$ arm depth by $\sim 30 \%$ arm length, thereafter narrowing to arm tip; lateral membrane on Arms IV 100\% arm width along entire arm length.

Tentacles robust, $55-69-80 \% \mathrm{ML}$; aboral surface of stalk with low keel, $\sim 15 \%$ tentacle thickness, extending from stalk base to level with carpus. Club (Figs 6D-F) proximally expanded, $21-27-38 \% \mathrm{ML}(42-54 \% \mathrm{TnL})$. Carpus ovoid to oblong, with seven to nine 
Table 3. Measures ( $\mathrm{mm}$ ) and counts of Onychoteuthis banksii (Leach, 1817) (arm and tentacle measures recorded from more complete side of specimen, indicated $\mathrm{R}$ or $\mathrm{L}$ ).

\begin{tabular}{|c|c|c|c|c|c|c|c|c|c|c|c|c|}
\hline Specimen ID & $\begin{array}{c}\text { BMNH } \\
1986266\end{array}$ & $\begin{array}{c}\text { UMML } \\
31.2965\end{array}$ & D4075XI & $\begin{array}{l}\text { UMML } \\
31.577\end{array}$ & $\begin{array}{l}\text { UMML } \\
31.577\end{array}$ & $\begin{array}{c}\text { USNM } \\
726945\end{array}$ & $\begin{array}{l}\text { USNM } \\
\mathbf{8 8 5 3 0 7}\end{array}$ & $\begin{array}{c}\text { USNM } \\
727512\end{array}$ & $\begin{array}{l}\text { USNM } \\
575114\end{array}$ & $\begin{array}{c}\text { USNM } \\
575945\end{array}$ & \multicolumn{2}{|c|}{ Mean Indices } \\
\hline Type Status & Holotype & None & None & None & None & None & None & None & None & None & \multirow{2}{*}{\multicolumn{2}{|c|}{ (ML 59-110) }} \\
\hline ML & 31 & 110 & 81 & 73 & 70 & 59 & 53 & 49 & 48 & 32 & & \\
\hline Sex & Indet. & $\mathrm{M}$ & $F$ & $\mathrm{~F}$ & $M$ & Indet. & Indet. & $\mathrm{F}$ & Indet. & Indet. & & \\
\hline MW & * & 21 & 20 & 18 & 16 & 16 & 13 & 12 & 11 & 10 & MWI & 23 \\
\hline $\mathrm{HL}$ & 8 & 20 & 15 & 16 & 18 & 17 & 15 & 10 & 12 & 9 & HLI & 21 \\
\hline HW & 7 & 15 & 18 & 17 & 17 & 17 & 11 & 12 & 11 & 6 & HWI & 21 \\
\hline FL & 18 & 60 & 46 & 42 & 40 & 34 & 30 & 28 & 29 & 19 & FLI & 57 \\
\hline FW & 16 & 77 & 57 & 55 & 54 & 46 & 47 & 32 & 38 & 28 & FWI & 73 \\
\hline Arms I & $16 \mathrm{~L}$ & $31 \mathrm{~L}$ & $29 R$ & $23 \mathrm{~L}$ & $22 \mathrm{~L}$ & $19 \mathrm{~L}$ & $20 \mathrm{~L}$ & $16 \mathrm{~L}$ & $16 \mathrm{~L}$ & $9 \mathrm{~L}$ & A1I & 32 \\
\hline Arms II & $20 \mathrm{~L}$ & $39 L$ & $39 R$ & $29 L$ & $32 \mathrm{~L}$ & $25 \mathrm{~L}$ & $22 \mathrm{~L}$ & $20 \mathrm{~L}$ & $21 \mathrm{~L}$ & $12 \mathrm{~L}$ & A2I & 42 \\
\hline Arms III & $19 \mathrm{~L}$ & $42 \mathrm{~L}$ & $37 R$ & $31 \mathrm{~L}$ & $32 \mathrm{~L}$ & $25 \mathrm{~L}$ & $21 \mathrm{~L}$ & $20 \mathrm{~L}$ & $18 \mathrm{~L}$ & $12 \mathrm{~L}$ & A3I & 43 \\
\hline Arms IV & $17 \mathrm{~L}$ & $42 \mathrm{~L}$ & $37 R$ & $29 L$ & $35 \mathrm{~L}$ & $26 \mathrm{~L}$ & $19 \mathrm{~L}$ & $20 \mathrm{~L}$ & $22 \mathrm{~L}$ & $11 \mathrm{~L}$ & A4I & 44 \\
\hline TnL & $43 R$ & $61 \mathrm{~L}$ & $65 R$ & $49 \mathrm{~L}$ & $48 \mathrm{~L}$ & $43 \mathrm{~L}$ & $37 \mathrm{~L}$ & $31 \mathrm{~L}$ & $31 \mathrm{~L}$ & $19 \mathrm{~L}$ & TnLI & 69 \\
\hline $\mathrm{CL}$ & $12 \mathrm{R}$ & $23 \mathrm{~L}$ & $24 \mathrm{R}$ & $20 \mathrm{~L}$ & $20 \mathrm{~L}$ & $18 \mathrm{~L}$ & $20 \mathrm{~L}$ & $14 \mathrm{~L}$ & $13 \mathrm{~L}$ & $9 \mathrm{~L}$ & CLI & 27 \\
\hline CS & 8 & 7 & 8 & 7 & 8 & 9 & 7 & $8 \mathrm{R}$ & 7 & 9 & & \\
\hline MH & 22 & 21 & 21 & 22 & 22 & 23 & 21 & $21 \mathrm{R}$ & 22 & 20 & & \\
\hline MS & * & 0 & 0 & 0 & 0 & 0 & 0 & 0 & 0 & 0 & & \\
\hline TPS & * & 13 & 14 & 12 & 15 & 14 & 13 & $13 R$ & 10 & 13 & & \\
\hline
\end{tabular}

* indicates damaged features.

suckers; manus with 20-23 hooks (usually 21 or 22 ) in two rows (Figs 7A, 7E, 7F); terminal pad with $10-15$ suckers. Hooks D1 and D2 each larger than paired ventral hooks; dorsal hooks thereafter decreasing in size to D4 and D5, which are slightly smaller than D3 and offset toward midline of club; D6 larger than D5; size of dorsal hooks thereafter decreasing distally. Ventral hooks V1-V6 increase in size distally, with V4 intermediate in size between small proximal hooks and large mid-club hooks; V6 or V7 largest (Fig. 7E), three to four times longer than paired dorsal hook and $\sim 20 \%$ club length; distal-most ventral hooks with ventral basal cusp slightly enlarged but not produced into spike (Fig. 7F) (to ML 121 mm). Necks of largest ventral hooks slender, straight in lateral profile; overall recurve of hook in profile $80-90^{\circ}$. Ventral membrane $\sim 30 \%$ club width along entire club length; dorsal membrane $\sim 20 \%$ club width, flanking hooks D1-D4 or D5; dorsal keel $~ 100 \%$ club width, flanking dorsal hooks from D3 to club tip.

Lateral profile of lower beak (Figs 7B-D, ML 88mm) longer than deep, with crest sloping steeply downward in nearly straight line, posterior hood edge well above crest, hood length $\sim 50 \%$ total crest length, and hood and crest together $\sim 75 \%$ baseline; jaw edge straight proximally, becoming slightly concave distally and forming small rostral point; jaw angle obtuse, slightly obscured laterally by low, rounded, indistinct wing fold. Shoulder ridge distinct, sharp; angle ridge well developed, spanning $\sim 80 \%$ lateral wall depth; angle point distinct, darkly pigmented; wings broaden distally, with greatest width $\sim 130 \%$ that at jaw angle, length $\sim 170 \%$ LRL, with small triangular insert of cartilage below jaw angle; crest broad, not thickened; lateral wall diagonally bisected 
by dark, prominent shelf anteriorly, becoming low, rounded ridge posteriorly, curving slightly and becoming indistinct by $\sim 75 \%$ distance between rostral tip and posterior lateral wall corner.
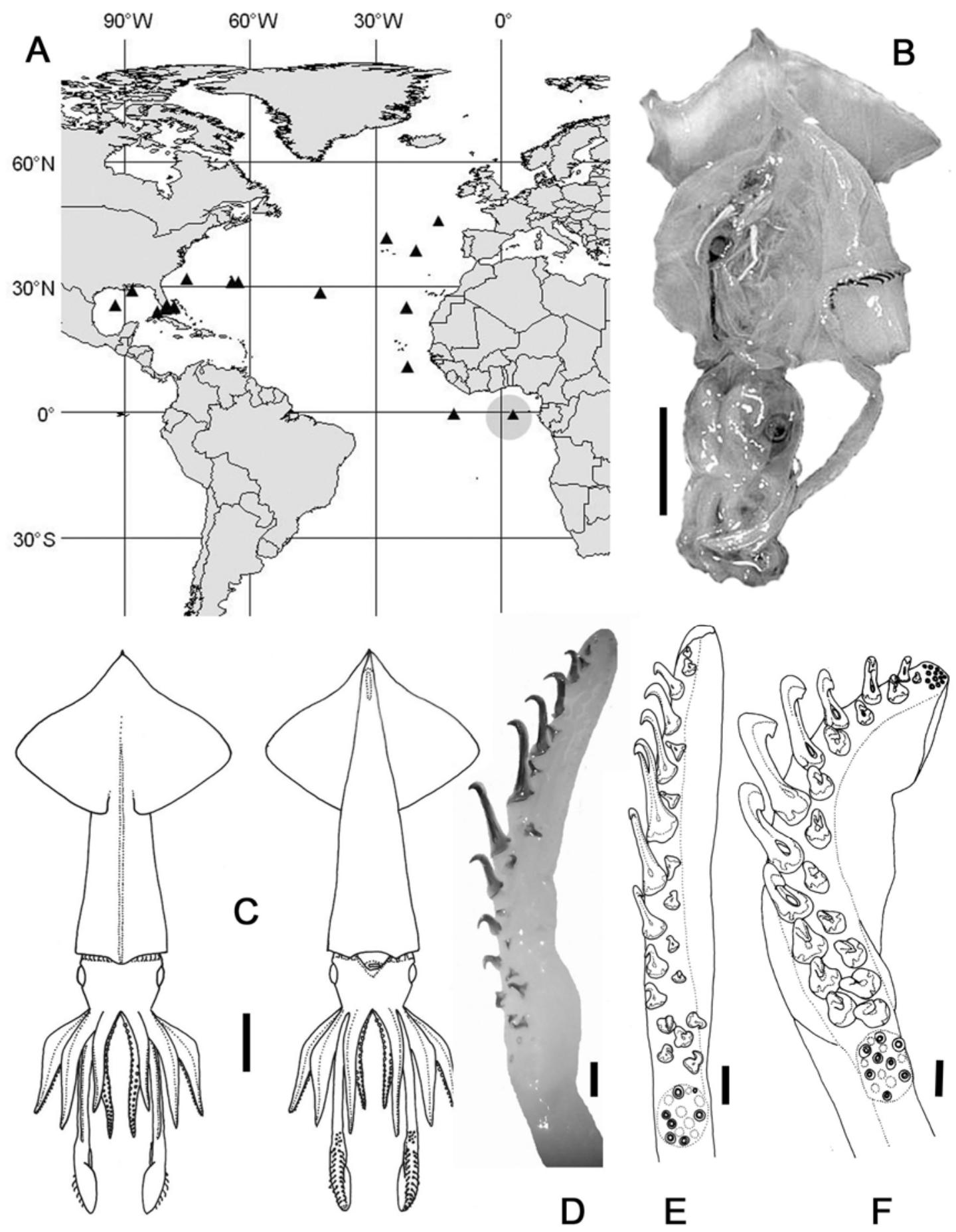

Fig. 6 - Onychoteuthis banksii. A) distribution (grey circle indicates type locality); B, D, E) BMNH 1986266, holotype, sex indet., ML 31 mm: (B) ventral view of whole animal, photo by M. Vecchione, (D, E) right tentacle club; C, F) USNM 727512, , ML 49mm: (C) whole animal, $(F)$ right tentacle club. Scale bars = B, C) $10 \mathrm{~mm}$; D-F) $1 \mathrm{~mm}$. 
Radula (Fig. 7G) with unicuspid rachidian, with broad triangular mesocone and low, broad, rounded base, its height $\sim 25 \%$ that of entire rachidian height. First lateral tooth unicuspid, slightly shorter and more narrow than rachidian, straight or slightly curved, slightly medially directed, with base shorter on inner edge of tooth than outer (sometimes indistinguishable on inner edge). Second lateral tooth simple, slender, slightly curved, $\sim 150 \%$ height of rachidian. Marginal tooth simple, slightly curved or straight proximally, curving only at tip, $\sim 200 \%$ height of rachidian. Palatine palp (Fig. $7 \mathrm{H}$ ) with $\sim 70$ long, robust teeth, each $70-230 \%$ rachidian height, densely distributed over palp.

Gladius (Fig. $7 \mathrm{I}$ ) with greatest width ( $\sim 7 \% \mathrm{GL}$ ) attained at $\sim 45 \% \mathrm{GL}$; free rachis $\sim 20 \%$ GL; vanes broaden rapidly between 20 and $50 \%$ GL, gradually narrowing thereafter; dorsal keel solid, deep, comprising $\sim 50 \%$ gladius depth at $\sim 80 \%$ GL. Rostrum $\sim 5 \%$ $\mathrm{GL}$, triangular in lateral profile, narrow in dorsal profile.

Remarks - The holotype of Os. banksii, from the Gulf of Guinea, is juvenile (ML $31 \mathrm{~mm}$ ), and its tissues appear somewhat stretched (Fig. 6B). The intestinal photophores on this specimen are intact, however, and the ventral hooks on the remaining (right) tentacle club (Figs 6D, 6E) are long and slender. These characters and their states are of considerable systematic value, and are shared with Atlantic specimens herein attributed to Os. banksii, one of which was collected near the type locality. This species is not currently known from the Pacific Ocean. It occurs commonly at the surface at night, with the majority of specimens quite remarkably having been found on ship decks. 

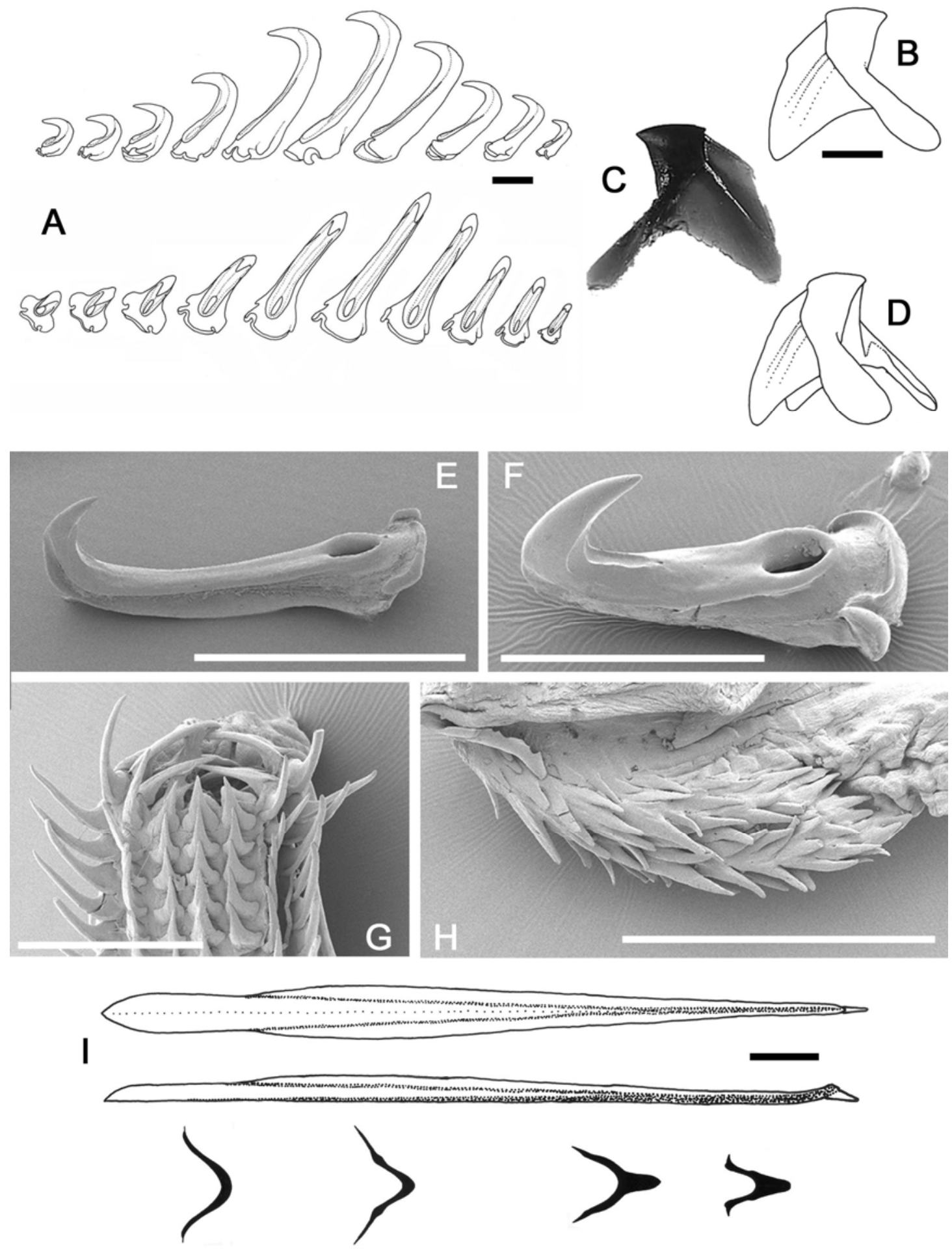

Fig. 7 - Onychoteuthis banksii. A, E-H) USNM 727512, q, ML 49mm; B-D, I) USNM 575113, o, $M L 88 \mathrm{~mm}$. A) right ventral tentacular hook series; B-D) lower beak: (B) left, (C) right, (D) left oblique profiles; E, F) right tentacular hooks: (E) V7, (F) V10; G) radula; H) palatine palp; I) gladius. Scale bars = A-C, H) $1 \mathrm{~mm}$; E) $2 \mathrm{~mm} ; \mathrm{F}, \mathrm{G}) 500 \mu \mathrm{m}$; l) $10 \mathrm{~mm}$. 
Onychoteuthis bergii Lichtenstein, 1818 (Tables 2, 4, Figs 8, 9)

Onychoteuthis bergii Lichtenstein, 1818: 1591, 1592; Férussac \& d'Orbigny (1835): Figs 1-3, (1848): 332, PI. 5; Köhler \& Glaubrecht 2004: 280.

Not Onychoteuthis bergii Lichtenstein, 1818 - Dall (1873): 484, 485 (= Ok. (Ok.) robusta).

Type material examined - MNB Moll. 121, lectotype (designated by Köhler \& Glaubrecht, 2004),, , ML 126mm, Cape of Good Hope [ $\left.33^{\circ} 24^{\prime} S, \sim 6^{\circ} 30^{\prime} E\right], 1817$, coll. Bergius, NFD; MNB Moll. 123, paralectotype, + , ML 128mm, Cape of Good Hope [ $\left.\sim 33^{\circ} 24^{\prime} \mathrm{S}, \sim 6^{\circ} 30^{\prime} \mathrm{E}\right]$, 1817, coll. Bergius, NFD.

Additional (unlocalised) material examined (6 specimens) - MNHN 3.2.639, $\uparrow$, ML 144mm, coll. Zelee, 1841, M. Leguillou, NFD; ZMBN 2400, 2ᄋ, ML 137mm, 81mm, India, NFD;

MNHN 630,, , ML 130mm, 'prix entre 'es Tropiques', 1834, coll. M. Dussumier, NFD; MNHN 636,,+ ML 105mm, Malabar [southwestern India], coll. Dussumier, NFD; SAM A30202, $q$, ML 92mm, 'S 10W, Vasco de Gama Pk,' 20.8km, 155m, PF2724.

Distribution — Southern Atlantic and Indian Oceans; one depth record from 155m.

Diagnosis - Intestinal photophores circular, well defined, with diameter of anterior 60$70 \%$ that of posterior; ocular photophore of uniform breadth, with rounded ends, spanning ventral $\sim 30 \%$ eye circumference; posterior portion of mantle remains broad beneath fins; tentacle club length $\sim 25 \% \mathrm{ML}$, with 21-23 short, robust, hooks; distalmost ventral hook bases with small spike by ML $92 \mathrm{~mm}$.

Description (Figs 8A-9E) - Mantle muscular, width 17-23-31\% ML, cylindrical anteriorly, widely conical posteriorly and remaining broad to tip; dorsal mantle surface with medial groove; gladius visible in groove through thin membrane but not raised above surface of mantle; ventral mantle surface with low, fleshy medial keel over posterior $\sim 15 \% \mathrm{ML}$; rostrum of gladius visible ventrally through posterior $3 \% \mathrm{ML}$; intestinal photophores circular, well defined; diameter of anterior $60-70 \%$ that of posterior. Fins rhombic, length $\sim 56 \% \mathrm{ML}$, width generally $64-70-86 \% \mathrm{ML}$; posterior fin margin slightly convex; anterior margin straight; anterior lobes small, rounded.

Head rounded, length 15-18-21\% ML, width 23\% ML, depth 15\% ML; not constricted anteriorly; occipital region with six secondary folds. Ocular photophore of uniform width, with rounded ends, spanning ventral $\sim 30 \%$ eye circumference. Funnel stout, muscular; funnel aperture opens level with posterior margin of eye aperture; 
Table 4. Measures $(\mathrm{mm})$ and counts of Onychoteuthis bergii Lichtenstein, 1818 (measurements taken from more complete side of specimen, indicated $\mathrm{R}$ or $\mathrm{L})$.

\begin{tabular}{|c|c|c|c|c|c|c|c|c|}
\hline Specimen ID & $\begin{array}{c}\text { MNB Moll. } \\
121\end{array}$ & $\begin{array}{c}\text { MNB Moll. } \\
123\end{array}$ & $\begin{array}{l}\text { MNHN } \\
3.2 .639\end{array}$ & ZMBN 2400 & MNHN 630 & ZMBN 2400 & \multicolumn{2}{|c|}{ Mean Indices } \\
\hline Type Status & Lectotype & Paralectotype & None & None & None & None & & \\
\hline ML & 126 & 128 & 144 & 137 & 130 & 81 & \multicolumn{2}{|c|}{ (ML 81-144) } \\
\hline Sex & $\mathrm{F}$ & $\mathrm{F}$ & $\mathrm{F}$ & $\mathrm{F}$ & $\mathrm{F}$ & $\mathrm{F}$ & & \\
\hline MW & 24 & 22 & 38 & 28 & 40 & 19 & MWI & 23 \\
\hline HL & 26 & $26^{*}$ & 32 & 30 & 30 & 21 & HLI & 23 \\
\hline HW & $23^{*}$ & 19 & * & 24 & 22 & 17 & HWI & 18 \\
\hline $\mathrm{FL}$ & 71 & 72 & 76 & 80 & 70 & 46 & FLI & 56 \\
\hline FW & 81 & 82 & 102 & 87 & 112 & 57 & FWI & 70 \\
\hline Arms I & $38 \mathrm{~L}$ & $41 \mathrm{~L}$ & $50 \mathrm{~L}$ & $45 \mathrm{~L}$ & $55 \mathrm{~L}$ & $22 \mathrm{~L}$ & A1I & 33 \\
\hline Arms II & $51 \mathrm{~L}$ & $53 \mathrm{~L}$ & $60 \mathrm{~L}$ & $56 \mathrm{~L}$ & $73 \mathrm{~L}$ & $40 \mathrm{~L}$ & A2I & 45 \\
\hline Arms III & $53 \mathrm{~L}$ & $59 \mathrm{~L}$ & $65 \mathrm{~L}$ & $56 \mathrm{~L}$ & $73 \mathrm{~L}$ & $41 \mathrm{~L}$ & A3I & 47 \\
\hline Arms IV & $54 \mathrm{~L}$ & $53 \mathrm{~L}$ & $70 \mathrm{~L}$ & $56 \mathrm{~L}$ & $66 \mathrm{~L}$ & $41 \mathrm{~L}$ & A4I & 46 \\
\hline TnL & 97L & $160 \mathrm{~L}$ & $143 \mathrm{~L}$ & $100 \mathrm{~L}$ & $165 \mathrm{~L}$ & $81 \mathrm{~L}$ & TnLI & 100 \\
\hline CL & $31 \mathrm{~L}$ & $33 \mathrm{~L}$ & $31 \mathrm{~L}$ & $30 \mathrm{~L}$ & $34 \mathrm{~L}$ & $23 \mathrm{~L}$ & CLI & 25 \\
\hline CS & $9 \mathrm{~L}$ & $8 \mathrm{~L}$ & 9 & 8 & 7 & 6 & & \\
\hline МH & $23 \mathrm{~L}$ & $21 \mathrm{~L}$ & 22 & 23 & 22 & 22 & & \\
\hline MS & 0 & 0 & 0 & 0 & 0 & 0 & & \\
\hline TPS & * & ${ }^{*} \mathrm{~L}, 14 \mathrm{R}$ & 14 & 14 & 14 & 14 & & \\
\hline
\end{tabular}

* indicates damaged features.

funnel groove appears broad, well defined (but distorted in most examined material); funnel component of locking apparatus 10-15\% ML; mantle component 25-30\% ML.

Arms $30-33,-46_{\| I I V}-56 \% \mathrm{ML}$, formula $\|I=\|=|\mathrm{V}>|$ or $\|||||=\mid \mathrm{V}>1$, robust at bases, narrowing rapidly to slender tips, each with $\sim 90$ suckers (ML $81-128 \mathrm{~mm}$ ); keel not apparent on Arms I; that on Arms II $\sim 40 \%$ arm depth over entire arm length; that on Arms III attaining $100 \%$ arm depth at $40 \%$ arm length, thereafter $30 \%$ arm depth; lateral membranes on Arms IV $100 \%$ arm width at base, thereafter $75 \%$ arm width.

Tentacles 73-100-127\% ML; stalk bases of comparable diameter to adjacent arm bases; aboral surface of stalk with low keel, $\sim 10 \%$ stalk thickness, terminating level with proximal manus hooks. Club (Figs 8C-F) somewhat expanded, 22-25-28\% ML; carpus circular to slightly ovoid in outline, with six to nine (usually eight or nine) suckers; manus with 21-23 hooks; terminal pad with $\sim 14$ suckers. Proximal-most hook (dorsal or ventral) smaller than paired hook; next two dorsal hooks variably slightly longer or shorter than paired ventral hooks; V3 and all subsequent ventral hooks consistently larger than D3 and subsequent dorsal hooks. Dorsal hooks decrease in size D3-D5 or D6, with D4 and D5 (or D5 and D6) markedly smaller than proximal dorsal hooks, and offset toward centre of club. Ventral hooks increase in size from V1 to V7 or V8 (Fig. 9D), with largest ventral hook four to five times larger than paired 


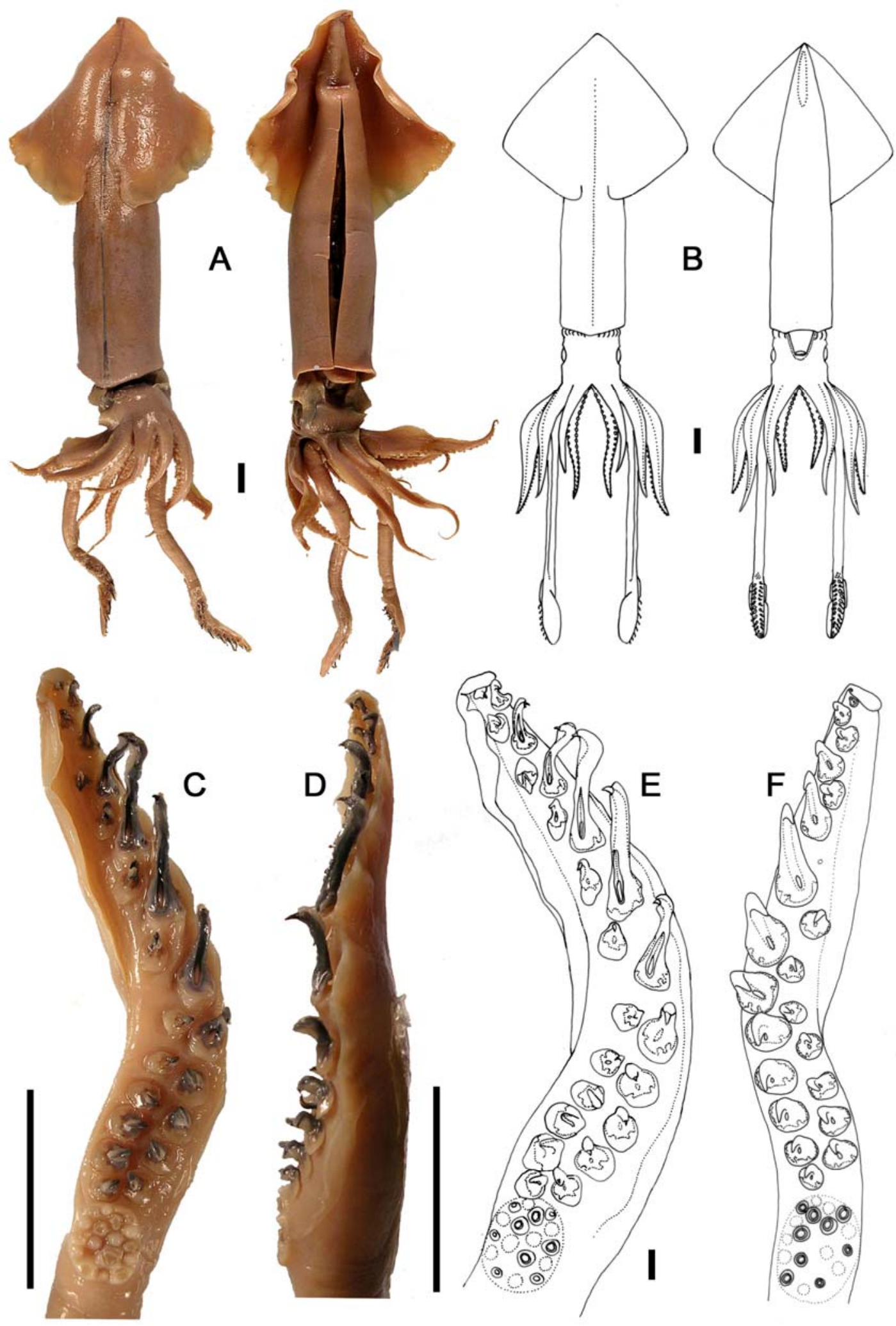

Fig. 8 - Onychoteuthis bergii. A-E) MNB Moll. 121, lectotype,, , ML 126mm; F) MNHN 3.2.639, o, ML 144mm. A, B) whole animal; C-E) left tentacle club; F) right tentacle club. Scale bars $=A-D) 10 \mathrm{~mm}$; E, F) $1 \mathrm{~mm}$. 
dorsal hook and $\sim 17 \% \mathrm{CL}$, thereafter decreasing in size distally. Hooks with long, slender apertures; bases asymmetrical, with large round basal notches and ventral cusp produced into low, rounded spike in distal-most hooks by ML $92 \mathrm{~mm}$. Ventral club membrane $\sim 20 \%$ club width, present along hooks V1-V5; dorsal membrane $\sim 10 \%$ club width, present along hooks D1-D5; dorsal keel $\sim 100 \%$ club width, present from opposite hook D4 to tip of club.

Lateral profile of lower beak (Figs 9A-C, ML 128mm) longer than deep, with hood length $\sim 50 \%$ crest length, and hood and crest together $\sim 80 \%$ baseline; jaw edge straight over proximal 50\% LRL, slightly curved over distal $50 \%$ LRL, produced distally into small pointed rostral tip. Crest broad; lateral walls not indented below crest; lateral wall with prominent, well-developed ridge anteriorly, reducing to broad indistinct fold posteriorly; wing length $\sim 200 \%$ LRL; wing width constant along wing length; angle ridge extends over $\sim 30 \%$ lateral wall; angle point indistinct.

Radula (Fig. 9E) with unicuspid rachidian; mesocone triangular, broad proximally, narrowing to slender, rounded tip; margins straight to slightly concave; base low, rounded, height $\sim 25 \%$ overall rachidian height; proximal margin of base slightly concave, distal margin slightly concave, with rounded corners. First lateral tooth unicuspid, $\sim 90 \%$ height of rachidian; overall form broadly triangular but varying greatly in breadth; base low, rounded. Second lateral tooth simple, $\sim 110 \%$ height of rachidian, triangular overall, medially directed, curved distally away from plane of radula. Marginal tooth simple, broad, dorso-ventrally compressed, rounded distally and (sometimes strongly) curved away from plane of radula; height $200-300 \%$ that of rachidian height.

Overall colouration (preserved) very pale pink, with fine, dark purple chromatophores on head, scattered densely over dorsal surface and occipital region, and sparsely over ventral surface.

Remarks - The type specimens of Os. bergii resemble specimens of similar sizes attributed to Os. banksii in many regards; the ocular and intestinal photophores are of similar shapes and relative sizes, and the club lengths and overall body proportions are similar. The chromatophores on the Os. bergii types are greatly faded, so their distribution is uncertain, and the tentacular hooks appear somewhat shrunken and deformed, prohibiting direct comparison of these characters. However, certain differences between the Os. bergii types (and other material attributed to Os. bergii) 
and Os. banksii specimens are consistent: where the posterior portion of the mantle in Os. banksii is consistently tapered (Fig. 6C), even in maturing specimens, in Os. bergii it remains widely conical even toward the posterior extremity (Figs 8A, 8B) in specimens of ML 92-144mm; the lower beak proportions are different, with the LRL longer relative to the wing length in Os. banksii (Figs 7B-D) than in Os. bergii (Figs 9A-C); the largest ventral tentacular hooks in Os. bergii appear more robust, shorter relative to club length and longer relative to paired dorsal hook length (Figs $8 \mathrm{C}-\mathrm{F}$ ) than those in Os. banksii (Figs 6D-F); and the ventral bases of the distal-most ventral hooks in Os. banksii are not produced into a spike, while those in Os. bergii do develop a small spike.

The type specimens of Os. bergii were collected at Cape Good Hope ( $34^{\circ} 25^{\prime} \mathrm{S}$, $\left.\sim 18^{\circ} 25^{\prime} \mathrm{E}\right)$; the type locality of Os. banksii is the Gulf of Guinea $\left(\sim 0^{\circ}, \sim 10^{\circ} \mathrm{E}\right)$; and limited material was available from any intermediate locality. Such material is required to more fully evaluate the ontogenetic and geographic variation in both Os. banksii and
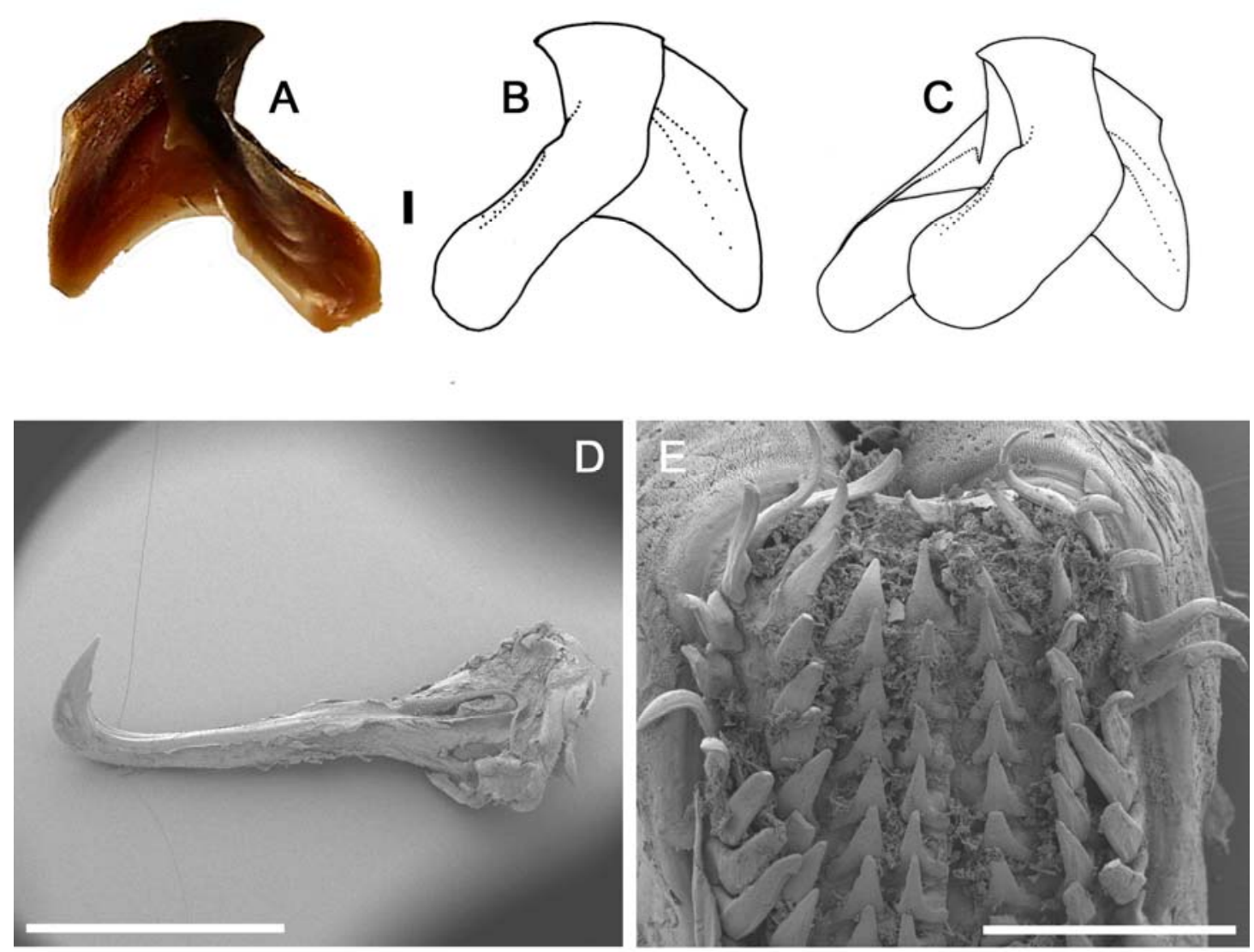

Fig. 9 - Onychoteuthis bergii. A-E) MNB Moll. 123, paralectotype, , ML 128mm. AC) lower beak: (A) left, (B) right, (C) right oblique profiles; $D$ ) right tentacular hook V7;

E) radula. Scale bars $=A$, B) $1 \mathrm{~mm}$; D) $2 \mathrm{~mm}$; E) $500 \mu \mathrm{m}$. 
Os. bergii, and accordingly the taxonomic validity of the latter name. It is currently considered a separate species whose distribution may be influenced by either of the two currents (the north-flowing Antarctic Benguela current and the warm, southwestflowing Agulhas current) that converge at Cape Good Hope, especially given that some specimens attributed to Os. bergii were taken in the Indian Ocean.

Lichtenstein's original (1818) description of Os. bergii is translated in Appendix A. Köhler \& Glaubrecht (2004) designated the lectotype to assist in stabilisation of this taxon.

Given the rarity of this species (no collection contained more than three specimens) dissection of the gladius and palatine palp was not justified. 
Onychoteuthis aequimanus Gabb, 1868 (Tables 2, 5, Figs 10-13)

Onychoteuthis aequimanus Gabb, 1868: 23, 24, PI. 2; Spamer \& Bogan (1992): 1; Bolstad (in press).

Onychoteuthis banksi [sic] (not Leach, 1817) — Dell (1951): 99; Imber \& Russ (1975): 30; Imber (1976): 124.

Onychoteuthis banksii (not Leach, 1817) — Dell (1951): 102, Fig. 6, (1952): 100, 101, Tables 27, 28, PI. 20.

? Onychoteuthis sp. C Young \& Harman, 1987: 313-321, Figs 1-4, 6, 8; Sweeney et al. (1992): 129, Fig. 181; Seki (2001): 44-50.

Onychoteuthis 'banksii' (not Leach, 1817) — Bolstad (2007): 308-314, Tables 1, 6, Figs 2-13.

Type material examined — ANSP $6444(2 \hat{\jmath})$, syntypes, ML 93mm, 80mm, Society Islands, NFD.

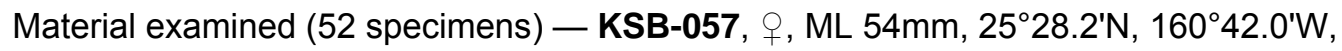
28/03/1992, 2113-2213h, CMT, TC 92-02 Stn 40, NMFS S-0408; KSB-026 [SBMNH], $\mathrm{O}^{\AA}$, ML 44, sex indet., ML 35mm, $22^{\circ} 50.4^{\prime} \mathrm{N}, 159^{\circ} 35.1^{\prime} \mathrm{W}, 26 / 03 / 1992,1955-2055 \mathrm{~h}$, TC 92-02, CMT, NMFS S-0309; KSB-005 [SBMNH], $q, \mathrm{ML} 70 \mathrm{~mm}, 21^{\circ} 43^{\prime} \mathrm{N}, 157^{\circ} 51^{\prime} \mathrm{W}$, off northeast O'ahu, 1-0m over 472m, 22/09/1992, 2247h, ST 26.5 $\mathrm{C}$, RV Wecoma, Tsuji

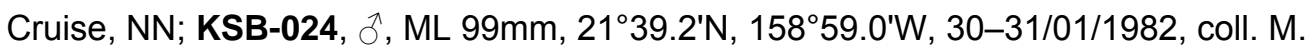
Seki, FTS Hokusei Maru 82-01 Stn 23 (HOG 8203), surface gill net, 30mm mesh, NMFS Ceph-0008; USNM 813414, $2{ }^{\lambda}$, ML 100, 96mm, O'ahu, Hawai'i [ $21^{\circ} 30^{\prime} \mathrm{N}$, $158^{\circ} \mathrm{W}$ ], surface, 29/04/1957, RV Hugh M. Smith Stn 39-[x], NL\&D; KSB-022, 今̃, ML $97 \mathrm{~mm}, 21^{\circ} 00.5^{\prime} \mathrm{N}, 158^{\circ} 51^{\prime} \mathrm{W}, 31 / 01 / 1982-01 / 02 / 1982$, surface gillnet, FTS Hokusei Maru 82-01 Stn 29 (HOG 8204), coll. M. Seki, NMFS Ref Ceph-0011; KSB-023, 2 q, ML 108, 106mm, 2059.4'N, 159 42.6'W, 28-29/01/1982, FTS Hokusei Maru 82-02 Stn 12 (HOG 8201), surface gillnet, coll. M. Seki, NMFS Ref Ceph-0006; USNM 813433, $q$, ML 65mm, Kaoko, Hawai'ian Islands, $19^{\circ} 21^{\prime} \mathrm{N}, 162^{\circ} 05^{\prime} \mathrm{W}$, surface, 23/07/1951, RV J.R. Manning 7-B-1, NL\&D; KSB-025, sex indet., ML 43mm, north coast of Palmyra Island [ $\left.5^{\circ} 53^{\prime} \mathrm{N}, 162^{\circ} 05^{\prime} \mathrm{W}\right], 1-0 \mathrm{~m}$ over $\sim 500 \mathrm{~m}, 16 / 12 / 1989,2156-2258 \mathrm{~h}$, NN, L/A-N9; NMNZ M.74553, sex indet., $\mathrm{ML} 43 \mathrm{~mm}, 2^{\circ} 08^{\prime} \mathrm{S}, 175^{\circ} 30^{\prime} \mathrm{W}, 348 \mathrm{~m}$ over $1000+\mathrm{m}, 08 / 12 / 1976$, 1833-1933h, FRV James Cook Stn J017/047/76; NMNZ M.91739, đ̂, ML 110mm, S. of Kermadec Islands [ $\left.29^{\circ} 17^{\prime} \mathrm{S}, 177^{\circ} 55^{\prime} \mathrm{W}\right]$, coll. R. Grace, NFD; NMNZ M.74494, 2 $94,81 \mathrm{~mm}, 31^{\circ} 04^{\prime} \mathrm{S}, 175^{\circ} 04^{\prime} \mathrm{W}, 70 \mathrm{~m}$ over 5000+m, 04/12/1976, FRV James Cook; NMNZ M.74145, 2ᄋ, ML 138, 137mm, 240km SW of Esperance Rock, Kermadec Islands [ $33^{\circ} \mathrm{S}, 179^{\circ} \mathrm{E}$ ], 01/04/1973, RV Acheron, flew/washed aboard; NMNZ M.74491, sex indet., ML $66 \mathrm{~mm}, 33^{\circ} 14^{\prime} \mathrm{S}, 179^{\circ} 14^{\prime} \mathrm{W}, 695 \mathrm{~m}$ over $3000+\mathrm{m}, 04 / 12 / 1976$, FRV James Cook; NMNZ M.91480 (25 sex indet.), ML 37-13mm, 34²0.6-19.5'S, 173 29.7-27.7'E, 150m over 1558-1453m, 25-26/10/1985, FRV James Cook Stn J16/32/85, FMMWT; NMNZ M.74537, 2 sex indet., ML 62, 46mm, 35어'S, $179^{\circ} 20^{\prime} \mathrm{W}$, 774m over 3000+m, 03/12/1976, FRV James Cook; NMNZ M.160477, §, ML 106mm, +, ML $90 \mathrm{~mm}$, sex indet., ML $79 \mathrm{~mm}, 37^{\circ} \mathrm{S}, 167^{\circ} \mathrm{E}$, winter 1960 , coll. N.W. Thomas, 
'flew' on board; NMNZ M.91594, + , ML 84mm, 3858.8-57.6'S, $178^{\circ} 38.7-40^{\prime} \mathrm{E}, 35 \mathrm{~m}$, 01/06/1987, FRV James Cook Stn J7/10/87, FMMWT; NMNZ M.91593, §, ML 100mm, 3909.7'S, 179³8.8'E, 35m, 31/05/1987, FRV James Cook; NIWA 32735, §ొ, ML $92 \mathrm{~mm}, 40^{\circ} 51^{\prime} \mathrm{S}, 178^{\circ} 20^{\prime} \mathrm{E}, 100-20 \mathrm{~m}$ over $3064 \mathrm{~m},-/ 02 / 2002$, RV Tangaroa Stn TAN9802/172, FMMWT; NMNZ M.160464, + , ML 79mm, 41ํ13.8'S, 17651.6'E, 800m over 1378-996m, 31/07/1985, FRV James Cook Stn J10/01/85; NMNZ M.160454, sex indet., ML 76mm, $41^{\circ} 41.2^{\prime} \mathrm{S}, 168^{\circ} 44.2^{\prime} \mathrm{E}, 1056-1043 \mathrm{~m}, 16 / 10 / 1983$, FV Arrow Stn A02/132/83.

Unlocalised material examined (5 specimens) - KSB-031, ठ̊, ML 50mm, off O'ahu, 800-0m, 1200-2000h, FTS Hokusei Maru Trawl \#1, Stn 1, NFD; KSB-028 2ᄋ, ML 108, 58mm, 1-Om over 500m, 31/10/1989, 0410-0500h, Tsuji IV, Tow 48B, NN, NFD; KSB-004, ㅇ, ML 62mm, 1-0m over 500m, 07/01/1992, 2213h, NN, MBC Cruise 3, Tow 3018, NFD; KSB-027, sex indet., ML 52mm, 1-0m over 800m, 26/10/1989, 2003h, Tsuji IV, Tow 19B, NN, NFD; NMNZ M.74508, + , ML 122mm, 3 sex indet., ML 110-61mm, FRV James Cook Stn J01/[x]/76, NFD.

Distribution (Fig. 10A) - Pacific Ocean, south of $30^{\circ} \mathrm{N}$; known from Hawai'ian and New Zealand waters, Palmyra Island, and the Society Islands.

Diagnosis - Intestinal photophores circular, well defined, with diameter of anterior $\sim 40-60 \%$ that of posterior; ocular photophores uniformly broad, spanning ventral $\sim 30 \%$ eye circumference; chromatophores forming reduced band across dorsal half to one-third tentacle stalk width orally, proximal to carpus; chromatophores absent from oro-ventral tentacle stalk ridge, and from small patch on ventral surface of mantle (posterior $\sim 15 \% \mathrm{ML}$ ); tentacle club length 28\% ML, with 19-23 long, slender hooks, shallowly recurved $\left(90-100^{\circ}\right)$ in lateral profile; distal-most ventral hook bases may develop into low spike above ML $90 \mathrm{~mm}$.

Description (Figs 10B-13C) - Mantle muscular, conical, width 17-24-31\% ML; gladius visible along anterior $\sim 85 \%$ of dorsal midline as dark, raised ridge; rostrum of gladius visible ventrally through posterior $\sim 5 \% \mathrm{ML}$; rostrum may pierce mantle posteriorly in specimens to $\mathrm{ML} \sim 70 \mathrm{~mm}$; low, narrow medial keel present along posterior $20 \%$ of ventral mantle surface. Fins rhombic in outline, length $\sim 55 \% \mathrm{ML}$, width $64-72-$ $86 \% \mathrm{ML}$ at $\mathrm{ML}>70 \mathrm{~mm}, \sim 80 \% \mathrm{ML}$ at $\mathrm{ML}<70 \mathrm{~mm}$, slightly narrowed and extended posteriorly; posterior margin concave over posterior third, thereafter straight to slightly convex; anterior margin straight to slightly convex; anterior lobes broad, shallow. Intestinal photophores well defined, circular in outline; diameter of anterior 
Table 5. Measures ( $\mathrm{mm}$ ) and counts of Onychoteuthis aequimanus Gabb, 1868 (arm and tentacle measures recorded from more complete side of specimen, indicated $\mathrm{R}$ or $\mathrm{L}$ ).

\begin{tabular}{|c|c|c|c|c|c|c|c|c|c|c|c|c|}
\hline Specimen ID & $\begin{array}{c}\text { ANSP } \\
6444\end{array}$ & $\begin{array}{c}\text { ANSP } \\
6444\end{array}$ & $\begin{array}{c}\text { NMNZ } \\
\text { M.74145 }\end{array}$ & KSB-028 & $\begin{array}{l}\text { USNM } \\
813414\end{array}$ & KSB-024 & KSB-005 & $\begin{array}{l}\text { USNM } \\
813433\end{array}$ & $\begin{array}{c}\text { KSB- } \\
026\end{array}$ & $\begin{array}{c}\text { NMNZ } \\
\text { M.91480 }\end{array}$ & \multicolumn{2}{|c|}{ Mean Indices } \\
\hline Type Status & Syntype & Syntype & None & None & None & None & None & None & None & None & \multirow{2}{*}{\multicolumn{2}{|c|}{ (ML 65-137) }} \\
\hline ML & 93 & 80 & 137 & 108 & 100 & 99 & 70 & 65 & 44 & 25 & & \\
\hline Sex & $M$ & M & $\mathrm{F}$ & $\mathrm{F}$ & $\mathrm{M}$ & $M$ & $\mathrm{~F}$ & $\mathrm{~F}$ & $M$ & Indet. & & \\
\hline MW & 19 & 17 & $35^{*}$ & 25 & 28 & 26 & 18 & 12 & 12 & 7 & MWI & 24 \\
\hline HL & 18 & 19 & $33^{*}$ & 22 & 24 & 16 & 22 & 17 & 10 & 12 & $\mathrm{HLI}$ & 24 \\
\hline HW & 18 & 15 & $37^{*}$ & 15 & 15 & $11^{*}$ & 17 & 15 & 9 & 16 & HWI & 18 \\
\hline $\mathrm{FL}$ & 49 & 48 & 75 & 60 & 53 & 54 & 38 & 38 & 24 & 5 & FLI & 55 \\
\hline FW & $56^{*}$ & 50 & 105 & 74 & 78 & 72 & 51 & 52 & 32 & 6 & FWI & 72 \\
\hline Arms I & $37 R$ & $27 R$ & $57 \mathrm{~L}$ & $32 \mathrm{~L}$ & $36 \mathrm{~L}$ & $33 \mathrm{~L}$ & $22 \mathrm{~L}$ & $26 \mathrm{R}$ & $12 R^{*}$ & $6 \mathrm{~L}$ & A1I & 35 \\
\hline Arms II & $49 \mathrm{R}$ & $32 R$ & $77 \mathrm{~L}$ & $46 \mathrm{~L}$ & $48 \mathrm{~L}$ & $46 \mathrm{~L}$ & $20 \mathrm{~L}$ & $33 R$ & $17 R$ & $8 \mathrm{~L}$ & A2I & 44 \\
\hline Arms III & $50 \mathrm{R}$ & $32 R$ & $81 \mathrm{~L}$ & $47 \mathrm{~L}$ & $46 \mathrm{~L}$ & $45 \mathrm{~L}$ & $31 \mathrm{~L}$ & $32 \mathrm{R}$ & $17 R$ & $8 \mathrm{~L}$ & A3I & 46 \\
\hline Arms IV & $39 \mathrm{R}$ & $31 R$ & $74 \mathrm{~L}$ & $54 \mathrm{~L}$ & $56 \mathrm{~L}$ & $51 \mathrm{~L}$ & $34 \mathrm{~L}$ & $34 \mathrm{R}$ & $18 \mathrm{R}$ & $8 \mathrm{~L}$ & A4I & 47 \\
\hline $\mathrm{TnL}$ & $95 R$ & $60 R$ & $160 \mathrm{~L}$ & $118 \mathrm{~L}$ & $94 \mathrm{~L}$ & $126 \mathrm{~L}$ & $73 \mathrm{~L}$ & $51 R$ & $41 R$ & $13 \mathrm{~L}$ & TnLI & 104 \\
\hline $\mathrm{CL}$ & $24 \mathrm{R}$ & $17 R$ & $41 \mathrm{~L}$ & $34 \mathrm{~L}$ & $30 \mathrm{~L}$ & $30 \mathrm{~L}$ & $21 \mathrm{~L}$ & $21 R$ & $14 \mathrm{R}$ & $6 \mathrm{~L}$ & CLI & 28 \\
\hline $\mathrm{CS}$ & 6 & 8 & $7 \mathrm{~L}$ & 7 & 7 & 8 & 7 & 9 & 7 & 6 & & \\
\hline MH & 23 & 23 & 22 & 21 & 19 & 20 & 21 & 21 & 21 & $\begin{array}{l}\text { V3-11, D } \\
\text { just forming }\end{array}$ & & \\
\hline MS & 0 & 0 & 0 & 0 & 0 & 0 & 0 & 0 & 0 & all & & \\
\hline TPS & * & * & 14 & 13 & 12 & 14 & 15 & 13 & 12 & 11 & & \\
\hline
\end{tabular}

* indicates damaged features.

$\sim 40-60 \%$ that of posterior; ink sac often swollen beneath posterior photophore, creating distinctive 'bulging' appearance (Fig. 12A).

Head boxy, length 16-24-31\% ML; width 14-18-24\% ML; occipital region with seven to ten secondary folds. Ocular photophore broad, rounded at both ends, of uniform breadth along its length, spanning ventral $\sim 30 \%$ eye circumference. Funnel groove deep, broadly V-shaped posteriorly, with acute anterior angle, bordered by low, fleshy ridge; funnel component of locking apparatus $\sim 15 \% \mathrm{ML}$; mantle component $\sim 26 \% \mathrm{ML}$.

Arms $30-35,-47_{I I-I V}-59 \% \mathrm{ML}$, formula $|I=| I|=| V>\mid$ (or IV $>||=|||>|$, mostly in specimens of ML $70-110 \mathrm{~mm}$ ); each arm with $90-110$ suckers by ML $70 \mathrm{~mm}$. Keels present on Arms I-III: that on Arms I 25\% arm width over distal 50\% arm length; that on Arms II $\sim 50 \%$ arm depth over proximal $25 \%$ arm length, thereafter $\sim 75 \%$ arm depth to tip of arm; that on Arms III attaining $~ 150 \%$ arm depth in medial 30\% arm length, thereafter narrowing to arm tip; lateral membrane on Arms IV 100\% arm width over proximal $25 \%$ arm length, narrowing thereafter to $\sim 75 \%$ arm width along remainder of arm length.

Tentacles $75-104-127 \%$ ML, with low aboral keel ( 10\% stalk thickness) along stalk. Clubs $21-28-31 \% \mathrm{ML}(25-40 \% \mathrm{TnL})$ (Figs 10C, 12E), with six to nine carpal suckers, 19-23 manus hooks (Figs 12B, 12C, 12I) and 11-15 terminal pad suckers. Proximal ventral hooks variably sized, subequal to, larger or smaller than paired dorsal hooks; largest ventral hook (V6 or V7, Fig. 12B) five to seven times larger than paired dorsal hook and $\sim 20 \% \mathrm{CL}$; longest ventral hooks slender, slightly curved in lateral profile; claw weakly recurved at $\sim 90-100^{\circ}$. Bases of distal-most ventral hooks asymmetrical, but not produced ventrally into spike in individuals of $\mathrm{ML}<90 \mathrm{~mm}$ (Fig. 12C); small 
spike may develop at larger MLs. Hooks D4 and D5 smaller than D1-D3 and offset toward centre of club. Ventral membrane $\sim 75 \%$ club width along entire club; dorsal membrane $\sim 5 \%$ club width, flanking hooks D1-D4; dorsal keel originates near hook D3 and attains $\sim 125 \%$ club width near hook D8.

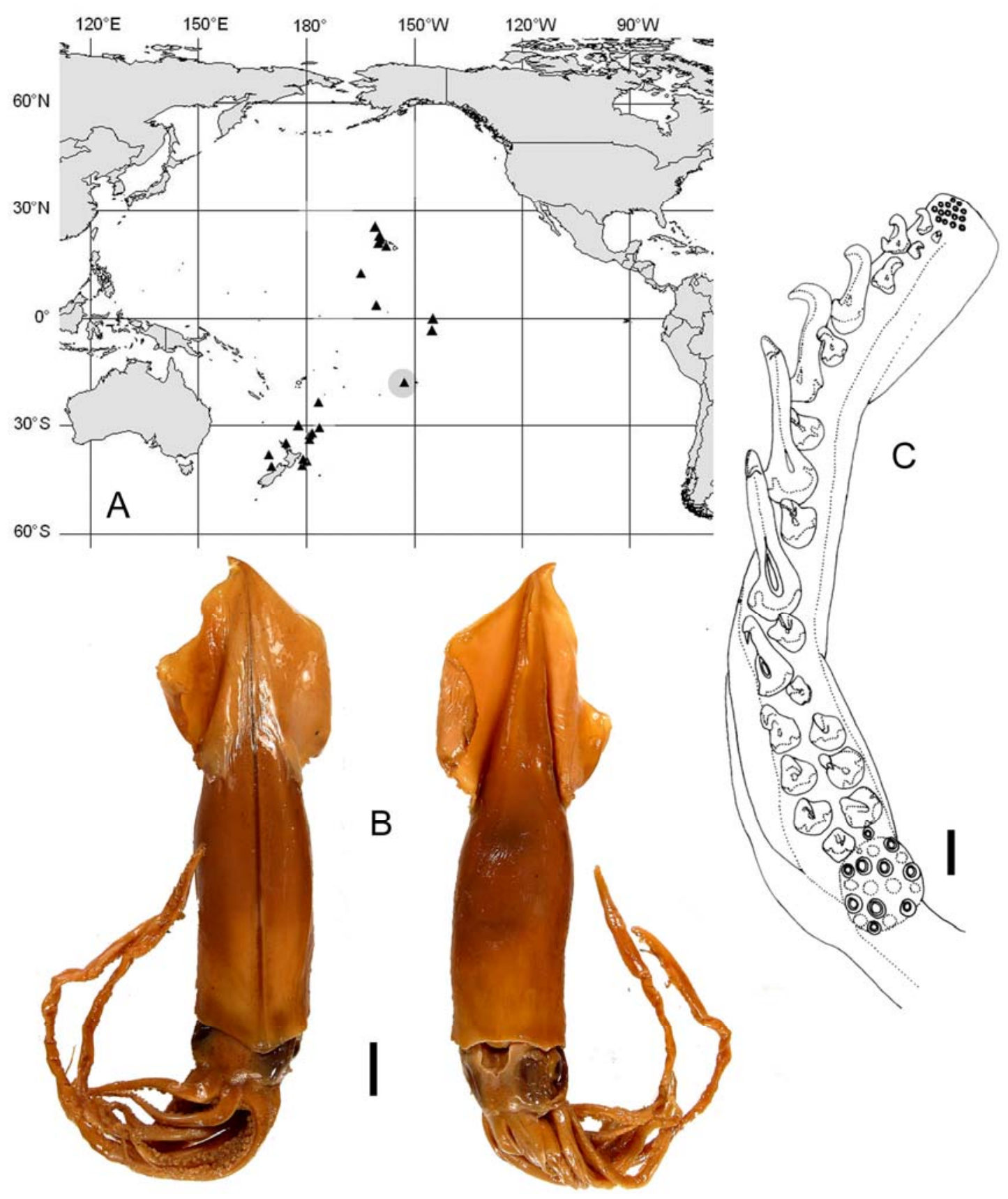

Fig. 10 - Onychoteuthis aequimanus. A) distribution (grey circle indicates type locality); B, C) ANSP 6444, syntype, $\hat{\sigma}$, ML 93mm: (B) whole animal, (C) right tentacle club. Scale bars = B) $10 \mathrm{~mm}$; C) $1 \mathrm{~mm}$. 
Lateral profile of lower beak (Figs 12F-H, ML 97mm) longer than deep, with crest sloping steeply downward in nearly straight line, posterior edge of hood close to crest, hood length $\sim 40 \%$ total crest length, and hood and crest together $\sim 80 \%$ baseline; jaw edge slightly concave; jaw angle obtuse, slightly obscured laterally by low, rounded, indistinct wing fold. Shoulder ridge distinct, sharp; angle ridge spans $40-60 \%$ lateral wall depth; angle point darkly pigmented, not raised; distal wing width $\sim 140 \%$ that at jaw angle, wing length $170-200 \%$ LRL, with small semicircular insert of cartilage below
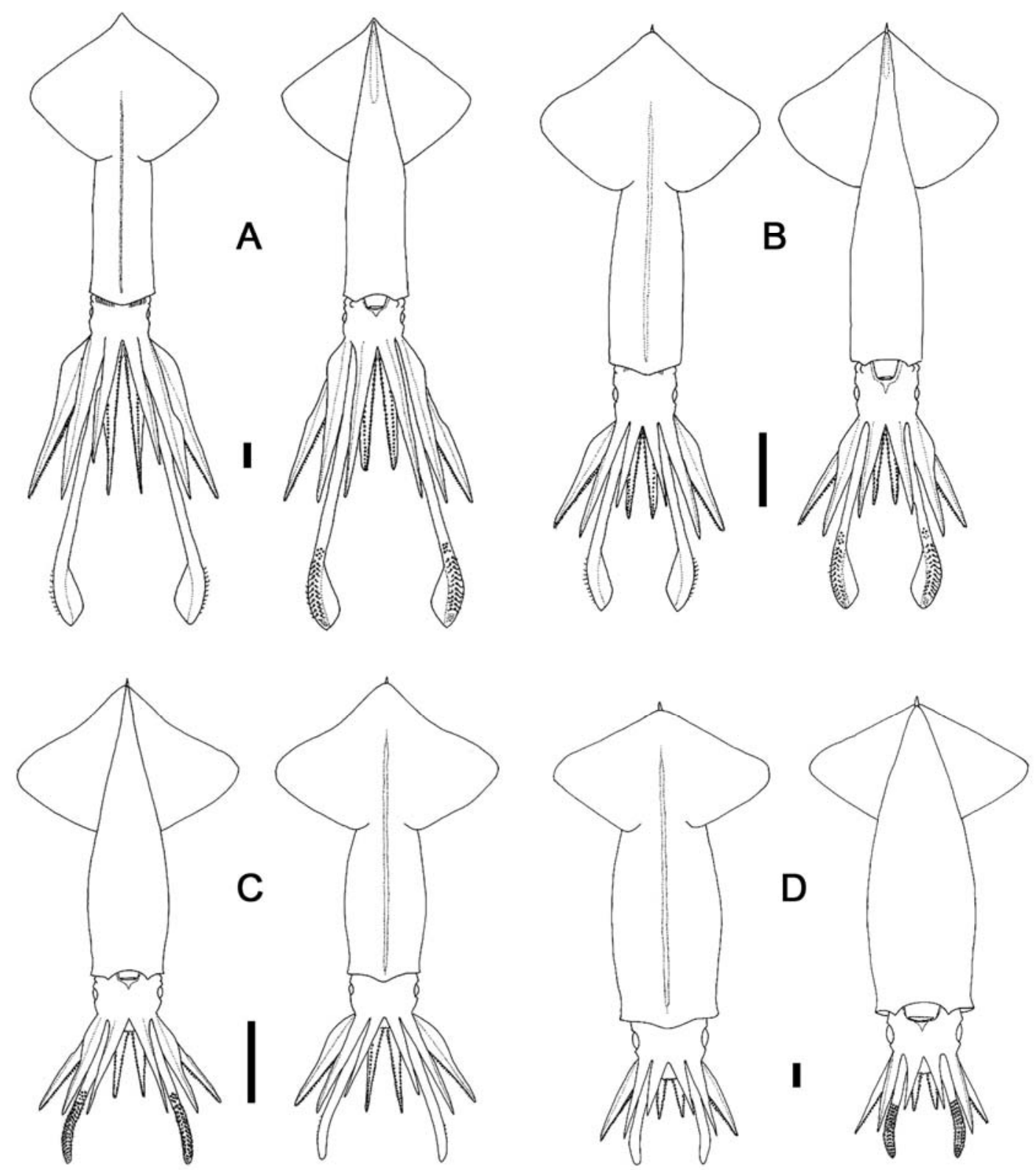

Fig. 11 - Onychoteuthis aequimanus. A) NMNZ M.74145, ․, ML 138mm; B) NMNZ M.74491, sex indet., ML 66mm; C, D) NMNZ M.91480, sex indet.: (C) ML 37mm, (D) ML $15 \mathrm{~mm}$. Scale bars = A) $10 \mathrm{~mm}$; B, C) $5 \mathrm{~mm}$; D) $1 \mathrm{~mm}$. 
jaw angle; crest broad, not thickened or only slightly thickened; lateral wall not indented below crest, diagonally bisected by dark, prominent ridge, remaining distinct to lateral wall corner.

Radula (Fig. 12D) with tricuspid rachidian, with narrow triangular mesocone and narrow, rounded lateral cusps, their height $\sim 25 \%$ that of mesocone height. First lateral tooth unicuspid to slightly bicuspid; inner cusp straight, slightly shorter than rachidian and of similar breadth, slightly medially directed; outer cusp (where present) low ( $25 \%$ height of inner cusp), pointed, slightly laterally directed. Second lateral tooth simple, robust, straight, short, $\sim 120 \%$ height of rachidian. Marginal tooth simple, straight proximally, curving distally to slightly laterally directed tip, $\sim 200 \%$ height of rachidian. Palatine palp (Fig. 13A) with $\sim 35$ robust, flat teeth, each $130-200 \%$ rachidian height, sparsely set in approximately transverse rows.

Gladius (Fig. 13C) with greatest width ( $8 \% \mathrm{GL})$ attained at $\sim 45 \% \mathrm{GL}$; free rachis $\sim 20 \% \mathrm{GL}$; vanes broaden and taper rapidly between 20 and $60 \% \mathrm{GL}$; dorsal keel solid, narrow. Rostrum $\sim 5 \% \mathrm{GL}$, triangular in lateral profile, narrow in dorsal profile.

Colour (preserved) deep purple-red, darkest dorsally on mantle and fins, surrounding very dark red-brown keel of gladius; small posterior portion of ventral mantle surface ( 15\% ML) unpigmented; head dark dorsally, paling laterally and ventrally apart from pronounced darkening on dorsal lobe of anterior eye sinus; chromatophores present over all external funnel surfaces and within groove; arms darkest on aboral surfaces, with few oral chromatophores; tentacle stalks dark aborally, pale orally; chromatophores absent from ventral stalk ridge and present only in small dorsal cluster proximal to carpus.

Paralarvae to small juveniles (ML 14-40mm, Figs 11C, 11D, 13B) differ from the above description as follows. Mantle spindle-shaped, widest ( $30 \% \mathrm{ML})$ at $\sim 40 \% \mathrm{ML}$; ventral medial mantle keel present by $\mathrm{ML} \sim 20 \mathrm{~mm} \mathrm{ML}$; intestinal photophores present by $\mathrm{ML} 15 \mathrm{~mm}$. Fin length $35-45 \% \mathrm{ML}$, width $\sim 65 \% \mathrm{ML}$, with straight anterior and posterior margins. Funnel component of locking apparatus $12-13 \% \mathrm{ML}$; mantle component $\sim 40 \% \mathrm{ML}$; secondary occipital folds not yet apparent at ML $37 \mathrm{~mm}$. Arms 20-30\% ML, each with 40-50 suckers by ML $37 \mathrm{~mm}$; keels on Arms I develop at ML $>40 \mathrm{~mm}$, those on Arms II at ML $>31 \mathrm{~mm}$, those on Arms III already apparent at ML $13 \mathrm{~mm}$. Tentacles $35-50 \% \mathrm{ML}$; carpus poorly defined; ventral hooks begin to develop at ML 12-13mm; first dorsal hooks develop at ML $\sim 27 \mathrm{~mm}$; hooks V3-11 fully formed 


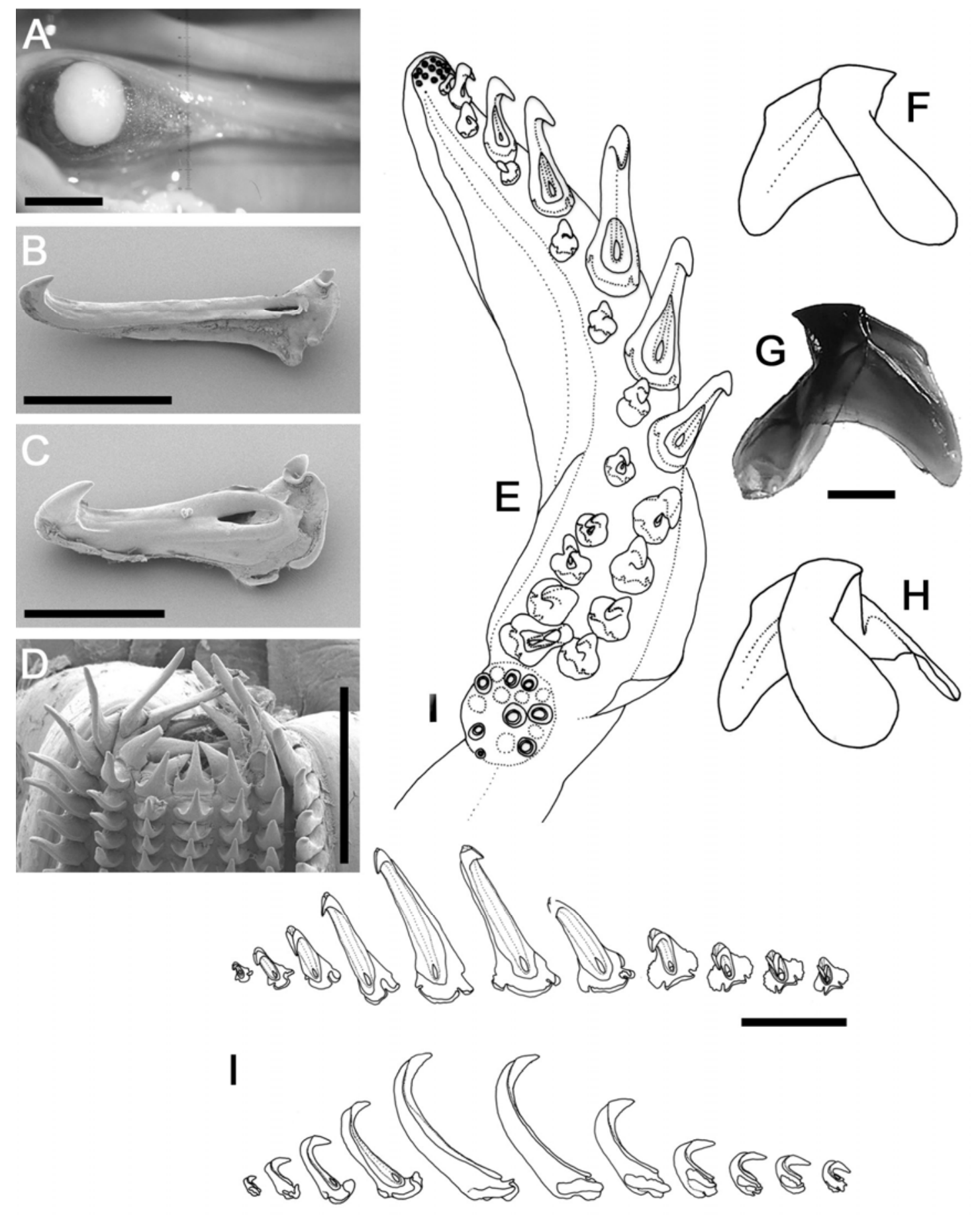

Fig. 12 - Onychoteuthis aequimanus. A) KSB-057, ㅇ, ML 54mm; B, C) KSB-031, „ิ, ML 50mm; D, F-H) KSB-022, ô, ML 97mm; E) KSB-005, ㅇ, ML 70mm; I) NMNZ M.74145,, , ML 138mm. A) posterior intestinal photophore; $B, C)$ left tentacular hooks: (B) V7, (C) V10; D) radula; E) left tentacle club; F-H) lower beak: (F) left, $(G)$ right, $(H)$ left oblique profiles; I) left ventral tentacular hook series. Scale bars = A, B) $2 \mathrm{~mm}$; C, D) $500 \mu \mathrm{m} ; \mathrm{E}-\mathrm{G}) 1 \mathrm{~mm}$; I) $5 \mathrm{~mm}$. 
by $\mathrm{ML} 37 \mathrm{~mm}$, with all others still developing; all marginal suckers lost by $\mathrm{ML} 40 \mathrm{~mm}$, with those flanking hooks V4, V5 and V11 retained latest. Tentacular suckers ovoid (Fig. 13B), broadest proximally, with small, adentate circular aperture ( $25 \%$ sucker diameter) offset toward proximal margin; proximal pegs largest, in two or three concentric rows, with faces of innermost pegs laterally compressed into oblong polygons, particularly in marginal suckers; distal pegs set in eight or nine dense, concentric rows, with faces of inner pegs circular, outer pegs polygonal. Colour (preserved) pale, with scattered pale to dark red-brown chromatophores; ventral mantle surface with row of small dark chromatophores along anterior margin; single pair of large, dark brown-red 'tail-spots' present beneath fins; dorsal surface of head with several large, pale chromatophores overlapped by several small, dark ones.

Remarks - Os. aequimanus was described from two specimens taken near the Society Islands (Gabb 1868), but was subsequently synonymised with Os. banksii by Adam (1952) and Voss (1962). However, Young \& Harman (1987) recognised the presence of at least three Onychoteuthis species in Hawai'ian waters, and Seki (2001) also concluded that each was distinct, in addition to Os. borealijaponica and a fourth species, Os. lacrima (his 'sp. D').
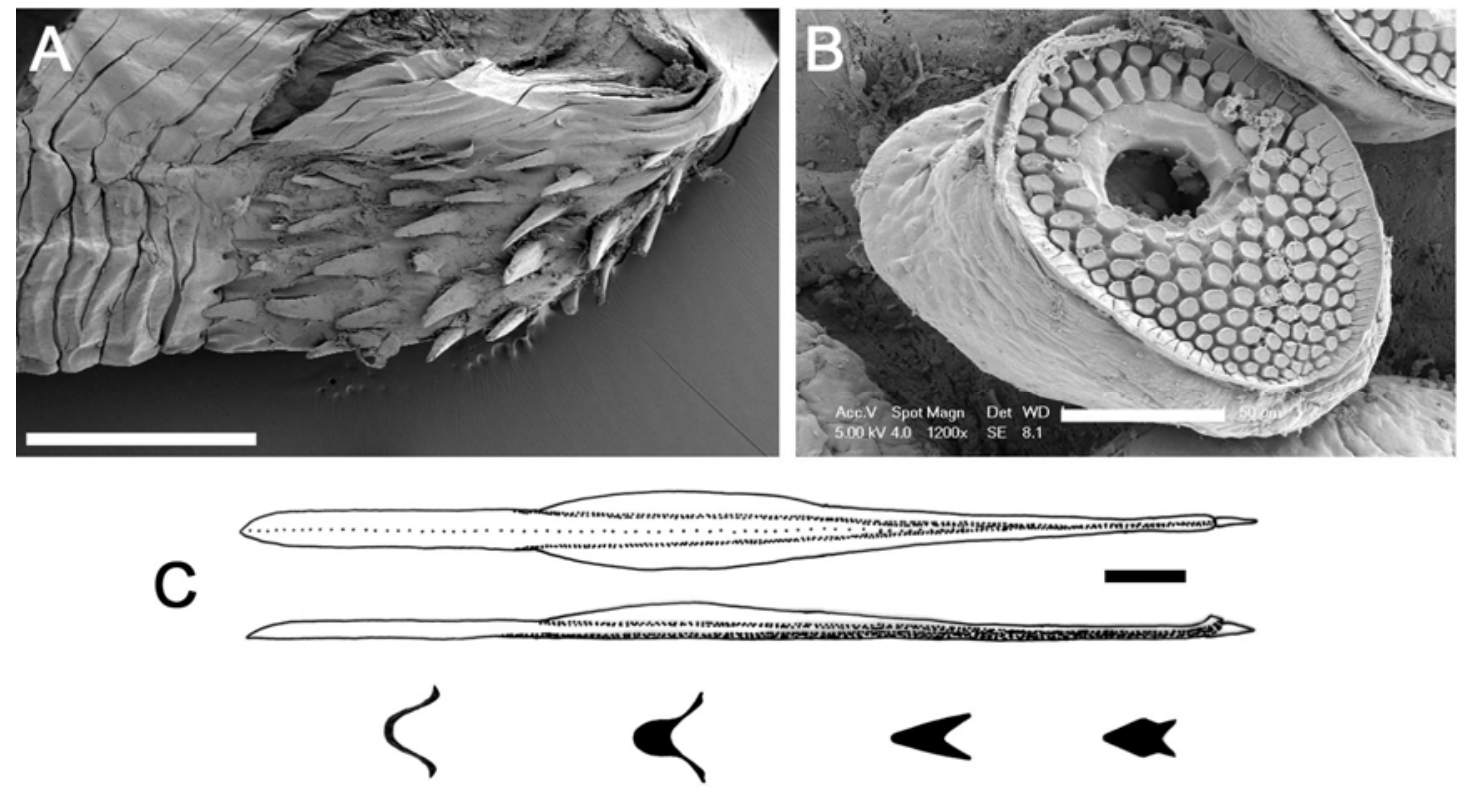

Fig. 13 - Onychoteuthis aequimanus. A, C) NMNZ M.74508, , ML 122mm; B) NMNZ M.91480, sex indet., ML 20mm. A) palatine palp; B) juvenile tentacular sucker; C) gladius. Scale bars = A, B) $50 \mu \mathrm{m}$; C) $10 \mathrm{~mm}$. 
Gabb (1868) noted that the Os. aequimanus syntypes had been preserved in alcohol for a significant amount of time before he received them, and consequently had lost much pigmentation. The soft tissues of the syntypes have also hardened, the tentacles appear stretched, and the hooks somewhat desiccated. Nevertheless, apart from chromatophore patterns, the important characters (photophores, club morphology (Fig. $10 \mathrm{C}$ ), overall body proportions, beak and radula) are in sufficient condition to permit comparison with material reported herein. Strong similarities in morphology are observed between the Os. aequimanus syntypes, similar-sized specimens from Hawai'i provisionally attributed to Young \& Harman's (1987) 'species C,' and Os. 'banksii' (sensu Bolstad 2007) specimens from New Zealand; the differences between these specimens and other Pacific species are also consistent. Therefore, Os. 'banksii' (sensu Bolstad 2007) from New Zealand, and provisionally 'species C' (Young \& Harman 1987), are attributed to the existing name Os. aequimanus Gabb, 1868.

The ink sac 'bulge' (see Fig. 12A) observed in the Os. aequimanus syntypes and in other material attributed to this species may be a preservation artefact, although it has not been reported or observed in any other taxon. 
Onychoteuthis mollis (Appellöf, 1891) (new comb.) (Table 2, Fig. 14)

? Onychoteuthis bartlingii (not Lesueur, 1821) — Férussac \& d'Orbigny (1835): PI. 3, (1848): 332.

Chaunoteuthis mollis Appellöf, 1891: 1-29, Pls 1-4 - Clarke (1986): 74, Fig. 32C.

Not Chaunoteuthis mollis Appellöf, 1891 - Toll (1982): PI. 5 Fig. C (= ? Onychoteuthis sp. indet.).

Type material examined — ZMBN Holotype 2428, spent 9 , ML 153mm (preserved; GL $181 \mathrm{~mm})$, Mediterranean, coll. Capt. Lampe, NFD.

Distribution - Mediterranean (based on sole known specimen).

Diagnosis - Posterior intestinal photophore slightly longer than broad, its diameter twice that of anterior photophore; ocular photophore elongate, bulbous posteriorly, narrow anteriorly, rounded at both ends; about eight secondary occipital folds present; arms $30-40 \% \mathrm{ML}$; free rachis of gladius $\sim 40 \% \mathrm{GL}$.

Description (ML 153mm, spent female, Fig. 14) - Mantle broadly conical, greatest width $\sim 40 \% \mathrm{ML}$ (at anterior margin), musculature flaccid; ventral surface with two longitudinal cuts near anterior margin, from which bulbs of embedded spermatangia protrude (Figs 14B, C); left-hand incision Y-shaped, with $\sim 60$ spermatangia in main cut and $\sim 20$ in side branch; right-hand incision simple, straight, with $\sim 50$ spermatangia. Intestinal photophores circular to slightly ovate, with diameter of anterior $\sim 55 \%$ that of posterior. Fins approximately rhombic, length $\sim 55 \% \mathrm{ML}$, width $\sim 100 \% \mathrm{ML}$, more muscular than mantle.

Head damaged, length $\sim 15 \% \mathrm{ML}$, with about eight secondary occipital folds. Ocular photophore rounded at both ends, bulbous posteriorly and more narrow anteriorly, spanning ventral $\sim 25 \%$ eye circumference. Funnel broad, stretched, groove damaged; funnel component of locking apparatus $\sim 12 \% \mathrm{ML}$, mantle component $\sim 30 \% \mathrm{ML}$.

Arms stubby, length $30-41 \% \mathrm{ML}$, formula IV=III>II>I, each with $\sim 110$ suckers. Aboral surfaces of Arms I-III with fleshy keels and Arms IV with lateral membrane; more detailed description prevented by specimen damage. Tentacles reduced to short, flabby stumps, 7\% ML; clubs absent. 
Beak not available for examination; radula (not extant) with unicuspid or slightly tricuspid rachidian (fide Appellöf 1891).

Gladius (Fig. 14D) narrow, its greatest width $\sim 4 \% \mathrm{GL}$, attained at $\sim 50 \% \mathrm{GL}$; free rachis extremely long, $\sim 40 \% \mathrm{GL}$; rostrum $\sim 4 \% \mathrm{GL}$, narrow in dorsal profile, triangular in lateral profile.

Remarks - Appellöf (1891), whose original description of Os. mollis is partially translated in Appendix B, considered this species allied to Onychoteuthis, specifically citing its nuchal folds and the form of the gladius as being similar to those of Os. bergii. (Although the occipital region of the holotype is damaged, Appellöf's original description states that Os. mollis possesses 11 occipital folds in total; these likely comprise eight secondary and three primary folds.) Appellöf excluded this species
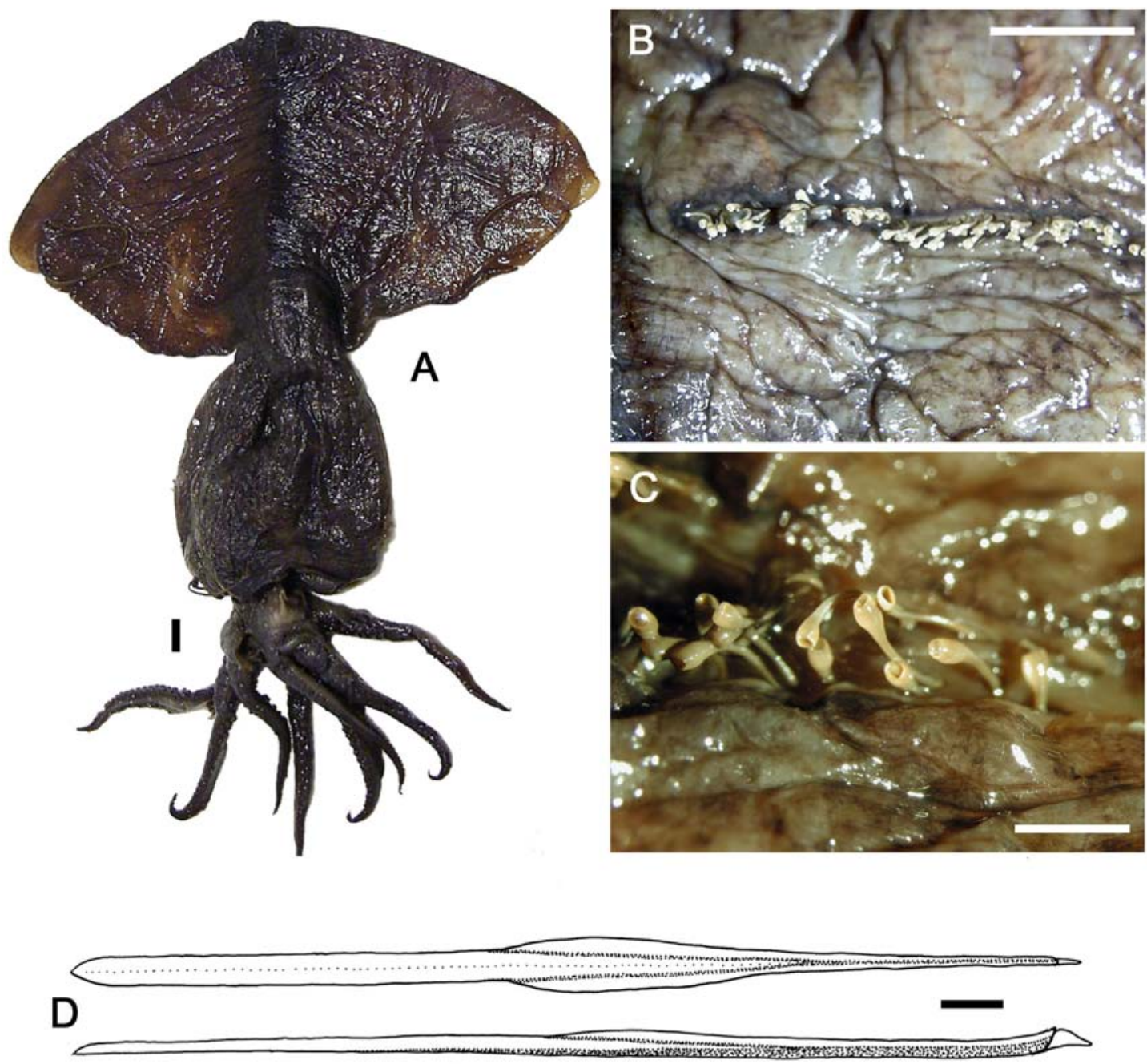

Fig. 14 - Onychoteuthis mollis, ZMBN 2428, holotype, spent $\odot$, ML 153mm. A) dorsal view, whole animal; $B$ ) ventral cut with implanted spermatangia; C) implanted spermatangia; D) gladius. Scale bars = A, B, D) $10 \mathrm{~mm}$; C) $5 \mathrm{~mm}$. 
from Onychoteuthis based on the lost tentacles and slack consistency of the mantle tissue (since recognised as degenerate conditions common to many spent onychoteuthids), and on the lack of photophores (which are, in fact, present, on both the intestine and eyes).

Other isolated specimens of 'Chaunoteuthis mollis' have been described from the Mediterranean (e.g. Grimpe 1921, Torchio 1967, Adam 1972, Bello 1998), and several of these authors have remarked that since the photophores and body proportions in their specimens were similar or identical to those in Os. 'banksii', specimens historically attributed to 'C. mollis' probably represent spent individuals of Onychoteuthis. One or more of these specimens may in fact have been attributable to Os. mollis (sensu stricto); however, in no instance is the gladius (the most distinctive feature in the holotype) described, so positive identification is not possible. ' $C$. mollis' has also been reported from isolated or mass-stranded specimens in other oceans (e.g. Nesis 1970, Okutani \& Ida 1986), but again in too little detail to retrospectively attribute this material to species.

Arkhipkin \& Nigmatullin (1997) used morphometrics, morphology, and life history patterns to analyse the relationship between two ' $C$. mollis' specimens from the southeastern Pacific and Os. 'banksii' (218 tropical specimens, representing most tropical water masses; these appear to have included a number of recently disambiguated taxa). They also concluded that ' $C$. mollis' represented mature to spent stages of Onychoteuthis and considered the former a junior synonym of Os. 'banksii.'

Each species of Onychoteuthis likely degenerates into a gelatinous state near the end of its lifespan, and Os. mollis may ultimately prove synonymous with an older species. However, the peculiar gladius of the Os. mollis holotype prevents its synonymy with any other known species of Onychoteuthis at present, including Os. banksii (sensu stricto). Toll (1982) noted that the relative length of the free rachis (the most peculiar feature of the Os. mollis holotype) changes ontogenetically in some species, so the unique gladius proportions of this holotype may appear less significant in the context of additional material. It is perhaps worth noting, however, that Férussac \& d'Orbigny (1835) illustrated a subadult or adult specimen of 'Os. bartlingii Lesueur' (PI. 3bis) which does not appear referable to Lesueur's species (Lesueur 1821, PI. 8, and Férussac \& d'Orbigny 1835 , PI. 3) - in which the free rachis comprises $33 \%$ GL and appears very similar to that of the Os. mollis holotype. The fins of the 'Os. bartlingii' specimen in PI. 3bis also bear more resemblance to those of the Os. mollis holotype 
(bearing in mind that the proportions of spent onychoteuthids become distorted) than those of other known central- to north-Atlantic Onychoteuthis species (Os. banksii and Os. compacta). Examination of an ontogenetic series of Onychoteuthis specimens from the Mediterranean, which was not possible in this study, is necessary to confirm the status of Os. mollis.

The beak of the holotype was not with the specimen at the time of examination in 2007. It was photographed by Clarke (1986 Fig. 32C), allowing the following description for the purposes of comparison. Lower beak, lateral profile: deeper than long; hood $\sim 50 \%$ crest length; hood and crest together $\sim 70 \%$ baseline; posterior edge of hood well above crest. In general: angle ridge extends over $\sim 100 \%$ depth of lateral wall (fide Clarke 1986). 
Onychoteuthis compacta (Berry, 1913) (Tables 2, 6, Figs 15, 16)

Teleoteuthis compacta Berry, 1913: 565 - Berry (1914): 324, Fig. 32; Roper \& Sweeney (1978): 5.

Onychoteuthis compacta (Berry, 1913) - Young \& Harman (1987): 313-321, Figs 1-6, Tables 1-3; Sweeney et al. (1992): 129, Fig. 179; Bizikov (1996): Figs 3, 4; Kubodera et al. (1998): 279, Fig. 5; Sweeney \& Roper (1998): 571; Seki (2001): 44-50; Okutani (2005): 154; Wakabayashi et al. (2007) 959-965, Table 2, Fig. 2; Bolstad (in press).

Onychoteuthis banksi [sic] (not Leach, 1817) — Hess (1987): 288, 289, 300, 301, Fig. 54a-c.

Type material examined - USNM 214318, holotype, sex indet., ML $21 \mathrm{~mm}, 22^{\circ} 00^{\prime} \mathrm{N}, 159^{\circ} 30^{\prime} \mathrm{W}$, off Kauai Island, Hawai'i, 915-704m, 11/06/1902, Albatross 3989.

Additional material examined (111 specimens) - UMML 31.1304, 2 sex indet., ML 68, 56mm, $37^{\circ} 24^{\prime} \mathrm{N}, 66^{\circ} 42^{\prime} \mathrm{W}, 12 / 07 / 1958$, MV Delaware, dipnet; USNM 813413, ㅇ, ML 88mm, $36^{\circ} 11^{\prime} \mathrm{N}, 149^{\circ} 00^{\prime} \mathrm{W}, 22 / 07 / 1955$, RV J.R. Manning Stn 26-7, NL\&D; USNM 813441, , ML $65 \mathrm{~mm}, 34^{\circ} 45^{\prime} \mathrm{N}, 71^{\circ} 49^{\prime} \mathrm{W}$, surface, 10/10/1957, RV Delaware, NL\&D; USNM 813395,, , ML $75 \mathrm{~mm}$, sex indet., $\mathrm{ML} 61 \mathrm{~mm}, 34^{\circ} 23^{\prime} \mathrm{N}, 150^{\circ} 07^{\prime} \mathrm{W}, 21 / 07 / 1955$, RV J.R. Manning Stn 26-5, CMT; NSMT Mo66881, + , ML $97 \mathrm{~mm}, 34^{\circ} 04^{\prime} \mathrm{N}, 140^{\circ} 06^{\prime} \mathrm{E}$, 24/07/1971, surface, 0900h, RV Soyo-Maru Stn B2, coll. N. Sato, HN; USNM Acc\#277764, 2 \%, ML 94, 67mm, 31 $24^{\circ} \mathrm{N}, 77^{\circ} 14^{\prime} \mathrm{W}$, western North Atlantic Ocean, surface, 10/11/1967, MV Search Tide, coll. B.J. Zahuranec, dipnetted at night; BMNH 20070479, ㅇ, ML $71 \mathrm{~mm}, 31^{\circ} \mathrm{N}, 125-129^{\circ} \mathrm{W}, 30 / 01 / 1981$, pres. P. Horsman; UMML 31.2345, 2 ㅇ, ML 62, $46 \mathrm{~mm}, 30^{\circ} 13.2^{\prime} \mathrm{N}, 53^{\circ} 21^{\prime} \mathrm{W}, 22-23 / 05 / 1963$, MV Delaware Cruise 63-4 Stn 19, coll. Collette \& de Sylva, NL\&D; KSB-021, 万ิ, ML 125mm, $\sim 30^{\circ} \mathrm{N}, 178^{\circ} \mathrm{W}$, on deck, 3-4/08/1991, 1900-0600h, TC 91-06, NMFS S-0272; USNM 813394, , ML 93mm, $29^{\circ} 59^{\prime} \mathrm{N}, 164^{\circ} 49^{\prime} \mathrm{W}$, Hawai'i, 25/08/1955, RV H.M. Smith, 3m IKMWT; NSMT Mo66888, 2 q, ML 97, 84mm, $29^{\circ} 00^{\prime} \mathrm{N}, 135^{\circ} 00^{\prime} \mathrm{E},-/ 06 / 1969$, NFD; USNM 813422,, , ML $112 \mathrm{~mm}$, ô, ML $108 \mathrm{~mm}, 3$ sex indet., ML $85-63 \mathrm{~mm}, 28^{\circ} 13^{\prime} \mathrm{N}, 177^{\circ} 22^{\prime} \mathrm{W}$, Fishing dock, Midway Island, 50-0m, 29/05/1956, RV J.R. Manning Stn 31-2, NL\&D; KSB-055, ․, ML $130 \mathrm{~mm}$, Holoikuaua, Pearl \& Hermes Atoll [ $\left.27^{\circ} 50^{\prime} \mathrm{N}, 175^{\circ} 50^{\prime} \mathrm{W}\right]$, on deck, 08/04/1980, TC 80-02; KSB-060, ㅇ, ML 125mm, Pearl \& Hermes Atoll [ $27^{\circ} 50^{\prime} \mathrm{N}$, $\left.175^{\circ} 50^{\prime} \mathrm{W}\right]$, on deck, 08/04/1980, TC 80-02; KSB-059, ${ }^{\wedge}, \mathrm{ML} 119 \mathrm{~mm}$, Pearl \& Hermes Atoll [ $\left.27^{\circ} 50^{\prime} \mathrm{N}, 175^{\circ} 50^{\prime} \mathrm{W}\right]$, on deck, 09/04/1980, TC 80-02; KSB-014, 2 \%, ML 88, $88 \mathrm{~mm}, 27^{\circ} 45.04^{\prime} \mathrm{N}, 167^{\circ} 30.02^{\prime} \mathrm{W}$, on deck, 13/05/1997, 0607-0637h, TC 97-04 Stn 138 , CTD cast 079 , coll. BCM, JFP; KSB-015, sex indet., ML $61 \mathrm{~mm}, 27^{\circ} 15^{\prime} \mathrm{N}, 172^{\circ} \mathrm{W}$, on deck, 01/05/1997, 1000h, coll. J. Palmer, TC 97-04 Stn 8; KSB-017, đ̂, ML 106mm, $27^{\circ} 15^{\prime} \mathrm{N}, 158^{\circ} \mathrm{W}$, on deck, 27/04/1998, TC 98-05 Stn 26; KSB-016, ๆ, ML 87mm, $27^{\circ} 15^{\prime} \mathrm{N}, 158^{\circ} \mathrm{W}, 27 / 04 / 1998$, TC 98-05 Stn 26, flew on deck; UMML 31.1042, 4 sex indet., ML 66-55mm, $25^{\circ} 41^{\prime} \mathrm{N}, 79^{\circ} 50^{\prime} \mathrm{W}, 26 / 05 / 1962$, RV Gerda Stn 11, dipnet; UMML 31.419, 7 sex indet., ML 80-63mm, 2521-52.5'N, 7941-31'W, 27/09/1962, RV Gerda Stn 75 ; KSB-012, $+, \mathrm{ML} 98 \mathrm{~mm}, 6 \mathrm{~km}$ north of Laysan Island [ $\left.25^{\circ} 46^{\prime} \mathrm{N}, 171^{\circ} 44^{\prime} \mathrm{W}\right]$, on deck, 06/06/1980, TC 80-03; UMML 31.987,, , ML $79 \mathrm{~mm}, 25^{\circ} 23^{\prime} \mathrm{N}, 79^{\circ} 41^{\prime} \mathrm{W}$, surface, 
27/09/1962, RV Gerda Stn 70; UMML 31.986, 17 sex indet., ML 74-65mm, 2520'N, 7942'W, surface, 22/06/1962, RV Gerda Stn 40; UMML 31.2347, 3ㅇ, ML 82, 77, 76mm, $25^{\circ} 20-24^{\circ} 17.6^{\prime} \mathrm{N}, 78^{\circ} 02-01.5^{\prime} \mathrm{W}, 19 / 04 / 1969,1 \mathrm{~m}$ over $1071 \mathrm{~m}, \mathrm{RV}$ Gerda Stn 1077, plankton net; UMML 31.2058, 16 sex indet., ML 118-66mm, $25^{\circ} 17^{\prime} \mathrm{N}, 77^{\circ} 55^{\prime} \mathrm{W}$, 17/08/1966, RV Tursiops Stn 324; UMML 31.881, ㅇ, ML 68mm, $25^{\circ} 00^{\prime} \mathrm{N}, 86^{\circ} 09^{\prime} \mathrm{W}$, surface, 10/05/1962, MV Oregon Stn 3531; UMML 31.1540, ㅇ, ML 73mm, 2458.558.0'N, 77³5.5-47.6'W, 25-26/09/1973, RV Columbus Iselin Stn 128, NL; UMML 31.2501,, , ML $90 \mathrm{~mm}, 24^{\circ} 48^{\prime} \mathrm{N}, 79^{\circ} 58^{\prime} \mathrm{W}, 14 / 06 / 1967$, RV Undaunted $6704 \mathrm{Stn} 3$, NL\&D; KSB-013, + , ML 118mm, $24^{\circ} 36^{\prime} \mathrm{N}, 170^{\circ} 53.6^{\prime} \mathrm{W}, 93 \mathrm{~km}$ NW of Maro, 03/07/1990,

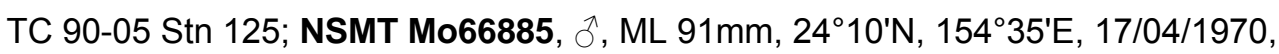
NFD; UMML 31.334, + , ML 88mm, $23^{\circ} 28.2^{\prime} \mathrm{N}, 76^{\circ} 40.7^{\prime} \mathrm{W}$, off Shark Spit, 25/07/1958, 2100-0000h, RV Gerda, coll. T. McKenney \& J. Alexander, NL\&D; USNM 813416, ð, ML 112mm, $21^{\circ} 24^{\prime} \mathrm{N}, 157^{\circ} 44^{\prime} \mathrm{W}$, off Kailua, 07/08/1970, RV H.M. Smith Stn 3-[x],

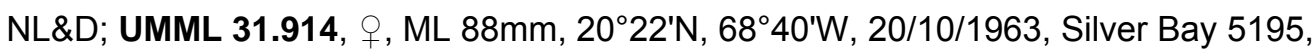
NFD; USNM 813428, + , ML 89mm, ${ }^{\circ}, \mathrm{ML} 63 \mathrm{~mm}, 20^{\circ} 09.5^{\prime} \mathrm{N}, 171^{\circ} 30^{\prime} \mathrm{W}$, Hawai'ian Islands [label says $71^{\circ}$ ], surface, NL\&D, 13/10/1963, RV Silver Bay; UMML 31.2346, , ML 86mm, ô, ML 79mm, 19 $42^{\prime} \mathrm{N}, 74^{\circ} 15^{\prime} \mathrm{W}, 23 / 05 / 1965$, RV Oregon Stn 5412, NL\&D; KSB-006, $\sigma^{\jmath}, \mathrm{ML} 123 \mathrm{~mm}, 18^{\circ} 54^{\prime} \mathrm{N}, 157^{\circ} 50^{\prime} \mathrm{W}$, on deck, 02/05/2001, 0700h, TC 01-04; USNM 813424, ㅇ, ML 75mm, $18^{\circ} 35^{\prime} \mathrm{N}, 159^{\circ} 18^{\prime} \mathrm{W}$, Hawai'i, 30/06/1951, coll. NMFS-HI, NL\&D; USNM 813365, + , ML 54mm, $18^{\circ} 22^{\prime} \mathrm{N}, 147^{\circ} 09^{\prime} \mathrm{W}, 20-0 \mathrm{~m},-/ 03 / 1959, \mathrm{CMT}$; UMML 31.663, ㅇ, ML $77 \mathrm{~mm}, 17^{\circ} 35^{\prime} \mathrm{N}, 8^{\circ} 22^{\prime} \mathrm{W}, 23 / 05 / 1965$, RV Geronimo Cruise 6 Stn 218, NL; KSB-020 [SBMNH], + , ML 59mm, $15^{\circ} \mathrm{N}, 159^{\circ} 16.8^{\prime} \mathrm{W}$, surface, 24/04/2001, TC 01-04 Stn 15 Sample 1; UMML 31.2343, §ิ, ML 90mm, 10²9'N, 11358.6'W, 03/02/1964, RV Shoyo Maru Cruise 13 Stn 247, coll. W. Klawe, NL; USNM 813385,, , ML $73 \mathrm{~mm}, 10^{\circ} \mathrm{N}, 158^{\circ} \mathrm{W}, 1006-0 \mathrm{~m},-/ 02 / 1950$, RV H.M. Smith Stn 2-36, CMT; USNM 813400, ㅇ, ML $96 \mathrm{~mm}, 05^{\circ} \mathrm{N}, 130^{\circ} \mathrm{W}$, Line Islands, $12 / 11 / 1952, \mathrm{RV}$ H.M. Smith Stn 18-33, CMT; USNM 813434, 2 sex indet., ML 61, 49mm, $03^{\circ} 11^{\prime} \mathrm{N}$, $168^{\circ} 07^{\prime} \mathrm{W}, 25 / 06 / 1951, \mathrm{NL}$ and dipnet, NFD; USNM 813415, ㅇ, $114 \mathrm{~mm}, 01^{\circ} 52^{\prime} \mathrm{N}$, $157^{\circ} 20^{\prime} \mathrm{W}$, off Christmas Island, Line Islands, 20/02/1967, RV C.H. Gilbert 98-[x], NL\&D; UMML 31.1300, sex indet., ML 48mm, 0007'S, 8655'W, 02/11/1961, coll. Joseph \& Forsbergh, NL; USNM 813423, 2 \%, ML 85, 47mm, $00^{\circ} 28^{\prime} \mathrm{N}, 169^{\circ} 54^{\prime} \mathrm{W}$, Line Islands, surface, 24/06/1951, coll. NMFS, NL\&D; USNM 812435, ㅇ, ML 47mm,

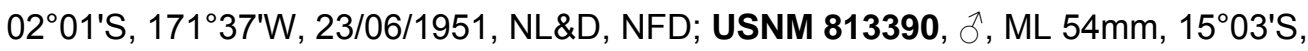
$170^{\circ} 47$ 'W, 13/03/1962, RV C.H. Gilbert Stn 55-103, CMT; USNM 813411,, , ML $76 \mathrm{~mm}, 17^{\circ} 46^{\prime} \mathrm{S}, 176^{\circ} 12^{\prime} \mathrm{E}, 21 / 02 / 1962$, CMT, RV C.H. Gilbert Stn 55-58; USNM 813410, $\widehat{\partial}$, ML 92mm, 3 sex indet., ML 59-52mm, 2052'S, 173³1'W, 10/03/1962, CMT, RV C.H. Gilbert Stn 55-93.

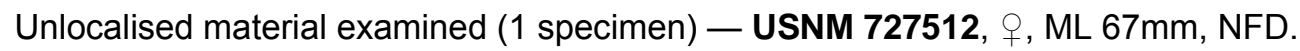


Distribution (Fig. 15A) - Central Pacific, primarily $30^{\circ} \mathrm{N}-30^{\circ} \mathrm{S}$, and western NorthCentral Atlantic, primarily $0-40^{\circ} \mathrm{N}$; probably worldwide in tropical to warm temperate oceans. Depth distribution unknown; majority of specimens collected at surface.

Diagnosis — Intestinal photophores circular, well defined, with diameter of anterior 30$50 \%$ that of posterior; ocular photophore broad posteriorly, narrow and rounded anteriorly; chromatophores absent from tentacle stalk proximal to carpus, from oroventral stalk ridge, and from large patch on ventral surface of mantle (posterior $\sim 40 \%$ $\mathrm{ML}$ ); tentacle club length 17-23\% ML, with 20-23 short, robust, strongly recurved (70$90^{\circ}$ ) hooks, with curved necks in lateral profile; bases of distal-most ventral hooks with large spike.

Description (Figs 15C-16K) - Mantle conical overall, widening slightly midway along ML, width 20-25-30\% ML at ML <100mm, 20-24\% ML at $\mathrm{ML}>100 \mathrm{~mm}$; gladius visible dorsally as prominent ridge along anterior $90 \% \mathrm{ML}$; rostrum visible ventrally through posterior $\sim 7 \% \mathrm{ML}$ and may pierce mantle posteriorly in individuals to $\mathrm{ML} 63 \mathrm{~mm}$. Intestinal photophores circular, with diameter of anterior $30-50 \%$ that of posterior. Posterior portion of mantle slender, with small, firm, oblong ventral keel, its length $\sim 10 \% \mathrm{ML}$. Fins rhombic, length 56\% ML; width 57-76-90\% ML; anterior and posterior margins straight, becoming slightly concave posteriorly.

Head rounded, length 16-19-25\% ML, width 16-20-24\% ML, depth 17\% ML, constricted anteriorly; occipital region with seven or eight secondary folds; funnel groove broad, well-defined V-shape; funnel aperture level with midpoint of eye. Ocular photophore somewhat broader posteriorly than anteriorly, rounded at both ends, spanning ventral $\sim 40 \%$ eye circumference. Funnel component of locking apparatus 13-15\% ML; mantle component 25-33\% ML.

Arms $29-32,-40_{I I-I V}-47 \% \mathrm{ML}$, formula II=III=IV>I, each with $70-100$ suckers at $\mathrm{ML}$ $\sim 100 \mathrm{~mm}, 90-110$ suckers at $\mathrm{ML}>100 \mathrm{~mm}$; oral faces of arms bordered on either side by trabeculate membrane; sucker diameter $\sim 40-50 \%$ arm width. Aboral keels present on Arms I-III: that on Arms I 10\% arm width over distal $60-70 \%$ arm length; that on Arms II $\sim 30 \%$ arm depth over proximal 30\% arm length, thereafter $\sim 100 \%$ arm depth; that on Arms III $\sim 25 \%$ arm depth over proximal $25 \%$ arm length, thereafter $\sim 100 \%$ arm depth; lateral membrane on Arms IV 100\% arm width proximally, narrowing to $75 \%$ arm depth by $25 \%$ arm length. 
Table 6. Measures (mm) and counts of Onychoteuthis compacta (Berry, 1913) (arm and tentacle measures recorded from more complete side of specimen, indicated $\mathrm{R}$ or $\mathrm{L}$ ).

\begin{tabular}{|c|c|c|c|c|c|c|c|c|c|c|c|}
\hline Specimen ID & $\begin{array}{l}\text { USNM } \\
\mathbf{8 1 3 4 2 2}\end{array}$ & $\begin{array}{l}\text { USNM } \\
813422\end{array}$ & $\begin{array}{c}\text { USNM Acc } \\
277764\end{array}$ & $\begin{array}{l}\text { USNM } \\
\mathbf{8 1 3 4 2 8}\end{array}$ & $\begin{array}{c}\text { USNM } \\
813395\end{array}$ & $\begin{array}{c}\text { USNM } \\
727512\end{array}$ & $\begin{array}{c}\text { USNM Acc } \\
277764\end{array}$ & $\begin{array}{l}\text { USNM } \\
\mathbf{8 1 3 4 2 8}\end{array}$ & $\begin{array}{c}\text { USNM } \\
813395\end{array}$ & \multicolumn{2}{|c|}{ Mean Indices } \\
\hline Type Status & None & None & None & None & None & None & None & None & None & \multirow{2}{*}{\multicolumn{2}{|c|}{ (ML 61-112) }} \\
\hline ML & 112 & 108 & 94 & 89 & 75 & 67 & 67 & 63 & 61 & & \\
\hline Sex & $\mathrm{F}$ & $M$ & $\mathrm{~F}$ & $\mathrm{~F}$ & $\mathrm{~F}$ & $\mathrm{~F}$ & Indet. & M & Indet. & & \\
\hline MW & 27 & 24 & 22 & 25 & 20 & 17 & 13 & 19 & 18 & MWI & 25 \\
\hline HL & 18 & 17 & 17 & 18 & 15 & 11 & 12 & 12 & 15 & $\mathrm{HLI}$ & 19 \\
\hline HW & 20 & 21 & 18 & 18 & 15 & 15 & 15 & 14 & 10 & HWI & 20 \\
\hline FL & 62 & 60 & 51 & 49 & 43 & 37 & 36 & 36 & 35 & FLI & 56 \\
\hline FW & 88 & 76 & 68 & 67 & 65 & 51 & 46 & 50 & 49 & FWI & 76 \\
\hline Arms I & $34 \mathrm{~L}$ & $35 \mathrm{~L}$ & $28 \mathrm{~L}$ & $29 L$ & $23 R$ & $23 \mathrm{R}$ & $18 \mathrm{R}^{*}$ & $21 \mathrm{~L}$ & $18 \mathrm{~L}$ & A1I & 32 \\
\hline Arms II & $45 \mathrm{~L}$ & $38 \mathrm{~L}$ & $36 \mathrm{~L}$ & $33 \mathrm{~L}$ & $31 \mathrm{R}$ & $26 \mathrm{R}$ & $24 R$ & $27 \mathrm{~L}$ & $22 \mathrm{~L}$ & A2I & 38 \\
\hline Arms III & $45 \mathrm{~L}$ & $39 L$ & $39 L$ & $33 \mathrm{~L}$ & $31 \mathrm{R}$ & $27 R$ & $26 \mathrm{R}$ & $29 L$ & $23 \mathrm{~L}$ & A3I & 40 \\
\hline Arms IV & $47 \mathrm{~L}$ & $45 \mathrm{~L}$ & $39 L$ & $35 \mathrm{~L}$ & $32 \mathrm{R}$ & $29 R$ & $25 R$ & $28 \mathrm{~L}$ & $25 \mathrm{~L}$ & A4I & 41 \\
\hline TnL & $64 \mathrm{~L}$ & $57 \mathrm{~L}$ & $46 \mathrm{~L}$ & $45 \mathrm{~L}$ & $45 R$ & $35 R$ & $33 R$ & $30 \mathrm{~L}$ & $34 \mathrm{~L}$ & TnLI & 53 \\
\hline $\mathrm{CL}$ & $24 \mathrm{~L}$ & $23 \mathrm{~L}$ & $18 \mathrm{~L}$ & $18 \mathrm{~L}$ & $17 R$ & $15 R$ & $13 R$ & $14 \mathrm{~L}$ & $13 \mathrm{~L}$ & CLI & 21 \\
\hline CS & 8 & 10 & 8 & 6 & 9 & 9 & 8 & 8 & 7 & & \\
\hline MH & 21 & 22 & 21 & 20 & 23 & 21 & 21 & 21 & 21 & & \\
\hline MS & 0 & 0 & 0 & 0 & 0 & 0 & 0 & 0 & 0 & & \\
\hline TPS & 14 & 14 & 11 & 13 & 13 & 12 & 14 & 12 & 13 & & \\
\hline
\end{tabular}

* indicates damaged features.

Tentacles robust, $47-53-61 \% \mathrm{ML}$; aboral surface of stalk with low keel ( $15 \%$ tentacle thickness) extending from stalk base to level with carpus. Club (Figs 15D, 25A) slightly expanded, 17-21-23\% ML (32-40\% TnL); carpus compact, nearly circular in outline, with six to ten suckers (usually eight or nine); manus with 20-23 hooks (usually 21 or 22) (Figs 16A-C); terminal pad with 11-14 suckers. Proximal ventral hooks variably sized (smaller than, equal to or slightly larger than paired dorsal hooks); hooks V1-V4 or V1-V5 small, subequal in size; V5 transitional between small proximal and large mid-club ventral hooks; V6 and V7 (Fig. 16B) largest on club, about four times longer than paired dorsal hooks and $\sim 17 \%$ club length. Largest ventral hooks robust, with thick necks, curved in lateral profile; distal ventral hooks (V7-V10 or V11) with ventral basal cusp produced into spike (Fig. 16C) by ML 60mm. Hook D2 largest in dorsal series; hook D5 smaller than hooks D1-D4 and slightly offset toward midline of club. Overall recurvature of hooks $70-90^{\circ}$. Ventral club membrane $\sim 30 \%$ club width along entire club length; dorsal membrane $\sim 10 \%$ club width, flanking hooks D1-D4; dorsal keel $\sim 75 \%$ club width, flanking dorsal hooks from D3 to club tip.

Lateral profile of lower beak (Figs 16E-I, ML 89-118mm) longer than deep, with crest sloping steeply toward baseline (may have noticeable 'hump' midway along its length in lateral profile (Figs 16H, 16I), more commonly seen in female beaks), posterior edge of hood close to crest, hood length $50-60 \%$ crest length, and hood and crest together $60-70 \%$ baseline; jaw edge short, concave along entire length or concave distally and straight proximally; jaw angle obtuse, slightly obscured laterally by low, rounded, indistinct wing fold. Shoulder ridge distinct; angle ridge poorly developed, spanning 
$\sim 50 \%$ lateral wall depth; angle point indistinct; distal wing width $\sim 200 \%$ that at jaw angle, wing length $200-230 \%$ LRL, with small triangular insert of cartilage below jaw angle; crest broad, slightly thickened; lateral wall diagonally bisected by dark, prominent ridge and fold, remaining distinct to lateral wall corner.

Radula (Fig. 16D) with tricuspid rachidian, with narrow, conical mesocone and low, rounded lateral cusps $25-30 \%$ mesocone height. First lateral tooth subequal to
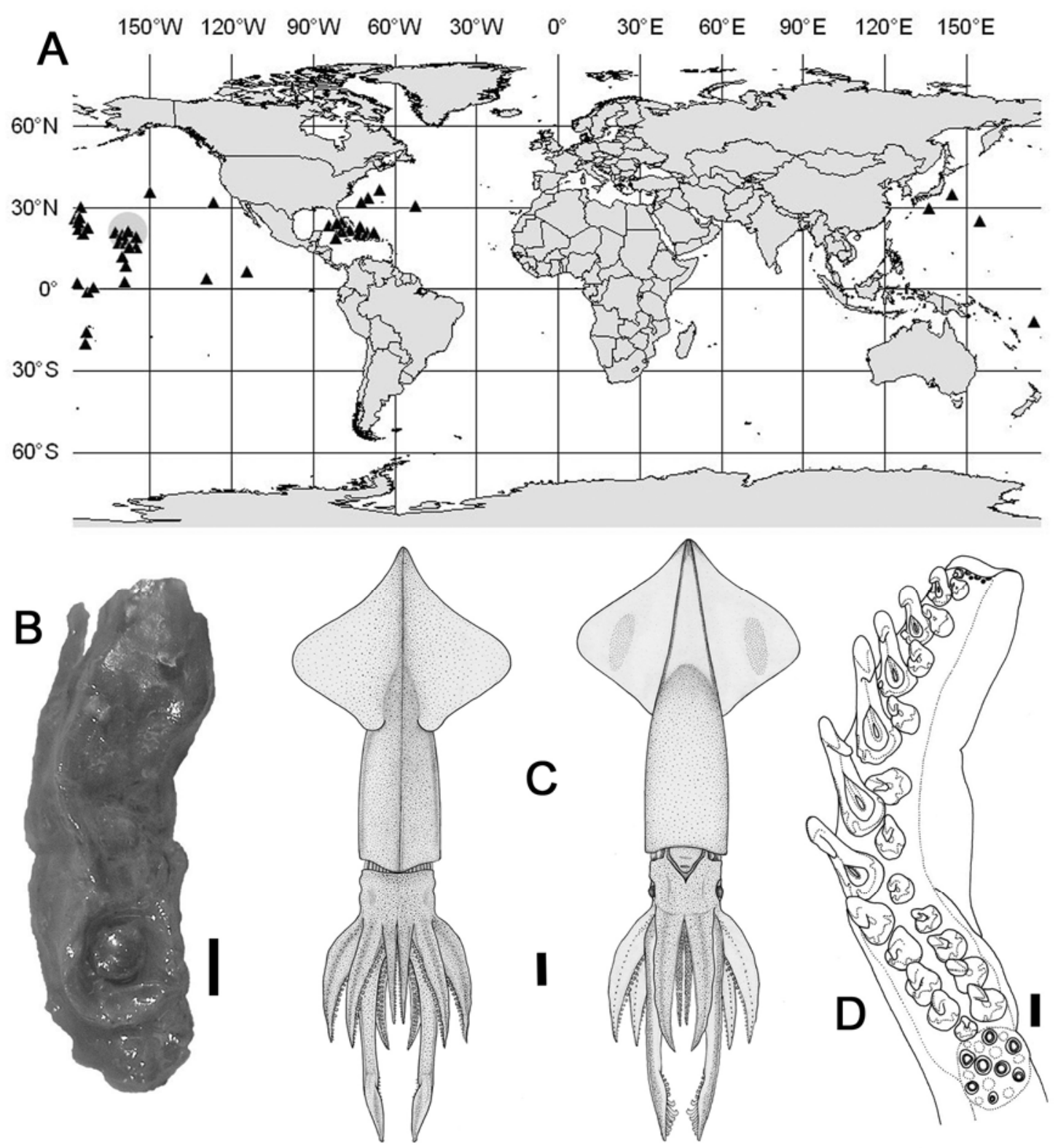

Fig. 15 - Onychoteuthis compacta. A) distribution (grey circle indicates type locality); B) USNM 214318, holotype, sex indet., ML $21 \mathrm{~mm}$; C) USNM 813428, , ML $89 \mathrm{~mm}$, illustration by K.H. Moore; D) USNM 813395, $\circ, \mathrm{ML} 75 \mathrm{~mm}$, right tentacle club. Scale bars = B, D) $1 \mathrm{~mm}$; C) $10 \mathrm{~mm}$. 
rachidian, with curved, slightly medially directed inner cusp; outer cusp barely produced, shorter than lateral cusps of rachidian. Second lateral tooth simple, slightly curved, $\sim 130 \%$ height of rachidian and first lateral. Marginal tooth simple, slightly curved, $\sim 170 \%$ height of rachidian and first lateral. Palatine palp (Fig. 16J) with $\sim 27$ mostly short, stubby teeth (with several longer, slender, curved teeth anteriorly), each $140-230 \%$ rachidian height, sparsely distributed over palp.

Gladius (Fig. 16K) with greatest width ( $7 \% \mathrm{GL}$ ) attained at $\sim 35 \% \mathrm{GL}$; free rachis $\sim 18 \% \mathrm{GL}$; vanes broaden and taper rapidly between 20 and $50 \% \mathrm{GL}$; dorsal keel solid, deep, comprising nearly entire gladius depth by $\sim 70 \%$ GL. Rostrum $\sim 5 \%$ GL; rostrum triangular in lateral profile, narrow, with nearly parallel margins in dorsal profile.

Overall colouration (preserved) pale creamy pink overlaid with dark reddish-brown chromatophores, darkest along visible ridge of gladius dorsally, becoming sparse but still distinct over dorsal and dorso-lateral mantle surfaces, small and nearly indistinguishable ventrally over anterior $\sim 60 \% \mathrm{ML}$; large ventral unpigmented patch present over posterior $\sim 40 \%$ ML (Fig. 15C). Dorsal and dorso-lateral surfaces of head dark red-brown; lateral surfaces iridescent. Aboral surfaces of Arms I and II dark; Arms II with coppery-green iridescence over proximal $40 \%$ arm length; tentacle stalks with same iridescence ventral to aboral keel; chromatophores absent from oro-ventral tentacle stalk ridge and across oral face of stalk proximal to carpus.

Remarks - Os. compacta was described by Berry (1913) based on a juvenile of ML $21 \mathrm{~mm}$. Due to paucity of material and the poor condition of the holotype (Fig. 15B), this species was largely overlooked until Young \& Harman (1987) attributed one of three paralarval Hawai'ian Onychoteuthis to it. Kubodera et al. (1998) accepted it as a taxonomically valid species, describing additional juvenile characters; Vecchione et al. (2003) added adult characters, including the unpigmented patch on the ventral mantle surface and the large basal spike on the distal-most ventral hooks.

This is the first report of Os. compacta from the Atlantic Ocean. Although the species' worldwide distribution now appears disjunct, Atlantic specimens herein attributed to Os. compacta share all salient morphological traits that distinguish this species from Pacific congeners. These traits include short tentacle clubs, large unpigmented patch on the posterior portion of the ventral mantle surface, pronounced 'hump' midway along the crest of the lower beak in lateral profile, and large basal spike on the distalmost ventral hooks. These character states also reliably separate Atlantic Os. 
compacta from the other local congeners (Os. banksii, Os. prolata and Os. bergii). The absence of chromatophores from the oral face of the tentacle stalk, proximal to the carpus, is also a reliable character state in all Atlantic and most Pacific Os. compacta specimens examined, with the exception of a small number of specimens from northern Hawai'ian waters, which possess a small cluster of chromatophores near the dorsal edge of the oral face of the tentacle stalk, proximal to the carpus.

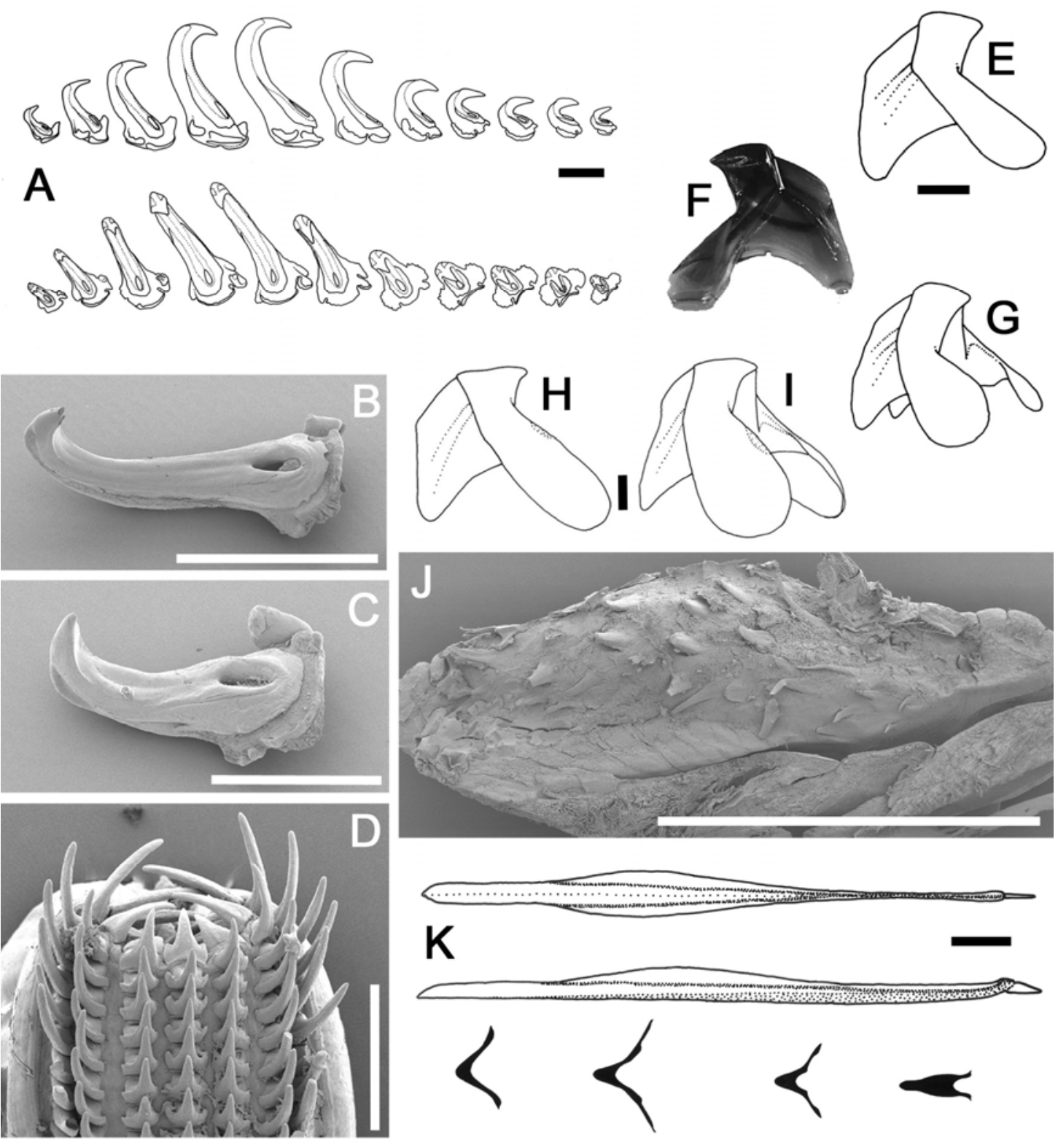

Fig. 16 - Onychoteuthis compacta. A-C, H-K) USNM 813428, +, ML 89mm; D-G) KSB-013, , ML 118mm. A-C) left ventral tentacular hooks: (A) entire series; (B) V7, (C) V10; D) radula; $E-I)$ lower beak: $(E, H)$ left, $(F)$ right, $(G, I)$ left oblique profiles; J) palatine palp; K) gladius. Scale bars = A, C, E, F, H) $1 \mathrm{~mm}$; B, J) 2mm; D) $500 \mu \mathrm{m} ; \mathrm{K}$ ) $10 \mathrm{~mm}$. 
Given the morphological similarity between many Onychoteuthis species, designation of a neotype to replace the damaged holotype may prove useful. The larger specimen registered under USNM 813428 ( 9 , ML 89mm, Table 6, Figs 15C, 16A-C, 16H-K, 25A) would be a suitable neotype, although it should be noted that the collection data read ' $20^{\circ} 09.5^{\prime} \mathrm{N}, 71^{\circ} 30^{\prime} \mathrm{W}$, Hawai'ian Islands' and should probably be corrected to '171 30 'W.' 
Onychoteuthis borealijaponica Okada, 1927 (Tables 2, 7, Figs 17-19)

Onychoteuthis borealijaponica Okada, 1927: 4-7; Okutani (1978): 144, 145; Murata et al.

(1982): 351-354; Okutani \& Murata (1983): 189-195; Roper et al. (1984): 129; Hess (1987): 290, 291, Fig. 54d-i; Okutani et al. (1987): 114, 115, Fig. 40; Sweeney et al. (1992): 129, Fig. 178; Bigelow (1994): 13-23, Tables 1-5, Figs 1-8; Gillespie (1997): 15-20, Tables 2, 3; Kubodera et al. (1998): 279, Fig. 2; Sweeney \& Roper (1998): 571; Okutani (2005): 153; Wakabayashi et al. (2007) 959-965, Table 2, Fig. 2; Bolstad (in press).

Onychoteuthis borealijaponicus [sic] Okada, 1927 - Young (1972): 61-64, Table 15, PI. 18A, 19A-G; Murata \& Ishii (1977): 1-23; Toll (1982): 55, 56, PI. 5B; Hess (1987): 290, 291, 300, 301, Fig. 54d-i; Filippova et al. (1997): 180, Figs 106Г [sic], E; Young \& Harman (1998): 257-271, Fig. 3b, Table 1.

Onychoteuthis banksii (not Leach, 1817) — Berry (1916): 50; Sasaki (1929): 228-230, PI. 20,

Fig. 12, PI. 30, Figs 1, 2; Akimushkin (1965): 166-168, Figs 48, 49.

Onychoteuthis boreali-japonicus [sic] Okada, 1927 — Iverson \& Pinkas (1971): Fig. 37; Clarke (1986): 72-74, Fig. 32b.

Onychoteuthis banksi borealijaponicus [sic] Okada, 1927 - Nesis (1987): 192, 193, Figs 48D, E.

Onychoteuthis banksi [sic] (not Leach, 1817) — Filippova et al. (1997): Fig. 106 [sic].

Type material — None (never designated).

Material examined (89 specimens) - USNM 1091107, $3{ }^{\hat{\prime}}, \mathrm{ML} 262-244 \mathrm{~mm}, 50^{\circ} 00^{\prime} \mathrm{N}$, $176^{\circ} 22^{\prime} \mathrm{W}, 15 / 08 / 1968$, RV Miller Freeman; NSMT Mo66606, 5 sex indet., ML 270$247 \mathrm{~mm}, 47^{\circ} 58.5^{\prime} \mathrm{N}, 174^{\circ} 59.7^{\prime} \mathrm{E}, 14 / 10 / 1985$, RV No. 1 Kanki-Maru, gill net; NSMT

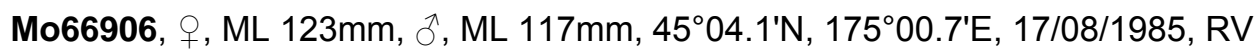
Kanki-Maru Stn 7, coll. T. Kubodera, gill net; KSB-043,, , ML 158mm, $45^{\circ} 01^{\prime} \mathrm{N}$, 179 $30^{\prime} \mathrm{W}, 14 / 08 / 1991,2120-2220 \mathrm{~h}$, TC 91-06 Stn 109, NMFS S0222; KSB-053, 2 \%, $\mathrm{ML} 80,79 \mathrm{~mm}, \hat{\sigma}$, ML $74 \mathrm{~mm}, 45^{\circ} 00.2^{\prime} \mathrm{N}, 174^{\circ} 30.5^{\prime} \mathrm{W}, 158-0 \mathrm{~m}, 18 / 08 / 1991,2042-$ 2142h, CMT, TC 91-06 Stn 135, NMFS S-0277; KSB-045, §, ML 175mm, 45N, 1743'ㄴ, 19/08/1991, TC 91-06, NMFS S-0281, squid jig; KSB-044, ㅇ, ML 165mm, $45^{\circ} \mathrm{N}, 179^{\circ} 30^{\prime} \mathrm{W}, 15 / 08 / 1991$, TC 91-06, NMFS S-0280, squid jig; KSB-054, Љ, ML $78 \mathrm{~mm}, 44^{\circ} 58^{\prime} \mathrm{N}, 174^{\circ} 35^{\prime} \mathrm{W}, 18 / 08 / 1991,2216-2316 \mathrm{~h}$, TC 91-06 Stn 136, NMFS S0203; UMML 31.1305, ․, ML 105mm, $44^{\circ} 39^{\prime} \mathrm{N}, 125^{\circ} 17^{\prime} \mathrm{W}$, off Oregon (State), 01/08/1961, coll. W. Pearcy; KSB-051, ô ML 59mm, 4312'N, 179³0'W, 12/08/1991, 1611-1837h, CMT, TC 91-06 Stn 83, NMFS S-0177; KSB-011 6 sex indet., ML 81$68 \mathrm{~mm}, 43^{\circ} 04^{\prime} \mathrm{N}, 179^{\circ} 30^{\prime} \mathrm{W}, 12 / 08 / 1991,2215-2315 \mathrm{~h}, \mathrm{CMT}$, TC $91-06$ Stn 85, NMFS S-0276; KSB-011, 7 sex indet., ML 81-68mm,, , ML 69mm, 430.' $\mathrm{N}, 179^{\circ} 30^{\prime} \mathrm{W}$,

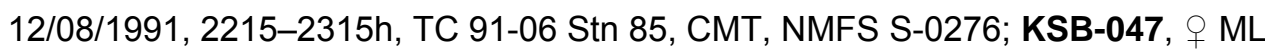

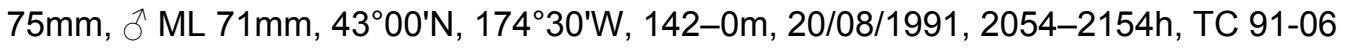
Stn 159, NMFS S-0279; NSMT Mo66905, , ML $121 \mathrm{~mm}, 42^{\circ} 45^{\prime} \mathrm{N}, 153^{\circ} 43^{\prime} \mathrm{E}$,

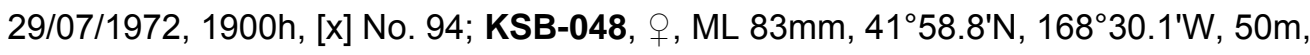


12/09/1983, 2012h, CMT, coll. WMM and VAH, TC 83-03, NMFS-Ref S-0057; KSB038, 4 sex indet., $M L$ 79-67mm, $41^{\circ} 55.2^{\prime} \mathrm{N}, 168^{\circ} 29.4^{\prime} \mathrm{W}, 150 \mathrm{~m}, 12 / 09 / 1983,2141 \mathrm{~h}$, CMT, coll. WMM and VAH, TC 83-03 Stn 26-2, NMFS-Ref S-0050; NSMT Mo71949, ${ }^{\lambda}$, ML 80mm, $41^{\circ} 21.2^{\prime} \mathrm{N}, 144^{\circ} 05.0^{\prime} \mathrm{E}$, off Sanriku, Japan, 550m, 25/07/1996, RV

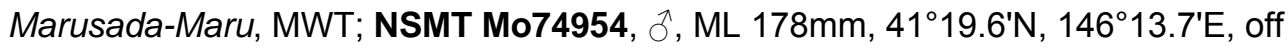
Sanriku, Japan, 550m, 09/07/1996, RV Marusada-Maru, Dymo Stn 1016, MWT; NSMT Mo74956, ô $^{\prime}$ ML $170 \mathrm{~mm}, 41^{\circ} 19.6^{\prime} \mathrm{N}, 146^{\circ} 13.7^{\prime} \mathrm{E}$, off Sanriku, Japan, 550m, 09/07/1996, RV Marusada-Maru, Dymo Stn 1018, MWT; NSMT Mo74957, ๆ, ML 163mm, 41⒚6'N, 146¹3.7'E, off Sanriku, Japan, 550m, 09/07/1996, RV MarusadaMaru, Dymo Stn 1019, MWT; NSMT Mo74955, o, ML 152mm, 41 ${ }^{\circ} 19.6^{\prime} \mathrm{N}, 146^{\circ} 13.7^{\prime} \mathrm{E}$, off Sanriku, Japan, 550m, 09/07/1996, RV Marusada-Maru, Dymo Stn 1017, MWT;

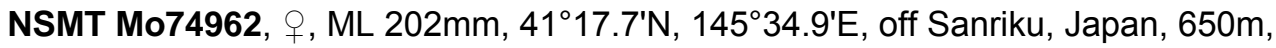
22/07/1996, RV Marusada-Maru, Dymo Stn 1027, MWT; NSMT Mo74964, , ML 200mm, 41 $17.7^{\prime} \mathrm{N}, 145^{\circ} 34.9^{\prime} \mathrm{E}$, off Sanriku, Japan, 650m, 22/07/1996, RV MarusadaMaru, Dymo Stn 1029, MWT; NSMT Mo74967, ồ, ML 185mm, 41 ${ }^{\circ} 17.7^{\prime} \mathrm{N}, 145^{\circ} 34.9^{\prime} \mathrm{E}$, off Sanriku, Japan, 650m, 22/07/1996, RV Marusada-Maru, Dymo Stn 1032, MWT;

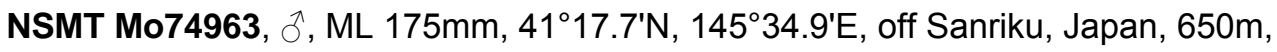
22/07/1996, RV Marusada-Maru, Dymo Stn 1028, MWT; NSMT Mo74966, §̊, ML $170 \mathrm{~mm}, 41^{\circ} 17.7^{\prime} \mathrm{N}, 145^{\circ} 34.9^{\prime} \mathrm{E}$, off Sanriku, Japan, 650m, 22/07/1996, RV Marusada-

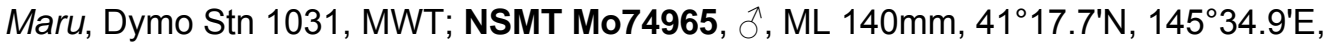
off Sanriku, Japan, 650m, 22/07/1996, RV Marusada-Maru, Dymo Stn 1030, MWT;

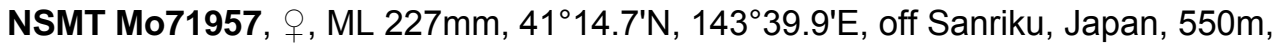
26/07/1996, RV Marusada-Maru, Dymo Stn 957, MWT; NSMT Mo71958, ふ̋, ML $190 \mathrm{~mm}, 41^{\circ} 14.7^{\prime} \mathrm{N}, 143^{\circ} 39.9^{\prime} \mathrm{E}$, off Sanriku, Japan, 550m, 26/07/1996, RV Marusada-

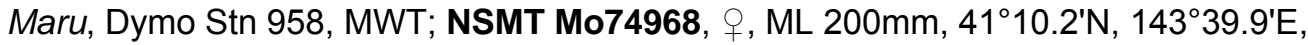
off Sanriku, Japan, 550m, 26/07/1996, RV Marusada-Maru, Dymo Stn 1009, MWT; NSMT Mo74969, sex indet., ML 175mm, 41 $14.7^{\prime} \mathrm{N}, 143^{\circ} 39.9^{\prime} \mathrm{E}$, off Sanriku, Japan, 550m, 26/07/1996, RV Marusada-Maru, Dymo Stn 1049, MWT; KSB-052, , ML 63mm, 41 $14^{\circ} \mathrm{N}, 174^{\circ} 29^{\prime} \mathrm{W}, 500-0 \mathrm{~m}, 22 / 08 / 1991,1610-1824 \mathrm{~h}, \mathrm{CMT}$, coll. KAB and J. Sinn; KSB-046, , , ML 104mm, $41^{\circ} 03.5^{\prime} \mathrm{N}, 176^{\circ} 33.9^{\prime} \mathrm{E}, 150 \mathrm{~m}, 22 / 08 / 1983,2133 \mathrm{~h}$, CMT, coll. WMM and VAH, TC 83-03 Stn 10-2, NMFS-Ref S-0056; NSMT Mo74960, ơ, ML $175 \mathrm{~mm}, 40^{\circ} 59.7^{\prime} \mathrm{N}, 143^{\circ} 34.0^{\prime} \mathrm{E}$, off Sanriku, Japan, 650m, 20/07/1996, RV Marusada-Maru, Dymo Stn 975, MWT; NSMT Mo74961, ô, ML 145mm, 4059.7'N, 14334.0'E, off Sanriku, Japan, 650m, 20/07/1996, RV Marusada-Maru, Dymo Stn 976,

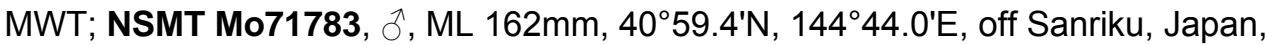
550m, 20/07/1996, RV Marusada-Maru, Dymo Stn 933, MWT; KSB-050, ð, ML 75mm, $40^{\circ} 06^{\prime} \mathrm{N}, 179^{\circ} 41^{\prime} \mathrm{W}, 08 / 08 / 1991, \mathrm{CMT}, \mathrm{TC}$ 91-06 Stn 39, NMFS S-0164; NSMT

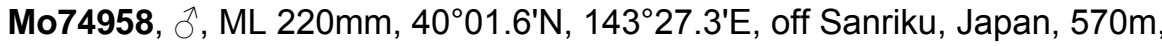
13/07/1996, RV Marusada-Maru, Dymo Stn 1005, MWT; NSMT Mo76092, + , ML $320 \mathrm{~mm}$, off Sanriku coast [ $\left.40^{\circ} 01.6^{\prime} \mathrm{N}, 143^{\circ} 27.3^{\prime} \mathrm{E}\right]$, coll. M. Sato, Tohoku Fish Reg., NFD; NSMT Mo76091, + , ML 262mm, off Sanriku coast [ 4001.6' $\left.\mathrm{N}, 143^{\circ} 27.3^{\prime} \mathrm{E}\right]$, coll. 
M. Sato, Tohoku Fish Reg., NFD; NSMT Mo72089, , ML $147 \mathrm{~mm}, 40^{\circ} 01.0^{\prime} \mathrm{N}$, 14350.3'E, off Sanriku, Japan, 530m, 13/07/1996, RV Marusada-Maru, Dymo Stn 944,

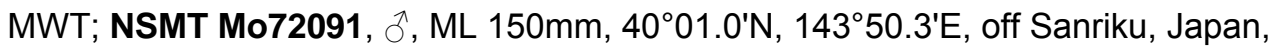
530m, 13/07/1996, RV Marusada-Maru, Dymo Stn 946, MWT; NSMT Mo72090, $170 \mathrm{~mm}, 40^{\circ} 01.0^{\prime} \mathrm{N}, 143^{\circ} 50.3^{\prime} \mathrm{E}$, off Sanriku, Japan, 530m, 13/07/1996, RV Marusada-

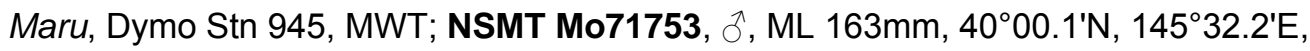
off Sanriku, Japan, 600m, 14/07/1996, RV Marusada-Maru, Dymo Stn 942, MWT; NSMT Mo71754, ô, ML 135mm, 4000.1'N, 145 32.2'E, off Sanriku, Japan, $600 \mathrm{~m}$, 14/07/1996, Marusada-Maru, Dymo Stn 943, MWT; NSMT Mo74959, , ML 135mm, $39^{\circ} 59.6^{\prime} \mathrm{N}, 143^{\circ} 32.6^{\prime} \mathrm{E}$, off Sanriku, Japan, 530m, 14/07/1996, RV Marusada-Maru, Dymo Stn 969, MWT; NSMT Mo72017, ㅇ, ML 148mm, 3951.1'N, 143²7.4'E, off Sanriku, Japan, 650m, 28/07/1996, RV Marusada-Maru, Dymo Stn 936, MWT; NSMT

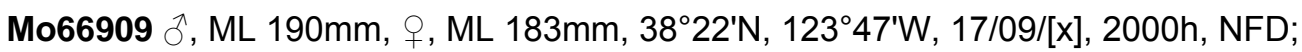

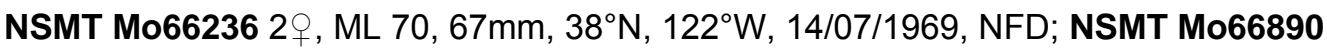
4 sex indet., ML $173-131 \mathrm{~mm}, 37^{\circ} 00.5^{\prime} \mathrm{N}, 142^{\circ} 58.8^{\prime} \mathrm{E}, 1 \mathrm{~m}, 27 / 07 / 1972,1000 \mathrm{~h}, \mathrm{RV}$ Soyo-Maru Stn 1-7, HN; CASIZ 061056, ㅇ, ML 123mm, 36 $46.4-47.0^{\circ} \mathrm{N}, 121^{\circ} 58.1-$ 55.6'W, 648-504m, 11/02/1975, Stn 38Ya, Tow 38, coll. M.E. Anderson; NSMT Mo66907, 今ึ, ML 148mm, ㅇ, ML 127mm, 36 $00^{\circ} \mathrm{N}, 125^{\circ} 35^{\prime} \mathrm{W}, 25 / 09 /[\mathrm{x}]$, NFD; USNM 727478, ${ }^{\top}, \mathrm{ML} 80 \mathrm{~mm}, 33^{\circ} 15^{\prime} \mathrm{N}, 118^{\circ} 25^{\prime} \mathrm{W}$, Southern California, 766m, 22/10/1962, RV Velero IV 8237, 3m IKMWT; CASIZ 057971,, , ML 270mm, off entrance to Newport Bay, Orange County, California [ $\left.33^{\circ} \mathrm{N}, 117^{\circ} \mathrm{W}\right]$, coll. J.H. Souder, NFD; CASIZ 030508, $\odot$, ML $65 \mathrm{~mm}$, Baja California, SW of Coronado Island $\left[\sim 32^{\circ} 40.68^{\prime} \mathrm{N}\right.$, $\left.117^{\circ} 10.35^{\prime} \mathrm{W}\right]$, on surface, 03/07/1941, Stn 2561, coll. J.C. Marr; KSB-013, , ML 92mm, $24^{\circ} 36^{\prime} \mathrm{N}, 170^{\circ} 53.6^{\prime} \mathrm{W}, 93 \mathrm{~km}$ NW of Maro, 03/07/1990, TC 90-05 Stn 125. Unlocalised material examined (4 specimens) - USNM 000199, + , ML 245mm, off salmon trawler, 11/07/1965, coll. D. Gotshall, Eureka Lab, A-125, NFD; BMNH 20070547 (3 sex indet.), ML 49, 37, 31mm, California, 1962, coll. P. Foxton, NFD.

Distribution (Fig. 17A) - North Pacific (generally north of $30^{\circ} \mathrm{N}$ ), $800-0 \mathrm{~m}$, in the Kuroshio, Alaskan, and California currents, and the subtropical countercurrent. Elsewhere recorded from Aleutian Islands to Japan and southern Baja California (Nesis 1987).

Diagnosis - Both intestinal photophores always discernible, oval or tear-drop-shaped, anterior $\sim 30 \%$ size of posterior, with somewhat indistinct edges, connected by a band of iridescent gold tissue; ocular photophore uniformly broad, spanning ventral $\sim 30 \%$ eye circumference; chromatophores absent from tentacle stalk proximal to carpus and along oro-ventral stalk ridge, but present over entire ventral surface of mantle; tentacle club $\sim 27 \% \mathrm{ML}$ in subadults, $\sim 23 \% \mathrm{ML}$ in specimens of ML $49-80 \mathrm{~mm}$, with $23-28$ hooks; bases of distal-most ventral hooks with small ventral spike. 
Table 7. Measures $(\mathrm{mm})$ and counts of Onychoteuthis borealijaponica Okada, 1927 (arm and tentacle measures recorded from more complete side of specimen, indicated $\mathrm{R}$ or $\mathrm{L}$ ).

\begin{tabular}{|c|c|c|c|c|c|c|c|c|c|c|c|c|c|}
\hline Specimen ID & $\begin{array}{c}\text { NSMT } \\
\text { Mo76092 }\end{array}$ & $\begin{array}{c}\text { NSMT } \\
\text { Mo76091 }\end{array}$ & $\begin{array}{c}\text { USNM } \\
1091107\end{array}$ & $\begin{array}{c}\text { USNM } \\
1091107\end{array}$ & $\begin{array}{c}\text { CASIZ } \\
061056\end{array}$ & \multicolumn{2}{|c|}{ Mean Indices } & $\begin{array}{l}\text { USNM } \\
727478\end{array}$ & KSB-011 & $\begin{array}{l}\text { CASIZ } \\
030508\end{array}$ & $\begin{array}{c}\text { BMNH } \\
20070547\end{array}$ & \multicolumn{2}{|c|}{$\begin{array}{c}\text { Mean } \\
\text { Indices }\end{array}$} \\
\hline Type Status & None & None & None & None & None & & & None & None & None & None & \multirow{2}{*}{\multicolumn{2}{|c|}{ (ML 49-80) }} \\
\hline $\mathrm{ML}$ & 320 & 262 & 244 & 245 & 123 & \multicolumn{2}{|c|}{ (ML 123-320) } & 80 & 69 & 65 & 49 & & \\
\hline Sex & $\mathrm{F}$ & $\mathrm{F}$ & $\mathrm{M}$ & $\mathrm{M}$ & $\mathrm{F}$ & & & $M$ & $\mathrm{~F}$ & $\mathrm{~F}$ & Indet. & & \\
\hline MW & 52 & 51 & 47 & 52 & 24 & MWI & 21 & 17 & 15 & 14 & 10 & MWI & 21 \\
\hline $\mathrm{HL}$ & 35 & 38 & 51 & 38 & 21 & $\mathrm{HLI}$ & 17 & 11 & 18 & 12 & 9 & $\mathrm{HLI}$ & 19 \\
\hline HW & 40 & 35 & 33 & 28 & 20 & HWI & 13 & 15 & 14 & 12 & 7 & HWI & 18 \\
\hline $\mathrm{FL}$ & 170 & 140 & 142 & 137 & 73 & FLI & 57 & 45 & 38 & 37 & 26 & FLI & 55 \\
\hline FW & 200 & 166 & 166 & 163 & 87 & FWI & 69 & 58 & 47 & 47 & 39 & FWI & 73 \\
\hline Arms I & $81 \mathrm{~L}$ & $72 \mathrm{~L}$ & $102 \mathrm{~L}$ & $92 \mathrm{~L}$ & $38 \mathrm{~L}$ & A1I & 36 & $21 \mathrm{R}$ & $20 \mathrm{~L}$ & $17 \mathrm{~L}$ & $14 \mathrm{~L}$ & A1I & 27 \\
\hline Arms II & $95 \mathrm{~L}$ & $81 \mathrm{~L}$ & $107 \mathrm{~L}$ & $101 \mathrm{~L}$ & $48 \mathrm{~L}$ & A2I & 41 & $31 \mathrm{R}$ & $27 \mathrm{~L}$ & $22 \mathrm{~L}$ & $16 \mathrm{~L}$ & A2I & 36 \\
\hline Arms III & $93 \mathrm{~L}$ & $89 \mathrm{~L}$ & $108 \mathrm{~L}$ & $104 \mathrm{~L}$ & $48 \mathrm{~L}$ & A3I & 41 & $30 R$ & $27 \mathrm{~L}$ & $20 \mathrm{~L}$ & $16 \mathrm{~L}$ & A3I & 35 \\
\hline Arms IV & $98 \mathrm{~L}$ & $81 \mathrm{~L}$ & $111 \mathrm{~L}$ & $105 \mathrm{~L}$ & $54 \mathrm{~L}$ & A4I & 42 & $31 \mathrm{R}$ & $25 \mathrm{~L}$ & $23 \mathrm{~L}$ & $15 \mathrm{~L}$ & $\mathrm{~A} 4 \mathrm{I}$ & 35 \\
\hline $\mathrm{TnL}$ & $166 \mathrm{~L}$ & $130 \mathrm{~L}$ & $265 \mathrm{~L}$ & $245 \mathrm{~L}$ & $88 \mathrm{~L}$ & TnLI & 93 & $37 \mathrm{R}$ & $66 \mathrm{~L}$ & $30 \mathrm{~L}$ & $27 \mathrm{~L}$ & TnLI & 61 \\
\hline $\mathrm{CL}$ & $64 \mathrm{~L}$ & $54 \mathrm{~L}$ & $66 \mathrm{~L}$ & $59 \mathrm{~L}$ & $32 \mathrm{~L}$ & CLI & 27 & $17 R$ & $18 \mathrm{~L}$ & $13 \mathrm{~L}$ & $12 \mathrm{~L}$ & CLI & 23 \\
\hline CS & 10 & 7 & 9 & 9 & 9 & & & 9 & 10 & 9 & 7 & & \\
\hline MH & 27 & 25 & 26 & 25 & 26 & & & 23 & 25 & 25 & 26 & & \\
\hline MS & 0 & 0 & 0 & 0 & 0 & & & 0 & 0 & 0 & 0 & & \\
\hline TPS & 16 & 14 & 13 & 13 & 14 & & & 15 & 10 & 14 & $7^{*}$ & & \\
\hline
\end{tabular}

* indicates damages features.

Description (Figs 17B-19C) - Mantle muscular, smooth, slender, conical, width 1821-25\% ML; gladius visible dorsally as dark subcutaneous line; dorsal keel not protruding through mantle musculature; rostrum barely visible ventrally through posterior $3 \% \mathrm{ML}$; posterior portion of mantle broadly attenuate; ventral surface of mantle without apparent keel. Fins rhombic, length 57\% ML, width 62-69-75\% ML, with large anterior lobes; posterior margins slightly concave medially, becoming convex laterally; anterior margins slightly convex. Intestinal photophores always discernible but with variably distinct margins, oval or tear-drop-shaped, each attenuated toward the other; each approximately twice as long as wide; size of anterior $\sim 30 \%$ that of posterior.

Head boxy, length 14-17-21\% ML, width 13\% ML, depth 11\% ML; occipital region with seven to nine secondary folds. Funnel broad; aperture level with anterior margin of eye aperture; funnel groove shallow, well defined, V-shaped, extending to point directly below midpoint of eye. Ocular photophore elongate, rounded at both ends, uniformly broad, spanning ventral $\sim 30 \%$ eye circumference. Mantle component of locking apparatus $20-25 \% \mathrm{ML}$, funnel component $\sim 12 \% \mathrm{ML}$.

Arms slender, 33-36,-41 $\left.\right|_{I I I V}-46 \% \mathrm{ML}$; formula II=III=IV>I; each with 90-100 suckers (ML 123-320mm); oral faces of arms bordered on each side by low protective membrane with indistinct trabeculae; diameter of largest suckers $50-60 \%$ arm width. Arms I without apparent keel; broad keels present on Arms II and III: that on Arms II $\sim 100 \%$ arm depth to $40 \%$ arm length, narrowing thereafter to $\sim 50 \%$ arm depth; that on Arms III $\sim 150 \%$ arm depth by $40 \%$ arm length, thereafter $\sim 100 \%$ arm depth to arm tip; 


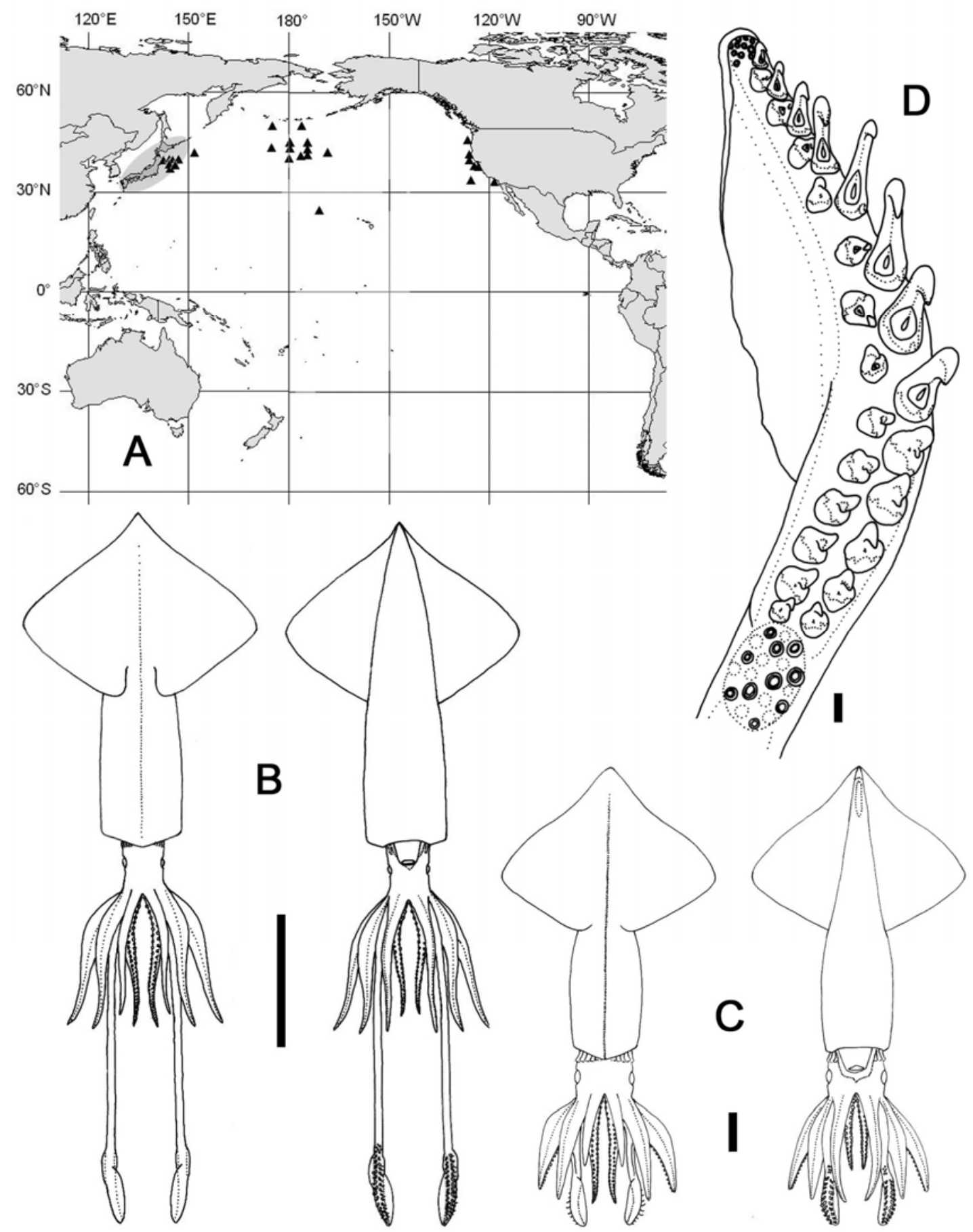

Fig. 17 - Onychoteuthis borealijaponica. A) distribution (grey oval indicates type locality); B) USNM 1091107, ô, ML 244mm; C) USNM 727478, ô, ML 80mm; D) NSMT Mo66905, , ML 121mm, left tentacle club. Scale bars = B) $100 \mathrm{~mm}$; C) $10 \mathrm{~mm}$; D) $1 \mathrm{~mm}$. 
lateral membrane on Arms IV $100 \%$ arm depth at base, gradually narrowing thereafter to $\sim 40 \%$ arm width along remainder of arm.

Tentacles slender, $70-93-110 \% \mathrm{ML}$; stalk bases $~ 75 \%$ thickness of adjacent arms; aboral surface of stalk with small longitudinal keel. Clubs (Fig. 17D) 24-27-31\% ML (25-40\% TnL), only slightly expanded. Carpus ovoid, well defined, with seven to 11 suckers (usually eight or nine); distal-most carpal suckers may crowd proximal hooks;
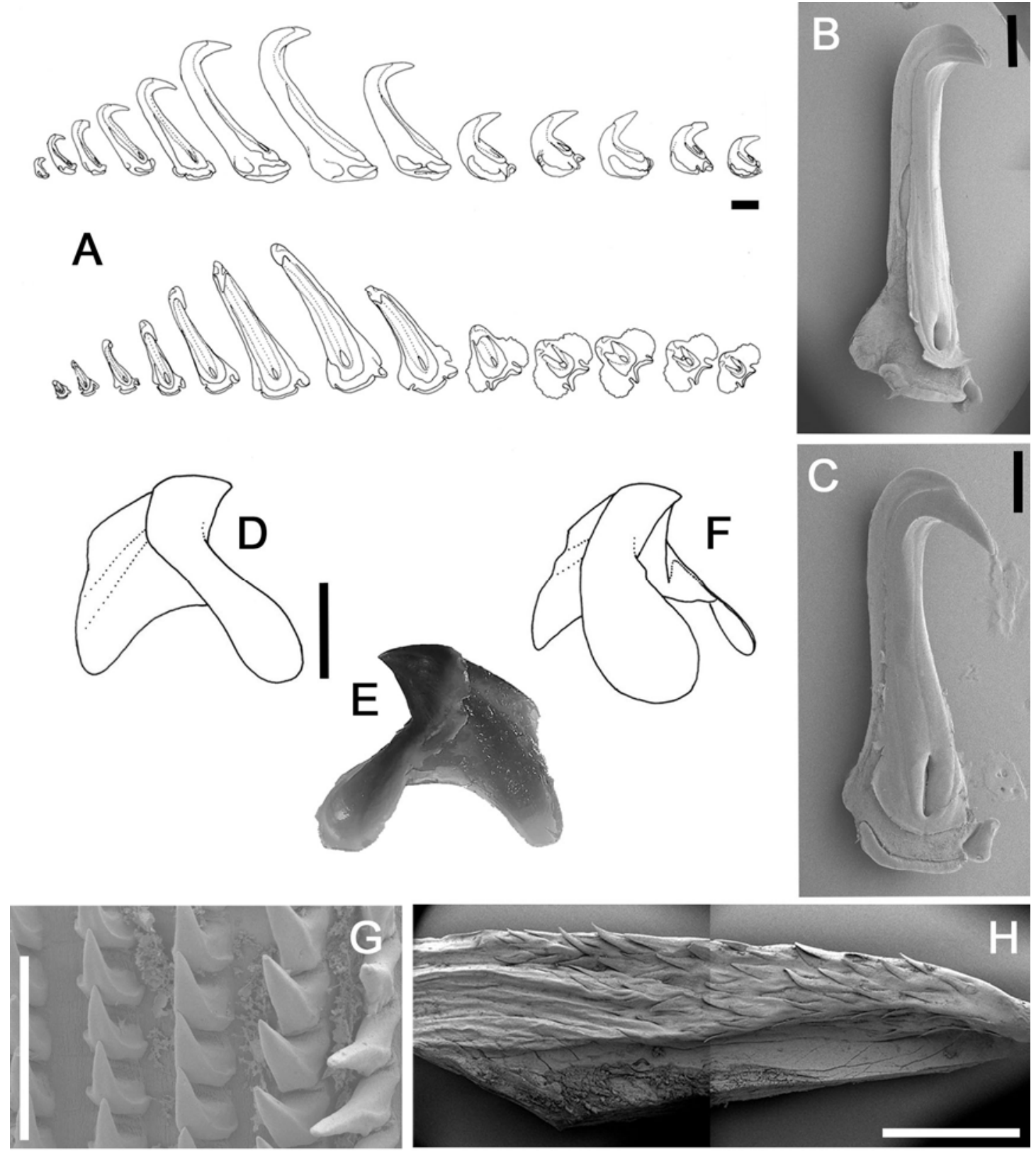

Fig. 18 - Onychoteuthis borealijaponica, USNM 1091107, 今, ML 262mm. A-C) left ventral tentacular hooks: (A) entire series, (B) V7, (C) V10; D-F) lower beak: D) left, E) right, F) left oblique profiles; $G$ ) radula; $H$ ) palatine palp. Scale bars $=A, B$ ) $1 \mathrm{~mm} ; C, G$ ) $500 \mu \mathrm{m} ; \mathrm{D}$, E) $5 \mathrm{~mm}$; H) $2 \mathrm{~mm}$. 
manus with 23-27 robust hooks (Figs 18A-C); terminal pad with 9-16 suckers (usually 13-15). Bases of large ventral hooks asymmetrical, with those of hooks V7-V13 enlarged ventrally into small spike (Fig. 18C). Ventral hooks all larger than corresponding dorsal hooks, increasing in size through hook V7 or V8 (Fig. 18B); largest ventral hook about five times longer than corresponding dorsal hook and 16$19 \%$ CL. Dorsal hooks decrease in size from D1 to D5, with D4 and D5 slightly offset toward centre of club; length of D6 subequal to D1, with hooks D7-D13 gradually decreasing in size distally. Ventral membrane $\sim 80 \%$ club width, flanking hooks V1-V6; dorsal membrane $\sim 20 \%$ club width, flanking hooks D1-D5; hooks D4-D13 bordered by dorsal keel, $\sim 120 \%$ club width.

Lateral profile of lower beak (Figs 18D-F, ML 262mm) longer than deep, with straight crest, sloping steeply downward, posterior edge of hood close to crest, hood length $\sim 50 \%$ total crest length, and hood and crest together $\sim 60 \%$ baseline; jaw edge weakly concave along entire length; jaw edge narrow and sharp; jaw angle obtuse, obscured by slight wing fold. Crest narrow, slightly thickened; lateral walls slightly indented below crest; shoulder ridge distinct, rounded; angle ridge distinct, spanning $\sim 75 \%$ lateral wall depth; angle point distinct; wing width distally $\sim 150 \%$ that at jaw angle, wing length $\sim 250 \%$ LRL; small insert of clear cartilage present on medial wing margin just below jaw angle; lateral wall fold broad, rounded, well-developed anteriorly, becoming broader and shallower but still distinct to posterior margin of lateral wall.

Radula (Fig. 18G) with unicuspid rachidian; mesocone robust, triangular, pointed distally; height of base $\sim 20 \%$ height of rachidian; proximal margin of base straight, distal margin with distinct lateral corners. First lateral tooth unicuspid, subequal to rachidian, robust, forming nearly right triangle, with straight to slightly concave inner margin and straight to slightly convex outer margin. Second lateral tooth simple, robust, $\sim 120 \%$ height of rachidian; margins of cusp straight to slightly convex. Marginal tooth simple, slender, with cusp somewhat twisted on some teeth; height $\sim 180 \%$ that of rachidian. Palatine palp (Fig. 18H) with $\sim 65$ large, flat, pointed teeth, each 150$300 \%$ rachidian height, evenly set over surface of palp.

Gladius (Fig. 19A) with greatest width ( $7 \% \mathrm{GL}$ ) attained at $\sim 40 \% \mathrm{GL}$; free rachis $\sim 21 \% \mathrm{GL}$; vanes broaden and taper rapidly between 20 and $75 \% \mathrm{GL}$; dorsal keel robust, narrow; rostrum $\sim 3 \% \mathrm{GL}$, triangular in lateral profile, narrow in dorsal profile. Chromatophores present over entire ventral surface of mantle, but not along oroventral tentacle stalk-ridge or across oral face of stalk, proximal to carpus. 
Smaller specimens (below ML $\sim 100 \mathrm{~mm}$, Figs 17C, 19B, 19C) deviate from the above description in having somewhat different body proportions: mantle slender, spindleshaped, tapering rapidly beneath fins; fin width $68-73-80 \% \mathrm{ML}$; head length $\sim 19 \%$ $\mathrm{ML}$, width $\sim 18 \% \mathrm{ML}$, depth $\sim 14 \% \mathrm{ML}$; arm length $26-27,-35_{I I I I V}-39 \% \mathrm{ML}$, each with 80-90 suckers bordered by trabeculate membrane; sucker diameter $\sim 50 \%$ arm width on Arms I-III, 30\% arm width on Arms IV; keel on Arms II 75\% arm depth from $\sim 20 \%$ arm length to arm tip; keel on Arms III 120\% arm depth by $\sim 30 \%$ arm length, thereafter $\sim 100 \%$ arm depth; lateral membrane on Arms IV $150 \%$ arm width at bases, narrowing thereafter to $\sim 100 \%$ arm width over remaining arm length; tentacles robust, 46-61-95\% ML, bases thicker than adjacent arms; club length 23\% ML ( 45\% TnL); adult club morphology present by ML 59mm. Marginal suckers in paralarvae and small juveniles (Fig. 19C) ovoid in outline, with small, proximally offset aperture ( $25 \%$ sucker diameter); pegs with flattened oral faces, set in two concentric rows, polygonal in outline proximally, set in six to seven concentric rows and ovoid to round in outline

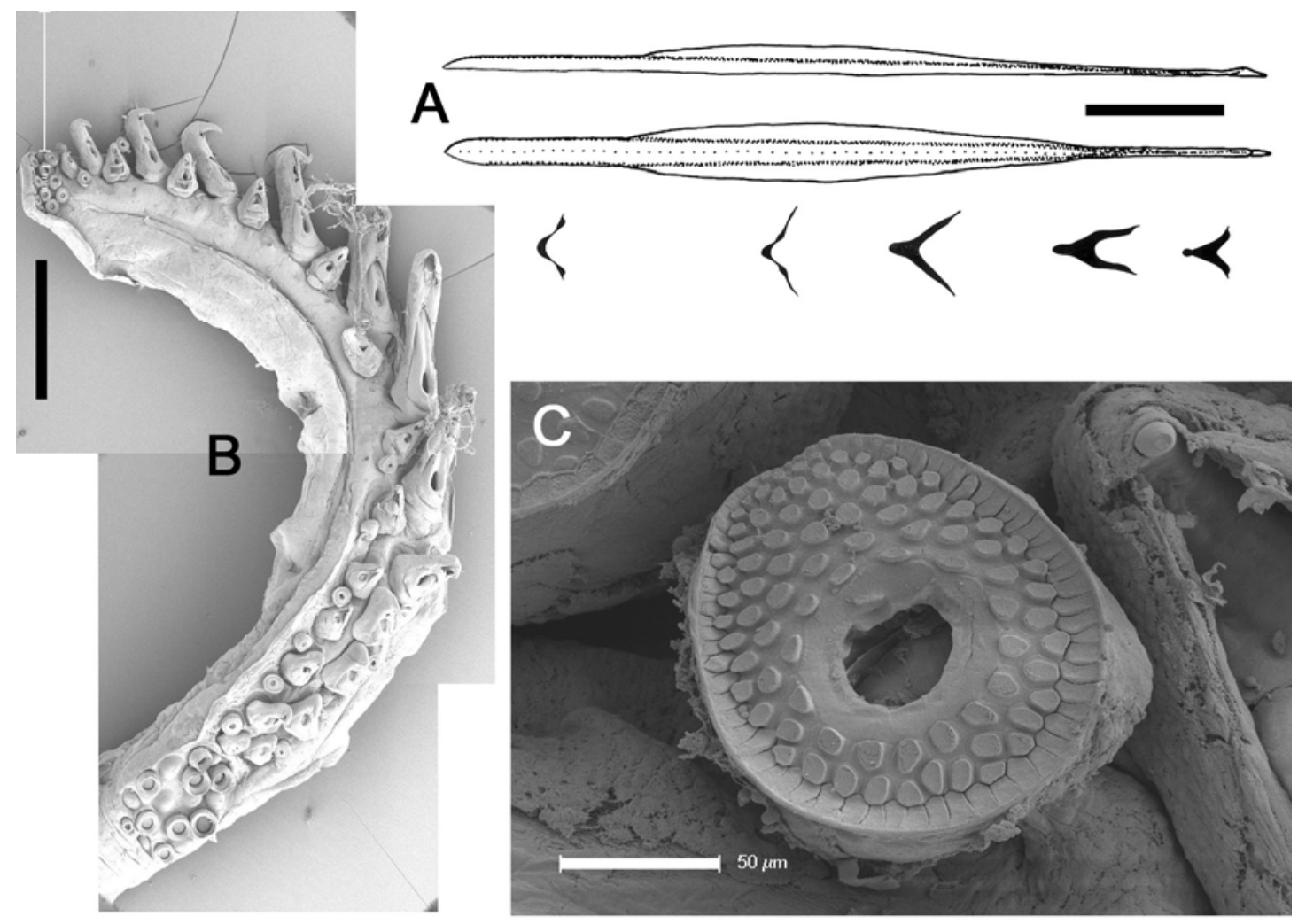

Fig. 19 - Onychoteuthis borealijaponica. A) USNM 1091107, ô, ML 262mm, gladius; B, C) BMNH 20070547, sex indet., ML 37mm: B) left tentacle club, C) marginal sucker. Scale bars = A) $50 \mathrm{~mm}$, B) $1 \mathrm{~mm}$, C) $50 \mu \mathrm{m}$. 
distally; full series of marginal suckers present on club at $\mathrm{ML} 31 \mathrm{~mm}$, with approximately half lost by ML $37 \mathrm{~mm}$ (Fig. 19B) and all absent by ML $49 \mathrm{~mm}$.

Remarks - No type specimen was originally or has subsequently been designated for Os. borealijaponica, but the original description, Okada (1927) clearly described the elongate photophores, contrasting them directly with the round photophores observed in Os. 'banksii,' and describes the band of iridescent tissue connecting the photophores, speculating on its possible photogenic nature. Adam (1952) and Nesis (1987) considered Os. borealijaponica to be a synonym and a subspecies of Os. 'banksii' respectively, but Young (1972) concluded that photophore shape, in addition to the number of hooks and the general robustness of the body, enabled differentiation of Os. borealijaponica from Os. 'banksii' at $\mathrm{ML}>12 \mathrm{~mm}$. Should questions about this species' identity arise, the specimen registered under NSMT Mo76092 ( $\%$, ML 320mm, Table 7) would be a suitable neotype.

Os. borealijaponica is the largest known Onychoteuthis species, reaching ML $>350 \mathrm{~mm}$. It is one of the few onychoteuthids fished commercially and as such, several studies have reported on aspects of its biology, growth and abundance (Okutani \& Murata 1983, Osako \& Murata 1983, Gillespie 1997). 
Onychoteuthis meridiopacifica Rancurel \& Okutani, 1990 (Tables 2, 8, Figs 20, 21)

Onychoteuthis sp. Rancurel, 1970: 23-29, Tables 6, 7, Figs 17, 18a, 19a; Nesis (1987): 193,

Fig. 48F; Okutani \& Tsukada (1988): 19-21, Table 5, Figs 10-12.

Onychoteuthis meridiopacifica Rancurel \& Okutani, 1990: 25-30, Figs 1-7; Kubodera et al. (1998): 280, Fig. 4; Sweeney \& Roper (1998): 571; Okutani (2005): 154; Bolstad (in press).

Type material (not available for examination in this study) - NSMT Mo67008, holotype, $\widehat{\partial}, \mathrm{ML}$ 62.5mm, $21^{\circ} 15^{\prime} \mathrm{S}, 153^{\circ} 23^{\prime} \mathrm{E},-/ 12 / 1975$, ex-lancetfish, RV Sagami-Maru; NSMT 67009, paratype, ${ }^{\prime}$, ML $60.8 \mathrm{~mm}, 20^{\circ} 10.5^{\prime} \mathrm{S}, 156^{\circ} 09^{\prime} \mathrm{E},-/ 12 / 1975$, ex-lancetfish, RV SagamiMaru; NSMT Mo67011, paratype, + , ML 55.3mm, 20¹6.1'S, 153²3.6'E, -/12/1975, exlancetfish, RV Sagami-Maru; NSMT Mo67010, paratype, ơ , ML 62mm, $22^{\circ} 10^{\prime} \mathrm{S}$, $156^{\circ} 09^{\prime} E$, - /12/1975, ex-lancetfish, RV Sagami-Maru; NSMT Mo67012, paratype, $q$, ML $55.7 \mathrm{~mm}, 22^{\circ} 10^{\prime} \mathrm{S}, 156^{\circ} 09^{\prime} \mathrm{E},-/ 12 / 1975$, ex-lancetfish, RV Sagami-Maru.

Material examined (63 specimens) - NSMT Mo76066, ô. ML 44mm, $19^{\circ} 49.5^{\prime} \mathrm{S}, 153^{\circ} 01.5^{\prime} \mathrm{E}$, 28/11/1975, RFRL RV Soyo-Maru Stn SP107; NSMT Mo76080, sex indet., ML 38mm, 1949.5'S, 15301.5'E, 28/11/1975, RFRL RV Soyo-Maru Stn SP106; NSMT Mo76079, ð, ML 54mm, $19^{\circ} 56.8^{\prime} \mathrm{S}, 153^{\circ} 19.0^{\prime} \mathrm{E}, 30 / 11 / 1975$, RFRL RV Soyo-Maru Stn SP42; NSMT Mo76064, 7 sex indet., ML 37-29mm, 1956.8'S, 153¹9.0'E, 30/11/1975, RFRL RV Soyo-Maru Stn SP38; NSMT Mo76070, 2 \%, ML 35, 33mm, 1956.8'S, 153⒚0'E, 30/11/1975, RFRL RV Soyo-Maru Stn SP45; NSMT Mo76061, 6 sex indet., ML 3530mm, 1956.8'S, 153⒚0'E, 30/11/1975, RFRL RV Soyo-Maru Stn SP43; NSMT

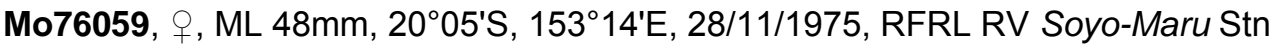
SP135, RFRL; NSMT Mo76088, 4 sex indet., ML 38-28mm, 2007.7'S, 15405.5'E, 23/11/1975, RFRL RV Soyo-Maru Stn SP89; NSMT Mo76067, ô, ML 59mm, 5 sex indet., ML 42-19mm, $20^{\circ} 10.5^{\prime} \mathrm{S}, 153^{\circ} 20^{\prime} \mathrm{E}, 25 / 11 / 1975$, RFRL RV Soyo-Maru Stn SP8;

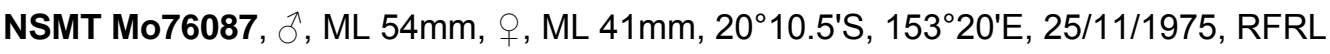

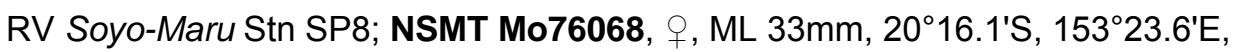
26/11/1975, RFRL RV Soyo-Maru Stn SP103; NSMT Mo76086, 6 sex indet., ML 48$38 \mathrm{~mm}, 20^{\circ} 21.5^{\prime} \mathrm{S}, 154^{\circ} 14^{\prime} \mathrm{E}, 22 / 11 / 1975$, RFRL RV Soyo-Maru Stn SP50; NSMT Mo76063, ㅇ, ML 44mm, 20²1.5'S, 15414'E, 22/11/1975, RFRL RV Soyo-Maru Stn SP54; NSMT Mo76072, , ML 44mm, 2025'S, 153³6'E, 30/11/1975, RFRL RV SoyoMaru Stn SP5; NSMT Mo76075, $q, \mathrm{ML} 38 \mathrm{~mm},{ }^{\AA}, \mathrm{ML} 32 \mathrm{~mm}, 20^{\circ} 31.5^{\prime} \mathrm{S}, 155^{\circ} 44^{\prime} \mathrm{E}$, 26/11/1975, RFRL RV Soyo-Maru Stn SP19; NSMT Mo76077, §ิ, ML 36mm, 2042.2'S,

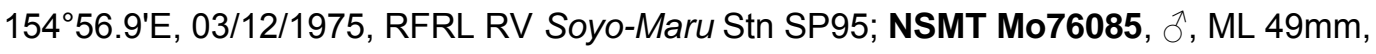
$21^{\circ} 15^{\prime} \mathrm{S}, 155^{\circ} 11.5^{\prime} \mathrm{E}, 03 / 12 / 1975$, RFRL RV Soyo-Maru Stn SP131; NSMT Mo76082 2へ, ML 47, 46mm, 21 ${ }^{\circ} 15^{\prime} \mathrm{S}, 155^{\circ} 11.5^{\prime} \mathrm{E}, 03 / 12 / 1975$, RFRL RV Soyo-Maru Stn SP131; NSMT Mo76084, 5 sex indet., ML 45-26mm, 21 ${ }^{\circ} 15^{\prime} \mathrm{S}, 155^{\circ} 11.5^{\prime} \mathrm{E}, 03 / 12 / 1975$, RFRL RV Soyo-

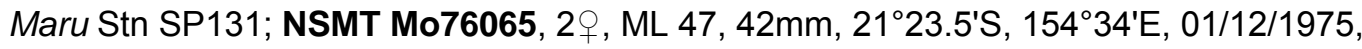
RFRL RV Soyo-Maru Stn SP37; NSMT Mo76081, ㅇ, ML 38mm, 22¹4.5'S, $156^{\circ} 24^{\prime} \mathrm{E}$, 04/12/1975, RFRL RV Soyo-Maru Stn SP60; NMNZ M.172941,, , ML 90mm, §̊, ML 
$59 \mathrm{~mm}, 26^{\circ} 31.42 \mathrm{~S}, 166^{\circ} 34.42^{\prime} \mathrm{E}$, NNW of Norfolk Island, 2000m, 18/05/2003, RV

Tangaroa, NORFANZ Stn 5; NMNZ M.172966, ${ }^{\top}$, ML 48mm, 29³1.75'S, $167^{\circ} 38.05^{\prime} \mathrm{E}$, Norfolk Ridge, 1200-200m, 15/05/2003, RV Tangaroa, NORFANZ Stn 23; NMNZ M.172989 (4 sex indet.), ML 26-16mm, $29^{\circ} 31.75^{\prime} \mathrm{S}, 167^{\circ} 38.05^{\prime} \mathrm{E}, \mathrm{S}$ of Norfolk Island, 1200-200m, 15/05/2003, RV Tangaroa, NORFANZ Stn 23; NMV F55013, ð, ML 65mm, $34^{\circ} 26.0^{\prime} \mathrm{S}, 151^{\circ} 25.0^{\prime} \mathrm{E}, 48 \mathrm{~km}$ east of Wollongong, Australia, 550-0m, 25/03/1981, Engels high-lift demersal trawl, CSIRO RV Soela; NMV F50968, , ML 56mm, 36²6-27'S, $151^{\circ} 24-23^{\prime} \mathrm{E}, 95 \mathrm{~km}$ east of Narooma, Australia, 600-475m, 01/12/1978, EMWT 300, CSIRO RV Courageous.

Distribution (Fig. 20A) - Southwest Pacific, off the eastern coast of Australia, 2000Om (based on three records; nearly all known specimens are ex-stomach-content), in the East Australian current. Elsewhere recorded from $15-30^{\circ} \mathrm{S}, 160^{\circ} \mathrm{E}$ to $170^{\circ} \mathrm{W}$ (Rancurel 1970) and the northern Indian Ocean (Rancurel \& Okutani 1990).

Diagnosis - Fins very short, length $\sim 40 \% \mathrm{ML}$; intestinal photophores tiny, may be poorly defined, with anterior only slightly smaller than posterior; ocular photophore uniformly broad, spanning ventral $\sim 25 \%$ eye circumference; chromatophores absent from tentacle stalk proximal to carpus; chromatophores present along oro-ventral stalk ridge; tentacle club length $\sim 19 \% \mathrm{ML}$, with 15-19 short, robust hooks; distal-most ventral hook bases not produced into spike.

Description (Figs 20B-21G) - Mantle muscular, spindle-shaped, width 21-26-32\% ML; gladius visible dorsally as wide, rounded, amber-coloured (in preserved specimens) ridge over anterior $85 \% \mathrm{ML}$; blunt conus visible ventrally through posterior $\sim 5 \% \mathrm{ML}$; ventral mantle surface with fleshy oval pad over posterior $15 \% \mathrm{ML}$, its width $\sim 4 \%$ ML. Fins broadly rhombic, length 35-40-44\% ML, width 69-80-90\% ML; posterior and anterior margins straight; anterior lobes shallow, broadly rounded.

Head slightly narrower than mantle, length $14-18-22 \% \mathrm{ML}$, width $\sim 19 \% \mathrm{ML}$, depth $\sim 17 \% \mathrm{ML}$; dorsal and lateral surfaces convex, ventral surface flat; occipital region with eight small secondary folds. Funnel stout, with aperture level with midpoint of eyes; funnel groove well defined, V-shaped, broad posteriorly, attenuating anteriorly to narrow $\mathrm{V}$, and reaching nearly to bases of Arms IV. Mantle component of locking apparatus $\sim 40 \% \mathrm{ML}$, funnel component $\sim 14 \% \mathrm{ML}$.

Arms $21-29_{I}-34_{\| I I V}-44 \% \mathrm{ML}$, formula $I I=|I|=|V>|$ or $I I|>| V>||>\mid$, each with $50-60$ suckers (ML 50mm), diameter 40-50\% arm width; oral arm faces bordered on each 
Table 8. Measures $(\mathrm{mm}$ ) and counts of Onychoteuthis meridiopacifica Rancurel \& Okutani, 1990 (arm and tentacle measures recorded from more complete side of specimen, indicated $\mathrm{R}$ or $\mathrm{L}$ ).

\begin{tabular}{|c|c|c|c|c|c|c|c|c|c|c|c|}
\hline Specimen ID & $\begin{array}{c}\text { NMNZ } \\
\text { M.172941 }\end{array}$ & $\begin{array}{c}\text { NMV } \\
\text { F55013 }\end{array}$ & $\begin{array}{c}\text { NMNZ } \\
\text { M.172941 }\end{array}$ & $\begin{array}{c}\text { NMV } \\
\text { F50968 }\end{array}$ & $\begin{array}{c}\text { NSMT } \\
\text { Mo76079 }\end{array}$ & $\begin{array}{c}\text { NSMT } \\
\text { Mo76087 }\end{array}$ & $\begin{array}{c}\text { NSMT } \\
\text { Mo76085 }\end{array}$ & $\begin{array}{c}\text { NMNZ } \\
\text { M.172966 }\end{array}$ & $\begin{array}{c}\text { NSMT } \\
\text { Mo76087 }\end{array}$ & \multicolumn{2}{|c|}{$\begin{array}{c}\text { Mean } \\
\text { Indices }\end{array}$} \\
\hline Type Status & None & None & None & None & None & None & None & None & None & \multirow{2}{*}{\multicolumn{2}{|c|}{ (ML 41-65) }} \\
\hline ML & 90 & 65 & 59 & 56 & 54 & 54 & 49 & 48 & 41 & & \\
\hline Sex & $\mathrm{F}$ & M & $M$ & $\mathrm{~F}$ & M & M & M & $M$ & $\mathrm{~F}$ & & \\
\hline MW & 35 & 15 & 13 & 15 & 16 & 15 & 11 & 10 & 13 & MWI & 26 \\
\hline HL & 13 & 10 & 11 & 8 & 9 & 11 & 8 & 8 & 9 & HLI & 18 \\
\hline HW & 16 & 13 & 10 & 11 & 10 & 11 & 9 & 10 & 8 & HWI & 19 \\
\hline $\mathrm{FL}$ & 32 & 23 & 23 & 23 & 23 & 22 & 20 & 19 & 18 & FLI & 40 \\
\hline FW & 71 & 45 & 49 & 42 & 48 & 44 & 39 & 35 & 37 & FWI & 80 \\
\hline Arms I & $24 \mathrm{R}$ & $17 \mathrm{R}$ & $19 \mathrm{~L}$ & $12 \mathrm{~L}$ & $17 \mathrm{~L}$ & $18 \mathrm{~L}$ & $12 \mathrm{~L}$ & $12 \mathrm{R}$ & $14 \mathrm{~L}$ & A1I & 29 \\
\hline Arms II & $29 R$ & $22 \mathrm{R}$ & $24 \mathrm{~L}$ & $15 \mathrm{~L}$ & $17 \mathrm{~L}$ & $18 \mathrm{~L}$ & $17 \mathrm{~L}$ & $13 R$ & $13 \mathrm{~L}$ & A2I & 32 \\
\hline Arms III & $28 \mathrm{R}$ & $24 \mathrm{R}$ & $23 \mathrm{~L}$ & $18 \mathrm{~L}$ & $20 \mathrm{~L}$ & $20 \mathrm{~L}$ & $14 \mathrm{~L}$ & $13 R$ & $13 \mathrm{~L}$ & A3I & 34 \\
\hline Arms IV & $27 \mathrm{R}$ & $23 R$ & $26 \mathrm{~L}$ & $17 \mathrm{~L}$ & $18 \mathrm{~L}$ & $21 \mathrm{~L}$ & $17 \mathrm{~L}$ & $12 \mathrm{R}$ & $13 \mathrm{~L}$ & A4I & 34 \\
\hline TnL & - & $34 \mathrm{R}$ & $31 \mathrm{~L}$ & $25 \mathrm{~L}$ & $28 \mathrm{~L}$ & $36 \mathrm{~L}$ & $21 \mathrm{~L}$ & $19 \mathrm{R}$ & $24 \mathrm{~L}$ & TnLI & 51 \\
\hline $\mathrm{CL}$ & - & $12 \mathrm{R}$ & $12 \mathrm{~L}$ & $9 \mathrm{~L}$ & $10 \mathrm{~L}$ & $11 \mathrm{~L}$ & $9 \mathrm{~L}$ & $8 \mathrm{R}$ & $9 \mathrm{~L}$ & CLI & 19 \\
\hline CS & - & 7 & 6 & 6 & $6^{*}$ & 7 & 8 & 7 & 7 & & \\
\hline MH & - & 18 & * & 19 & $14^{*}$ & 15 & 17 & 19 & 16 & & \\
\hline MS & - & D1-4, V1-4 & * & D1-4, V1-4 & D1-3, V1, 2 & D1-3, V1, 2 & D1-3, V1 & D1-4, V1-4 & $\mathrm{D} 1-4, \mathrm{~V} 1,2$ & & \\
\hline TPS & - & 11 & * & 9 & * & 7 & * & ${ }^{*}$ & 8 & & \\
\hline
\end{tabular}

- indicates missing features, ${ }^{*}$ indicates damaged features.

side by low trabeculate membrane. Keels present on Arms I-III: those on Arms I and II $\sim 10 \%$ arm width/depth along entire arm length; those on Arms III attaining $90 \%$ arm depth at $25 \%$ arm length, thereafter tapering to arm tips; Arms IV with lateral membrane $30 \%$ arm width over entire arm length.

marginal suckers bordering proximal two to five hook pairs through at least ML $65 \mathrm{~mm}$; terminal pad with seven to 11 suckers. Hooks (Figs 20E, 21A) robust, close-set, with thick necks and long, strongly recurved claws. Proximal hook pair subequal in size, with ventral hook larger than paired dorsal hook in all subsequent pairs; hooks D4 and D5 not markedly smaller or offset toward midline. Largest ventral hooks (V4 and V5) two to three times longer than paired dorsal hooks and $\sim 16 \% \mathrm{CL}$; bases of largest ventral hooks slightly asymmetrical but lacking ventral spikes. Dorsal club membrane narrow, $\sim 10 \%$ club width, bordering hooks D1-D3; ventral membrane scarcely evident, $\sim 15 \%$ club width, extending along entire club length; dorsal keel $\sim 80 \%$ club width, originating near hook D3 and continuing to club tip.

Lateral profile of lower beak (Figs 21B-D, ML 90mm) longer than deep, with crest sloping gently toward baseline; hood domed, with posterior edge close to crest; hood length $\sim 60 \%$ crest length, and hood and crest together $\sim 60 \%$ baseline; jaw edge straight proximally, concave distally near rostral tip; jaw angle obtuse, slightly obscured by low, indistinct wing fold. Crest moderately broad; shoulder ridge distinct; angle ridge well developed but short, spanning $\sim 40 \%$ lateral wall depth; angle point distinct, not raised, darkly pigmented; distal wing width $\sim 200 \%$ that at jaw angle, wing length 
$\sim 200 \%$ LRL, with small insert of cartilage below jaw angle; lateral wall diagonally bisected by dark, prominent ridge and fold, remaining distinct to just above posterior corner of lateral wall.

Radula (Fig. 20E) with tricuspid rachidian; mesocone triangular, slender distally; lateral cusps low, $15-20 \%$ total rachidian height, slightly laterally directed. First lateral tooth
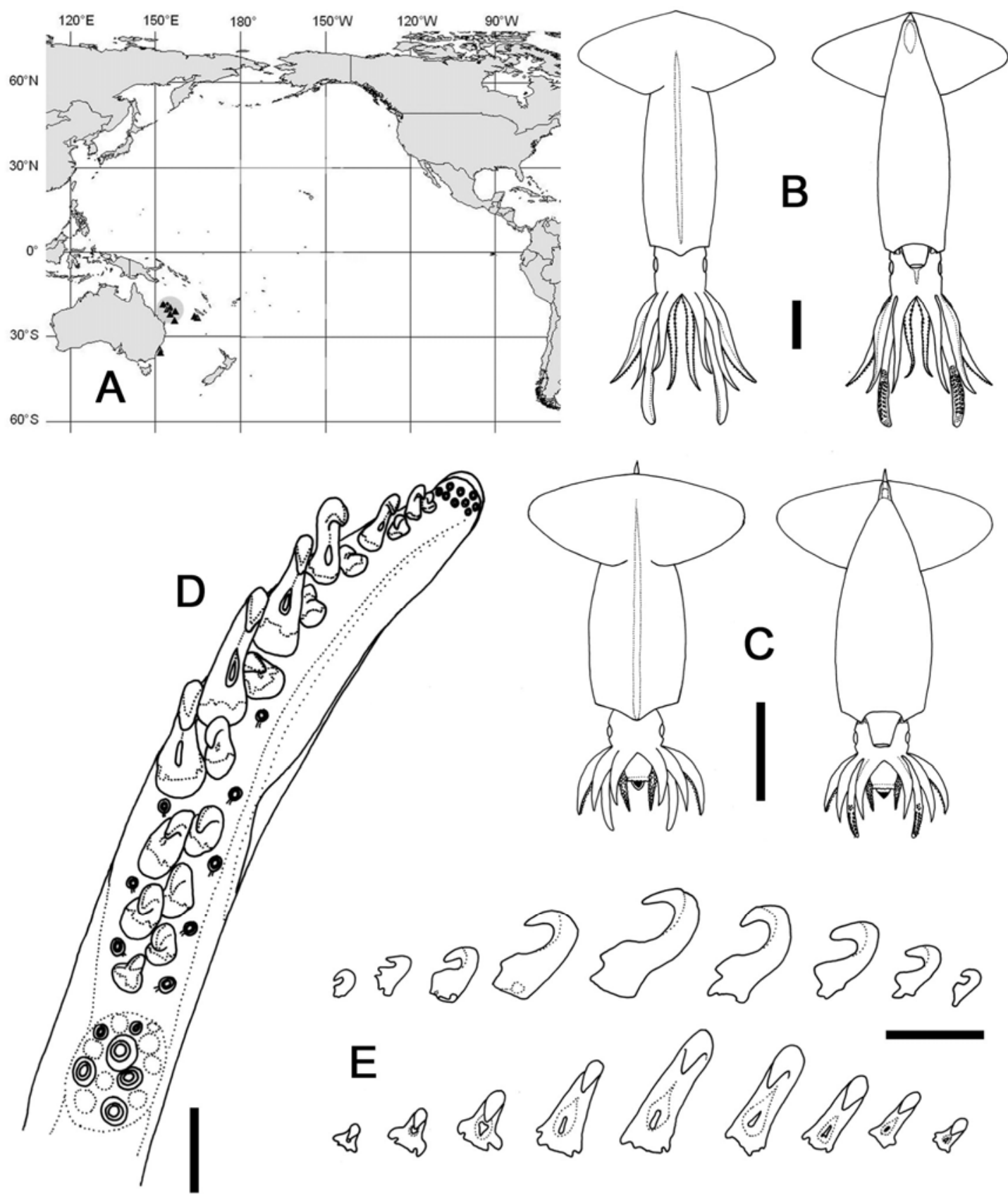

Fig. 20 - Onychoteuthis meridiopacifica. A) distribution (grey circle indicates type locality); B) NMNZ M.172941, ô, ML 59mm; C) NMNZ M.172989, sex indet., ML 26mm; D, E) NSMT Mo76059,,+ ML 48mm: D) right tentacular club, E) right ventral tentacular hook series. Scale bars = B, C) $10 \mathrm{~mm}$; D, E) $1 \mathrm{~mm}$. 
subequal to rachidian, with single straight or slightly curved cusp oriented toward rachidian, with distal margin of base concave, forming small outer corner. Second lateral tooth simple, slightly curved, only slightly taller than rachidian. Marginal tooth simple, slightly curved, $\sim 130 \%$ height of rachidian. Palatine palp (Fig. $21 \mathrm{~F}$ ) with $\sim 45$ robust, flat teeth, each $130-310 \%$ rachidian height; those near middle of palp largest, those near edges of palp more slender, curved outward away from surface of palp and set more densely.
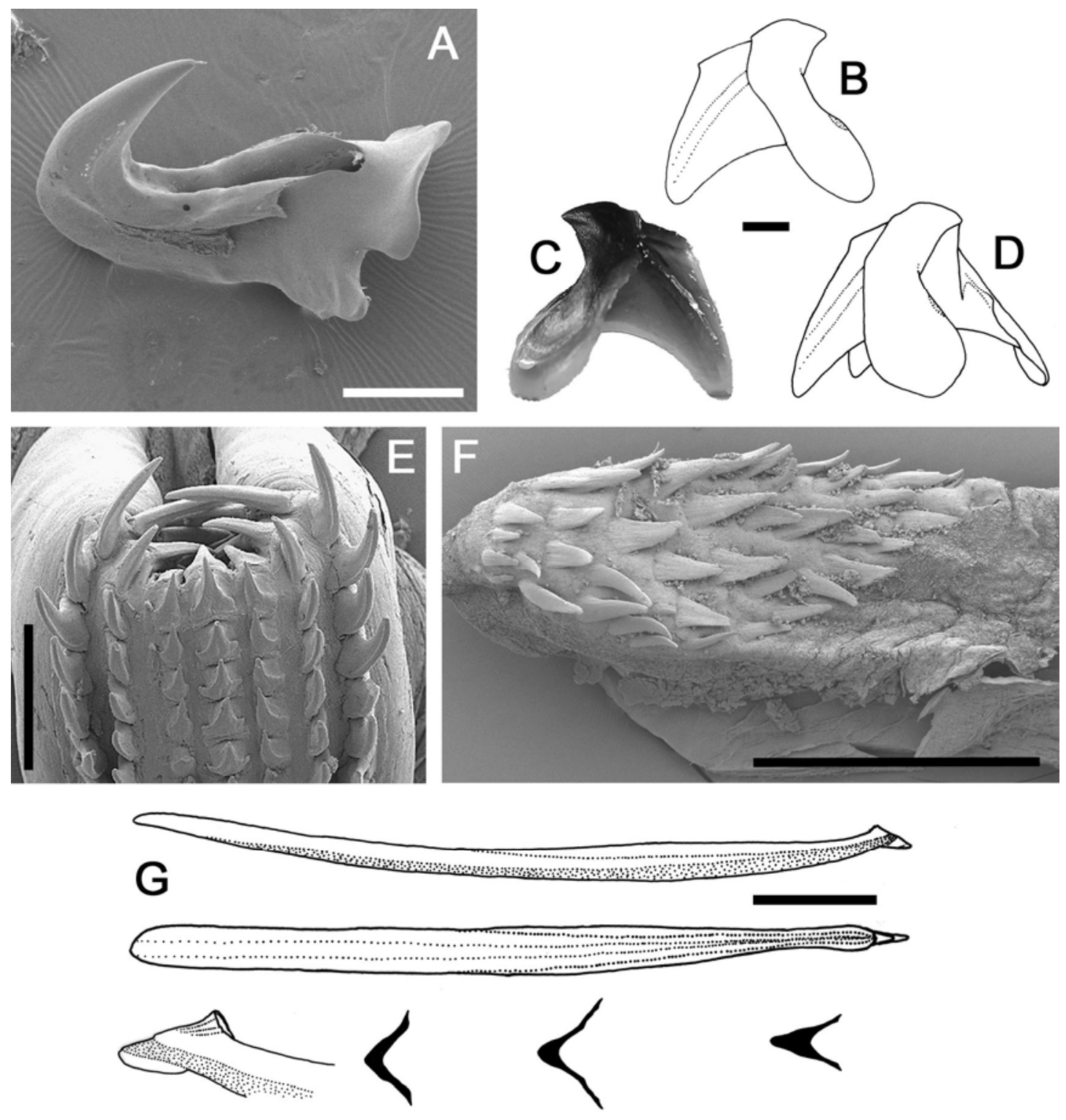

Fig. 21 - Onychoteuthis meridiopacifica. A) NMV F50968, q, ML 56mm; B-F) NMNZ M.172941, ᄋ, ML 90mm; G) NSMT Mo76067, ô, ML 59mm. A) right tentacular hook V3; B-D) lower beak, B) left, C) right, D) left oblique profiles; E) radula; F) right palatine palp; G) gladius. Scale bars = A) $200 \mu \mathrm{m}, \mathrm{B}, \mathrm{C}$, F) $1 \mathrm{~mm}$; E) $500 \mu \mathrm{m}$; F) $10 \mathrm{~mm}$. 
Gladius (Fig. 21G) with greatest width ( $6 \% \mathrm{GL}$ ), attained at $\sim 50 \% \mathrm{GL}$; free rachis $\sim 40 \% \mathrm{GL}$; vanes broaden slightly between 40 and $50 \% \mathrm{GL}$, then taper gradually until $85 \%$ GL; dorsal keel comprises $\sim 50 \%$ gladius depth at $\sim 85 \%$ GL. Rostrum $\sim 4 \%$ GL, stubby, triangular in lateral and ventral profiles, with conus recurved to form small, flared, pleated cup.

Overall colouration dark reddish-purple, darkest medially on dorsal surface of mantle, fins and head; lateral surfaces of mantle and head silvery to coppery iridescent. Arms and tentacles darkest aborally, paling laterally, palest on oral surfaces.

Smaller specimens (ML 15-25mm, Fig. 20C) deviate from the above description only in having somewhat different bodily proportions: mantle bulbous anterior to fins, width $35-40 \% \mathrm{ML}$, thereafter tapering rapidly and pierced posteriorly by rostrum of gladius; no fleshy ventral keel present; fins together ovate in outline, width $\sim 90 \% \mathrm{ML}$, posterior margins forming nearly straight line across posterior extremity of mantle; head length, width and depth all $\sim 20 \% \mathrm{ML}$; secondary occipital folds not developed, primary folds present; mantle component of locking apparatus $\sim 46 \% \mathrm{ML}$, funnel component $\sim 19 \%$ ML; buccal bulb protruding from arm crown; arm length 25-35\%, each with $~ 30$ closeset suckers; aboral keels forming only on Arms III; tentacles slender, appearing flimsy, length $\sim 35 \% \mathrm{ML}$; club length $\sim 15 \% \mathrm{ML}$ ( $\sim 33 \% \mathrm{TnL})$; suckers and hooks damaged or absent in all specimens examined (sheaths present); one or two dark 'tail spots' present beneath fins on either side of mantle at $M L 16-20 \mathrm{~mm}$, but indistinct by $\mathrm{ML}$ $26 \mathrm{~mm}$.

Spent female (ML $90 \mathrm{~mm}$ ) with proportions similar to those of subadult and adult specimens as described above, but with tissues stretched, flabby; mid-portion of ventral mantle surface with two slightly diagonal longitudinal cuts, $\sim 15 \% \mathrm{ML}$, nearly symmetrical across midline, each with 25-30 embedded spermatangia, anchored within mantle wall; tentacles absent (short stumps remaining).

Remarks - Rancurel (1970) recognised Os. meridiopacifica (his 'Onychoteuthis sp.') as distinct from Os. 'banksii' based on its small size at maturity and its broad, short fins. Additional distinctive character states include the small intestinal photophores, and robust tentacular hooks, lacking the more pronounced asymmetry and ventral spikes seen in congeners. 
Onychoteuthis lacrima Bolstad \& Seki, in press (Tables 2, 9, Figs 22, 23)

Onychoteuthis sp. (undescribed new species) - Bonnaud et al. (1998): 1761-1770, Fig. 6.

Onychoteuthis sp. D Seki, 2001: 44-50, Figs 14, 15.

Onychoteuthis lacrima Bolstad \& Seki, in press.

Type material examined - SBMNH 423106, holotype, $q$, ML 94mm, $37^{\circ} 59^{\prime} \mathrm{N}, 179^{\circ} 28^{\prime} \mathrm{W}, 154-$ Om, 06/08/1991, 2142-2242h, TC 91-06, Stn 15, CMT, NMFS S-0188, KSB-007; KSB-

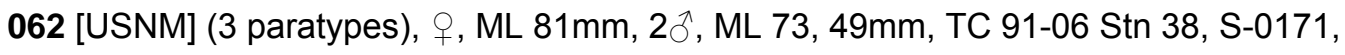
NFD; SBMNH 423108, paratype, $\hat{\partial}, \mathrm{ML} 72 \mathrm{~mm}$, NW Hancock Seamount [ $29^{\circ} 47^{\prime} \mathrm{N}$, $179^{\circ} 04^{\prime} E$ ], 10/07/1983, TC 83-02, CMT, NMFS S-0528, KSB-009; SBMNH 423107, paratype, + , ML 54mm, $37^{\circ} 59^{\prime} \mathrm{N}, 179^{\circ} 28^{\prime} \mathrm{W}, 154-0 \mathrm{~m}, 06 / 08 / 1991,2142-2242 \mathrm{~h}$, TC $91-$ 06, Stn 15, CMT, NMFS S-0188, KSB-007.

Material examined (39 specimens) - KSB-042, 8 sex indet., ML $82-54 \mathrm{~mm}, 40^{\circ} 06^{\prime} \mathrm{N}$, $179^{\circ} 41^{\prime} \mathrm{W}, 08 / 08 / 1991$, TC 91-06 Stn 39, CMT, NMFS S-0164; NSMT Mo75319, 8 sex indet., ML 91-38mm, 40 $01.3^{\prime} \mathrm{N}, 144^{\circ} 44.3^{\prime} \mathrm{E}$, off Sanriku, Japan, 15m, 16/07/1996, RV Marusada-Maru, MWT; NSMT Mo66701, ㅇ, ML 79mm, 39¹3.2' N, $147^{\circ} 08.7^{\prime} \mathrm{E}$, 30/09/1988, 1800-1830h, Stn SR-72; NSMT Mo75326, sex indet., ML 46mm, $39^{\circ} 00.6^{\prime} \mathrm{N}, 143^{\circ} 32.1^{\prime} \mathrm{E}$, off Sanriku, Japan, 650m, 29/07/1996, RV Marusada-Maru,

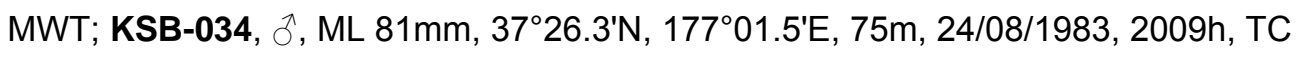
83-03 Stn 12-1, CMT, coll. WMM and VAH, NMFS S-0051; KSB-040, 5 sex indet., ML 89-70mm, 37²3.0'N, 176 57.7'E, 150m, 24/08/1983, 2200h, TC 83-03 Stn 12-2, CMT, coll. WMM and VAH, NMFS S-0031; NSMT Mo66889, 4 sex indet., ML 76-63mm, $37^{\circ} 00.5^{\prime} \mathrm{N}, 142^{\circ} 58.8^{\prime} \mathrm{E}, 1 \mathrm{~m}, 27 / 07 / 1972,1000 \mathrm{~h}, \mathrm{RV}$ Soyo-Maru Stn 1-7, HN; KSB-036, ㅇ, ML 93mm, 36 $59^{\circ} \mathrm{N}, 179^{\circ} 29^{\prime} \mathrm{W}, 182-0 \mathrm{~m}, 05 / 08 / 1991,2308-2358 \mathrm{~h}$, TC $91-06 \mathrm{Stn} 6$, CMT, NMFS S-0161; NSMT Mo66608, $0^{\top}$, ML 112mm, 3501. $8^{\prime} \mathrm{N}, 174^{\circ} 58.7^{\prime} \mathrm{E}$, 30/08/1985, Stn 27, hand-jig; NSMT Mo66883, + , ML 76mm, 34 $58.8^{\prime} \mathrm{N}, 157^{\circ} 06.8^{\prime} \mathrm{E}$, surface, 21/05/1972, 2010-2039h, RV Soyo-Maru Stn 78, HN; KSB-049, , , ML $108 \mathrm{~mm}, 34^{\circ} 00.0^{\prime} \mathrm{N}, 177^{\circ} 30.5^{\prime} \mathrm{E}, 75 \mathrm{~m}, 26 / 08 / 1983$, 2008h, TC 83-03 Stn 14-1, CMT, coll. WMM and VAH, NMFS S-0042; KSB-039, 5 sex indet., ML $81-53 \mathrm{~mm}, 33^{\circ} 56.5^{\prime} \mathrm{N}$, $177^{\circ} 28.8^{\prime} \mathrm{W}, 150 \mathrm{~m}, 26 / 08 / 1983,2140 \mathrm{~h}, \mathrm{TC}$ 83-03 Stn 14-2, CMT, coll. WMM and VAH, NMFS S-0038; NSMT Mo66887, ㅇ, ML 92mm, 3006'N, 142¹1'E, 13/11/1971, 0130h, RV Soyo-Maru Stn B6, coll. G. Honma; NSMT Mo66888, $\partial^{\jmath}, \mathrm{ML} 91 \mathrm{~mm}, 29^{\circ} 00^{\prime} \mathrm{N}$, $135^{\circ} 00^{\prime} \mathrm{E},-/ 06 / 1969, \mathrm{NFD}$.

Unlocalised material examined (4 specimens) - KSB-041, 4 sex indet., ML 80-49mm, TC 9106 Stn 38, S-0171, NFD.

Distribution (Fig. 22A) — Shallow, north-central Pacific waters (200-0m), primarily 30$45^{\circ} \mathrm{N}$ and $170^{\circ} \mathrm{W}$ to $170^{\circ} \mathrm{E}$; also found off eastern Japan. 
Table 9. Measures and counts of Onychoteuthis lacrima Bolstad \& Seki, in press (arm and tentacle measures recorded from more complete side of specimen, indicated $\mathrm{R}$ or $\mathrm{L}$ ).

\begin{tabular}{|c|c|c|c|c|c|c|c|c|c|c|}
\hline Specimen ID & $\begin{array}{l}\text { SBMNH } \\
423106\end{array}$ & $\begin{array}{c}\text { KSB-062 } \\
\text { [USNM] }\end{array}$ & $\begin{array}{c}\text { KSB-062 } \\
\text { [USNM] }\end{array}$ & $\begin{array}{l}\text { SBMNH } \\
423108\end{array}$ & $\begin{array}{c}\text { SBMNH } \\
423107\end{array}$ & $\begin{array}{c}\text { KSB-062 } \\
\text { [USNM] }\end{array}$ & KSB-036 & KSB-034 & \multicolumn{2}{|c|}{$\begin{array}{c}\text { Mean } \\
\text { Indices }\end{array}$} \\
\hline Type Status & Holotype & Paratype & Paratype & Paratype & Paratype & Paratype & None & None & \multirow{2}{*}{\multicolumn{2}{|c|}{ (ML 49-94) }} \\
\hline ML & 94 & 81 & 73 & 72 & 54 & 49 & 93 & 81 & & \\
\hline Sex & $\mathrm{F}$ & $\mathrm{F}$ & $\mathrm{M}$ & $M$ & $\mathrm{~F}$ & $M$ & $\mathrm{~F}$ & $M$ & & \\
\hline MW & 18 & 16 & 15 & 15 & 12 & 11 & 19 & 18 & MWI & 21 \\
\hline HL & 22 & 16 & 17 & 16 & 12 & 11 & 18 & 18 & HLI & 24 \\
\hline HW & 17 & 13 & 13 & 13 & $11^{*}$ & 9 & 22 & 15 & HWI & 20 \\
\hline FL & 55 & 48 & 41 & 42 & 31 & 27 & 57 & 49 & FLI & 58 \\
\hline FW & 63 & 60 & 55 & 51 & 43 & 41 & 66 & 54 & FWI & 73 \\
\hline Arms I & $33 R$ & $26 \mathrm{~L}$ & $25 \mathrm{~L}$ & $26 \mathrm{R}$ & $17 \mathrm{~L}$ & $15 \mathrm{~L}$ & $30 \mathrm{~L}$ & $22 \mathrm{~L}$ & A1I & 32 \\
\hline Arms II & $45 R$ & $33 \mathrm{~L}$ & $32 \mathrm{~L}$ & $32 R$ & $25 \mathrm{~L}$ & $18 \mathrm{~L}$ & $44 \mathrm{~L}$ & $31 \mathrm{~L}$ & A2I & 43 \\
\hline Arms III & $43 R$ & $34 \mathrm{~L}$ & $32 \mathrm{~L}$ & $31 \mathrm{R}$ & $22 \mathrm{~L}$ & $22 \mathrm{~L}$ & $41 \mathrm{~L}$ & $33 \mathrm{~L}$ & A3I & 43 \\
\hline Arms IV & $42 \mathrm{R}$ & $37 \mathrm{~L}$ & $33 \mathrm{~L}$ & $26 \mathrm{R}$ & $21 \mathrm{~L}$ & $21 \mathrm{~L}$ & $42 \mathrm{~L}$ & $32 \mathrm{~L}$ & A4I & 42 \\
\hline TnL & 87R & $61 \mathrm{~L}$ & $56 \mathrm{~L}$ & $61 \mathrm{R}$ & $41 \mathrm{~L}$ & $47 \mathrm{~L}$ & $80 \mathrm{~L}$ & $65 \mathrm{~L}$ & TnLI & 88 \\
\hline CL & $30 \mathrm{R}$ & $21 \mathrm{~L}$ & $20 \mathrm{~L}$ & $20 \mathrm{R}$ & $15 \mathrm{~L}$ & $15 \mathrm{~L}$ & $30 \mathrm{~L}$ & $23 \mathrm{~L}$ & CLI & 29 \\
\hline CS & 9 & 8 & 9 & 9 & 9 & 7 & 9 & 9 & & \\
\hline MH & 22 & 21 & 21 & 20 & 22 & 22 & 24 & 22 & & \\
\hline MS & 0 & 0 & 0 & 0 & 0 & 0 & 0 & 0 & & \\
\hline TPS & 14 & 15 & 13 & 15 & 14 & 13 & 12 & 16 & & \\
\hline
\end{tabular}

* indicates damaged features.

Diagnosis - Posterior intestinal photophore oval or tear-drop-shaped, usually well defined; anterior intestinal photophore circular, minute, ill-defined and occasionally indiscernible; ocular photophore broad posteriorly, tapering to point anteriorly; chromatophores absent from tentacle stalk proximal to carpus and along oro-ventral stalk ridge, but present over entire ventral surface of mantle; tentacle club $26-32 \% \mathrm{ML}$, with 20-24 long, slender hooks; distal-most ventral hooks with large, ventral, orolaterally directed basal spike.

Description (Figs 22B-23H) - Mantle anteriorly cylindrical, width 19-21-25\% ML; keel of gladius raised through dorsal midline, forming dark ridge; rostrum of gladius visible ventrally through posterior $\sim 5 \% \mathrm{ML}$; mantle robust posteriorly, with small fleshy keel, $\sim 15 \%$ ML. Posterior intestinal photophore oval or tear-drop-shaped, occasionally with indistinct borders; anterior intestinal photophore greatly reduced, generally circular in outline but often indistinct and occasionally indiscernible. Fins rhombic, length generally $54-58-61 \% \mathrm{ML}$, width $69-73-84 \%$ at $\mathrm{ML}>70 \mathrm{~mm}$, broader at $\mathrm{ML} 49-69 \mathrm{~mm}$, occasionally $85-90 \% \mathrm{ML}$; posterior margins straight to slightly concave distally, anterior margins gently convex; lateral 'corners' sharp; anterior lobes small, rounded.

Head cylindrical, length 15-24-32\% ML, width 16-20-25\% ML; depth 15-18\% ML; not constricted anterior to brachial crown; occipital region with seven to nine secondary folds. Funnel groove V-shaped, broad posteriorly, becoming acute anteriorly, reaching 
to point directly below midpoint of eyes. Ocular photophore broad, rounded anteriorly, tapering to narrow point posteriorly, spanning ventral $\sim 40 \%$ eye circumference. Funnel component of locking apparatus 13-21\% ML; mantle component 26-32\% ML.

Arms muscular, $27-32-43_{I I-I V}-48 \% \mathrm{ML}$, formula II=III=IV>I; each with $80-90$ suckers (ML 72-94mm; $\sim 60$ at ML 49mm), each with a small fleshy distal knob. Aboral keels present on Arms I-III: that on Arms I 10\% arm width along entire arm length; that on Arms II $\sim 30 \%$ arm depth along entire arm length; that on Arms III $\sim 130 \%$ arm depth at

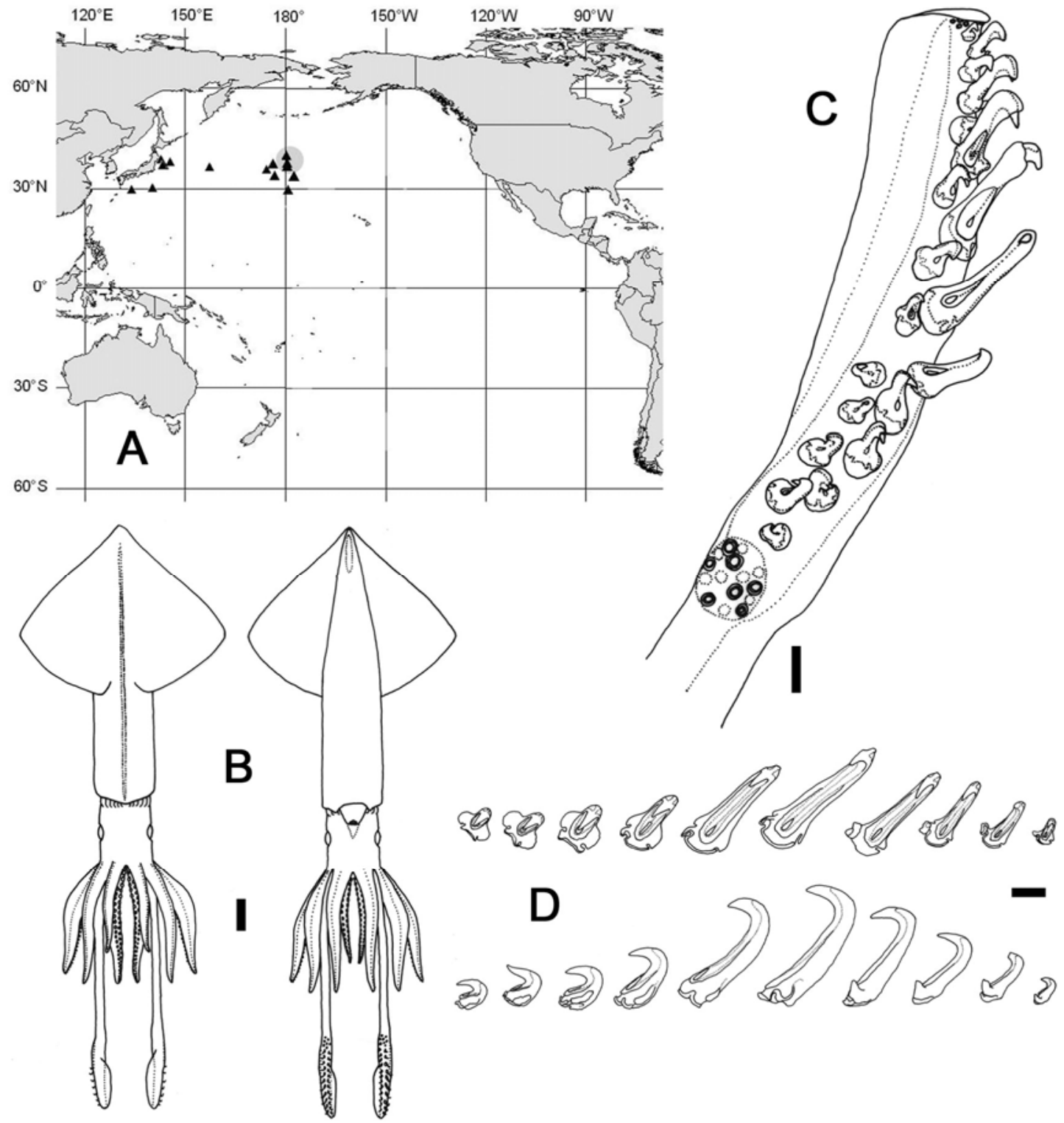

Fig. 22 - Onychoteuthis lacrima. A) distribution (grey circle indicates type locality); B) SBMNH 423106, holotype, , , ML 94mm; C) SBMNH 423109, paratype, ô, ML 72mm, left tentacular club, D) KSB-062,, , ML $81 \mathrm{~mm}$, right ventral tentacular hook series. Scale bars = B) $10 \mathrm{~mm}$; C, D) $1 \mathrm{~mm}$. 
$\sim 40 \%$ arm length, narrowing thereafter; lateral membrane on Arms IV $\sim 100 \%$ arm width along entire arm length.

Tentacles robust, length $72-88-128 \% \mathrm{ML}$; stalk bases comparably thick to adjacent arms; aboral surface of stalk with low keel, $\sim 10 \%$ stalk thickness. Club (Fig. 22C) slightly expanded, length $26-29-32 \% \mathrm{ML}(24-38 \% \mathrm{TnL})$; carpus circular to slightly ovoid in outline, with seven to ten suckers (usually nine); manus with 20-24 hooks (usually 21 or 22) (Figs 22D, 23A, 23B); terminal pad with 12-16 suckers (usually 13 or 14). Hooks D1 and D2 subequal to paired ventral hooks; dorsal hooks diminish in size thereafter through hook D5 or D6, with D4 and D5 (or D5 and D6) slightly off-set toward centre of club; hook D6 or D7 suddenly enlarged (similar in size to proximalmost dorsal hooks), with dorsal hooks thereafter decreasing in size distally. Largest ventral hooks (V6-V8) (Fig. 23A) with slightly curved necks and acutely recurved claws $\left(80-90^{\circ}\right)$ in lateral profile; longest hook 18-25\% club length (mean 20\%) and four to six times longer than paired dorsal hook; hooks V7 or V8 through distal-most ventral hook with large, oro-laterally directed spike on ventral side of hook base (Fig. 23B). Ventral club membrane $\sim 50 \%$ club width along hooks $\mathrm{V} 1-\mathrm{V} 5$, narrowing thereafter to $\sim 10 \%$ club width along remainder of club length; dorsal membrane $\sim 30 \%$ club width, flanking hooks D1-D4; dorsal keel $\sim 100 \%$ club width, from hook D2 or D3 through tip of club.

Lateral profile of lower beak (Figs 23C-E, ML 81mm) longer than deep, with crest sloping steeply toward baseline; posterior edge of hood well above crest; hood length $\sim 45 \%$ total crest length, and hood and crest together $\sim 80 \%$ baseline; jaw edge straight proximally, with rostrum produced into sharp or slightly rounded tip; jaw angle obtuse, not obscured laterally by wing fold; shoulder ridge sharp. Crest narrow, rounded, slightly thickened; lateral walls slightly indented between crest and lateral wall fold; angle ridge well defined but short, spanning $\sim 50 \%$ lateral wall depth; distal wing width $\sim 120 \%$ that at jaw angle, wing length $\sim 200 \%$ LRL, with small triangular insert of cartilage below jaw angle; lateral wall diagonally bisected by dark, prominent ridge and fold, remaining distinct to lateral wall corner.

Radula (Fig. 23F) with tricuspid rachidian, with short, narrow, triangular mesocone and narrow, rounded, slightly laterally directed lateral cusps, their height $\sim 40 \%$ that of mesocone height. First lateral tooth unicuspid, straight, slightly shorter than rachidian and of similar breadth, with base shorter on inner edge of tooth than outer, with margins of base often indistinguishable from margins of cusp. Second lateral tooth 
simple, robust, straight, short, $\sim 120 \%$ rachidian height. Marginal tooth simple, curved, $\sim 250 \%$ rachidian height. Palatine palp (Fig. 23G) with $\sim 65$ short, flat, poorly defined teeth, each $70-160 \%$ rachidian height, curving slightly away from surface of palp, densely distributed over palp.

Gladius (Fig. $23 \mathrm{H})$ with greatest width ( $5 \% \mathrm{GL})$ attained at $\sim 35 \% \mathrm{GL}$; free rachis $\sim 23 \% \mathrm{GL}$; vanes broaden rapidly from 25 to $35 \% \mathrm{GL}$, then taper gradually until $85 \%$ GL; dorsal keel solid, deep, comprising nearly entire gladius depth by $\sim 70 \% \mathrm{GL}$.
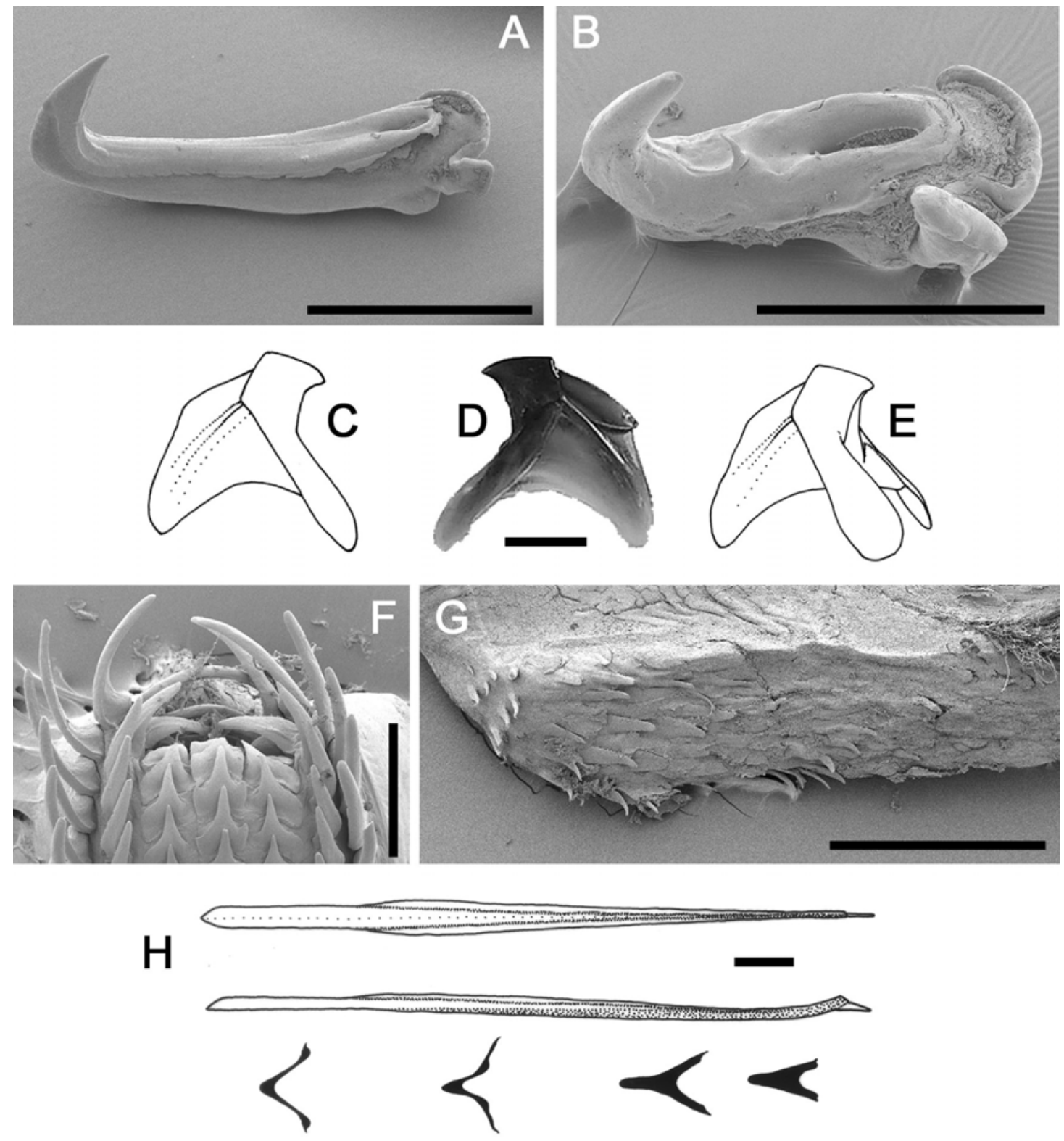

Fig. 23 - Onychoteuthis lacrima, KSB-062, paratype,, , ML $81 \mathrm{~mm}$. A) right tentacular hooks: (A) V6, (B) V10; C-E) lower beak: (C) left, (D) right, (E) left oblique profiles; F) radula; G) palatine palp; H) gladius. Scale bars = A) $2 \mathrm{~mm}$; B, F) $500 \mu \mathrm{m} ; \mathrm{C}, \mathrm{D}, \mathrm{G}) 1 \mathrm{~mm}$; H) $10 \mathrm{~mm}$. 
Conus (including rostrum) $\sim 4 \% \mathrm{GL}$; rostrum triangular in lateral profile, narrow in dorsal profile.

Remarks - The paralarva of Os. lacrima was described and illustrated by Seki (2001, 'sp. D'), based on character states (most obviously the absence of chromatophores on the posterior ventral region ('belly') of the mantle) that distinguished it from paralarvae of three other Onychoteuthis species - Os. compacta, Os. spp. B and C recognised from Hawai'ian waters by Young \& Harman (1987)). Ontogenetic series of specimens attributed to sp. D can be tracked from paralarval to subadult stages, characterised by the absent or minute anterior intestinal photophore and oval- to teardrop-shaped posterior intestinal photophore, the presence of chromatophores over the entire ventral mantle surface, and other subadult characters (see Table 2). Of locally occurring congeners, Os. lacrima is most similar to Os. aequimanus and Os.

borealijaponica, but it is readily separable from the former by the shape and size of the intestinal photophores and by the presence of large ventral basal spikes on the distalmost ventral tentacular hooks, and generally from the latter by the number of tentacular hooks. The full complement of tentacular hooks has earlier been described for this species by ML 70mm (Seki 2001), but herein is reported for specimens by ML $49 \mathrm{~mm}$.

All available material has been collected from a relatively small area within the North Pacific Transition Zone (approximately $30-40^{\circ} \mathrm{N}$ and $170^{\circ} \mathrm{E}-170^{\circ} \mathrm{W}$, Fig. $22 \mathrm{~A}$ ), and off Japan. The distribution of Os. lacrima thus overlaps with that of Os. borealijaponica (Fig. 17A) and Os. compacta (Fig. 15A), but not that currently recognised for Os. aequimanus (Fig. 10A).

Os. lacrima was included in Bonnaud et al.'s (1998) phylogenetic study of the Onychoteuthidae, as 'Onychoteuthis sp. (undescribed new species)' (R. Young, University of Hawai'i, pers. comm.). It appeared less closely related to Os. compacta and Os. prolata than the latter two were to each other, but more closely related to them than was the next closest taxon, Gen. nov. 1 knipovitchi. 
Onychoteuthis prolata Bolstad, Vecchione \& Young, in press (Tables 2, 10, Figs $24,25)$

? Onychoteuthis sp. B Young \& Harman, 1987 - 313-321, Figs 1-4, 7, Tables 1-3; Sweeney et al. (1992): 129, Fig. 180; Bonnaud et al. (1998): 1761-1770, Fig. 6; Seki (2001): 4450 .

Onychoteuthis prolata Bolstad, Vecchione \& Young (in press).

Type material examined - SBMNH 423101, holotype,, , ML 135mm, off Kona Coast of Hawai'i [ 19³8.6'N, 15959.39'W], surface, 10/09/1980, 0145h, RV Kana Keoki, dipnet; USNM 1102755, paratype,, , ML 155mm, Honolulu, HI, 19632, Stn CHG-7, coll. C. Gilbert, NFD.

Additional material examined (10 specimens) — UMML 31.1513, sex indet., ML 71mm, 29²7$25^{\prime} \mathrm{N}, 70^{\circ} 08-09^{\prime} \mathrm{W}$, surface, 06/08/1964, RV Pillsbury Stn 161; UMML 31.92, 今̂, ML

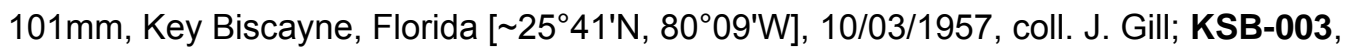

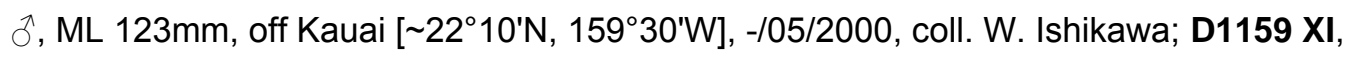
sex indet., $\mathrm{ML} 76 \mathrm{~mm}, 17^{\circ} 55^{\prime} \mathrm{N}, 24^{\circ} 35^{\prime} \mathrm{W}$, surface, 30/10/1921, 0145h, Dana Expedition Stn 1159 IX; UMML 31.2959, ㅇ, ML 59mm, $13^{\circ} 36^{\prime} \mathrm{N}, 60^{\circ} 45^{\prime} \mathrm{W}, 1298 \mathrm{~m}, 09 / 03 / 1966$, RV Oregon Stn 5953; USNM 813418,, , ML $127 \mathrm{~mm}, 00^{\circ} 52^{\prime} \mathrm{S}, 139^{\circ} 52^{\prime} \mathrm{W}, 200-0 \mathrm{~m}$, 25/09/1956, RV H.M. Smith, NL\&D; MNHN 637, o, ML 83mm, Bahia, Brazil [ 15S, $38^{\circ} \mathrm{W}$ ], 1844, coll. Lemesle, NFD; BMNH 20070532, , ML 78mm, $18^{\circ} 27^{\prime} \mathrm{S}, 03^{\circ} 29^{\prime} \mathrm{E}$, 12/06/1937, 'Discovery' Expedition, on deck; SAM S299 2ㅇ, ML 109, $91 \mathrm{~mm}, 23^{\circ} 20^{\prime} \mathrm{S}$, $53^{\circ} 56^{\prime} \mathrm{E}, 02 / 12 / 1975$, coll. P. Best, squid jig.

Unlocalised material examined (1 specimen) - KSB-002, , ML 110mm, TC-52-15, NFD.

Distribution (Fig. 24A) - Worldwide in temperate and tropical oceans, 1300-0m.

Diagnosis - Intestinal photophores circular, well defined, diameter of anterior $50-75 \%$ that of posterior; ocular photophore uniformly broad, spanning ventral $\sim 50 \%$ eye circumference; chromatophores present on tentacle stalk proximal to carpus and along oro-ventral stalk ridge, and over entire ventral surface of mantle; tentacle club $\sim 36 \%$ ML, with 20-23 long, sharply recurved hooks, without ventral basal spikes in distalmost ventral hooks.

Description (Figs 24B-25I) - Mantle muscular, width 18-22-27\% ML; gladius visible dorsally as dark line, but keel does not protrude above dorsal mantle surface; rostrum of gladius visible ventrally through posterior $\sim 10 \% \mathrm{ML}$; mantle robust posteriorly, without apparent fleshy ventral keel. Intestinal photophores circular, well defined, diameter of anterior $50-75 \%$ that of posterior. Fins large, rhombic, length $54-60-63 \%$ ML, width $60-68-75 \% \mathrm{ML}$; posterior margins straight, becoming slightly 
Table 10. Measures $(\mathrm{mm})$ and counts of Onychoteuthis prolata Bolstad, Vecchione \& Young, in press (arm and tentacle measures recorded from more complete side of specimen, indicated $R$ or $L$ ).

\begin{tabular}{|c|c|c|c|c|c|c|c|c|c|c|c|}
\hline Specimen ID & $\begin{array}{c}\text { SBMNH } \\
423101\end{array}$ & $\begin{array}{c}\text { USNM } \\
1102755\end{array}$ & $\begin{array}{c}\text { USNM } \\
813418\end{array}$ & KSB-003 & KSB-002 & $\begin{array}{c}\text { UMML } \\
31.92\end{array}$ & $\begin{array}{c}\text { Dana } \\
1159 \mid X\end{array}$ & $\begin{array}{c}\text { UMML } \\
31.1513\end{array}$ & $\begin{array}{c}\text { UMML } \\
\mathbf{3 1 . 2 9 5 9}\end{array}$ & \multicolumn{2}{|c|}{ Mean Indices } \\
\hline Type Status & Holotype & Paratype & None & None & None & None & None & None & None & \multirow{3}{*}{\multicolumn{2}{|c|}{ (ML 59-155) }} \\
\hline ML & 135 & 155 & 127 & 123 & 110 & 101 & 76 & 71 & 59 & & \\
\hline Sex & $\mathrm{F}$ & $\mathrm{F}$ & $F$ & $\mathrm{M}$ & $F$ & $\mathrm{M}$ & Indet. & Indet. & $\mathrm{F}$ & & \\
\hline MW & 28 & 28 & 32 & 26 & 28 & 21 & 14 & 19 & 13 & MWI & 22 \\
\hline HL & 33 & 24 & 28 & 22 & 25 & 25 & 17 & 17 & 11 & HLI & 21 \\
\hline HW & 27 & 23 & 17 & 22 & 20 & 20 & 15 & 12 & 9 & HWI & 17 \\
\hline FL & 81 & 93 & 80 & 76 & 64 & 63 & 45 & 41 & 32 & FLI & 60 \\
\hline FW & 89 & 95 & 85 & 86 & 66 & 75 & 57 & 52 & 41 & FWI & 68 \\
\hline Arms I & $53 \mathrm{~L}$ & $78 \mathrm{~L}$ & $71 \mathrm{~L}$ & $60 \mathrm{~L}$ & $51 \mathrm{R}$ & $46 \mathrm{~L}$ & $31 \mathrm{~L}$ & $29 R$ & $24 \mathrm{~L}$ & A1I & 45 \\
\hline Arms II & $68 \mathrm{~L}$ & $98 \mathrm{~L}$ & $76 \mathrm{~L}$ & $79 \mathrm{~L}$ & $61 \mathrm{R}$ & $59 \mathrm{~L}$ & $40 \mathrm{~L}$ & $35 R$ & $31 \mathrm{~L}$ & A2I & 56 \\
\hline Arms III & $69 \mathrm{~L}$ & $95 \mathrm{~L}$ & $70 \mathrm{~L}$ & $76 \mathrm{~L}$ & $59 R$ & $60 \mathrm{~L}$ & $39 \mathrm{~L}$ & $35 R$ & $31 \mathrm{~L}$ & A3I & 55 \\
\hline Arms IV & 71L & 93L & $80 \mathrm{~L}$ & $81 \mathrm{~L}$ & $61 \mathrm{R}$ & $57 \mathrm{~L}$ & $42 \mathrm{~L}$ & $31 \mathrm{R}$ & $32 \mathrm{~L}$ & A4I & 56 \\
\hline TnL & $98 \mathrm{~L}$ & $153 \mathrm{~L}$ & $154 \mathrm{~L}$ & 147L & $115 \mathrm{R}$ & 94L & $83 \mathrm{~L}$ & $69 R$ & $65 \mathrm{~L}$ & TnLI & 103 \\
\hline $\mathrm{CL}$ & $54 \mathrm{~L}$ & $61 \mathrm{~L}$ & $52 \mathrm{~L}$ & $46 \mathrm{~L}$ & $31 \mathrm{R}$ & $37 \mathrm{~L}$ & $27 \mathrm{~L}$ & $23 R$ & $22 \mathrm{~L}$ & CLI & 36 \\
\hline CS & 9 & 8 & 8 & 9 & $8 \mathrm{~L}$ & 8 & 7 & 8 & 7 & & \\
\hline $\mathrm{MH}$ & 23 & 22 & 22 & 23 & $22 \mathrm{~L}$ & 22 & 23 & 22 & 20 & & \\
\hline MS & 0 & 0 & 0 & 0 & $\mathrm{OL}$ & 0 & 0 & 0 & 0 & & \\
\hline TPS & 13 & 14 & 16 & 18 & $14 \mathrm{~L}$ & 12 & 16 & 13 & 13 & & \\
\hline
\end{tabular}

concave posteriorly; anterior margins varying from slightly convex to slightly concave, lateral 'corners' sharp, forming approximate right angle.

Head approximately cylindrical, length $15-21-25 \% \mathrm{ML}$, width $13-17-20 \% \mathrm{ML}$, depth $11-19 \% \mathrm{ML}$, widening slightly proximal to arm bases; occipital region with seven to nine secondary folds; funnel groove well defined, triangular, narrowing to acute anterior point below midpoint of eyes. Ocular photophore broad, rounded at both ends and of uniform breadth along its length, spanning ventral $~ 50 \%$ eye circumference. Funnel component of locking apparatus $11-15 \% \mathrm{ML}$; mantle component 21-28\% ML.

Arms slender, 39-45,-56 $6_{I I-I V}-66 \% \mathrm{ML}$, formula $I I=|I|=|\mathrm{V}>|$, attenuating to narrow tips; suckers on each arm number 90-110 (ML 71-155mm); sucker diameter $~ 50 \%$ arm width on Arms I-III, $\sim 40 \%$ arm width on Arms IV. Keels present on Arms I-III: that on Arms I 5\% arm width along entire arm length; that on Arms II $\sim 50 \%$ arm depth along entire arm length; that on Arms III $\sim 150 \%$ arm depth at $\sim 40 \%$ arm length, narrowing distally; lateral membrane on Arms IV $\sim 100 \%$ arm width at base, narrowing thereafter to $\sim 75 \%$ arm width along remainder of arm length.

Tentacles robust, length $73-103-121 \% \mathrm{ML}$; thickness of stalk at base comparable to that of adjacent arms; aboral keel $\sim 10 \%$ stalk thickness proximally, widening to $20 \%$ distally. Club (Figs 24C, 24D, 25B) 28-36-40\% ML (27-55\% TnL), expanded; carpus broad, ovoid, with seven to nine suckers; manus with 20-23 long, robust, widely spaced hooks, with V5 or V6 longest (Fig. 25C), three to five times longer than paired dorsal hook and $16-21 \% \mathrm{CL}$; terminal pad with 12-18 suckers. Largest ventral hooks 
slender, with nearly straight necks and acutely recurved claws in lateral profile; ventral bases of distal-most ventral hooks not produced into spike (Fig. 25D).

Lateral profile of lower beak (Figs 25F-H, ML 123mm) longer than deep, with crest sloping steeply toward baseline; posterior edge of hood well above crest; hood length
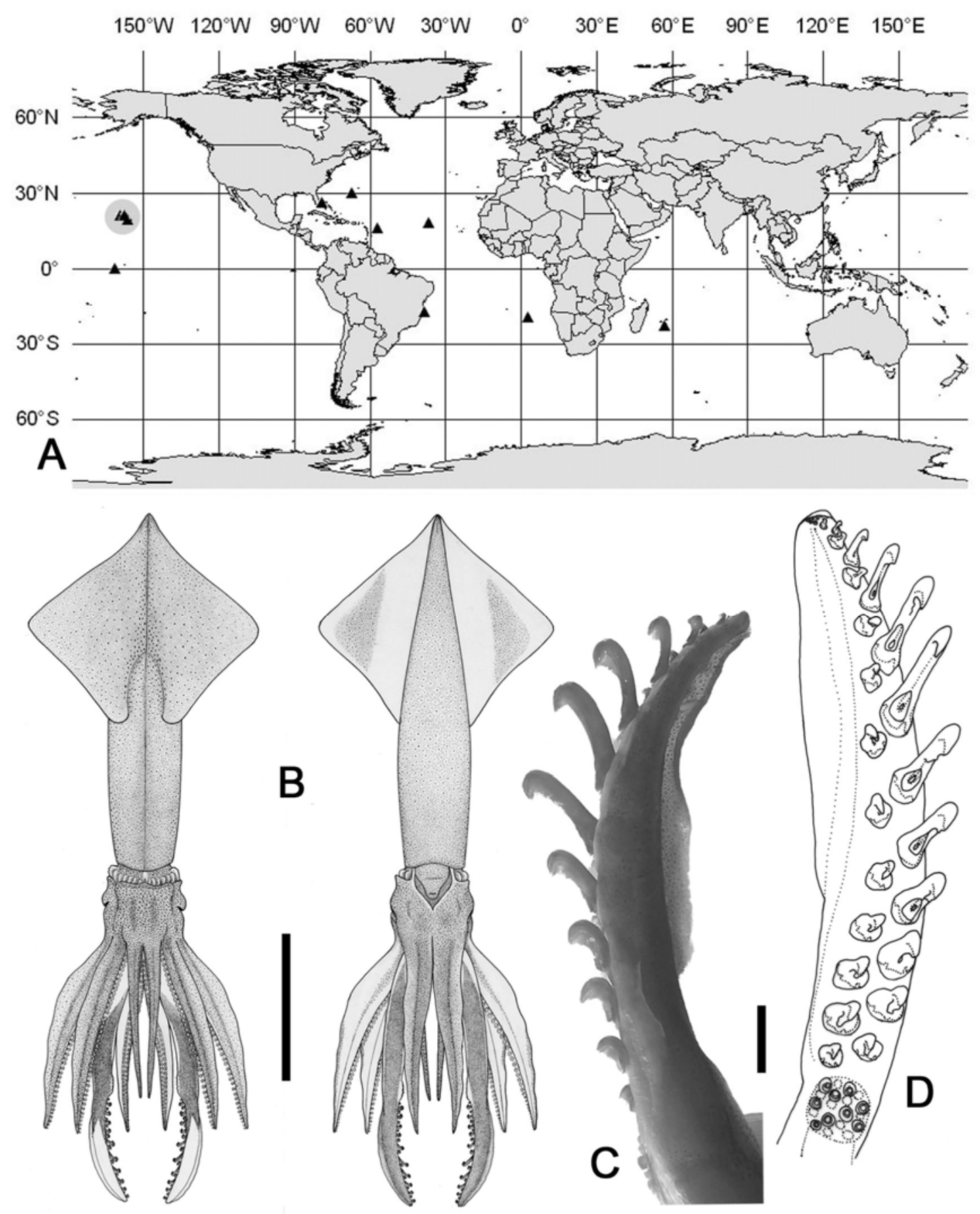

Fig. 24 - Onychoteuthis prolata. A) distribution (grey circle indicates type locality); BD) SBMNH 423101, holotype, , ML 135mm: (B) whole animal (illustration by K.H. Moore), (C, D) left tentacle club. Scale bars = B) $50 \mathrm{~mm}$; C, D) $5 \mathrm{~mm}$. 
$\sim 50 \%$ total crest length, and hood and crest together $\sim 65 \%$ baseline; jaw edge short, slightly concave along entire length; jaw angle obtuse, slightly obscured by low, rounded wing fold. Crest broad, rounded, slightly thickened; lateral wall not indented between crest and lateral wall fold; shoulder ridge rounded; angle ridge well defined but short, spanning $\sim 40 \%$ lateral wall depth; angle point indistinct; distal wing width $\sim 200 \%$ that at jaw angle, wing length $275-300 \%$ LRL, with small triangular insert of cartilage below jaw angle; lateral wall diagonally bisected by dark, prominent ridge and fold, becoming indistinct by lateral wall corner.

Radula (Fig. 25E) with tricuspid rachidian, with broad triangular mesocone and narrow, rounded, slightly laterally directed lateral cusps, their height $\sim 30 \%$ of total rachidian height. First lateral tooth bicuspid, inner cusp straight or slightly curved, subequal or slightly shorter than rachidian and slightly narrower, medially directed; outer cusp low, rounded, slightly laterally directed. Second lateral tooth simple, robust, curved, $\sim 150 \%$ height of rachidian. Marginal tooth simple, curved, $\sim 180 \%$ rachidian height. Palatine palp not examined.

Gladius (Fig. 25I) with greatest width ( $7 \%$ GL) attained at $\sim 40 \% \mathrm{GL}$; free rachis $\sim 27 \%$ $\mathrm{GL}$; vanes broaden and taper rapidly between 30 and $65 \% \mathrm{GL}$; dorsal keel robust, narrow. Rostrum short, $4 \% \mathrm{GL}$, triangular in lateral profile, narrow in dorsal profile.

Overall colouration (preserved) pale pink overlaid with dark purple-brown chromatophores, darkest along dorsal midline, becoming sparse but still distinct over dorsal and dorso-lateral mantle surfaces and dorsal surface of fins, fine but distinct over entire ventral mantle surface. Dorsal and dorso-lateral surfaces of head purple; lateral and ventral surfaces with faint silver iridescence. Aboral surfaces of arms pinkish-purple, slightly iridescent; tentacle stalks with same iridescence ventral to aboral keel; chromatophores present proximal to carpus (Fig. 25B) in band from oroventral tentacle stalk ridge and across oral face of stalk proximal to carpus, and along oro-ventral stalk ridge.

Remarks - The overall appearance of this species is quite robust, with long, broad tentacle clubs and large, widely spaced, strongly recurved hooks (Figs 25C, 25D) being its most distinctive characters. Of all congeners, Os. aequimanus is the most similar, although Os. aequimanus appears more slight of body, its tentacular clubs are generally shorter, and its hooks are more slender and less recurved. 

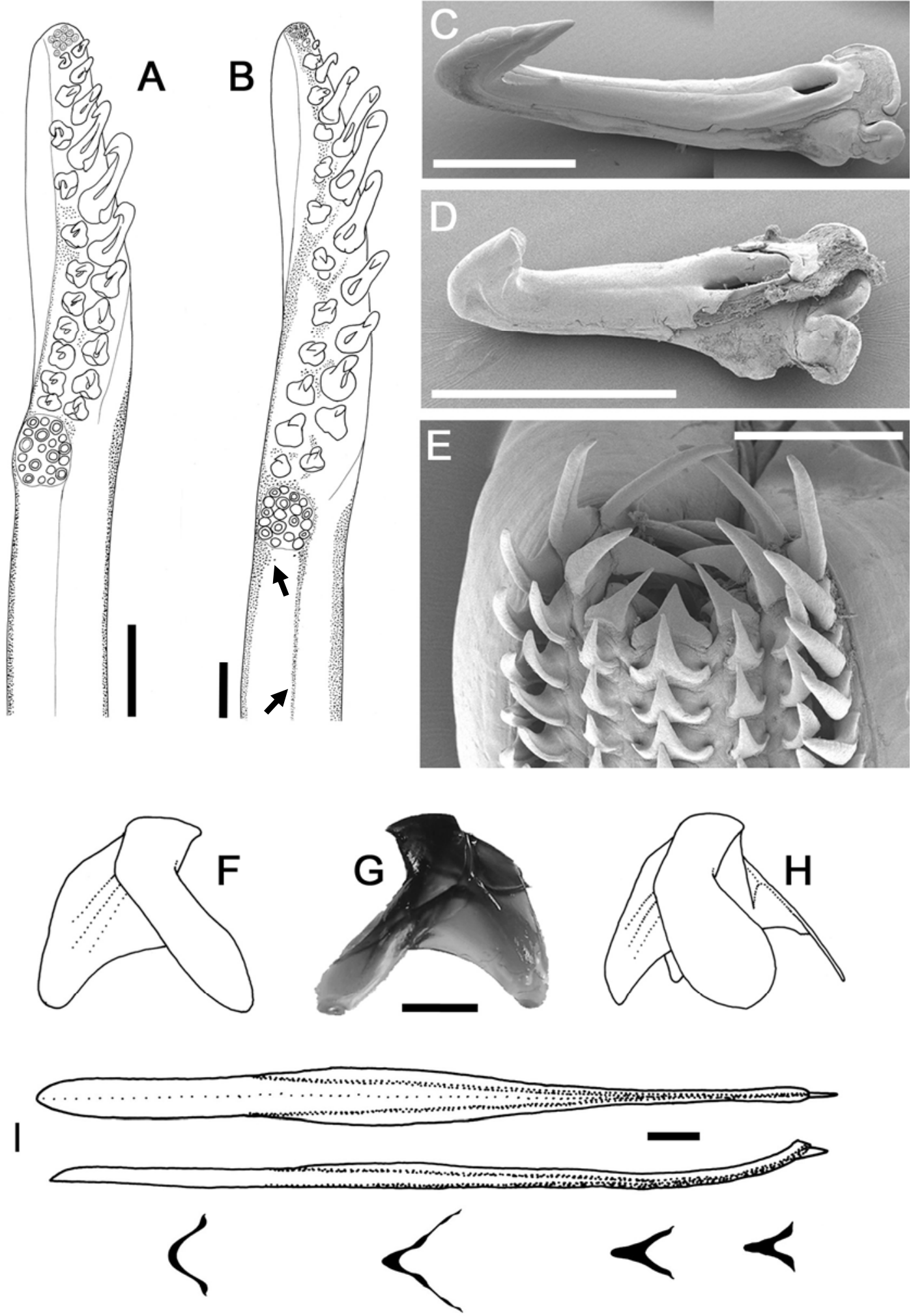

Fig. 25 - Onychoteuthis compacta (A only) and Onychoteuthis prolata (B-I). A) USNM 813428, , , ML 89mm; B-D) SBMNH 423101, holotype, , ML 135mm; E-H) KSB-003, 万. ML 123mm; I) USNM 1102755, paratype,, , ML 155mm. A, B) chromatophores across tentacle stalk proximal to carpus and along oro-ventral stalk ridge in: (A) Os. compacta (absent), (B) Os. prolata (present — arrows) (illustrations by K.H. Moore); C, D) right tentacular hooks of Os. prolata: (C) hook V7, (D) hook V11; E) radula of Os. prolata; $(\mathrm{F}-\mathrm{H})$ lower beak of Os. prolata: $(\mathrm{F})$ left, $(\mathrm{G})$ right, $(\mathrm{H})$ left oblique profiles; I) gladius of Os. prolata. Scale bars = A, B, F, G) $5 \mathrm{~mm}$; C) $2 \mathrm{~mm}$; D) $1 \mathrm{~mm}$; E) $500 \mu \mathrm{m}$; I) $10 \mathrm{~mm}$. 
Genetic material from Young \& Harman's (1987) 'species B' paralarvae (herein tentatively attributed to Os. prolata) was included by Bonnaud et al. (1998) in their phylogenetic study of the Onychoteuthidae, as 'Onychoteuthis sp. B' (R. Young, University of Hawai'i, pers. comm.). 'Os. sp. B' was found to be closely related to Os. compacta and somewhat more distantly to Os. lacrima. 


\section{Onykia Lesueur, 1821}

Onychia [sic] Lesueur, 1821 - Latreille, 1825, fide Gray, 1849 (proposed emendation). Moroteuthis Verrill, 1881: 298. Type species Ommastrephes robustus Verrill, 1876, by original designation.

Teleoteuthis Verrill, 1882: 280. Type species Onykia carriboea Lesueur, 1821, by original designation.

Steenstrupiola Pfeffer, 1884: 16. Type species Steenstrupiola chilensis Pfeffer, 1884, by subsequent designation (Hoyle 1910).

Type species — Onykia carriboea Lesueur, 1821, by monotypy.

Diagnosis - Large-bodied squids, observed ML at maturity $300 \mathrm{~mm}-1.97 \mathrm{~m}$, elsewhere reported to $2.3 \mathrm{~m}$ (Nesis 1987); photophores and secondary occipital folds absent; epidermis of mantle and sometimes head rugose or ridged in all species where subadult to adult specimens are known; funnel groove U-shaped or trapezoidal, with Y-shaped ridge in most species; arms generally $50-130 \% \mathrm{ML}$ at maturity; tentacle clubs with 24-36 hooks, with strong lateral grooves on hook claw in most species; longest ventral hooks approximately twice as long as paired dorsal hooks and $5-11 \%$ club length; marginal suckers variably present on tentacle clubs; rostrum of gladius posteriorly directed, $10-30 \% \mathrm{GL}$, free rachis $15-20 \% \mathrm{GL}$; rachidian strongly tricuspid.

Remarks - The type species of Onykia - Ok. (Ok.) carriboea - was described from a juvenile specimen (the holotype) that is no longer extant. Onykia subsequently became a cosmopolitan catch-all name for juvenile onychoteuthids not obviously belonging to other genera, generally characterised by dark bluish-purple to nearly black pigmentation on the dorsal mantle surface, ovate fins, two full rows of marginal suckers in addition to the hooks on the tentacle clubs, and the absence of photophores or secondary occipital folds. Unfortunately, in most species of Onykia, the former three character states are present only in juveniles, making correct species attribution of larger specimens difficult and causing many of these to be described as new species.

Tsuchiya \& Okutani (1992) recognised that species historically attributed to 'Moroteuthis' were often known from large juvenile to subadult stages only, while Onykia comprised only species known from paralarvae to small juveniles. 'Moroteuthis' was synonymised with Onykia, supported by a thorough description of an ontogenetic series of Ok. (Ok.) robusta specimens ('Ommastrephes robustus' being the type species of 'Moroteuthis'). Wakabayashi et al. (2007) subsequently confirmed that 
north-Pacific paralarvae of classic Onykia morphology were young individuals of $O K$. (Ok.) robusta (new comb.), supporting the earlier synonymy.

Two species of Onykia remain known solely from early ontogenetic stages - Ok. (Ok.) carriboea and $O k$. (Ok.) indica - and three are known solely from later ontogenetic stages - Ok. (Ok.) aequatorialis (new comb.), Ok. (Ok.) loennbergii (new comb.), and Ok. (Ok.) robsoni (new comb.). It is likely that at least one of the latter will prove synonymous with one of the former, but confirming which is which is not yet possible, given the absence of specimens of intermediate ontogenetic stages.

Recent morphological and genetic findings (herein and Wakabayashi et al. 2007, respectively) suggest that $O k$. (M.) ingens (new comb.) is more different from the other six Onykia species than they are from each other. For this reason, Pfeffer's (1908b) subgenus Moroteuthopsis is revived for Ok. (M.) ingens, diagnosed herein as lacking a Y-shaped ridge in the funnel groove and lateral grooves on the claw of the tentacular hooks. The remaining Onykia species all possess these characters and are grouped into the subgenus Onykia (Onykia).

The generic name Steenstrupiola was proposed by Pfeffer (1884) for two species, S. chilensis (later designated the type species by Hoyle (1910)) and S. atlantica. Pfeffer later (1912) recognised that the character states affiliated with his Steenstrupiola were simply early ontogenetic phases of Onykia, and he accordingly placed Steenstrupiola into synonymy with Onykia and S. atlantica in synonymy with Ok. (Ok.) carriboea. He retained ' $S$.' chilensis as a distinct species at the time, but the loss of the type material and non-diagnostic original description caused Nesis et al. (1998) to declare it a nomen dubium.

Key character states of all species of Onykia are given in Table 11, with $K$. longimana (pp. 175-182) and Gen. nov. 1 knipovitchi (pp. 215-221) for comparison. 


\begin{tabular}{|c|c|c|c|c|c|c|c|c|c|}
\hline 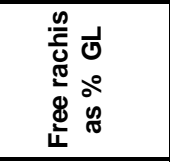 & $\stackrel{*}{\stackrel{*}{\imath}}$ & $\stackrel{m}{i}$ & $\stackrel{\Upsilon}{i}$ & $\stackrel{*}{\stackrel{*}{ }}$ & $\stackrel{\llcorner}{i}$ & $\stackrel{k}{i}$ & $\stackrel{\circ}{\zeta}$ & ণิ & $\hat{i}$ \\
\hline 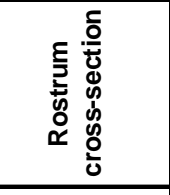 & 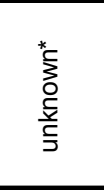 & $\begin{array}{l}\frac{\bar{\pi}}{\bar{J}} \\
\frac{.0}{0}\end{array}$ & 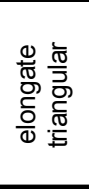 & 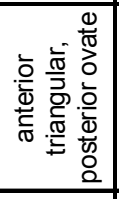 & 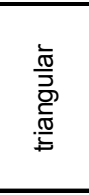 & 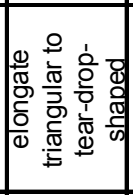 & 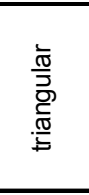 & 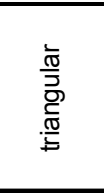 & 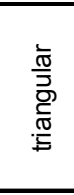 \\
\hline 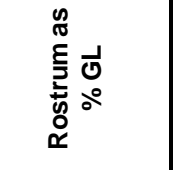 & $\stackrel{*}{\underset{T}{T}}$ & $\stackrel{\text { iิ }}{\text { in }}$ & ণ্ & $\begin{array}{l}\stackrel{*}{*} \\
\stackrel{+}{上}\end{array}$ & 怘 & $\stackrel{*}{i}$ & $\frac{m}{i}$ & $\stackrel{0}{i}$ & $\stackrel{ \pm}{i}$ \\
\hline 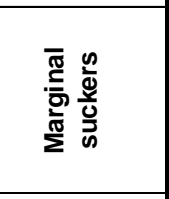 & 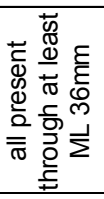 & 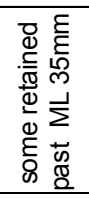 & 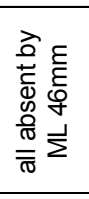 & 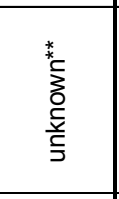 & 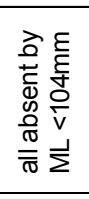 & 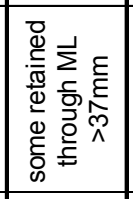 & 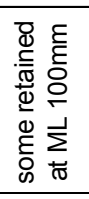 & 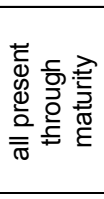 & 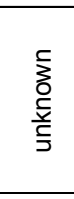 \\
\hline 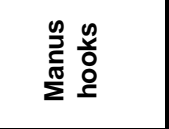 & $\begin{array}{l}\stackrel{O}{N} \\
\stackrel{N}{N}\end{array}$ & $\begin{array}{l}\infty \\
\tilde{m} \\
\infty\end{array}$ & 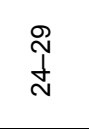 & 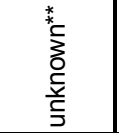 & $\begin{array}{l}\stackrel{\infty}{N} \\
\stackrel{\sim}{\sim}\end{array}$ & $\begin{array}{l}\stackrel{\infty}{N} \\
\stackrel{\omega}{\omega}\end{array}$ & $\begin{array}{l}\text { Nิ } \\
\text { @્ }\end{array}$ & 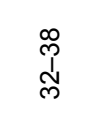 & స్ \\
\hline 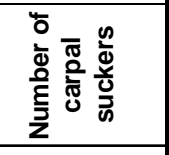 & i & $\stackrel{ }{\grave{1}}$ & $\stackrel{P}{i}$ & 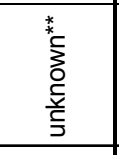 & $\underset{\Gamma}{\Gamma}$ & $\frac{0}{1}$ & $\frac{N}{3}$ & $\stackrel{N}{\frac{1}{\circ}}$ & $\stackrel{m}{\frac{m}{\sigma}}$ \\
\hline 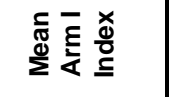 & $\stackrel{*}{*}$ & $\bar{\sigma}$ & 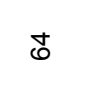 & F & $\tilde{\sigma}$ & 음 & $\Xi$ & 8 & \& \\
\hline 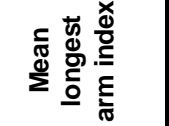 & $\stackrel{*}{\stackrel{*}{\circ}}$ & $\hat{\imath}$ & $\hat{\wedge}$ & $\bar{\sigma}$ & ஜீ & $\stackrel{p}{=}$ & $\stackrel{\infty}{\infty}$ & $\bar{\infty}$ & in \\
\hline 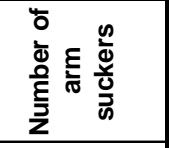 & 莡 & $\frac{R}{i}$ & $\stackrel{ }{\frac{0}{i}}$ & $\frac{8}{i}$ & $\frac{9}{i}$ & $\begin{array}{l}\stackrel{*}{*} \\
\stackrel{0}{0} \\
i\end{array}$ & $\frac{\text { Tิ }}{i}$ & $\frac{q}{i}$ & $\stackrel{\leftrightarrow}{i}$ \\
\hline 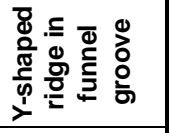 & 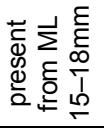 & 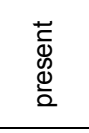 & 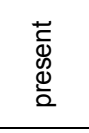 & 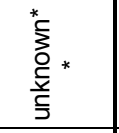 & 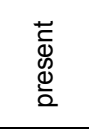 & 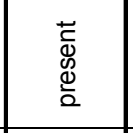 & 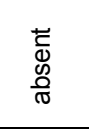 & 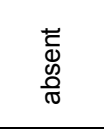 & 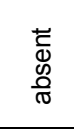 \\
\hline 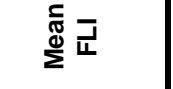 & $\stackrel{*}{q}$ & 员 & $\bar{n}$ & 员 & 용 & 员 & $\stackrel{\infty}{\stackrel{\infty}{+}}$ & ষे & 요 \\
\hline 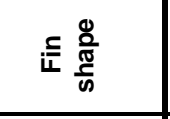 & $\frac{\frac{*}{\pi / n}}{\partial 0}$ & 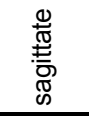 & 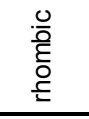 & $\begin{array}{l}.0 \\
\frac{0}{0} \\
\text { है } \\
\underline{\underline{E}}\end{array}$ & 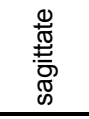 & $\begin{array}{l}\frac{*}{\sqrt{\pi}} \\
\partial\end{array}$ & 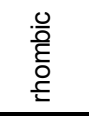 & 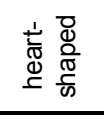 & $\begin{array}{l}.0 \\
\frac{0}{0} \\
\bar{E} \\
\frac{E}{\Sigma}\end{array}$ \\
\hline 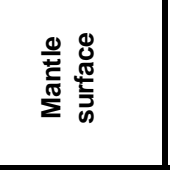 & 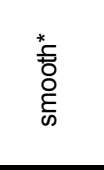 & 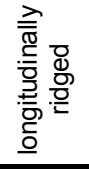 & 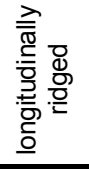 & $\begin{array}{l}0 \\
\stackrel{0}{0} \\
\stackrel{9}{9}\end{array}$ & $\begin{array}{l}0 \\
\stackrel{0}{0} \\
\stackrel{9}{9}\end{array}$ & 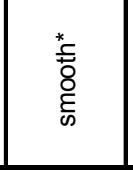 & $\begin{array}{l}0 \\
\stackrel{0}{0} \\
\stackrel{9}{2}\end{array}$ & 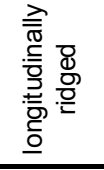 & 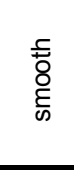 \\
\hline 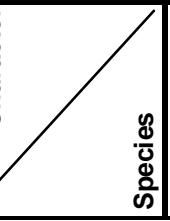 & 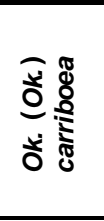 & 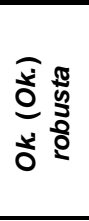 & 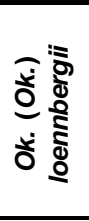 & 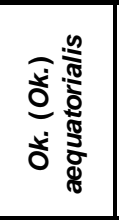 & 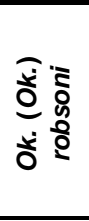 & 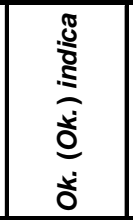 & 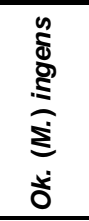 & 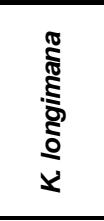 & 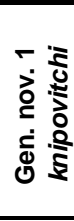 \\
\hline
\end{tabular}




\section{Onykia (Onykia) Lesueur, 1821}

Diagnosis - Funnel groove with Y-shaped ridge; manus hooks with one deep lateral groove on either side of hook claw.

Onykia (Onykia) carriboea Lesueur, 1821 (Tables 11, 12, Figs 26-28)

Onykia carriboea Lesueur, 1821: 98, 99, PI. 9 Figs 1, 2; Guerra 1992: 141-143, Fig. 44;

Kubodera et al. (1998): 282, Fig. 7; Lu et al. (1995): 313; Sweeney \& Roper (1998): 572.

Teleoteuthis agilis Verrill, 1885: 400, 401, PI. 42, Figs 2, 2a; Roper \& Sweeney (1978): 3.

Teleoteuthis caribaea [sic] Lesueur, 1821 - Pfeffer (1912): 37-43 [English translation], PI. 1

Figs 1-26.

Onykia carribaea [sic] Lesueur, 1821 — Lu et al. (1995): 313.

Type material - formerly ANSP holotype (no longer extant, see Voss 1962); USNM 40121, holotype (Teleoteuthis agilis, see above), sex indet., ML $36 \mathrm{~mm}$ (preserved; pre-fix $46 \mathrm{~mm}$ ), $36^{\circ} 05.5^{\prime} \mathrm{N}, 69^{\circ} 51.75^{\prime} \mathrm{W}$, Cape Hatteras, North Carolina, surface, 09/09/1884, coll. Albatross (examined).

Additional material examined (27 specimens) - BMNH 20070571, sex indet., ML 8mm, $32^{\circ} 32^{\prime} \mathrm{N}, 17^{\circ} 14.8^{\prime} \mathrm{W}, 20 / 10 / 1986$, RRS Challenger Stn C86/36, RMT and 150W light; USNM 728071, sex indet., ML 13mm, 32 ${ }^{\circ} 17^{\prime} \mathrm{N}, 63^{\circ} 50^{\prime} \mathrm{W}$, Bermuda, surface, 24/02/1972, Acre 13-9-L, $1 \mathrm{~m}$ NN; USNM 575092, sex indet., ML $10 \mathrm{~mm}, 25^{\circ} 30^{\prime} \mathrm{N}, 92^{\circ} 00^{\prime} \mathrm{W}$, Marsh Island, south of Louisiana, 10/05/1954, 3162m [sic], RV Oregon, drift net; UMML 31.672, 19 sex indet., ML $15-7 \mathrm{~mm}, 13^{\circ} 49^{\prime} \mathrm{N}, 39^{\circ} 03^{\prime} \mathrm{W}$, Drifter Cruise, NL\&D; NMNZ M.74163, 2 sex indet., $M L 13,11 \mathrm{~mm}, 20 \mathrm{~km}$ from $31^{\circ} 24^{\prime} \mathrm{S}, 179^{\circ} 00^{\prime} \mathrm{E}, 3108-2742 \mathrm{~m}, 25 / 07 / 1962$, 1354-1700h, Tui Oceanographic Cruise, dip net; USNM 816685, sex indet., ML 18mm, $34^{\circ} 25^{\prime} \mathrm{S}, 14^{\circ} 47^{\prime} \mathrm{E}$, SW of Cape Town, 112-0m, 21/04/1971, RV Walther Herwig, 1600mm mesh EMWT; USNM 576021, sex indet., ML 34mm, $41^{\circ} 22^{\prime} \mathrm{S}$, $148^{\circ} 20^{\prime} \mathrm{E}$, St Helena Island, Jamestown, 06/08/1965, fairy tern stomach, coll. A. Loveridge; NMNZ M.74374, sex indet., ML 9mm, 42 $59^{\circ} \mathrm{S}, 178^{\circ} 00.1^{\prime} \mathrm{E}, 15 / 12 / 1977$, 0645-0655h, sex indet; NMNZ M.279053, ML 8mm, 4344.8'S, 17609.6'E, 1913-1923h, RV Kaiyo Maru.

Unlocalised material examined (3 specimens) - BMNH 89.4.24.105, sex indet., ML 15mm, North Atlantic, surface, Challenger Expedition, NFD; NMNZ M.74372, sex indet., ML 13mm, G. Patchell Plankton Sample \#1 [New Zealand], NFD; NMNZ M.74457, ML 7mm, NFD.

Distribution (Fig. 26A) - Central to North Atlantic including Gulf of Mexico, South Atlantic, South Pacific. Generally at surface; one dubious report of $3162 \mathrm{~m}$.

Diagnosis (known only from small juveniles, to $\mathrm{ML} 36 \mathrm{~mm}$ ) - Fins ovate to rhombic by $\mathrm{ML} 36 \mathrm{~mm}$, length $\sim 40 \% \mathrm{ML}$; dorsal surface of mantle deep reddish-purple to bluish- 
Table 12. Measures $(\mathrm{mm}$ ) and counts of Onykia (Onykia) carriboea Lesueur, 1821 (arm and tentacle measures recorded from more complete side of specimen, indicated $\mathrm{R}$ or $\mathrm{L}$ ).

\begin{tabular}{cccccc}
\hline Specimen ID & USNM 40121 & USNM 576021 & USNM 816685 & UMML 31.672 & USNM 575092 \\
\hline Type Status & $\begin{array}{c}\text { T. agilis } \\
\text { holotype }\end{array}$ & None & None & None & None \\
ML & 36 & 34 & 18 & 15 & 10 \\
Sex & Indet. & Indet. & Indet. & Indet. & Indet. \\
MW & 9 & 12 & 7 & 5 & 4 \\
HL & 7 & 10 & 6 & 5 & 4 \\
HW & 8 & 9 & 7 & 5 & 4 \\
FL & 14 & 16 & 7 & 6 & 4 \\
FW & 20 & 28 & 16 & 12 & 8 \\
Arms I & $22 R$ & $33 \mathrm{~L}$ & $12 \mathrm{R}$ & $10 \mathrm{R}$ & $7 \mathrm{~L}$ \\
Arms II & $27 R$ & $38 \mathrm{~L}$ & $15 \mathrm{R}$ & $13 \mathrm{R}$ & $9 \mathrm{~L}$ \\
Arms III & $25 \mathrm{R}$ & $36 \mathrm{~L}$ & $14 \mathrm{R}$ & $12 \mathrm{R}$ & $10 \mathrm{~L}$ \\
Arms IV & $26 \mathrm{R}$ & $38 \mathrm{~L}$ & $15 \mathrm{R}$ & $12 \mathrm{R}$ & $10 \mathrm{~L}$ \\
TnL & $32 \mathrm{R}$ & $53 \mathrm{~L}$ & $17 \mathrm{R}$ & $13 \mathrm{R}$ & $12 \mathrm{~L}$ \\
CL & $17 \mathrm{R}$ & $18 \mathrm{~L}$ & $8 \mathrm{R}$ & $6 \mathrm{R}$ & $5 \mathrm{~L}$ \\
CS & 8 & 7 & 8 & 8 & 9 \\
MH & 25 & 26 & 24 & 10 prox. V, 6 prox. D & 0 \\
MS & All & All & All & All & All \\
TPS & 11 & 13 & 14 & 11 & Undifferentiated \\
\hline
\end{tabular}

* indicates damaged features.

black; Y-shaped ridge present in funnel groove from ML 15-18mm; arms generally 60$80 \% \mathrm{ML}$; manus with 24-26 hooks and two complete series of marginal suckers; proximal dorsal and distal ventral marginal suckers larger than medial suckers/hooks through ML $15 \mathrm{~mm}$; first ventral hooks begin to differentiate at $\mathrm{ML} 10-11 \mathrm{~mm}$ and first dorsal at ML 16-18mm; tentacular suckers with five to seven long, sharp teeth around distal inner margin of sucker ring, two concentric rows of pegs proximally and laterally, and five to seven rows of pegs distally, with innermost row of distal pegs intermediate in morphology between teeth and pegs, resembling second row of teeth.

Description (ML 8-36mm, Figs 26B-28F) - Mantle slender (width 25\% ML), generally conical but narrowing slightly between midpoint and anterior margin; middorsum slightly ridged, but gladius not visible dorsally through tissue; rostrum visible ventrally through posterior $\sim 5 \% \mathrm{ML}$. Fins rhombic in outline, length $\sim 40 \%$, width $\sim 55 \%$ $\mathrm{ML}$; posterior margins convex, anterior margins straight.

Head rounded, length $\sim 20 \% \mathrm{ML}$, width at least equal to that of mantle (25-30\% ML), depth $\sim 25 \% \mathrm{ML}$; all three primary occipital folds readily apparent; dorsal occipital region with low transverse crest; funnel slender, slightly bulbous; groove rounded into broad U-shape, with $\mathrm{Y}$-shaped ridge forming by $\mathrm{ML} 15-18 \mathrm{~mm}$; funnel aperture level with posterior margin of lens; head constricted anteriorly at base of brachial crown. Funnel component of locking apparatus $\sim 20 \% \mathrm{ML}$, mantle component $\sim 30 \% \mathrm{ML}$. 
Arms slender, $60-75 \% \mathrm{ML}$, arm formula II>IV>III>I, each with $\sim 70$ suckers (ML

$36 \mathrm{~mm}$ ), diameter of largest $\sim 50 \%$ arm width; Arms II and III with keels; that on Arms II $\sim 15 \%$ arm depth over entire arm length; that on Arms III 30\% arm depth over entire arm length; lateral membrane on Arms IV $\sim 150 \%$ arm width at base, narrowing to nonexistent by $30 \%$ arm length.

Tentacles short, $\sim 90 \% \mathrm{ML}$, stout, diameter of stalks at bases similar to that of adjacent arms; low aboral keel present over distal $50 \%$ of stalk length. At ML $36 \mathrm{~mm}$, club (Fig. $26 \mathrm{C}$ ) slightly expanded, length $\sim 50 \% \mathrm{ML}(\sim 35 \% \mathrm{TnL})$; carpus elongate oval, with

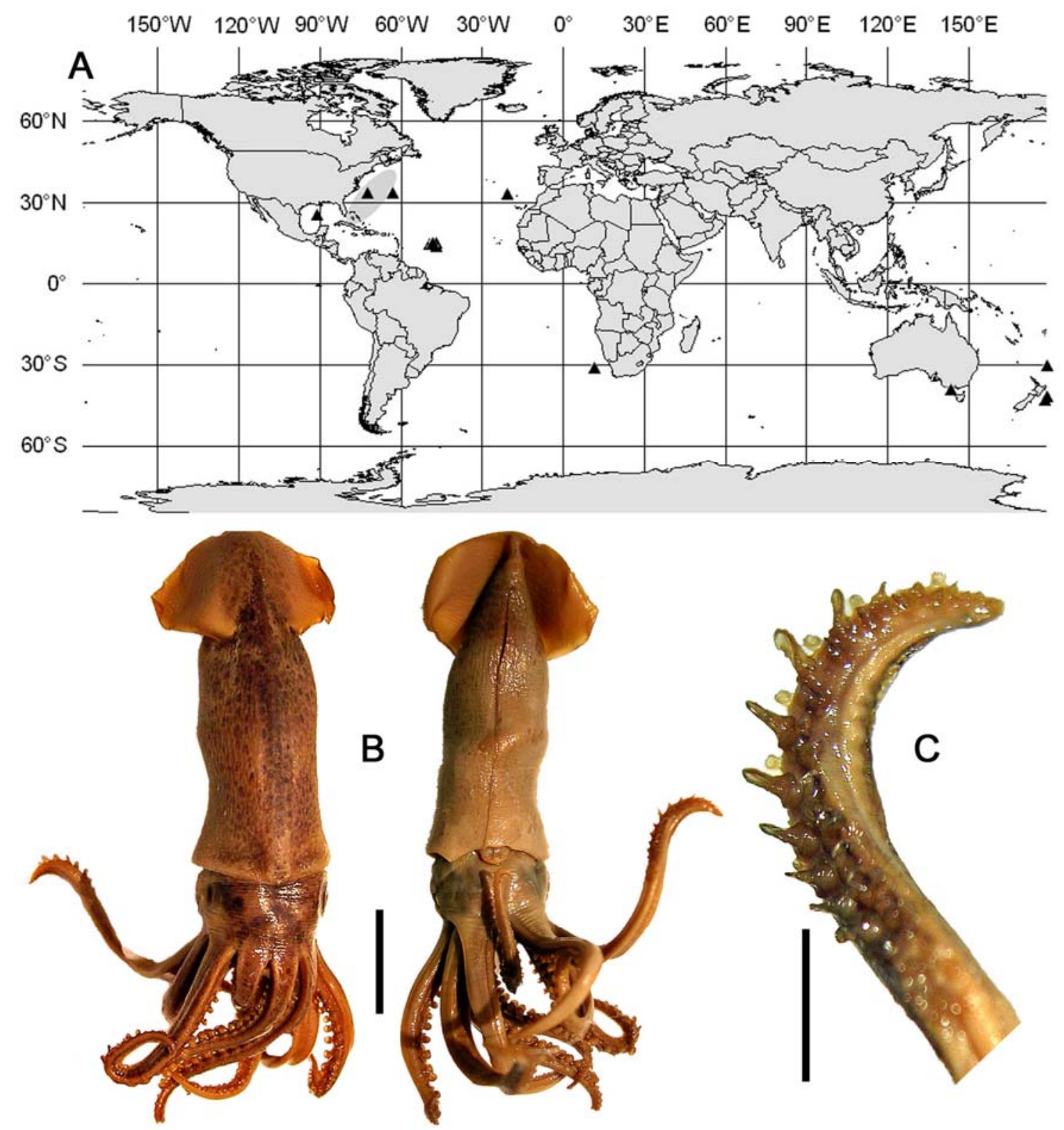

Fig. 26 - Onykia (Onykia) carriboea. A) distribution (grey oval indicates type locality); B, C) USNM 40121, holotype of 'Teleoteuthis agilis,' ML 36mm: (B) whole animal, (C) right tentacle club. Scale bars = B) $10 \mathrm{~mm}$; C) $5 \mathrm{~mm}$. 
seven to nine suckers; manus with 24-26 slender, symmetrical, sharply recurved hooks, and two complete rows of large marginal suckers (diameter of those bordering hooks D1-D8 nearly $80 \%$ hook length); terminal pad with nine to 14 suckers; each ventral hook larger than corresponding dorsal hook, with V6 (largest on club) about twice as long as D6 and $\sim 11 \% \mathrm{CL}$; both hook series increase, then decrease in size steadily with no abrupt enlargements or reductions; no membranes apparent on club. At ML 18mm (Fig. 28D), all ventral and dorsal hooks forming, with medial ventral hooks well developed, and larger than adjacent marginal suckers; proximal and distal ventral hooks and all dorsal hooks just differentiating, with proximal dorsal marginal suckers larger than future hook bearers. At ML 13-16mm (Fig. 28C), medial hooks in dorsal series beginning to form; all marginal suckers larger than all medial suckers, with proximal dorsal and distal ventral marginal suckers particularly large. At ML $10 \mathrm{~mm}$ (Fig. 28B), ventral medial hooks begin to change from round to distally pointed, but do
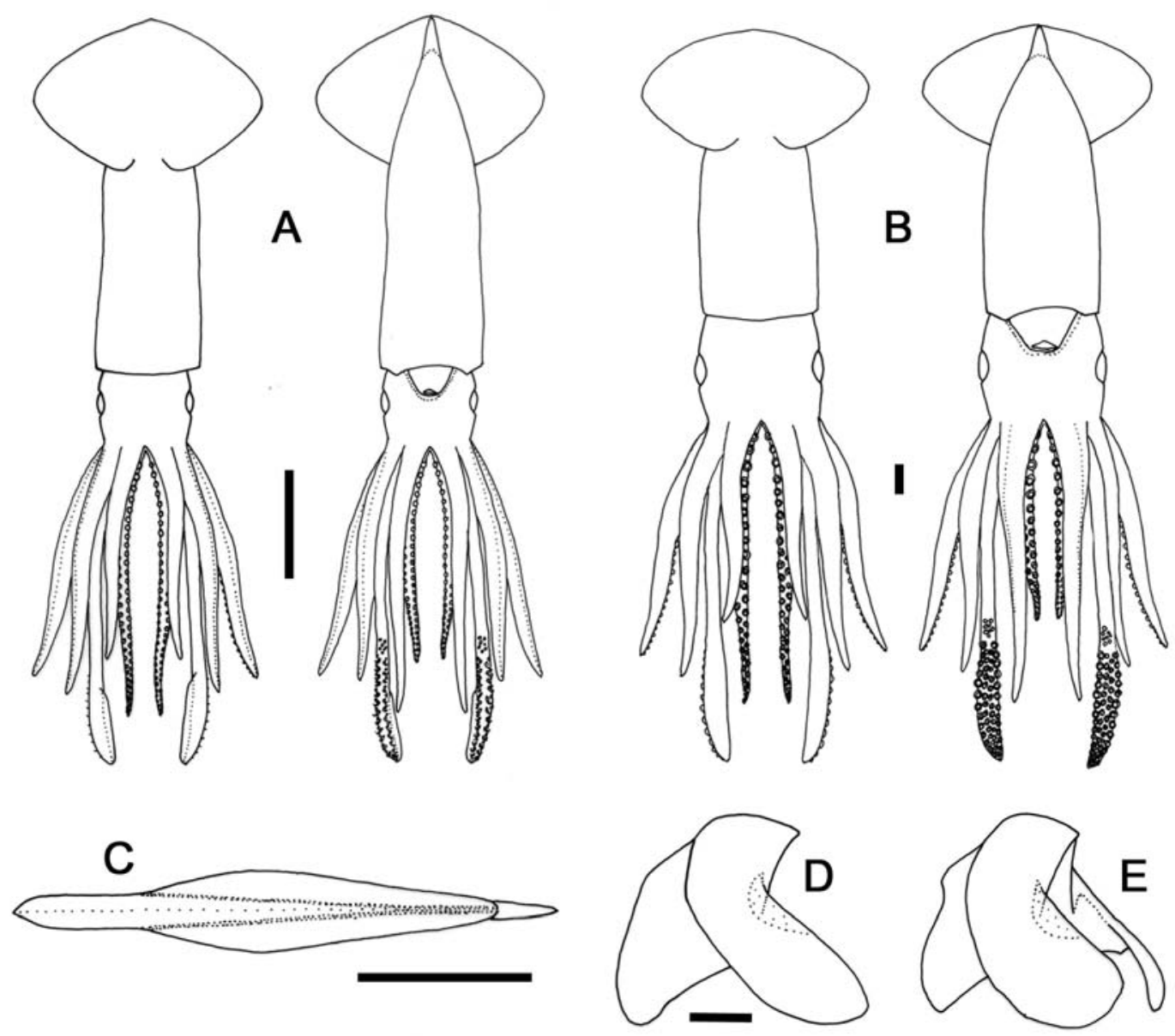

Fig. 27 - Onykia (Onykia) carriboea. A) USNM 40121, Teleoteuthis agilis holotype, sex indet., ML 36mm; B) USNM 525072, sex indet., ML 10mm; C-E) USNM 576021, sex indet., ML 34mm: (C) gladius; ( $D, E)$ lower beak: (D) left, $(E)$ left oblique profiles. Scale bars $=A, C) 10 \mathrm{~mm} ; B, D) 1 \mathrm{~mm}$. 
not form hooks. At ML 7-8mm (Fig. 28A), medial suckers have not begun to change into hooks; diameter of medial suckers $50-75 \%$ that of marginals. Paralarval to juvenile tentacular suckers (Figs $28 \mathrm{E}, 28 \mathrm{~F}$ ) with widely spaced, orally pointed to flattened or slightly concave pegs, set in two concentric rows proximally and laterally, increasing distally to five to seven rows, resembling a triangular pad of pegs; inner margin of sucker ring with five to seven long, sharp teeth distally; innermost row of distal pegs of transitional morphology between teeth and pegs, resembling a second row of teeth.

Gladius (Fig. 27C) with greatest width ( 15\% GL) attained at $\sim 45 \%$ GL; vanes broaden rapidly between 25 and $45 \% \mathrm{GL}$, thereafter tapering gradually to $\sim 90 \% \mathrm{GL}$; free rachis $\sim 23 \% \mathrm{GL}$, rostrum $\sim 11 \% \mathrm{GL}$.

Overall colouration pale pinkish with dark purple to bluish-black chromatophores, largest and densest over dorsal surface of head, mantle and between fins; chromatophores on ventral mantle surface pale, densest posteriorly without apparent 'tail spots; arms dark aborally, and with large, dark, alternating biserial chromatophores between suckers on oral surface.

Remarks - The loss of the Ok. (Ok.) carriboea holotype specimen is particularly unfortunate, since Ok. (Ok.) carriboea specimens span one of the widest geographic ranges of any species treated herein. However, the holotype of 'Teleoteuthis agilis' Verrill, 1885 is extant (USNM 40129, Figs 26B, 26C, 27A); it is of comparable mantle length $(36 \mathrm{~mm})$ to Lesueur's original specimen $(\mathrm{ML} 25 \mathrm{~mm})$ and its morphology closely matches Lesueur's (1821) original description and illustrations, and it was collected from the Atlantic Ocean at $36^{\circ} 05.5^{\prime} \mathrm{N}, 69^{\circ} 51.75^{\prime} \mathrm{W}$ (within the Gulf Stream, given as type locality for Ok. (Ok.) carriboea). The description given above for Ok. (Ok.) carriboea is therefore based primarily on the ' $T$. agilis' holotype, augmented with that of a number of other specimens from diverse locations.

Tsuchiya \& Okutani (1992) considered most nominal Onykia species to represent early ontogenetic stages of taxa generally attributed to the better-known genus 'Moroteuthis.' Many distinguishing features of the latter first develop in juvenile to subadult stages. Complete ontogenetic series of specimens bridging the gap between Ok. (Ok.) carriboea and larger juveniles or adults of any younger Onykia species are not available; at present, Ok. (Ok.) carriboea remains known solely from small juveniles and paralarvae, while several younger Onykia species - Ok. (Ok.) 
loennbergii, aequatorialis and robsoni - are known exclusively from later ontogenetic stages.

Juveniles of Ok. (Ok.) robusta, Ok. (M.) ingens and Ok. (Ok.) indica are identifiable from early ontogenetic stages. Ok. (Ok.) robusta possesses 16 pairs of developing tentacular hooks by ML $25 \mathrm{~mm}$, whereas Ok. (Ok.) carriboea has 12 or 13 pairs at ML
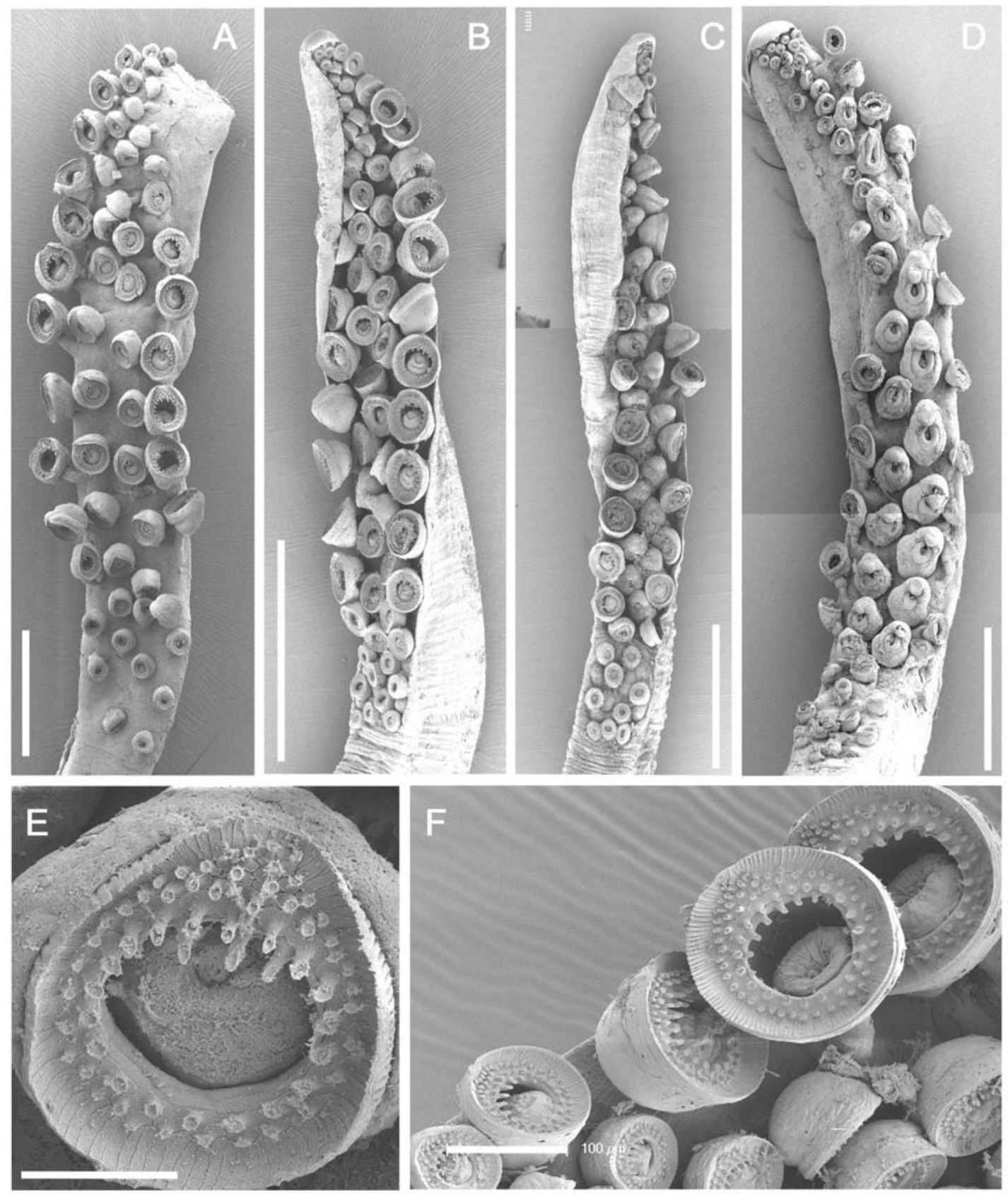

Fig. 28 - Onykia (Onykia) carriboea, paralarval and juvenile tentacular morphology. A, E) BMNH 20070571, ML 8mm; B, F) UMML 31.672, ML 10mm; C) UMML 31.672, ML 16mm; D) USNM 816685, ML 18mm. A) right, B-D) left club; E, F) marginal suckers. Scale bars = A) $500 \mu \mathrm{m}$; B-D) $1 \mathrm{~mm}$; E) $50 \mu \mathrm{m}$; F) $100 \mu \mathrm{m}$. 
$36 \mathrm{~mm}$; the paralarval to juvenile tentacular suckers of $O k$. (M.) ingens bear three distinct concentric rows of densely set, orally flattened pegs proximally, while OK. (OK.) carriboea suckers bear two proximal rows of widely spaced, pointed pegs; additionally, Ok. (M.) ingens lacks a Y-shaped pad in the funnel groove at any ontogenetic stage, while this feature appears in OK. (OK.) carriboea by ML 15-18mm. The arms and tentacles of Ok. (Ok.) indica are long, on mean 110-130\% ML, while those of Ok. (Ok.) carriboea are shorter, generally $60-80 \% \mathrm{ML}$. The known geographic ranges of these three species also do not overlap or even border on the type locality of Ok. (Ok.) carriboea (western North Atlantic). Given that Ok. (Ok.) loennbergii is known solely from the Indian and western Pacific Oceans, it too is unlikely to represent later ontogenetic stages of Ok. (Ok.) carriboea, although it is possible that small juveniles are found in waters where later ontogenetic stages do not occur.

Both Ok. (Ok.) aequatorialis and Ok. (Ok.) robsoni are known only from relatively late ontogenetic stages; both species' distributions also include the central Atlantic and Gulf of Mexico. Given the relative abundance of Ok. (Ok.) carriboea and Ok. (Ok.) robsoni specimens in collections, both from the Atlantic and Pacific Oceans, and the paucity of $O k$. (Ok.) aequatorialis specimens, it seems likely that $O k$. (Ok.) robsoni is synonymous with Ok. (Ok.) carriboea. Small subadult Ok. (Ok.) robsoni specimens were examined in the hope of comparing any remaining marginal suckers with those of Ok. (Ok.) carriboea, but no Ok. (Ok.) robsoni marginal suckers were found in a sufficient state of preservation to permit detailed morphological analysis. Therefore, until OK. (OK.) carriboea can be confirmed as the older name for one of these (or a different) species, all three names are retained and each diagnosed by their most distinctive features. 
Onykia (Onykia) robusta (Verrill, 1876) (new comb.) (Tables 11, 13, Figs 29-33)

Onychoteuthis bergii (not Lichtenstein, 1818) — Dall (1873): 484, 485.

Ommastrephes robustus [sic] Verrill, 1876: 236, 237; Roper \& Sweeney (1978): 12.

Onychoteuthis robusta (Verrill, 1876) - Verrill (1879): 195, (1880): 246-250, PI. 23 Figs 1-6.

Ancistroteuthis robusta (Verrill, 1876) - Steenstrup (1882): 150 (fide Pfeffer 1908a) -

Thompson (1900): 992-998, Figs 1, 2.

Moroteuthis robusta (Verrill, 1876) - Verrill (1882): 65, 209, PI. 13, 14 (fide Pfeffer 1908a) -

Pfeffer (1908a): 68, Figs 78, 79 — Berry (1912): 314, 315 - Sasaki (1929) 230-235,

Text-figs 171, 172, PI. 30, Fig. 7 - Phillips (1961): 416, 417, Fig. 1 - Akimushkin

(1965): 168, 169 - Clarke (1966): 147, Fig. 21, (1986): 77, Fig 14.33D — Iverson \&

Pinkas (1971): Fig. 38 - Zuev \& Nesis (1971): 180-182 — Okutani (1978): 148-150 —

Nesis (1987): 193, Fig. 49G - Okutani et al. (1987): 120, 121, Fig. 43 - Hess (1987):

294-297, 300, 301 — Fiscus et al. (1989): 4, 5, Fig. 1 - Tsuchiya \& Okutani (1992):

137-147, Figs 1-22, 27 - Bizikov (1996): Fig. 10C-E — Filippova et al. (1997): 188, Fig.

111 - Kubodera et al. (1998): 284, 285, Fig. 11 - Sweeney \& Roper (1998): 572 -

Okutani (2005): 156 - Wakabayashi et al. (2007): 959-965, Table 2, Figs 2-6.

Onykia japonica Taki, 1964: 277-281, Text-figs 1-10 — Toll (1982): 62-64, PI. 6 Fig. I —

Sweeney et al. (1992): 136.

Moroteuthis robustus [sic] (Verrill, 1876) - Toll (1982): 64-70, PI. 7 Fig. A, PI. 8 - Roper et al. (1984): 134.

Moroteuthis pacifica Okutani, 1983: 105-112, Table 1, Figs 1-10, PI. 1 - Sweeney et al. (1992): 136.

Type material - USNM 576952, syntype (buccal mass, beaks, rostrum of gladius and arm fragment only), $\mathrm{ML} 1.55 \mathrm{~m}, 53^{\circ} 45^{\prime} \mathrm{N}, 166^{\circ} 45^{\prime} \mathrm{W}, 1872$, Unalaska, lliuliuk, Aleutian Islands, coll. W. Dall (examined); USNM 576974, syntype, (buccal bulb, arm fragment only), ML unknown, $53^{\circ} 45^{\prime} \mathrm{N}, 166^{\circ} 45^{\prime} \mathrm{W}, 1872$, Unalaska, Iliuliuk, Aleutian Islands, coll. W. Dall (examined); NSMT Mo61211, paratype (Moroteuthis pacifica, see above), , ML 113mm, $35^{\circ} 59^{\prime} \mathrm{N}, 170^{\circ} 03^{\prime} \mathrm{E}, 13 / 06 / 1979$, RV No. 1 Ryoun-Maru (JAMARC) (examined); NSMT Mo61212, paratype (Moroteuthis pacifica), sex indet., ML $90 \mathrm{~mm}, 35^{\circ} 54^{\prime} \mathrm{N}, 175^{\circ} 39^{\prime} \mathrm{E}$, 26/06/1978, RV No. 1 Koei-Maru (JAMARC) (examined); NSMT Mo61213, paratype (Moroteuthis pacifica), sex indet., ML $90 \mathrm{~mm}, 35^{\circ} 38^{\prime} \mathrm{N}, 142^{\circ} 30^{\prime} \mathrm{E}, 20 / 12 / 1978,1700 \mathrm{~h}, \mathrm{RV}$ No. 1 Kyowa-Maru (examined); Unregistered holotype (Onykia japonica, see above), collection of I. Taki, Kyoto, Japan, collected 'western part of Tosa Bay to Bungo Channel,' Japan, 230-120m, -/04/1961, trawl, coll. K. Kurohara (not examined).

Additional material examined (19 specimens) - USNM 816870 Specimen A, sex indet., ML 1.00m, 56 56.9'N, 178 09.2'E, 350m, 02/07/1983, RV Miller Freeman Cruise 83-3 Stn 31; USNM 730000 Specimen B, , ML 1.07m, 52 $08.4^{\prime} \mathrm{N}, 175^{\circ} 09.8^{\prime} \mathrm{E}, 137 \mathrm{~m}, 07 / 08 / 1983$, RV Miller Freeman Cruise 83-3, Stn 63; USNM 816870 Specimen B, sex indet., ML 978mm, $51^{\circ} 57.6^{\prime} \mathrm{N}, 178^{\circ} 11.8^{\prime} \mathrm{E}, 269 \mathrm{~m}, 02 / 07 / 1983$, RV Miller Freeman Cruise 83-3 Stn 32; USNM 730000 Specimen A, sex indet., ML $1.4 \mathrm{~m}, 51^{\circ} 56.2^{\prime} \mathrm{N}, 177^{\circ} 00.8^{\prime} \mathrm{E}, 92 \mathrm{~m}, 05 / 08 / 1983$, RV 
Miller Freeman Cruise 83-3, Stn 45; USNM 816870 Specimen C, sex indet., ML 991mm, 51 $1^{\circ} 53.6^{\prime} \mathrm{N}, 178^{\circ} 09.4^{\prime} \mathrm{E}, 185 \mathrm{~m}$, RV Miller Freeman Cruise 83-3 Stn 36; USNM 730000 Specimen C, $\widehat{O}, \mathrm{ML} 1.07 \mathrm{~m}$, Andreanof Island north of entrance to Sequam Pass [ 51 $\left.51^{\circ} 3^{\prime} \mathrm{N}, 176^{\circ} 38^{\prime} \mathrm{W}\right], 128 \mathrm{~m}, 25 / 07 / 1980$, MV Arctic Trawler, coll. P. Richardson; USNM 816872, sex indet., ML $1.97 \mathrm{~m}, 51^{\circ} 46.9^{\prime} \mathrm{N}, 177^{\circ} 39.7^{\prime} \mathrm{E}, 134 \mathrm{~m}$ over $192 \mathrm{~m}, 04 / 08 / 1983$, RV Miller Freeman Cruise 83-3 Stn 43; USNM 816871, 2 sex indet., Specimens C, D, ML $1.07 \mathrm{~m}, 991 \mathrm{~mm}, 51^{\circ} 39.6^{\prime} \mathrm{N}, 179^{\circ} 00.8^{\prime} \mathrm{E}, 428 \mathrm{~m}, 03 / 07 / 1983$, RV Miller Freeman Cruise 833 Stn 37; USNM 816871 Specimen A, sex indet., ML 1.33m, $51^{\circ} 37.8^{\prime} \mathrm{N}, 178^{\circ} 08.8^{\prime} \mathrm{E}$, 411m, 29/07/1983, RV Miller Freeman Cruise 83-3 Stn 12; USNM 816870 Specimen D, sex indet., $\mathrm{ML} 1.12 \mathrm{~m}, 51^{\circ} 36.3^{\prime} \mathrm{N}, 176^{\circ} 22.25^{\prime} \mathrm{E}, 274 \mathrm{~m}, 12 / 08 / 1983$, RV Miller Freeman Cruise 83-3 Stn 87; USNM 816871 Specimen B, sex indet., ML 1.02m, 51ำ $14.5^{\prime} \mathrm{N}$, 179 12.4'E, 262m, 31/07/1983, RV Miller Freeman Cruise 83-3 Stn 19; CASIZ 084613, ㅇ, ML $970 \mathrm{~mm}, 41^{\circ} 03^{\prime} \mathrm{N}, 124^{\circ} 28^{\prime} \mathrm{W}, \mathrm{W}$ of Trinidad Head, California, 549m, 28/07/1962, City of Eureka, coll. G. Alley; CASIZ 084618, + , ML $820 \mathrm{~mm}$, W of Trinidad Head, California [ $\left.41^{\circ} 03^{\prime} \mathrm{N}, 124^{\circ} 28^{\prime} \mathrm{W}\right], 366 \mathrm{~m}, 17-21 / 10 / 1962$, City of Eureka, coll. G. Alley; CASIZ 035031, $\sigma^{\top}, \mathrm{ML} \sim 330 \mathrm{~mm}$ (damaged), W of Trinidad Head, California [ $\sim 41^{\circ} 03^{\prime} \mathrm{N}$, $\left.124^{\circ} 28^{\prime} \mathrm{W}\right], 402 \mathrm{~m}, 11 / 07 / 1950$, Johnny P; NSMT Mo67834,, , ML 90mm, 40¹0'N,

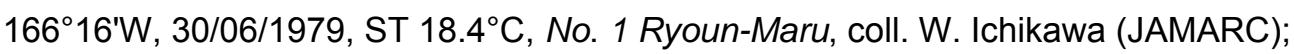
USNM 817578,, , ML 299mm, $37^{\circ} 53.1^{\prime} \mathrm{N}, 123^{\circ} 19.1^{\prime} \mathrm{W}$, Farallon Islands, $32 \mathrm{~m}$ over $85 \mathrm{~m}$, 06/11/1989, 0212h, Stn 160-2; CASIZ 061191, đ̊, ML 790mm, 30km W of Princeton, CA [ 37.5 $\left.\mathrm{N}, \sim 123^{\circ} \mathrm{W}\right], 366 \mathrm{~m}, 03 / 01 / 1986$; NSMT Mo67199, sex indet., ML 160mm, $37^{\circ} 45.0^{\prime} \mathrm{N}, 158^{\circ} 08.7^{\prime} \mathrm{W}, 15 / 06 / 1980$, ST $17.7^{\circ} \mathrm{C}$, coll. W. Ichikawa (JAMARC); CASIZ 112259, ㅇ, ML 830mm, California, 'probably off Big Sur' [36 $06.45^{\prime} \mathrm{N}, 121^{\circ} 37.55^{\prime} \mathrm{W}$ ], 15/10/1997, caught while live spot prawn fishing aboard Nita $H$.

Unlocalised material examined (11 specimens) - CASIZ 084617,, , ML 1.09m, NFD; CASIZ 084616, + , ML 790mm, NFD; NMV F109458, $\odot, ~ M L ~ 730 m m$, British Columbia, NFD; USNM 1102752, $\widehat{O}$, ML 514mm, Pacific Raider Stn 41, NFD; USNM 000542 (7 sex indet.) ML 35-8mm, NL, 67S4-7, NFD.

Distribution (Fig. 29A) - North Pacific, 450-30m; paralarvae reported from north of Hawai'i $\left(26^{\circ} 30^{\prime}-37^{\circ} \mathrm{N}, 155^{\circ}-162^{\circ} 30^{\prime} \mathrm{W}\right.$ (Wakabayashi et al. 2007).

Diagnosis — to $\mathrm{ML} \sim 2 \mathrm{~m}$; fins sagittate, length $\sim 55 \% \mathrm{ML}$, width $\sim 45 \% \mathrm{ML}$, drawn out posteriorly; mantle epidermis with fleshy longitudinal ridges; 31-36 hooks present on adult tentacle club; rostrum of gladius $\sim 20 \% \mathrm{GL}$.

Description, syntypes (Figs 29B-G) - Buccal bulb and arm fragment only (USNM 576974), buccal mass, beaks, rostrum of gladius and arm fragment (USNM 576952). LRL 13mm, URL 13mm (USNM 576952). Lower beak (Figs 29B, 29F, LRL 13mm), lateral profile: longer than deep; hood length $\sim 30 \%$ crest length; jaw angle barely 
obscured by low wing fold. Jaw edge sharp; angle ridge extends over $25 \%$ lateral wall; lateral wall not indented between fold and crest; fold reaches to posterior margin of lateral wall; wing length $\sim 200 \%$ LRL; anterior wing corner proximal to jaw angle with small insert of amber-coloured cartilage. Upper beak with deep notch and groove extending over $40-50 \%$ lateral wall. Rostrum of gladius (Fig. 29C) amber-coloured, $414 \mathrm{~mm}$, rubbery, laminate with concentric layers offset toward ventral surface, oval in cross-section (laterally compressed). Arm fragment (Fig. 29E) diameter 40mm; oral surface $30 \mathrm{~mm}$ wide, with $11 \mathrm{~mm}$ protective membrane; aboral surface $50 \mathrm{~mm}$ wide with $15 \mathrm{~mm}$ swimming keel; suckers smooth-ringed, diameter of each $\sim 7 \mathrm{~mm}$.

Description (other material examined, Figs 30-33) - Mantle fleshy, thick-muscled, broadly spindle-shaped, width 16-23-27\% ML; posterior tip of mantle fleshy, narrow ventrally; epidermis with fleshy longitudinal ridges. Gladius not visible through mantle tissue dorsally or ventrally. Fins elongate heart-shaped to sagittate, smooth-skinned, thick, length $51-55-60 \% \mathrm{ML}$, width $37-45-60 \% \mathrm{ML}$; posterior margins straight to slightly concave, anterior margins straight to slightly convex; anterior lobes rounded.

Head broad, smooth-skinned, dorso-ventrally compressed, length 11-15-20\% ML, width $9-13-19 \% \mathrm{ML}$, depth $~ 8 \% \mathrm{ML}$; not constricted anterior to brachial crown; dorsal occipital region with pronounced transverse crest. Funnel groove broad, shallow Ushape with Y-shaped ridge; groove extends to directly below anterior margin of eye aperture; funnel aperture level with midpoint of eye. Funnel component of locking apparatus $\sim 10 \% \mathrm{ML}$; mantle component $\sim 15 \% \mathrm{ML}$.

Arms smooth-skinned, tips blunt, length $49-61_{I}-77_{I I-I V}-106 \% \mathrm{ML}$, formula IV=II>III>| or IV>||>III>I, each with 140-180 suckers (ML 299-970mm) bordered by strong trabeculate membranes; keels present on Arms I-III: that on Arms I and II 25\% arm width/depth along entire arm length; that on Arms III 75\% arm depth at $~ 50 \%$ arm length, thereafter narrowing along remainder of arm length; lateral membrane on Arms IV $\sim 80 \%$ arm width at base, narrowing to $\sim 50 \%$ arm depth by $\sim 50 \%$ arm length. Sucker diameter $\sim 40 \%$ arm width.

Tentacles 105-165-206\% ML, robust; thickness of stalk bases similar to that of adjacent arms; aboral surface of stalk with low keel. Clubs (Figs 31A-D) 15-18-37\% ML (12-30\% TnL, mean 32\%), not expanded. Carpus well defined, elongate oval with ten to 12 suckers; manus with 31-36 hooks; marginal suckers absent; terminal pad with 11-18 suckers. Hooks (Figs 31F, 32A) short, robust, with deep lateral grooves on 

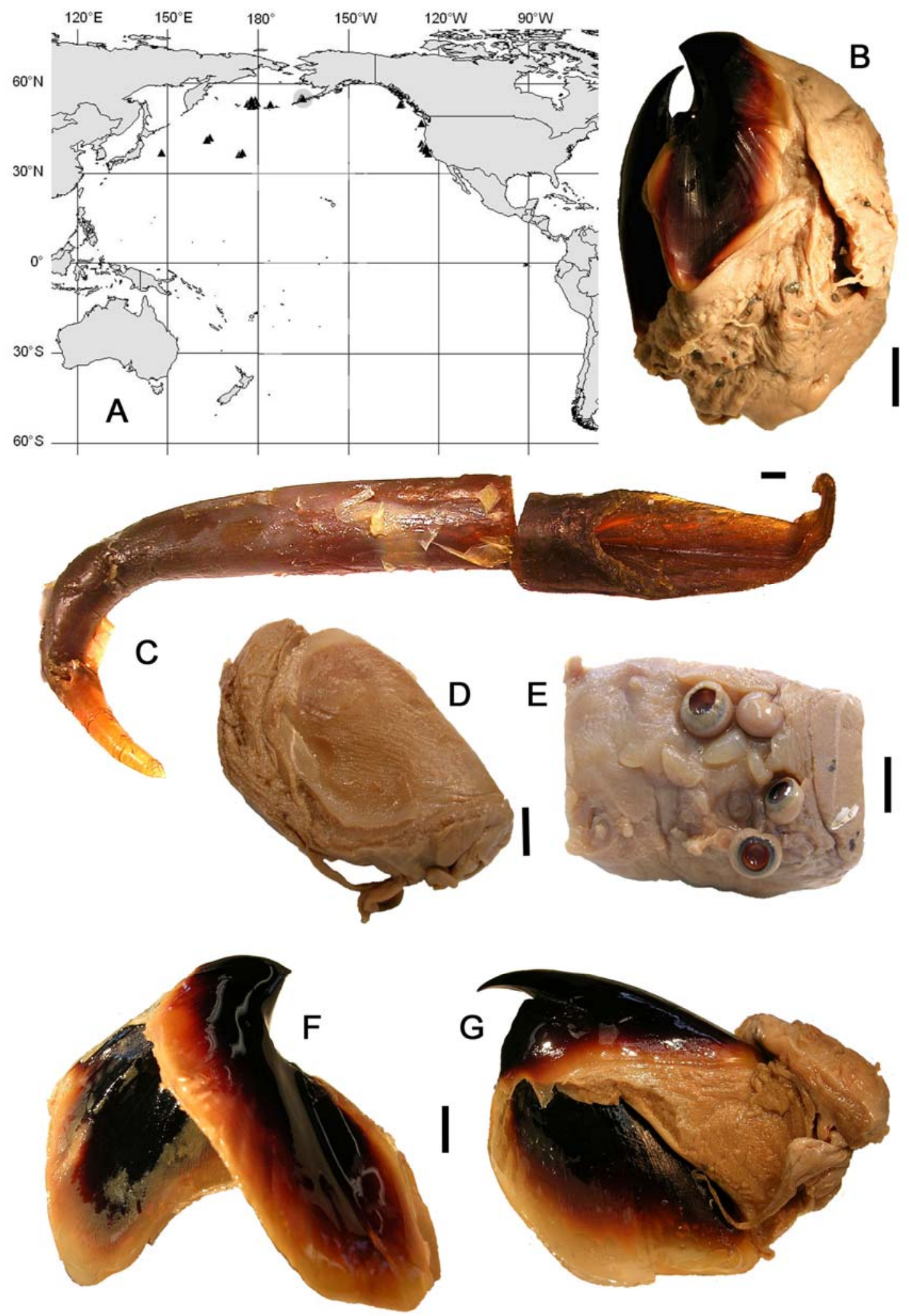

Fig. 29 - Onykia (Onykia) robusta. A) distribution (grey circle indicates type locality); B) USNM 576974, syntype, ML unknown, buccal bulb; C-G) USNM 576952, syntype, ML 1.55m: (C) rostrum of gladius, (D) buccal mass, lateral view, $(E)$ arm fragment, $(F)$ lower beak, $(G)$ upper beak. Scale bars $=10 \mathrm{~mm}$. 
Table 13. Measures ( $\mathrm{mm}$ ) and counts of Onykia (Onykia) robusta (Verrill, 1876) (new comb.) (arm and tentacle measures recorded from more complete side of specimen, indicated $\mathrm{R}$ or $\mathrm{L}$ ).

\begin{tabular}{|c|c|c|c|c|c|c|c|c|c|c|c|c|}
\hline Specimen ID & $\begin{array}{c}\text { CASIZ } \\
084613 \\
\end{array}$ & $\begin{array}{c}\text { CASIZ } \\
084618 \\
\end{array}$ & $\begin{array}{l}\text { CASIZ } \\
061191 \\
\end{array}$ & $\begin{array}{c}\text { USNM } \\
1102752\end{array}$ & $\begin{array}{c}\text { CASIZ } \\
035031 \\
\end{array}$ & $\begin{array}{l}\text { USNM } \\
817578 \\
\end{array}$ & $\begin{array}{c}\text { NSMT } \\
\text { Mo67199 }\end{array}$ & $\begin{array}{c}\text { USNM } \\
000542\end{array}$ & $\begin{array}{c}\text { USNM } \\
000542\end{array}$ & $\begin{array}{l}\text { USNM } \\
050042 \\
\end{array}$ & \multicolumn{2}{|c|}{ Mean Indices } \\
\hline Type Status & None & None & None & None & None & None & None & None & None & None & & \\
\hline ML & 970 & 820 & 790 & 514 & $330^{*}$ & 299 & 160 & 35 & 33 & 12 & \multicolumn{2}{|c|}{ (ML 299-970) } \\
\hline Sex & $\mathrm{F}$ & $\mathrm{F}$ & $\mathrm{M}$ & $\mathrm{M}$ & M & $\mathrm{F}$ & Indet. & Indet. & Indet. & Indet. & & \\
\hline MW & 260 & 220 & 140 & 82 & $70^{*}$ & 81 & 33 & 10 & 10 & 4 & MWI & 23 \\
\hline HL & 105 & 125 & 95 & 57 & 45 & 60 & 33 & 7 & 8 & 4 & $\mathrm{HLI}$ & 15 \\
\hline HW & 100 & 110 & 70 & 69 & 39 & 52 & 22 & 10 & 9 & 4 & HWI & 13 \\
\hline $\mathrm{FL}$ & 492 & 470 & 420 & 292 & $150^{*}$ & 175 & 94 & 17 & 16 & 3 & FLI & 55 \\
\hline FW & 460 & 365 & 290 & 216 & 143 & 152 & 84 & 29 & 23 & 9 & FWI & 45 \\
\hline Arms I & $600 \mathrm{~L}$ & $520 R^{*}$ & $355 L^{*}$ & $254 \mathrm{~L}$ & $206 R^{*}$ & $219 \mathrm{~L}$ & $110 \mathrm{~L}$ & $22 \mathrm{R}^{*}$ & $27 \mathrm{~L}$ & $9 \mathrm{~L}$ & $\mathrm{~A} 1 \mathrm{I}$ & 61 \\
\hline Arms II & $625 \mathrm{~L}^{*}$ & $690 R^{*}$ & $260 L^{*}$ & $343 \mathrm{~L}$ & $297 R^{*}$ & $240 L^{*}$ & $140 \mathrm{~L}$ & $34 \mathrm{R}$ & $31 \mathrm{~L}$ & $12 \mathrm{~L}$ & A2I & 78 \\
\hline Arms III & $580 L^{*}$ & $590 R^{*}$ & $480 L^{*}$ & $356 \mathrm{~L}$ & $305 \mathrm{R}$ & $282 \mathrm{~L}$ & $145 \mathrm{~L}$ & $33 R$ & $32 \mathrm{~L}$ & $11 \mathrm{~L}$ & A3I & 76 \\
\hline Arms IV & $625 \mathrm{~L}^{*}$ & $670 R^{*}$ & $545 \mathrm{~L}$ & $368 \mathrm{~L}$ & $340 \mathrm{R}$ & $290 \mathrm{~L}$ & $145 \mathrm{~L}$ & $32 \mathrm{R}$ & $32 \mathrm{~L}$ & $11 \mathrm{~L}$ & A4I & 78 \\
\hline TnL & $1800 \mathrm{~L}$ & 1690R & $1450 \mathrm{~L}$ & $730 \mathrm{~L}$ & 1040R & $579 \mathrm{~L}$ & $225 \mathrm{~L}$ & $42 \mathrm{R}$ & $34 \mathrm{~L}$ & $13 \mathrm{~L}$ & TnLI & 165 \\
\hline $\mathrm{CL}$ & $220 \mathrm{~L}$ & $255 \mathrm{R}$ & $220 \mathrm{~L}$ & 137L & $165 \mathrm{R}$ & $125 \mathrm{~L}$ & $58 \mathrm{~L}$ & $13 R$ & $14 \mathrm{~L}$ & $6 \mathrm{~L}$ & CLI & 18 \\
\hline cs & 10 & 12 & 12 & 11 & 11 & 12 & 11 & 11 & 11 & 12 & & \\
\hline MH & 36 & 34 & 36 & 33 & 35 & $36^{*}$ & 34 & 33 & 34 & None & & \\
\hline MS & 0 & 0 & 0 & 0 & 0 & 0 & 0 & All & All & All & & \\
\hline TPS & 12 & 16 & 11 & 15 & 13 & * & 12 & 15 & 15 & Undifferentiated & & \\
\hline
\end{tabular}

* indicates damaged features.

claw, most densely set proximally, becoming widely spaced distally; proximal three or four hook pairs all of similar size, thereafter ventral hooks gradually increase in size through hooks V8 and V9; largest ventral hooks (Fig. 32A) 5\% CL, twice length of paired dorsal hooks. Ventral membrane $\sim 50 \%$ club width, flanking hooks V1-V7; dorsal membrane not apparent; dorsal keel 150-200\% club width, flanking hooks D7D17.

Lateral profile of lower beak (Figs 32C-E, ML 730mm) longer than deep, with straight crest, sloping moderately steeply downward; posterior edge of hood well above crest; hood length $\sim 60 \%$ total crest length, and hood and crest together $\sim 60 \%$ baseline; jaw edge straight along entire length, produced distally into small rostral tip; jaw angle obtuse, obscured by slight wing fold. Crest narrow, slightly thickened, with lateral walls markedly indented below; jaw edge narrow and sharp; shoulder ridge indistinct; angle ridge short, spanning $\sim 40 \%$ lateral wall depth, moderately well developed; angle point indistinct; wings broaden somewhat distally, width $\sim 150 \%$ that at jaw angle, length $\sim 300 \%$ LRL; small insert of cartilage present on medial wing margin just below jaw angle; lateral wall fold rounded, well developed along entire length and remaining distinct to posterior margin of lateral wall.

Radula (Fig. 32F) with narrow mesocone, conical to nearly cylindrical, pointed distally; lateral cusps $\sim 50 \%$ mesocone height, slightly laterally directed or gently recurved toward rachidian; proximal margin of base concave, distal margin between mesocone 
and lateral cusps roundly concave. First lateral tooth bicuspid; inner cusp broad, conical, straight but directed slightly toward rachidian, its height $\sim 90 \%$ that of rachidian; outer cusp somewhat pointed, slightly laterally directed, its height $\sim 50 \%$ that of inner cusp. Second lateral tooth forms slender isosceles triangle, $\sim 150 \%$ rachidian height. Marginal tooth simple, slender, curved away from plane of radula; height
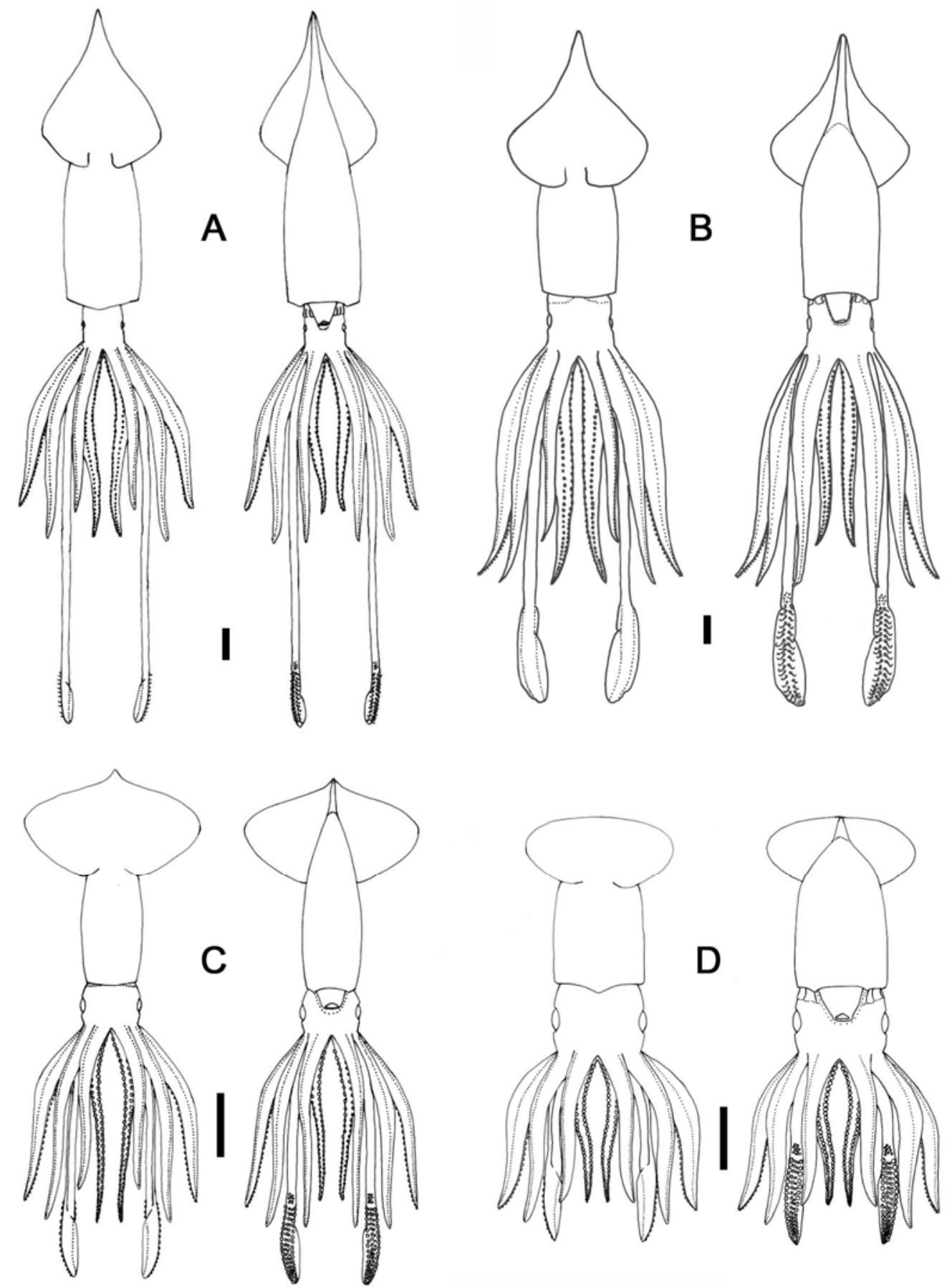

Fig. 30 - Onykia (Onykia) robusta. A) USNM 730000, Specimen B, +, ML 1.07m; B) NSMT Mo61211, paratype of 'Moroteuthis pacifica,' 9, ML 113mm; C, D) USNM 000542: (C) sex indet., ML 25mm, (D) sex indet., ML 13mm. Scale bars = A) $100 \mathrm{~mm}$; B, C) $10 \mathrm{~mm}$; D) $1 \mathrm{~mm}$. 
$\sim 160 \%$ rachidian height. Palatine palp (Fig. 32G) broad, greatest width of toothbearing plane $\sim 35 \%$ its length, with $\sim 50$ short, stubby, flattened triangular teeth, each 100-200\% rachidian height, sparsely distributed over surface of palp.

See Remarks regarding gladius.

Smaller specimens (ML 25-100mm, Figs 30C, 32B, 33A) resemble subadults but deviate from the above description in several respects: mantle smooth through at least $\mathrm{ML} 35 \mathrm{~mm}$, ridged by $\mathrm{ML} 90 \mathrm{~mm}$; rostrum of gladius visible ventrally through posterior 15-30\% ML; fins more heart-shaped, width 70-83\% ML; head width comparable to that of mantle ( 30\% ML), length $20-24 \% \mathrm{ML}$, depth $\sim 20 \% \mathrm{ML}$; Y-shaped ridge in funnel groove apparent by ML $26 \mathrm{~mm}$; funnel component of locking apparatus $\sim 15 \%$ ML, mantle component 26-29\% ML; arms with $\sim 100$ suckers at $M L ~ \sim 100 \mathrm{~mm}$; keels present on Arms I-III: that on Arms I $10 \%$ arm width along entire arm length; that on Arms II $\sim 20 \%$ arm depth along entire arm length; that on Arms III $\sim 75 \%$ arm depth at $\sim 40 \%$ arm length, narrowing to $30 \%$ arm depth along remainder of arm length; lateral membrane on Arms IV 100\% arm width at bases, narrowing thereafter to $\sim 50 \%$ arm width along remainder of arm length; tentacles 105-120\% ML, bases approximately equal in thickness to adjacent arm bases; aboral surface of stalk with low keel, $20 \%$ tentacle width, from base to opposite sixth hook pair on manus; tentacle club (Fig. $33 \mathrm{~A})$ somewhat expanded, length $37-42 \% \mathrm{ML}(31-41 \% \mathrm{TnL}$, mean $42 \%)$.

By ML 33mm: carpus well defined, circular to oval in outline, with $\sim 11$ suckers; manus with 33 or 34 hooks, each bordered by large marginal sucker; terminal pad with $\sim 15$ suckers. By ML 30mm, mid-series ventral hooks are longer than adjacent marginal suckers. By ML 26mm all hooks are forming. Gladius (Fig. 32B) broad, greatest width $(\sim 12 \% \mathrm{GL})$ attained at $\sim 55 \% \mathrm{GL}$; free rachis $\sim 20 \% \mathrm{GL}$, with vanes widening and then narrowing rapidly between 20 and $85 \%$ GL; rostrum $\sim 14 \%$ GL, cartilaginous, narrow when viewed ventrally, triangular in lateral profile.

Overall colouration (preserved) pinkish with dark reddish- to bluish-purple chromatophores, darkest on dorsal surfaces of head and mantle and between fins; ventral surface of mantle with a single large, dark-brown 'tail-spot' on either side; dorso-lateral mantle surfaces iridescent blue-green; lateral surfaces of head iridescent coppery-blue; aboral surfaces of arms iridescent green-gold over proximal half, with large, dark chromatophores in longitudinal rows along entire aboral surface; oral faces 

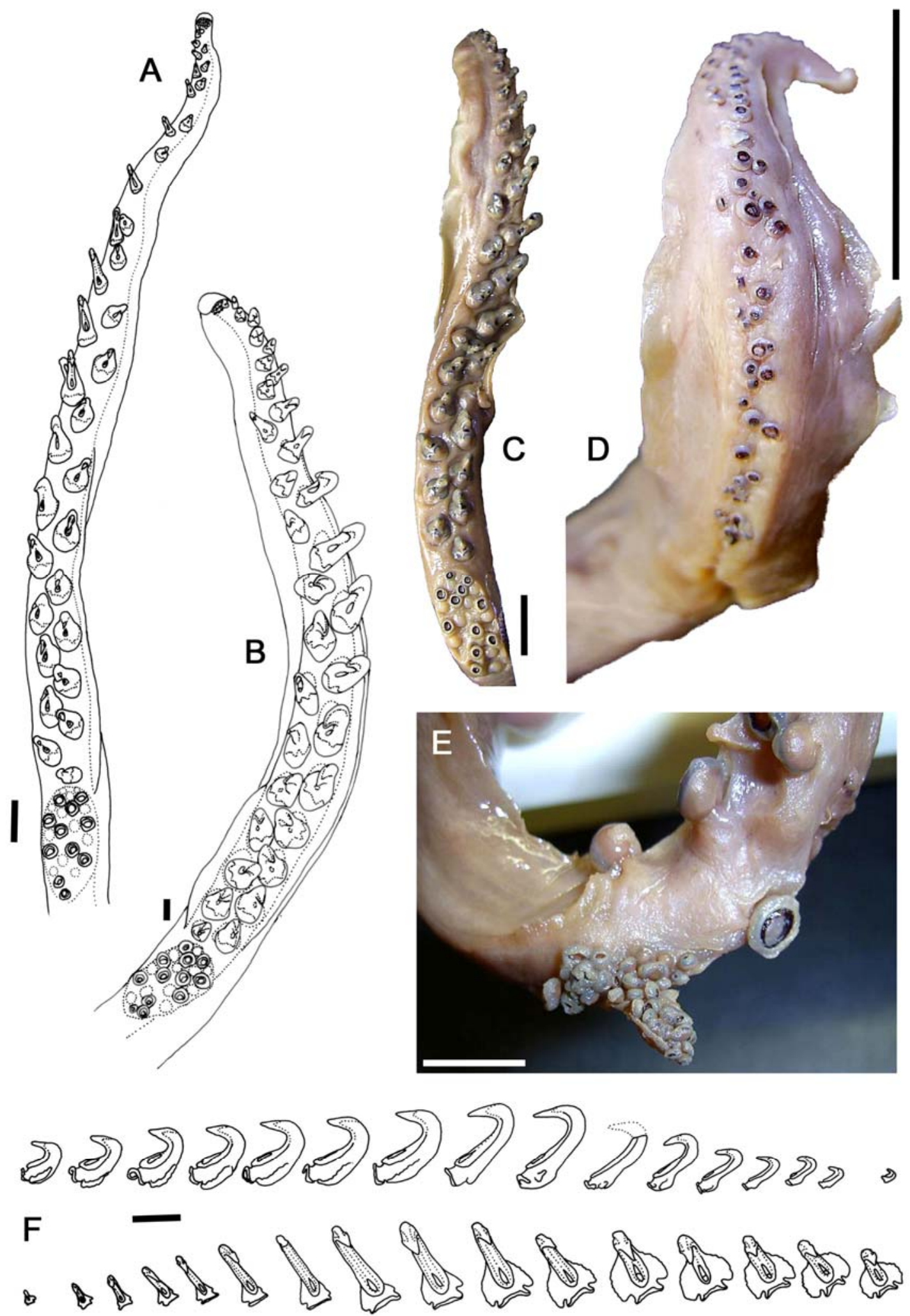

Fig. 31 - Onykia (Onykia) robusta. A) CASIZ 61191, đૈ, ML 790mm; B) NSMT Mo61211, paratype of 'Moroteuthis pacifica,' 9, ML 113mm; C-E) USNM 1102752, 0 , $M L 514 \mathrm{~mm}$. A) right, $B, C$ ) left tentacle club; D) regenerating right tentacle club; $E$ ) abnormal growth on regenerating Arm IIR; F) right ventral hook series. Scale bars $=A$, C-E ) $10 \mathrm{~mm}$; B) $1 \mathrm{~mm}$; F) $5 \mathrm{~mm}$. 
of arms with single row of large dark brown chromatophores interspersed with each sucker row; aboral surfaces of tentacle stalks and club with several longitudinal rows of large chromatophores, becoming finer on lateral and oral surfaces.

Description, paralarva (ML 8-13mm, Figs 30D, 33B, 33C) - Mantle broad, spindleshaped, stubby, width 33-50\% ML; epidermis smooth; gladius not visible dorsally; rostrum visible ventrally through posterior $\sim 13 \% \mathrm{ML}$; posterior portion of mantle very broad anteriorly, somewhat attenuate posteriorly where supported by rostrum of gladius. Fins broad, oval in combined outline, length $25-40 \% \mathrm{ML}$, width $75-80 \% \mathrm{ML}$;
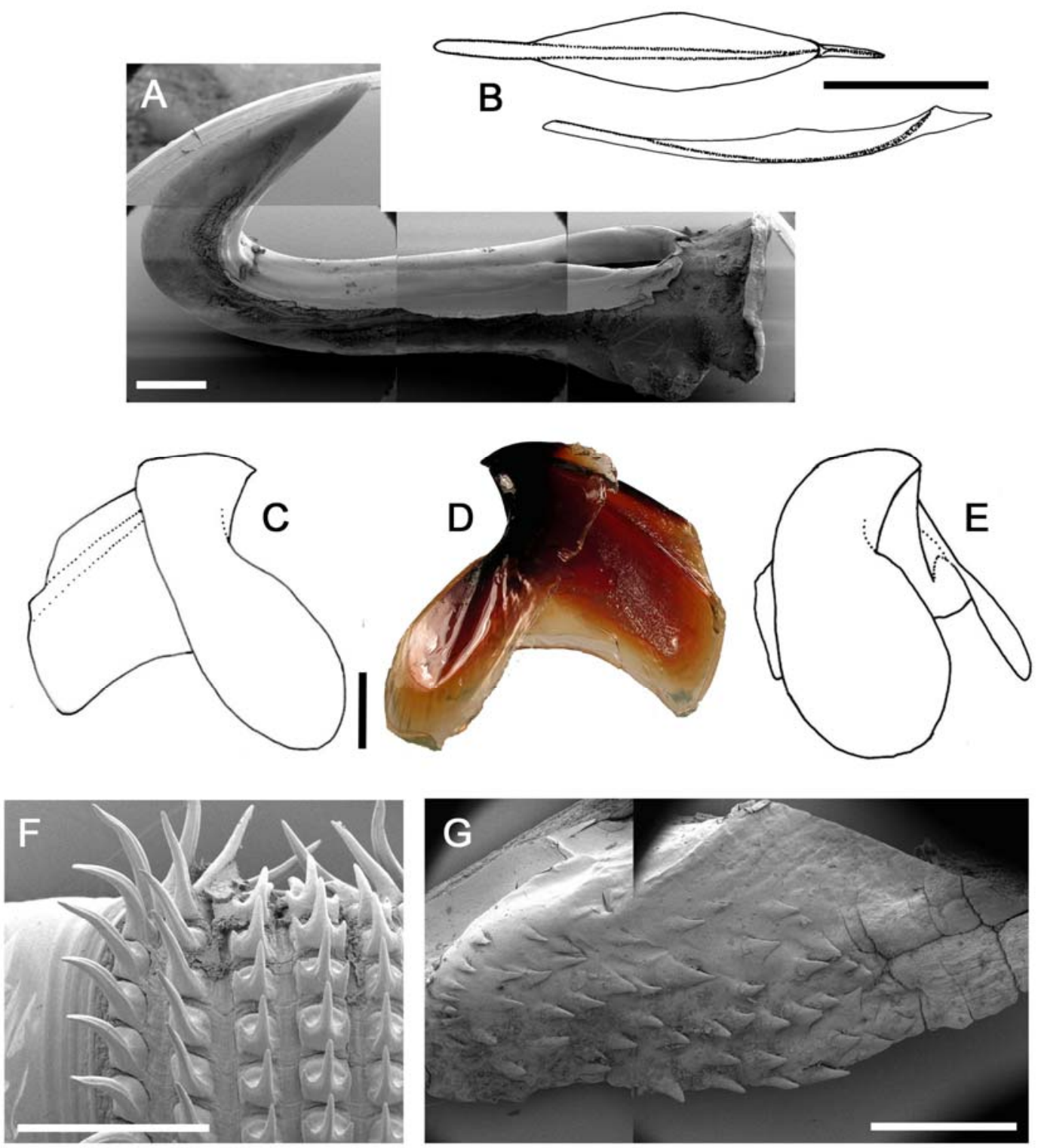

Fig. 32 - Onykia (Onykia) robusta. A) CASIZ 61191, ô, ML 790mm; B) USNM 000542, sex indet., ML 25mm; C-E) NMV F109458, +, ML 730mm; F, G) USNM 1102752, O, ML 514mm. A) right tentacular hook V9; B) gladius; C-E) lower beak: (C) left, $(D)$ right, $(E)$ left oblique profiles; $F$ ) radula; $G$ ) left palatine palp. Scale bars = A-D, F) $10 \mathrm{~mm}$; B) $10 \mathrm{~mm}$; G) $2 \mathrm{~mm}$. 
posterior margins gently convex, together rounded, forming nearly straight single margin across midline; lateral 'corners' broadly rounded; anterior margins convex; anterior lobes shallow, barely apparent. Head width comparable to that of mantle (33$50 \% \mathrm{ML}$ ), length $25-40 \% \mathrm{ML}$; eyes slightly anteriorly directed; dorsal transverse ridge not well defined; ventral occipital region with three well-defined primary folds; head slightly constricted anteriorly. Funnel groove broad, U-shaped, Y-shaped ridge not yet well developed; funnel short, bulbous, with aperture at level below posterior margin of eye aperture; funnel component of locking apparatus $15-17 \% \mathrm{ML}$, mantle component 25-35\% ML. Arms slender, length 75-100\% ML, formula II=III>IV>I, each with 70-80 suckers bordered on either side by well-developed trabeculate membrane; keels on Arms I-III as in ML 25-35mm; lateral membrane on Arms IV $~ 75 \%$ arm width at bases, narrowing thereafter to $\sim 50 \%$ arm width along remainder of arm length. Tentacles $105-115 \% \mathrm{ML}$, with bases more slender than adjacent arms; aboral surface of stalk with low keel, $\sim 10 \%$ tentacle width, from base to opposite sixth hook pair on manus. Club (Fig. 33C) somewhat expanded, length 50-56\% ML (46-50\% TnL). Carpus not well defined, with ten to 12 suckers merging somewhat into proximal manus region; manus with $\sim 17$ transverse rows of four suckers; suckers in medial two rows (future hook-bearers) recessed into surface of club, just beginning to form distal points at $\mathrm{ML}$
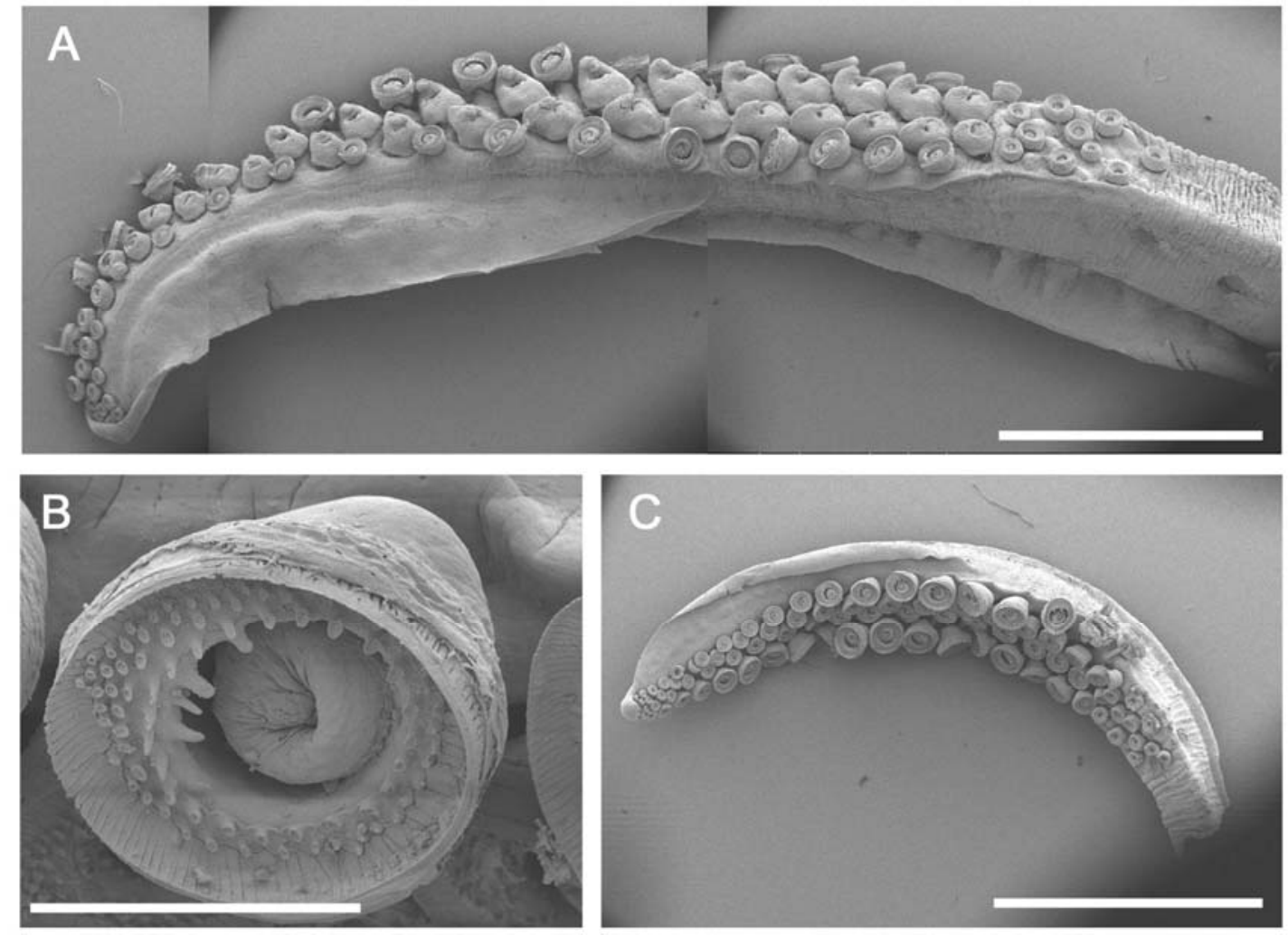

Fig. 33 - Onykia (Onykia) robusta, USNM 000542. A) sex indet., ML 25mm, left tentacle club; B, C) sex indet., ML 12mm: (B) marginal tentacular sucker, (C) left tentacle club. Scale bars = A, C) $2 \mathrm{~mm}$; B) $200 \mu \mathrm{m}$. 
$12 \mathrm{~mm}$; diameter of all ventral marginal suckers greater than that of adjacent suckers in ventral median row; diameter of marginal suckers D1-D8 greater than that of adjacent dorsal median suckers, thereafter dorsal median and marginal suckers subequal in size; terminal pad not yet differentiated. Marginal suckers (Fig. 33B) with large, circular aperture, diameter $\sim 50 \%$ sucker diameter, with about seven low, indistinct bumps around proximal portion of inner margin; pegs set in two concentric rows proximally and about five concentric rows distally; two proximal-most rows of distal pegs ( 15 pegs in total) elongate, with concave oral surfaces, directed toward centre of sucker, forming 'teeth.'

Remarks - Verrill, in his original description (1876) and later more detailed description of this species (1882), referred to three specimens of Ok. (Ok.) robusta. The label of USNM 576952 indicates that it is Verrill's Specimen 2 and calls it the holotype. However, a second specimen (USNM 576974), comprised of a buccal mass and arm fragment, has the same collection data as the holotype and is labelled 'Part of Type Series,' although Verrill's specimen number is not given. As Verrill states that the 'pharynx, beak and odontophore' of Specimen 1 were preserved, but does not mention preservation of any fragments of Specimen 3, it is likely that USNM 576974 represents the remains of Specimen 1. Since neither of these specimens is formally designated as holotype by Verrill or as lectotype by any subsequent publication, both are in fact syntypes.

Tsuchiya \& Okutani (1992) placed the Moroteuthis into synonymy of Onykia, based on a thorough examination of growth series of the type species of Moroteuthis, 'Ommastrephes robustus' - a synonymy supported by Wakabayashi et al. (2007), following molecular analysis of north Pacific Onykia and 'Moroteuthis' specimens, wherein most Onykia specimens were identified as paralarvae of Ok. (Ok.) robusta.

One specimen of Ok. (Ok.) robusta (USNM 1102752) was observed with several arm tips and the right tentacle club regenerating; the left club was entirely normal in all respects (Fig. 31C). The entire right tentacle was $20 \mathrm{~cm}$ long, with $\sim 70$ tiny suckers set in irregular clusters along the oral face over the distal 4cm (Fig. 31D), not yet differentiated into distinct regions. The tips of Arms IL and IIR were also regenerating, and midway along Arm IIR, an abnormal growth about 10mm long projected laterally from the oral face of the arm, densely set with $\sim 35$ suckers (Fig. 31E). It appears that the regrowth triggered by the loss of brachial tissue occasionally goes awry; in this 
case, a small, malformed arm-like appendage had begun to grow at the site of a lost arm sucker.

No adult gladius of Ok. (Ok.) robusta was available for examination during this study; for completeness and comparison, the following description is based on Sasaki 1929, Text-fig, 172: greatest width ( $8 \% \mathrm{GL})$ attained at $25-35 \% \mathrm{GL}$; free rachis $\sim 13 \% \mathrm{GL}$; vanes broaden rapidly between 15 and $30 \% \mathrm{GL}$, gradually narrowing thereafter; dorsal keel shallow, broad. Rostrum $20 \%$ GL, with circular cross-section. 
Onykia (Onykia) loennbergii (Ishikawa \& Wakiya, 1914) (new comb.) (Tables 11, 14, Figs 34-36)

Moroteuthis lönnbergii [sic] Ishikawa \& Wakiya, 1914: 445-459, Pls 45, 46; Sasaki (1929): 235-237, Text-fig. 119, PI. 20 Fig. 13; Clarke (1966): 147, Fig. 21; Sweeney \& Roper (1998): 572; Wakabayashi et al. (2007): 959-965, Table 1, Figs 2, 3.

Moroteuthis lömbergi [sic] (Ishikawa \& Wakiya, 1914) — Roper et al. (1984): 132.

Moroteuthis loennbergi [sic] (Ishikawa \& Wakiya, 1914) - Nesis (1987) 193, Fig. 49H; Okutani et al. (1987): 118, 119, Fig. 42; Wadley (1990): 4; Filippova et al. (1997): 188.

Moroteuthis lonnbergii [sic] (Ishikawa \& Wakiya, 1914) — Sweeney et al. (1992): 135, 136;

Kubodera et al. (1998): 283-286, Table 2, Fig. 10.

Moroteuthis lonbergi [sic] (Ishikawa \& Wakiya, 1914) — Okutani (1978): 150-152.

Moroteuthis loennbergii (Ishikawa \& Wakiya, 1914) — Tsuchiya \& Okutani (1992): 137-147,

Fig. 23; Okutani (2005): 157.

Type material - None (never designated).

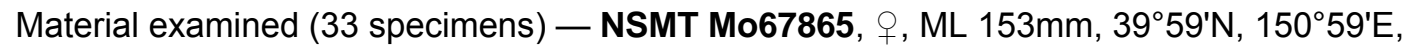
24/06/1993, RV Wakashio-Maru squid jig; NSMT Mo67866, sex indet., ML 105mm, 3901'N, 14958'E, 26/06/1993, RV Wakashio-Maru, squid jig; NSMT Mo71583, §̊, ML 295mm, 37040.9'N, 14147.2'E, 343m, 08/06/1999, RV Wakataka-Maru, coll. D. Kitagawa; NSMT Mo67328, $2 \hat{\circ}$, ML 240, 240mm, $1 \mathrm{~km}$ off Kambara [ $\sim 35^{\circ} 07^{\prime} \mathrm{N}$, 138 36'E], 100-80m, 2050-2225h, 17/05/1978, RV Inari-Maru, SrT; JAMSTEC HD521SS4, sex indet., ML $61 \mathrm{~mm}, 34^{\circ} 59.4^{\prime} \mathrm{N}, 140^{\circ} 15.5^{\prime} \mathrm{E}$, off Kamogawa, $481 \mathrm{~m}$, 04/03/2006, 1135h, Cruise NT06-03; NSMT Mo67451, ڤ̂, ML 285mm, Suruga Bay [ 34 $\left.{ }^{\circ} 51^{\prime} \mathrm{N}, 138^{\circ} 33^{\prime} \mathrm{E}\right], 20 / 05 / 1978$, RV Inari-Maru, SrT; NSMT Mo67330, 3ㅇ, ML 265, 216, $210 \mathrm{~mm}$, Suruga Bay [ $\left.34^{\circ} 51^{\prime} \mathrm{N}, 138^{\circ} 33^{\prime} \mathrm{E}\right]$, 09/05/1978, RV Inari-Maru, SrT; NSMT M067329, , ML 253mm, Suruga Bay [ $\left.34^{\circ} 51^{\prime} \mathrm{N}, 138^{\circ} 33^{\prime} \mathrm{E}\right], 03 / 05 / 1978$, RV Inari-Maru, SrT; NSMT Mo67453,, , ML 236mm, Suruga Bay [ $\left.34^{\circ} 51^{\prime} \mathrm{N}, 138^{\circ} 33^{\prime} \mathrm{E}\right]$, 20/05/1978, RV Inari-Maru, SrT; NSMT Mo67330, 3ㅇ, ML 265, 215, 210mm, Suruga

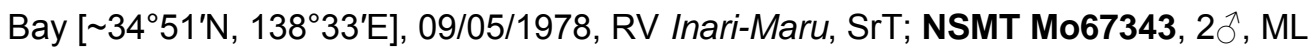
199, 175mm, Suruga Bay [ 3451'N, 138³3'E], 24/05/1978, RV Inari-Maru, SrT;

NSMT Mo67809, sex indet., ML 196mm, Suruga Bay [ $\left.34^{\circ} 51^{\prime} \mathrm{N}, 138^{\circ} 33^{\prime} \mathrm{E}\right]$, 03/06/1978, RV Daikoku-Maru; NSMT Mo67452, + , ML 179mm, Suruga Bay [ 34 $\left.51^{\circ} \mathrm{N}, 138^{\circ} 33^{\prime} \mathrm{E}\right], 20 / 05 / 1978, \mathrm{RV}$ Inari-Maru, SrT; NSMT Mo72205, sex indet., ML $45 \mathrm{~mm}$, Suruga Bay [ $\left.34^{\circ} 51^{\prime} \mathrm{N}, 138^{\circ} 33^{\prime} \mathrm{E}\right]$, Shizuoka Pref., 26/07/1976; NSMT Mo67344, ㅇ, ML 245mm, Ô, ML $201 \mathrm{~mm}$, off Atawa, Kumano-Nada (S. off Mikisaki) [ $\left.\sim 33^{\circ} 50^{\prime} \mathrm{N}, 136^{\circ} 15^{\prime} \mathrm{E}\right], 300-200 \mathrm{~m}, 07-08 / 05 / 1982$, coll. M. Uchida (Mie University);

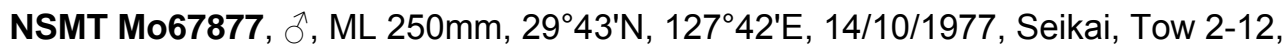
coll. Kitajima; NMV F71690, sex indet. (viscera removed), ML 230mm; $17^{\circ} 34^{\prime} \mathrm{S}$, 118 $44^{\prime} \mathrm{E}, 450 \mathrm{~m}, 03 / 02 / 1984$, NFD; NMV F109452, 2ð, ML 305, 225mm, 2ㅇ, ML 271, $269 \mathrm{~mm}, 17^{\circ} 53^{\prime} \mathrm{S}, 118^{\circ} 26-30^{\prime} \mathrm{E}$, of Broome, Western Australia, 460-442m, 05/02/1983, 


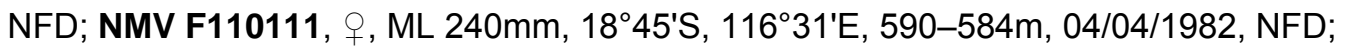

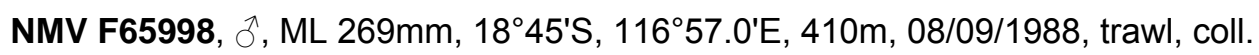
CSIRO Marine Research; NSMT Mo76089, $0^{\top}, \mathrm{ML} 71 \mathrm{~mm}, 24^{\circ} 58.0^{\prime} \mathrm{S}, 108^{\circ} 59^{\prime} \mathrm{W}$, surface, 26/10/1979, $22.6^{\circ} \mathrm{C}$, lancetfish stomach, RV Kaki-Maru 1, ST 22.6 ${ }^{\circ} \mathrm{C}$; NMV F110115, sex \& ML unknown (beak only), 2904.8'S, 101²43.8'E, 19/10/1992, NFD; SAM S2188, + , ML $222 \mathrm{~mm}, 34^{\circ} 47.1^{\prime} \mathrm{S}, 18^{\circ} 03.9^{\prime} \mathrm{E}, 684 \mathrm{~m}$ over $699 \mathrm{~m}, 13 / 03 / 1988$, 1140h, RS Africana Mesopelagic Cruise Stn A7029-060-13-03M, EMWT.

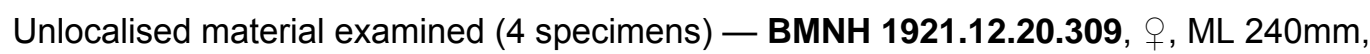
Japan, NFD; NSMT Mo67251, , ML 223mm, ô, ML 127mm, 15/04/1983, RV SoyoMaru Stn 41, NFD; NSMT Mo66918, §̂, ML 54mm, Sagami Bay, coll. Kubota (Tokai University).

Distribution (Fig. 34A) - North- and south-west Pacific and eastern Indian Oceans, $1100-0 \mathrm{~m}$. Occasional reports from eastern South Pacific and southern Atlantic.

Diagnosis $-M L$ to $\sim 350 \mathrm{~mm}$; epidermis of mantle with narrow longitudinal ridges; fins rhombic, length and width subequal, $\sim 52 \% \mathrm{ML}$; manus with 24-29 hooks; free rachis and rostrum of gladius each $\sim 20 \% \mathrm{GL}$.

Description (Figs 34B-36F) - Mantle cylindrical anteriorly, width 15-22-28\% ML, slender in subadult specimens, narrowing rapidly at $\sim 50 \% \mathrm{FL}$, with fine longitudinal ridges (Fig. 35E) over all surfaces apart from ventral posterior 15\% ML (over rostrum of gladius). Gladius not visible dorsally or ventrally through mantle tissue. Fins rhombic; length and width subequal, 48-52-56\% ML; posterior margins straight laterally, becoming slightly concave toward midline and forming small distal point; anterior margins straight, with large anterior lobes.

Head cylindrical, slightly dorso-ventrally compressed, length $\sim 16 \% \mathrm{ML}$, width 13-16$20 \% \mathrm{ML}$, depth $12-18 \% \mathrm{ML}$, not constricted anteriorly; skin on head with less pronounced longitudinal ridges than those on mantle; dorsal occipital region with pronounced transverse crest; funnel robust, aperture at level below posterior margin of eye aperture; funnel groove U-shaped with $Y$-shaped pad, extending over ventral surface of head to point level with midpoint of eye; funnel component of locking apparatus $\sim 11 \% \mathrm{ML}$, mantle component $\sim 17 \% \mathrm{ML}$.

Arms slender, length $53-64-74_{\|-I V}-85 \% \mathrm{ML}$, formula IV>III=II>I, attenuating to very slender tips, each with 100-120 suckers (ML 61-295mm), their maximum diameter 
Table 14. Measures ( $\mathrm{mm}$ ) and counts of Onykia (Onykia) loennbergii (Ishikawa \& Wakiya, 1914) (new comb.) (arm and tentacle measures recorded from more complete side of specimen, indicated $\mathrm{R}$ or $\mathrm{L}$ ).

\begin{tabular}{|c|c|c|c|c|c|c|c|c|c|c|c|c|}
\hline Specimen ID & $\begin{array}{c}\text { NSMT } \\
\text { Mo71583 }\end{array}$ & NMV F65998 & $\begin{array}{c}\text { BMNH } \\
1921.12 .20 . \\
309\end{array}$ & $\begin{array}{c}\text { NSMT } \\
\text { Mo67251 }\end{array}$ & $\begin{array}{l}\text { SAM } \\
\text { S2188 }\end{array}$ & $\begin{array}{c}\text { NSMT } \\
\text { Mo67330 }\end{array}$ & $\begin{array}{c}\text { NSMT } \\
\text { Mo67865 }\end{array}$ & $\begin{array}{c}\text { NSMT } \\
\text { Mo67251 }\end{array}$ & $\begin{array}{l}\text { JAMSTEC } \\
\text { HD521SS4 }\end{array}$ & $\begin{array}{c}\text { NSMT } \\
\text { Mo72205 }\end{array}$ & \multicolumn{2}{|c|}{ Mean Indices } \\
\hline Type Status & None & None & None & None & None & None & None & None & None & None & \multirow{2}{*}{\multicolumn{2}{|c|}{ (ML 127-295) }} \\
\hline $\mathrm{ML}$ & 295 & 269 & 240 & 223 & 222 & 215 & 152 & 127 & 61 & 46 & & \\
\hline Sex & $\mathrm{M}$ & $M$ & $\mathrm{~F}$ & $\mathrm{~F}$ & $\mathrm{~F}$ & $\mathrm{~F}$ & $\mathrm{~F}$ & $M$ & Indet. & Indet. & & \\
\hline MW & 65 & 75 & 55 & 45 & 54 & 49 & 33 & 24 & 14 & 18 & MWI & 22 \\
\hline $\mathrm{HL}$ & 40 & 42 & 33 & 39 & 37 & 35 & 26 & 23 & Unknown & 14 & $\mathrm{HLI}$ & 16 \\
\hline HW & 43 & 35 & 40 & 36 & 45 & 34 & 27 & 24 & 14 & 12 & HWI & 16 \\
\hline FL & 153 & 130 & 122 & 125 & 111 & 112 & 77 & 61 & 27 & 19 & FLI & 51 \\
\hline FW & 150 & 140 & 123 & 125 & 108 & 109 & 83 & 70 & 40 & 34 & FWI & 53 \\
\hline Arms I & $152 \mathrm{~L}$ & $125 L^{*}$ & $140 R$ & $119 \mathrm{~L}$ & $140 R$ & $162 \mathrm{~L}$ & $105 \mathrm{~L}$ & $85 \mathrm{~L}$ & $36 R$ & $41 \mathrm{~L}$ & A1I & 64 \\
\hline Arms II & $190 \mathrm{~L}$ & $150 \mathrm{~L}^{*}$ & $161 R$ & $158 \mathrm{~L}$ & $166 \mathrm{R}$ & $170 \mathrm{~L}$ & $130 \mathrm{~L}$ & $102 \mathrm{~L}$ & $45 R$ & $51 \mathrm{~L}$ & A2I & 76 \\
\hline Arms III & $172 \mathrm{~L}$ & $155 \mathrm{~L}$ & $158 \mathrm{R}$ & $154 \mathrm{~L}$ & $160 R$ & $150 \mathrm{~L}^{*}$ & $120 \mathrm{~L}$ & $96 \mathrm{~L}$ & $41 R$ & $48 \mathrm{~L}$ & A3I & 70 \\
\hline Arms IV & $195 \mathrm{~L}$ & $195 \mathrm{~L}$ & $181 R$ & $160 \mathrm{~L}$ & $165 R$ & $178 \mathrm{~L}$ & $126 \mathrm{~L}$ & $103 \mathrm{~L}$ & $46 R$ & $50 \mathrm{~L}$ & A4I & 77 \\
\hline TnL & $360 \mathrm{~L}$ & $380 \mathrm{~L}$ & $355 R$ & $318 \mathrm{~L}$ & $380 R$ & $332 \mathrm{~L}$ & $245 \mathrm{~L}$ & $204 \mathrm{~L}$ & $60 R$ & $102 \mathrm{~L}$ & TnLI & 150 \\
\hline $\mathrm{CL}$ & $78 \mathrm{~L}$ & $62 \mathrm{~L}$ & $66 \mathrm{R}$ & $68 \mathrm{~L}$ & $71 R$ & $70 \mathrm{~L}$ & $58 \mathrm{~L}$ & $46 \mathrm{~L}$ & $20 R$ & $27 \mathrm{~L}$ & $\mathrm{CLI}$ & 30 \\
\hline cs & 8 & 9 & 8 & 9 & 7 & 9 & $6^{*}$ & 7 & 8 & 8 & & \\
\hline MH & 26 & 24 & 29 & 26 & 25 & 26 & 27 & 27 & 25 & 26 & & \\
\hline MS & 0 & 0 & 0 & 0 & 0 & 0 & 0 & 0 & 0 & 0 & & \\
\hline TPS & 13 & 10 & 11 & 13 & 13 & 14 & * & 12 & 9 & 11 & & \\
\hline
\end{tabular}

* indicates damaged features

$\sim 50 \%$ arm width; keels present on Arms II and III, that on Arms II $\sim 30 \%$ arm depth along entire arm length, that on Arms III $\sim 100 \%$ arm depth at $\sim 40 \%$ arm length, narrowing thereafter; lateral membranes on Arms IV $\sim 50 \%$ arm width at base, narrowing thereafter to $\sim 30 \%$ arm width along remainder of arm length.

Tentacle length 122-150-171\% ML; stalk bases slightly narrower than those of adjacent arms, with low aboral ridge, $\sim 10 \%$ tentacle thickness. Clubs (Figs 35B-D) slightly expanded, length $23-30-38 \% \mathrm{ML}(16-43 \% \mathrm{TnL})$; carpus squarish in outline, with seven to nine (usually eight) suckers; manus with 24-29 (usually 26 or 27) hooks, with deep lateral grooves on claw (Fig. 35A); terminal pad with nine to 14 (usually 1113) suckers. Ventral hooks (Figs 35A, 35F) all subequal to or larger than paired dorsal hooks, increasing in size from hook V1 to V8, with V6-V8 (Fig. 35A) largest (10-12\% $\mathrm{CL}$, two to four times longer than paired dorsal hooks), with claws strongly recurved at $\sim 60^{\circ}$, thereafter decreasing in size distally. Hooks D1-D6 approximately subequal, thereafter decreasing in size distally. Ventral membrane, $\sim 50 \%$ club width, present along entire ventral hook series; dorsal membrane, $\sim 30 \%$ club width, flanking hooks D1-D5; dorsal keel, $\sim 100 \%$ club width, present from hook D5 to tip of club.

Lateral profile of lower beak (Figs 36A-C, ML 305mm) longer than deep, with nearly straight crest sloping shallowly downward, posterior edge of hood close to crest, hood length $\sim 45 \%$ total crest length, and hood and crest together $\sim 65 \%$ baseline; jaw edge straight proximally, only becoming concave distally near rostral tip; jaw angle obtuse, obscured by slight wing fold. Crest moderately broad, slightly thickened, lateral walls 
rounded; angle ridge short, spanning $\sim 30 \%$ lateral wall depth, poorly developed; angle point indistinct; wings broaden somewhat distally, width $~ 125 \%$ that at jaw angle, length $\sim 300 \%$ LRL; no cartilage insert apparent on wings; lateral wall fold broad and shallow, indistinct by posterior margin of lateral wall.

Radula (Fig. 36D) with narrow, triangular mesocone on rachidian; lateral cusps $\sim 50 \%$ mesocone height, slightly laterally directed; proximal margin of base concave. First
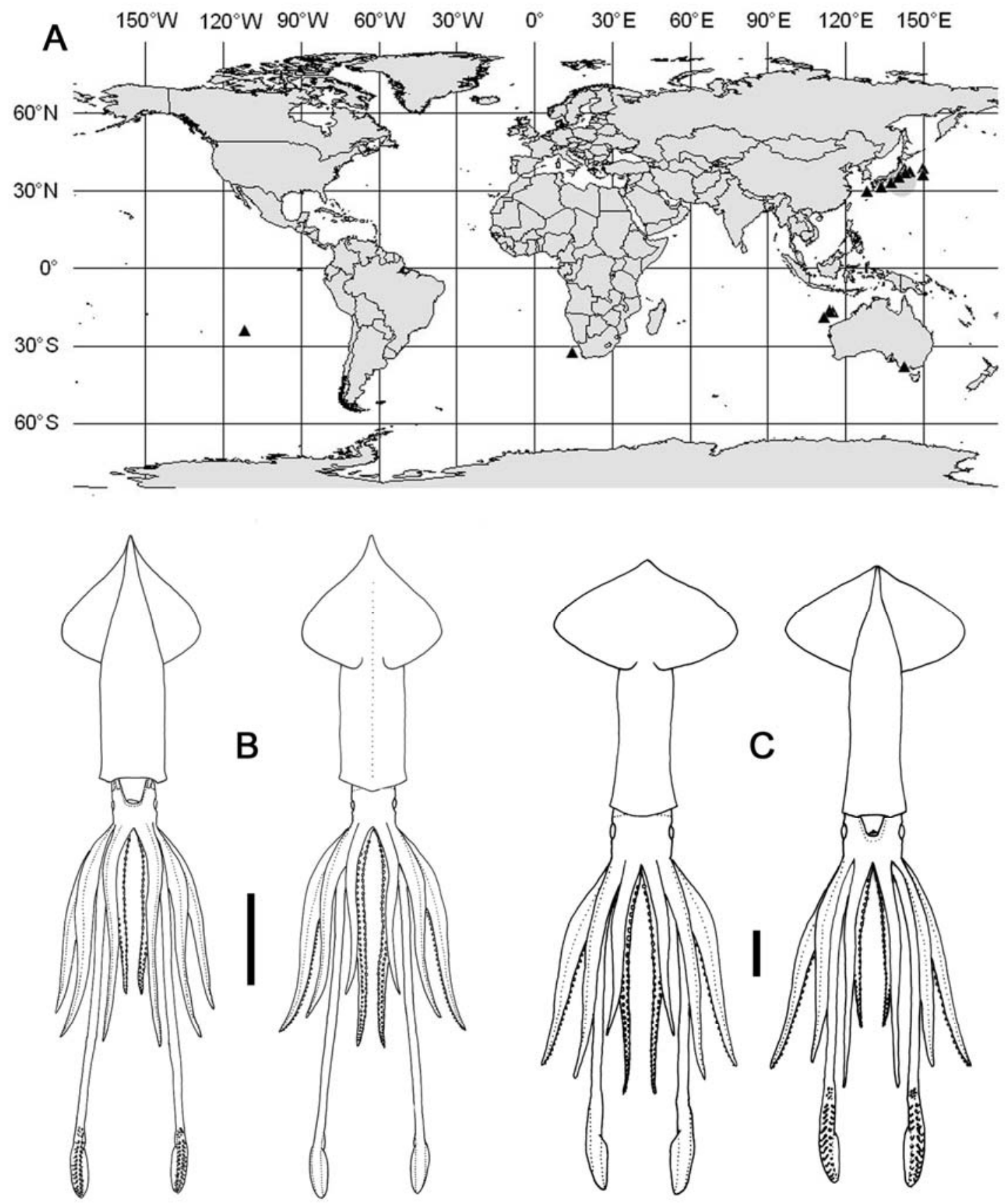

Fig. 34 - Onykia (Onykia) loennbergii. A) distribution (grey circle indicates type locality); B) NMV F110111, +, ML 240mm; C) JAMSTEC HD521SS4, sex indet., ML $61 \mathrm{~mm}$. Scale bars $=$ A) $100 \mathrm{~mm}$, B) $10 \mathrm{~mm}$. 
lateral tooth bicuspid; inner cusp broad, triangular, slightly curved toward rachidian, its height $\sim 80 \%$ that of rachidian; outer cusp sharply pointed to slightly hooked, slightly laterally directed, its height $\sim 50 \%$ that of inner cusp. Second lateral tooth forms slender isosceles triangle, $\sim 125 \%$ rachidian height. Marginal tooth simple, slender, with cusp somewhat twisted on some teeth; height $\sim 150 \%$ that of rachidian. Palatine palp (Fig. $36 \mathrm{E}$ ) with greatest width of tooth-bearing plane $\sim 35 \%$ its length, with $\sim 55$ long, slender, conical teeth, each $100-200 \%$ rachidian height, densely set over surface of palp, most concentrated toward posterior portion of palp.

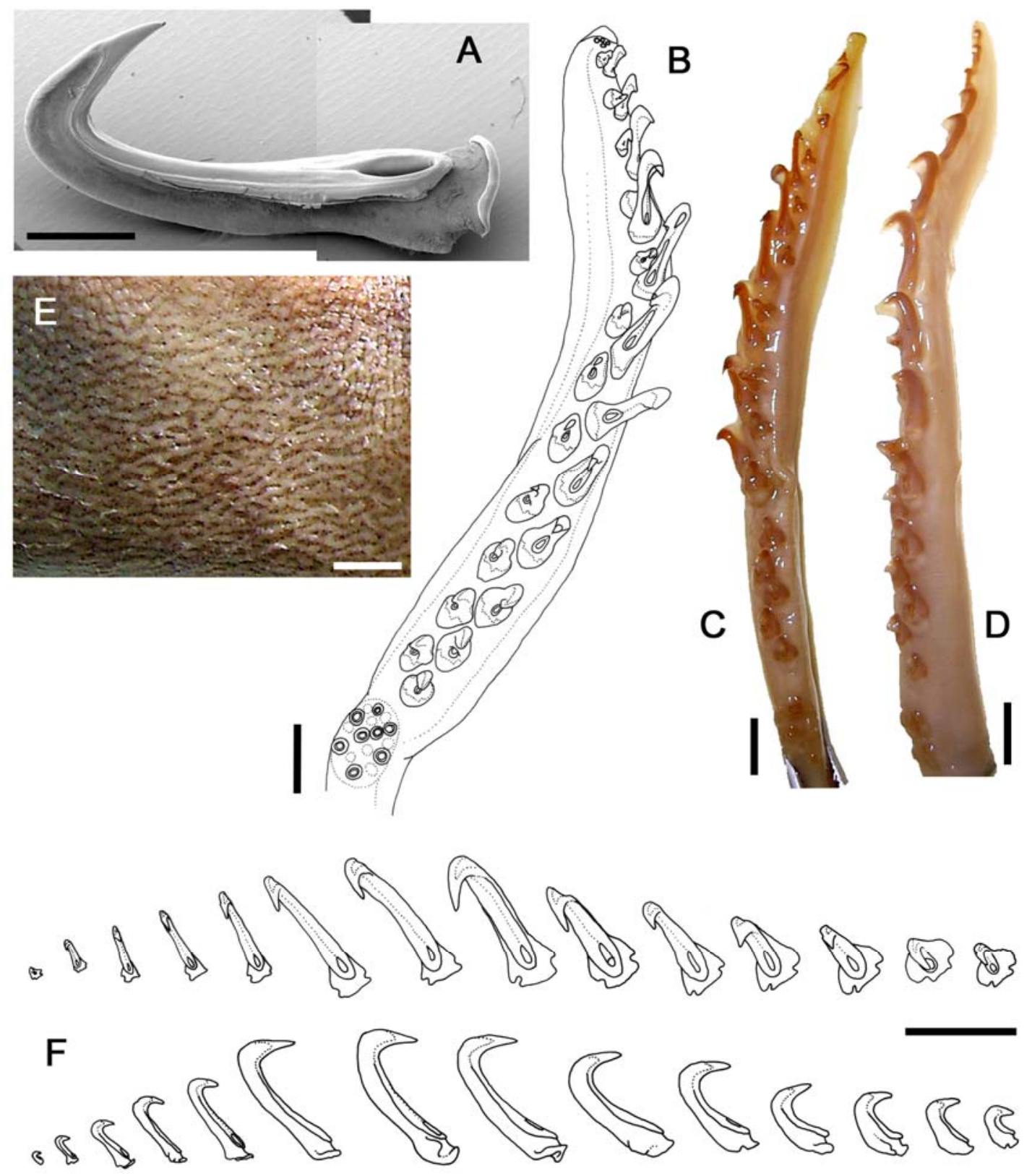

Fig. 35 - Onykia (Onykia) loennbergii. A, C-F) NSMT Mo67865, q, ML 153mm; B) BMNH 1920.12.20.309, o, ML 240mm; E) SAM S2188, , ML 222mm. A) left tentacular hook V7; B) left tentacle club; C) right tentacle club; D) left tentacle club; (E) sculpture on mantle surface; $(F)$ ventral hook series from left tentacle club. Scale bars = A) $1 \mathrm{~mm}$; B-D, F) $5 \mathrm{~mm}$; E) $10 \mathrm{~mm}$. 
Gladius (Fig. 36F) with greatest width ( 9\% GL) attained at 35-40\% GL; free rachis $\sim 20 \%$ GL; vanes broaden rapidly between 20 and $30 \%$ GL, gradually narrowing thereafter. Rostrum 20\% GL; anterior cross-section generally elongate triangular or keyhole-shaped, expanded and rounded ventrally; posterior cross-section ovate to tear-drop-shaped.

Smaller specimens (below ML $100 \mathrm{~mm}$, Fig 34C) deviate from the above description in having somewhat different body proportions: mantle bulging slightly midway along length, width $\sim 27 \% \mathrm{ML}$, with all surfaces smooth (no ridges), and ventro-lateral and ventral surfaces coppery iridescent; fins shorter and broader, length $\sim 42 \% \mathrm{ML}$, width
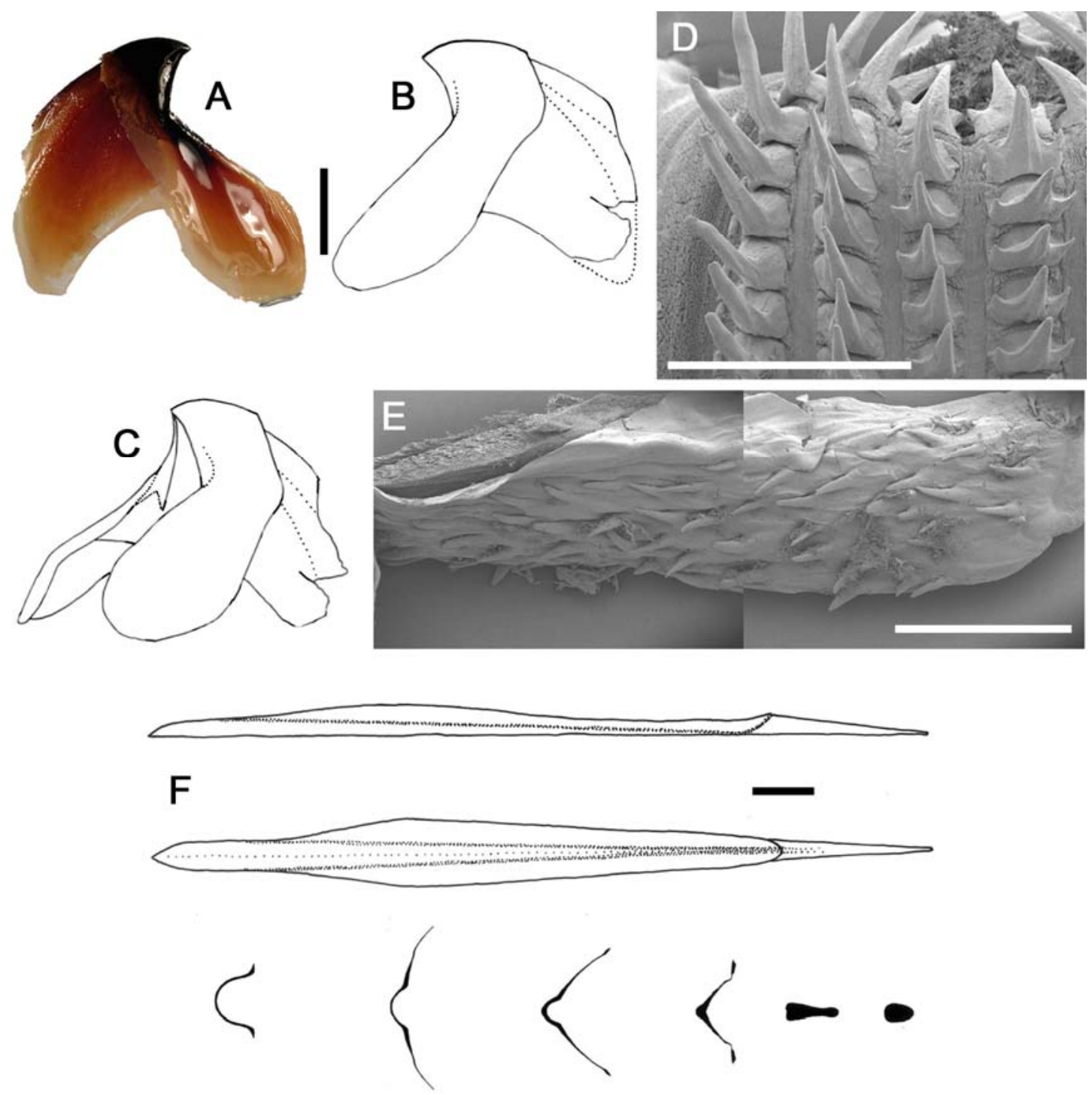

Fig. 36 - Onykia (Onykia) loennbergii. A-C) NMV F109452, đ, ML 305mm; D, E) NMV F110115, sex and ML unknown; F) NSMT Mo67251, ${ }^{\lambda}$, ML 127mm. A-C) lower beak: $(A)$ left, $(B)$ right, $(C)$ right oblique profiles; $D)$ radula; $E$ ) palatine palp; $F$ ) gladius. Scale bars = A, B, D) $1 \mathrm{~mm}$; E) $2 \mathrm{~mm}$; F) $10 \mathrm{~mm}$. 
$\sim 62 \% \mathrm{ML}$; head relatively wider, $\sim 21 \% \mathrm{ML}$; funnel component of locking apparatus $\sim 14 \% \mathrm{ML}$, mantle component $\sim 21 \% \mathrm{ML}$; Y-shaped ridge in funnel distinct by at least ML 46mm; arms 70-110\% ML (Arms I mean 80\%, Arms II-IV 95\%), with 90 suckers and iridescent aboral surfaces; tentacles 100-220\% ML (mean 166\%) and clubs 35-60\% ML (mean $\sim 45 \% \mathrm{ML}, \sim 30 \% \mathrm{TnL}$ ); no marginal suckers observed on specimens from ML $46 \mathrm{~mm}$.

Remarks - Ok. (Ok.) loennbergii is generally considered a western Pacific to eastern Indian Ocean species, but while the majority of material examined herein had been collected within these regions, one unmistakeable specimen was also encountered from the southern Atlantic and one from the southeastern Pacific (see Fig. 34A). It appears that the range of $O k$. (Ok.) loennbergii may be greater than previously thought, and that it may occur throughout subtropical waters in the Southern Hemisphere.

No type specimen of Ok. (Ok.) loennbergii was originally or has been subsequently designated. Should questions about this species' identity arise, the larger specimen registered under NSMT Mo67251 ( $\circ$, ML 223mm, Table 14) would be a suitable neotype. 
Onykia (Onykia) aequatorialis (Thiele, 1920) (new comb.) (Tables 11, 15, Figs 37, 38)

Moroteuthis aequatorialis Thiele, 1920: 442-444, PI. 53 Fig. 17, PI. 54 Figs 1-5; Clarke (1966): 145, Fig. 21; Sweeney et al. (1992): 136; Glaubrecht \& Salcedo-Vargas (2000): 271.

Type material examined — ZMB Holotype Moll.-110000, spent + , ML 380mm (preserved; ML $400 \mathrm{~mm}$ fresh according to original description), $0^{\circ} 16 \mathrm{~N}, 18^{\circ} 07 \mathrm{~W}, 21 / 09 / 1903$, floating dead at surface, Deutsche Südpolar Expedition.

Additional material examined (1 specimen) - UMML 31.3148, spent $q$, ML 545mm, $24^{\circ} 34 \mathrm{~N}$, $77^{\circ} 31$ W, 1742-1707m, 19/08/1975, coll. Cl-354.

Distribution (Fig. 37A) - Equatorial Atlantic and Gulf of Mexico; one record from the surface, one from 1742-1707m.

Diagnosis - (known exclusively from spent females) Fins rhombic, length $\sim 55 \% \mathrm{ML}$, width 55-68\% ML; epidermis of mantle rugose; arms 50-60\% ML and remaining robust at tips, each with $\sim 100$ suckers; rostrum of gladius $>17 \% \mathrm{GL}$. Tentacle clubs unknown.

Description, ML 380mm (spent female, Figs 37B-38H) - Mantle approximately conical, width $28-36 \% \mathrm{ML}$, tissue thin, slack in consistency; remnants of round to irregular dermal warts apparent on anterior third of dorsal and ventral surfaces (Fig. $38 \mathrm{~B})$; posterior $15 \%$ of mantle indistinct from ventral surface fins. Mantle studded with $\sim 80$ implanted spermatangia in middle third of right side, dorsally and ventrally (Figs $37 \mathrm{C}, 38 \mathrm{~A}$ ); implantation sites characterised by $5-10 \mathrm{~mm}$ transverse cut (or small round pore) in epidermis, darkened and puckered tissue, and hard subcutaneous knot of implanted spermatangium. Fins rhombic in outline, length $55 \% \mathrm{ML}$, width $55-68 \%$ $\mathrm{ML}$, not drawn out posteriorly; anterior and posterior margins convex.

Head boxy, small, length $\sim 15 \% \mathrm{ML}$, width $\sim 13 \% \mathrm{ML}$, depth $\sim 10 \% \mathrm{ML}$. Dorsal occipital region with weak transverse crest. Eye apertures tightly contracted in holotype. Funnel cylindrical; aperture at level below eye apertures. Funnel groove indistinct (ventral surface of head dissected previously). Mantle component of locking apparatus indistinct past anterior $40 \mathrm{~mm}(\sim 10 \% \mathrm{ML})$; funnel component $\sim 13 \% \mathrm{ML}$.

Arms fleshy, soft, 38-58\% ML, formula IV>III=II>I; arm tips largely intact, but most suckers missing over proximal $40-50 \%$ of each arm's length; estimated original number of suckers (based on peduncles) 100 per arm. Keels present on Arms I-III; 
Table 15. Measures ( $\mathrm{mm}$ ) and counts of Onykia (Onykia) aequatorialis (Thiele, 1920) (new comb.) (arm and tentacle measures recorded from more complete side of specimen, indicated $\mathrm{R}$ or $\mathrm{L}$ ).

\begin{tabular}{ccccc}
\hline Specimen ID & \multicolumn{2}{c}{ ZMB Moll.-110000 } & \multicolumn{2}{c}{ UMML 31.3148 } \\
\hline Type Status & (Absolute) & (Indices) & (Absolute) & (Indices) \\
ML & Holotype & & None & 28 \\
Sex & 380 & & 545 & 15 \\
MW & F & 36 & 150 & 12 \\
HL & 135 & 16 & 80 & 55 \\
HW & 62 & 14 & 65 & 68 \\
FL & 52 & 55 & 300 & $38^{*}$ \\
FW & 210 & 55 & 370 & 58 \\
Arms I & 210 & 48 & $205 R^{*}$ & 50 \\
Arms II & $181 R$ & 58 & $315 R^{*}$ \\
Arms III & $221 R$ & $275 R$ & $7^{*}$ \\
Arms IV & $207 R^{*}$ & $54^{*}$ & $280 R^{*}$ & \\
TnL & $233 R$ & 61 & $40 R^{*}$ & - \\
CL & $30 R^{*}$ & $8^{*}$ & - & \\
CS & - & & - & \\
MH & - & & - & \\
MS & - & & - & \\
TPS & - & & - &
\end{tabular}

* indicates damaged features, - indicates missing features.

that on Arms I apparent only as slightly thickened fleshy ridge, $\sim 10 \%$ arm width, along entire arm length; that on Arms II fleshy, thick, $\sim 30 \%$ arm depth along entire arm length; that on Arms III attaining $\sim 100 \%$ arm depth at $\sim 40 \%$ arm length, thereafter reducing to $\sim 25 \%$ arm depth along remainder of arm; lateral membrane on Arms IV $\sim 75 \%$ arm width at base, reducing to $\sim 25 \%$ arm width by $\sim 40 \%$ arm length.

Tentacles lost; remains of stalks short, narrow, flaccid; severed plane of stalk covered by puckered but not entirely healed epidermis.

Lateral profile of lower beak (Figs 38C-E, ML 380mm) with length and depth approximately equal, posterior margin of hood close to crest, hood length $\sim 45 \%$ crest length, and hood and crest together $\sim 60 \%$ baseline; jaw angle obtuse, slightly obscured by low wing fold. Lateral wall sharply indented below narrow crest; lateral fold well-developed, becoming broader and lower but still visible at posterior lateral wall margin; wing length $\sim 200 \% \mathrm{LRL}$; wing width distally $\sim 150 \%$ that at jaw angle; angle ridge well-developed, spanning $\sim 40 \%$ of distance to lateral wall margin.

Radula (Fig. 38F) with nearly rectangular rachidian base, its height $\sim 40 \%$ total rachidian height; mesocone short, narrow, triangular, sharply pointed; lateral cusps 

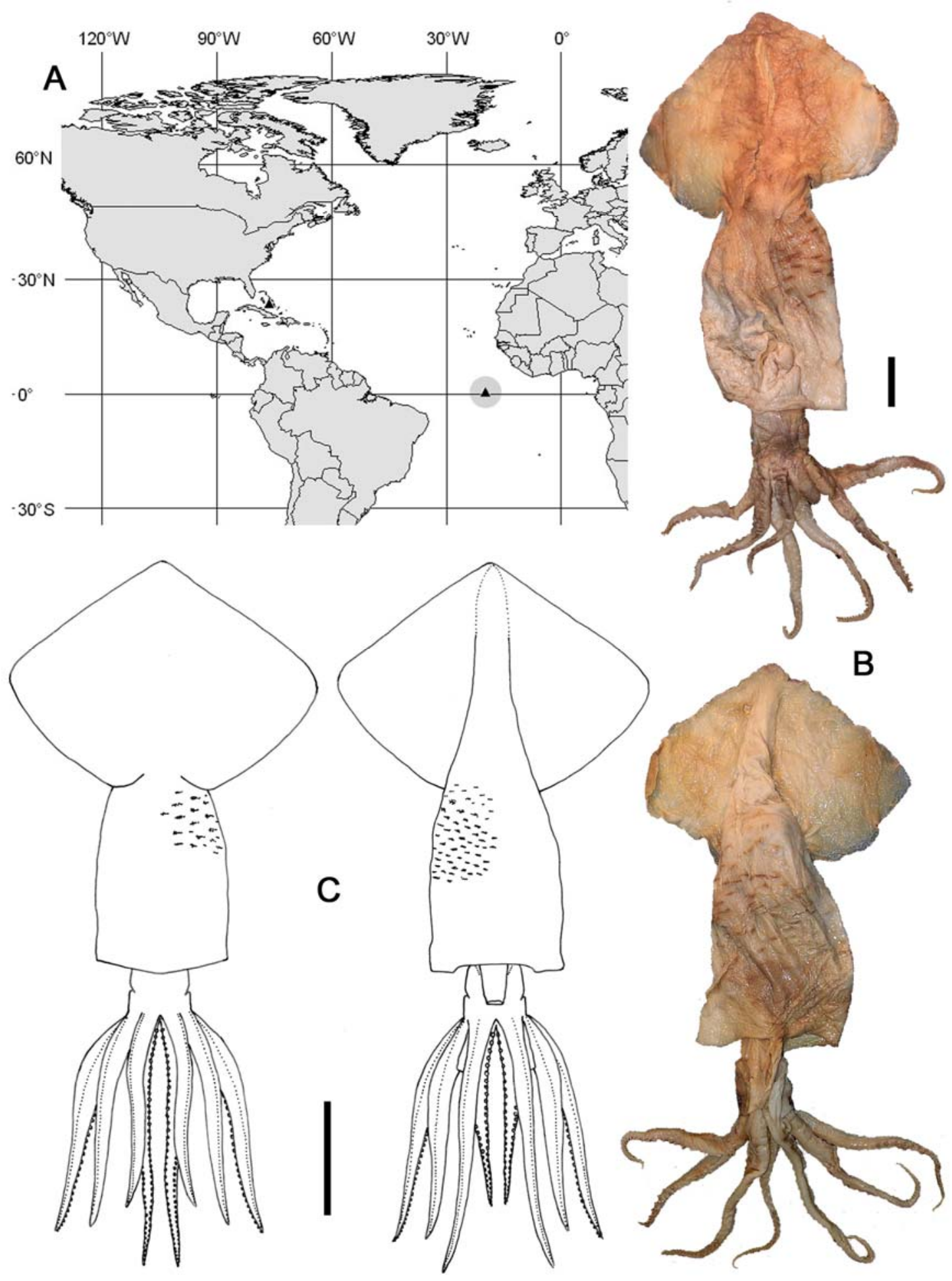

Fig. 37 - Onykia (Onykia) aequatorialis. A) distribution (grey circle indicates type locality); B, C) ZMB Moll.-110000, holotype, spent + , ML 380mm. Scale bars = B) 40mm; C) $100 \mathrm{~mm}$. 
short, triangular, laterally directed. First lateral tooth bicuspid, its inner and outer cusps resembling mesocone and lateral cusp of rachidian in shape and height, with outer cusp slightly laterally directed. Second lateral tooth robust, triangular, with rounded base, its height subequal to or slightly longer than rachidian. Marginal tooth robust, with straight inner edge and curved outer edge, its height $\sim 130 \%$ that of rachidian. Palatine palp (Fig. 38G) breadth $\sim 30 \%$ its length, with $60+$ short teeth in approximately transverse rows, each $160-230 \%$ rachidian; those near outer edge of palp wide, flat, blunt, those situated inward becoming narrow, pointed.

Gladius (Fig. $38 \mathrm{H}$ ) broken, with mid-section ( 45mm) missing (ML therefore substituted for GL in calculating proportions). Vanes broad, greatest width $\sim 13 \% \mathrm{ML}$; free rachis short $(\sim 12 \% \mathrm{ML})$; dorsal keel not well developed. Tip of rostrum missing; remaining portion $\sim 17 \% \mathrm{ML}$, flexible, anterior cross-section approximately triangular with rounded corners, posterior cross-section ovate.

Overall colouration of mantle pale dorsally, darkest at spermatangium implantation sites; ventral surface slightly darker, with several large, dark chromatophores between implantation sites. Fins flesh-coloured, somewhat darker than overall mantle, with small, dark purple-brown chromatophores densest medially on dorsal surface; no pigmentation apparent on ventral surface of fins. Dorsal surface of head with many small, dark-brown chromatophores; epidermis surrounding contracted eye apertures nearly black. Arms darkest aborally, particularly Arms I and II, with few chromatophores apparent on lateral and oral surfaces.

Remarks - Thiele (1920; see translation in Appendix C) separated Ok. (Ok.) aequatorialis from the two other then-known 'Moroteuthis' (= Onykia) species - Ok. $(M$.$) ingens and Ok. (Ok.) robusta - based on its size, the rostrum of the gladius, and$ several other bodily proportions. More recent information on the latter two and the other Onykia species allows for a somewhat more detailed comparison, although the absence of certain systematic characters (tentacle clubs in particular) on the two known specimens of $O k$. (Ok.) aequatorialis precludes a complete evaluation of its relationship to congeneric taxa. Its rugose skin separates it readily from the ridgeskinned Ok. (Ok.) robusta and Ok. (Ok.) loennbergii (and its known distribution does not overlap or even border on that of these two species), and its relatively short arms make it unlikely to represent the adult form of Ok. (Ok.) indica, in which arm length in all known (juvenile) specimens exceeds $100 \% \mathrm{ML}$. It is possible that Ok. (Ok.) aequatorialis may prove synonymous with Ok. (Ok.) carriboea, since the latter is 
known exclusively from paralarval and small juvenile specimens, but without specimens of intermediate ontogenetic stages it is unwise to speculate further. The remaining two Onykia species, Ok. (M.) ingens and Ok. (Ok.) robsoni, both possess rugose skin on the mantle. The short arms in Ok. (Ok.) aequatorialis (48-68\% ML, on mean $20 \%$ shorter relative to the ML than the those of Ok. (M.) ingens and Ok. (Ok.) robsoni) separate it readily from both. Although the non-attenuation of the mantle posteriorly and the form of the dermal warts (Fig. 38B) ally Ok. (Ok.) aequatorialis more closely with Ok. (M.) ingens (see Fig. 45A) than Ok. (Ok.) robsoni, in which the warts are mostly round in outline and well separated from each other (Fig. 40B), OK. (Ok.) aequatorialis is further separable from Ok. (Ok.) ingens by the oval cross-section of the rostrum of the gladius, form of the beak, and distinctly longest fourth arms.

The subgeneric position of Ok. (Ok.) aequatorialis is unconfirmed since the funnel groove is damaged and the tentacle clubs are absent from both known specimens. It is provisionally attributed to Onykia (Onykia) at present.

Most of the other large Onykia species are encountered relatively frequently in the waters they inhabit, but the paucity of $O k$. (Ok.) aequatorialis specimens may be due in part to the absence of deep-water fishing activities in the tropics. However, this species may also be morphologically similar enough to Ok. (M.) ingens at younger life stages that other existing specimens have been overlooked. Nesis (1987) suggested that Voss' (1956) Gulf of Mexico specimen and Rancurel's (1970) New Caledonian material, both attributed to Ancistroteuthis 'lichtensteini', may be Ok. (Ok.) aequatorialis, given their lack of secondary occipital folds. None of these specimens was available for re-examination in this study, but in both Voss' (1956) and Rancurel's (1970) illustrations, the arms appear much longer (85-90\% ML) than those observed on either Ok. (Ok.) aequatorialis specimen examined herein. 

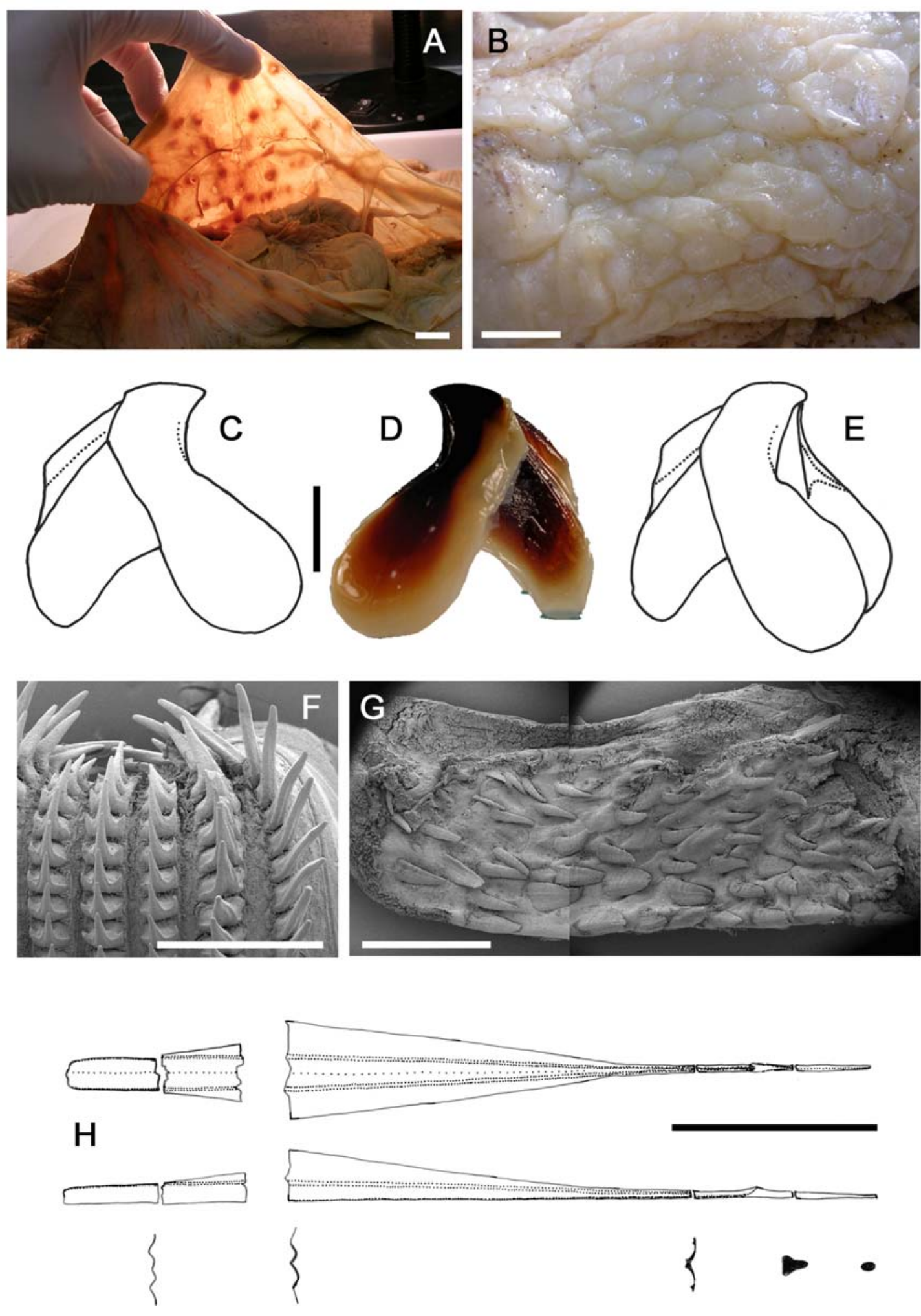

Fig. 38 - Onykia (Onykia) aequatorialis. A-E, H) ZMB Moll.-110000, holotype, spent q, ML 380mm; F, G) UMML 31.3148, spent 9 , ML 545mm. A) implanted spermatangia; $B$ ) rugose surface of mantle; C-E) lower beak: (C) left, (D) right, (E) left oblique profiles; F) radula; G) left palatine palp; H) gladius. Scale bars = A-D) $10 \mathrm{~mm}$; F) $2 \mathrm{~mm}$; G) $10 \mathrm{~mm}$; H) $40 \mathrm{~mm}$. 
Onykia (Onykia) robsoni (Adam, 1962) (new comb.) (Tables 11, 16, Figs 39-41)

Moroteuthis sp. A. Robson, 1924 - 595-599, Text-figs 1, 2.

Moroteuthis robsoni Adam, 1962: 24-30, Figs 2, 3, Pl. 1 Figs 1-4; Clarke (1966): 147, Fig. 21 , (1980): 81-128, Table 25, Text-figs 56-67, 76, 77, 80-89, PI. 1 Fig 2, (1986): 74-77, Fig. 33a; Imber (1975): 29, 30; Okutani (1978): 155, 156; Roper et al. (1984): 133, (1985): 181, 188, 189; Sánchez \& Molí (1984): 12, 13, Fig. 10; Okutani \& Clarke (1985): 15, 16; Nesis (1987): 193, Figs 49E, F; Kubodera (1990): 337, Fig. 267; Sweeney et al. (1992): 134, 135; Filippova et al. (1997): 183, Fig. 110; Anderson et al. (1998) (unpaginated); Bonnaud et al. (1998): 1761-1770, Figs 5, 6; Kubodera et al. (1998): 284, Tables 1, 2 , Fig. 8; Sweeney \& Roper (1998): 572; Okutani (2005): 157; Bolstad (2007): 319-322, 332, 333, Tables 3, 6, Figs 28-37; Haimovici et al. (2007): Fig. 34. Ancistroteuthis lichtensteinii (not Férussac, 1835) - Toll (1982) (partim): 58, 59, PI. 5 Fig. D, USNM 730690, 730691, 730692; Hess (1987): 292, 293, 300, 301, Fig. 55.

? Onychoteuthis banksii (not Leach, 1817) — Haimovici et al. (2007): Fig. 36.

Type material - Deposition of holotype unknown (see Remarks).

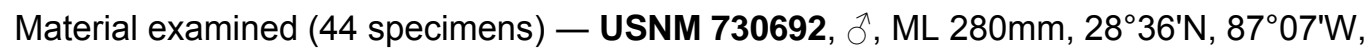

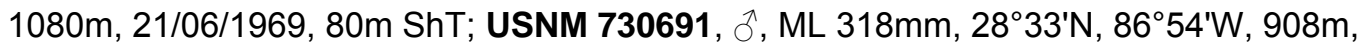
21/06/1969, 80m ShT; USNM 730690, ô. ML 239mm, $21^{\circ} 41^{\prime} \mathrm{N}, 96^{\circ} 55^{\prime} \mathrm{W}, 897 \mathrm{~m}$,

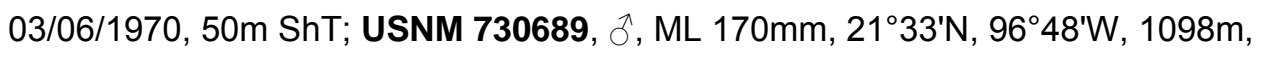

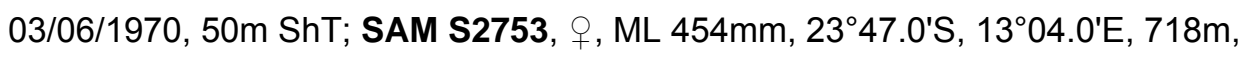
15/01/1989, 2210h, RV Africana III, SFRI WCHBS Stn A8374-069-DT-01, BTT; NIWA

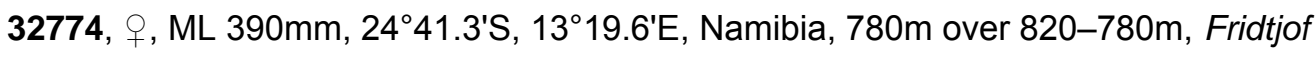
Nansen Stn 2217, coll. M. Clark; SAM S2795,, , ML 505mm, 26²40.6'S, $13^{\circ} 31.8^{\prime} \mathrm{E}$, 1005m, 21/01/1989, 1350h, RV Africana III, SFRI WCHBS Stn A8414-069-DT-05, BTT; SAM S2376, + , ML $417 \mathrm{~mm}, 27^{\circ} 22.2^{\prime} \mathrm{S}, 14^{\circ} 16.2^{\prime} \mathrm{E}, 475 \mathrm{~m}, 19 / 02 / 1988,1700 \mathrm{~h}, \mathrm{RV}$ Africana III, SFRI WCHBS Stn A6950-059-E019, BTT; SAM S3687, 2ᄋ, ML 542, $520 \mathrm{~mm}, \hat{O}^{\lambda}, \mathrm{ML} 375 \mathrm{~mm}, 28^{\circ} 35.1^{\prime} \mathrm{S}, 1^{\circ} 23.3^{\prime} \mathrm{E}, 849 \mathrm{~m}, 20 / 02 / 1988,2020 \mathrm{~h}, \mathrm{RV}$ Africana III, SFRI WCHBS Stn A6956-059-E024, BTT; SAM S1872, đ̊, ML 622mm, 2855.5'S, 14ำ15.1'E, 778m, 25/01/1987, 0000h, RV Africana III, SFRI WCHBS Stn A050-E-22, BTT; SAM S2375, + , ML 623mm, 29³8.8'S, 14²5.4'E, 861m, 13/01/1990, 1843-2135h, RV Africana III, SFRI WCHBS Stn A9812-079-DT-001, BTT; SAM S3957, đૈ, ML 595mm, ․, ML 490mm, 3003'S, $14^{\circ} 23^{\prime} \mathrm{E}, 986 \mathrm{~m}, 11 / 07 / 1998$, MT Iris trawl \#12, BTT, coll. B. Rose \& R. Leslie; SAM S3955, + , ML 410mm, 3003'S, 14²3'E, 986m, 11/07/1998, MT Iris trawl \#12, BTT, coll. B. Rose \& R. Leslie; SAM S2588, ${ }^{\lambda}$, ML 505mm, 30³8.0'S, 31 $39.0^{\prime} E, 07 / 07 / 1973$, SFRI Stn U73/1363, ex-sperm-whale, coll. P. Best; SAM S2520,

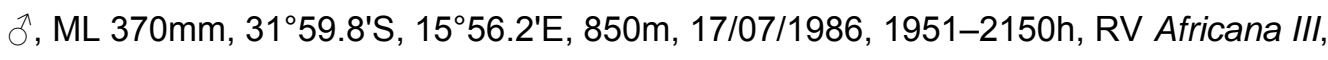
SFRI WCHBS Stn A4361-046-E12, trawl; SAM S2518, 2 + , ML 630, 570mm, off Cape Columbine [ $\left.32^{\circ} \mathrm{S}, 17.5^{\circ} \mathrm{E}\right], 300 \mathrm{~m},-/ 12 / 1991$, Coll. Don. B. Rose, I\&J; NMV F110375, ㅇ, ML 620mm, $34^{\circ} 15^{\prime} \mathrm{S}, 114^{\circ} 20^{\prime} \mathrm{E}, 825 \mathrm{~m}$, NFD; SAM S775, $q$, ML 552mm, $34^{\circ} 22.0^{\prime} \mathrm{S}$, $17^{\circ} 44.8^{\prime} \mathrm{E}, 432 \mathrm{~m}, 10 / 03 / 1981$, RV Africana, SFRI Stn 33, 110m otter trawl; SAM S2521, 


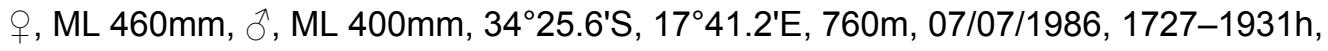
trawl, RV Africana III, SFRI WCHBS Stn A4310-046-E01; NMV F109457, ๆ, ML 575mm, $35^{\circ} 03^{\prime} \mathrm{S}, 114^{\circ} 45^{\prime} \mathrm{E}, 970-950 \mathrm{~m}$, NFD; NMNZ M.71003, ㅇ, ML 93mm, 3857.48'S, 178 16.55'E, 30m, 02/10/1979, FRV James Cook; NMNZ M.118359, ㅇ, ML 547mm, $40^{\circ} 00^{\prime} \mathrm{S}, 168^{\circ} 00^{\prime} \mathrm{E}$, Challenger Plateau, 900m, NFD; NSMT Mo67259, ㅇ, ML 156mm, $41^{\circ} 16^{\prime} \mathrm{S}, 152^{\circ} 00^{\prime} \mathrm{E}$, east of Tasmania, 50m, ST $14.8^{\circ} \mathrm{C}$; NMV F110109, ${ }^{\wedge}$, ML 350mm, $41^{\circ} 36.4^{\prime} \mathrm{S}, 148^{\circ} 42.2^{\prime} \mathrm{E}, 990-970 \mathrm{~m}$, NFD; USNM unaccessioned, ${ }^{\lambda}$, ML $127 \mathrm{~mm}$, $41^{\circ} 47^{\prime} \mathrm{S}, 39^{\circ} 58^{\prime} \mathrm{W}, \mathrm{WH} 105$, MT1600; NSMT Mo76090, ㅇ, ML 104mm, $41^{\circ} 52.5^{\prime} \mathrm{S}$,

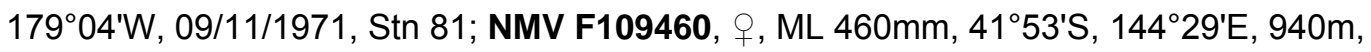
NFD; NIWA 32741, , , ML 419mm, 30ิ', ML 397, 351, 327mm, 4246.93-99'S, 178 18.61-22.72'W, 735-733m, 04/01/2002, RV Tangaroa Stn TAN0201/40, Z10997,

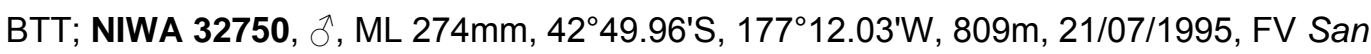
Waitaki Stn SWA9501, Z8339; NIWA 32745, , ML 274mm, FV San Waitaki Stn SWA9501/73, Z8331, NFD [nearby station Z8336: $42^{\circ} 50.12 ' \mathrm{~S}, 176^{\circ} 54.36^{\prime} \mathrm{W}$ ]; NIWA

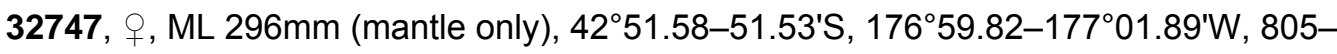
804m, 15/07/1995, FV San Waitaki Stn SWA9501/56, Z8338; NIWA 32737, ๆ, 77mm, 4252.05-07'S, 176 34.94-33.58'W, 855-848m, 15/07/1996, RV Tangaroa Stn

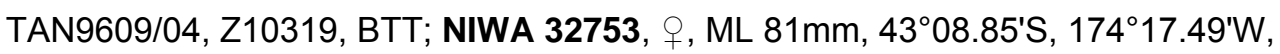

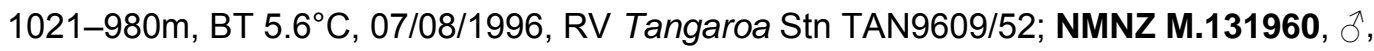
ML 595mm, 43⒓12'S, 169¹7.01'E, 700-350m over 850-724m, 21/08/1980, RV

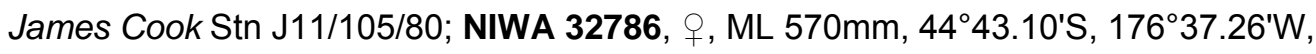
20/10/1998, RV Tangaroa Stn TAN9812/70, Z9460; NMV F109459, §, ML 490mm, $43^{\circ} 59^{\prime} \mathrm{S}, 146^{\circ} 59^{\prime} \mathrm{E}$, NFD; NIWA 32779, ${ }^{\top}$, ML 526mm, $47^{\circ} 19.00^{\prime} \mathrm{S}, 148^{\circ} 23.00^{\prime} \mathrm{E}$, 01/09/1998, Stn 1152/02, Z9282.

Unlocalised material examined (2 specimens) - NMNZ M.126577, ๆ, ML 619mm, NFD; AUT Unaccessioned, $\hat{O}, \mathrm{ML} 500 \mathrm{~mm}$, NFD.

Distribution (Fig. 39A) - Subtropical to sub-Antarctic waters in the Southern Hemisphere, generally $20-50^{\circ} \mathrm{S}$, and in the Gulf of Mexico. Depth range 1200-30m, adults most commonly $1100-700 \mathrm{~m}$, frequently captured by bottom trawl. Elsewhere recorded from sub-Antarctic waters throughout the Southern Hemisphere (Nesis 1987, Kubodera et al. 1998).

Diagnosis - ML to $\sim 630 \mathrm{~mm}$; epidermis of mantle with large, soft, round, wellseparated, blister-like warts; epidermis of head and arm-bases smooth; head noticeably narrower than mantle; fins sagittate, length $\sim 60 \% \mathrm{ML}$, width $\sim 45 \% \mathrm{ML}$, attenuate posteriorly; 26-28 hooks present on adult tentacle club; free rachis of gladius $\sim 15 \% \mathrm{GL}$, rostrum $25-30 \% \mathrm{GL}$. 
Table 16. Measures (mm) and counts of Onykia (Onykia) robsoni (Adam, 1962) (new comb.) (arm and tentacle measures recorded from more complete side of specimen, indicated $\mathrm{R}$ or $\mathrm{L}$ ).

\begin{tabular}{|c|c|c|c|c|c|c|c|c|c|c|c|c|}
\hline Specimen ID & $\begin{array}{c}\text { NMNZ } \\
\text { M.126577 }\end{array}$ & $\begin{array}{l}\text { SAM } \\
\text { S3957 }\end{array}$ & $\begin{array}{c}\text { NMNZ } \\
\text { M.118359 }\end{array}$ & $\begin{array}{l}\text { NIWA } \\
32741\end{array}$ & $\begin{array}{l}\text { NIWA } \\
32741 \\
\end{array}$ & $\begin{array}{l}\text { NIWA } \\
32741 \\
\end{array}$ & $\begin{array}{l}\text { NIWA } \\
32745\end{array}$ & $\begin{array}{c}\text { NSMT } \\
\text { Mo76090 }\end{array}$ & $\begin{array}{c}\text { NMNZ } \\
\text { M.71003 }\end{array}$ & $\begin{array}{l}\text { NIWA } \\
32737 \\
\end{array}$ & \multicolumn{2}{|c|}{ Mean Indices } \\
\hline Type Status & None & None & None & None & None & None & None & None & None & None & \multirow{2}{*}{\multicolumn{2}{|c|}{ (ML 274-619) }} \\
\hline ML & 619 & 595 & 547 & 419 & 397 & 327 & 274 & 104 & 93 & 77 & & \\
\hline Sex & $\mathrm{F}$ & $\mathrm{M}$ & $\mathrm{F}$ & $\mathrm{F}$ & $\mathrm{M}$ & $M$ & $\mathrm{~F}$ & $\mathrm{~F}$ & $\mathrm{~F}$ & $\mathrm{~F}$ & & \\
\hline MW & 115 & 125 & 105 & 70 & 90 & 75 & 70 & 32 & 24 & 20 & MWI & 21 \\
\hline HL & 80 & 53 & 80 & 60 & 65 & 60 & 53 & 28 & 21 & 17 & $\mathrm{HLI}$ & 15 \\
\hline HW & 60 & 60 & 58 & 45 & 58 & 52 & 43 & 26 & 18 & 19 & HWI & 12 \\
\hline $\mathrm{FL}$ & 380 & 330 & 297 & 250 & 225 & 203 & 180 & 66 & 51 & 35 & FLI & 59 \\
\hline FW & 270 & 240 & 250 & 180 & 175 & 160 & 135 & 66 & 56 & 50 & FWI & 45 \\
\hline Arms I & $262 R^{*}$ & $230 L^{*}$ & $265 R^{*}$ & 255 & $240 \mathrm{~L}$ & $170 L^{*}$ & $140 R^{*}$ & $92 \mathrm{R}$ & $53 \mathrm{~L}$ & $57 R^{*}$ & A1I & 61 \\
\hline Arms II & $270 L^{*}$ & $240 L^{*}$ & $327 \mathrm{R}$ & $230 L^{*}$ & $280 \mathrm{~L}$ & $245 \mathrm{~L}$ & $203 R^{*}$ & $102 R$ & $73 \mathrm{~L}$ & $65 R$ & A2I & 68 \\
\hline Arms III & $290 \mathrm{R}$ & $240 L^{*}$ & $300 R^{*}$ & $255 L^{*}$ & $250 \mathrm{~L}$ & $290 \mathrm{~L}$ & $260 \mathrm{R}^{*}$ & $101 \mathrm{R}$ & $70 \mathrm{~L}$ & $63 R$ & A3I & 66 \\
\hline Arms IV & $320 \mathrm{R}$ & $345 \mathrm{~L}$ & $353 R$ & $252 L^{*}$ & $250 \mathrm{~L}$ & $275 \mathrm{~L}$ & $190 R^{*}$ & $111 R$ & $76 \mathrm{~L}$ & $62 R$ & A4I & 64 \\
\hline TnL & $742 \mathrm{R}$ & $670 \mathrm{~L}$ & $776 \mathrm{R}$ & $566 \mathrm{~L}$ & $565 \mathrm{~L}$ & $644 \mathrm{~L}$ & $460 \mathrm{R}$ & $202 R$ & $147 \mathrm{~L}$ & $140 \mathrm{R}$ & TnLI & 145 \\
\hline $\mathrm{CL}$ & $135 R$ & $130 \mathrm{~L}$ & $148 \mathrm{R}$ & $116 \mathrm{~L}$ & $116 \mathrm{~L}$ & $125 \mathrm{~L}$ & $108 \mathrm{R}$ & $45 \mathrm{R}$ & $33 \mathrm{~L}$ & $35 \mathrm{R}$ & CLI & 29 \\
\hline CS & 11 & 9 & 9 & 9 & 9 & 10 & 9 & 7 & 10 & 10 & & \\
\hline MH & 26 & 28 & 27 & 26 & 28 & 27 & 27 & 27 & 28 & 27 & & \\
\hline MS & 0 & 0 & 0 & 0 & 0 & 0 & 0 & 0 & * & * & & \\
\hline TPS & 16 & 18 & 12 & 13 & 14 & 15 & 14 & 12 & 12 & 13 & & \\
\hline
\end{tabular}

* indicates damaged features.

Description (Figs 39B-41G) - Mantle fleshy, spindle-shaped, greatest width 17-21$26 \% \mathrm{ML}$, attained at $\sim 20 \% \mathrm{ML}$, thereafter tapering to long, narrow cone over posterior $\sim 40 \% \mathrm{ML}$; dorsal and ventral surfaces studded with soft, fleshy, well-separated, blisterlike warts (Fig. 40B), round to irregular in outline, largest along dorsal midline; gladius may be visible dorsally as dark medial groove. Fins sagittate, length including tail 5459-66\% ML, width 40-45-49\% ML, posteriorly attenuate; greatest fin width attained within anterior $20-30 \%$ of fin length; posterior margin concave from lateral extremity to midpoint of posterior margin, then straight or slightly convex; anterior margin slightly convex; anterior lobes semicircular, separated by triangular inset of warty skin; fins otherwise smooth over entire surface.

Head smooth, rounded, narrower than mantle; length 9-15-19\% ML, width 12\% ML, depth $\sim 15 \% \mathrm{ML}$; funnel broad posteriorly, narrow anteriorly; funnel groove U-shaped or broadly trapezoidal; funnel aperture at level below posterior margin of eye lens; $Y$ shaped ridge present in funnel groove, extending posteriorly to surround funnel bridles; funnel component of locking apparatus 6-8\% ML; mantle component 15-18\% ML.

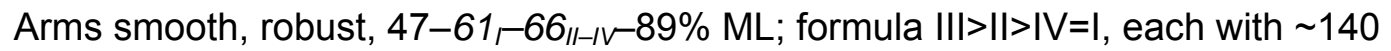
suckers (ML 274-619mm); arm bases fleshy, narrowing distally to very thin; oral faces bordered on each side by low trabeculate protective membrane; Arms I noticeably thinnest, without keel; Arms II and III with keels: that on Arms II 15\% arm depth along entire arm length, that on Arms III 30-50\% arm depth over anterior 30\% arm length, 
becoming indistinct thereafter; lateral membrane on Arms IV 15\% arm width along entire arm length; arm tips often lost or severely damaged in adult to spent females.

Tentacles 113-145-197\% ML; stalk thickness subequal to that of arms at bases; club length 22-29-39\% ML ( 20\% TnL); club unexpanded (Figs 40C-E); carpus well defined, ovoid, with seven to 11 suckers; manus with 26-28 hooks (Figs 40F, 41A), without marginal suckers by ML $93 \mathrm{~mm}$; terminal pad with $12-18$ small suckers. All hooks with deep lateral grooves on claw, forming strong distal ridge (Fig. 41A); proximal dorsal hooks variably subequal to, slightly larger or smaller than corresponding ventral hooks, attaining greatest size in hooks D2-D4, thereafter diminishing distally; ventral hooks steadily increasing in size distally, largest at V8 or V9, three to four times longer than paired dorsal hooks and $\sim 11 \% \mathrm{CL}$, thereafter diminishing distally; dorsal and ventral rows nearly merge distally as club narrows; bases of hooks symmetrical, with broad semicircular lateral lobes of equal size. Ventral membrane, $\sim 100 \%$ club width, present along entire club; dorsal membrane, $\sim 15 \%$ club width, flanks hooks D1-D5; dorsal keel, $100 \%$ club width, present from hook D4 to tip of club.

Lateral profile of lower beak (Figs 41B-D, ML 370-619mm) with hood length $\sim 40-50 \%$ crest length, and hood and crest together $\sim 55 \%$ baseline; rostrum nearly straight, length $\sim 50 \%$ wing length. Crest narrow, only slightly thickened; lateral walls indented below crest; lateral wall with wide, rounded but only slightly thickened lateral fold anteriorly, reducing posteriorly to broad, very slight thickening; angle ridge extends from jaw angle over $\sim 60 \%$ of distance to lateral wall margin.

Radula (Fig. 41E) with conical mesocone on rachidian; lateral cusps $\sim 25 \%$ mesocone height, somewhat laterally directed. First lateral tooth bicuspid, slightly shorter than rachidian; inner cusp straight or slightly medially directed; outer cusp small, $\sim 30 \%$ height of inner cusp, curved, slightly medially directed. Second lateral tooth simple, triangular, height comparable to or slightly longer than rachidian. Marginal tooth long, slender, $\sim 150 \%$ height of rachidian. Palatine palp (Fig. 41F) with long, narrow teeth ( 30 observed on available sample, comprising approximately one-half of palp), each $110-260 \%$ height of rachidian, arranged in approximately transverse rows; largest teeth broad, straight, laterally compressed; smaller teeth narrow, slightly curved away from palp. 
Gladius (Fig. 41G) with greatest width (5-8\% GL) attained at $~ 35 \% \mathrm{GL}$; free rachis $\sim 15 \% \mathrm{GL}$; vanes broaden quickly, then taper gradually between 15 and $75 \%$ GL; keel not thickened; rostrum 25-30\% GL, with triangular to inverted-T-shaped cross-section and triangular lateral profile.

Overall colouration (preserved) dark reddish-purple, with warts on mantle appearing pale pink beneath dark epidermis; fins darkest medially, with small dark chromatophores distributed in several epidermal layers; individual chromatophores
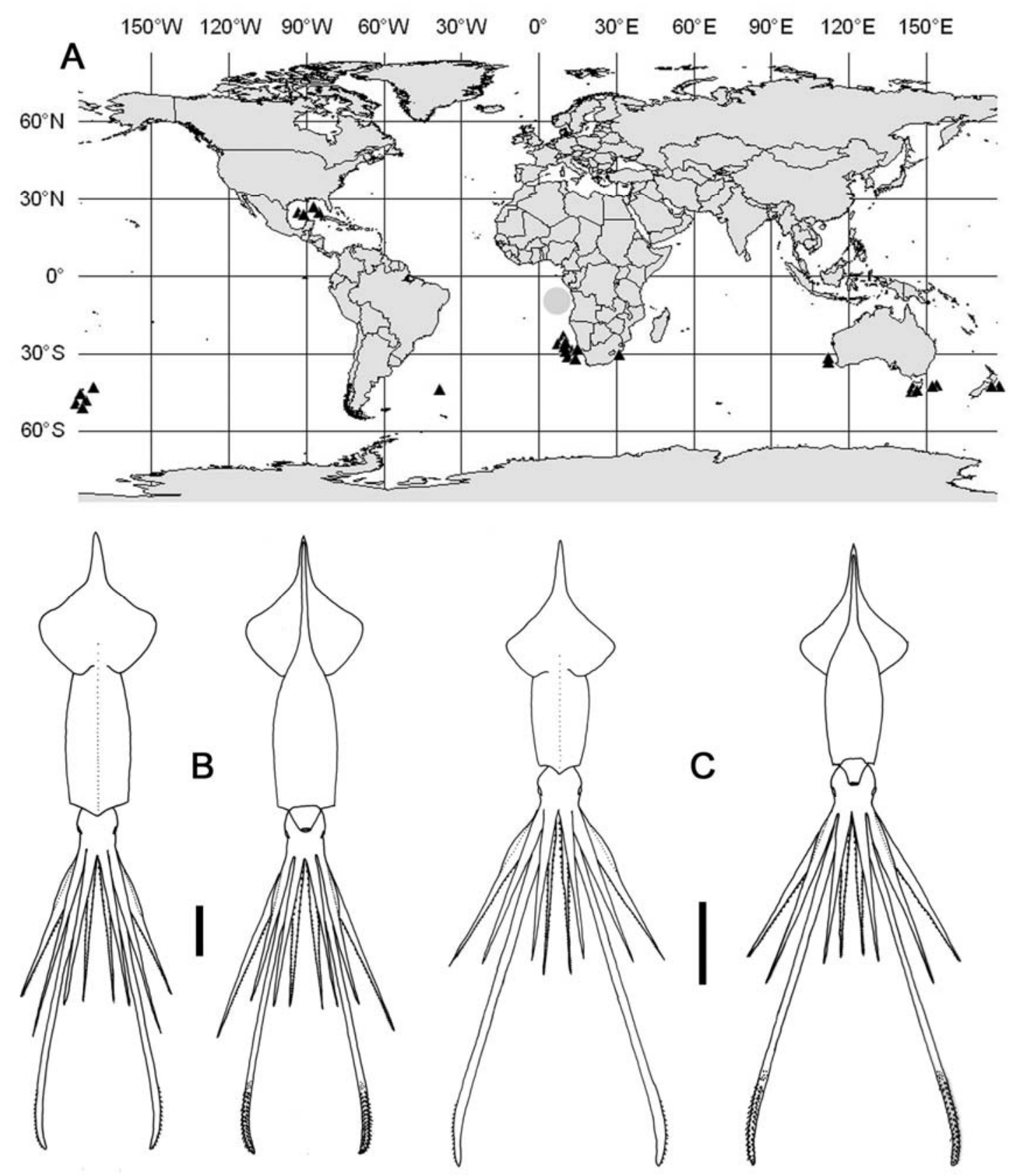

Fig. 39 - Onykia (Onykia) robsoni. A) distribution (grey circle indicates type locality); B) NIWA 32786, , ML 570mm; C) NIWA 32745, $q$, ML 274mm. Scale bars $=100 \mathrm{~mm}$. 
most discernible along dorsal midline and at margins, becoming blotchy and indistinct over central portion of fins; head paler than mantle, darkest dorsally; arms dark pinkish-red aborally, paler on lateral surfaces and palest orally, with individual chromatophores scattered between suckers.

Smaller specimens (ML 77-93mm, Fig. 40A) differ from the above description as follows: mantle fleshy, roundly conical anterior to fins, width $\sim 25 \% \mathrm{ML}$, studded with small fleshy warts from at least ML $77 \mathrm{~mm}$; gladius not visible dorsally; rostrum visible

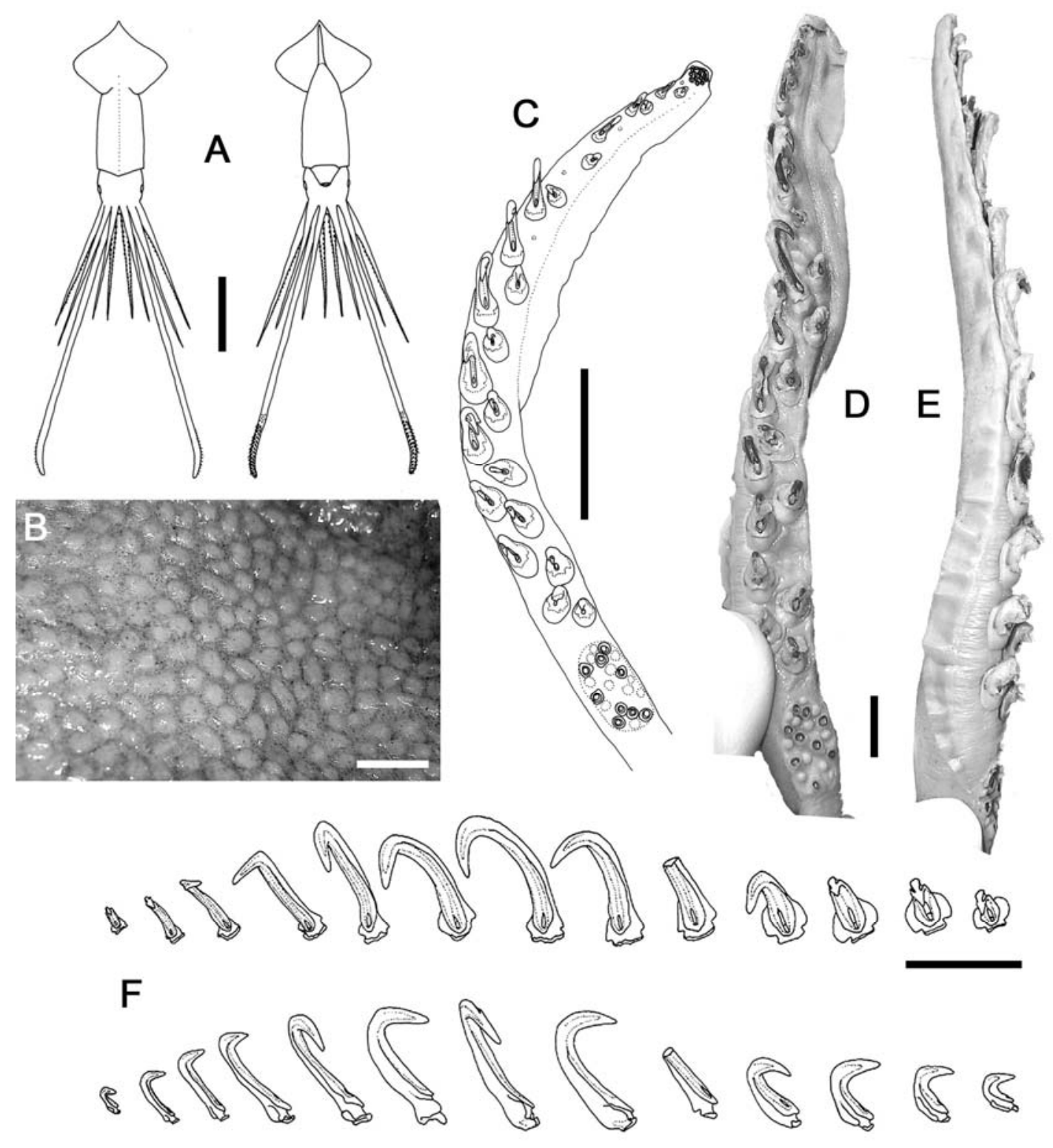

Fig. 40 - Onykia (Onykia) robsoni. A) NMNZ M.71003, q, ML 93mm; B) NIWA 32774 , क, ML 390mm; C) NIWA 32786, + , ML 570mm; D, E) NMNZ M.126577, , ML 619mm; F) NMNZ M.131960, Õ, ML 595mm. Scale bars = A, C) 50mm; B, D-F) $10 \mathrm{~mm}$. 
ventrally through translucent skin of mantle over posterior $\sim 20 \% \mathrm{ML}$; fins rhombic in outline, length $45-55 \% \mathrm{ML}$, width $60-65 \% \mathrm{ML}$, beginning to attenuate posteriorly by ML $77 \mathrm{~mm}$; head only slightly narrower than mantle; arms fleshy, $75-85 \% \mathrm{ML}$, formula II $>$ III $>I V=I$, each with $~ 100$ suckers; keels indistinct or very narrow; club $35-45 \% \mathrm{ML}$ (20-25\% TnL); marginal suckers flank several distal ventral hooks to at least ML $93 \mathrm{~mm}$; dorsal keel developing along distal half of club, $\sim 60 \%$ club width; dorsal and ventral membranes not apparent; overall preserved colouration dark purplish blue, darkest dorsally on mantle and head; head and bases of Arms I and II with faint coppery iridescence; arms darkest aborally, paling laterally; oral surfaces with two rows of distinct, dark, paired chromatophores often alternating with suckers over length of arm; tentacles darkest aborally, paling laterally and orally; oral surface of club and membranes without apparent pigmentation.

No specimens below ML $77 \mathrm{~mm}$ were positively identified.

Remarks - Ok. (Ok.) robsoni is one of the most distinctive onychoteuthids at maturity. Its long sagittate fins, characteristic round, well-separated mantle warts (Fig. 40B), lateral grooves on the manus hook claws, and the presence of the $Y$-shaped ridge in the funnel easily separate it from OK. (M.) ingens, the other most commonly encountered notalian Onykia species. Given its broad distribution, it is remarkable that juvenile specimens of Ok. (Ok.) robsoni have not been described to date; it seems most likely that small specimens exist in collections but have not yet been recognised as such. Attempts were made in this study to identify likely paralarvae and juveniles using SEMs of their tentacular suckers, but no marginal suckers from larger Ok. (Ok.) robsoni specimens were available for comparison, and no paralarva or small juvenile specimen displayed characters or character states allying it strongly with larger specimens attributed to Ok. (Ok.) robsoni.

Since the name Ok. (Ok.) robsoni is relatively young and a large number of older Onykia species have been described based solely on paralarval to small juvenile specimens, it is possible that $O k$. (Ok.) robsoni will eventually be recognised as a junior synonym of an earlier taxon. Its presence in the Gulf of Mexico, near the type locality of Ok. (Ok.) carriboea, may be relevant; paralarvae and juveniles closely resembling Ok. (Ok.) carriboea have been examined from other areas where Ok. (Ok.) robsoni occurs, including New Zealand, South Africa, and the central Pacific. However, Ok. (Ok.) aequatorialis also occurs in the tropical Atlantic and Gulf of Mexico, and is 
known only from spent females, so it is not possible to identify either nominal species as the adult form of Ok. (Ok.) carriboea without further material.

Reproduction in Ok. (Ok.) robsoni may follow a similar pattern to that hypothesised for Ok. (M.) ingens (Bolstad 2006). The dorsal surface of the mantle in several examined adult to spent females of $O k$. (Ok.) robsoni was scored with deep, broad, longitudinal scratches, with spermatangia inserted into mantle musculature near the bases of gills and protruding into the gladius sac. Lower beak damage similar to that occurring in OK. (M.) ingens was also observed in several large specimens, but other sexual dimorphism in the beaks (either in morphology or size relative to the weight or $M L$ ) is unknown.
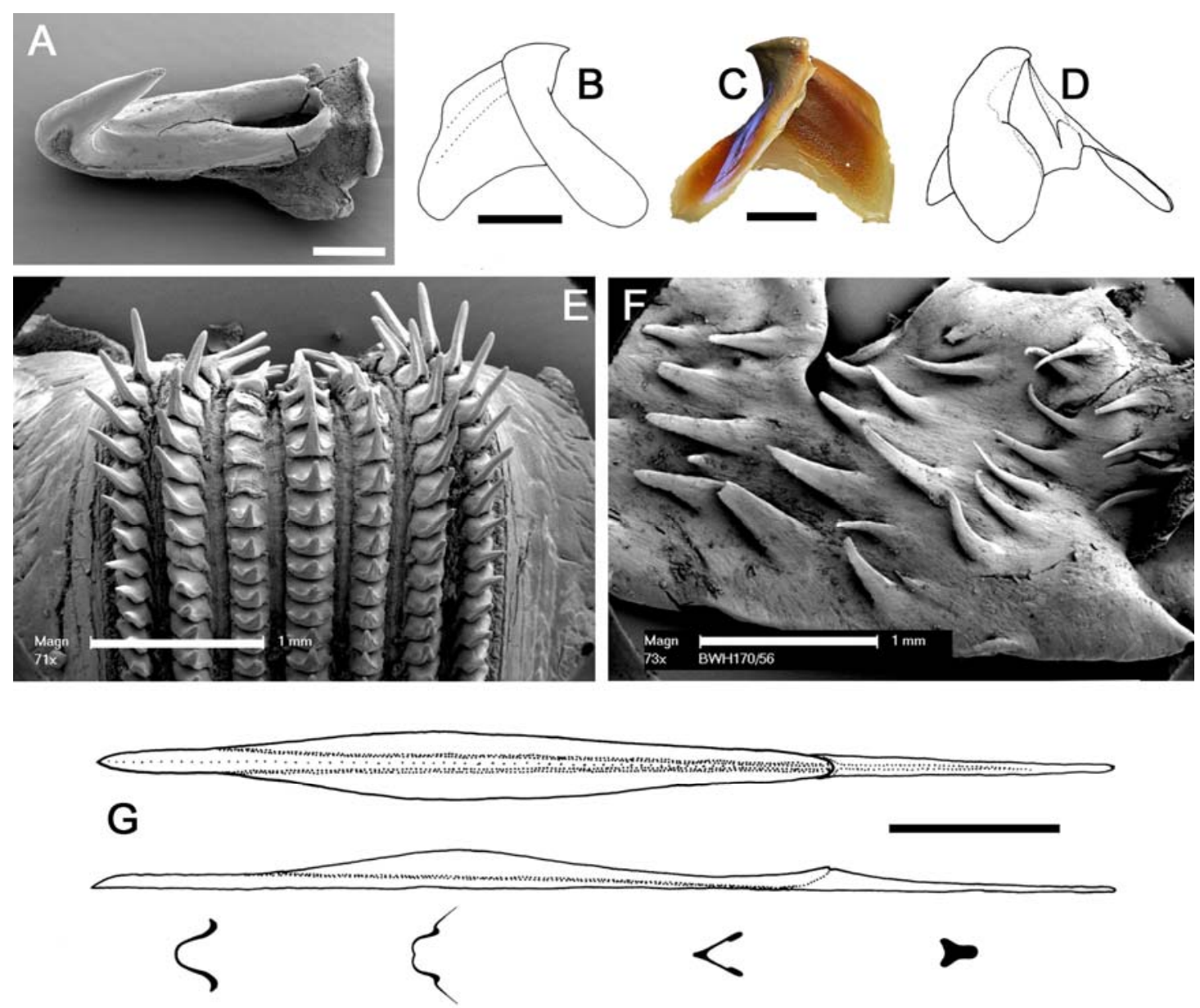

Fig. 41 - Onykia (Onykia) robsoni. A) NMNZ M.131960, ô, ML 595mm; B) AUT unaccessioned, ô, ML 500mm; C) SAM S2520,, , ML 370mm; D-F) NMNZ

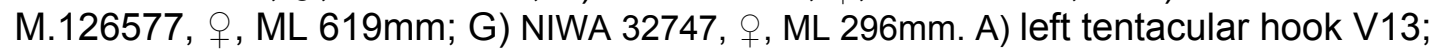
$B-D)$ lower beak: (B) left, (C) right, (D) left oblique profiles; E) radula; $F$ ) half of palatine palp; G) gladius. Scale bars = A) $50 \mu \mathrm{m}$; B, C) $10 \mathrm{~mm}$; E, F) $1 \mathrm{~mm}$; G) $50 \mathrm{~mm}$. 
Adam (1962, see translation in Appendix D) designated a holotype for Ok. (Ok.) robsoni ('MBM - 1957 NO, M 7'), but its deposition is unknown and recent authors (e.g. Sweeney \& Young 2003) have considered it lost. Should questions about this species' identity arise, the male specimen registered under SAM S3957 (ML 595mm) would be a suitable neotype.

The alimentary tracts of several adult to spent females examined were infested with nematode worms, most often concentrated within the stomach caecum walls. 
Onykia (Onykia) indica Okutani, 1981 (Tables 11, 17, Figs 42, 43)

Onykia indica Okutani, 1981: 159-162, Table 2, Figs 10-14; Sweeney et al. (1992): 133; Sweeney \& Roper (1998): 572.

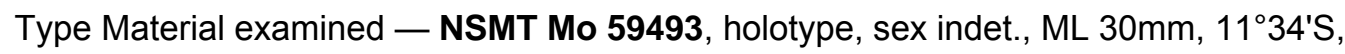
$109^{\circ} 45.5^{\prime} E, 26 / 12 / 1975$, RV Shonan-Maru Sample No. El42, coll. K. Fujita; NSMT Mo59494, paratype, sex indet., ML $23.5 \mathrm{~mm}, 12^{\circ} 50^{\prime} \mathrm{S}, 108^{\circ} 53^{\prime} \mathrm{E}$, eastern Indian Ocean, from stomach of $A$. ferox ( $661 \mathrm{~mm} \mathrm{FL}$ ), fished $17 / 12 / 1975$ by RV Shonan-Maru; NSMT

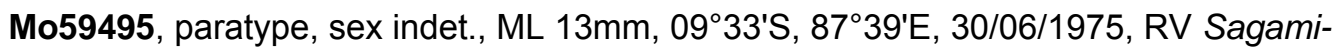
Maru Sample No. Cl35-44, coll. K. Fujita.

Additional (unlocalised) material examined (4 specimens) - NSMT Mo76151, sex indet., ML $52 \mathrm{~mm}$, ex lancetfish stomach, NFD; MNHN 3.2.650, sex indet., ML $37 \mathrm{~mm}$, Indian Ocean, 1841, coll. Dussumier, NFD; ZMBN 2417, 2 sex indet., ML 30, 27mm, Eastern India, NFD.

Distribution (Fig. 42A) — Indian Ocean, depth unknown (most specimens ex-gutcontent).

Diagnosis - Fins ovate, length $\sim 37 \% \mathrm{ML}$, width $\sim 74 \% \mathrm{ML}$; epidermis of mantle smooth; length of Arms II-IV >100\% ML; tentacles 140-220\% ML; length of tentacle clubs $50-70 \% \mathrm{ML}$, with $25-28$ hooks flanked proximally by tiny marginal suckers (barely larger than hook apertures) through at least $\mathrm{ML} 37 \mathrm{~mm}$; rostrum of gladius $\sim 17 \%$ GL.

Description (Figs 42B-43C) - Mantle fleshy, broad, width 30-38-46\% ML, stubby in appearance, remaining broad posteriorly beneath fins; gladius not visible dorsally or ventrally through mantle tissue; skin smooth. Fins broadly ovate, length $26-37-47 \%$ $\mathrm{ML}$, width $60-74-90 \% \mathrm{ML}$, not at all produced posteriorly, forming nearly straight line across tip of mantle; anterior and posterior fin margins convex.

Head boxy, length 25-32-38\% ML, width 22-27-42\% ML, depth 30\% ML; broader dorsally than ventrally. Funnel narrow, with aperture level with posterior margin of eye aperture; funnel groove with Y-shaped pad by $\mathrm{ML} 27 \mathrm{~mm}$; groove margins indistinct on specimens examined. Funnel component of locking apparatus $\sim 15 \% \mathrm{ML}$, mantle component $\sim 30 \% \mathrm{ML}$. 
Table 17. Measures $(\mathrm{mm})$ and counts of Onykia (Onykia) indica Okutani, 1981(arm and tentacle measures recorded from more complete side of specimen, indicated $\mathrm{R}$ or $\mathrm{L}$ ).

\begin{tabular}{|c|c|c|c|c|c|c|c|c|c|}
\hline Specimen ID & $\begin{array}{c}\text { NSMT } \\
\text { Mo59493 }\end{array}$ & $\begin{array}{c}\text { NSMT } \\
\text { Mo59494 }\end{array}$ & $\begin{array}{c}\text { NSMT } \\
\text { Mo59495 }\end{array}$ & $\begin{array}{c}\text { NSMT } \\
\text { Mo76151 }\end{array}$ & $\begin{array}{l}\text { MNHN } \\
3.2 .650\end{array}$ & $\begin{array}{c}\text { ZMBN } \\
2417\end{array}$ & $\begin{array}{c}\text { ZMBN } \\
2417\end{array}$ & \multicolumn{2}{|c|}{$\begin{array}{c}\text { Mean } \\
\text { Indices }\end{array}$} \\
\hline Type Status & Holotype & Paratype & Paratype & None & None & None & None & & \\
\hline ML & 30 & 23.5 & 13 & 52 & 37 & 30 & 27 & & \\
\hline Sex & Indet. & $\mathrm{F}$ & Indet. & Indet. & Indet. & Indet. & Indet. & & \\
\hline MW & 12 & 10 & 6 & 17 & 15 & 10 & 8 & MWI & 38 \\
\hline $\mathrm{HL}$ & 9 & 8 & 5 & 13 & 12 & 10 & 8 & HLI & 32 \\
\hline HW & 8 & 6 & 5.5 & 13 & 11 & 6 & 6 & HWI & 27 \\
\hline $\mathrm{FL}$ & 14 & 6 & 4 & 24 & 18 & 9 & 9 & FLI & 37 \\
\hline FW & 27 & 19 & 8 & 34 & 34 & 18 & 18 & FWI & 74 \\
\hline Arms I & $36 \mathrm{~L}$ & $31 \mathrm{~L}$ & $17 \mathrm{~L}$ & $45 \mathrm{~L}$ & $41 \mathrm{R}$ & $26 \mathrm{~L}$ & $26 \mathrm{~L}$ & A1I & 109 \\
\hline Arms II & $42 \mathrm{~L}$ & $39 \mathrm{~L}$ & $19 \mathrm{~L}$ & $55 \mathrm{~L}$ & $48 R^{*}$ & $34 \mathrm{~L}$ & $31 \mathrm{~L}$ & A2I & 132 \\
\hline Arms III & $43 \mathrm{~L}$ & $38 \mathrm{~L}$ & $19 \mathrm{~L}$ & $52 \mathrm{~L}$ & $50 \mathrm{R}$ & $32 \mathrm{~L}$ & $30 \mathrm{~L}$ & A3I & 130 \\
\hline Arms IV & $46 \mathrm{~L}$ & $35 \mathrm{~L}$ & $20 \mathrm{~L}$ & $56 \mathrm{~L}$ & $48 R^{*}$ & $33 \mathrm{~L}$ & $29 L$ & A4I & 131 \\
\hline TnL & $65 \mathrm{~L}$ & $45 \mathrm{~L}$ & $25 \mathrm{~L}$ & $88 \mathrm{~L}$ & 71R & $44 \mathrm{~L}$ & $44 \mathrm{~L}$ & TnLI & 182 \\
\hline $\mathrm{CL}$ & $18 \mathrm{~L}$ & $15 \mathrm{~L}$ & $9 \mathrm{~L}$ & $26 \mathrm{~L}$ & $23 R$ & $15 \mathrm{~L}$ & 14 & CLI & 58 \\
\hline CS & 8 & 7 & 7 & 8 & 9 & 9 & 10 & & \\
\hline MH & 28 & 25 & 28 & 26 & $24^{*}$ & $26^{*}$ & $24^{*}$ & & \\
\hline MS & D1-6, V1-3 & * & * & 0 & All & * & * & & \\
\hline TPS & 13 & * & $6^{*}$ & $10^{*}$ & * & * & * & & \\
\hline
\end{tabular}

* indicates damaged features.

Arms slender, extremely long, 85-109,-130 ${ }_{I I I V}-170 \% \mathrm{ML}$, each with $80-100$ widely spaced suckers (ML 37-52mm) bordered by low trabeculate membrane; arm formula II=III=IV>I. Arms I-III without apparent keels; Arms IV with lateral membrane, $\sim 75 \%$ arm width at base, thereafter reducing to $\sim 15 \%$ arm depth along remainder of arm length.

Tentacles narrow, 147-182-217\% ML; bases more slender than those of adjacent arms. Club (Fig. 42C) unexpanded, length 50-58-69\% ML (30-40\% TnL); carpus with seven to ten suckers, manus damaged in most specimens examined, with 25-28 hooks; terminal pad damaged in most specimens examined, with $\sim 13$ suckers. Hooks with symmetrical bases; all dorsal hooks smaller than paired ventral hooks; hooks D2D6 largest in dorsal series; hooks in ventral series increase gradually in size through hook V7 or V8, with length of longest hook approximately twice that of paired dorsal hook and $\sim 8 \% \mathrm{CL}$; marginal suckers comparatively tiny, flanking proximal two or three and distal two or three ventral hooks, and proximal four to six dorsal hooks. No membranes apparent bordering club.

Lateral profile of lower beak (Figs 42D, 42E, ML52mm) longer than deep, with crest straight or very gently convex and sloping steeply downward, posterior edge of hood well above crest, hood length $\sim 40 \%$ total crest length, and hood and crest together $\sim 70 \%$ baseline; jaw edge straight proximally, weakly concave distally; jaw edge sharp; jaw angle obtuse, slightly obscured by low wing fold. Crest broad, slightly thickened; lateral walls not indented below crest; shoulder ridge distinct, sharp; angle ridge 
distinct but not raised, spanning $\sim 60 \%$ lateral wall depth; wing width distally $\sim 130 \%$ that at jaw angle, length $\sim 170 \%$ LRL; small insert of clear cartilage present on medial wing margin just below jaw angle; lateral wall fold shallow, rounded, remaining distinct to posterior margin of lateral wall.

Radula (Fig. 43A) with triangular mesocone on rachidian; lateral cusps $\sim 50 \%$ mesocone height, slightly laterally directed; proximal margin of base straight to slightly
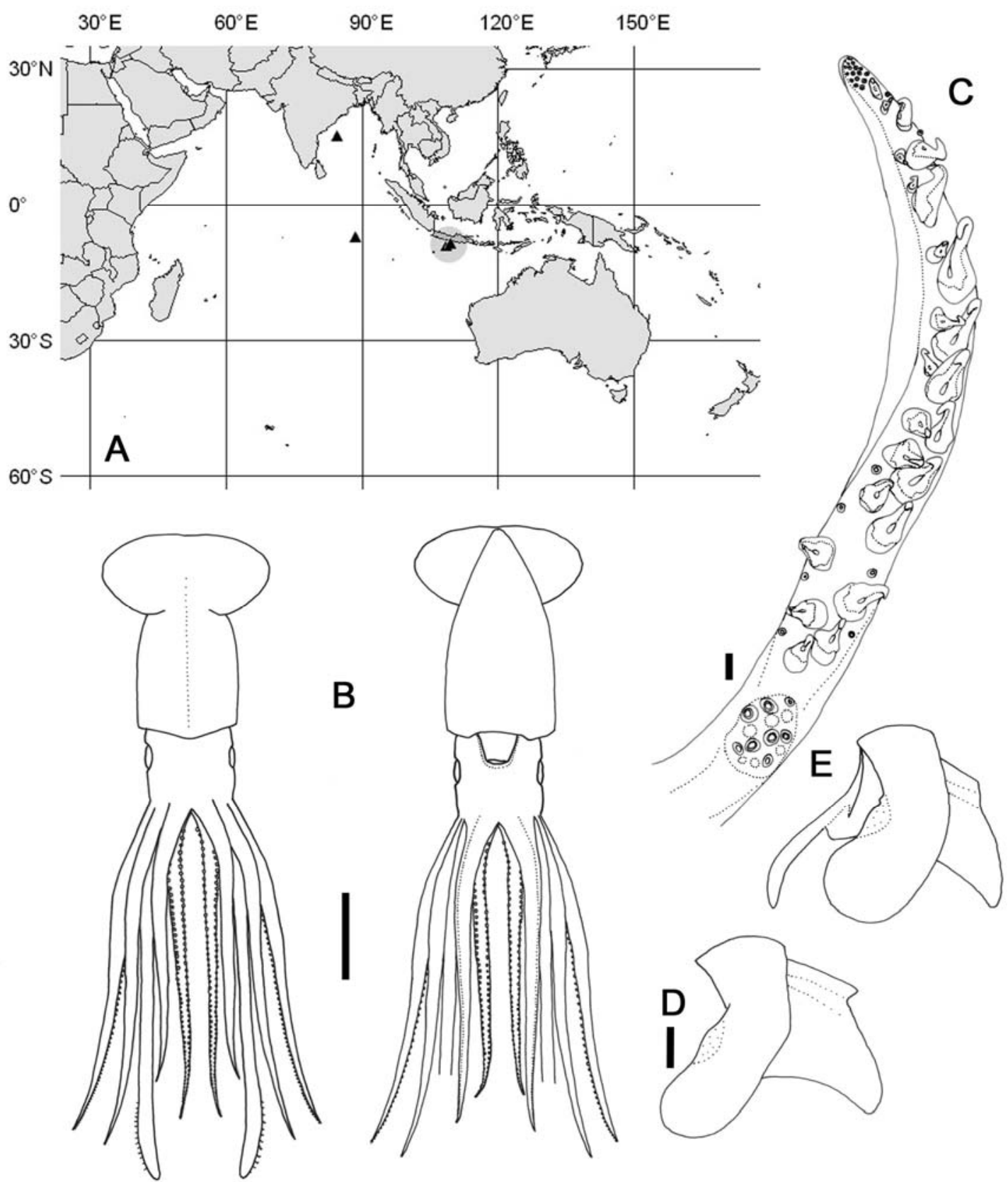

Fig. 42 - Onykia (Onykia) indica. A) distribution (grey circle indicates type locality); B, C) NSMT 59494, paratype, ML 23.5mm: B) whole animal, C) left tentacle club; D, E) NSMT Mo76151, sex indet., ML 52mm, lower beak: D) right, E) right oblique profiles. Scale bars = B) $10 \mathrm{~mm}$; C, D) $1 \mathrm{~mm}$. 
convex, distal margin between mesocone and lateral cusps roundly concave. First lateral tooth bicuspid; inner cusp moderately broad, triangular, straight and slightly directed toward rachidian, subequal to rachidian in height; outer cusp slightly laterally directed, its height $\sim 50 \%$ that of inner cusp. Second lateral tooth robust, slightly curved, $\sim 125 \%$ rachidian height. Marginal tooth simple, slender, height $\sim 200 \%$ that of rachidian. Palatine palp (Fig. 43B) broad, greatest width of tooth-bearing plane $\sim 25 \%$ of its length, with $\sim 40$ short, slender teeth, flattened into palp surface, each $50-130 \%$ rachidian height, sparsely and evenly distributed in no discernible pattern over palp surface.

Gladius (Fig. 43C) with greatest width ( 9\% GL) attained at $\sim 50 \% \mathrm{GL}$; free rachis $\sim 17 \%$ GL; vanes broaden and taper gradually between 20 and $85 \%$ GL. Rostrum $\sim 11 \% \mathrm{GL}$, of elongate triangular to tear-drop-shaped cross-section.

Small, pale red chromatophores scattered over entire surface of mantle (preserved). No tail-spots apparent beneath fins. Head with pale red chromatophores in several layers dorsally, smaller and more isolated over ventral surface; small and sparse over funnel; funnel groove with one large chromatophore lateral to each bridle.
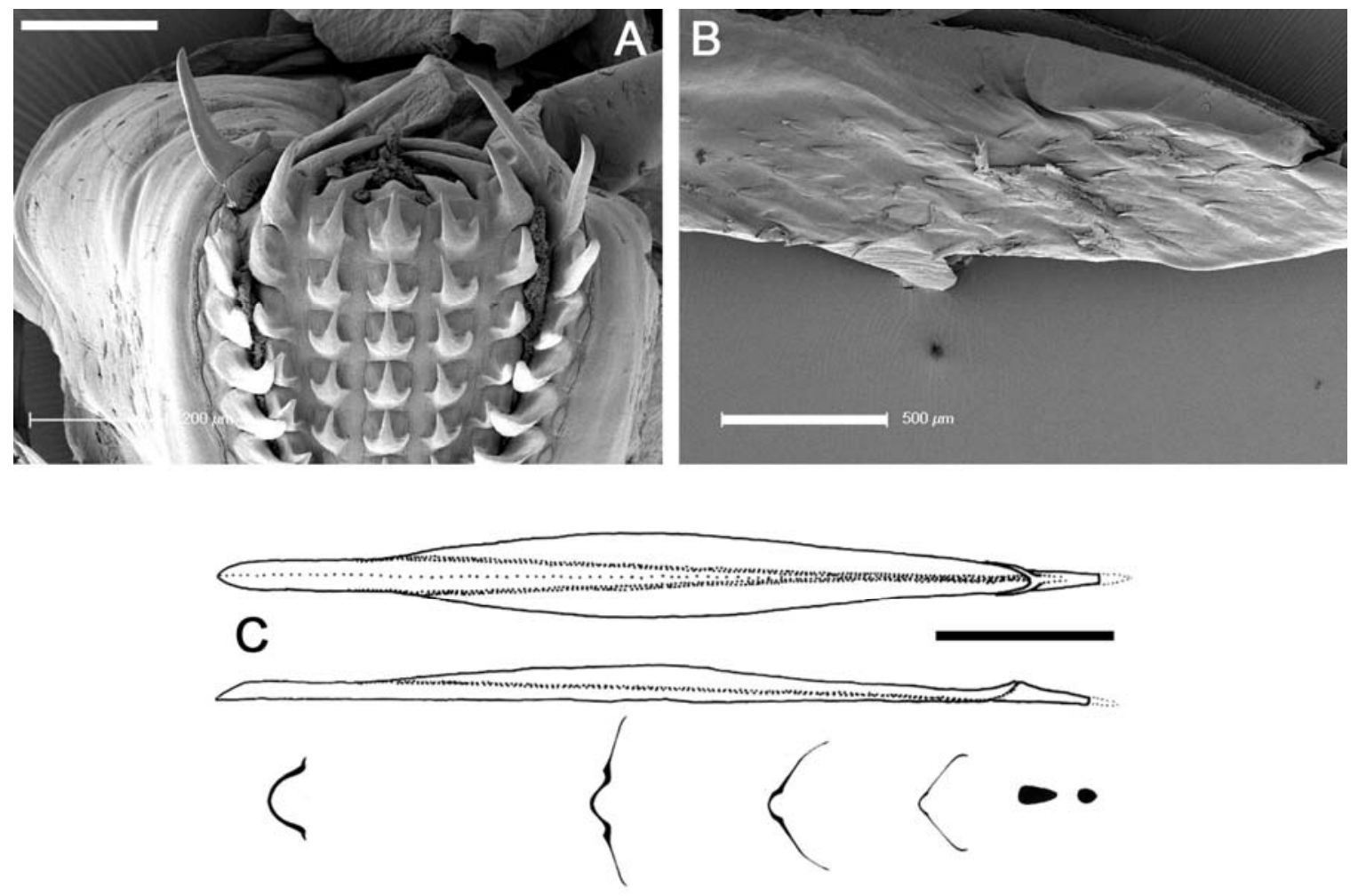

Fig. 43 - Onykia (Onykia) indica. A-C) NSMT Mo76151, ML 52mm: A) radula, B) palatine palp, C) gladius. Scale bars = A) $200 \mu \mathrm{m}$; B) $500 \mu \mathrm{m}$; C) $10 \mathrm{~mm}$. 
Remarks - Onychoteuthids from the Indian Ocean were scarce in collections. The largest Ok. (Ok.) indica specimen examined was only ML $52 \mathrm{~mm}$; however, at this size Ok. (Ok.) indica already appears distinct from other Onykia species at similar sizes. The large size of the head and brachial crown relative to the mantle, the lack of ventral 'tail-spots' on the posterior mantle surface, and the very long arms and tentacles combine to create a distinctive appearance in specimens from $\mathrm{ML} 13 \mathrm{~mm}$.

Given that five of the seven examined specimens were ex-gut-content samples, the superlative length of the arms and tentacles may be partially a preservation artefact caused by either loosening of the tissues or contraction of the mantle due to digestive processes, or the physical response of the squid to predation and subsequent mortality. (The arm indices of the two non-gut-content specimens were 87-92-131 1 $I^{-115 \%}$ ML.) However, the tentacular morphology of Ok. (Ok.) indica (Fig. 42C) does appear distinct from that of other Onykia species for which small individuals are known. The comparatively tiny marginal suckers relative to the tentacular hooks (the former barely larger than the apertures of the latter, already at $\mathrm{ML} 23.5 \mathrm{~mm}$ ) are unique; Okutani also considered the low number of carpal suckers important (six to eight in the type series, up to ten in additional specimens examined). This low number is indeed rare within the genus, although Ok. (Ok.) carriboea and Ok. (Ok.) loennbergii generally have seven to nine and $O k$. (OK.) robsoni may also occasionally have as few as seven carpal suckers.

Since the material herein attributed to $O k$. (Ok.) indica appears distinct from other small Onykia, and no intermediate-sized material links it to subadults of a known species, and especially given that the Indian Ocean is poorly represented in onychoteuthid collections, Ok. (Ok.) indica is presently considered a distinct species. 


\section{Onykia (Moroteuthopsis) Pfeffer, 1908b}

Diagnosis - Y-shaped ridge absent from funnel groove; ventral manus hooks with flat lateral 'blades' on claw, meeting distally at midline of claw; lateral grooves absent from claw.

Onykia (Moroteuthopsis) ingens (Smith, 1881) (new comb.) (Tables 11, 18, 19, Figs 44-48)

Onychoteuthis ingens Smith, 1881: 25, PI. 3 Figs 1-1d; Lönnberg (1898): 55-63, Pls 4, 5; Pfeffer (1900): 159, 160; Lipinski et al. (2000): 107.

Moroteuthopsis ingens (Smith, 1881) — Pfeffer (1908b): 295.

Moroteuthis ingens (Smith, 1881) — Pfeffer (1912): Pls 11, 12; Dell (1951): 99; Clarke (1965): 287-307, Table 21 (not column 3), (1966): 145-147, Fig. 21, (1980): 81-128, Table 21 (columns 1 and 2 only), Text-fig. 53, (1986): 74-77, Figs 10.17F, 14.35F, 34, 36B, C; Zuev \& Nesis (1971): 182, Fig. 54; Imber \& Russ (1975): 29, 30; Imber (1976): 126; Okutani (1978): 153, 154; Toll (1982): 70, 71, PI. 7 Fig. B; Roper et al. (1984): 130, (1985): 181, 183, 184; Okutani \& Clarke (1985): 13, 14; Lipinski \& Linkowski (1986): $97-$ 105, Figs 1-5; Nesis (1987): 193, Fig. 49A-D; Kubodera (1990): 336, Fig. 266; Tsuchiya \& Okutani (1992): 137-147, Figs 26, 35; Sweeney et al. (1992): 129, 130; Jackson (1993): 1-9, Figs 1-4, (1995): 9-14, Table 1, Figs 1-5, (1997): 268-274; Jackson \& Lu (1994): 195-200; Jackson \& Mladenov (1994): 189-201, Figs 1-3, Pls 1-4; Bizikov (1996): Figs 10F, 10G, 114; Filippova et al. (1997): 188, Fig. 112; Jackson et al. (1997): 1235-1238; Anderson et al. (1998) (unpaginated); Clarke \& Roper (1998): 129-133; Kubodera et al. (1998): 283, Fig. 12; Sweeney \& Roper (1998): 572; Sands et al. (2003): 166-170; Jackson \& Jackson (2004): 783, 784; Okutani (2005): 158; Bolstad (2006): 317-327, Figs 1-18, (2007): 314-319, 332, 333, Tables 2, 6, Figs 14-27; Wakabayashi et al. (2007) 959-965, Table 2, Fig. 2.

Moroteuthis (Moroteuthopsis) ingens (Smith, 1881) - Pfeffer (1912): 83-87; Dell (1952): 102. Moroteuthis sp. (types Aii, Aiii) Gaskin \& Cawthorn, 1967a - 168, 169, 176, (1967b): 62, 63, 69, Fig. 2.

Moroteuthis sp. A Clarke, 1980 - 121-126, Text-figs 56-67, 77, 90-93, PI. 1 Figs 3, 4. Moroteuthis [sic] (not Verrill, 1881) - Sweeney et al. (1992): Fig. 183c.

Type material examined - BMNH 1880.10.8.1, holotype (head \& brachial crown only), Port Riofrio, west coast of Patagonia, NFD.

Additional material examined (125 specimens) - UMML 31.2946, ㅇ, ML 98mm, 37¹1'S, $114^{\circ} 41$ 'W, from Alepisaurus stomach, 10/11/1963, RV Shoyo Maru Stn F6; NMNZ M.160471, sex indet., ML 73mm, 37²7.80'S, $169^{\circ} 55.80^{\prime} \mathrm{E}, 945-937 \mathrm{~m}, 26 / 10 / 1983$, FV

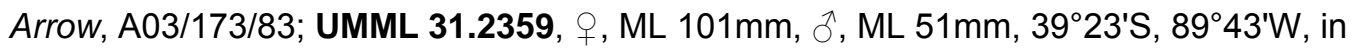
fish stomach, 08/12/1963, RV Shoyo Maru Cruise 13, Stn 13; NSMT Mo67547, ô, ML 
290mm, 40²8'S, 74¹9'W, 600-594m, 24/09/1977, RV Akebono-Maru Stn 1-195, JAMARC; SAM S3997,, , ML 365mm, 4044'S, 4321'E, "Knobby Hill," 978-847m, 30/01/2000, MT Iris, coll. R. Leslie, BTT; NMNZ M.12952, sex indet., ML 90mm, Waikanae Beach [ $\left.\sim 0^{\circ} 53^{\prime} \mathrm{S}, 175^{\circ} 04^{\prime} \mathrm{E}\right], 20 / 06 / 1954$, coll. M. Porter, washed ashore; NMNZ M.131972, ô, ML 365mm, 41 ${ }^{\circ} 23.6^{\prime} \mathrm{S}, 176^{\circ} 21.7^{\prime} \mathrm{E}, 1143-982 \mathrm{~m}, 03 / 04 / 1984$, FRV

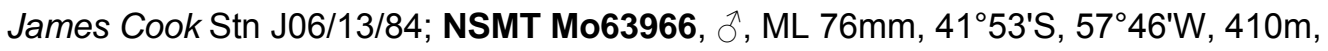
05/10/1984, JAMARC RV Banshu-Maru, MWT; NMNZ M.17889, §̂, ML 260mm, off Kaikoura, NZ [ $\left.42^{\circ} 25^{\prime} S, 173^{\circ} 54^{\prime} E\right]$, sperm whale stomach, $25 / 10 / 1963$, pres. NZ Marine

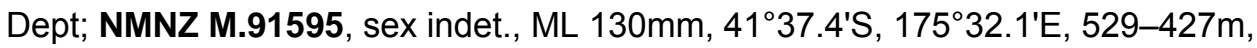
11/04/1981, FRV James Cook; NSMT Mo67938, 12 sex indet., ML 92-47mm, 4203'S, 57 58'W, 440m, 05/10/1984, JAMARC RV Banshu-Maru MWT; NSMT Mo67524,, , ML 373mm, 42 25'S, 745' W, 609-283m, 29/12/1977, RV Akebono-Maru Stn 4-7, JAMARC; NMNZ M.183111, sex indet., ML 12mm, 42³6.97'S, $176^{\circ} 43.40^{\prime} \mathrm{E}, 25 \mathrm{~m}$,

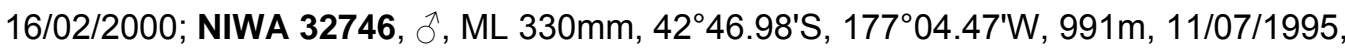
FV San Waitaki Stn SWA9501/8, Z8337; NIWA 32773, , ML 470mm, 42 46.54 46.45'S, $179^{\circ} 59.14-59.00^{\prime} E, 1070-942 \mathrm{~m}, 01 / 06 / 1997$, RV Tangaroa Stn TAN0219/12,

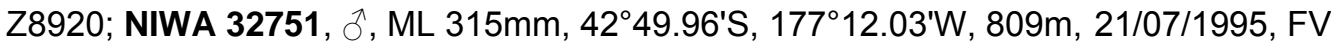
San Waitaki Stn SWA9501/56, Z8339; NIWA 32748, , ML 450mm, ô, ML 330mm, 4251.58-51.53'S, 17659.82-01.89'W, 805-804m, 15/07/1995, FV San Waitaki Stn SWA9501/56, Z8338; NIWA 32754, ㅇ, ML 188mm, 4304-12'S, 175³9-53'E, 460m, 01/10/1996, FV Drysdale, Z8566/1; NIWA 32755, ㅇ, ML 150mm, 434-12'S, 175³953'E, 460m, 01/10/1996, FV Drysdale, Z8566/2; NIWA 32756, ठ̊. ML 127mm, 430412'S, 175 39-53'E, 460m, 01/10/1996, FV Drysdale, Z8566/3; NIWA 32794, †, ML 180mm, 43⒙56-20.71'S, 17402.35-01.68'E, 646-609m, 20/01/1999, RV Tangaroa Stn TAN9901/101, Z9663; NIWA 32795, §ิ, ML 138mm, 3 sex indet., ML 142-116mm, 431․56-20.71'S, 17402.35-01.68'E, 646-609m, 20/01/1999, RV Tangaroa Stn TAN9901/101; NIWA 32799, + , ML 395mm, 4336.84-35.18'S, 175⒕32-10.88'W, 578-569m, 09/01/1999, RV Tangaroa Stn TAN9901/35, Z7933; NIWA 32796, 2 sex indet., ML 127, $112 \mathrm{~mm}, 43^{\circ} 44.15-46.73^{\prime} \mathrm{S}, 1^{\circ} 4^{\circ} 32.74-30.60^{\prime} \mathrm{E}, 569-528 \mathrm{~m}, 20 / 01 / 1999$, RV Tangaroa Stn TAN9901/98, Z9664; NIWA 32793, ㅇ, ML 429mm, 4346.84'S, 17539.25'W, 320-297m, 09/01/1999, RV Tangaroa Stn TAN9901/33, Z9662; NIWA 32705, $\widehat{\jmath}$, ML 270mm, 4348.16-49.83'S, 175²7.19-30.66'E, 417m, 24/01/2001, RV Tangaroa Stn TAN0101/138; NIWA 32798, 2 , , ML 320, 195mm, 4354.15-52.15'S, 178 44.50-41.39'E, 567-522m, 13/01/1999, RV Tangaroa Stn TAN9901/54, Z9732; NIWA 32797, $q$, ML 300mm, 4354.15-52.15'S, 17844.50-41.39'E, 567-522m, 13/01/1999, RV Tangaroa Stn TAN9901/54, Z9732; NIWA 32792, 2ᄋ, ML 300, 280mm, 435⒌41'S, 177³2.86'E, 740-687m, 16/01/1999, RV Tangaroa Stn TAN9901/72, Z9661; NIWA 32789, , ML 370mm, 4357.95-59.37'S, 17349.84-47.89'E, 193-190m, 30/12/1998, RV Kaharoa Stn KAH9809/66, Z9550; NSMT Mo67534, ㅇ, ML 373mm, 4402'S, 75¹2'W, 355m, 22/09/1977, RV Akebono-Maru Stn 1-176, JAMARC; NIWA

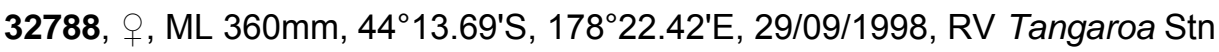


TAN9815/02, Z9471; NIWA 32759, 2 sex indet., ML 40, 25mm, 44ํำ.50'S, 176³0.15'E, 300-50m, 16/02/1992, RV Tangaroa Stn TAN9202/43, FMMWT, NZOI Z8722; NIWA 32785, $q$, ML 370mm, 44⒙77'S, 178 $13.35^{\prime} \mathrm{W}, 668-660 \mathrm{~m}, 25 / 10 / 1998$, RV Tangaroa Stn TAN9812/83; NMNZ M.160449, 3 sex indet., ML 41, 34, 31mm, 44²7.50'S, $173^{\circ} 54.60^{\prime} \mathrm{E}$, off Banks Peninsula, New Zealand, 30m, -/12/1987; NIWA TAN0101/111

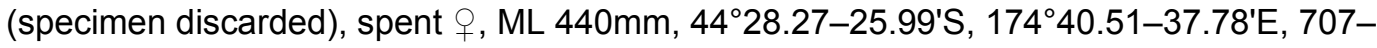
694m, 19/01/2001, RV Tangaroa Stn TAN0101/111; NIWA 32783, ô, ML 280mm, $44^{\circ} 28.85^{\prime} \mathrm{S}, 178^{\circ} 30.97^{\prime} \mathrm{W}, 956-940 \mathrm{~m}, 25 / 10 / 1998$, RV Tangaroa Stn TAN9812/85; NIWA

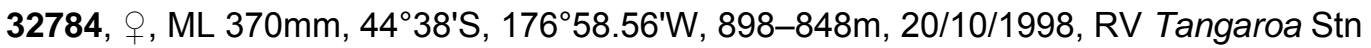

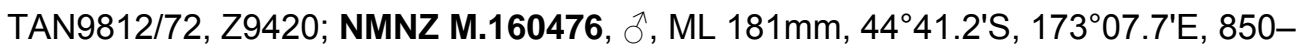
842m, 10/06/1984, FRV James Cook Stn J10/17/84; NMNZ M.160469, sex indet., ML $78 \mathrm{~mm}, 45^{\circ} 02.50^{\prime} \mathrm{S}, 173^{\circ} 59.90^{\prime} \mathrm{E}, 200 \mathrm{~m}$ over $1251-1225 \mathrm{~m}, 10 / 12 / 1984$, FMMWT, FRV James Cook Stn J21/13/84; NIWA 32760, sex indet., ML 19mm, 45²1.98'S, 177²9.60'E, 300-30m, 17/02/1992, RV Tangaroa Stn TAN9202/48, Z8727; NSMT

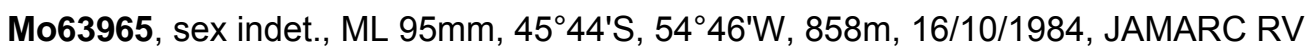
Banshu-Maru, BTT; NSMT Mo67546, ô, ML 380mm, 46º9'S, 75³0'W, 212-202m, 17/10/1977, RV Akebono-Maru Stn 2-64, JAMARC; NMNZ M.183083, 3 sex indet., ML 48, 42, 30mm, 46¹0.20'S, 17201.90'E, 30m, 11/12/1987, FRV James Cook, Stn J15/30/87, FMMWT; NSMT Mo63964, ô, ML 92mm, 46¹7'S, 6003'W, 668m, 16/10/1984, JAMARC RV Banshu-Maru, BTT; NMNZ M.74296, , ML 487mm, 46 32 'S, 16609'E, 852-814m, NFD; NMNZ M.117885, đ', ML 305mm, 46³7.14'S, 166¹6.32'E, 852-814m, 04/12/1993, RV Tangaroa Stn TAN9310/67; NMNZ M.74256, ㅇ, ML 335mm, 46 $55.8-46^{\circ} 53.4^{\prime} \mathrm{S}, 1^{\circ} 9^{\circ} 44.5-169^{\circ} 45.3^{\prime} \mathrm{E}, 470 \mathrm{~m}, 14 / 02 / 1977$, FRV James Cook Stn J9/008/77; NMNZ M.74285, , ML 205mm, 46 $55.8^{\circ}-46^{\circ} 53.4^{\prime}$ S, $169^{\circ} 44.5-169^{\circ} 45.3^{\prime} \mathrm{E}$, 470m, 14/02/1977, FRV James Cook Stn J9/008/77; MNHN 2769, 21 sex indet., ML $\sim 70 \mathrm{~mm}$ (rotten), $47^{\circ} 07^{\prime} \mathrm{S}, 70^{\circ} 19^{\prime} \mathrm{E}$, Kerguelen, $190 \mathrm{~m}, 13 / 02 / 1995$, chalutier 'Mys ostrovskogo'; MNHN 2770, 6 sex indet., ML 95-87mm, 47 $17^{\circ} \mathrm{S}, 70^{\circ} 37^{\prime} \mathrm{E}$, Kerguelen, 190m, 23/02/1995, chalutier 'Mys ostrovskogo'; NMNZ M.277905 (10 specimens), ML 12-25mm, 47³9.4'S, 169³5.4'E, 30m over 626m, FRV James Cook Stn J15/37/87; NMNZ M.118344, ․, ML 148mm, 47²40.68'S, 17800.72'E, 846-840m, 09/12/1990, FV

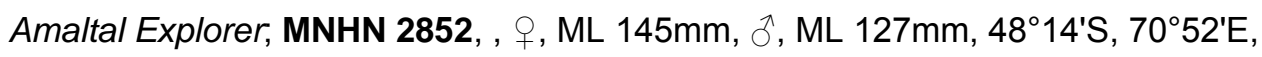
Kerguelen, 150-145m, 29/02/1988, chalutier 'Skif'; NMNZ M.67830, ð, ML 100mm, 48ㄴㄷ․03'S, $170^{\circ} 25.66^{\prime} \mathrm{E}, 857 \mathrm{~m}, 26 / 09 / 1979$, FV Wesermünde; NIWA 32738, 2 \% , ML 386, 372mm, 49¹4.65-11.95'S, 170¹5.10-13.09'E, 704-668m, 16/12/2000, RV Tangaroa Stn TAN0012/85, Z10627; NMNZ M.118754, ㅇ, ML 105mm, 50³8.51'S,

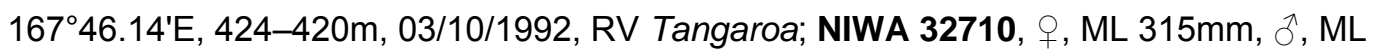
225mm, 51 ${ }^{\circ} 05.77-03.75^{\prime} \mathrm{S}, 171^{\circ} 13.69-17.25^{\prime} \mathrm{S}$, 535-534m, 29/11/2002, RV Tangaroa

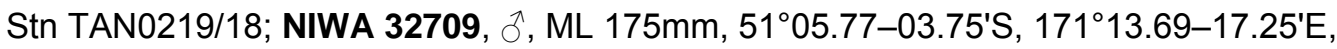
535-534m, 29/11/2002, RV Tangaroa Stn TAN0219/18; NMNZ M.74116, ㅇ, ML 178mm, $52^{\circ} 26^{\prime} \mathrm{S}, 170^{\circ} 31$ 'E, 482-480m, 18/01/1977, FRV James Cook; NIWA 32712, , ML 155mm, 52 $30.97-33.97 ' \mathrm{~S}, 172^{\circ} 34.53-34.36 ' \mathrm{E}, 563-538 \mathrm{~m}, 30 / 11 / 2002$, RV Tangaroa 


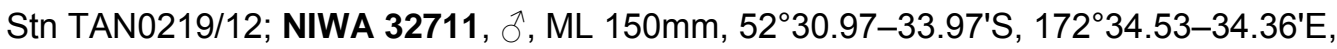
563-538m, 30/11/2002, RV Tangaroa Stn TAN0219/12; NIWA 32707, 今̊, ML 225mm, , ML 213mm, 53⒔84-13.06'S, 170¹7.25-22.12'E, 470m, 01/12/2002, RV Tangaroa Stn

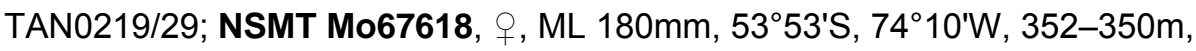
27/11/1977, RV Akebono-Maru Stn 3-32, JAMARC.

Unlocalised material examined (3 specimens) - NIWA 32776, §, ML 250mm, 1118-974m, FV Margaret Phillipa trip 1124/50, Z9211, coll J. Wills, NFD; BMNH 20070487, ð, ML 87mm, 'Discovery' Investigations Stn 234, 207-195m, 05/07/1928, N7-T; NMNZ M.279110, ML $7 \mathrm{~mm}, 07 / 04 / 85$, FV Kalinovo, NFD.

Additional material examined (beaks only) - stomach contents (ex Physeter macrocephalus): NMNZ unaccessioned, 140 male Ok. (M.) ingens lower beaks and 3 male Ok. (M.) ingens buccal bulbs, LRL 9.75-8.68mm; 386 female Ok. (M.) ingens lower beaks and 7 female Ok. (M.) ingens buccal bulbs, LRL 12.85-9.13mm.

Distribution (Fig. 44A) - Sub-tropical and sub-Antarctic waters throughout the Southern Hemisphere, primarily $30-60^{\circ} \mathrm{S}$; adults usually $900-200 \mathrm{~m}$.

Diagnosis - Skin of mantle, head, and bases of arms with small, firm, irregular warts; head nearly as wide as mantle; fins rhombic, not drawn out posteriorly, length $\sim 48 \%$ $\mathrm{ML}$, width $\sim 71 \% \mathrm{ML}$; funnel groove without Y-shaped ridge; 26-31 hooks present on adult tentacle club; hooks without deep lateral grooves on claw; rostrum of gladius $\sim 15 \%$ GL.

Description (Figs 44B-47E) - Mantle muscular, cylindrical anteriorly, width 20-26$30 \% \mathrm{ML}$, rugose, with small, fleshy, irregular 'warts' over all surfaces (Fig. 45A); dorsal midline with shallow groove and underlying dark line indicating gladius; rostrum of gladius visible ventrally through posterior $\sim 10 \%$ of mantle. Fins broad, smooth, rhombic in outline, length $40-48-52 \% \mathrm{ML}$, width $62-71-84 \% \mathrm{ML}$; posterior margin straight to slightly convex; anterior margin gently convex; anterior lobes pronounced.

Head rounded, length $\sim 18 \% \mathrm{ML}$, width $14-17-24 \% \mathrm{ML}$, depth $\sim 13 \% \mathrm{ML}$. Epidermis rugose, with similar warts to those on mantle; funnel aperture at level below posterior margin of eye lens; funnel groove U-shaped or broadly trapezoidal, without Y-shaped ridge; funnel component of locking apparatus $\sim 15 \% \mathrm{ML}$; mantle component $\sim 20 \% \mathrm{ML}$. 
Table 18. Measures ( $\mathrm{mm}$ ) and counts of Onykia (Moroteuthopsis) ingens (Smith, 1881) (new comb.) (arm and tentacle measures and counts recorded from more complete side of specimen, indicated $R$ or $L$ ).

\begin{tabular}{|c|c|c|c|c|c|c|c|c|c|c|c|c|}
\hline Specimen ID & $\begin{array}{c}\text { BMNH } \\
1880.10 .8 .1\end{array}$ & $\begin{array}{l}\text { NIWA } \\
32773\end{array}$ & $\begin{array}{c}\text { NMNZ } \\
\text { M.74256 }\end{array}$ & $\begin{array}{c}\text { NMNZ } \\
\text { M.117885 }\end{array}$ & $\begin{array}{l}\text { NIWA } \\
32707\end{array}$ & $\begin{array}{c}\text { NMNZ } \\
\text { M.67830 }\end{array}$ & $\begin{array}{c}\text { NMNZ } \\
\text { M.160471 }\end{array}$ & $\begin{array}{c}\text { NMNZ } \\
\text { M.183083 }\end{array}$ & $\begin{array}{c}\text { NMNZ } \\
\text { M.183083 }\end{array}$ & $\begin{array}{l}\text { NIWA } \\
32760\end{array}$ & \multicolumn{2}{|c|}{ Mean Indices } \\
\hline Type Status & Holotype & None & None & None & None & None & None & None & None & None & \multirow{2}{*}{\multicolumn{2}{|c|}{ (ML 100-470) }} \\
\hline ML & - & 470 & 335 & 305 & 213 & 100 & 73 & 48 & 30 & 19 & & \\
\hline Sex & Indet. & $\mathrm{F}$ & $\mathrm{F}$ & $\mathrm{M}$ & $\mathrm{F}$ & $M$ & Indet. & Indet. & Indet. & Indet. & & \\
\hline MW & - & 130 & 66 & 65 & 59 & 30 & 20 & 12 & 9 & 6 & MWI & 26 \\
\hline $\mathrm{HL}$ & 48 & 70 & 65 & 52 & 35 & 20 & 13 & 12 & 8 & 5 & HLI & 18 \\
\hline HW & 59 & 65 & 52 & 43 & 42 & 24 & 13 & 12 & 8 & 5 & HWI & 17 \\
\hline FL & - & 200 & 175 & 157 & 108 & 50 & 30 & 20 & 13 & 7 & FLI & 48 \\
\hline FW & - & 324 & $195 \mathrm{~L}$ & 202 & 133 & 74 & 61 & 34 & 23 & 12 & FWI & 71 \\
\hline Arms I & $212 R$ & $190 R^{*}$ & $212 L$ & $196 \mathrm{R}$ & $139 \mathrm{~L}$ & $80 \mathrm{R}$ & $52 R$ & $28 \mathrm{R}$ & $17 \mathrm{~L}$ & $8 \mathrm{~L}$ & $\mathrm{~A} 1 \mathrm{I}$ & 64 \\
\hline Arms II & $299 R$ & $310 \mathrm{R}$ & $272 L$ & $192 R^{*}$ & $189 \mathrm{~L}$ & $106 \mathrm{R}$ & $75 \mathrm{R}$ & $29 R$ & $24 \mathrm{~L}$ & $12 \mathrm{~L}$ & A2I & 85 \\
\hline Arms III & $220 R^{*}$ & $280 R^{*}$ & $244 \mathrm{~L}$ & $207 R$ & $175 \mathrm{~L}$ & $90 \mathrm{R}$ & $66 \mathrm{R}$ & $35 R$ & $24 \mathrm{~L}$ & $11 \mathrm{~L}$ & A3I & 77 \\
\hline Arms IV & $248 R$ & $280 \mathrm{R}$ & $212 \mathrm{~L}$ & $200 R$ & $175 \mathrm{~L}$ & $93 R$ & $70 \mathrm{R}$ & $36 \mathrm{R}$ & $23 \mathrm{~L}$ & $11 \mathrm{~L}$ & A4I & 77 \\
\hline TnL & $365 \mathrm{~L}$ & $395 R$ & $442 \mathrm{~L}$ & $371 \mathrm{R}$ & $320 \mathrm{~L}$ & $165 R$ & $83 R$ & $60 \mathrm{R}$ & $39 \mathrm{~L}$ & $18 \mathrm{~L}$ & TnLI & 128 \\
\hline $\mathrm{CL}$ & $73 \mathrm{~L}$ & $80 \mathrm{R}$ & $89 \mathrm{~L}$ & $79 R$ & $66 \mathrm{~L}$ & $35 R$ & $27 R$ & $17 R$ & $11 \mathrm{~L}$ & $7 \mathrm{~L}$ & CLI & 29 \\
\hline cs & $10 \mathrm{R}$ & 9 & 12 & 12 & 10 & 11 & * & 10 & 12 & 10 & & \\
\hline MH & $29 R$ & 28 & 28 & 28 & 29 & 28 & 26 & 27 & 26 & V3-12 & & \\
\hline MS & 0 & 0 & 0 & 0 & 0 & D4, V9-14 & D1-6, V1-2 & All & All & All & & \\
\hline TPS & $14 \mathrm{R}$ & * & 14 & 13 & 15 & 13 & 13 & 16 & 16 & 14 & & \\
\hline
\end{tabular}

- indicates missing features, ${ }^{*}$ indicates damaged features.

Arms $40-64,-80_{I I-I V}-106 \% \mathrm{ML}$, formula II>III>IV>I, each with $110-130$ suckers (ML $100-470 \mathrm{~mm}$ ); aboral epidermis of arm-bases rugose, with smaller, less distinct warts than on head; keels absent from Arms I and II; Arms III with keel over medial $30 \%$ of arm length, attaining maximum $100 \%$ arm depth; lateral membrane on Arms IV 15\% arm width along entire arm length.

Tentacles 84-128-165\% ML, with stalk thickness at base comparable to that of adjacent arms. Club slightly expanded (Figs 45C-E), 17-29-37\% ML (20-25\% TnL); carpus elongate-ovoid, well defined, with nine to 12 suckers; manus with 26-29 hooks (Figs 45B, 45F); terminal pad with 13-16 suckers. Hooks V6-V8 largest (Fig. 45B), $\sim 10 \% \mathrm{CL}$ and two to three times longer than paired dorsal hooks; marginal suckers lost between ML 100 and 200mm. Ventral membrane $\sim 40 \%$ club width, from hook V1 to tip; dorsal membrane $\sim 10 \%$ club width, flanking hooks D1-D5; dorsal keel $\sim 100 \%$ club width, from hook D4 to tip of club. Large ventral hooks with flat lateral 'blades' on claw, meeting distally at midline of claw.

Lower beaks sexually dimorphic (Table 19 , Figs $46 \mathrm{~A}-\mathrm{L}$ ). Hood in both sexes $\sim 30 \%$ crest length, apart from adult females. Male beak with moderately developed lateral wall fold anteriorly, reducing posteriorly to scarcely apparent thickening, disappearing altogether well before posterior lateral wall corner; crest barely thickened; lateral walls not indented below crest; rostrum slightly curved, length $\sim 80 \%$ wing length. Subadult male beak (LRL to $\sim 8.6 \mathrm{~mm}$, Figs $46 \mathrm{G}-\mathrm{l}$ ) with wide band of clear cartilage across wing from jaw angle to posterior margin; angle ridge extends over $50 \%$ of distance to lateral 


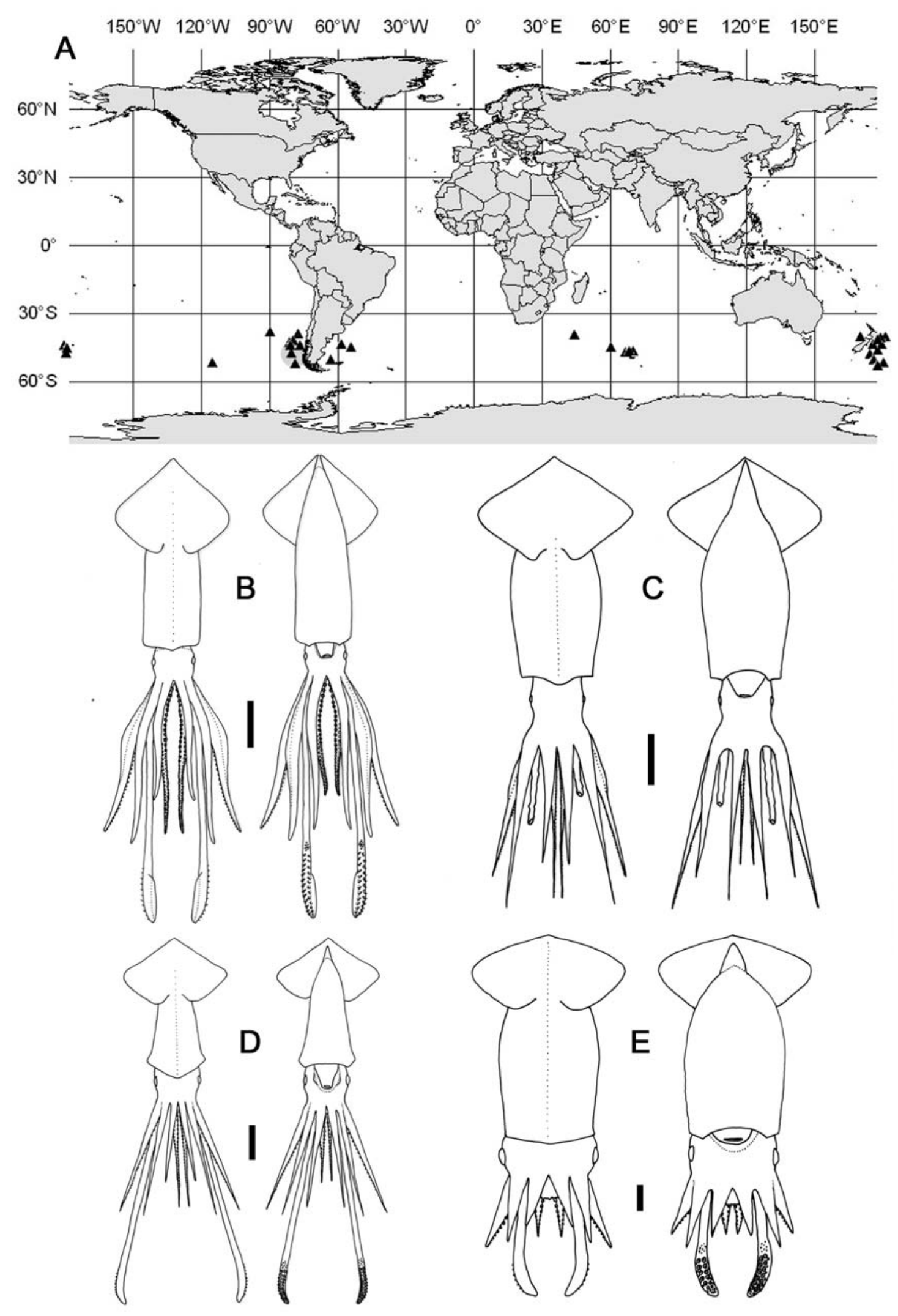

Fig. 44 - Onykia (Moroteuthopsis) ingens. A) distribution (grey circle indicates type locality); B) NIWA 32707,, , ML 213mm; C) NIWA TAN0101/111, spent $q$, ML 440mm; D) NMNZ M.183083, sex indet., ML 42mm; E) NMNZ M.279110, sex indet., ML $7 \mathrm{~mm}$. Scale bars = B) $50 \mathrm{~mm}$; C) $100 \mathrm{~mm}$; D) $10 \mathrm{~mm}$; E) $1 \mathrm{~mm}$. 
wall margin. Adult male beak ( $L R L \sim 8.6-9.8 \mathrm{~mm}$, Figs $46 \mathrm{~J}-\mathrm{L}$ ) often with pronounced excavation of jaw angle, which removes angle ridge. Female lower beaks (Figs 46A-F) darker and more robust, with well-developed, very thick lateral wall fold and ridge anteriorly, reducing to slight thickening of lateral wall posteriorly; crest very thick; lateral walls not indented below crest; rostrum slightly curved, length $\sim 60 \%$ wing length; hood often greatly eroded in adult females ( $L R L \sim 9.1-12.9 \mathrm{~mm}$, Figs 46D-F), extending $\sim 10 \%$ crest length; angle ridge extends $70-75 \%$ distance to lateral wall margin.

Radula (Fig. 47A) with long, triangular mesocone on rachidian; lateral cusps $\sim 30 \%$ mesocone height, slightly laterally directed. First lateral tooth bicuspid, slightly shorter than rachidian; inner cusp straight or somewhat medially to laterally directed; outer cusp $\sim 40 \%$ height of inner cusp, straight or slightly medially directed. Second lateral tooth simple, triangular, subequal to or slightly taller than rachidian. Marginal tooth slender, $\sim 150 \%$ height of rachidian. Palatine palp (Fig. 47B) with $65+$ small teeth, each $150-300 \%$ rachidian height, densely arranged in approximately transverse rows.

Gladius (Fig. 47E) attaining greatest width ( 11\% GL) at $~ 50 \%$ GL; vanes broaden and taper gradually between 15 and $75 \% \mathrm{GL}$; free rachis $\sim 15 \% \mathrm{GL}$; rostrum $\sim 13 \% \mathrm{GL}$, triangular to inverted T-shaped in cross-section and triangular in lateral profile.

Overall colour (preserved) dark reddish, darkest on dorsal surface of mantle and fins; head pale pinkish-red with small purple or red-brown chromatophores in several layers; arms dark reddish aborally, paler laterally and palest orally.

Smaller specimens (ML 31-105mm, Figs 44D, 47D) deviate from the above description as follows. Mantle conical; fin length $\sim 40 \% \mathrm{ML}$, width $70-85 \% \mathrm{ML}$; arms 60-95\% ML, formula ||$>|||>| V>\mid$ or $|I=|||>|V>|$, each with 50-80 suckers; tentacles 130-140\% ML; club length 35-40\% ML (20-30\% TnL); all manus hooks developing by $\mathrm{ML} 40 \mathrm{~mm}$; ventral membrane present along hooks $\mathrm{V} 1-\mathrm{V} 3$, up to $\sim 50 \%$ club width; overall colouration (preserved) dark pinkish-red, darkest dorsally on mantle and fins; dorsal and lateral epidermis of mantle, head and proximal $20 \%$ of arms may have silvery to coppery iridescence; one large dark brown chromatophore ('tail-spot') present posteriorly on either side of mantle, ventral to fins.

Paralarval to small juveniles (ML 7-30mm, Figs 44E, 47C) with spindle-shaped mantle, often broadly flared at anterior margin, then narrowing slightly before widening 
to $\sim 30 \% \mathrm{ML}$ at midpoint; skin rugose by ML $25 \mathrm{~mm}$; fins small, length $25-40 \% \mathrm{ML}$, width $45-75 \% \mathrm{ML}$, ovate or paddle-shaped; anterior and posterior margins convex; head rounded, width comparable to that of mantle; eyes slightly anteriorly directed; funnel groove rounded, edges not clearly defined; funnel component of locking apparatus 15\% ML; mantle component 20-25\% ML; arms 40-80\% ML, formula II>III>IV>I, each with 40-50 suckers; no keels apparent; tentacles 60-130\% ML, stalk thickness subequal to bases of Arms III and IV; club slightly expanded, 30-40\% ML, ( $30-50 \% \mathrm{TnL})$, with four longitudinal rows of suckers at ML $7-12 \mathrm{~mm}$; ventral hooks begin to develop from ML $\sim 15 \mathrm{~mm}$. Tentacular suckers roughly circular (Fig. 47C), with
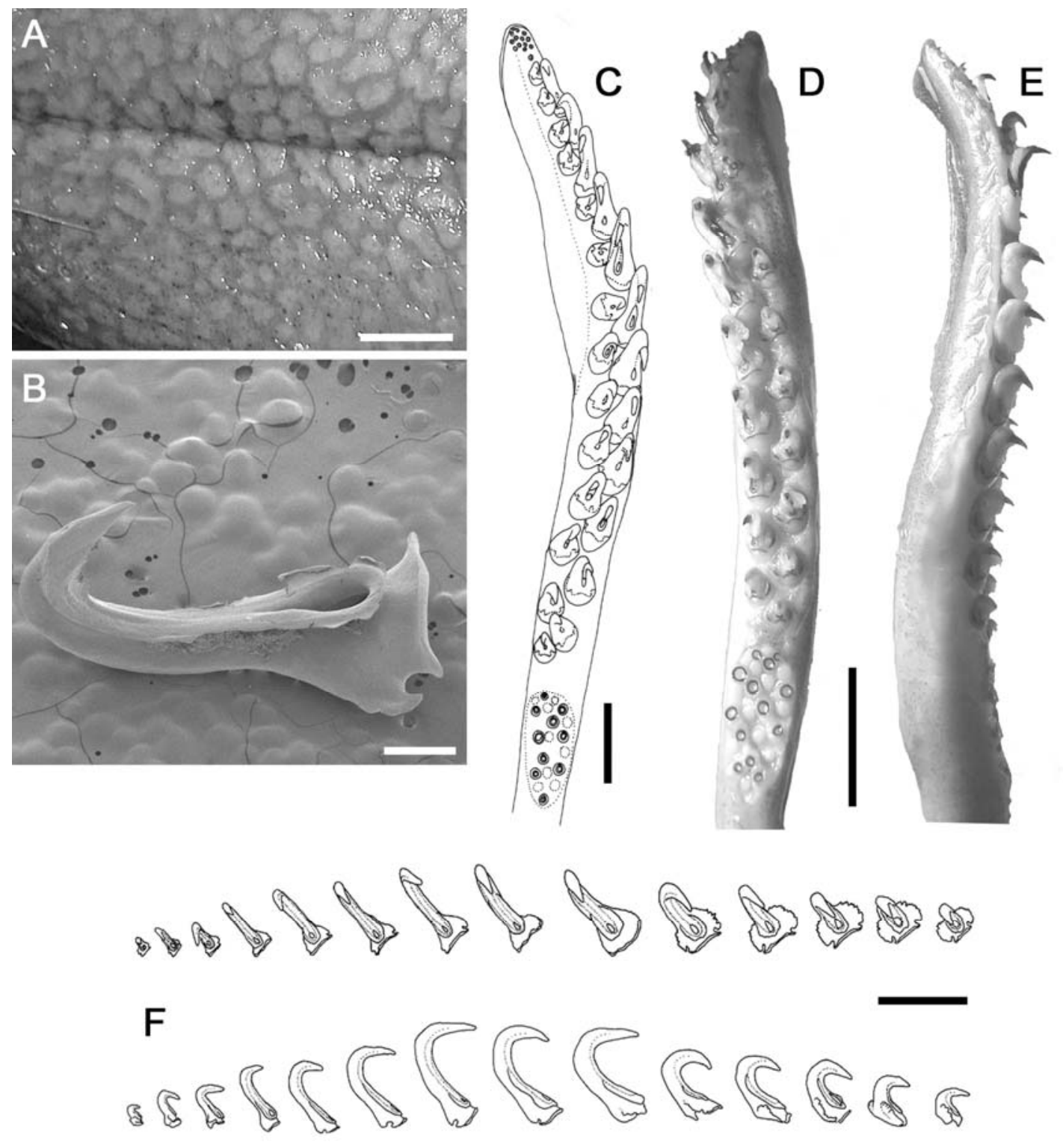

Fig. 45 - Onykia (Moroteuthopsis) ingens. A) NIWA 32789, ๆ, ML 370mm; B) NMNZ M.12952, sex indet., ML 90mm; C) NIWA 32795, ô, ML138mm; D, E) NIWA 32711, ô, ML 150mm; F) NMNZ M.118754, , ML 105mm. A) epidermis of mantle; B) left tentacular hook V7; C-E) tentacle clubs: (C) left, (D, E) right; F) left ventral tentacular hook series. Scale bars $=A, D-F) 10 \mathrm{~mm}$; B) $500 \mu \mathrm{m}$; C) $5 \mathrm{~mm}$. 
large, circular central aperture (diameter $\sim 40 \%$ sucker diameter; ten to 12 teeth present around inner margin, longest distally, wide and blunt proximally. Proximal pegs largest, in three concentric rows, with faces of innermost pegs circular or slightly ovoid; distal pegs round to polygonal, somewhat sparsely set in five or six concentric rows.

Remarks - Pfeffer (1908b) erected the genus Moroteuthopsis for 'M.' ingens, uncertain of its relationship to 'Moroteuthis' (= Onykia) robusta, given morphological differences between the gladius and skin sculpture in these two species. Pfeffer later (1912) suggested Moroteuthopsis as a subgenus of 'Moroteuthis,' should additional material prove separation of ' $M$. .' robusta and 'M.' ingens necessary. Morphologically, Ok. (M.) ingens shares many characters with the other Onykia species (see Table 11), including general body proportions, gladius shape and proportions, tentacular morphology, and epidermal sculpture (in all species where adults are known).

However, Ok. (M.) ingens lacks two characters common to adults of all other Onykia species - a Y-shaped ridge in the funnel groove, and deep lateral grooves on the claw of the ventral tentacular hooks. Wakabayashi et al. (2007), investigating the genetics
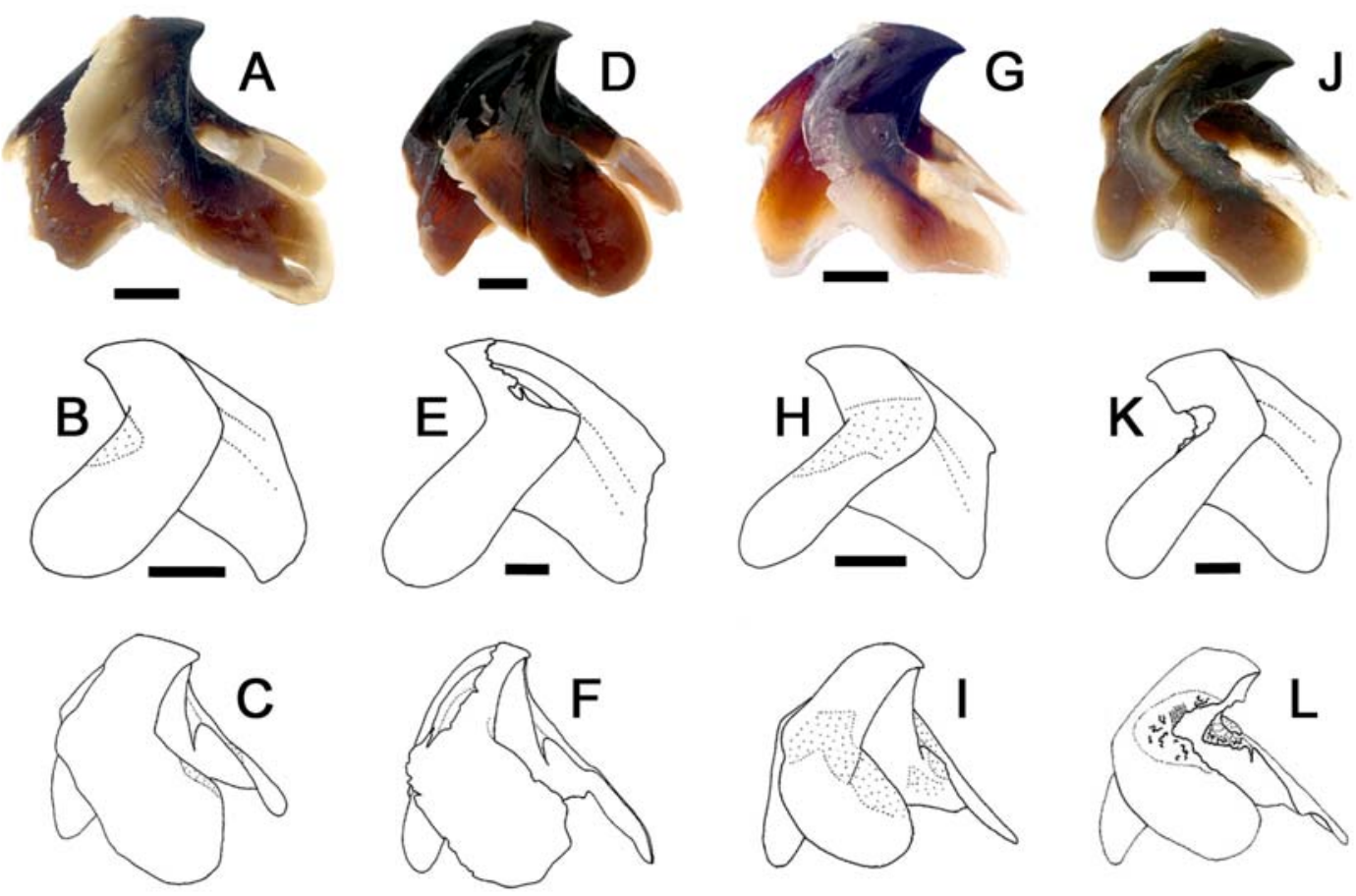

Fig. 46 - Onykia (Moroteuthopsis) ingens, lower beaks: $\mathrm{A}-\mathrm{C}$ ) maturing $\bigcirc$; D-F) adult क; G-I) maturing $\delta^{\prime}$; J-L) adult $\delta$. A, C) NIWA 32785, क, ML 370mm; B) NIWA 32710, ․, ML 315mm; D, F) NMNZ M.74296, , ML 487mm; E) NIWA 32773, 9 , ML 470mm;

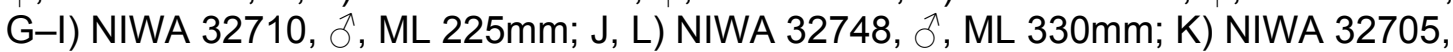
J, ML $270 \mathrm{~mm}$. A, D, G, J) left (photos by I. Anderson), (B, E, H, K) right, (C, F, I, L) left oblique profiles. Scale bars $=5 \mathrm{~mm}$. 
of several Onykia species, found that $O k .(M$.$) ingens was genetically much more$ distantly related to Ok. (Ok.) robusta, Ok. (Ok.) loennbergii and Ok. sp. than these latter three were to each other; in fact, Ok. (M.) ingens was related to the other three Onykia species only marginally more closely than Gen. nov. 1 knipovitchi was related to the three Onychoteuthis species examined in the same study. Bonnaud et al. (1998) also found the genetic relationships between Ok. (M.) ingens and Ok. (Ok.) robusta to be somewhat distant, although Ok. (M.) ingens and Ok. (Ok.) robsoni (not included by Wakabayashi et al. 2007) were closely related.

Ok. (M.) ingens is also the only Onykia species in which sexual dimorphism has been observed, although a similar sex-specific relationship between $M L$ and beak LRL may exist in Kondakovia longimana. The sculpture of the hook claw in K. longimana, with
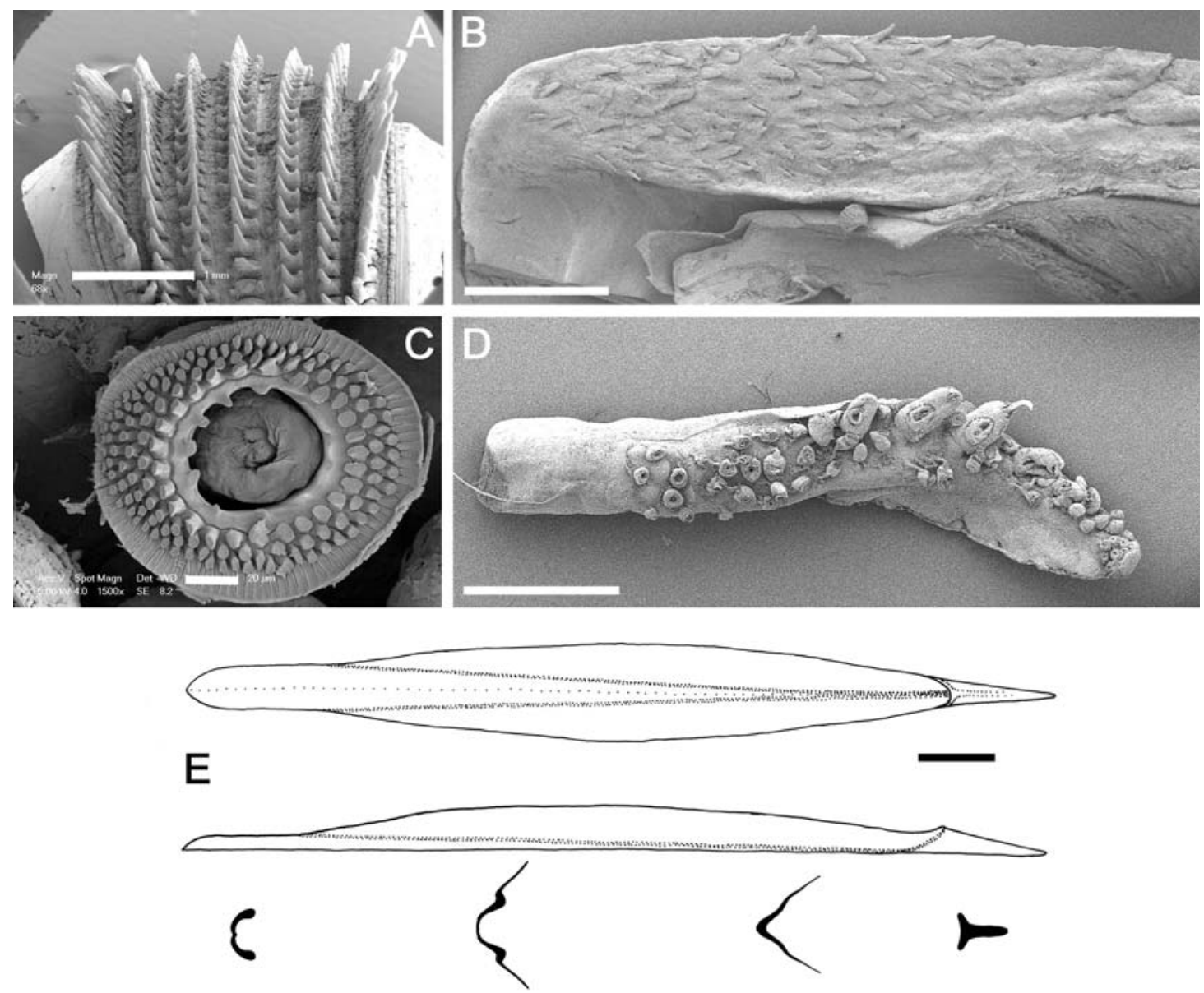

Fig. 47 - Onykia (Moroteuthopsis) ingens. A) NIWA 32773, , ML 470mm; B) NMNZ M.67830, ग, ML 100mm; C) NMNZ M.183111, sex indet., ML 12mm; D) NMNZ M.277905, sex indet., ML 34mm; E) NMNZ M.118754, , ML 105mm. A) radula, B) palatine palp, C) juvenile tentacular sucker, D) right tentacular club, E) gladius. Scale bars = A, B, D) $1 \mathrm{~mm}$; C) $20 \mu \mathrm{m}$; E) $10 \mathrm{~mm}$. 
lateral blades meeting at the distal midline of the claw (Fig. 54G), is also similar to that seen in Ok. (M.) ingens (Fig. 45B).

Ok. (M.) ingens manifests somewhat unusual morphological and genetic characters/states. In light of these differences and similarities outlined above, it is retained in the genus Onykia, but Pfeffer's subgenus Moroteuthopsis is revived. Further information on $O k$. (Ok.) aequatorialis, which appears to possess similar dermal sculpture (Fig. 38B) to Ok. (M.) ingens, and in which the presence/absence of the $\mathrm{Y}$-shaped ridge in the funnel groove and the lateral grooves on the tentacular hooks are unknown, may reveal that it too belongs in the subgenus Moroteuthopsis.

Where it occurs, Ok. (M.) ingens is a major prey item for sperm whales and other teuthophagous cetaceans (Gaskin \& Cawthorn 1967a, 1967b; Clarke \& Roper 1998, Gómez-Villota 2007). Bolstad (2006) reported that sexual dimorphism in beak morphology affected calculations of prey biomass, requiring that beaks be sexed and equations specific to each sex be used. The relationship between $L R L$ and $M L$ is linear for both sexes (Fig. 48A). For females, $(M L)=40.192(L R L)-12.174$; for males $(M L)=$ 32.023 $(L R L)+8.31$. The relationship between $L R L$ and weight is exponential for both sexes (Fig. 48B). For females, weight $=1.9976\left(\mathrm{LRL}^{2.8899}\right)$; for males weight $=$ 3.3332( $\left(\mathrm{LRL}^{2.4067}\right)$. The weight of females with $\mathrm{LRL}>6 \mathrm{~mm}(\mathrm{ML}>250 \mathrm{~mm})$ varies somewhat according to differing states of maturity. The greatest weight is attained at the height of maturity, prior to spawning, likely due to the development of nidamental glands and eggs.

More than 500 beaks attributable almost exclusively to adult Ok. (M.) ingens were found in the stomach of a single sperm whale. All beaks were of a size consistent with adult to spent OK. (M.) ingens (male LRLs 8.68-9.75, female LRLs 9.13-12.85mm), and nearly all possessed those character states (excavation of the rostrum in males and erosion of the hood in females) associated with mated individuals. Moreover, two buccal bulbs from this sample, one male and one female, were locked together in a mutual bite (Figs 48D, 48E).

Head-to-head mating in OK. (M.) ingens has been postulated by Nesis (1995) and Bolstad (2006), and Jackson \& Jackson (2004) reported the capture of a male-female pair of Ok. (M.) ingens locked together, beak to beak. The damage observed in both male and female beaks at maturity could result from beak-to-beak mating (the cartilaginous insert seen in subadult male beaks would be damaged, or completely 
excavated, by prolonged grinding against the hardened lower rostrum of the female). Nearly all individuals represented in this stomach content sample would therefore have mated, suggesting that the foraging sperm whale found a spawning or post-spawning aggregation of Ok. (M.) ingens, consuming many individuals and at least one pair mating in a head-to-head position.

The inverted orientation of the locked buccal bulbs (Figs 48D, 48E), and the primarily dorsal and dorso-lateral implantation of spermatophores reported by Jackson \& Jackson (2004), also suggest that one mating individual may commonly be positioned 'upside down' relative to the other (i.e. the female's Arms I positioned against the male's Arms IV and vice-versa, resulting in the implantation of spermatophores in the dorsal surface of the female's mantle, by way of the male's extraordinarily long penis, its total length up to $130 \% \mathrm{ML}$ (Fig. 48C). The hood damage observed in adult females is consistent with the position of the male's upper rostral tip during an inverted bite, while the female's longer upper rostrum passes over the male's lower hood, leaving it intact. The 'dislocation' observed frequently in adult male beaks (and infrequently in females), with the upper rostrum positioned outside the lower (Fig. $48 \mathrm{H}$ ), may result from the extended gape required to accommodate the other's beak during mating, preventing the beaks from returning to their natural position as the bite is released. In several adult males, a pronounced swelling in the lower mandibular muscle had developed, forming a hard, spherical, uniformly muscular knot of tissue directly below the lower hood (Figs 48F, 48G). Although the cause of this swelling is unknown, its position is consistent with the position of the female's upper rostrum during an inverted bite.

A number of studies have also been conducted on trophic interactions between $O k$. (M.) ingens and its prey (Jackson et al. 1998; Phillips et al. 2001, 2002, 2003a, 2003b).

The alimentary tracts of several adult to spent females examined were infested with nematodes, most often concentrated in the stomach caecum walls. 
Table 19. Sexually dimorphic character states in lower beaks of subadult and adult/spent Onykia (Moroteuthopsis) ingens (new comb.) (reproduced from Bolstad 2006).

\begin{tabular}{|c|c|c|c|c|}
\hline $\begin{array}{l}\text { Beak } \\
\text { character }\end{array}$ & Subadult female & Adult female & Subadult male & Adult male \\
\hline $\begin{array}{l}\text { Length vs. } \\
\text { depth in } \\
\text { lateral profile }\end{array}$ & Deeper than long & $\begin{array}{l}\text { Length and depth } \\
\text { subequal }\end{array}$ & Longer than deep & Longer than deep \\
\hline Pigmentation & $\begin{array}{l}\text { Nearly black } \\
\text { anteriorly, fading } \\
\text { rapidly to } \\
\text { brown/amber on } \\
\text { wing margins }\end{array}$ & $\begin{array}{l}\text { Nearly black, } \\
\text { fading to dark } \\
\text { brown only at wing } \\
\text { margins }\end{array}$ & $\begin{array}{l}\text { Nearly black } \\
\text { anteriorly, fading } \\
\text { rapidly to pale } \\
\text { amber on wing } \\
\text { margins }\end{array}$ & $\begin{array}{l}\text { Nearly black, } \\
\text { fading to dark } \\
\text { brown only at wing } \\
\text { margins }\end{array}$ \\
\hline Cartilage & $\begin{array}{l}\text { Narrow anterior } \\
\text { wing insertion }\end{array}$ & None & $\begin{array}{l}\text { Broad band across } \\
\text { wing from jaw } \\
\text { angle, wide } \\
\text { anterior insert into } \\
\text { wing }\end{array}$ & $\begin{array}{l}\text { Narrow anterior } \\
\text { wing insert; thin } \\
\text { veneer covers } \\
\text { broken / excavated } \\
\text { chitin surfaces }\end{array}$ \\
\hline $\begin{array}{l}\text { Relative } \\
\text { length of LRL }\end{array}$ & $50 \%$ wing length & $40 \%$ wing length & $\begin{array}{l}80-90 \% \text { wing } \\
\text { length }\end{array}$ & $\begin{array}{l}\text { Excavated; not } \\
\text { accurately } \\
\text { measurable }\end{array}$ \\
\hline $\begin{array}{l}\text { Shape of jaw } \\
\text { edge in lateral } \\
\text { profile }\end{array}$ & $\begin{array}{l}\text { Slightly curved } \\
\text { along entire length }\end{array}$ & $\begin{array}{l}\text { Proximal } 80 \% \\
\text { straight, distal } 20 \% \\
\text { curved }\end{array}$ & $\begin{array}{l}\text { Proximal } 60 \% \\
\text { straight, distal } 40 \% \\
\text { curved }\end{array}$ & $\begin{array}{l}\text { Remaining portion } \\
\text { slightly curved }\end{array}$ \\
\hline $\begin{array}{l}\text { Hood length } \\
\text { (\% of } \\
\text { baseline) }\end{array}$ & $30 \%$ baseline & $\begin{array}{l}\text { Greatly eroded; } \\
\text { remaining portion } \\
\sim 10 \% \text { baseline }\end{array}$ & $30 \%$ baseline & $40 \%$ baseline \\
\hline $\begin{array}{l}\text { Visible crest } \\
\text { length ( } \% \text { of } \\
\text { baseline) }\end{array}$ & $30 \%$ baseline & $50 \%$ baseline & $50 \%$ baseline & $40 \%$ baseline \\
\hline Wing width & $\begin{array}{l}\text { Uniform along wing } \\
\text { length }\end{array}$ & $\begin{array}{l}\text { Uniform along wing } \\
\text { length }\end{array}$ & $\begin{array}{l}\text { Narrowest at jaw } \\
\text { angle, widening } \\
\text { distally }\end{array}$ & $\begin{array}{l}\text { Narrowest at jaw } \\
\text { angle, widening } \\
\text { distally }\end{array}$ \\
\hline $\begin{array}{l}\text { Crest } \\
\text { thickening }\end{array}$ & Pronounced & Pronounced & Very slight & Very slight \\
\hline $\begin{array}{l}\text { Lateral wall } \\
\text { indented } \\
\text { below crest }\end{array}$ & No & No & Yes & Yes \\
\hline $\begin{array}{l}\text { Lateral wall } \\
\text { fold }\end{array}$ & $\begin{array}{l}\text { Semicircular in } \\
\text { cross-section; } \\
\text { somewhat } \\
\text { thickened }\end{array}$ & $\begin{array}{l}\text { Semicircular in } \\
\text { anterior cross- } \\
\text { section; thickened } \\
\text { into ridge }\end{array}$ & $\begin{array}{l}\text { Broad, low, only } \\
\text { slightly thickened }\end{array}$ & $\begin{array}{l}\text { Broad, low, only } \\
\text { slightly thickened }\end{array}$ \\
\hline
\end{tabular}



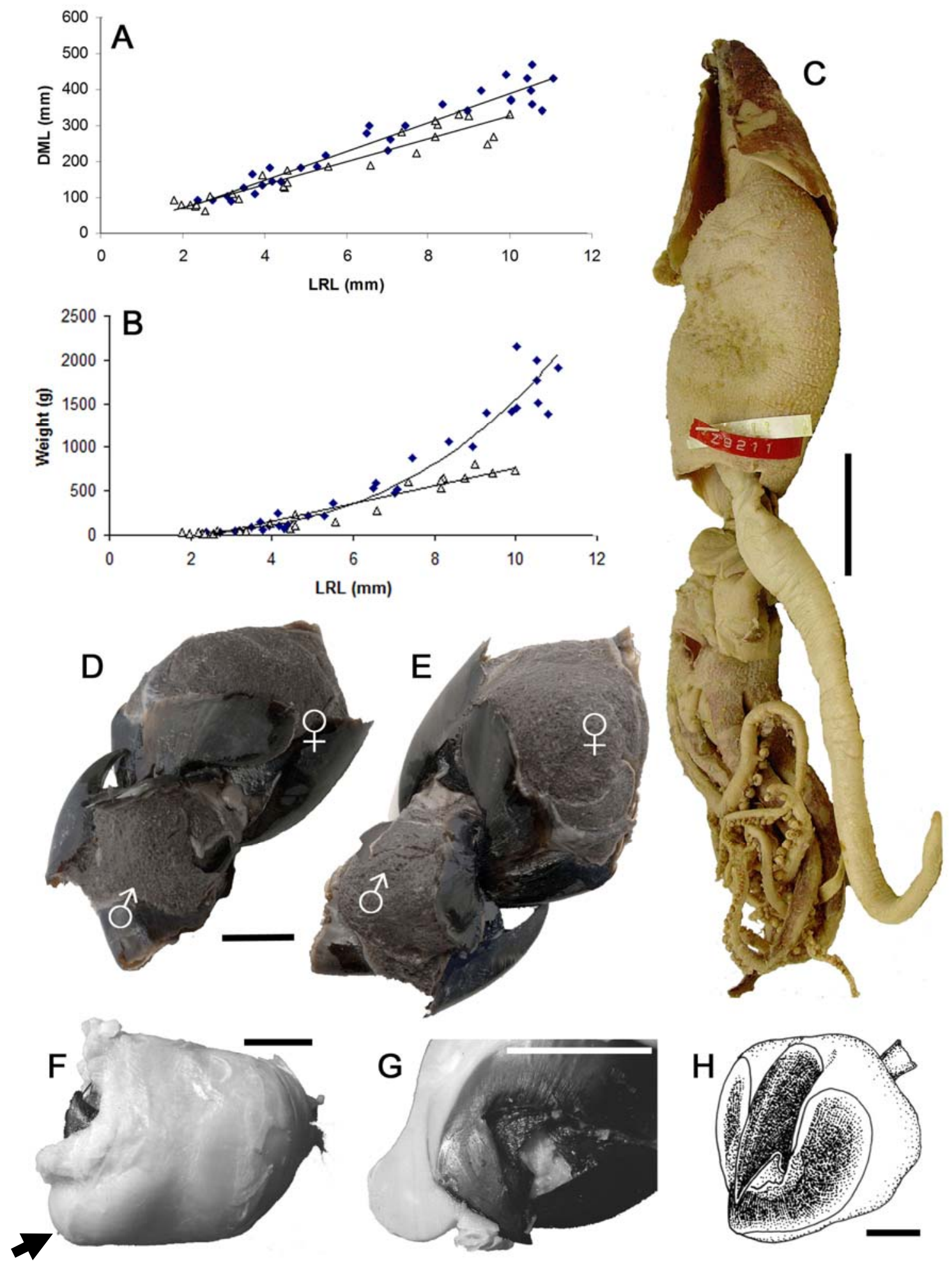

Fig. 48 - Onykia (Moroteuthopsis) ingens. A) relationship between DML and LRL; B) relationship between weight and LRL ( $\Delta$ male, $n=30$, female, $n=36$ ); C) NIWA 32776, $\widehat{O}$, ML 250mm; D, E) interlocked male and female beaks from stomach of sperm whale (photos by I. Anderson); F, G) NIWA 32746, ô, ML 330mm, swelling of lower mandibular muscle (observed in some males with excavated beaks): (F) left lateral view of intact swelling, $(G)$ right lateral view of cross-section through swelling; (H) NMNZ M.131972, §, ML 365mm, buccal bulb with 'dislocated' beaks inverted from natural position. Scale bars $=$ C) $50 \mathrm{~mm}$; D-H) $10 \mathrm{~mm}$. (Reproduced from Bolstad 2006.) 


\section{Ancistroteuthis Gray, 1849}

Diagnosis - Skin smooth; photophores absent from mantle cavity, may be present but indistinct on ventral surface of eye; six or seven secondary occipital folds present; epidermis of mantle smooth; funnel groove V-shaped; manus with 21-24 hooks, with asymmetrical bases, without spike, with deep lateral grooves on claw; terminal pad with 16-19 suckers; lateral profile of lower beak deeper than long; gladius without free rachis, not visible through dorsal mantle musculature; rostrum of gladius posteriorly directed, $\sim 12 \% \mathrm{GL}$.

Type species - Ancistroteuthis lichtensteinii Gray, 1849, by monotypy.

Remarks - This genus has been considered monotypic since its description, although several authors have suggested the presence of a second species in the central to southern Atlantic Ocean. Adam (1952) described but did not illustrate a specimen attributed to Ancistroteuthis from the coast of Angola, with somewhat different body proportions from A. lichtensteinii (e.g. fin width $90 \%$ ML, Arms $61-68 \% \mathrm{ML}$; see Appendix D). Kubodera et al. (1998) described 'A. lichtensteinii, Central Atlantic Form,' with broader (and not posteriorly attenuate) fins than $A$. lichtensteinii (sensu stricto). This Central Atlantic Form was additionally distinguished from A. lichtensteinii (s.s.) by eight or nine secondary occipital folds, 19 or 20 hooks on the manus, and 15-17 suckers on the terminal pad. Finally, Young et al. (2003a) recognised a form referred to as 'A. lichtensteinii Type B' from the southern central Atlantic $\left(21^{\circ} 35^{\prime} \mathrm{S}, 02^{\circ} 00^{\prime} \mathrm{W}\right)$, giving its most distinctive feature as rhomboidal rather than sagittate fins.

Within this study, material from the central and southern Atlantic was somewhat scarce, and included no Ancistroteuthis. Several Mediterranean Ancistroteuthis were observed with widely varying fin shapes, from broad and rhombic, without pronounced attenuation, to the more narrow, elongate and attenuate shape classically attributed to A. lichtensteinii. Consequently, additional material is required to determine the reliability of fin shape and degree of attenuation as character states for differentiating Ancistroteuthis species, should more than one species exist.

Three small Ancistroteuthis specimens (ML 35-41mm, Figs 52B, 52C, 52E) possessed extremely broad, rhombic fins, and may represent the other form observed by other authors; however, their location of capture is unknown but likely somewhere in the North Atlantic, since the research vessel that collected them was near the 
Shetland Islands several days earlier. These specimens are damaged and little external morphological detail is discernible apart from the overall body proportions; the gladius and radula are illustrated in Figs $52 \mathrm{C}$ and 52E, respectively.

It is possible that a second, broad-finned species of Ancistroteuthis is present in the central to southern Atlantic, but due to lack of material it cannot be more fully diagnosed or described here, and it is not known whether the three small North Atlantic specimens are affiliated with it, given their probably disjunct distributions with the other specimens observed herein (from the Mediterranean and north central Atlantic).

Kubodera et al. (1998) mentioned two additional taxa that they considered regional morphological variants of $A$. lichtensteinii. These are now recognised as separate species belonging to the genus Notonykia Nesis, Roeleveld \& Nikitina, 1998: 'A. lichtensteinii, South Atlantic Form,' as described by Kubodera et al. (1998), was attributed by Nesis et al. (1998) to N. africanae Nesis et al., 1998; specimens examined by Kubodera et al. (1998) and attributed to 'A. lichtensteinii, South Pacific form' have been re-examined and described as N. nesisi Bolstad, 2007.

The genera Ancistroteuthis and Notonykia are similar in many respects, but the lower beak of Ancistroteuthis is distinctly deeper than long; the gladius is unique amongst the Onychoteuthidae in lacking a free rachis and in the form of the vanes (merged with the ribs into a single lateral thickening); and the arms in Ancistroteuthis are very rarely shorter than $50 \% \mathrm{ML}$, while those in the Notonykia species are rarely longer than $45 \%$ ML. Moreover, the number of hooks in A. lichtensteinii is generally $21-24$, while 16-18 (rarely 19) hooks are found in Notonykia; and the number of suckers on the terminal pad in Ancistroteuthis falls within the more common onychoteuthid range (16-19), while neither Notonykia species has been observed with fewer than 30 terminal pad suckers. 


\section{Ancistroteuthis lichtensteinii (Férussac in Férussac \& d'Orbigny, 1835) (Table}

20, Figs 49-51, 52A, 52D)

Onychoteuthis lichtensteinii Férussac, 1835 (in Férussac \& d'Orbigny (1835): PI. 8, (18391842): PI. 14 Figs 1-3, (1848): 334, 335; Vérany (1851): 78, PI. 29 (fide Pfeffer 1912]); Lu et al. (1995): 319.

Ancistroteuthis lichtensteinii (Férussac, 1835) - Gray (1849): 55; Pfeffer (1912): 71-76, Pls 9 , 10; Toll (1982) (partim): 58, 59, USNM 730595; Guerra Sierra (1992): 139-141, Fig. 43; Kubodera et al. (1998) (partim): 280, 281, Fig. 1; Sweeney \& Roper (1998): 572; Okutani (2005): 155.

Onychoteuthis hamatus Risso, 1854: 35, pl. 16 Figs 1, 2 - Adam (1942): 15.

Ancistroteuthis lichtensteini [sic] (Férussac, 1835); Naef (1921/1923): 306-313, Figs 154b, 159-162; Clarke (1966): 148, 149; Zuev \& Nesis (1971): 184, Fig. 55; Okutani (1978): 146, 147; Roper et al. (1984): 127; Nesis (1987): 192, Fig. 48I; Sweeney et al. (1992): 133; Filippova et al. (1997): 182, Fig. 108.

Not Ancistroteuthis lichtensteinii (Férussac, 1835) - Voss (1956): 130-132, Table 14, Fig. 13a (see Remarks).

Not Ancistroteuthis lichtensteinii (Férussac, 1835) - Rancurel (1970): 29-33, Table 8, Figs 2022 (see Remarks).

Not Ancistroteuthis lichtensteinii (Férussac, 1835) - Toll (1982) (partim): 58, 59, PI. 5 Fig. D, USNM 730690, 730691, 730692 (= Ok. (Ok.) robsoni); Hess (1987): 292, 293, 300, 301, Fig. 55 (= Ok. (Ok.) robsoni).

Not Ancistroteuthis lichtensteinii (Férussac, 1835), South Atlantic Form - Kubodera et al. (1998): 281, 282 (= Notonykia africanae).

Not Ancistroteuthis lichtensteinii (Férussac, 1835), Pacific Form — Kubodera et al. (1998): 282 (= Notonykia nesisi).

Type material examined - MNHN 3.1.620, Holotype, $\odot$, ML 147mm, Nice, NFD; MNHN 3.1.621, gladius of MNHN 3.1.620, Nice.

Additional material examined (21 specimens) - ZMBN 73696, sex indet., ML 33mm, $47^{\circ} 49.50^{\prime} \mathrm{N}, 29^{\circ} 12.66^{\prime} \mathrm{W}, 825-600 \mathrm{~m}, 25 / 06 / 2004$, Mar-Eco Stn 26-355-1106; ZMBN 77742, sex indet., $M L 36 \mathrm{~mm}, 47^{\circ} 48.62^{\prime} \mathrm{N}, 29^{\circ} 11.25^{\prime} \mathrm{W}, 603-250 \mathrm{~m}$ over $3095 \mathrm{~m}$, 25/06/2004, Mar-Eco Stn 26-355-1107; ZMBN 73698, ô, ML 76mm, sex indet., ML $65 \mathrm{~mm}, 42^{\circ} 49.14^{\prime} \mathrm{N}, 27^{\circ} 56.98^{\prime} \mathrm{W}, 800-0 \mathrm{~m}, 27 / 06 / 2004$, Mar-Eco Stn 28-357-1116; ZMBN 77741, 2 sex indet., ML 44, 25mm, 42 $37.99^{\prime} \mathrm{N} 30^{\circ} 10.00^{\prime} \mathrm{W}, 800-50 \mathrm{~m}$ over $2175 \mathrm{~m}$, 29/06/2004, Mar-Eco Stn 32-361-1127; ZMBN 73722, 3 sex indet., ML 22, 15, 14mm, $41^{\circ} 41.06^{\prime} \mathrm{N}, 2^{\circ} 59.966^{\prime} \mathrm{W}, 30 / 06 / 2004$, Mar-Eco Stn 34-363-1137; USNM 730595, 3 sex indet., ML $110-75 \mathrm{~mm}, 41^{\circ} 41^{\prime} \mathrm{N}, 02^{\circ} 48^{\prime} \mathrm{E}$, -/05/1979, NFD; ZMBN 73800,, , ML 72mm, $41^{\circ} 33.59^{\prime} \mathrm{N}, 29^{\circ} 55.44$ 'W, 30/06/2004, Mar-Eco Stn 34-364; ZMBN 77740, ðَ, ML 42mm, $41^{\circ} 31.02^{\prime} \mathrm{N} 29^{\circ} 54.51^{\prime} \mathrm{W}, 1800-800 \mathrm{~m}$ over 2230m, 30/06/2004, Mar-Eco Stn 34-3641139; ZMBN 73699, 2 sex indet., ML 26, $14 \mathrm{~mm}, 41^{\circ} 28.25^{\prime} \mathrm{N}, 29^{\circ} 53.74 ' \mathrm{~W}, 800-0 \mathrm{~m}$, 
30/06/2004 Mar-Eco Stn 34-364-1140; ZMBN 73799, ô, ML 70mm, 41²8.25'N,

2953.74'W, 800-0m, 30/06/2004, Mar-Eco Stn 34-364-1140; ZMBN 73697, 4 sex indet., ML 52-17mm, 26⒋74' N, 2909.96'W, 250-0m, 25/06/2004, Mar-Eco Stn 26-355-1108. Unlocalised material examined (27 specimens) - ; ZMH WH1982/99 (3ㅇ), ML 161, 132, 89mm, NFD; ZMBN 2509 (2), ML 161, 100mm, Naples, NFD; ZMBN 2468, , ML 148mm, Naples, NFD; MNHN 618, + , ML 130, Mediterranean, 1841, coll. M. Coste, NFD; MNHN 3305, sex indet., ML 121mm, Corse, -/10/1991, NFD; MNHN 3.1.622, + , ML 112mm, Mediterranean, 1841, coll. M. Coste, NFD; MNHN 3.1.623, sex indet., ML 102mm, Nice, NFD; BMNH 20070468, + , ML 95mm, 'Discovery' Expedition Stn 6190, 120-0m, 18/11/1966, EMWT, NFD; ZMH WH1982/54, 2 9 , ML 78, 78mm, ơ, ML 66mm, NFD; ZMH WH 1982/64, 2ð, ML 74, 66mm, NFD; BMNH 20070466 (11 sex indet.), ML 47-11mm, 15/05/1967, Boris Trawl, NFD; USNM unaccessioned, sex indet., ML 16mm, Amsterdam Mid-Atlantic Plankton Expedition Stn 16-6.

Additional Ancistroteuthis material examined (3 sp. indet.) - BMNH 20070490, 3 sex indet., ML 41, 35mm, 35mm [mantle only], 500m, 11/03/1982, RMT100/Otter, RRS Frederick Russell Stn FR 82/74, NFD [but RRS Frederick Russell sampled near the Shetlands on 06/03/1982].

Distribution (Fig. 49A) - North Atlantic, primarily $20-60^{\circ} \mathrm{N}$, including Mediterranean Sea, $800-0 \mathrm{~m}$.

Diagnosis - As for genus.

Description (Figs 49B-51E) - Mantle muscular, conical, width 17-21-27\% ML, becoming very slender posteriorly, ventral to fins. Gladius not visible dorsally; visible ventrally through posterior $15 \% \mathrm{ML}$. Fins sagittate, length $53-61-67 \% \mathrm{ML}$, width $53-$ $61-68 \% \mathrm{ML}$; posterior margins straight to slightly concave; anterior margins straight to slightly convex. Anterior lobes not pronounced; semicircular indentation in mantle visible between fin insertions.

Head boxy, length $\sim 23 \% \mathrm{ML}$, width 12-17-21\% ML, depth 15\% ML. Occipital region with six or seven secondary folds; ventral-most primary fold borders on funnel groove; primary and ventral-most secondary occipital folds joined posteriorly by low occipital membrane. Funnel groove well-defined V-shape, extending to level below midpoint of eye aperture; funnel stout, aperture at level below posterior margin of eye aperture. Funnel component of locking apparatus $\sim 11 \% \mathrm{ML}$, mantle component $\sim 22 \% \mathrm{ML}$. 
Table 20. Measures (mm) and counts of Ancistroteuthis lichtensteinii (Ferussac, 1835) (arm and tentacle measures recorded from more complete side of specimen, indicated $\mathrm{R}$ or $\mathrm{L}$ ).

\begin{tabular}{|c|c|c|c|c|c|c|c|c|c|c|c|c|}
\hline Specimen ID & $\begin{array}{r}\text { MNHN } \\
3.1 .620 \\
\end{array}$ & $\begin{array}{c}\text { ZMH } \\
\text { WH1982/99 }\end{array}$ & $\begin{array}{c}\text { ZMBN } \\
2468\end{array}$ & $\begin{array}{c}\text { BMNH } \\
20070468\end{array}$ & $\begin{array}{c}\text { ZMH } \\
\text { wH1982/54 }\end{array}$ & $\begin{array}{c}\text { ZMH } \\
\text { WH1982/54 }\end{array}$ & $\begin{array}{c}\text { ZMH } \\
\text { WH1982/64 }\end{array}$ & $\begin{array}{c}\text { ZMH } \\
\text { WH1982/54 }\end{array}$ & $\begin{array}{c}\text { ZMH } \\
\text { WH1982/64 }\end{array}$ & $\begin{array}{c}\text { BMNH } \\
20070466\end{array}$ & \multicolumn{2}{|c|}{ Mean Indices } \\
\hline Type Status & Holotype & None & None & None & None & None & None & None & None & None & & \\
\hline $\mathrm{ML}$ & 147 & 161 & 148 & 95 & 78 & 78 & 74 & 66 & 66 & 47 & \multicolumn{2}{|c|}{ (ML 66-147) } \\
\hline Sex & $\mathrm{F}$ & $\mathrm{F}$ & $\mathrm{F}$ & $\mathrm{F}$ & $\mathrm{F}$ & $\mathrm{F}$ & $M$ & $M$ & $M$ & Indet. & & \\
\hline MW & 25 & 27 & 28 & 18 & 18 & 15 & 15 & 14 & 18 & 11 & MWI & 21 \\
\hline HL & $35^{\star}$ & 30 & 32 & 21 & 14 & 16 & 16 & 15 & 15 & 10 & HLI & 23 \\
\hline HW & 23 & 20 & 22 & 17 & 10 & 10 & 14 & 10 & 14 & 11 & $\mathrm{HWI}$ & 17 \\
\hline FL & 92 & 99 & 90 & 60 & 52 & 48 & 40 & 37 & 37 & 25 & FLI & 61 \\
\hline FW & 80 & 85 & 79 & 59 & 46 & 53 & 50 & 44 & 45 & 35 & FWI & 61 \\
\hline Arms I & $65 \mathrm{~L}$ & $73 \mathrm{~L}$ & $80 \mathrm{R}$ & $40 R$ & $26 \mathrm{~L}^{*}$ & $34 \mathrm{~L}$ & $34 \mathrm{R}$ & $28 \mathrm{R}$ & 28 & $18 \mathrm{~L}$ & A1I & 46 \\
\hline Arms II & $82 \mathrm{~L}$ & $90 \mathrm{~L}$ & $103 R$ & $50 R$ & $40 \mathrm{~L}$ & $44 \mathrm{~L}$ & $42 R$ & $35 R$ & 38 & $23 \mathrm{~L}$ & A2I & 60 \\
\hline Arms III & $80 \mathrm{~L}$ & $78 \mathrm{~L}$ & $100 R$ & $48 R$ & $39 \mathrm{~L}$ & $43 \mathrm{~L}$ & $40 R$ & $35 R$ & 33 & $21 \mathrm{~L}$ & A3I & 58 \\
\hline Arms IV & $85 \mathrm{~L}$ & $81 \mathrm{~L}$ & $87 \mathrm{R}$ & $50 R$ & $36 \mathrm{~L}$ & $42 \mathrm{~L}$ & $33 R$ & $32 \mathrm{R}$ & 35 & $21 \mathrm{~L}$ & A4I & 54 \\
\hline TnL & $218 \mathrm{~L}$ & $200 \mathrm{~L}$ & $204 R$ & $100 \mathrm{R}$ & 97L & $83 \mathrm{~L}$ & $72 \mathrm{R}$ & $72 \mathrm{R}$ & 61 & $35 \mathrm{~L}$ & TnLI & 128 \\
\hline $\mathrm{CL}$ & $52 \mathrm{~L}$ & $54 \mathrm{~L}$ & $54 \mathrm{R}$ & $34 \mathrm{R}$ & $32 \mathrm{~L}$ & $25 \mathrm{~L}$ & $24 R$ & $25 R$ & 25 & $15 \mathrm{~L}$ & CLI & 37 \\
\hline CS & 10 & 9 & 9 & 9 & 9 & 10 & 11 & 9 & 8 & 9 & & \\
\hline MH & 24 & 21 & 21 & 21 & 21 & 22 & 22 & 22 & 21 & 21 & & \\
\hline MS & 0 & 0 & 0 & 0 & V9, 10 & 0 & $\mathrm{~V} 10,11$ & $\mathrm{~V} 10,11$ & v9, 10 & $\begin{array}{c}\text { D3, } 11,12, \\
\text { V7-10 }\end{array}$ & & \\
\hline TPS & 16 & 16 & 17 & 17 & 17 & 17 & 17 & 17 & 17 & 19 & & \\
\hline
\end{tabular}

Arms $42-46,-57_{I I-I V}-70 \% \mathrm{ML}$, formula $|I=| I|=| \mathrm{V}>\mid$ or IV $>||=|||>|$, each with $90-110$ suckers (ML 66-161mm), bordered on each side of oral face by trabeculate membranes. Keels present on Arms I-III; that on Arms I 15\% arm width along entire arm length; that on Arms II $\sim 25 \%$ arm depth at base, expanding to $\sim 60 \%$ arm depth at $30 \%$ arm length, narrowing thereafter to $20 \%$ arm depth; that on Arms III broadest of all, fleshy, attaining $150 \%$ arm depth at $40 \%$ arm length, narrowing thereafter to arm tip; lateral membrane on Arms IV $150 \%$ arm width at base, narrowing thereafter to $75 \%$ arm width.

Tentacles $92-128-148 \% \mathrm{ML}$, with low keel, $10 \%$ stalk depth, along aboral surface of stalk from base to proximal portion of club. Club (Figs 49C, 49D, 50C) 32-37-41\% ML (24-41\% TnL), only slightly expanded. Carpus a well-defined oval with eight to 11 suckers; manus with 21-24 hooks (usually 21 or 22); terminal pad with 16-19 suckers. Hooks (Figs 50D, 51A, 51B) with slightly asymmetrical bases; each side of claw with deep lateral groove. All ventral hooks larger than corresponding dorsal hooks; hooks V1-V3 increase gradually in size, with V4 suddenly larger; V6 or V7 largest of all (Fig. $51 \mathrm{~A}$ ), four to fives times longer than paired dorsal hook and $15-20 \% \mathrm{CL}$. Distal-most dorsal and ventral hooks may still be forming at ML $161 \mathrm{~mm}$; marginal suckers flank two distal-most ventral hooks through $\mathrm{ML} \sim 80 \mathrm{~mm}$. Ventral membrane $\sim 40 \%$ club width, present along entire club margin, with trabeculae flanking hooks V6-V11; dorsal membrane, $\sim 10 \%$ club width, present along hooks D1-D3; dorsal keel $\sim 100 \%$ club width, present from D3 to tip of club.

Lateral profile of lower beak (Figs 50E-H, ML 132mm) deeper than long, appearing to 'lean backwards' when set on baseline, with posterior edge of hood well above crest; 
hood length $\sim 50 \%$ crest length, and hood and crest together $\sim 65 \%$ of baseline; jaw angle obtuse and curved, slightly obscured from the side by indistinct wing fold; jaw edge nearly $65 \%$ wing length. Crest narrow, with lateral wall indented below; posterior portion of crest with several small diagonal ridges nearly parallel to slope of lateral wall fold; hood and wings slender; wings narrowest at jaw angle; shoulder ridge distinct, slightly rounded; lower rostral edge curved and produced into a distinct, sharp rostral

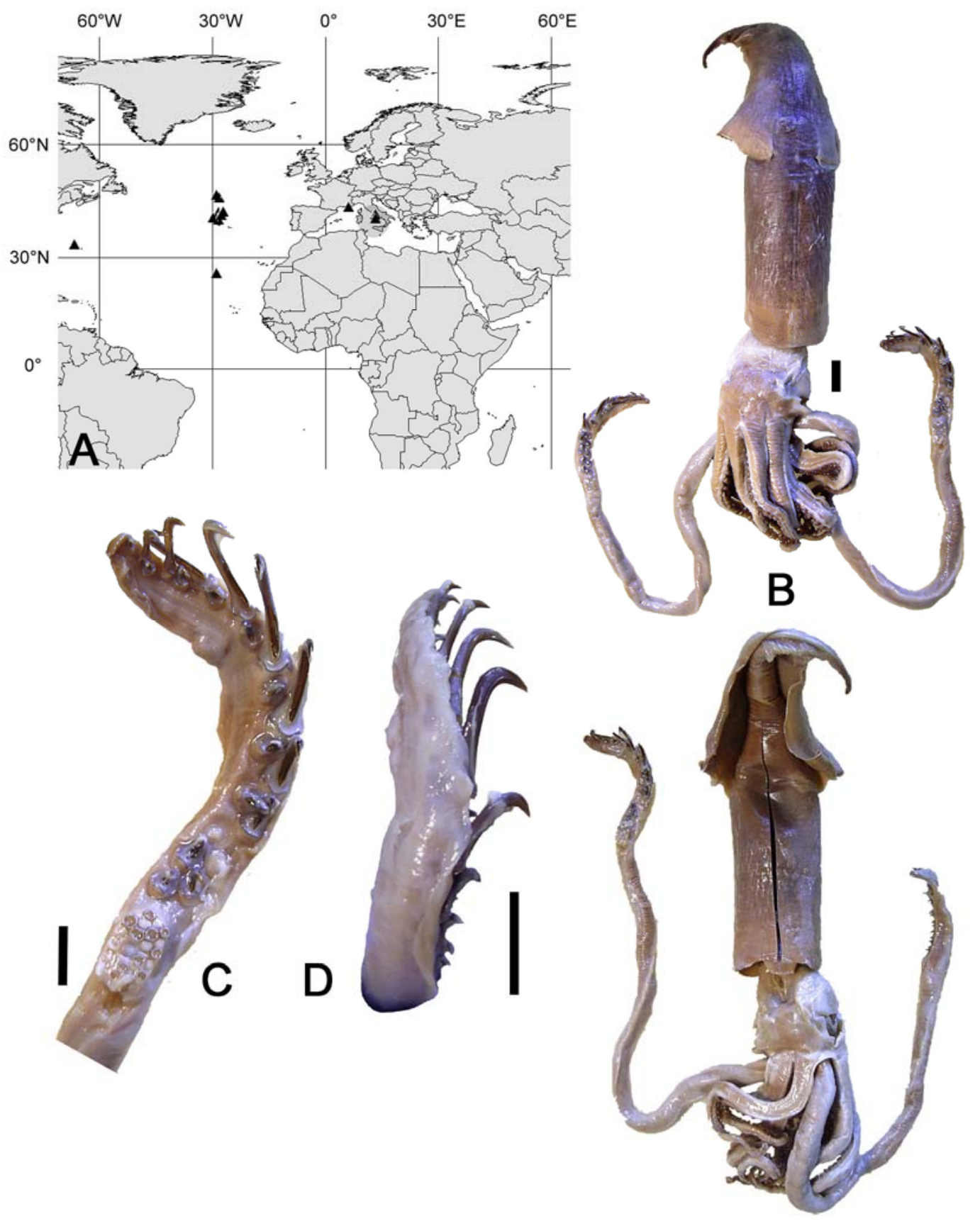

Fig. 49 - Ancistroteuthis lichtensteinii. A) distribution (grey circle indicates type locality); B-D) MNHN 3.2.646, holotype, $\odot$, ML 147mm: (B) whole animal, (C) left tentacle club, oral view, (D) left tentacle club, ventral view. Scale bars $=B, D) 10 \mathrm{~mm}$; C) $5 \mathrm{~mm}$. 


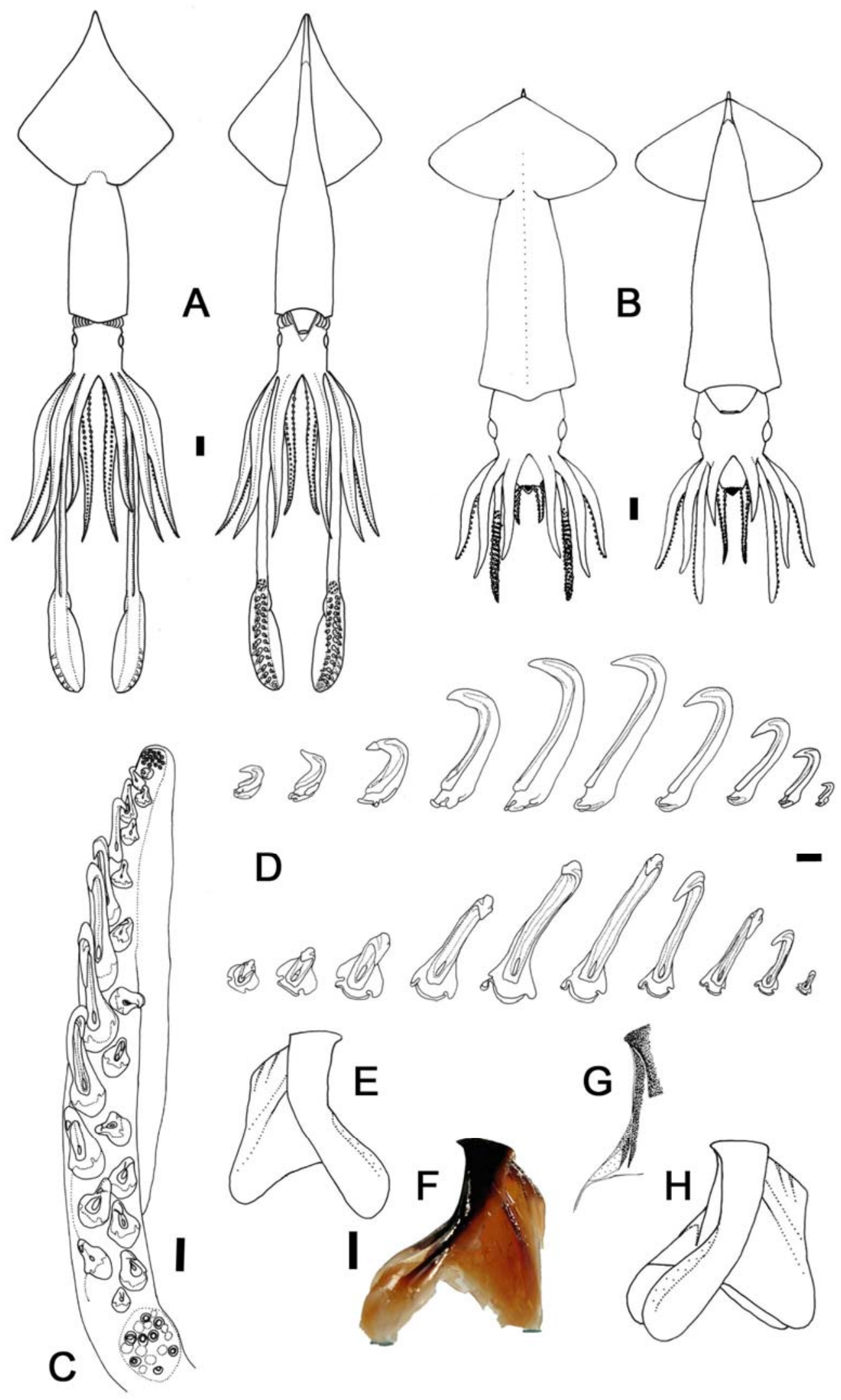

Fig. 50 - Ancistroteuthis lichtensteinii. A, C) ZMH WH1982/64, Ô, ML 66mm; B) USNM unaccessioned, sex indet., ML 16mm; D-H) ZMH WH1982/99, , ML 132mm. $A, B)$ whole animal; C) right tentacle club; D) right ventral tentacular hook series; E-H) lower beak: $(E)$ left, $(F)$ right profiles, $(G)$ angle point and 'false' angle point, $(H)$ right oblique profile. Scale bars = A) $10 \mathrm{~mm}$; B-F) $1 \mathrm{~mm}$. 
tip; angle ridge poorly developed, marked by very narrow wedge of dark pigmentation; second narrow wedge, nearly identical, but not raised, present just anterior to first, delineating the cartilage section of the shoulder and giving the impression of two angle points (Fig. 50G); lateral wall fold and ridge prominent, traversing lateral wall in steep, slightly convex downward slope from tip of rostrum to posterior corner of lateral wall.

Radula (Fig. 51C) with tricuspid rachidian, with narrow, triangular, distally rounded mesocone; lateral cusps curved, inward-hooking, 25-30\% total rachidian height. First lateral tooth bicuspid, $\sim 75 \%$ height of rachidian, with broad, triangular, slightly medially directed inner cusp and low, rounded, variably distinct outer cusp. Second lateral tooth simple, slightly curved, $\sim 150 \%$ height of rachidian and first lateral. Marginal tooth simple, slightly curved, $\sim 200 \%$ height of rachidian and first lateral. Palatine palp (Fig.
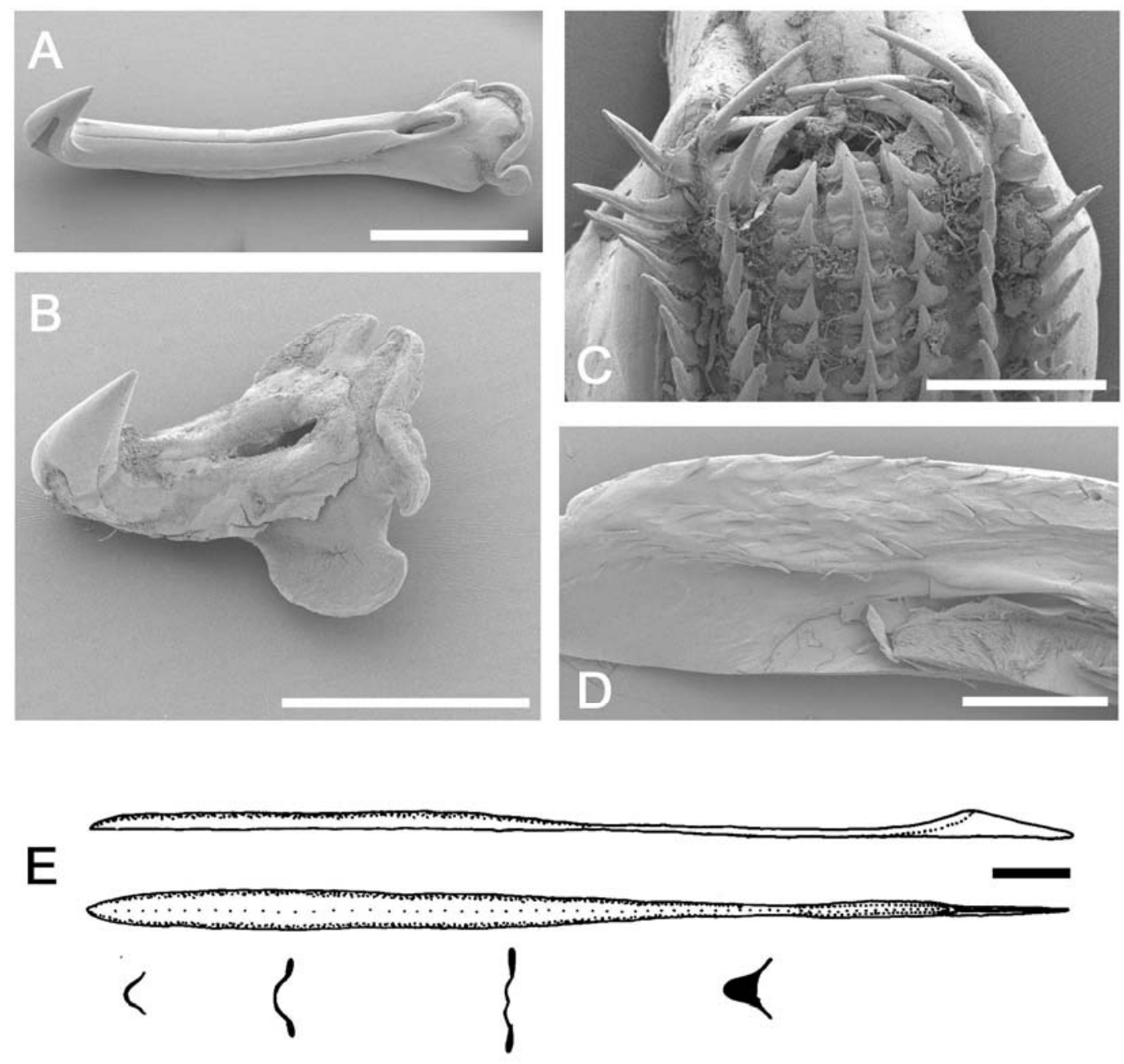

Fig. 51 - Ancistroteuthis lichtensteinii. A-D) ZMH WH1982/99, q, ML 132mm; E) ZMH WH1982/99, , , ML 132mm. A, B) right tentacular hooks: (A) V6, (B) D6; (C) radula; D) right palatine palp; E) gladius. Scale bars $=A) 2 \mathrm{~mm} ; B$, D) $1 \mathrm{~mm}$; C) $500 \mu \mathrm{m}$; E) $10 \mathrm{~mm}$. 
51D) slender, its greatest width $\sim 25 \%$ of its length; with $\sim 35$ slender, variably sized, sparsely distributed triangular teeth, each $100-200 \%$ rachidian height.

Gladius (Fig. 51E) with greatest width ( 4\% GL), attained within anterior 33\% GL. No free rachis; vanes and ribs merged into single lateral thickening, beginning at anterior margin and merged into dorsal keel by $\sim 70 \% \mathrm{GL}$. Rostrum $\sim 12 \% \mathrm{GL}$, triangular in
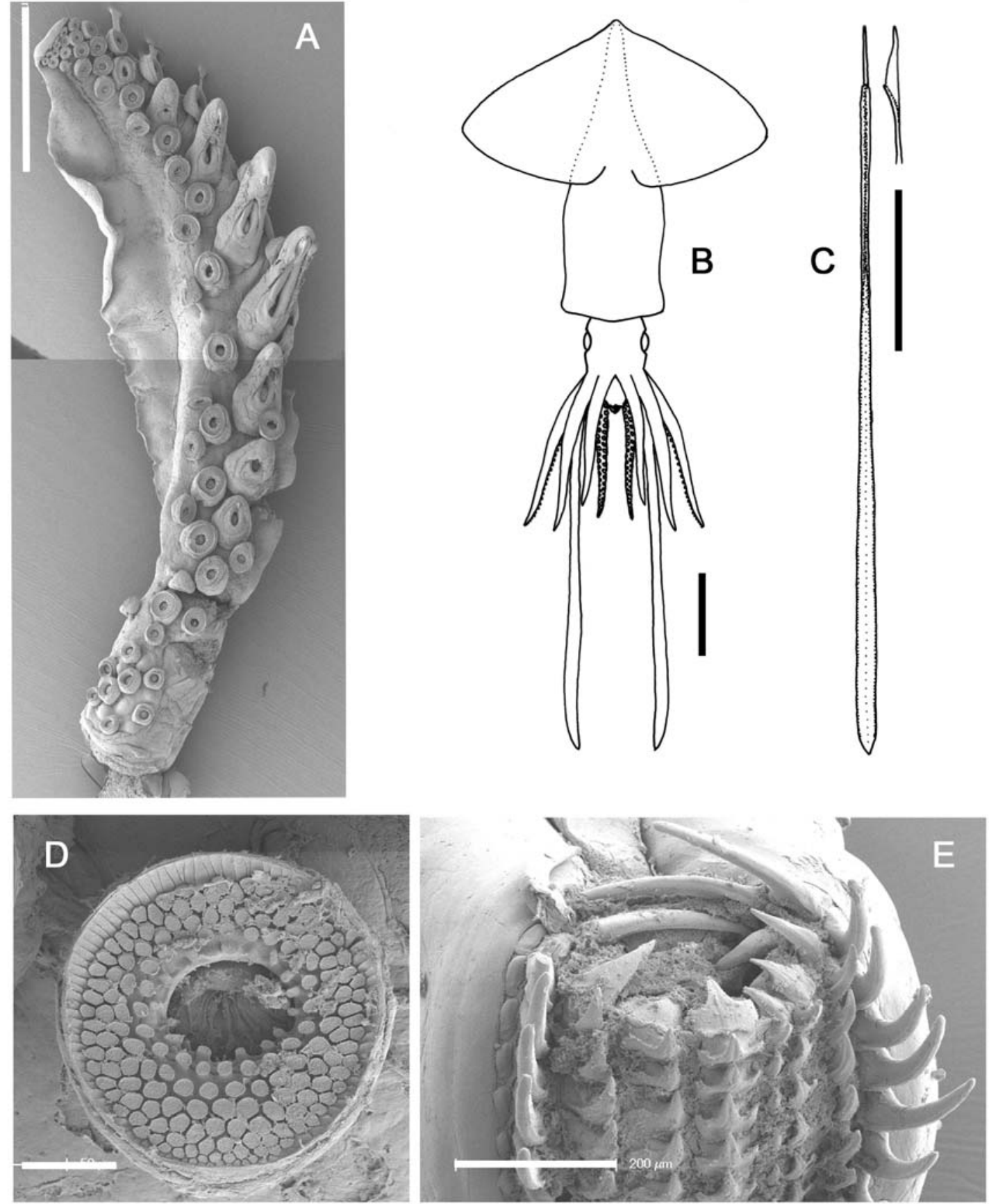

Fig. 52 - Ancistroteuthis spp. A, D) A. cf. lichtensteinii, USNM Unaccessioned, sex indet., ML 16mm; B, C, E) BMNH 20070547, Ancistroteuthis sp., sex indet., ML 35mm. A) left tentacle club; B) whole animal; C) gladius; D) juvenile marginal tentacular sucker; E) radula. Scale bars = A) $1 \mathrm{~mm}$; B, C) $10 \mathrm{~mm}$; D) $50 \mu \mathrm{m}$; E) $200 \mu \mathrm{m}$. 
lateral profile; elongate isosceles triangle in anterior cross-section; elongate ovoid in posterior cross-section; ventral edge of rostrum thickens from narrow spine anteriorly to broad, rounded ridge posteriorly.

Dorsal surface of mantle (preserved) iridescent copper-gold medially, overlaid with dark purple-red chromatophores, largest along midline and between fins. Dorsal surface of head and proximal aboral surfaces of arms iridescent greenish-pink, with similar chromatophores to mantle.

Small juvenile specimens (ML 16-35mm, Figs 50B, 52A, 52D) deviate from the above description as follows. Mantle width $\sim 31 \% \mathrm{ML}$; rostrum of gladius visible ventrally through posterior $\sim 8 \% \mathrm{ML}$ and piercing tip of mantle. Fins rhombic in outline or very slightly attenuate posteriorly, length $\sim 31 \% \mathrm{ML}$, width $\sim 63 \% \mathrm{ML}$; posterior margins straight, anterior margins convex. Head rounded, length $\sim 19 \% \mathrm{ML}$, width and depth $\sim 25 \% \mathrm{ML}$; eyes somewhat anteriorly directed; no folds apparent in occipital region. Funnel rounded, short; groove not well defined; funnel component of locking apparatus $\sim 19 \% \mathrm{ML}$; mantle component $\sim 38 \% \mathrm{ML}$. Arm length $25-44 \% \mathrm{ML}$, formula II=III $>|>| \mathrm{V}$, each with $\sim 50$ suckers (ML $16 \mathrm{~mm}$ ) to $\sim 70$ suckers (ML $35 \mathrm{~mm}$ ). No keels apparent on any arms; lateral membrane on Arms IV 50\% arm width at base, reduced to unapparent by $10 \%$ arm length. Tentacles barely longer than arms at $\mathrm{ML} 16 \mathrm{~mm}$, length $\sim 50 \% \mathrm{ML}$; stalk thickness at bases similar to that of adjacent arms. Clubs (Fig. $52 \mathrm{~A}$ ) unexpanded to slightly expanded, length $\sim 31 \% \mathrm{ML}(\sim 63 \% \mathrm{TnL})$; carpus not yet well defined at ML $16 \mathrm{~mm}$, distinctly comprised of about eight suckers by ML $21 \mathrm{~mm}$; manus with six recognisable ventral hooks midway along series, and no dorsal hooks at $\mathrm{ML} 16 \mathrm{~mm}$, with first dorsal hooks starting to form around ML $23 \mathrm{~mm}$ and both hook series mostly formed by $\mathrm{ML} \sim 27 \mathrm{~mm}$; two full series of marginal suckers present through $\mathrm{ML} \sim 30 \mathrm{~mm}$, with several lost midway along each series by $\mathrm{ML} 32 \mathrm{~mm}$; terminal pad with $\sim 16$ suckers. Tentacular suckers (Fig. 52D) circular in outline, with small aperture, its diameter $\sim 30 \%$ sucker diameter, circular, situated slightly proximally, with seven to nine low, blunt teeth present around distal to lateral inner margin; pegs set densely in three or four concentric rows proximally and somewhat less densely in six or seven rows distally; proximal and distal pegs of similar size; inner pegs roughly circular, with those in outer rings more polygonal. Overall colouration pale creamy pink with several isolated, small dark chromatophores on dorsal surface of mantle and fins; mantle with dorso-lateral pinkish-copper iridescence; dorso-lateral surfaces of head with pale blue iridescence; aboral surfaces of Arms I and II with blue-green-gold iridescence. 
Remarks - The holotype of $A$. lichtensteinii (MNHN 3.1.620) is in good condition (Figs 49B-D). However, it is labelled 'Syntype,' as is the gladius dissected from this specimen and separately accessioned (MNHN 3.1.621). Since these are parts of the same specimen, it is in fact the holotype.

Photophores have not been widely reported in the genus Ancistroteuthis. Kubodera et al. (1998) mentioned a small photogenic patch on the ventral eye surface, which Vecchione et al. (2003) considered iridescent, but not photogenic. Examination of the A. lichtensteinii holotype revealed a small square patch of tissue on the ventral surface of each eyeball that is possibly photogenic; several additional specimens, but not the majority, possessed a similar patch. The ventral ocular region of live or fresh specimens must be examined to resolve the nature of this tissue.

Voss (1956) reported A. 'lichtensteini' from the Gulf of Mexico, and Rancurel (1970) also reported very similar-looking A. 'lichtensteini' from New Caledonia, all of ML 61$80 \mathrm{~mm}$. In both accounts the specimens lacked secondary occipital folds and possessed many hooks (28-30). Nesis (1987) suggested that these specimens might represent juvenile 'Moroteuthis' aequatorialis (which is possible - see Remarks for Ok. (Ok.) aequatorialis); given historical confusion between A. lichtensteinii and OK. (Ok.) robsoni, they may also be attributable to the latter. 
Diagnosis - Photophores and secondary occipital folds absent; epidermis of mantle longitudinally ridged; fins heart-shaped, length $\sim 35 \% \mathrm{ML}$, width $\sim 40 \% \mathrm{ML}$; arms $\sim 80 \%$ $\mathrm{ML}$; manus of tentacle club with 30-36 hooks and full series of marginal suckers through maturity; rostrum of gladius posteriorly directed, $\sim 10 \% \mathrm{ML}$.

Type species - Kondakovia longimana Filippova, 1972, by monotypy.

Remarks - Kondakovia has been monotypic until recently, and although Filippova (1972) remarked that $K$. longimana resembled 'Moroteuthis' (= Onykia) in many aspects, she considered the differences great enough (in particular the marginal suckers retained through maturity) to warrant description of this species in a separate genus.

Laptikhovsky et al. (2008) (subsequent to the cutoff date for material treated in this study) described a second species of Kondakovia, K. nigmatullini, from sub-Antarctic waters, with somewhat different bodily proportions and epidermal sculpture from $K$. longimana. However, as specimens of this second species are not currently available for examination, its morphology cannot be described here. The generic diagnosis above is based solely on $K$. longimana and may require revision once information on the second species becomes available. 
Kondakovia longimana Filippova, 1972 (Tables 11, 21, Figs 53-55)

Kondakovia longimana Filippova, 1972: 395-398, Table 1, Figs 4, 5; Clarke (1980): 81-128, Table 23, Text-figs 53, 55, 56, 68-77, PI. 1 Fig. 1, (1986): 77, Figs 15D, 35A, 35B; Nemoto et al. (1985): 415-420, Tables 1-3, Figs 1-3, (1988): 292-296, Tables 1, 2, Figs 1-3; Toll (1982): 72, 73, PI. 7 Fig. D; Okutani \& Clarke (1985): 19-21; Roper et al. (1985): 181, 182; Nesis (1987): 192, Figs 49L-O; Hess (1987): 297, 300, 301; Engeser \& Clarke (1988): Fig. 6f-i; Sweeney et al. (1992): 136, Fig. 184b; Jackson \& Lu (1994): 196, Table 1, Fig. 1a; Lu \& Williams (1994): 231-234, Table 1, Fig. 1; Vacchi et al. (1994): 283, Table 1; Bizikov (1996): 10, 20, 45, 182, 185-191, 232, 234, 237, Figs 26, 108-112; Filippova et al. (1997): 182, Fig. 107; Kubodera et al. (1998): 285-286, Table 2, Fig. 13; Sweeney \& Roper (1998): 572; Okutani (2005): 159.

Type material (not available for examination in this study) - ZMMGU holotype (registration unknown), , ML 260mm, Scotia Sea, Om, 20/03/1967, RV Academician Knipovitch Stn 970, NFD; ZMMGU paratype (registration unknown), + , ML 210mm, Scotia Sea, 50m, 20/03/1967, RV Academician Knipovitch Stn 969, NFD; ZMMGU paratype (registration unknown), + , ML 138mm, Scotia Sea, 50m, 17/02/1967, RV Academician Knipovitch Stn 835, NFD.

Material examined (8 specimens) - BMNH 20070538, $\partial$, ML $\sim 320 \mathrm{~mm}$ (brachial crown only, AIV $395 \mathrm{~mm}$ ), $55^{\circ} 42^{\prime} \mathrm{S}, 00^{\circ} 02^{\prime} \mathrm{W}, 20 / 11 / 1947$, from male sperm whale \#1; SAM S300,, , ML 98mm, 56 $04^{\circ} \mathrm{S}, 50^{\circ} 56$ 'W, 50-0m, 21/12/1965, 1655-1704h GMT, 1216 krill net; BMNH 20070521, sex indet., $\mathrm{ML} \sim 240 \mathrm{~mm}$, Signy Island [ $\left.60^{\circ} 43^{\prime} \mathrm{S}, 45^{\circ} 36^{\prime} \mathrm{W}\right]$, 18/03/1982, NFD; NMV F109447, + , 'est. ML 1.5m' (damaged), LRL 21mm, 6304.72'S, $62^{\circ} 56.02^{\prime} \mathrm{E}, 14 / 10 / 1985$, coll. D. Williams; NMV F71538, ô, ML 449mm, 66³2.11'S, 6901.89'E, 48-33m over 1733-1726m, 11/02/1991, IYGPT; NMNZ M.160622, , ML $720 \mathrm{~mm}, 71^{\circ} 08^{\prime} \mathrm{S}, 179^{\circ} 49^{\prime} \mathrm{E}, 29 / 01 / 2003$, attacking hooked toothfish, MFish SOP trip

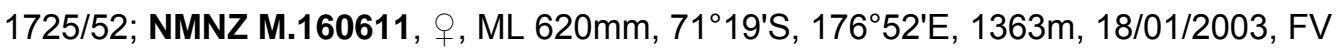
San Aotea, MFish SOP trip 1725/48, hooked on toothfish line; NMNZ M.160621,, , ML $\sim 700 \mathrm{~mm}$ (damaged), $71^{\circ} 34^{\prime} \mathrm{S}, 176^{\circ} 56^{\prime} \mathrm{E}$, Ross Sea, toothfish line over $1123 \mathrm{~m}$ bottom depth, 03/12/2002, FV Janus, MFish SOP trip1725/48, coll. T. Brunning.

Unlocalised material examined (8 specimens) - BMNH 20070522, sex indet., ML 700mm, Antarctic, 01/11/1963, A734, M.R. Clarke, Acc. \#2366, NFD; BMNH 20070524, $\odot$, ML

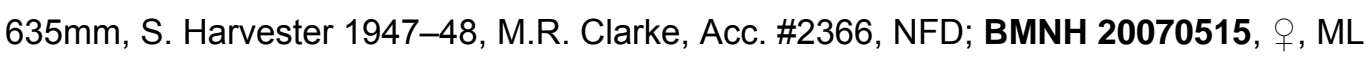
600mm, South Georgia 1964-5, M.R. Clarke, Acc. \#2366, NFD; BMNH 20070543,, , ML $520 \mathrm{~mm}$, Antarctic 'Area IV,' 1947-48, Southern Harvester, Pres. R. Clarke; BMNH 20070510, 9 , ML 500mm, South Georgia 1964-5, M.R. Clarke, Acc. \#2366, NFD; USNM 20070508, sex indet., ML 470mm, coll. M.R. Clarke, Acc. \#2366, NFD; BMNH 20070533, sex indet., ML $\sim 200 \mathrm{~mm}$ (brachial crown only, AIV 250mm) 06/12/1935, from blue whale stomach, S. Empress, NFD; USNM 1091106, 今̄, ML 189mm, ELT 1392 21-IV-64 IKMWT $10 \%$ BFS beaks \#31, NFD. 
Table 21. Measures ( $\mathrm{mm}$ ) and counts of Kondakovia longimana Filippova, 1972 (arm and tentacle measures recorded from more complete side of specimen, indicated $\mathrm{R}$ or $\mathrm{L}$ ).

\begin{tabular}{|c|c|c|c|c|c|c|c|c|c|c|}
\hline Specimen ID & $\begin{array}{c}\text { NMNZ } \\
\text { M.160622 }\end{array}$ & $\begin{array}{c}\text { NMNZ } \\
\text { M.160621 }\end{array}$ & $\begin{array}{c}\text { NMNZ } \\
\text { M.160611 }\end{array}$ & $\begin{array}{c}\text { BMNH } \\
20070543\end{array}$ & $\begin{array}{c}\text { BMNH } \\
20070510\end{array}$ & $\begin{array}{c}\text { NMV } \\
\text { F71538 }\end{array}$ & $\begin{array}{c}\text { USNM } \\
1091106\end{array}$ & SAM S300 & \multicolumn{2}{|c|}{$\begin{array}{l}\text { Mean } \\
\text { Indices }\end{array}$} \\
\hline Type Status & None & None & None & None & None & None & None & None & \multirow{3}{*}{\multicolumn{2}{|c|}{ (ML 449-720) }} \\
\hline $\mathrm{ML}$ & 720 & $700^{*}$ & 620 & 520 & 500 & 449 & 189 & 98 & & \\
\hline Sex & $\mathrm{F}$ & $\mathrm{F}$ & $\mathrm{F}$ & $\mathrm{F}$ & $\mathrm{F}$ & $\mathrm{F}$ & $M$ & $\mathrm{~F}$ & & \\
\hline MW & 230 & $230^{*}$ & $190^{*}$ & 120 & 120 & 95 & 35 & 20 & MWI & 27 \\
\hline $\mathrm{HL}$ & 80 & 80 & 80 & 70 & 68 & 55 & 34 & 18 & $\mathrm{HLI}$ & 12 \\
\hline HW & 100 & 110 & 90 & 75 & 85 & 55 & 34 & 18 & HWI & 15 \\
\hline FL & 257 & 255 & 230 & 203 & 175 & 85 & 79 & 45 & FLI & 34 \\
\hline FW & 295 & 300 & 280 & 195 & 180 & 155 & 87 & 53 & FWI & 40 \\
\hline Arms I & $490 \mathrm{R}$ & $535 R$ & $434 \mathrm{R}$ & $360 L^{*}$ & $325 \mathrm{R}$ & $295 \mathrm{~L}$ & $115 \mathrm{~L}$ & $62 \mathrm{R}$ & A1I & 69 \\
\hline Arms II & $560 R$ & $637 R$ & $530 \mathrm{R}$ & $490 \mathrm{~L}$ & $335 R^{*}$ & $305 \mathrm{~L}$ & $139 \mathrm{~L}$ & $78 \mathrm{R}$ & A2I & 83 \\
\hline Arms III & $540 R$ & $560 R$ & $495 R^{*}$ & $450 \mathrm{~L}$ & $391 \mathrm{R}$ & $280 \mathrm{~L}$ & $132 \mathrm{~L}$ & $72 \mathrm{R}$ & A3I & 77 \\
\hline Arms IV & $540 \mathrm{R}$ & $565 R$ & $515 \mathrm{R}$ & $485 \mathrm{~L}$ & $395 \mathrm{R}$ & $345 \mathrm{~L}$ & $142 \mathrm{~L}$ & $74 \mathrm{R}$ & A4I & 81 \\
\hline TnL & $770 \mathrm{R}$ & $860 \mathrm{R}$ & 790R & $688 \mathrm{~L}$ & $490 \mathrm{R}$ & $389 L^{*}$ & 198L & $108 \mathrm{R}$ & TnLI & 112 \\
\hline CL & $215 R$ & $240 \mathrm{R}$ & $200 R$ & $195 \mathrm{~L}$ & $145 R$ & $112 \mathrm{~L}$ & $52 \mathrm{~L}$ & $28 \mathrm{R}$ & CLI & 28 \\
\hline CS & 11 & 10 & 10 & 12 & 10 & 10 & 10 & 10 & & \\
\hline MH & 36 & 37 & 36 & 38 & 38 & 32 & 33 & 28 & & \\
\hline MS & All & All & All & All & All & All & All & All & & \\
\hline TPS & $19^{*}$ & $16^{*}$ & $18^{*}$ & $16^{*}$ & * & 30 & 29 & 36 & & \\
\hline
\end{tabular}

* indicates damaged features.

Distribution (Fig. 53A) - circum-Antarctic, 1370-0m.

Diagnosis - As for genus.

Description (Figs 53B-55G) - Mantle conical, width 21-27-33\% ML, fleshy, thickwalled, epidermis with narrow longitudinal ridges (Fig. 54B); dorsal midline with dark longitudinal depression over gladius; rostrum of gladius visible ventrally through posterior $\sim 13 \%$ ML. Fins heart-shaped, small, length 19-34-39\% ML, width 35-40$45 \% \mathrm{ML}$; posterior margins concave medially, becoming convex laterally; anterior margins convex.

Head boxy, length $\sim 15 \% \mathrm{ML}$, width $\sim 12 \% \mathrm{ML}$, depth $\sim 14 \% \mathrm{ML}$, not constricted anteriorly; epidermis with longitudinal ridges; occipital region with pronounced dorsal transverse crest. Funnel narrow, short, with one finger-like, laterally compressed lappet on each side (Fig. 54A). Funnel groove well defined, narrowly trapezoidal in outline, with aperture opening at point directly below midpoint of eye. Funnel component of locking apparatus $10-12 \% \mathrm{ML}$, mantle component $15 \% \mathrm{ML}$.

Arms $65-69,-81_{\| I I V}-94 \% \mathrm{ML}$, formula IV=III=|I>I, each with 140-160 small suckers (diameter 20-30\% arm width) at ML 449-720mm; oral arm faces bordered by strong membranes with well-defined trabeculae. Keels present on Arms I-III: that on Arms I $25 \%$ arm width over proximal $10 \%$ arm length, thereafter reducing to $\sim 10 \%$ arm width over remainder of arm; that on Arms II $\sim 30 \%$ arm depth by $40 \%$ arm length, thereafter 
narrowing distally; that on Arms III 50\% arm depth by $40 \%$ arm length, thereafter narrowing distally; lateral membrane on Arms IV $\sim 60 \%$ arm width at bases, narrowing thereafter to $\sim 15 \%$ arm depth along remainder of arm length.

Tentacles $87-112-132 \% \mathrm{ML}$; stalks narrow, width $\sim 40 \%$ that of adjacent arms; aboral stalk surface with low keel. Clubs (Figs 54C, 54D) 25-28-38\% ML (25-30\% TnL), barely expanded. Carpus a well-defined oval with ten to 12 suckers; manus with 32-38
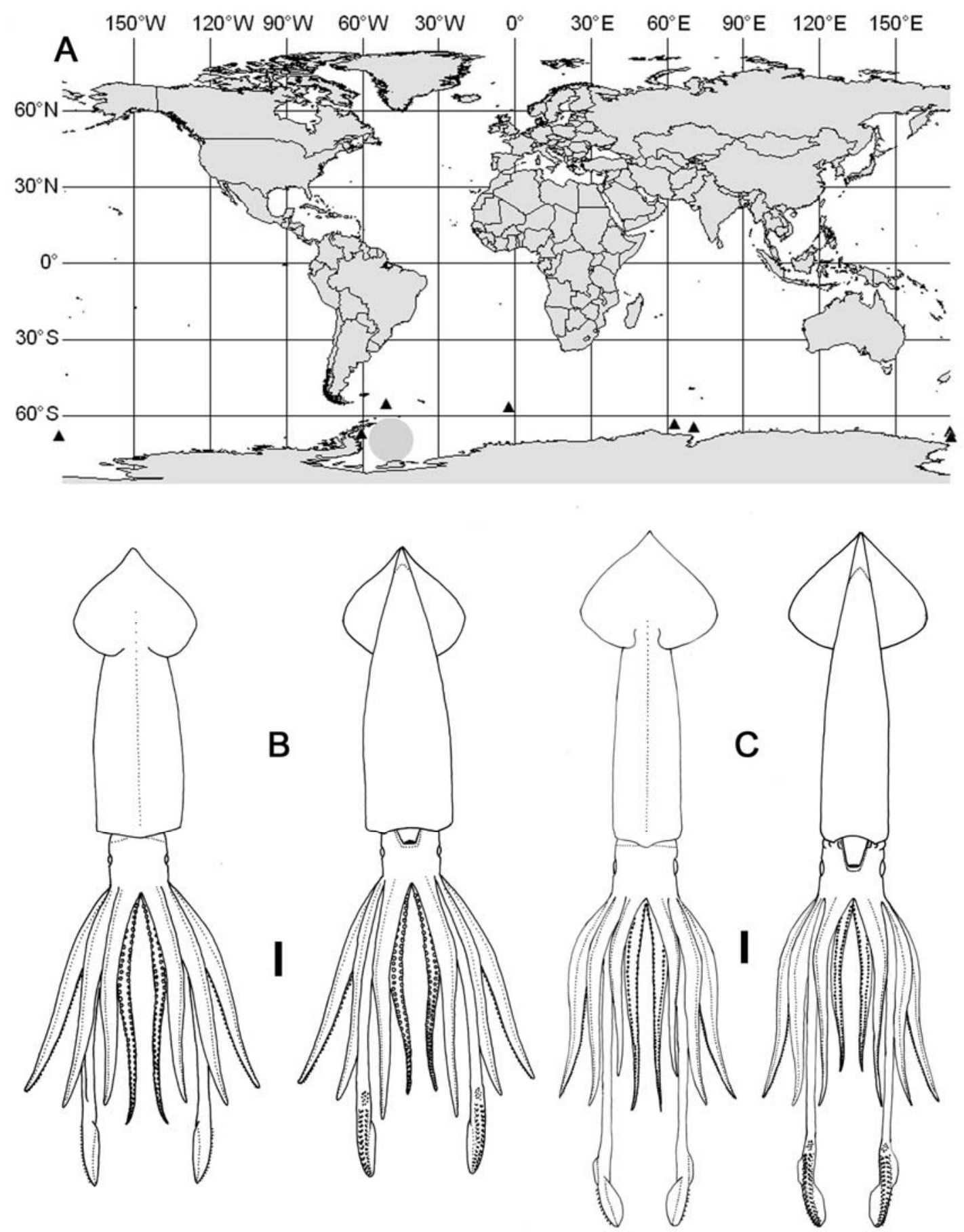

Fig. 53 - Kondakovia longimana. A) distribution (grey circle indicates type locality); B) NMNZ M.160621, o, ML 700mm; C) USNM 1091106, ô, ML 189mm. Scale bars = B) $100 \mathrm{~mm}$; C) $20 \mathrm{~mm}$. 
hooks (Figs 54E-G) and two complete rows of marginal suckers; terminal pad with 1636 suckers (usually 28-36). Ventral hooks increase in size gradually through $\mathrm{V} 7$, with V6-V8 largest (Fig. 54G), about twice as long as paired dorsal hooks and $\sim 7 \% \mathrm{CL}$, thereafter reducing gradually. Dorsal hooks increase in size gradually through D5, thereafter reducing in size. Ventral club membrane, $\sim 50 \%$ club width, borders hooks V1-V7; dorsal membrane not apparent; dorsal keel, $\sim 150 \%$ club width, borders hooks D4-D17.

Lower beak in females (Figs 55A-C, ML 1.5m) longer than deep in lateral profile, with crest straight and sloping steeply downward, posterior edge of hood well above crest, hood length $\sim 50 \%$ total crest length, and hood and crest together $\sim 60 \%$ baseline; jaw edge roundly concave along entire length; jaw angle obtuse, obscured by slight wing fold. Crest narrow, slightly thickened; lateral walls markedly indented below crest; jaw edge narrow and sharp; shoulder ridge rounded, indistinct; angle ridge indistinct, spanning $\sim 50 \%$ lateral wall depth, angle point indistinct; wings broaden somewhat distally, width $\sim 150 \%$ that at jaw angle, length $\sim 250 \%$ LRL; no cartilage apparent on medial wing margin in adult beaks; lateral wall fold rounded, well-developed along entire length and remaining distinct to posterior margin of lateral wall; minor breakage observed along posterior margin of hood in some adult beaks. Lower beak in males (Fig. 55D, ML 449mm): as above with the following exceptions: jaw angle obscured in lateral profile by large, rounded cartilaginous wing fold; shoulder ridge rounded, distinct, cartilaginous; distal wing width $\sim 125 \%$ that at jaw angle; wing length $\sim 170 \%$ $\mathrm{LRL}$; shoulder ridge and wings with broad, rounded, cartilaginous insert; lateral wall fold rounded, well-developed but less distinct than in females.

Radula (Fig. 55E) with unicuspid rachidian; mesocone forms isosceles triangle, broad at base, tapering to slender point. First lateral tooth unicuspid, subequal to rachidian in height, forming slightly more slender but otherwise similar isosceles triangle; may be slightly curved, with inner face gently concave and outer face gently convex. Second lateral tooth conical, height $\sim 125 \%$ that of rachidian. Marginal tooth simple, slender, nearly cylindrical, $\sim 150 \%$ rachidian height. Palatine palp (Fig. 55F) with greatest width of tooth-bearing plane $\sim 40 \%$ its length, with $\sim 90$ flat, somewhat blunt teeth, each 60 $180 \%$ rachidian height, almost evenly distributed over palp surface.

Gladius not available for examination in adult material; see Remarks and description for smaller specimens below. 

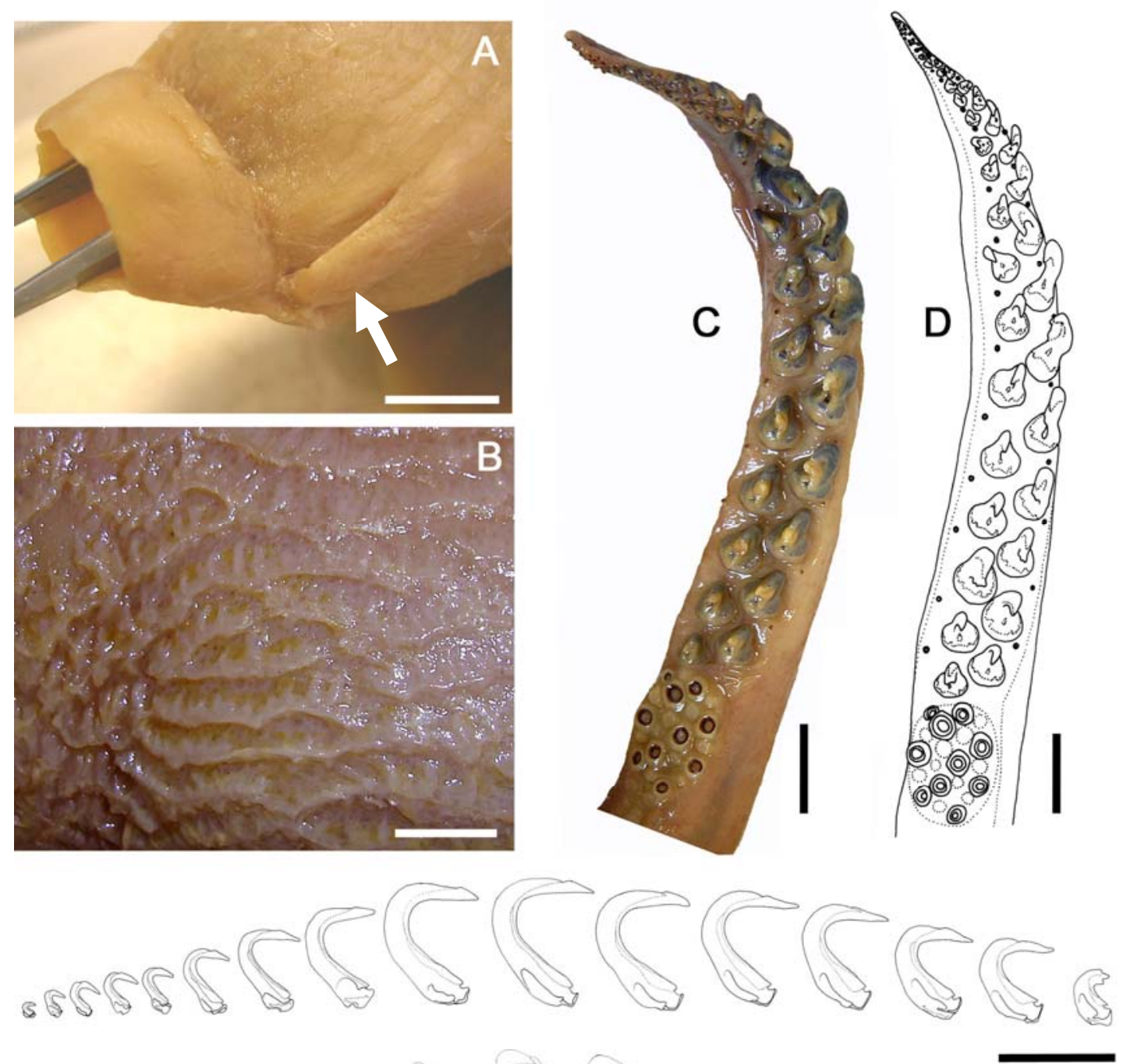

\section{$E$}
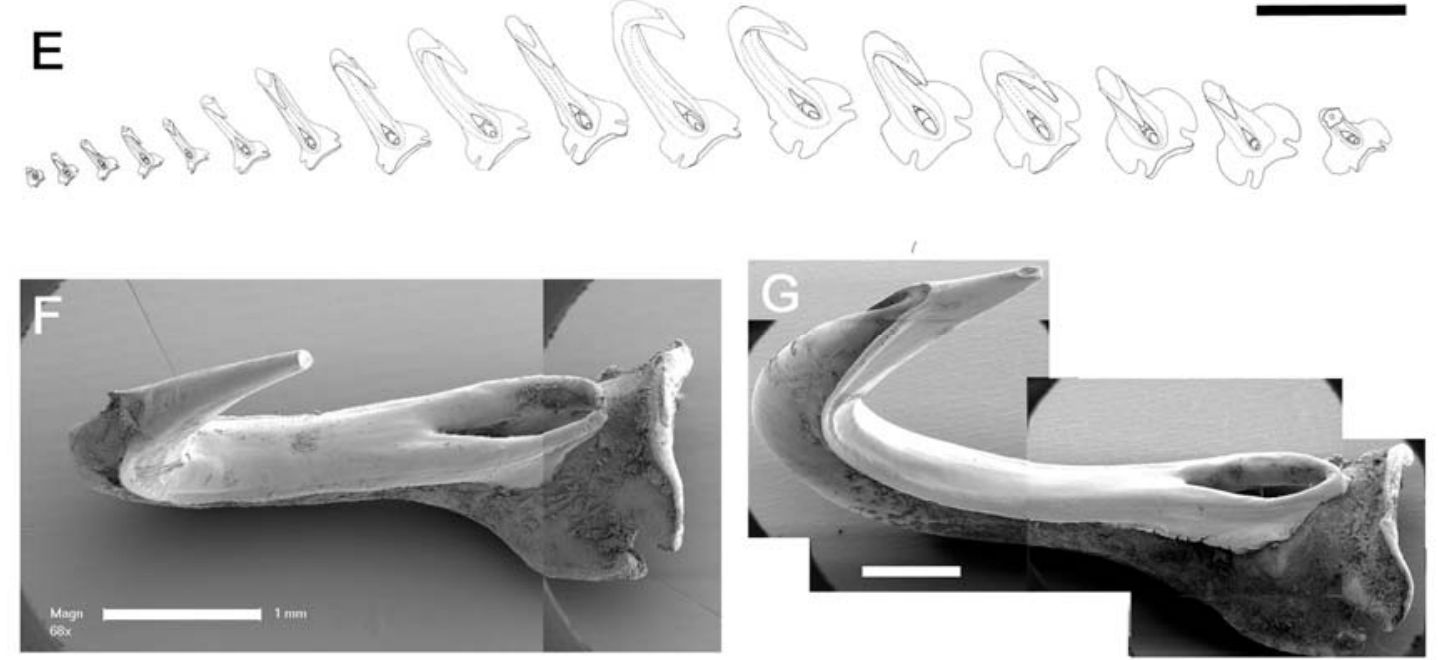

Fig. 54 - Kondakovia longimana. A) NMV F71538, ô, ML 449mm, lateral 'lappets' on funnel (arrow); B) NMNZ M.160622, , ML 720mm, epidermis of mantle; C, D), BMNH 20070521, sex indet., ML 240mm, left tentacle club; E-G) NMNZ M.160622, 9 , ML $720 \mathrm{~mm}$, tentacular hooks: (E) left ventral hook series, $(F)$ left hook V13, $(G)$ left hook V8. Scale bars = A, B) $10 \mathrm{~mm}$; C-E) $5 \mathrm{~mm} ; \mathrm{F}, \mathrm{G}) 1 \mathrm{~mm}$. 
Smaller specimens (ML 100-200mm, Fig. 53C, 55G) deviate from the above description as follows: mantle more slender, nearly cylindrical, width 18-20\% ML; fins larger, length $40-45 \% \mathrm{ML}$, width 55\% ML; head length and width 18\% ML; arms $\sim 70 \% \mathrm{ML}$, each with $\sim 110$ suckers at ML $98 \mathrm{~mm}$ and $\sim 140$ by ML $189 \mathrm{~mm}$; keels not developed on Arms I or II at ML 98mm, but all present by ML 189mm; marginal suckers much larger relative to manus hooks; dorsal club membrane not yet evident by ML 189mm. Gladius (Fig. 55G) with greatest width ( 8\% GL) attained at $\sim 40 \% \mathrm{GL}$; free rachis $\sim 20 \% \mathrm{GL}$; vanes broaden rapidly between 20 and $30 \% \mathrm{GL}$, gradually narrowing thereafter. Rostrum $\sim 8 \% \mathrm{GL}$, with triangular cross-section.

Remarks - The beak dimorphism in K. longimana resembles that observed in OK. (M.) ingens (Bolstad 2006); the difference in beak proportions in the two sexes, the large cartilaginous shoulder ridge and wing insert observed in the male, and the breakage observed in the hood region in adult females suggest that mating behaviour in K. longimana may also involve beak-to-beak copulation, as hypothesised for OK. (M.) ingens (Bolstad 2006). It is not known whether mating individuals orient themselves 'upside down' relative to each other, although observed female hood erosion is consistent with such a strategy.

The gladius of no subadult or adult specimen was in suitable condition to report morphology; for completeness and comparison, the following description is based on Bizikov (1996, Fig. 26): greatest width ( 9\% GL) attained at 30-40\% GL; free rachis $\sim 20 \% \mathrm{GL}$; vanes broaden rapidly between 20 and $30 \% \mathrm{GL}$, gradually narrowing thereafter. Rostrum $\sim 10 \% \mathrm{GL}$, with triangular cross-section.

Kondakovia appears most similar to the genus Onykia. Several adult characters/states unique to $K$. longimana (see Table 11) are observed in juveniles of various Onykia species, but disappear or change ontogenetically. These include marginal suckers on the manus and short fins ( $35 \% \mathrm{ML})$; both Kondakovia and young Onykia lack the distinctive $\mathrm{Y}$-shaped ridge in the funnel groove that all known Onykia species (except Ok. (M.) ingens) later develop. Adults of $K$. longimana also share several character states with some adult Onykia species, including sculptured skin on the mantle, relatively long arms and tentacles, the absence of secondary occipital folds, the form of the gladius, and apparent sexual dimorphism in the lower beaks. Strugnell et al. (2004) reported instances of close relationships between neotenous and polar cephalopod genera. The above-mentioned combinations of characters/states considered typical for both juveniles and adults in other genera, plus the predominance 
of krill in the diet of $K$. longimana (Nemoto et al. 1988) may also indicate neoteny, since some Onykia species are known to undergo an ontogenetic diet shift from crustaceans in early life stages to squid and myctophids nearer adulthood (Jackson et al. 1998, Phillips et al. 2003b).

Several authors have reported aspects of this species' biology and ecology, including growth (Jarre et al. 1991) and trophic interactions (Clarke 1980, Nemoto et al. 1988).
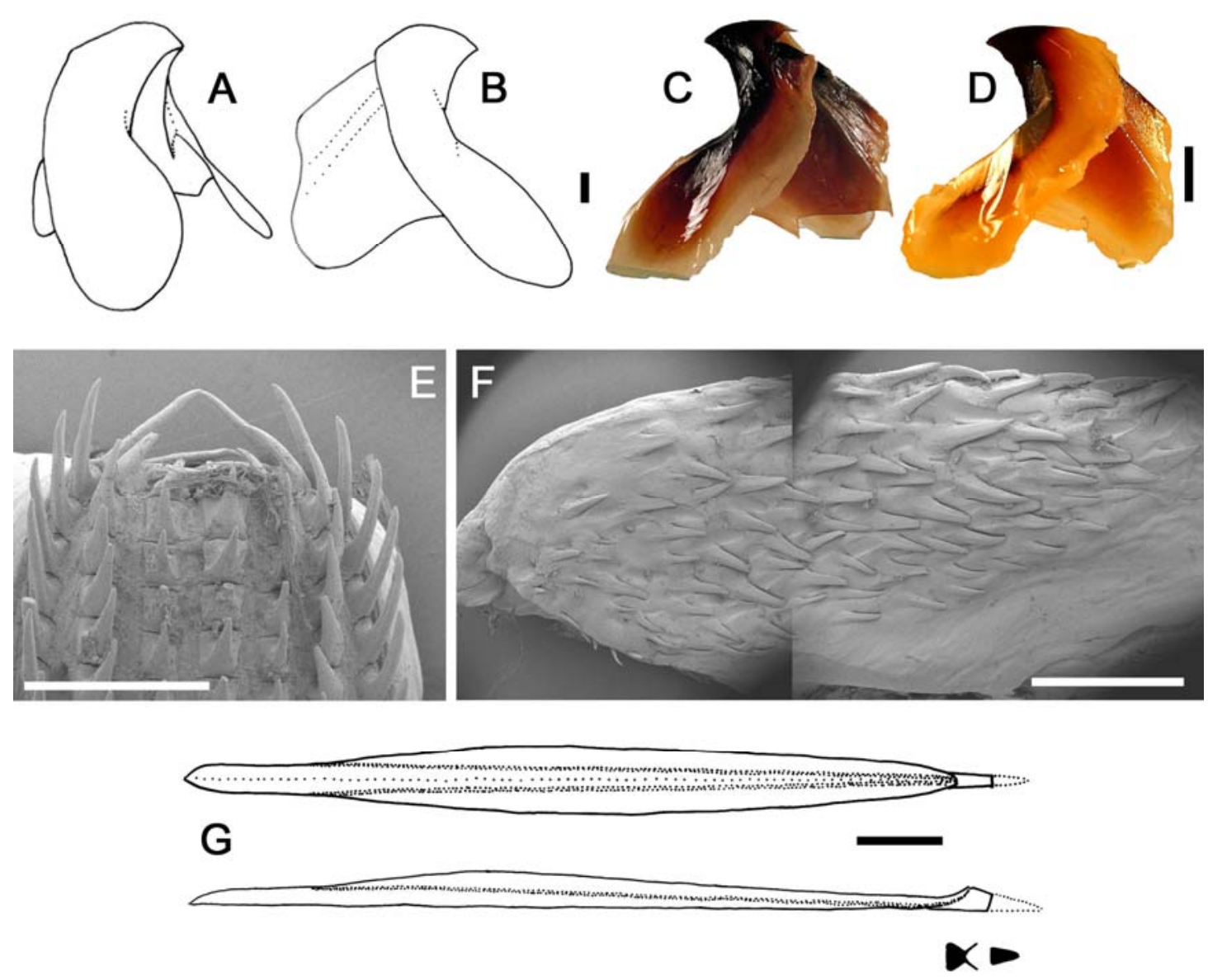

Fig. 55 - Kondakovia longimana. A-C) NMV F109447,, , ML 1.5m (damaged), LRL $21 \mathrm{~mm}$; D-F) NMV F71538, ô, ML 449mm; G) SAM S300, ․ ML 98mm. A-D) lower beak: $(A)$ left oblique, $(B)$ left, $(C, D)$ right profiles; $(E)$ radula, $(F)$ palatine palp, $(G)$ gladius. Scale bars = B-D, G) $10 \mathrm{~mm}$; E) $1 \mathrm{~mm}$; F) $2 \mathrm{~mm}$. 
Diagnosis - Skin smooth, fins rhombic in outline, length $\sim 52 \% \mathrm{ML}$, width $\sim 72 \% \mathrm{ML}$; gladius visible through dorsal mantle tissue between fins only; three secondary folds present in dorsal occipital region; rostrum of gladius with small sub-terminal rostrum, diverging from true rostrum into distinct separate point; fully developed tentacle club with 16-18 hooks; base of largest ventral hooks asymmetrical; rostrum of gladius posteriorly directed, $\sim 5 \% \mathrm{GL}$.

Type species - Notonykia africanae Nesis, Roeleveld \& Nikitina, 1998, by original designation.

Remarks - The two known species in the genus Notonykia were initially reported as southern morphological variants of Ancistroteuthis lichtensteinii (Kubodera et al. 1998). The two genera are somewhat similar but are readily separable. Notonykia has rhombic fins, fewer secondary occipital folds (three), fewer hooks on the manus (1618) and far more terminal pad suckers (30-40), a distinct free rachis on the gladius and shorter rostrum ( $\sim 5 \% \mathrm{GL})$, and unicuspid to slightly tricuspid rachidian tooth on the radula. In contrast, Ancistroteuthis generally has sagittate fins, more secondary occipital folds (six or seven), 20-24 hooks on the manus and only 15-19 terminal pad suckers, no free rachis on the gladius and a longer rostrum $(\sim 12 \% \mathrm{GL})$, and a distinctly tricuspid rachidian tooth on the radula.

The recognised distributions of these two genera are also disjunct, with Ancistroteuthis known from the central to North Atlantic and Mediterranean, and Notonykia known only from subtropical to sub-Antarctic waters of the southern Indian, Pacific and Atlantic Oceans. 
Notonykia africanae Nesis, Roeleveld \& Nikitina, 1998 (Table 22, Figs 56-58)

Notonykia africanae Nesis, Roeleveld \& Nikitina, 1998: 153-168, Tables 1, 2, Figs 1-5; Okutani (2005): 155; Bolstad (2007): 322-327, Tables 4, 6, Figs 38-50.

Ancistroteuthis lichtensteinii (not Férussac, 1835) 'South Atlantic Form' - Kubodera et al. (1998): 281, 282.

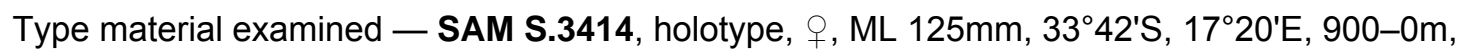
09/03/1988, FRV Africana Stn A7010-060-08-04M; SAM S3421, paratype, , ML 95mm, 31ํ⒕6'S, $15^{\circ} 35.5^{\prime} \mathrm{E}, 796 \mathrm{~m}, 26 / 01 / 1990$, FRV Africana Stn A9827-079-DT002, BTT; SAM S3412, paratype, + , ML 82mm, 33⒙1'S, 17²4.1'E, 990-910m, 07/03/1988, FRV Africana Stn A6996-060-03-04M, MWT; SAM S3413, paratype, + , ML 130mm (mantle only), 33²42.5'S, 17'21.9'E, 972m, 07/03/1988, FRV Africana Stn A6997-060-04-04M,

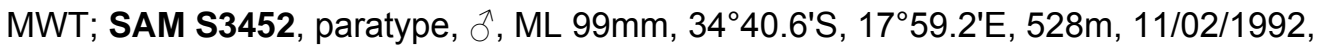
FRV Africana Stn A13007-100-M008, MWT; SAM S.3419, paratype, $\widehat{\jmath}$, ML 53mm, 3440.9'S, 18¹3.60'E, 489-479m, 13/03/1988, FRV Africana Stn A7030-060-13-02M,

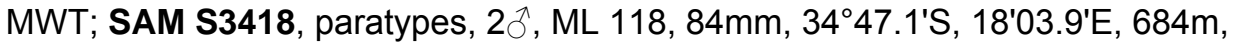
13/03/1988, FRV Africana Stn A7029-060-13-03M, MWT; SAM S3416, paratypes, 3 $\widehat{\jmath}$, ML 110, 109, $101 \mathrm{~mm}, 34^{\circ} 48.8^{\prime} \mathrm{S}, 1^{\circ} 08.1^{\prime} \mathrm{E}, 700 \mathrm{~m}, 11 / 03 / 1988$, FRV Africana Stn A7015-060-09-03M, MWT; SAM S3415, paratype, ô, ML 122mm, 34 ${ }^{\circ} 53.0^{\prime} \mathrm{S}, 18^{\circ} 05.1^{\prime} \mathrm{E}$, 918-913m, 11/03/1988, FRV Africana Stn A7014-060-09-04M, MWT; SAM S3420, paratype,, , ML $120 \mathrm{~mm}, 34^{\circ} 54.9^{\prime} \mathrm{S}, 18^{\circ} 12.1^{\prime} \mathrm{E}, 917 \mathrm{~m}, 14 / 03 / 1988$, FRV Africana Stn A7038-060-16-04B, BTT; SAM S3417, paratype,, , ML 107mm, 34ํ56.0'S, $18^{\circ} 12.1^{\prime} \mathrm{E}$, 906m, 13/03/1988, FRV Africana Stn A7028-060-13-04M, MWT.

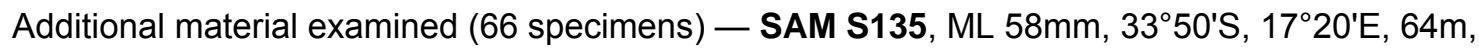
-/12/1961, coll. DSF, MWT100; NMNZ M.74523, sex indet., ML 16mm, 3453'S, $179^{\circ} 26^{\prime} \mathrm{W}, 101 \mathrm{~m}$ over 3000+m, 04/12/1976, FRV James Cook; NMNZ M.74550, sex indet., ML 50mm, 37 $27.36-29.30^{\prime} \mathrm{S}, 178^{\circ} 26.18-28.42^{\prime} \mathrm{W}, 277 \mathrm{~m}$ over $1460-1080 \mathrm{~m}$, 16/08/1975, J012/056/75; NMNZ M.74426, 2 sex indet., ML 43, 35mm, 37³2'S, 178 29.5'E, 70-Om over 606m, 2100 hours, 03/08/1976, FMMWT, FRV James Cook Stn J11/097/76; NMNZ M.74411, 5 sex indet., ML 44-35mm, 37²8.7'S, 178³4.7'E, 174m over 1320m, 03/08/1976, FRV James Cook, FMMWT; NIWA 32781, ô, ML 122mm, $38^{\circ} 00^{\prime} \mathrm{S}, 179^{\circ} 20^{\prime} \mathrm{E}, 120-100 \mathrm{~m}$ over $2536 \mathrm{~m}$, -/02/1998, FMMWT, RV Tangaroa Stn TAN9802/108, Z9290; NMNZ M.67836, ô. ML 92mm, 38²2.16'S, 179¹5.69'E, 30m, 01/10/1979, FRV James Cook; NMNZ M.160475, ․, ML 177mm, 3859.50'S, 167¹9.90'E, 1016m, 22/02/1983, FRV James Cook Stn J4/35/83; NMNZ M.67833, sex indet., ML 92mm, 3901.01'S, 178 27.16'E, 30m, 01/10/1979, FRV James Cook; NMNZ M.160450, sex indet., ML 38mm, 3905.2'S, 178 38.0'E, 30m over 1500m, 19/11/1979, FRV James Cook Stn J16/05/79, FMMWT; NMNZ M.71009, 2§, ML 102, 95mm, ๆ, ML 85mm, 3905.43'S, 178 38.90'E, 30m, 01/10/1979, FRV James Cook; NMNZ M.160448, sex indet., ML 48mm, 39 $9^{\circ} 16.3^{\prime} \mathrm{S}, 178^{\circ} 34.6^{\prime} \mathrm{E}, 30 \mathrm{~m}, 06 / 12 / 1987$, FRV James Cook Stn J15/05/87, FMMWT; NMNZ M.160461, sex indet., ML 63mm, 39¹6.3'S, 178³4.6'E, 
30m, 06/12/1987, FRV James Cook Stn J15/06/87, FMMWT; NMNZ M.277914, ðૈ, ML $61 \mathrm{~mm}, 39^{\circ} 47.8^{\prime} \mathrm{S}, 167^{\circ} 57.9^{\prime} \mathrm{E}, 905 \mathrm{~m}$ over 910m, 15/11/1984, RV James Cook Stn J19/18/84; NMNZ M.91598, 11 sex indet., ML 82-13mm, 4008.3'S, 160¹4.9'E, 45-35 m over 4700+m, 16/10/1985, FRV James Cook; NIWA 32732, $q$, ML 116mm, 40²4'S, 177³0'E, 100-20m over 1748m, -/02/1998, RV Tangaroa Stn TAN9802/156, FMMWT, Z10313; NMNZ M.183104, 9 sex indet., ML 30-18mm, 40 $55.40^{\prime} S, 176^{\circ} 58.00^{\prime} \mathrm{E}$, off Cape Turnagain, 30m, 09/12/1987, FMMWT, FRV James Cook Stn J15/18/87; NMNZ M.277902, 8 sex indet., ML 36-13mm, 40 $56.1^{\prime} \mathrm{S}, 177^{\circ} 25.6^{\prime} \mathrm{E}$, off Cape Turnagain, 30m, 08/12/1987, FRV James Cook Stn J15/16/87, FMMWT; ; NIWA 32728, sex indet., 25mm, $41^{\circ} 36^{\prime} \mathrm{S}, 177^{\circ} 09^{\prime} \mathrm{E}, 120-100 \mathrm{~m}$ over $2878 \mathrm{~m}, 24 / 02 / 1998$, RV Tangaroa Stn TAN9802/187, Z10143; NMNZ M.74555, 8 sex indet., ML 36-25mm, 4155.7-57.9'S, 175 39.8-42.1'E, 101m over 2000m, 28/06/1975NIWA 32782, $q$, ML 118mm, 42³0'S, $175^{\circ} 30^{\prime} \mathrm{E}, 100-20 \mathrm{~m}$ over 1967m, 21/02/1998, RV Tangaroa Stn TAN9802/196,

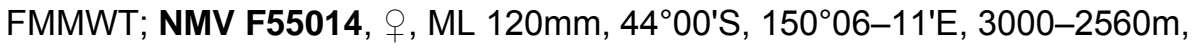

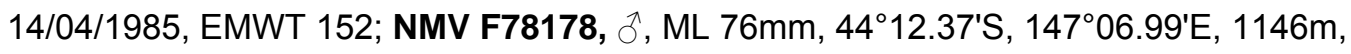
11/04/1983, MIDOC net; NMNZ M.109066, spent $\odot$, ML 200mm, 44⒘53'S, 178 55.46'E, 1176-1120m, 06/11/1991, RV Tangaroa Stn TAN9104/134; NIWA 32765,

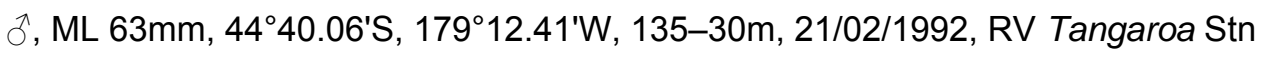

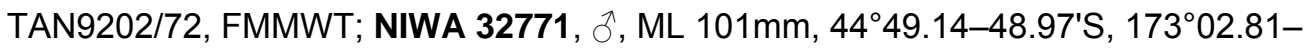
02.33'W, 1053-1052m, 13/04/1997, RV Tangaroa Stn TAN9705/03, FMMWT; NMNZ M.91652, , , ML 92mm, 46²8.70'S, $163^{\circ} 31.90^{\prime} \mathrm{E}, 490-70 \mathrm{~m}$ over $4330 \mathrm{~m}, 29 / 07 / 1985$, FV Kaiyo Maru.

Unlocalised material examined (21 specimens) - BMNH 20070497, 21 sex indet., ML 2114mm, 'Discovery' Expedition Stn 6637, 100-10m, 05/02/1968, TMT8/5.

Distribution (Fig. 56A) - Subtropical waters of the Southern Hemisphere, primarily $30-40^{\circ} \mathrm{S}$ off the coasts of South Africa and New Zealand; $1200-0 \mathrm{~m}$.

Diagnosis - Largest ventral hooks of tentacle club curved in broad, scimitar-like 'C'shape in lateral profile; carpus with nine to 12 suckers; tentacular hooks D1 and V1, and D2 and V2 of subequal size.

Description (Figs 56B-58I) - Mantle muscular, width 17-22-28\% ML, slightly flared at anterior margin; gladius visible along dorsal midline only through mid $60 \%$ of fin length, otherwise unapparent; rostrum not visible ventrally, obscured by low keel on mantle surface, extending over posterior $\sim 15 \% \mathrm{ML}$. Fins large, broadly rhombic in outline, length 55-58-62\% ML, width 68-73-85\% ML; posterior margins nearly straight or very slightly concave medially, slightly convex laterally; anterior margins straight to very slightly convex. 
Table 22. Measures ( $\mathrm{mm}$ ) and counts of Notonykia africanae Nesis, Roeleveld \& Nikitina, 1998 (arm and tentacle measures recorded from more complete side of specimen, indicated $\mathrm{R}$ or $\mathrm{L}$ ).

\begin{tabular}{|c|c|c|c|c|c|c|c|c|c|c|c|c|}
\hline Specimen ID & SAM S.3414 & $\begin{array}{c}\text { SAM } \\
\text { S.3419 }\end{array}$ & $\begin{array}{c}\text { NMNZ } \\
\text { M.109066 }\end{array}$ & $\begin{array}{c}\text { NMNZ } \\
\text { M.160475 } \\
\end{array}$ & $\begin{array}{l}\text { NIWA } \\
32781 \\
\end{array}$ & $\begin{array}{c}\text { NMNZ } \\
\text { M.71009 }\end{array}$ & $\begin{array}{l}\text { NIWA } \\
32782 \\
\end{array}$ & $\begin{array}{c}\text { NMNZ } \\
\text { M.74426 }\end{array}$ & $\begin{array}{c}\text { NMNZ } \\
\text { M.183104 } \\
\end{array}$ & $\begin{array}{c}\text { NMNZ } \\
\text { M.74523 }\end{array}$ & \multicolumn{2}{|c|}{$\begin{array}{c}\text { Mean } \\
\text { Indices }\end{array}$} \\
\hline Type Status & Holotype & Paratype & None & None & None & None & None & None & None & None & \multirow{3}{*}{\multicolumn{2}{|c|}{ (ML 80-177) }} \\
\hline ML & 125 & 53 & 200 & 177 & 122 & 102 & 80 & 43 & 30 & 16 & & \\
\hline Sex & $\mathrm{F}$ & $M$ & $\mathrm{~F}$ & $\mathrm{~F}$ & $M$ & $\mathrm{M}$ & $M$ & Indet. & Indet. & Indet. & & \\
\hline MW & 30 & 15 & 60 & 36 & 21 & 24 & 17 & 11 & 9 & 5 & MWI & 22 \\
\hline $\mathrm{HL}$ & 21 & 15 & 30 & 36 & 20 & 22 & 15 & 8 & 6 & 2 & $\mathrm{HLI}$ & 20 \\
\hline HW & $24^{*}$ & $9^{*}$ & 25 & 27 & 21 & 25 & 18 & 11 & 8 & 4 & HWI & 20 \\
\hline $\mathrm{FL}$ & 69 & 31 & 100 & 99 & 71 & 63 & 45 & 22 & 19 & 5 & FLI & 58 \\
\hline FW & 85 & 45 & 190 & 120 & 81 & 77 & 60 & 32 & 23 & 10 & FWI & 73 \\
\hline Arms I & $47 \mathrm{~L}$ & $22 \mathrm{~L}$ & $79 \mathrm{~L}$ & $65 R$ & $45 \mathrm{~L}$ & $38 \mathrm{R}$ & $29 \mathrm{~L}$ & $17 \mathrm{~L}$ & $9 \mathrm{~L}$ & $4 \mathrm{R}$ & A11 & 38 \\
\hline Arms II & $47 \mathrm{~L}$ & $26 \mathrm{~L}$ & $95 \mathrm{~L}$ & $83 R$ & $56 \mathrm{~L}$ & $46 \mathrm{R}$ & $36 \mathrm{~L}$ & $20 \mathrm{~L}$ & $12 \mathrm{~L}$ & $5 R$ & A2I & 45 \\
\hline Arms III & $52 \mathrm{~L}$ & $26 \mathrm{~L}$ & $100 \mathrm{~L}$ & $79 R$ & $53 \mathrm{~L}$ & $44 \mathrm{R}$ & $31 \mathrm{~L}$ & $21 \mathrm{~L}$ & $11 \mathrm{~L}$ & $4 \mathrm{R}$ & A3I & 41 \\
\hline Arms IV & $55 \mathrm{~L}$ & $27 \mathrm{~L}$ & $91 \mathrm{~L}$ & 75 & $52 \mathrm{~L}$ & $45 R$ & $36 \mathrm{~L}$ & $19 \mathrm{~L}$ & $9 \mathrm{~L}$ & $3 R$ & A4I & 45 \\
\hline TnL & $152 \mathrm{~L}$ & $40 \mathrm{~L}^{*}$ & - & $155 \mathrm{~L}$ & $125 \mathrm{~L}$ & $112 R$ & $87 \mathrm{~L}$ & $31 \mathrm{~L}$ & $21 \mathrm{~L}$ & $6 \mathrm{R}$ & TnLI & 106 \\
\hline $\mathrm{CL}$ & $34 \mathrm{~L}$ & - & - & $38 \mathrm{~L}$ & $30 \mathrm{~L}$ & $29 R$ & $20 \mathrm{~L}$ & $13 R$ & $8 \mathrm{~L}$ & $3 R$ & CLI & 25 \\
\hline $\mathrm{CS}$ & 10 & - & - & 11 & 10 & 9 & 10 & 11 & 10 & 12 & & \\
\hline MH & 17 & - & - & 18 & 17 & 17 & 16 & 16 & 16 & V2-7, OD & & \\
\hline MS & V7, 8 & - & - & V8, 9 & V7, 8 & V7, 8 & V7, V8 & V7, v8 & $\begin{array}{c}\text { all but } \\
\text { V3-5, D } \\
4-5\end{array}$ & * & & \\
\hline TPS & 39 & - & - & 40 & 35 & 38 & $36 \mathrm{~L}$ & $33 R$ & $36 \mathrm{~L}$ & * & & \\
\hline
\end{tabular}

- indicates missing features, * indicates damaged features.

Head squarish, length 15-20-28\% ML, width 15-20-25\% ML, depth 15\% ML, narrower than mantle; occipital region with three small secondary folds; ventral-most primary fold small, adjoining posterior border of funnel groove. Funnel muscular, short; funnel groove V-shaped, bordered by low, fine ridge of tissue; funnel component of locking apparatus $10-15 \% \mathrm{ML}$; mantle component $15-20 \% \mathrm{ML}$.

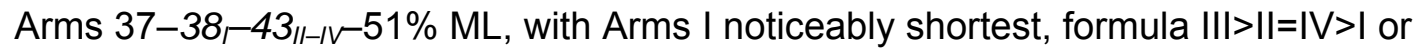
III=II>IV>I; each with 90-100 suckers (ML 102-177mm), bordered by fine trabeculate protective membrane. Keels present on Arms I-III: that on Arms I 25\% arm depth over distal $\sim 50 \%$ arm length; that on Arms II $\sim 30 \%$ arm depth over proximal $25 \%$ arm length, thereafter $\sim 100 \%$ arm depth; that on Arms III narrow proximally and distally but broadening to $\sim 150 \%$ arm depth at $30 \%$ arm length; lateral membrane on Arms IV $\sim 100 \%$ arm width at base, thereafter narrowing to and maintaining $25 \%$ arm width along remainder of arm length.

Tentacles 88-106-112\% ML; stalk diameter slightly less than that of adjacent arms at bases. Club only slightly expanded (Figs 57C, 57D), 21-25-38\% ML (20-25\% TnL); carpus well defined, with nine to 12 suckers (usually ten or 11), square to oblong in shape and bordered by low membrane; manus with 16-18 hooks (Fig. 57B, 58A); terminal pad with 33-40 small suckers set in diagonal rows of four. Proximal three ventral hooks of subequal size to paired dorsal hooks; thereafter ventral hooks markedly larger than corresponding dorsal hooks; D4 and all subsequent distal hooks smaller than proximal three dorsal hooks; hook V5 largest, (Fig. 58A), about five times longer than D5 and $\sim 20 \%$ CL; overall curve of large ventral hooks scimitar-like 'C'- 
shape, some nearly semicircular; base of large ventral hooks asymmetrical, enlarged ventrally. Marginal suckers may flank hooks V8 and V9 to at least ML $177 \mathrm{~mm}$. Ventral membrane $\sim 30 \%$ club width, along entire manus; dorsal membrane indistinct; dorsal keel narrow along D1-3, thereafter $\sim 100 \%$ club width along manus and terminal pad.
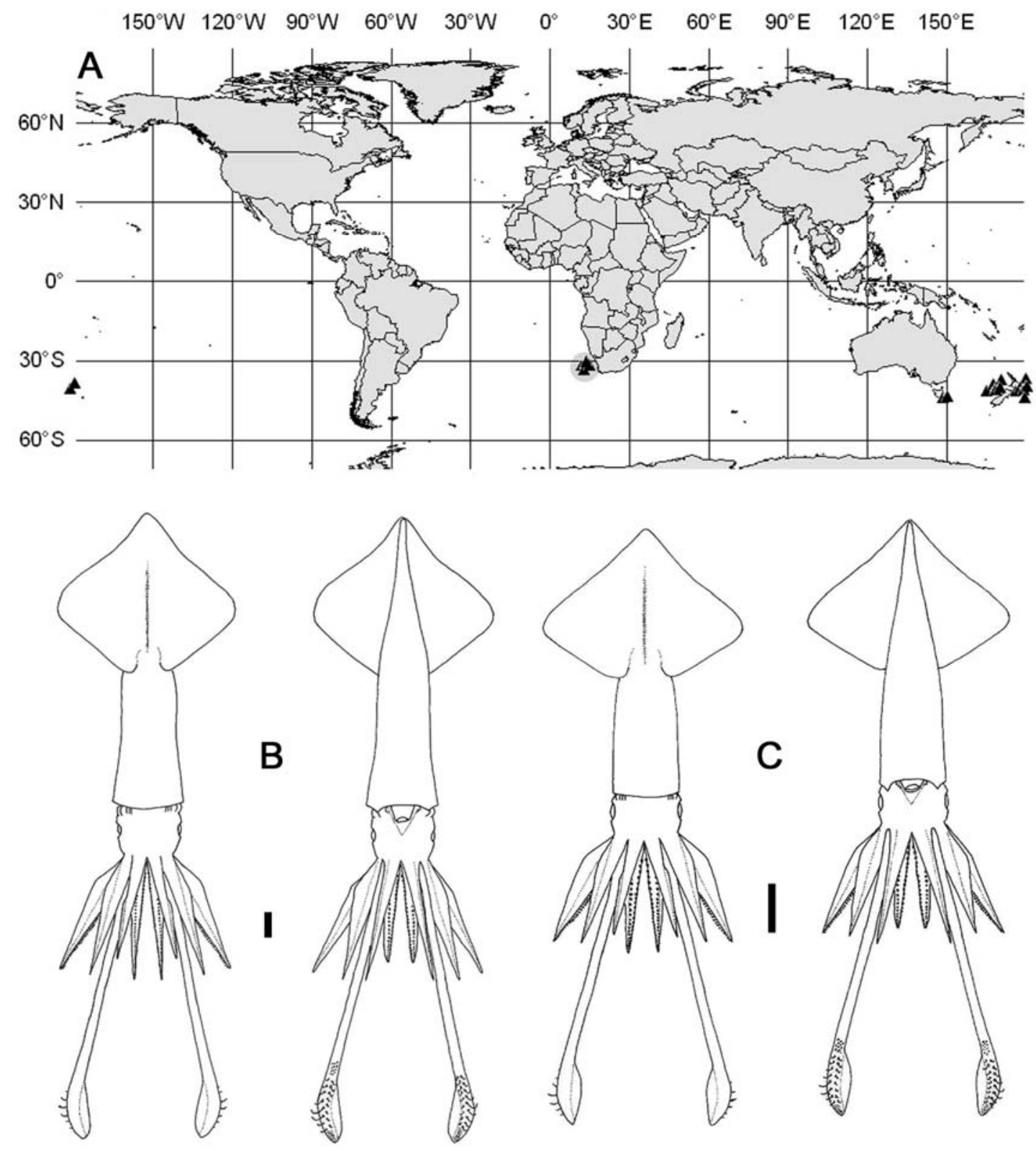

Fig. 56 - Notonykia africanae. A) distribution (grey circle indicates type locality); B) NIWA 32781, ô, 122mm; C) NMNZ M.74426, sex indet., ML 43mm. Scale bars = $10 \mathrm{~mm}$. 
Lateral profile of lower beak (Figs 58B-D, ML 177mm) longer than deep, with hood length $\sim 40 \%$ crest length, and hood and crest together $\sim 70 \%$ baseline; jaw angle obscured by low wing fold; rostrum nearly straight. Crest narrow, thickened from lateral fold to crest; lateral walls not indented below crest; angle ridge extending over $\sim 80 \%$ of distance to lateral wall margin; wing length $\sim 250 \%$ LRL; wing width distally $\sim 150 \%$ that level with LRL; lateral wall with small, thickened fold and ridge anteriorly, reducing posteriorly to slight fold and thickening.

Radula (Fig. 58E) with slender, unicuspid to slightly tricuspid rachidian, with distal corners of base forming small lateral cusps $\sim 25 \%$ mesocone height. First lateral tooth comparable to rachidian in size or slightly taller; unicuspid; triangular or slightly curved in shape, with straight medial margin and concave lateral margin. Second lateral tooth simple, curved, height nearly $150 \%$ that of rachidian. Marginal tooth long, at least $200 \%$ rachidian height, slender, nearly straight. Palatine palp (Fig. 58F) with $\sim 45$ short, stout teeth, each $80-170 \%$ rachidian height, flattened or slightly curved away from palp, sparsely arranged in no discernible pattern.

Gladius (Fig. 58I) broad, greatest width $\sim 10 \% \mathrm{GL}$; free rachis $\sim 18 \% \mathrm{GL}$; vanes broaden quickly and taper gradually between 20 and $80 \%$ GL; keel not solidified until distal $20 \%$ GL. Rostrum narrow, $\sim 5 \%$ GL, oval in cross-section, projecting dorsally from conus at slight angle, with tip of conus forming distinct, separate point ventral to rostrum.

Mantle colouration (preserved) deep bluish-purple, darkest along dorsal median line; 'tail-spots' (seen in juveniles) not apparent above ML $\sim 50 \mathrm{~mm}$. Small, dark chromatophores densely cover dorsal and ventral surfaces of fins, funnel and head, with those on the head darkest dorsally in several overlapping layers, paling and thinning laterally and ventrally. Arms darkest aborally, paling laterally; larger, dark, individual chromatophores present on oral face of arms, between suckers. Tentacles with chromatophores over aboral and lateral surfaces of stalk, present orally only on manus.

Earlier ontogenetic stages (ML 16-43mm, Figs 57A, 58G, 58H) differ from the above as follows. Mantle conical, width $25-30 \% \mathrm{ML}$ at $\mathrm{ML}>20 \mathrm{~mm}$, to slightly bulbous at $\mathrm{ML}$ 16-18mm, width 30-40\% ML. Fins rhombic, length 50-55\% ML, width $~ 75 \% \mathrm{ML}$ at ML $>20 \mathrm{~mm}$; at $\mathrm{ML} 16-18 \mathrm{~mm}$, width $\sim 65 \% \mathrm{ML}$, length $\sim 30 \% \mathrm{ML}$, rounded, lacking apparent anterior lobes. Head squarish at $\mathrm{ML}>20 \mathrm{~mm}$, rounded at $\mathrm{ML} 16-18 \mathrm{~mm}$. Arms 
$25-45 \% \mathrm{ML}$ at $43 \mathrm{~mm}, 20-30 \% \mathrm{ML}$ at $\mathrm{ML} 16-18 \mathrm{~mm}$, each with $60-80$ suckers; keel on Arms II $\sim 30 \%$ arm depth along entire arm length, keel on Arms III $\sim 100 \%$ arm depth at $30 \%$ arm length, thereafter $75 \%$ arm depth; lateral membrane on Arms IV 60\% arm width at bases, narrowing at $30 \%$ arm length to $50 \%$ arm width. Tentacles $\sim 70 \% \mathrm{ML}$ at $\mathrm{ML}>20 \mathrm{~mm}, \sim 40 \% \mathrm{ML}$ at ML 16-18mm; clubs slightly expanded, $230 \% \mathrm{ML}(\sim 40 \%$ $\mathrm{TnL})$ at $\mathrm{ML}>20 \mathrm{~mm}, \sim 20 \% \mathrm{ML}(\sim 50 \% \mathrm{TnL})$ at $\mathrm{ML} 16-18 \mathrm{~mm}$; carpus not well defined at $\mathrm{ML} 18 \mathrm{~mm}$; first ventral hooks begin to develop by $\mathrm{ML} 18.5 \mathrm{~mm}$; all hooks formed by $\mathrm{ML}$ $31-36 \mathrm{~mm}$. Tentacular suckers round proximally, squarish distally (Fig. 58G), with small circular aperture (its diameter $\sim 25 \%$ overall sucker diameter), situated slightly proximally; marginal suckers with five low, blunt teeth present around distal inner margin. Proximal pegs small, circular to polygonal, densely set in four concentric rows; distal pegs polygonal, very densely set in seven or eight concentric rows, with those in second to fourth rows largest. Dorsal keel width equal to club width along entire manus; ventral membrane width $\sim 30 \%$ club width, also present along entire manus. Dorsal surface of mantle (preserved) dark purple-red or bluish purple medially, paling laterally; ventral surface pale, with double tail spots clearly visible ventral to fins.

Remarks - Very little is known of the biology or ecology of $N$. africanae. It is one of the most recently described onychoteuthid taxa and has been reported only three times in the literature. It appears to be common in subtropical waters off South Africa and New Zealand. 


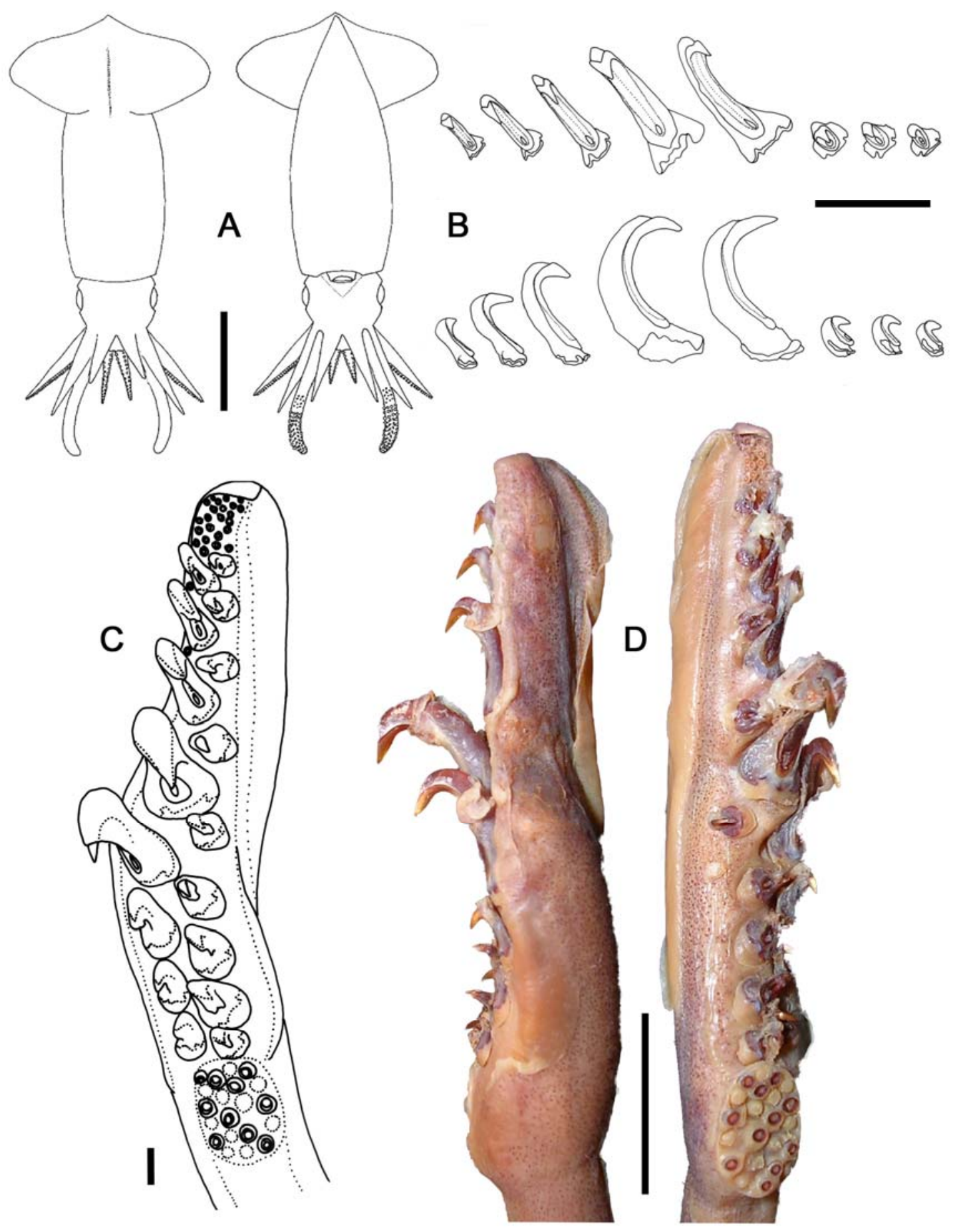

Fig. 57 - Notonykia africanae. A) NMNZ M.183104, sex indet., ML 18mm; B) NMNZ M.71009 ô, ML 102mm; C) NIWA 32782, ô, ML 80mm; D) NMNZ M.160475, , , ML $177 \mathrm{~mm}$. A) whole animal, B) ventral hook series from left tentacle club, C) right tentacle club, D) left tentacle club. Scale bars = A) $5 \mathrm{~mm}$; B, D) $10 \mathrm{~mm}$; C) $1 \mathrm{~mm}$. 

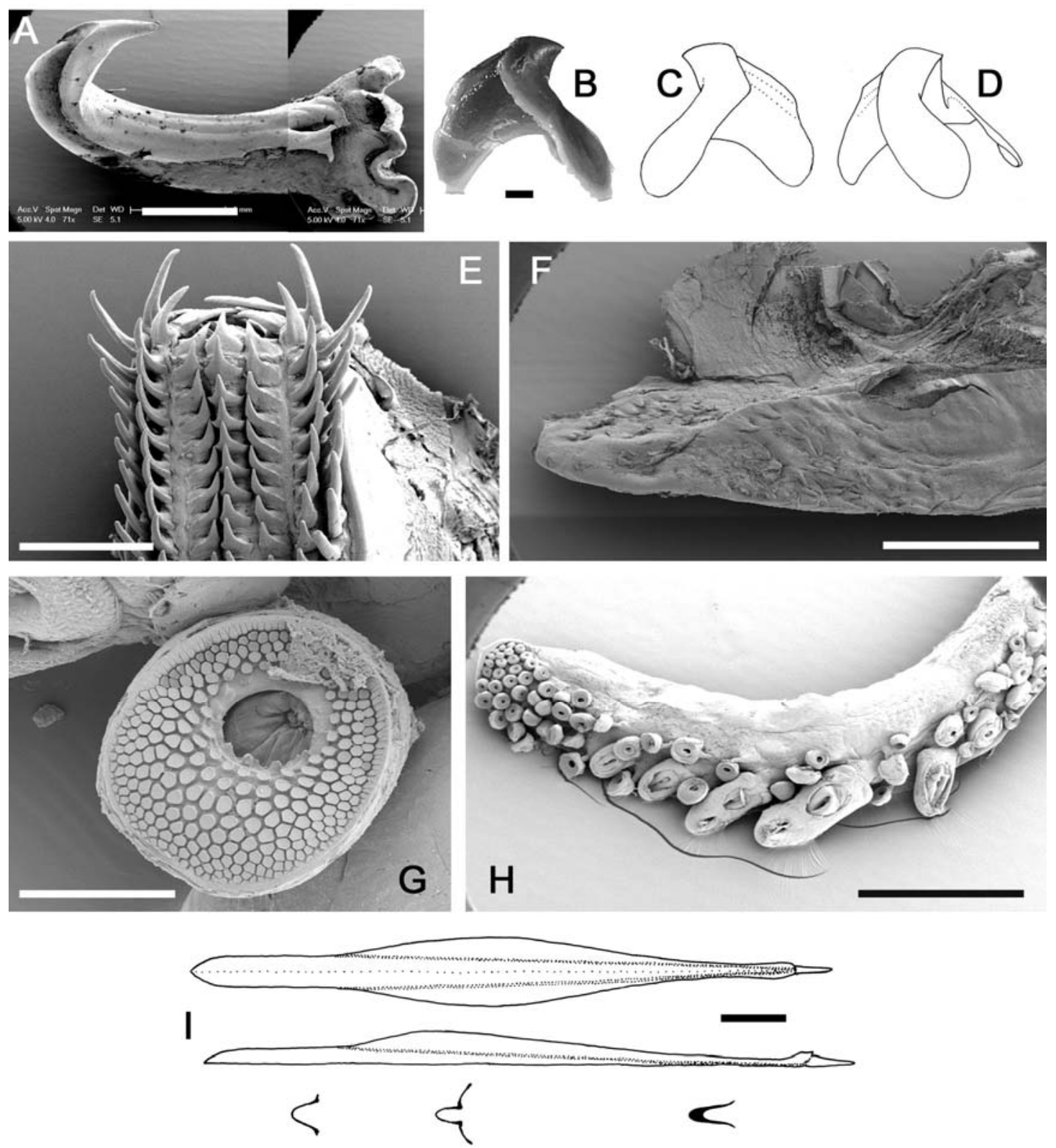

Fig. 58 - Notonykia africanae. A) NMNZ M.71009, ô, ML 102mm; B-D) NMNZ M.160475, , ML 177mm; E, F, I) NMNZ M.91652, , ML 92mm; G, H) NMNZ M.183104, sex indet., ML 22mm. A) left tentacular hook V5; B-D) lower beak: (B) left, (C) right (D) left oblique profiles; E) radula; F) palatine palp; G) marginal tentacular sucker; $H$ ) right tentacle club; I) gladius. Scale bars = A-C, F, H) 1mm; E) 2mm; G) $500 \mu \mathrm{m} ; \mathrm{H}) 100 \mu \mathrm{m}$; I) $10 \mathrm{~mm}$. 
Notonykia nesisi Bolstad, 2007 (Table 23, Figs 59, 60)

Notonykia nesisi Bolstad, 2007 - 327-333, Tables 5, 6, Figs 51-60.

Ancistroteuthis lichtensteinii (not Férussac, 1835) Pacific form — Kubodera et al. (1998): 282;

Nesis et al. (1998): 164.

Type material examined - NMNZ M.91597, holotype, + , $85 \mathrm{~mm}, 46^{\circ} 06.80^{\prime} \mathrm{S}, 171^{\circ} 52.00^{\prime} \mathrm{E}, 30 \mathrm{~m}$ over 1333m, 10/05/1985, FRV James Cook; NMNZ M.183110, paratypes, 3, ML 65, $61,59 \mathrm{~mm}, 46^{\circ} 06.80^{\prime} \mathrm{S}, 171^{\circ} 52.00^{\prime} \mathrm{E}, 30 \mathrm{~m}$ over $1333 \mathrm{~m}, 10 / 05 / 1985$, FRV James Cook. Additional material examined (26 specimens) - NIWA 32727, sex indet., ML 45mm, 41 ${ }^{\circ} 14^{\prime} \mathrm{S}$, 176 17 'E, 100-20m over 1202-1131m, -/02/1998, RV Tangaroa Stn TAN9802/179, FMMWT, Z10142; NMNZ M.183112, sex indet., $10 \mathrm{~mm}$, 42 $2^{\circ} 26.40^{\prime} \mathrm{S}, 174^{\circ} 44.20^{\prime} \mathrm{E}, 50 \mathrm{~m}$, 23/02/2000; NIWA 32733, sex indet., ML 60mm, 42³5'S, $178^{\circ} 00^{\prime} \mathrm{E}, 100-20 \mathrm{~m}$ over 1420m, -/02/1998, RV Tangaroa Stn TAN9802/190, FMMWT, Z10314; NMV F55012,

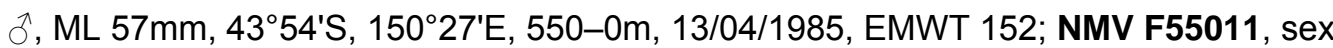

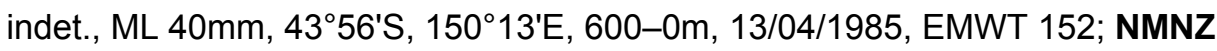

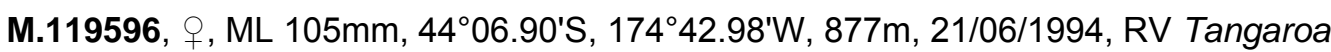
Stn TAN9406/223; NIWA 32769, sex indet., ML 57mm, 443․ $38^{\prime} \mathrm{S}, 177^{\circ} 32.33^{\prime} \mathrm{W}, 700$ 300m, 23/02/1992, RV Tangaroa Stn TAN9202/95, FMMWT, Z8774; NMNZ M.94091 (2 specimens), ô, ML $71 \mathrm{~mm}$, $+, 48 \mathrm{~mm}, 46^{\circ} 03.5^{\prime} \mathrm{S}, 171^{\circ} 37.30^{\prime} \mathrm{E}, 30 \mathrm{~m}$ over $1318 \mathrm{~m}$, 09/05/1985, FRV James Cook Stn J7/20/85; NMNZ M.160463, 5 sex indet., ML 63$41 \mathrm{~mm}, 46^{\circ} 01.90^{\prime} \mathrm{S}, 165^{\circ} 54.40^{\prime} \mathrm{E}$, Chalky Inlet, 30m, 06/03/1988, FRV James Cook Stn J4/38/88; NMNZ M.91466, 2 q, ML 57, 52mm, 46ํำ.80'S, $172^{\circ} 05.10^{\prime} \mathrm{E}, 25 \mathrm{~m}$ over 1400-1395m, 10/05/1985, FRV James Cook; NMNZ M.94090, ๆ, ML 84mm, 46 $48.50^{\prime}$ S, $166^{\circ} 46.10^{\prime} \mathrm{E}, 32 \mathrm{~m}$ over 945m, 08/05/1985, FRV James Cook; NMNZ

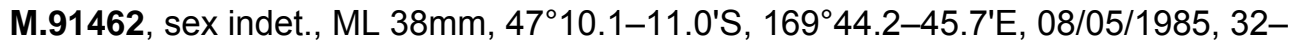
30m over 670-661m, FRV James Cook Stn J7/10/85; NMV F55015, 7 sex indet., ML 77-35mm, 47ำ'S, $149^{\circ} 15^{\prime} \mathrm{E}, 1444-0 \mathrm{~m}, 23 / 03 / 1986$, EMWT 152; NMNZ M.91706, sex indet., ML 40mm, $47^{\circ} 24.2-25.3^{\prime} \mathrm{S}, 170^{\circ} 02.5-03.8^{\prime} \mathrm{E}$, 30m over $1040-998 \mathrm{~m}$, 09/05/1985, James Cook Stn J7/13/85, FMWWT.

Distribution (Fig. 59A) - Sub-Antarctic, primarily over and south of Chatham Rise, off the coast of New Zealand, 950-0m, most commonly in the upper 30m. No confirmed distribution records outside the South Pacific.

Diagnosis - Neck of largest ventral tentacular hooks slender, straight in lateral profile, forming overall 'J' shape; carpus with seven to nine suckers; tentacular hooks D1 and D2 noticeably larger than hooks V1 and V2. 
Description (Figs 59B-60H) - Mantle muscular, width 22\% ML, nearly cylindrical anterior to fins, very slightly flared at anterior margin, widening slightly at $\sim 40 \% \mathrm{ML}$; mantle very narrow ventrally over posterior $60 \%$ of fin length; gladius visible in dorsal midline only as dark line over medial $\sim 60 \%$ fin length, not visible ventrally through skin; posterior portion of mantle ( $17 \% \mathrm{ML})$ with low, solid, fleshy median ventral keel, widening into soft bulbous 'blister' in posterior $10 \% \mathrm{ML}$. Fins rhombic, length $\sim 57 \% \mathrm{ML}$, width $\sim 70 \% \mathrm{ML}$; posterior fin margin slightly concave medially, becoming slightly convex laterally; anterior margin slightly convex.

Head rounded, length 16-22-24\% ML, width 20\% ML, depth 15\% ML; occipital region with three small secondary dorsal folds; ventral-most primary fold strong, well defined, triangular, adjoining posterior border of funnel groove; funnel set in deep, welldefined V-shaped groove; aperture level with posterior $30 \%$ of eye, anterior point of groove level with midpoint of eye; funnel groove bordered by low muscular ridge; posterior end of ridge widened into ventral-most primary occipital fold; funnel component of locking apparatus 10-15\% ML; mantle component $\sim 20 \% \mathrm{ML}$.

Arms $25-33_{-}-42_{I I-I V}-52 \% \mathrm{ML}$, formula $I I|>||=| \mathrm{V}>\mid$ or $I I I=||>|\mathrm{V}>|$, each with $\sim 70$ suckers (ML 85-105mm), bordered by trabeculate protective membrane; keels present on Arms I-III: that on Arms I present from $25 \%$ arm length to arm tip, attaining $75 \%$ arm width at $50 \%$ arm length; that on Arms II $\sim 75 \%$ arm depth along proximal $75 \%$ of arm, thereafter narrowing to tip; that on Arms III to $\sim 150 \%$ arm depth at $40 \%$ arm width; lateral membrane on Arms IV $\sim 100 \%$ arm width from base to tip.

Tentacles $75-90-119 \% \mathrm{ML}$, base diameter narrower than that of adjacent arm bases. Club length $\sim 30 \% \mathrm{ML}$ (25-35\% TnL), slightly expanded (Figs 60A, 60B); carpus with seven to nine suckers (most commonly seven or eight); manus with 17 or 18 hooks (Figs 59E, 60C); terminal pad with 31-38 small suckers set in diagonal rows of four. One or two distal marginal suckers present along ventral hook series through at least ML $177 \mathrm{~mm}$. Hooks D1 and D2 noticeably larger than V1 and V2, D3 and V3 subequal; thereafter ventral hooks much larger than dorsal, with hooks V5 and V6 largest of all (Fig. 60C), about five times longer than paired dorsal hooks and 20-24\% CL; D4 and D5 smaller than D1-D3 and slightly offset toward centre of club; largest hooks approximately ' $\mathrm{J}$ '-shaped in lateral profile, with straight neck, and claw recurved toward hook base at right or somewhat acute angle. Ventral membrane $\sim 30 \%$ club width along entire manus; dorsal membrane $\sim 15 \%$ club width along hooks D1-D3; dorsal keel nearly $\sim 100 \%$ club width from hook D3 to tip of club. 
Table 23. Measures $(\mathrm{mm})$ and counts of Notonykia nesisi Bolstad, 2007 (arm and tentacle measures recorded from more complete side of specimen, indicated $\mathrm{R}$ or $\mathrm{L}$ ).

\begin{tabular}{|c|c|c|c|c|c|c|c|c|c|c|}
\hline Specimen ID & $\begin{array}{c}\text { NMNZ } \\
\text { M.91597 }\end{array}$ & $\begin{array}{c}\text { NMNZ } \\
\text { M.183110 }\end{array}$ & $\begin{array}{c}\text { NMNZ } \\
\text { M.183110 }\end{array}$ & $\begin{array}{c}\text { NMNZ } \\
\text { M.183110 }\end{array}$ & $\begin{array}{c}\text { NMNZ } \\
\text { M.119596 }\end{array}$ & $\begin{array}{c}\text { NMNZ } \\
\text { M.91466 }\end{array}$ & $\begin{array}{c}\text { NMNZ } \\
\text { M.91466 }\end{array}$ & $\begin{array}{c}\text { NMNZ } \\
\text { M.160463 }\end{array}$ & \multicolumn{2}{|c|}{$\begin{array}{c}\text { Mean } \\
\text { Indices }\end{array}$} \\
\hline Type Status & Holotype & Paratype & Paratype & Paratype & None & None & None & None & & \\
\hline ML & 85 & 65 & 61 & 59 & 105 & 57 & 51 & 41 & (ML 51 & $-105)$ \\
\hline Sex & $\mathrm{F}$ & $\mathrm{F}$ & $\mathrm{F}$ & $\mathrm{F}$ & $\mathrm{F}$ & $\mathrm{F}$ & $\mathrm{F}$ & Indet. & & \\
\hline MW & 16 & 14 & 14 & 13 & 23 & 13 & 11 & 12 & MWI & 22 \\
\hline $\mathrm{HL}$ & 14 & 15 & 14 & 13 & 21 & 13 & 12 & 9 & HLI & 22 \\
\hline HW & 16 & 15 & 13 & 12 & $13^{*}$ & 11 & 9 & 11 & HWI & 20 \\
\hline FL & 50 & 37 & 34 & 32 & 60 & 32 & 29 & 25 & FLI & 57 \\
\hline FW & 58 & 46 & 44 & 40 & 72 & 41 & 35 & 38 & FWI & 70 \\
\hline Arms I & $29 L$ & $16 \mathrm{~L}$ & $19 \mathrm{~L}$ & $19 \mathrm{~L}$ & $44 \mathrm{~L}$ & $18 \mathrm{~L}$ & $18 \mathrm{R}$ & $17 \mathrm{R}$ & A1I & 33 \\
\hline Arms II & $36 \mathrm{~L}$ & $25 \mathrm{~L}$ & $22 \mathrm{~L}$ & $23 \mathrm{~L}$ & $53 \mathrm{~L}$ & $24 \mathrm{~L}$ & $22 R$ & $22 R$ & A2I & 42 \\
\hline Arms III & $44 \mathrm{~L}$ & $25 \mathrm{~L}$ & $24 \mathrm{~L}$ & $25 \mathrm{~L}$ & $52 \mathrm{~L}$ & $23 \mathrm{~L}$ & $22 \mathrm{R}$ & $21 R$ & A3I & 44 \\
\hline Arms IV & $36 \mathrm{~L}$ & $25 \mathrm{~L}$ & $22 \mathrm{~L}$ & $22 \mathrm{~L}$ & $49 \mathrm{~L}$ & $23 \mathrm{~L}$ & $21 \mathrm{R}$ & $18 \mathrm{R}$ & A4I & 40 \\
\hline TnL & $84 \mathrm{~L}$ & $49 \mathrm{~L}$ & $52 \mathrm{~L}$ & $51 \mathrm{~L}$ & $125 \mathrm{~L}$ & $50 \mathrm{~L}$ & $41 R$ & $40 R$ & TnLI & 90 \\
\hline $\mathrm{CL}$ & $24 \mathrm{~L}$ & $18 \mathrm{~L}$ & $19 \mathrm{~L}$ & $18 \mathrm{~L}$ & $34 \mathrm{~L}$ & $17 \mathrm{~L}$ & $15 R$ & $14 \mathrm{R}$ & CLI & 30 \\
\hline CS & $7 \mathrm{~L}$ & 8 & 8 & 8 & 7 & 7 & 7 & 8 & & \\
\hline MH & 18 & 18 & 17 & 17 & 18 & 18 & 18 & 17 & & \\
\hline MS & V8, 9 & V7-9 & V6-8 & V6-8 & V8, 9 & V7-9 & V7-9 & * & & \\
\hline TPS & $33 \mathrm{~L}$ & 34 & 38 & 33 & 34 & 33 & 31 & $33 R$ & & \\
\hline
\end{tabular}

* indicates damaged features.

Lateral profile of lower beak (Figs 60D, 60E, ML 105mm) with hood length $\sim 50 \%$ crest length, and hood and crest together $\sim 75 \%$ baseline; rostrum nearly straight; jaw angle obscured by low wing fold. Crest moderately broad, thickened from lateral fold to crest; lateral walls not indented below crest; angle ridge extends over $\sim 60 \%$ of distance to lateral wall margin; wing length $\sim 200 \%$ LRL, distal width $\sim 140 \%$ that level with jaw angle; lateral wall with small fold and ridge anteriorly, broadly thickened, reducing posteriorly to flat, broad thickening.

Radula (Fig. 60F) with slender, unicuspid rachidian. First lateral tooth subequal to rachidian; triangular, unicuspid; medial margin straight, lateral margin concave from base to tip. Second lateral tooth simple, curved, height nearly $150 \%$ that of rachidian. Marginal tooth long, at least $200 \%$ rachidian height, slender, nearly straight. Palatine palp (Fig. 60G) with $\sim 45$ short, sparsely distributed teeth, each $40-150 \%$ rachidian height, flattened or slightly curved away from palp.

Gladius (Fig. $60 \mathrm{H})$ with greatest width ( $7 \% \mathrm{GL}$ ) attained within anterior $30 \% \mathrm{GL}$; free rachis $\sim 28 \% \mathrm{GL}$; vanes broaden rapidly to $\sim 30 \% \mathrm{GL}$, thereafter tapering gradually to $80 \% \mathrm{GL}$; keel not thickened until posterior $20 \% \mathrm{GL}$; rostrum narrow, short, $<5 \% \mathrm{GL}$, oval in cross-section, with conus projecting dorsally from rostrum at slight angle, with tip forming distinct, separate point ventral to rostrum.

Dorsal surface of mantle and fins (preserved) purple, with pink tissue overlaid by many small, dark purple chromatophores, ventral surface paler; fins darkest medially and 

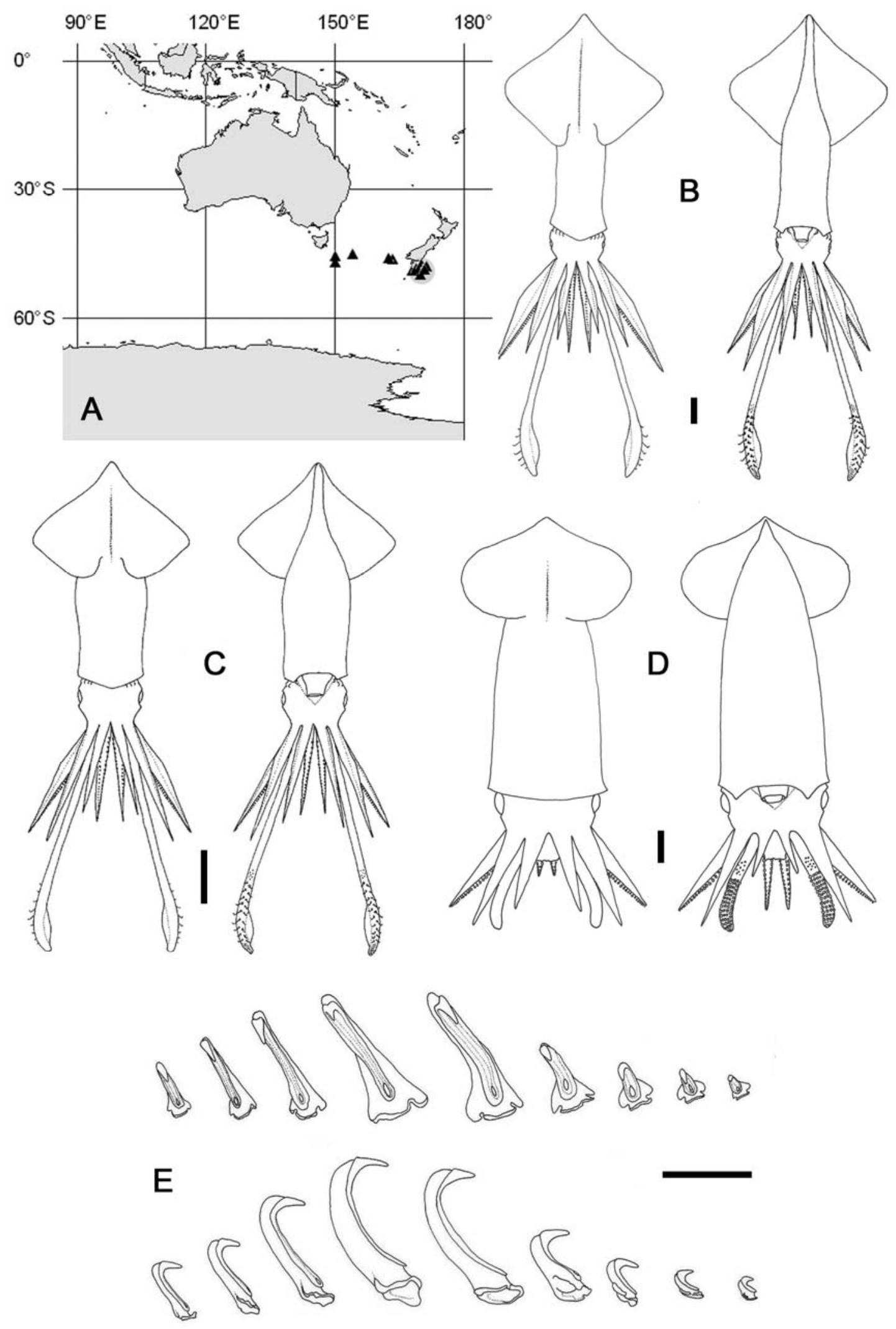

Fig. 59 - Notonykia nesisi. A) distribution (grey circle indicates type locality); B, E) NMNZ M.91597, holotype,, , 85mm; C) NMNZ M.160463, sex indet., ML 41mm; D) NMNZ M.183112, sex indet., ML 10mm. B-D) whole animal; E) ventral hook series. Scale bars = B, C, E) $10 \mathrm{~mm}$; D) $1 \mathrm{~mm}$. 
paling laterally; ventral surface with small pale chromatophores over lateral portions of fins. Dorsal surface of head slightly iridescent, with dark chromatophores in several layers; ventral surface paler. Arms darkest aborally but with chromatophores present over all surfaces of arms. Tentacles with chromatophores on all surfaces; club with chromatophores on all surfaces apart from oral faces of lateral membranes.

Earlier ontogenetic stages (ML 33-44mm, Fig. 59C) differ from the above description as follows. Mantle nearly conical, bulging midway along length to $\sim 30-35 \% \mathrm{ML}$. Fins rhombic, width $80-90 \% \mathrm{ML}$, length $50-60 \% \mathrm{ML}$, somewhat drawn out posteriorly. All occipital folds present by $\mathrm{ML} 33 \mathrm{~mm}$, with eyes slightly anteriorly directed, becoming laterally directed by $41 \mathrm{~mm}$; funnel component of locking apparatus $15-20 \% \mathrm{ML}$; mantle component 25-30\% ML. Arms at ML 41 mm 40-55\% ML, each with 70 suckers bordered by trabeculate protective membrane; keels absent from Arms I; keels on Arms II $\sim 30 \%$ arm depth along entire arm length; keels on Arms III to $\sim 75 \%$ arm depth at 30\% arm length; lateral membranes on Arms IV 30\% arm width from base to tip of arms. Tentacles $90-100 \%$ ML, diameter at bases comparable to that of adjacent arm bases. Clubs unexpanded or only slightly expanded, $\sim 35 \% \mathrm{ML}$ and $\mathrm{TnL}$; six to eight carpal suckers present; terminal pad with 33-36 small suckers in diagonal rows of four; manus at ML $41 \mathrm{~mm}$ with 16 hooks. Dorsal surface of mantle with large, irregular, faded red chromatophores; posterior $30 \%$ of ventral mantle surface marked with distinctive diamond pattern of $\sim 16$ small, dark red-brown chromatophores; two pairs of small, dark brown-black 'tail-spots' present along mantle posteriorly, beneath fins; chromatophores not visible on fins; lateral surfaces of mantle faintly iridescent.

Paralarva (ML 10mm, Fig. 59D) with conical mantle, width 35\% ML at anterior margin; tip of mantle forms small ventral tail, projecting ventrally, away from fins. Fins heartshaped, small, length $\sim 25 \% \mathrm{ML}$, width $\sim 50 \% \mathrm{ML}$; anterior and posterior margins convex. Head rounded, width $\sim 30 \% \mathrm{ML}$, depth $\sim 25 \% \mathrm{ML}$, length $\sim 20 \% \mathrm{ML}$; occipital folds not apparent. Eyes anteriorly directed; funnel groove well defined, V-shaped. Arms 30-35\% ML, I-III subequal; Arms IV markedly shorter, 15\% ML; each with 34 suckers. Tentacles of similar length and breadth to arms, with long clubs ( $65 \%$ tentacle length, $20-25 \% \mathrm{ML}$ ) comprised of four longitudinal rows of suckers.

Remarks - The overall morphology of $N$. nesisi is similar to that of $N$. africanae, and identification requires inspection of tentacular morphology; in specimens where the hooks are damaged, carpal sucker count is of taxonomic value. The lower beaks also differ, with the LRL longer relative to wing length in $N$. nesisi than in $N$. africanae. 

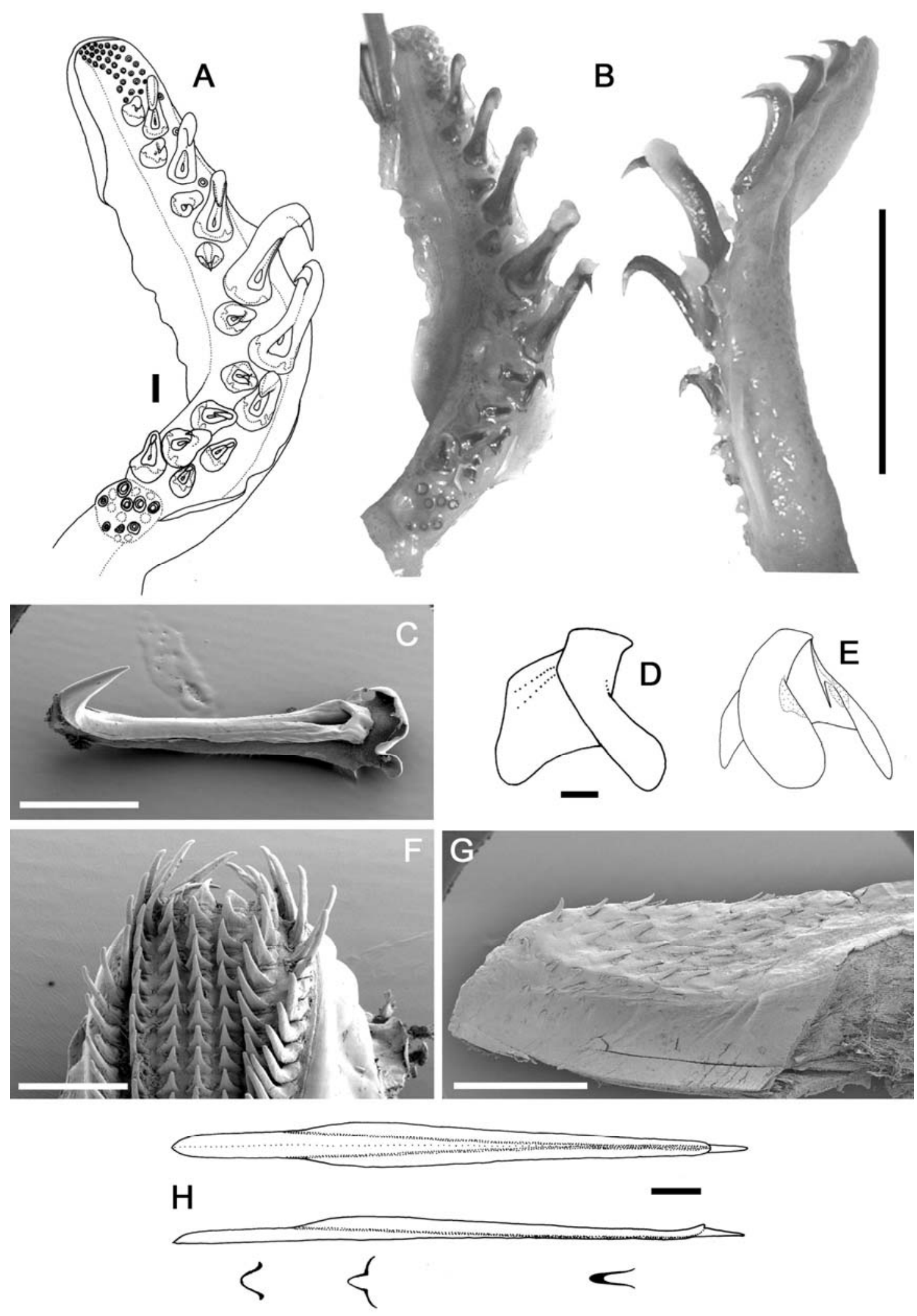

Fig. 60 - Notonykia nesisi. A) NMNZ M.183110, paratype, ๆ, ML 65mm; B)

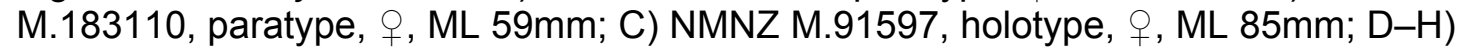
NMNZ M.119596,, , ML 105mm. A, B) left tentacle club; C) right tentacular hook V6; $D-F)$ lower beak: (D) left, (E) left oblique profiles; F) radula; $G$ ) palatine palp; $H$ ) gladius. Scale bars = A, C, D, G) $1 \mathrm{~mm} ; \mathrm{B}, \mathrm{H}) 10 \mathrm{~mm}$; F) $500 \mu \mathrm{m}$. 


\section{Gen. nov. 1}

Diagnosis - Occipital folds and photophores absent; skin of mantle, head and arms smooth; fins rhombic, length $\sim 60 \% \mathrm{ML}$, width $\sim 74 \% \mathrm{ML}$; head much narrower than mantle opening in subadults to adults; funnel groove U-shaped, without $Y$-shaped ridge; arms subequal, $~ 50 \% \mathrm{ML}$; bases of large ventral hooks asymmetrical; rostrum of gladius posteriorly directed, $\sim 15 \% \mathrm{GL}$; rachidian unicuspid.

Type species - Moroteuthis knipovitchi Filippova, 1972, by monotypy.

Remarks - Filippova (1972) initially placed Gen. nov. 1 knipovitchi in the genus Moroteuthis (= Onykia) based on the absence of photophores and secondary occipital folds, the proportions of the gladius, and the rounded funnel groove. While Gen. nov. 1 knipovitchi does appear allied to Onykia species in these respects, other character states are closer to those observed in the genera Onychoteuthis, Ancistroteuthis and Notonykia. These include the smooth skin of the mantle; the large, broad, wellmuscled fins; the relatively short arm length (all $\sim 50 \% \mathrm{ML}$ ); the asymmetrical bases of the largest ventral hooks; and the unicuspid rachidian tooth. Gen. nov. 1 knipovitchi is unique within the Onychoteuthidae in having all arms subequal (Arms I may be $\sim 5 \%$ shorter, and Arms IV 5\% longer, than Arms II and III).

Gen. nov. 1 knipovitchi has been included in three recent molecular studies. It was the only onychoteuthid species examined by Lindgren et al. (2004), but was one of eight examined by Bonnaud et al. (1998) and nine examined by Wakabayashi et al. (2007). The results in both of the latter studies placed Gen. nov. 1 knipovitchi outside the genus Onykia. Bonnaud et al. (1998) found that 'Onykia sp.' (= Gen. nov. 2 sp. 1, from Hawai'ian waters) was more closely related than Gen. nov. 1 knipovitchi to the three true Onykia species - Ok. (Ok.) 'robusta,' Ok. (M.) ingens, and Ok. (Ok.) robsoni and that Gen. nov. 1 knipovitchi was in fact most closely related to the Onychoteuthis group. Wakabayashi et al. (2007) also found that Gen. nov. 1 knipovitchi fell out with the Onychoteuthis clade rather than the 'Moroteuthis'/Onykia clade.

Since no genetic study has included all onychoteuthid species (or even all genera), it is impossible to fully evaluate the genetic position of Gen. nov. 1 knipovitchi within the Onychoteuthidae. However, Bonnaud et al. (1998) and Wakabayashi et al. (2007) concluded that Gen. nov. 1 knipovitchi was more distantly related to species in the genus Onykia than previously thought; in the former study it grouped with 
Onychoteuthis species, while in the latter, it grouped loosely with the Onykia species, but more distantly than did Gen. nov. 2.

Most onychoteuthid genera follow one of two body plans. Onychoteuthis, Ancistroteuthis, Notonykia and Gen. nov. 2 remain relatively small as adults, and possess smooth skin on the mantle, relatively large fins, and relatively short arms; all except Gen. nov. 2 possess secondary occipital folds; and Onychoteuthis and possibly Ancistroteuthis also possess photophores. Onykia and Kondakovia attain relatively large sizes, and possess rugose or otherwise sculptured skin on the mantle as adults (in all species where adults are known), relatively small fins, and relatively long arms and tentacles; these two genera lack photophores and secondary occipital folds. Gen. nov. 1 knipovitchi is morphologically anomalous within the family, in possessing character states allying it with, or intermediate between, each of the two general body plans: it attains adult sizes comparable to those attained by smaller Onykia species, and lacks secondary occipital folds and photophores, yet it also possesses large fins, short arms and smooth skin on the mantle in adults. Its gladius resembles the gladius of most Onykia species, while its tentacular hooks are asymmetrical, a character state associated with the smaller, smooth-skinned genera.

Gen. nov. 1 knipovitchi therefore does not fit within the morphological patterns considered to define any of the other onychoteuthid genera, and available genetic data corroborate its position somewhere between the two groups of related genera. At present the most useful designation for this species appears to be within a unique genus; however, lack of available material precludes a formal generic description at present. 
Gen. nov. 1 knipovitchi (Filippova, 1972) (new comb.) (Tables 11, 24, Figs 61, 62) ? Onychoteuthis ingens (not Smith, 1881) - Hoyle (1912): 281, 282, Fig. 9.

Moroteuthis knipovitchi Filippova, 1972: 392-395, Figs 2, 3; Clarke (1980): 107-111, Table 24, Figs 53, 56-67, 76, 78-80, (1986): 77, Fig. 33C; Toll (1982): 71, 72, PI. 7 Fig. C; Okutani \& Clarke (1985): 17, 18; Roper et al. (1984): 131, (1985): 186; Nesis (1987): 193, Fig. 49I; Engeser \& Clarke (1988): Fig. 6b-e; Filippova et al. (1997): 183, Fig. 109; Bonnaud et al. (1998): 1761-1770, Figs 5, 6; Kubodera et al. (1998): 283, Fig. 9; Sweeney \& Roper (1998): 572; Lindgren et al. (2004): 454-486, Table 3, Figs 1-6; Okutani (2005): 158; Wakabayashi et al. (2007) 959-965.

Moroteuthis knipovitch [sic] Filippova, 1972 - Wakabayashi et al. (2007) Table 2, Fig. 2.

Type material (not available for examination in this study) - ZMMGU holotype (registration unknown), đ, ML 212mm, Scotia Sea, 17/03/1965, 50-30m, RV Academician Knipovitch Stn 203, NFD.

Material examined ( 1 specimen) - USNM 1021102, + , ML 231, 61 ${ }^{\circ} 48.1^{\prime} \mathrm{S}, 59^{\circ} 30.88^{\prime} \mathrm{W}-$

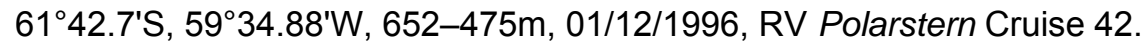

Unlocalised material examined (6 specimens) - BMNH Ref 1636, + , ML 320mm, Area IV, Antarctic Season 1947/48, Southern Harvester, Discovery Expedition 'WS,' pres. R. Clarke; BMNH 20070576, +, ML 315mm, NFD; IASOS HMK1, + , ML 315mm, Heard Island, NFD; BMNH 20070526, 2 , ML 275, 275mm, Antarctic, 1958-59, stomach of sperm whale; IASOS HMK2,, , ML 235mm, Heard Island, NFD.

Distribution (Fig. 61A) - Antarctic to sub-Antarctic waters. Depth unknown.

Diagnosis - As for genus.

Description (Figs 61B-62G) - Mantle conical or spindle-shaped, width 24-28-32\% $\mathrm{ML}$, muscular, thick-walled, attenuating beneath fins to broadly conical. Gladius not visible through dorsal mantle tissue; rostrum of gladius visible ventrally through posterior $\sim 10 \% \mathrm{ML}$. Fins rhombic, smooth, length $55-59-62 \% \mathrm{ML}$, width $\sim 74 \% \mathrm{ML}$; posterior margins straight laterally, becoming slightly concave medially; anterior margins straight, with small anterior lobes.

Head boxy, small, length 13-18-23\% ML, width and depth 14\% ML, with transverse dorsal occipital crest; not constricted anteriorly. Funnel slender; aperture opens at point below posterior eye margin; funnel groove broadly $\mathrm{U}$-shaped, without $\mathrm{Y}$-shaped ridge; funnel component of locking apparatus 9-15\% ML; mantle component 13-20\% ML. 
Table 24. Measures (mm) and counts of Gen. nov. 1 knipovitchi (Filippova, 1972) (new comb.) (arm and tentacle measures recorded from more complete side of specimen, indicated $\mathrm{R}$ or $\mathrm{L}$ ).

\begin{tabular}{|c|c|c|c|c|c|c|c|c|c|}
\hline Specimen ID & $\begin{array}{c}\text { BMNH Ref } \\
1636\end{array}$ & $\begin{array}{c}\text { BMNH } \\
20070576\end{array}$ & $\begin{array}{l}\text { IASOS } \\
\text { HMK1 }\end{array}$ & $\begin{array}{c}\text { BMNH } \\
20070526\end{array}$ & $\begin{array}{c}\text { BMNH } \\
20070526\end{array}$ & $\begin{array}{l}\text { IASOS } \\
\text { HMK2 }\end{array}$ & $\begin{array}{c}\text { USNM } \\
1021102\end{array}$ & \multicolumn{2}{|c|}{$\begin{array}{c}\text { Mean } \\
\text { Indices }\end{array}$} \\
\hline Type Status & None & None & None & None & None & None & None & & \\
\hline ML & 320 & 315 & 315 & 275 & 275 & 235 & 231 & & \\
\hline Sex & $\mathrm{F}$ & $\mathrm{F}$ & $\mathrm{F}$ & $\mathrm{F}$ & $\mathrm{F}$ & $\mathrm{F}$ & $\mathrm{F}$ & & \\
\hline MW & 90 & 100 & $85^{*}$ & 65 & 75 & $73^{*}$ & $59^{*}$ & MWI & 28 \\
\hline $\mathrm{HL}$ & 75 & 55 & 42 & $50^{*}$ & 40 & $34^{*}$ & 44 & HLI & 18 \\
\hline HW & 40 & 50 & 39 & 45 & 43 & 28 & 33 & HWI & 14 \\
\hline $\mathrm{FL}$ & 195 & 187 & 172 & * & * & 145 & 143 & FLI & 59 \\
\hline FW & 225 & 244 & 235 & * & * & $170^{*}$ & 150 & FWI & 74 \\
\hline Arms I & $140 \mathrm{~L}$ & $133 R$ & $128 \mathrm{~L}$ & $121 \mathrm{~L}$ & $120 \mathrm{~L}$ & $135 \mathrm{~L}$ & $133 \mathrm{~L}$ & A1I & 45 \\
\hline Arms II & $140 \mathrm{~L}$ & $145 R$ & $144 \mathrm{~L}$ & $130 \mathrm{~L}$ & $145 \mathrm{~L}$ & $149 \mathrm{~L}$ & $125 \mathrm{~L}$ & A2I & 50 \\
\hline Arms III & $140 \mathrm{~L}$ & $145 R$ & $147 \mathrm{~L}$ & $122 \mathrm{~L}$ & $142 \mathrm{~L}$ & $152 \mathrm{~L}$ & $110 \mathrm{~L}$ & A3I & 49 \\
\hline Arms IV & $155 \mathrm{~L}$ & $155 \mathrm{R}$ & $151 \mathrm{~L}$ & $130 \mathrm{~L}$ & $150 \mathrm{~L}$ & $154 \mathrm{~L}$ & $125 \mathrm{~L}$ & A4I & 52 \\
\hline TnL & $365 \mathrm{~L}$ & $240 R$ & $300 \mathrm{~L}$ & $210 \mathrm{~L}$ & $280 \mathrm{~L}$ & $300 \mathrm{~L}$ & $222 \mathrm{~L}$ & TnLI & 98 \\
\hline $\mathrm{CL}$ & $101 \mathrm{~L}$ & $107 R$ & $84 \mathrm{~L}$ & $92 \mathrm{~L}$ & $101 \mathrm{~L}$ & 87L & $82 \mathrm{~L}$ & CLI & 34 \\
\hline CS & 10 & 13 & 10 & 12 & 12 & $6^{*}$ & 11 & & \\
\hline MH & * & 30 & 27 & 28 & 31 & 26 & 25 & & \\
\hline MS & * & 0 & 0 & 0 & 0 & 0 & 0 & & \\
\hline TPS & * & 13 & 13 & * & $6^{*}$ & * & 17 & & \\
\hline
\end{tabular}

* indicates damaged features.

Arms $41-45,-50_{I I-I V}-66 \% \mathrm{ML}$, length subequal or with Arms IV marginally longer, muscular, robust along entire length, each with 90-100 suckers (ML 231-320mm), their diameter $40-50 \%$ arm width; oral faces bordered on either side by low protective trabeculate membrane. Keels present on Arms II and III, that on Arms II low, 10\% arm depth from base to tip; that on Arms III attaining 75\% arm depth at 30\% arm length, thereafter $20 \%$ arm depth; lateral membrane on Arms IV 15\% arm width over entire arm length.

Tentacles robust, $76-98-128 \% \mathrm{ML}$, with low aboral ridge along stalk; stalk thickness at base $\sim 75 \%$ that of adjacent arms and of fleshier consistency. Club (Fig. $61 \mathrm{C}$ ) slightly expanded, $27-34-37 \%$ ML (28-45\% TnL, mean 35\%). Carpus well-defined oval with ten to 13 suckers; manus with $24-31$ hooks (Figs 62A, 62B), with shallow lateral grooves nearly meeting distally; terminal pad with 13-17 suckers. Ventral hook V7 largest (Fig. 62A), its length three to four times that of $D 7$, and $\sim 12 \% \mathrm{CL}$; bases of larger ventral hooks asymmetrical, claw with . Ventral membrane $\sim 25 \%$ club width, flanking hooks V1-V4; Dorsal membrane damaged in examined specimens; dorsal keel $\sim 100 \%$ club width, flanking hooks D3-D13.

Lateral profile of lower beak (Figs 62E-G, ML $231 \mathrm{~mm}$ ) longer than deep, with crest nearly straight and sloping moderately steeply downward; posterior edge of hood close to crest; hood length $\sim 35 \%$ crest length, and hood and crest together $\sim 65 \%$ baseline; jaw edge straight; jaw angle obtuse, obscured by low wing fold. Crest moderately 
broad, rounded, not thickened; lateral walls slightly indented below crest; shoulder ridge distinct, rounded; angle ridge spans $\sim 50 \%$ lateral wall depth, indistinct by angle point; wings broaden somewhat distally, width $\sim 140 \%$ that at jaw angle, length $\sim 250 \%$ LRL; narrow insert of cartilage present on anterior margin of wings; lateral wall fold broad and shallow, indistinct by posterior margin of lateral wall.
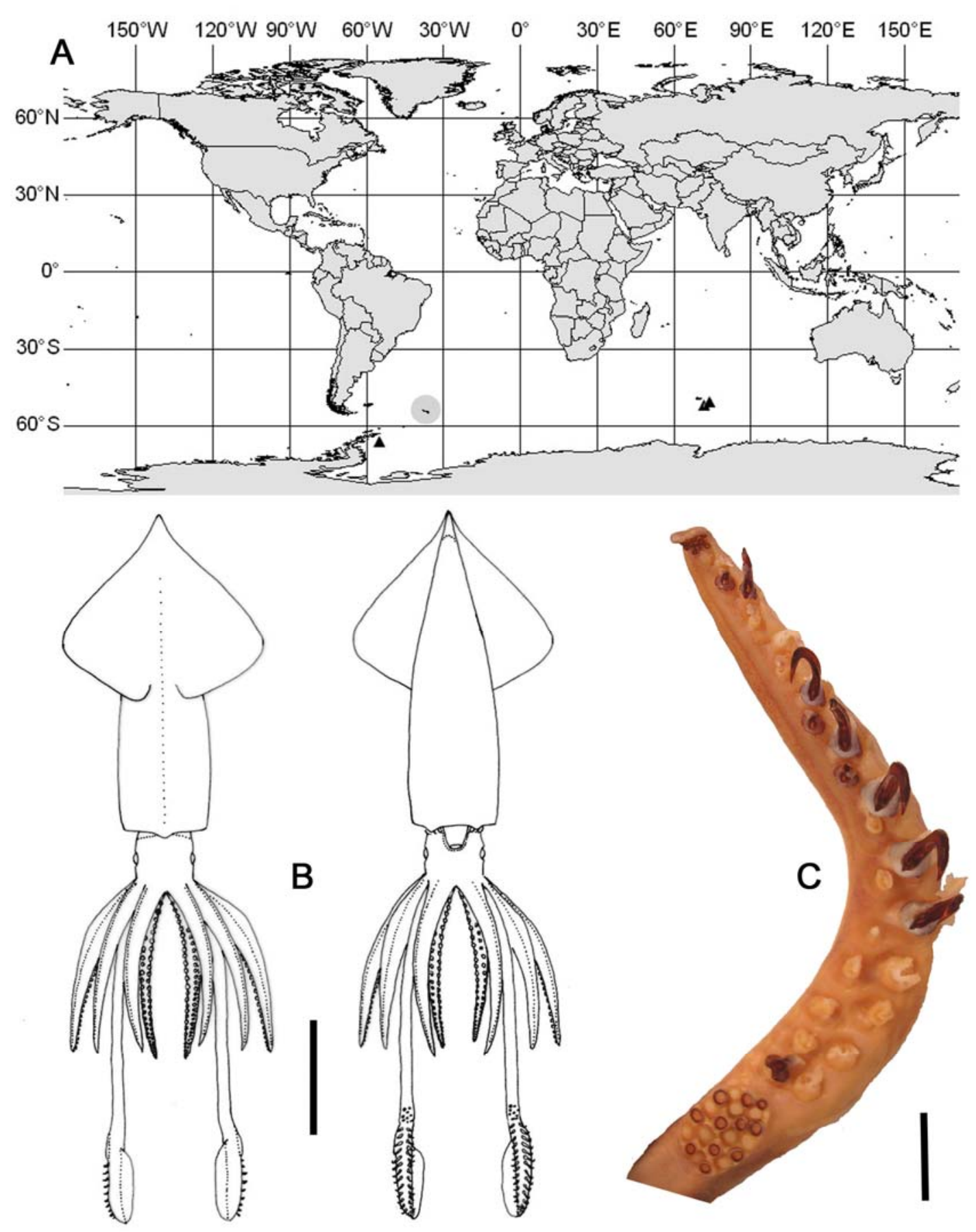

Fig. 61 - Gen. nov. 1 knipovitchi. A) distribution (grey circle indicates type locality); B, C) USNM 1021102, o, ML 231mm: (B) whole animal, (C) left tentacle club. Scale bars = B) $100 \mathrm{~mm}$; C) $10 \mathrm{~mm}$. 
Radula (Fig. 62C) with unicuspid rachidian; mesocone narrow, rounded distally; base squarish to rounded, $\sim 25-30 \%$ mesocone height. First lateral tooth forms approximate right triangle, $\sim 80 \%$ height of rachidian; inner margin straight, perpendicular to base; outer margin nearly straight, forming hypotenuse. Second lateral tooth robust, slightly curved, $\sim 150 \%$ height of rachidian. Marginal tooth simple, slightly curved, $\sim 200 \%$ height of rachidian. Palatine palp (Fig. 62D) with greatest width of tooth-bearing plane $\sim 25 \%$ of its length; with $\sim 65$ small, stubby, rounded teeth, each $120-150 \%$ rachidian height, sparsely distributed over surface of palp.
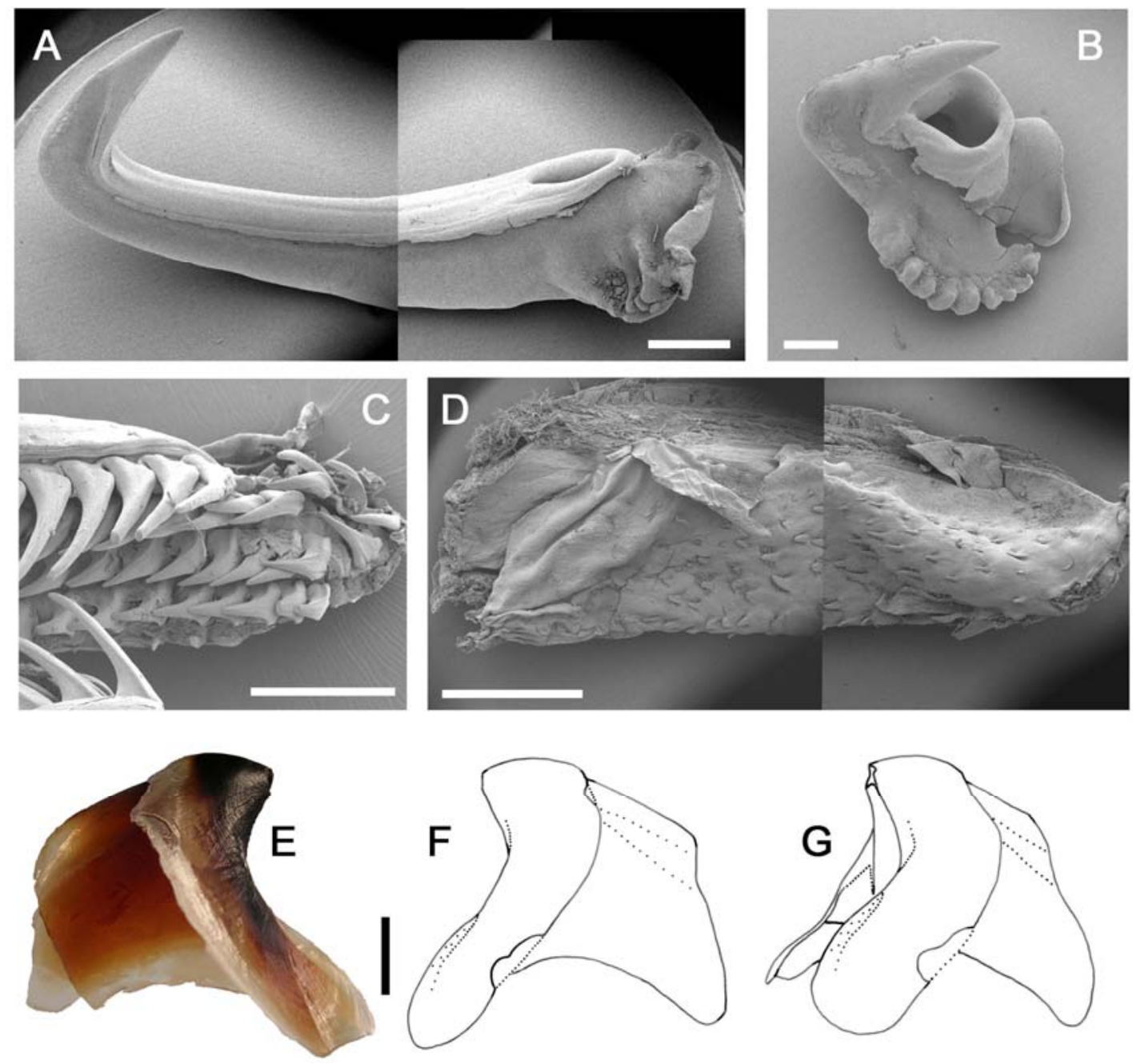

Fig. 62 - Gen. nov. 1 knipovitchi. A, B) USNM 1021102, क, ML 231mm; C-G) IASOS HMK1, , ML 315mm. A) right tentacular hook V7; B) right tentacular hook D1; C) radula, $D)$ palatine palp, $E-G)$ lower beak: $(E)$ left, $(F)$ right, $(G)$ right oblique view. Scale bars = A) $1 \mathrm{~mm}$; B, C) $500 \mu \mathrm{m}$; D) $2 \mathrm{~mm}$; E-G) $5 \mathrm{~mm}$. 
Remarks - Gen. nov. 1 knipovitchi is one of the most poorly represented onychoteuthids in collections (the number of publications citing this taxon far exceeds the number of specimens available for examination in this study), and specimens in good condition are extremely rare. As a consequence, information on several morphological features remains incomplete or could not be illustrated to the same detail as in other species. No gladius of the Gen. nov. 1 knipovitchi specimens examined was in sufficiently good condition to permit description; for completeness and comparison, the following description is based on Filippova (1972, Fig. 3): gladius with greatest width $(\sim 10 \% \mathrm{GL})$ attained at $\sim 40 \% \mathrm{GL}$; free rachis $\sim 18 \% \mathrm{GL}$; vanes broaden rapidly between 20 and $40 \%$ GL, gradually narrowing thereafter; dorsal keel robust, triangular; rostrum $\sim 15 \% \mathrm{GL}$, with tear-drop or triangular-shaped cross-section. 


\section{Gen. nov. 2}

Diagnosis - Squat-bodied squids to $M L>150 \mathrm{~mm}$; secondary occipital folds and photophores absent; epidermis of mantle smooth; fins ovate or paddle-shaped, with posterior fin margin variably produced beyond posterior tip of mantle; gladius visible as dark, broad ridge along dorsal midline; tentacle clubs with 24-28 hooks; rachidian strongly tricuspid; gladius with poorly defined free rachis (40-45\% GL) and elongaterhombic vanes, attaining greatest width within posterior $30 \% \mathrm{GL}$; rostrum of gladius low, rounded, dorsally directed.

Type species - 'Onykia' rancureli Okutani, 1981.

Remarks - Okutani (1981) originally attributed Gen. nov. 2 rancureli to Onykia, based on its overall external resemblance to juveniles of that genus (ovate to paddle-shaped fins, presence of marginal suckers on the tentacle club). However, its retention of these features into maturity, its small size, smooth skin and the unique elongaterhombic gladius separate it from Onykia.

Young et al. (2003b) observed that Walvisteuthis virilis (known from the South Atlantic and South Pacific (Nesis \& Nikitina 1986, 1992)) shares several of its distinguishing character states (ovate fins, rhombic gladius, broad protective membranes bordering the oral faces of some arms) with Gen. nov. 2 rancureli (known from the Indian Ocean), and suggested that Walvisteuthis virilis may be a junior synonym. However, several other characters/states reported for Walvisteuthis virilis are unlike those observed in Gen. nov. 2 or any other onychoteuthid to date, including the dentate arm suckers, the enlarged distal suckers on Arms III, and the club morphology (comprised solely of sparse, small, irregularly set suckers), and based on these features, Nesis \& Nikitina (1986) described Walvisteuthis virilis in its own new family, Walvisteuthidae. W. virilis is presently known from the holotype (adult male, ML $71 \mathrm{~mm}$ ) and one single other adult male in the USNM collections (ML and registration number unknown), lacking tentacle clubs, provisionally attributed to the genus (Young et al. 2003b). Neither specimen could be located prior to or during collection visits made for this study, although the holotype was later found and detailed photographs were supplied by ZMMGU (Fig. 63); the original illustrations (Nesis \& Nikitina 1986) also depict most of the salient morphological characters. 
Since $W$. virilis is known only from adult male specimens, while Gen. nov. 2 rancureli is known from subadult and spent females, and smaller individuals of both sexes, the morphological differences observed to date could conceivably represent sexual dimorphism. The unusual tentacle clubs on the $W$. virilis holotype may have been regenerating at the time of capture, since their overall appearance is similar to that of a regenerating club observed in Onykia robusta (Fig. 31D). However, given that the characteristic onychoteuthid hooks are absent from the holotype of $W$. virilis, while at least one character that would be unique within the Onychoteuthidae is present (the strongly enlarged distal suckers on Arms III), and that no material of $W$. virilis was available for examination in this study, Walvisteuthidae is retained as a separate family at present.

Gen. nov. 2 rancureli and putative spp. 1-3 are certainly onychoteuthids, but cannot be accommodated within any existing onychoteuthid genus as presently recognised. The most conservative course is to describe these species in a new onychoteuthid genus, pending additional material.

Tentacular club morphology varies widely among the limited number of Gen. nov. 2 specimens examined (see Figs 65A-D), suggesting that several morphological 'forms' different from Gen. nov. 2 rancureli (each known from single subadult specimens), occur in separate geographic regions outside the Indian Ocean. Further material is needed from all regions in order to clarify the systematic status of Gen. nov, 2 rancureli, the apparent other Gen. nov. 2 'forms,' and W. virilis.
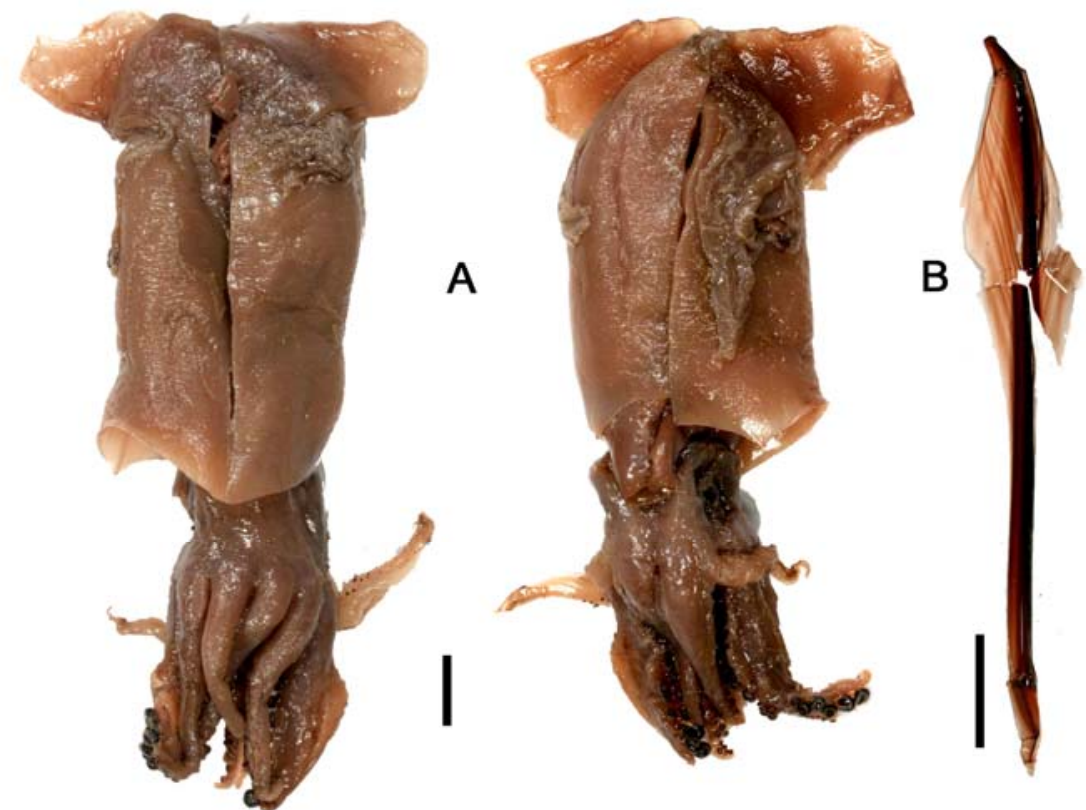

Fig. 63 - Walvisteuthis virilis, ZMMGU holotype, $\widehat{0}$, ML $71 \mathrm{~mm}$, photos by A. Syosoev. A) whole animal, B) gladius. Scale bars $=10 \mathrm{~mm}$. 
Gen. nov. 2 rancureli (Okutani, 1981) (new comb.) (Table 25, Figs 64A-C, 65A, 65E, 65F, 66A-C, 66J, 66M, 67D)

Onykia rancureli Okutani, 1981: 155-159, Table 1, Figs 1-9; Toll (1982): 63, 64, PI. 6G, H; Kubodera et al. (1998): 282, 283, Fig. 6; Sweeney \& Roper (1998): 572; Okutani (2005): 156.

Distribution (Fig 64A) - Indian Ocean, 1136-150m.

Type material examined - NSMT Mo59489, holotype, ${ }^{+}, \mathrm{ML} 71 \mathrm{~mm}, 07^{\circ} 51.5^{\prime} \mathrm{S}, 88^{\circ} 02.5^{\prime} \mathrm{E}$, 03/08/1975, RV Sagami-Maru sample Cl46, coll. K. Fujita; NSMT Mo59490, paratype,, , ML 46mm, $09^{\circ} 15^{\prime}$ S, $83^{\circ} 45^{\prime} \mathrm{E}, 28 / 07 / 1975$, RV Sagami-Maru, gut contents of $A$. ferox; NSMT Mo59491, paratype, ㅇ, ML 59mm, 09²1'S, 81ํㄴ'E, 06/08/1975, RV SagamiMaru sample Cl186, coll. K. Fujita; NSMT Mo59492, paratype, + , ML 65mm, 11¹6.2'S, 109²9.5'E, 25/12/1975, RV Shonan-Maru Sample No. El72, coll. K. Fujita.

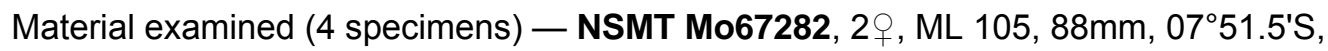
8802.5'E, 150-120m, 03/08/1975, RV Sagami-Maru, coll. K. Fujita, ex lancetfish (1.2m)

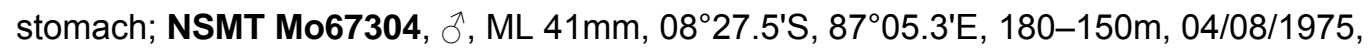
RV Sagami-Maru, coll. K. Fujita, ex lancetfish stomach; NMV F65985, ㅇ, 83mm, 29³5.4-34.0'S, $113^{\circ} 44.9-44.7^{\prime} \mathrm{E}, 115 \mathrm{~km}$ SW of Geraldton, Western Australia, $1136-$ 1132m, 07/02/1991, RV Southern Surveyor, coll. M.F. Gorman, trawl.

Unlocalised material examined (1 specimens) - NMV F51101,,+ ML 150mm, Victoria, Australia, NFD.

Diagnosis — Fin length $\sim 42 \% \mathrm{ML}$, fin width 93\% ML; tentacle clubs with $24-27$ robust, strongly recurved hooks, with curved necks in lateral profile; longest hooks $\sim 15 \%$ club length and about three times that of paired dorsal hooks; jaw angle of lower beak obscured by low wing fold in lateral profile; gladius elongate-rhombic, with poorly defined free rachis (40-45\% GL), attaining greatest width within posterior $30 \% \mathrm{GL}$.

Description (Figs 64B, 64C, 65A, 65E, 65F, 66A-C, 66J, 66M, 67D) - Mantle conical, muscular, width 32-36-38\% ML, remaining broad posteriorly to blunt tip; gladius visible dorsally as broad, dark ridge along midline; rostrum of gladius visible ventrally through distal $\sim 3 \% \mathrm{ML}$; mantle otherwise smooth ventrally. Fins broadly ovate in outline, length 32-42-49\% ML, width 70-93-100\% ML; posterior margins gently convex, anterior margins strongly convex; anterior lobes large, rounded.

Head squat, rounded, length 16-20-25\% ML, width 25\% ML, depth $~ 23 \% \mathrm{ML}$, not constricted anteriorly. Funnel broad, aperture at level below posterior margin of eye 
Table 25. Measures (mm) and counts of Gen. nov. 2 rancureli (Okutani, 1981) (new comb.) and Gen nov. 2 spp. 1-3 (measurements recorded from more complete side of specimen, indicated $\mathrm{R}$ or $\mathrm{L}$ ).

\begin{tabular}{|c|c|c|c|c|c|c|c|c|c|c|c|c|}
\hline Specimen ID & $\begin{array}{c}\text { NSMT } \\
\text { Mo59489 }\end{array}$ & $\begin{array}{c}\text { NSMT } \\
\text { Mo59492 }\end{array}$ & $\begin{array}{c}\text { NSMT } \\
\text { Mo59491 }\end{array}$ & $\begin{array}{c}\text { NSMT } \\
\text { Mo59490 }\end{array}$ & $\begin{array}{c}\text { NSMT } \\
\text { Mo67282 }\end{array}$ & $\begin{array}{c}\text { NSMT } \\
\text { Mo67282 }\end{array}$ & $\begin{array}{c}\text { NSMT } \\
\text { Mo67304 }\end{array}$ & KSB-063 & $\begin{array}{l}\text { USNM } \\
814618\end{array}$ & $\begin{array}{c}\text { UMML } \\
31.1614\end{array}$ & \multicolumn{2}{|c|}{ Mean Indices } \\
\hline Species & rancureli & rancureli & rancureli & rancureli & rancureli & rancureli & rancureli & sp. 1 & sp. 2 & sp. 3 & \multicolumn{2}{|c|}{ (rancureli) } \\
\hline Type Status & Holotype & Paratype 3 & Paratype 2 & Paratype 1 & None & None & None & None & None & None & \multirow{2}{*}{\multicolumn{2}{|c|}{ (ML41-105) }} \\
\hline ML & 71 & 65 & 59 & 46 & 105 & 88 & 41 & 63 & 35 & 45 & & \\
\hline Sex & $\mathrm{F}$ & $\mathrm{F}$ & $\mathrm{F}$ & $\mathrm{F}$ & $\mathrm{F}$ & $\mathrm{F}$ & $M$ & $M$ & M & $\mathrm{F}$ & & \\
\hline MW & 25 & 25 & 22 & 17 & 40 & 28 & 20 & 20 & 11 & 16 & MWI & 36 \\
\hline HL & 12 & 16 & 11 & 11 & 17 & 15 & 13 & 11 & 7 & 9 & $\mathrm{HLI}$ & 20 \\
\hline HW & 20 & 17 & 16 & 11 & 28 & 15 & $10^{*}$ & $17^{*}$ & 11 & 14 & HWI & 25 \\
\hline FL & 33 & 32 & 25 & 16 & 49 & 28 & 19 & 20 & 15 & 20 & $\mathrm{FLI}$ & 42 \\
\hline FW & 69 & 65 & 56 & 32 & 100 & 87 & 38 & 57 & 28 & 36 & FWI & 93 \\
\hline Arms I & $45 \mathrm{~L}$ & $40 R$ & $38 \mathrm{~L}$ & $21 \mathrm{~L}$ & $70 \mathrm{~L}$ & $62 R$ & $24 R$ & $30 \mathrm{~L}$ & $17 R$ & $24 \mathrm{R}$ & A1I & 62 \\
\hline Arms II & $59 \mathrm{~L}$ & $45 R$ & $45 \mathrm{~L}$ & $28 \mathrm{~L}$ & $86 \mathrm{~L}$ & $71 R$ & $26 R$ & $33 \mathrm{~L}$ & $22 \mathrm{R}$ & $28 \mathrm{R}$ & A2I & 75 \\
\hline Arms III & $57 \mathrm{~L}$ & $52 \mathrm{R}$ & $48 \mathrm{~L}$ & $25 \mathrm{~L}$ & $30 L^{*}$ & $76 R$ & $28 \mathrm{R}$ & $35 \mathrm{~L}$ & $24 R$ & $27 R$ & A3l & 76 \\
\hline Arms IV & $57 \mathrm{~L}$ & $42 R$ & $45 \mathrm{~L}$ & $28 \mathrm{~L}$ & $84 \mathrm{~L}$ & $70 R$ & $24 R$ & $35 \mathrm{~L}$ & $21 R$ & $28 \mathrm{R}$ & A4I & 73 \\
\hline TnL & $68 \mathrm{~L}$ & $65 R$ & $56 \mathrm{~L}$ & $39 L$ & $108 \mathrm{~L}$ & $93 R$ & $40 R$ & $56 \mathrm{~L}$ & $28 \mathrm{R}$ & $40 R$ & TnLI & 97 \\
\hline $\mathrm{CL}$ & $25 \mathrm{~L}$ & $31 R$ & $23 \mathrm{~L}$ & $15 \mathrm{~L}$ & $36 \mathrm{~L}$ & $33 R$ & $16 \mathrm{R}$ & $22 \mathrm{~L}$ & $13 R$ & $18 \mathrm{R}$ & CLI & 38 \\
\hline CS & 9 & 8 & 10 & 8 & 8 & 7 & 9 & 9 & 9 & 9 & & \\
\hline MH & 24 & $24^{*}$ & 24 & $26^{*}$ & 24 & 27 & 24 & 26 & 27 & 26 & & \\
\hline MS & D1-3 & * & V2, D1-4 & * & V1, D1-3 & D1-4 & V1-2, D1-5 & D1-2, V1-4 & $\begin{array}{l}\text { V1-3, } \\
9-14 \\
\text { D1-5, }\end{array}$ & $\begin{array}{c}\text { V1-5, } \\
\text { D1-4, } 12\end{array}$ & & \\
\hline TPS & * & $8^{*}$ & 9 & * & 11 & 10 & 9 & 8 & * & 10 & & \\
\hline
\end{tabular}

* indicates damaged features.

lens; funnel groove sharp V-shape in specimens $\mathrm{ML}<70 \mathrm{~mm}$, shallow, ill-defined in larger specimens; funnel component of locking apparatus 19\% ML, mantle component $\sim 30 \%$.

Arms 46-62--75 II-IV-86\% ML; formula II=III=IV>I; robust at bases, narrowing quickly to slender tips, each with 70-90 large suckers (ML 46-105mm), their diameter $\sim 60 \%$ arm width on Arms I-III, $50 \%$ arm width on Arms IV; oral faces of arms bordered by broad trabeculate protective membranes. Keels present on Arms II and III: that on Arms II $\sim 20 \%$ arm depth along entire arm length; that on Arms III $~ 30 \%$ arm depth along entire arm length; lateral membrane on Arms IV 100\% arm width at base, narrowing to $\sim 30 \%$ arm width by $25 \%$ arm length and continuing so to arm tips.

Tentacles robust, $85-97-105 \% \mathrm{ML}$; stalk thickness at base $\sim 80 \%$ that of adjacent arms, with low aboral keel from base to opposite carpus, $\sim 10 \%$ stalk depth. Clubs (Fig. $65 \mathrm{~A}$ ) slightly expanded, 33-38-48\% ML (33-41\% TnL, mean 39\%). Carpus ovate, with seven to ten suckers; manus with 24-27 hooks in two rows; up to five proximal dorsal and ventral hooks flanked by marginal suckers, through at least ML $105 \mathrm{~mm}$; terminal pad with eight to 11 suckers. Hooks (Figs 65E, 65F) robust, strongly recurved in profile, with curved necks; bases of ventral hooks approximately symmetrical. Dorsal hooks increase gradually in size through hook D4, thereafter decreasing gradually. Ventral hooks increase rapidly in size through V5 or V6 (Fig. 65F), with longest $\sim 15 \%$ club length and about three times as long as paired dorsal hooks, thereafter decreasing gradually. Ventral membrane low, inconspicuous, $\sim 20 \%$ club width along 

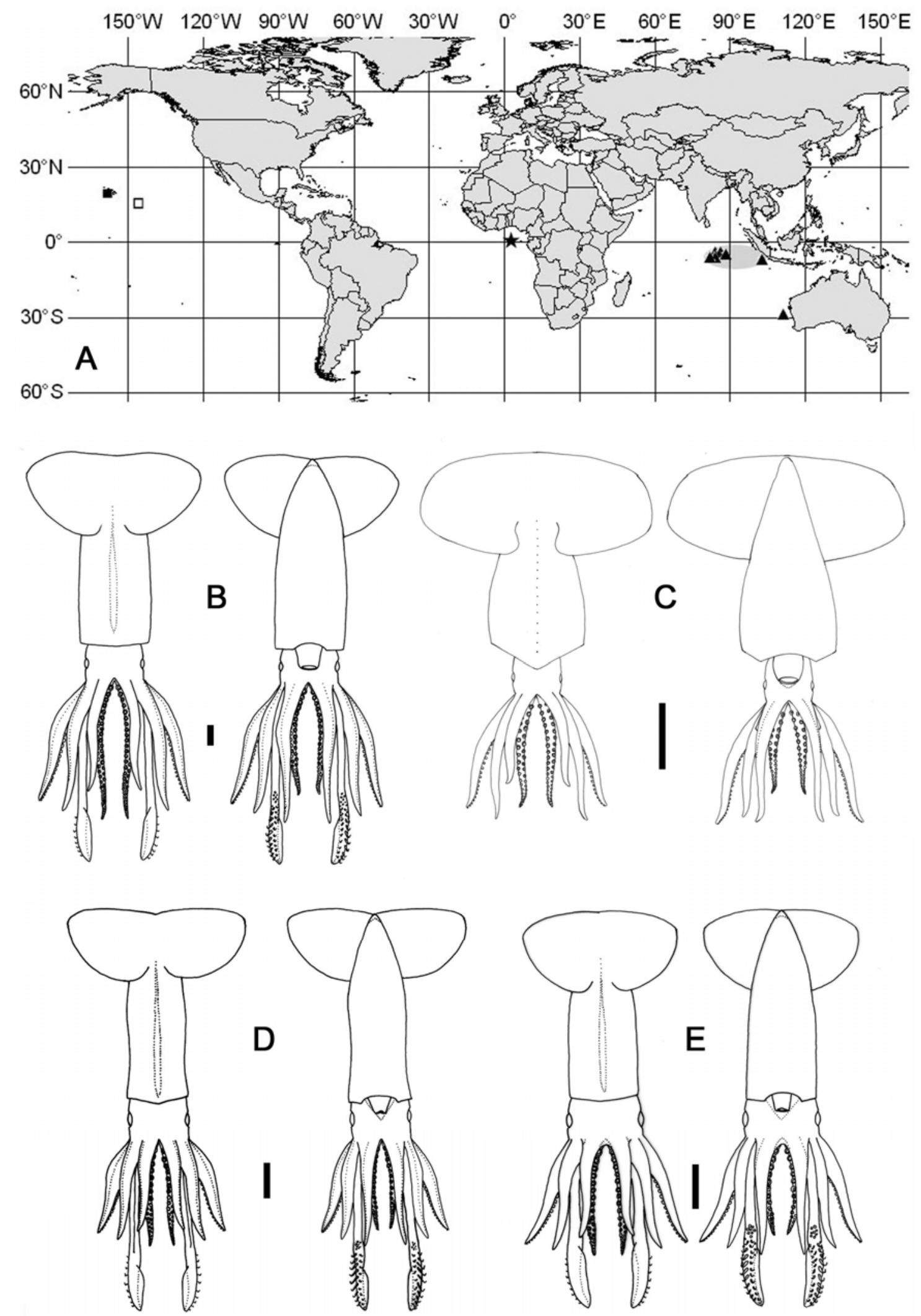

Fig. $64-$ Gen. nov. 2 spp. A) distribution of Gen. nov. 2 spp. ( $\Delta$ Gen. nov. 2 rancureli - grey oval indicates type locality, $\square$ Gen. nov. 2 sp. 1, $\square$ Gen. nov. 2 sp. 2 , $\star$ Gen. nov. 2 sp. 3); B) Gen. nov. 2 rancureli NSMT Mo67282, q, ML 105mm; C) Gen. nov. 2 rancureli NMV F51101, spent $Q$, ML 150mm; D) Gen. nov. 2 sp. 1, KSB-063, $\partial^{\lambda}, \mathrm{ML}$ $63 \mathrm{~mm}$; E) Gen. nov. 2 sp. 3, UMML 31.1614, o, ML 45mm. Scale bars = B, D, E) $10 \mathrm{~mm}$; C) $50 \mathrm{~mm}$. 
entire length; proximal dorsal membrane not apparent; dorsal keel originates near hook D4, its width $\sim 80 \%$ corresponding club width over remaining club length.

Lateral profile of lower beak (Figs 66A-C, ML 150mm) markedly longer than deep, with crest gently convex and sloping shallowly downward: posterior edge of hood well above crest; hood length $\sim 60 \%$ total crest length, and hood and crest together $\sim 60 \%$ baseline; jaw edge straight proximally, becoming slightly concave distally; jaw angle obtuse, obscured by slight wing fold. Crest broad, not thickened, jaw edge broad but sharp; shoulder ridge indistinct, rounded; angle ridge long (spanning 80-90\% lateral wall depth), poorly developed but darkly pigmented; wings broaden distally, width $\sim 200 \%$ that at jaw angle, wing length $\sim 300 \%$ LRL, with small triangular insert of cartilage below jaw angle; lateral wall fold broad and shallow, indistinct by posterior margin of lateral wall.

Radula (Fig. 66J) with slender, conical mesocone, with nearly parallel sides; lateral cusps $\sim 33 \%$ mesocone height, pointed, slightly laterally directed; height of base $\sim 25 \%$ rachidian height; proximal margin of base concave, distal margin concave between cusps. First lateral tooth bicuspid, $\sim 60 \%$ rachidian height; inner cusp slender, straight or slightly directed away from rachidian; outer cusp small, triangular, pointed, somewhat laterally directed. Second lateral tooth simple, slender, conical, $\sim 125 \%$ rachidian height. Marginal tooth simple, slender, conical; height $\sim 200 \%$ rachidian height. Palatine palp (Fig. 66M) with greatest width of tooth-bearing plane $\sim 25 \%$ of its length; with $\sim 60$ moderately long, flat teeth, evenly distributed over palp surface, each $\sim 100-200 \%$ rachidian height.

Gladius elongate rhombic, with small, dorsally directed, rounded rostrum. Greatest width $(\sim 17 \% \mathrm{GL})$ attained at $\sim 70 \% \mathrm{GL}$; vanes originate within anterior $20 \% \mathrm{GL}$ but remain narrow until $\sim 50 \% \mathrm{GL}$, thereafter widening and tapering rapidly.

Overall colouration (preserved) pale pink with silvery iridescence on mantle, head and aboral surfaces of arms and tentacles, with sparse dark reddish-purple chromatophores; pigmentation darkest dorsally on midline of mantle, fins, and head; ventrally on posterior portion of mantle.

Spent female, ML 150mm (Fig. 64C), as above, with the following deviations. Mantle widely conical, width $\sim 40 \% \mathrm{ML}$, flabby, loose; posterior mantle tip blunt, somewhat stiffened ventrally into nearly cartilaginous pouch enclosing tip of gladius. Fins large, 
oval in outline, length $\sim 55 \% \mathrm{ML}$, width $\sim 125 \% \mathrm{ML}$ with posterior margins level with or surpassing tip of mantle; tissue flabby but margins intact. Head broad, widely ovate, dorso-ventrally compressed (length and width 25\% ML, depth 15\% ML); two primary occipital folds barely discernible posterior to eye; funnel broad, rounded, bulbous, reaching to level below posterior margin of eye lens; retractor muscles stretched, flaccid; funnel groove shallow, indistinct, reaching to midpoint of eye; arms stubby, flabby, $\sim 60 \% \mathrm{ML}$, each with $\sim 50$ large, widely spaced, orally compressed suckers remaining. Tentacles reduced to short, flaccid stumps. Gladius (Fig. 67D) elongate rhombic, with small dorsal-directed, rounded cartilaginous rostrum. Greatest width $\sim 13 \% \mathrm{GL}$, attained at $70 \% \mathrm{GL}$. External tissues (preserved) without apparent pigmentation apart from ventral mantle tip. Several faded chromatophores visible over reproductive organs internally.

Remarks - Material conforming to the overall morphology generally attributed to Gen. nov. 2 rancureli was examined from a range of geographic locations, but differences are apparent in several specimens (see below). The previous description of Gen. nov. 2 rancureli (sensu stricto) is based on the type series, and on material that closely resembles the type series and illustrations (particularly in tentacle club morphology, including hook size and shape), taken primarily from the Indian Ocean, near the type locality.

Gen. nov. 2 sp. 1, North Pacific (Table 25, Figs 64A, 64D, 65B, 65G, 66D-F, 66K)

Onychia sp. Rancurel, 1970: 18-23, Tables III-V, Figs 12-16.

Onykia sp. — Bonnaud et al. (1998): 1761-1770, Fig. 6.

Material examined ( 1 specimen) - KSB-063, ô $, \mathrm{ML} 63 \mathrm{~mm}, \sim 21^{\circ} 23^{\prime} \mathrm{N}, \sim 158^{\circ} 18^{\prime} \mathrm{W}$, bottom depth 1400m, 01/11/1989, Tsuji IV Stn 52A, 40m² trawl.

Description, ML 63mm (Figs 64D, 65B, 65G, 66D-F, 66K) - Mantle approximately cylindrical, widening midway along $\mathrm{ML}$ to $\sim 32 \% \mathrm{ML}$, remaining broad and blunt posteriorly; gladius visible along dorsal midline as broad amber-brown-coloured ridge, widest anteriorly, becoming narrower and less distinct posteriorly and no longer visible within anterior $\sim 30 \%$ of fin length; rostrum of gladius visible ventrally through posterior $\sim 3 \% \mathrm{ML}$. Fins ovate, length $\sim 30 \% \mathrm{ML}$, width $\sim 90 \% \mathrm{ML}$; posterior margins gently convex, anterior margins strongly convex, anterior lobes large. 

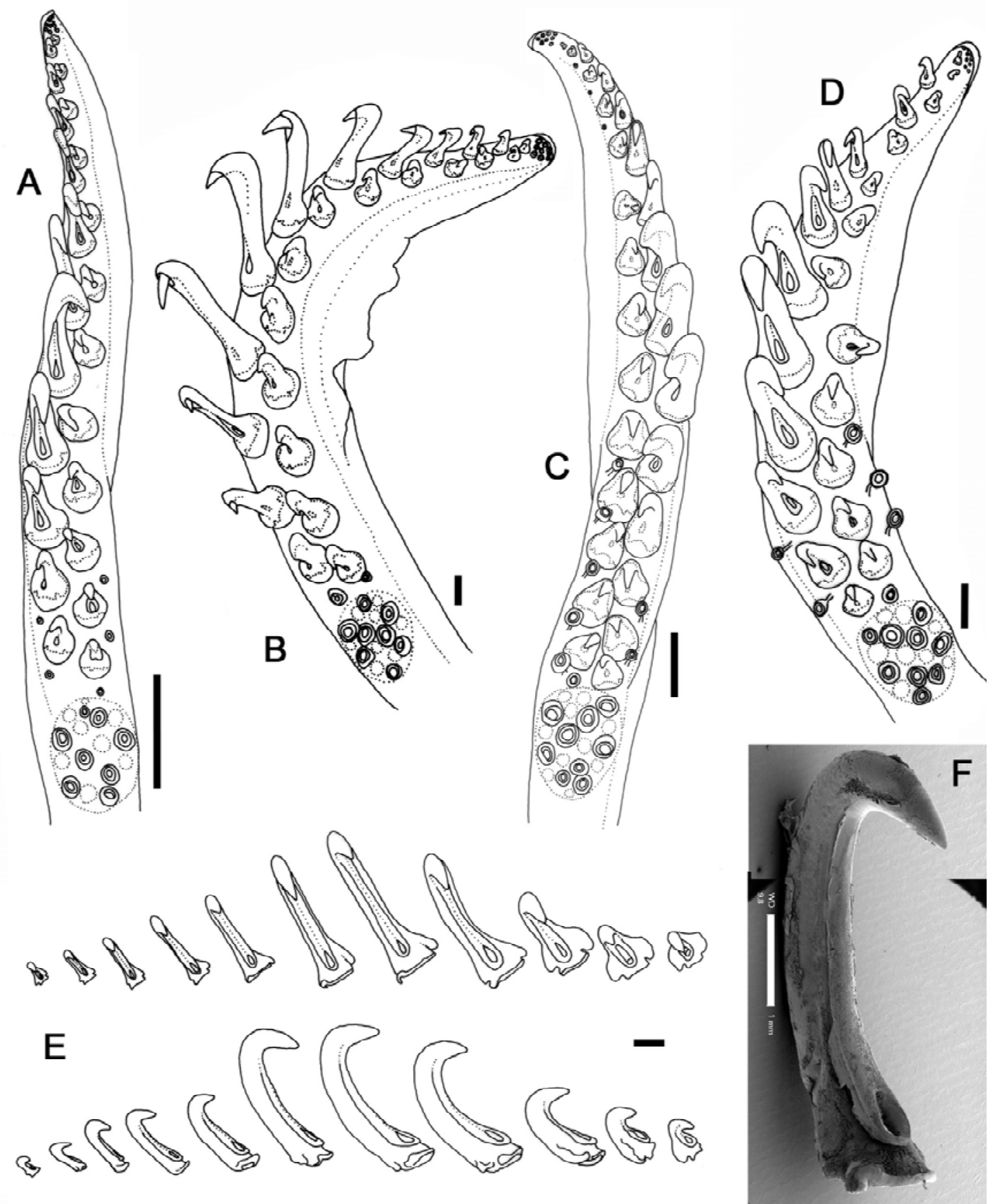

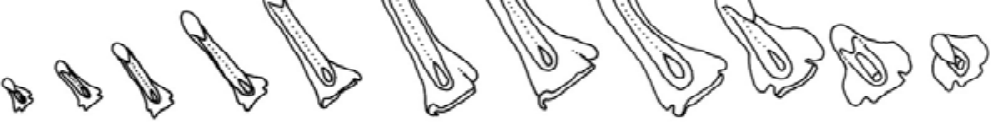

E
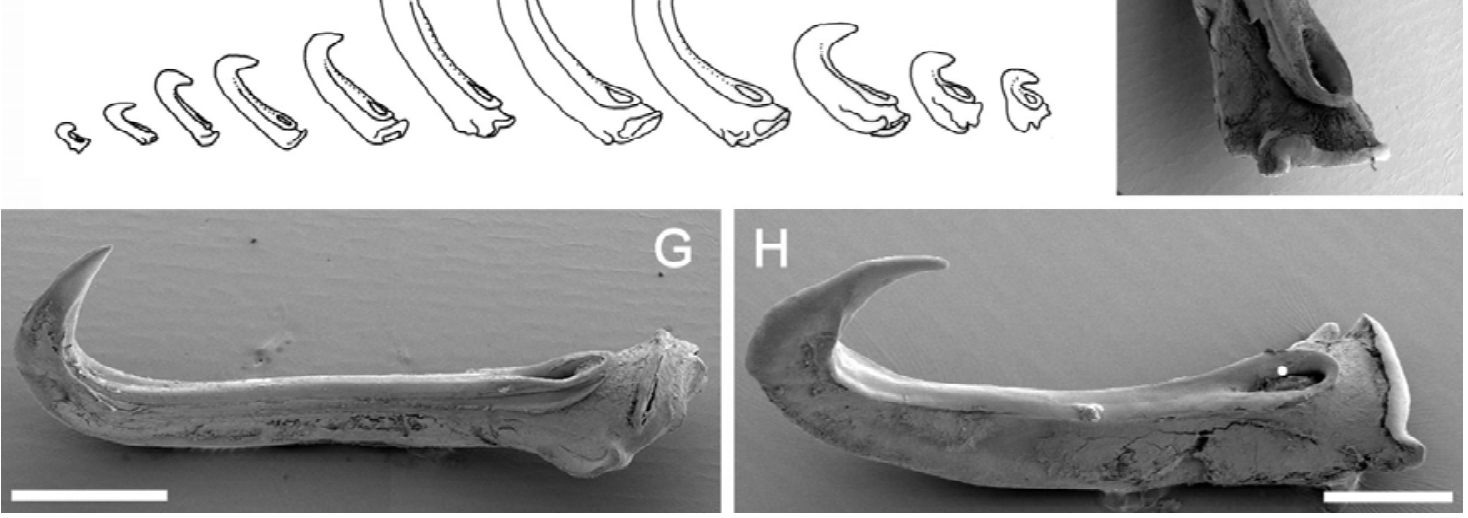

Fig. 65 - Gen. nov. 2 spp. A, E, F) Gen. nov. 2 rancureli NSMT Mo67282,, , ML $105 \mathrm{~mm}$; B, G) Gen. nov. 2 sp. 1, KSB-063, ô, ML 63mm; C) Gen. nov. 2 sp. 2, USNM 814618, ô, ML 35mm; D, H) Gen. nov. 2 sp. 3, UMML 31.1614, , ML 45mm. A, B, D) right tentacle club; $C$ ) left tentacle club; $E-G$ ) left ventral tentacular hooks: (E) entire series, $(F)$ V5, (G) V5; $(H)$ right hook V5. Scale bars = A) $5 \mathrm{~mm}$; B-G) $1 \mathrm{~mm}$; H) $500 \mu \mathrm{m}$. 
Head rounded, with convex dorsal and ventral surfaces, length $\sim 17 \% \mathrm{ML}$, width $\sim 30 \%$ $\mathrm{ML}$, depth $\sim 20 \% \mathrm{ML}$. Funnel groove sharp V-shape, extending anteriorly to level below midpoint of eye; funnel stubby, aperture opening at point directly below posterior margin of eye aperture. Funnel component of locking apparatus $\sim 15 \% \mathrm{ML}$, mantle component $\sim 35 \% \mathrm{ML}$.

Arms stocky, with tips remaining robust, $48-56 \% \mathrm{ML}$, formula $I I=I I=\mid \mathrm{V}>\mathrm{I}$, each with $\sim 70$ suckers, their diameter $\sim 50 \%$ arm width. Keels present on Arms I-III: that on Arms I $~ 5 \%$ arm width along entire arm length; that on Arms II $\sim 20 \%$ arm depth along entire arm length; that on Arms III $\sim 60 \%$ arm depth by $\sim 40 \%$ arm length, narrowing along remainder of arm; lateral membrane on Arms IV $~ 75 \%$ arm width at bases, narrowing to $\sim 40 \%$ arm width along remainder of arm length. Broad membranes with strong trabeculae border oral faces of all arms, particularly broad dorsally on Arms II and III.

Tentacles $\sim 90 \% \mathrm{ML}$; stalks $\sim 80 \%$ thickness of adjacent arms at bases, with low aboral keel ( $10 \%$ stalk thickness) reaching to level with carpus. Clubs (Fig. $65 \mathrm{~B}) \sim 35 \% \mathrm{ML}$, slightly expanded, with eight or nine carpal suckers, 25 or 26 long, spindly hooks, and eight terminal pad suckers. Dorsal hooks increase gradually in size through hook D4, with proximal one or two hooks flanked by marginal suckers; hooks decrease gradually in size from D5 to tip of club. All ventral hooks larger than corresponding dorsal hooks. Hooks V1-V5 increase rapidly in size, with marginal suckers flanking proximal four or five hooks; hooks V5 and V6 largest on club (Fig. 65G, 20\% club length and about three times as long as paired dorsal hooks); thereafter ventral hooks gradually decrease in size distally. Ventral club membrane $\sim 20 \%$ club width along entire club length; dorsal membrane scarcely discernible, $\sim 5 \%$ club width, flanking hooks D1-D3; dorsal keel originates level with hook D3, widening to $\sim 100 \%$ club width by hook D5 and remaining $\sim 100 \%$ of corresponding club width to club tip.

Lateral profile of lower beak (Figs 66D-F) longer than deep, with crest slightly convex and sloping gently downward, posterior edge of hood well above crest, hood length $\sim 40 \%$ total crest length, and hood and crest together $\sim 80 \%$ baseline; jaw edge weakly concave along entire length, produced distally into small pointed rostral tip; jaw angle obtuse, not obscured by wing fold. Crest broad; lateral walls not indented below crest; shoulder ridge distinct, sharp; angle ridge distinct, spanning $\sim 70 \%$ lateral wall depth; angle point distinct; wings broaden distally, width $\sim 130 \%$ that at jaw angle, length $\sim 180 \%$ LRL; jaw edge narrow and sharp; small crescent-shaped insert of clear cartilage present on medial wing margin just below jaw angle; lateral wall fold low, 
indistinct, closer to crest than dorsal margin of lateral wall, indistinct by posterior lateral wall margin.

Radula (Fig. 66K) with slender, conical, distally pointed mesocone; lateral cusps $\sim 50 \%$ mesocone height, pointed, slightly laterally directed or hooked; height of base $\sim 33 \%$ total rachidian height; proximal margin of base straight to slightly concave, distal margin concave between cusps. First lateral tooth bicuspid, $\sim 60 \%$ rachidian height; inner cusp slender, straight; outer cusp large, nearly as broad as inner cusp and $\sim 70 \%$ its height, with concave inner margin and convex outer margin giving hooked appearance. Second lateral tooth simple, slender, conical, $\sim 125 \%$ rachidian height. Marginal tooth simple, slender, conical; height $\sim 200 \%$ rachidian height. Palatine palp with greatest width of tooth-bearing plane $\sim 25 \%$ of its length; with $\sim 55$ short, flat teeth, evenly distributed over palp surface, each $\sim 75-140 \%$ rachidian height.

Remarks - Gen. nov. 2 sp. 1 is the most easily distinguishable of the regional 'forms,' appearing overall more elongate and slender in body, with relatively shorter arms, than Gen. nov. 2 rancureli, and possessing long, slender and less strongly recurved tentacular hooks. The lower rostral length is also slightly shorter relative to the wings, and the rachidian and first marginal tooth are more compact and robust than those of the other forms for which buccal morphology could be observed.

Gen. nov. 2 sp. 2, North Pacific (Table 25, Figs 64A, 65C)

Material examined ( 1 specimen) - USNM 814618, $0^{\lambda}, \mathrm{ML} 35 \mathrm{~mm}, 18^{\circ} 00^{\prime} \mathrm{N}, 147^{\circ} 00^{\prime} \mathrm{W}, 60-0 \mathrm{~m}$, 31/03/1959, RV H.M. Smith 51-25.

Description, ML 35mm (Fig. 65C) - Mantle muscular, conical, width 30\% ML, broad and blunt posteriorly; gladius visible along dorsal midline as broad amber-browncoloured ridge, widest anteriorly, becoming narrower and less distinct posteriorly and no longer visible within anterior $30 \%$ of fin length. Fins ovate, length $\sim 45 \% \mathrm{ML}$, width $\sim 80 \% \mathrm{ML}$; anterior and posterior margins convex; anterior lobes broad and shallow.

Head somewhat boxy, length $\sim 20 \% \mathrm{ML}$, width $\sim 30 \% \mathrm{ML}$, depth $25-30 \% \mathrm{ML}$; funnel groove broadly $\mathrm{V}$-shaped; funnel narrow, rounded, nearly cylindrical, with aperture at level below posterior margin of eye lens. Funnel component of locking apparatus $\sim 17 \% \mathrm{ML}$, mantle component $\sim 35 \%$. 
Arms robust, stubby, $50-70 \% \mathrm{ML}$, formula $\mathrm{II}>\mathrm{II}=\mathrm{IV}>\mathrm{I}$, each with $\sim 50$ large suckers, their diameter $50-55 \%$ arm width on Arms I-III, 30\% arm width on Arms IV; oral faces of arms bordered by broad trabeculate protective membranes. Keels present on Arms II and III: that on Arms II forming low ridge, $~ 15 \%$ arm depth along entire arm length; that on Arms III $\sim 75 \%$ arm depth from $30 \%$ arm length to arm tip; lateral membrane on Arms IV 150\% arm width at base, narrowing to $~ 15 \%$ arm width by $50 \%$ arm length.

Tentacles $\sim 80 \% \mathrm{ML}$ at $35 \mathrm{~mm}$; stalk diameter $\sim 70 \%$ diameter of adjacent arms, with low aboral keel from base to level with carpus. Club (Fig. 65C) slightly expanded, length $\sim 40 \% \mathrm{ML}(\sim 50 \% \mathrm{TnL})$. Carpus nearly circular in outline, with seven to ten suckers; manus with $\sim 27$ hooks, with distal-most pair nearly fully formed; marginal suckers flank all hooks except V4-V10; terminal pad incomplete but appears comprised of ten suckers in two irregular longitudinal rows. Ventral hooks increase steadily in size distally through hooks V5 and V6 (length of largest ventral hooks $\sim 10 \%$ $\mathrm{CL}$, about twice as long as paired dorsal hooks), thereafter steadily decreasing in size. Ventral membrane $\sim 20 \%$ corresponding club width along entire ventral club margin; proximal dorsal membrane not apparent; dorsal keel $\sim 120 \%$ club width, originating near hook D4 and continuing to tip of club.

Buccal mass absent from sole known specimen.

Remarks - Gen. nov. 2 sp. 2 appears very similar to Gen. nov. 2 sp. 3 in body proportions (Fig. 64E, but lacking the short membranes at the arm bases). It differs from to Gen. nov. 2 rancureli in possessing smaller, more slender tentacular hooks (largest ventral hooks $\sim 10 \% \mathrm{CL}$ and about twice as long as paired dorsal hooks in Gen. nov. 2 sp. 2, compared to $\sim 20 \%$ CL and about three times as long as paired dorsal hooks in Gen. nov. 2 rancureli), although the delicate condition of the hooks on the sole known specimen prevented closer observation via SEM. These differences may represent ontogenetic variability. Additional material is required to more fully evaluate the specific status of this 'form,' presently known from a single, juvenile, incomplete specimen. 

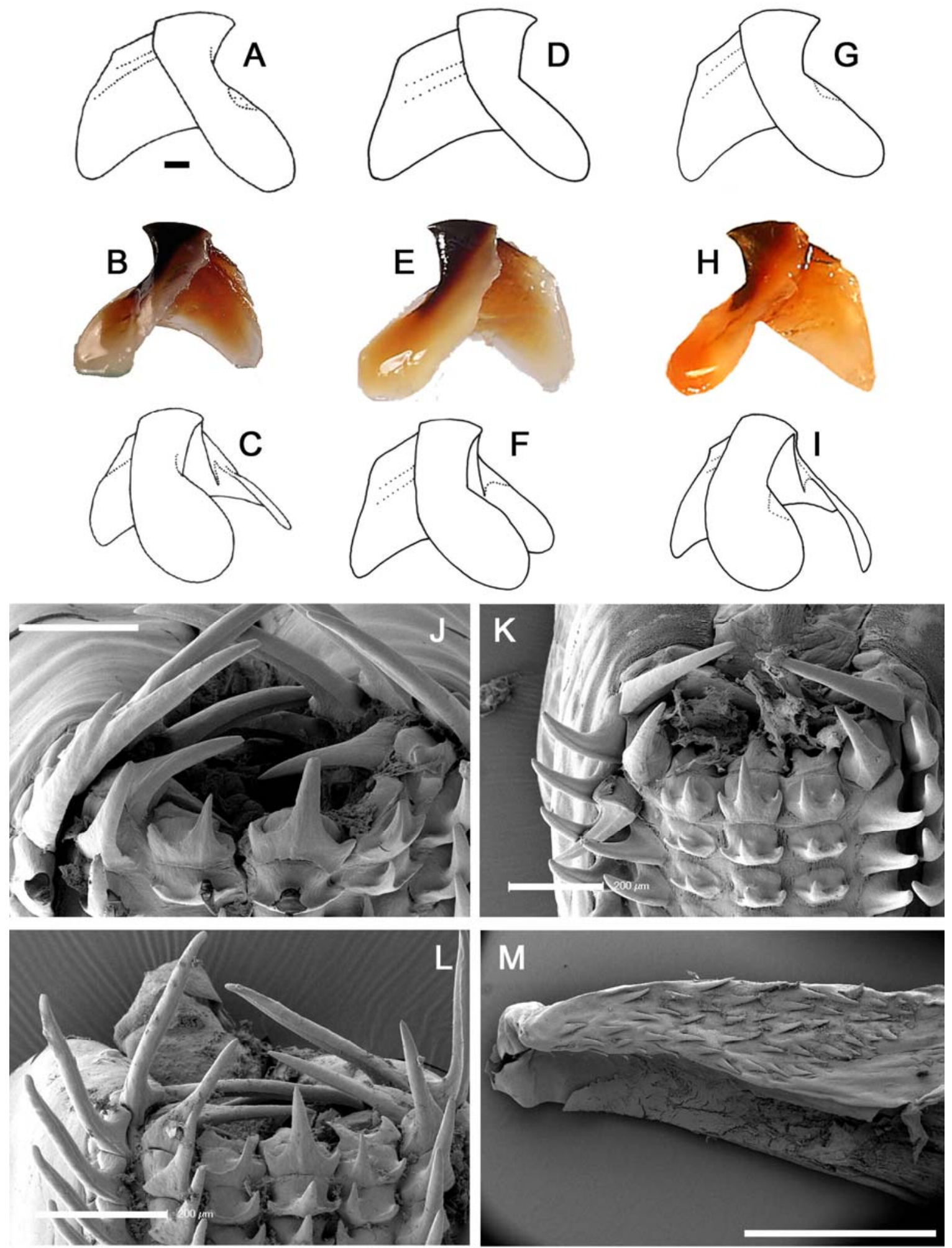

Fig. 66 - Gen. nov. 2 spp. A-C) Gen. nov. 2 rancureli, NMV F51101, spent 9 , ML $150 \mathrm{~mm}$; D-F, K) Gen. nov. 2 sp. 1, KSB-063, §, ML 63mm; G-I, L) Gen. nov. 2 sp. 3, UMML 31.1614, +, ML 45mm; J) Gen. nov. 2 rancureli, NSMT Mo67304, §., ML $41 \mathrm{~mm}$; M) Gen. nov. 2 rancureli, NMV F65985, ㅇ, 83mm. A-I) lower beak: (A, D, G) left, $(B, E, H)$ right, $(C, F, I)$ left oblique profiles; J-L) radula; $M)$ palatine palp. Scale bars $=$ A-I) $1 \mathrm{~mm}$; J-L) $200 \mu \mathrm{m} ;$ M) $2 \mathrm{~mm}$. 
Gen. nov. 2 sp. 3, North Atlantic (Table 25, Figs 64A, 64E, 65D, 65H, 66G-I, 66L)

Material examined ( 1 specimen) - UMML 31.1614, $q, \mathrm{ML} 45 \mathrm{~mm}, 05^{\circ} 57^{\prime} \mathrm{N}, 02^{\circ} 49^{\prime} \mathrm{E}, 114-92 \mathrm{~m}$, 25/05/1964, RV Pillsbury, IKMWT.

Description, ML 45mm (Figs 64E, 65D, 65H, 66G-I, 66L) - Body proportions as for Gen. nov. 2 sp. 2, with the following exceptions. Mantle slightly wider ( 36\% ML). Fine membranes present at arm bases, $\sim 5 \%$ arm length, joining each arm to adjacent arm. Tentacle clubs (Fig. 65D) stubby and broad, with robust, close-set hooks. Carpus with about nine suckers, manus with $\sim 26$ hooks, terminal pad with about ten suckers. Hooks strongly recurved, with slightly curved necks in lateral profile. Dorsal hooks increase gradually in size through hook D4, thereafter decreasing gradually. Ventral hooks increase rapidly in size through V5 or V6 (Fig. 65H), with largest ventral hook $\sim 15 \%$ club length and about three times as long as paired dorsal hook, thereafter decreasing gradually. Ventral membrane $\sim 30 \%$ corresponding club width along entire ventral club margin; dorsal membrane scarcely discernible, $\sim 5 \%$ club width, flanking hooks D1-D3; dorsal keel originates level with hook D3, widening to $\sim 100 \%$ club width by hook D5 and remaining $100 \%$ of corresponding club width to club tip.

Lateral profile of lower beak (Figs 66G-I) longer than deep, with crest straight and sloping gently downward; posterior edge of hood close to crest; hood length $\sim 55 \%$ total crest length, and hood and crest together $\sim 70 \%$ baseline; jaw edge weakly concave along entire length; jaw angle obtuse, not obscured by wing fold. Crest broad; lateral walls not indented below crest; shoulder ridge distinct, sharp; angle ridge distinct, spanning $\sim 70 \%$ lateral wall depth; angle point distinct; wings broaden distally, width $\sim 170 \%$ that at jaw angle, length $\sim 200 \%$ LRL; jaw edge narrow and sharp; small triangular insert of clear cartilage present on medial wing margin just below jaw angle; lateral wall fold low, indistinct, closer to crest than dorsal margin of lateral wall, remaining distinct to posterior lateral wall margin.

Radula (Fig. 66L) with conical mesocone on rachidian; lateral cusps $\sim 30 \%$ mesocone height, pointed, slightly laterally directed; height of base $\sim 25 \%$ height of rachidian; proximal margin of base straight to slightly concave; distal margin of base concave between cusps. First lateral tooth bicuspid; inner cusp slender, straight, $\sim 60 \%$ height of rachidian; outer cusp small, triangular, straight or slightly laterally directed. Second lateral tooth simple, slender, conical, $\sim 125 \%$ height of rachidian. Marginal tooth simple, slender, conical; height $\sim 200 \%$ height of rachidian. Palatine palp slender; greatest width of tooth-bearing plane $\sim 33 \%$ of its length; with $\sim 55$ short, flat teeth, some curved 
outward away from palp surface, each $\sim 35-130 \%$ rachidian height, evenly distributed over palp.

Overall colouration (preserved) dark purple-grey, with small, pale red chromatophores evenly distributed over dorsal and ventral mantle and head surfaces. Eyes strongly iridescent; keels on Arms II weakly iridescent.
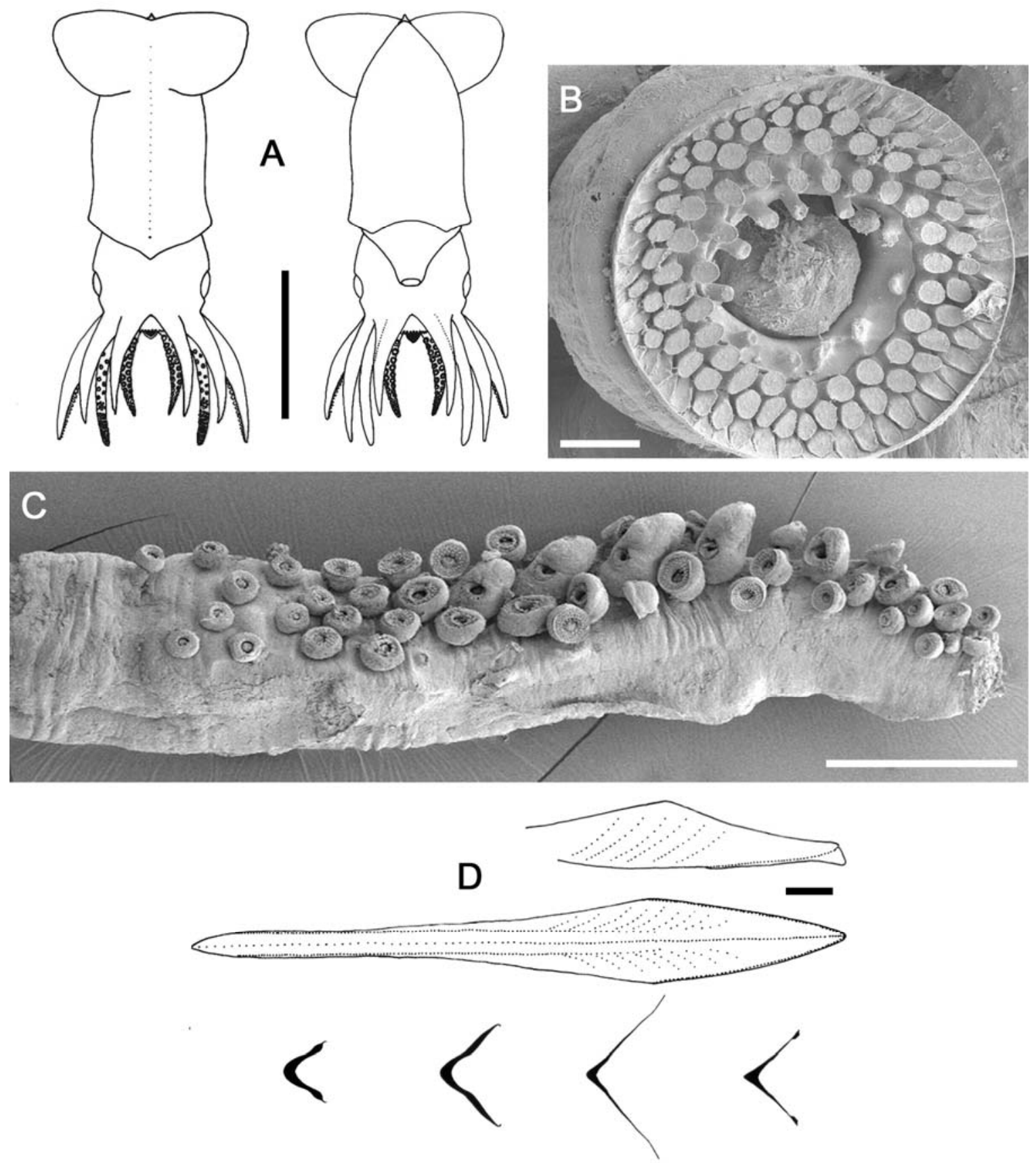

Fig. 67 - Gen. nov. 2 spp. A) Gen. nov. 2 sp. indet., USNM unaccessioned, sex indet., ML 8.5mm; B, C) BMNH 20070495, sex indet., ML 12mm: (B) juvenile tentacular sucker, (C) right tentacle club; D) Gen. nov. 2 rancureli, NMV F51101, spent +, ML 150mm, gladius. Scale bars = A) $5 \mathrm{~mm}$; B) $20 \mu \mathrm{m}$; C) $500 \mu \mathrm{m}$; D) $10 \mathrm{~mm}$. 
Remarks - Gen. nov. 2 sp. 3 is very similar to Gen. nov. 2 sp. 2 and Gen. nov. 2 rancureli in overall morphology. Its tentacle clubs are the most similar (of any of these three putative taxa) to those of Gen. nov. 2 rancureli, with robust, strongly recurved hooks (largest ventral hook $\sim 15 \% \mathrm{CL}$ and about three times larger than paired dorsal hook). Its radular and lower beak morphology are also similar to those of Gen. nov. 2 rancureli. However, the short inter-arm membranes have not been observed on any other Gen. nov. 2 specimen, and the Atlantic origin of the sole known specimen of Gen. nov. 2 sp. 3 (far from the type locality of Gen. nov. 2 rancureli) requires that additional material be examined before deciding on the specific status of this problematic taxon.

Gen. nov. 2 spp. indet.

Material examined - BMNH 20070495, ML 12mm, RRS Challenger Stn C83/47, 45m, 22/10/1983, RMT50, NFD; USNM unaccessioned, ML 8.5mm, Amsterdam North Atlantic Plankton Expedition Stn 65, Haul 6, 19/02/1982, RMT1.

Paralarvae to small juveniles (ML 8.5, 12mm, Fig. 67A-C, coll. northern Atlantic) with broad, slightly bulbous mantle, width $\sim 47 \% \mathrm{ML}$, blunt posteriorly and pierced by rostrum of gladius; dorsal anterior margin strongly produced into broad point. Fins paddle-shaped; length $\sim 35 \% \mathrm{ML}$, width $\sim 82 \% \mathrm{ML}$; posterior margins gently convex, produced beyond tip of mantle; anterior margins rounded, strongly convex, with broad shallow anterior lobes. Head broad, rounded; length $\sim 35 \% \mathrm{ML}$, width $\sim 53 \% \mathrm{ML}$, depth $\sim 25 \% \mathrm{ML}$; constricted anteriorly; eyes large, anteriorly directed, somewhat bulging. Funnel broad posteriorly, narrowing to slender cylinder anteriorly, aperture extending anteriorly to below midpoint of eyes; funnel groove shallow, broad, ill defined; funnel component of locking apparatus $\sim 25 \% \mathrm{ML}$, mantle component $\sim 50 \% \mathrm{ML}$.

Arms stubby; length 40-60\% ML; formula III>II>IV>I; each with $\sim 30$ suckers. No keels apparent on Arms I-III; Arms IV with low lateral membrane, $50 \%$ arm width at base, narrowing to $25 \%$ arm width by $25 \%$ arm length and becoming indistinct distally. Tentacle length $\sim 70 \% \mathrm{ML}$; stalk bases $\sim 75 \%$ thickness of adjacent arms; oral surface with $\sim 10$ small suckers in alternating rows along entire stalk length. Club not expanded, delineated from stalk by small close-set patch of eight carpal suckers; manus with four longitudinal rows of $\sim 12$ small suckers; no hooks present at ML $8 \mathrm{~mm}$; four ventral hooks present my ML 12mm (Fig. 67C); terminal pad indistinct. Tentacular suckers circular (Fig. 67B), with circular aperture, its diameter $\sim 30 \%$ overall sucker 
diameter, situated medially; marginal suckers with about five low, stumpy teeth present around proximal inner margin and about six longer, cylindrical teeth around distal inner margin. Proximal pegs and distal pegs of subequal size, circular to polygonal, densely set in two or three proximal and two to four concentric rows.

Overall colouration pale yellowish-pink; no chromatophores apparent.

Remarks - The paralarva and small juvenile described above could not be identified beyond genus, but are included herein to assist in identifying onychoteuthid paralarvae to at least the generic level. Paralarvae of Gen. nov. 2 'rancureli' have also been described and illustrated by Young et al (2003b).

General remarks - Gen. nov. 2 is poorly represented in worldwide collections, material is mostly ex-gut-content, and collection data are often incomplete. The majority of available specimens originate from near the type locality and can be attributed to Gen. nov. 2 rancureli, but spp. 1-3 described above are found in locations far from the Indian Ocean and each exhibit morphological differences from the type series and illustrations of Gen. nov. 2 rancureli. The most immediately apparent differences are observed on the tentacle clubs (Figs 65A-D), and in the morphology of the largest ventral hooks (Figs 65F-H), but in some instances, in the lower beak (Figs 66A-I) and radula (Figs 66J-L), differences are also apparent. Since specimens attributed to spp. 1-3 represent both sexes and a variety of ontogenetic stages, it is possible that all belong to a single variable species. However, some of their distinctive features can already be observed in the work of earlier authors (Rancurel (1970) appears to have had specimens of Gen. nov. $2 \mathrm{sp.} \mathrm{1,} \mathrm{from} \mathrm{the} \mathrm{south} \mathrm{central} \mathrm{Pacific;}$ his illustrations of the tentacle club and radula closely match those given here, Figs $65 \mathrm{~B}$ and $66 \mathrm{~K}$, respectively). Should further material reveal consistent regional morphological trends, the preliminary descriptions here may prove useful in further diagnosis of these taxa. 


\section{DISCUSSION}

Systematic value of characters and character states

Onychoteuthids are an interesting and challenging group; the number of taxonomically valid species is certainly lower than the 62 nominal taxa attributed to the family since 1807 , yet certainly higher than the 12-14 names most commonly recognised in recent literature (Nesis 1987, Kubodera et al. 1998, Vecchione et al. 2003). In order to make detailed comparisons between species and to critically evaluate relationships within the family, a reliable set of characters and states was required to diagnose taxa at the generic and specific (and in one genus, subgeneric) levels.

The descriptions and illustrations above encompass both traditionally reported and more newly recognised characters and character states. With all taxa now reported to the same level of detail, it is possible to assess the importance and usefulness of these features in differentiating onychoteuthid taxa. Some characters and their states (Table 1) are most helpful for generic separation, while others vary enough between species to distinguish congeners reliably (Tables 2,11 ).

\section{Generic characters}

No single character or state is sufficient on its own to distinguish between all onychoteuthid genera, although several character states, even individually, can diagnose most genera reliably. The species within almost every genus share at least one unique adult character state: intestinal photophores (Onychoteuthis); the absence of a free rachis on the gladius (Ancistroteuthis); the presence of an entire series of marginal suckers on the club in all ontogenetic stages (Kondakovia); the low number of secondary occipital folds (Notonykia); and the elongate-rhombic gladius (Gen. nov. 2).

Most genera can be diagnosed using a combination of two or three external features, such as fin size/shape, presence/number of secondary occipital folds, and number of tentacular hooks and terminal pad suckers. Additional external characters that are consistent among congenerics (or encompass an intrageneric range that excludes several other genera) include: structure (if any) of the mantle epidermis; body proportions, especially length of the arms relative to the mantle; and shape of the funnel groove. 
Of those internal characters reported herein, the gladius proves most useful for distinguishing most genera, although the gladii of several genera resemble each other, e.g. Notonykia and Onychoteuthis, and Onykia, Kondakovia and Gen. nov. 1 knipovitchi.

Presence/absence of photophores and number of cusps on the rachidian tooth of the radula (unicuspid vs tricuspid) also proved largely consistent at the generic level (although the rachidian is tricuspid in some Onychoteuthis species and unicuspid in others). Nesis (2000) also reported that the tissues of 'Moroteuthis' (= Onykia) and Kondakovia species contain high concentrations of ammonium ions, while those of Onychoteuthis, Ancistroteuthis, Notonykia and, oddly, Onykia species do not, and are negatively buoyant. It remains unknown whether ammonium ion concentrations increase with ontogeny in Onykia species; the tissue chemistry for species in Gen. nov. 1 knipovitchi and Gen. nov. 2 also remains unreported.

Specific characters

Three onychoteuthid genera are presently monospecific - Ancistroteuthis, Kondakovia and Gen. nov. 1 knipovitchi (although an additional species of Kondakovia is known, material has not yet been available for examination); Gen. nov. 2 includes one formally described species and several putative new 'forms' or species. Notonykia includes two described species. The remaining two genera are the most diverse, with nine species in Onychoteuthis (Table 2) and seven in Onykia (Table 11). Good specific characters are therefore most necessary in the latter two genera, while the specific and generic characters are the same in Ancistroteuthis, Kondakovia and Gen. nov. 1 knipovitchi at present.

Tentacular morphology has been closely examined in this study and has provided reliable specific characters in all genera. Important adult tentacular character states include club length relative to mantle length; number of carpal suckers; number, shape, sculpture, symmetry and relative size of hooks; number of marginal suckers, retention through ontogeny and size relative to the hooks; and number of terminal pad suckers. In the genus Onychoteuthis, the presence or absence of certain chromatophore patterns from parts of the club also appears consistent within species (see Figs 25A, 25B), although chromatophores are a tenuous character in general, susceptible to damage and fading during capture, fixing and storage of specimens. 
The presence or absence of lateral grooves on the hook claw in Onykia is also important, being diagnostic at the subgeneric level.

Some proportions of the gladius also prove useful for differentiating species, although in Onychoteuthis and Notonykia the relative length of the free rachis to total GL is more diagnostic, while in Onykia it is the relative length of the rostrum. Toll (1982) reported some ontogenetic variation in relative gladius proportions, however, so these character states require further investigation, particularly since relative length of the free rachis is considered one of the more important systematic character states in some species known from limited material (e.g. Os. mollis).

Other specific characters vary by genus. In Onychoteuthis (Table 2) shape and relative size of the photophores are valuable in distinguishing taxa. In Onykia (Table 11) sculpture of the mantle epidermis in large juvenile to spent specimens is important, as are fin size and shape, presence/absence of a Y-shaped ridge within the funnel groove, arm length relative to the mantle and number of arm suckers. Separating the two Notonykia species is reliant almost entirely on tentacular morphology, while the presence of a possible second Ancistroteuthis species is suggested most strongly by reported differences in fin size and shape (Adam 1952, Kubodera et al. 1998).

While identification and description of paralarval and small juvenile specimens has not been a primary focus of this study (given historical difficulties in disambiguating even the later, more reliably recognisable stages of some taxa), early ontogenetic stages are reported where material has permitted. As in larger specimens, identification of small onychoteuthids relies partly on presence/absence of photophores, fin size and shape, and arm length; and partly on several additional aspects of overall appearance, including mantle shape, chromatophore patterns (dependent on condition of specimens) and whether the tip of the mantle is pierced posteriorly by the rostrum of the gladius. However, as in later ontogenetic stages, tentacular morphology provides the most useful basis for comparison of small specimens. The form of the suckers (both marginal and future-hook-bearing) in paralarvae and small juveniles is useful, including: overall sucker morphology; number, distribution and morphology of pegs on the oral surface; presence/absence and number of teeth around the inner margin of the sucker ring; and size of the aperture relative to overall sucker diameter. The absolute size $(\mathrm{ML})$ at which certain ontogenetic changes are observed is also important, including development of first ventral and first dorsal hooks, completion of ventral and dorsal hook series development, and loss (if any) of marginal suckers. 
Since two Onykia species - Ok. (Ok.) carriboea and Ok. (Ok.) indica - remain known solely from early ontogenetic stages, tracing developments in their tentacular morphology as more intermediate-sized material becomes available will facilitate identification of adult forms.

Inter- and intrafamilial relationships

Morphologically, onychoteuthids are generally associated with the other hook-bearing families (e.g. Gray 1849, Young \& Harman 1998), but in fact, there appears to be no consensus among recent studies regarding the family's phylogenetic position. Young \& Harman (1998) used 13 characters to analyse relationships between ten teuthoid families and found the Onychoteuthidae equally closely related to the Octopoteuthidae, Gonatidae, Histioteuthidae and Ommastrephidae. In Carlini \& Graves' (1999) genetic study (using COI), Onychoteuthis consistently grouped with Ommastrephes and Sthenoteuthis, despite representatives of each of the other families included by Young \& Harman (1998) also being analysed. Lindgren et al. (2004), also using molecular data (18S, 28S, $\mathrm{H} 3$ and $\mathrm{COI}$ ), in synthesis with morphological characters, found the Onychoteuthidae most closely related to the Ancistrocheiridae, followed by the Architeuthidae and Gonatidae; the Ommastrephidae fell out nearly as distantly as possible within the Teuthida.

Several genetic studies have also addressed relationships within the family. Bonnaud et al. (1998) conducted a molecular analysis (16S) using eight onychoteuthid species from four genera (including Gen. nov. 1 knipovitchi), and concluded that the genera Onychoteuthis and 'Moroteuthis' (= Onykia) each comprised groups of closely related species. Their 'Onykia sp.' (= Gen. nov. 2 sp. 1) was most closely related to the 'Moroteuthis' group - more closely related, in fact, than was Gen. nov. 1 knipovitchi, which fell out between Gen. nov. 2 sp. 1 and the Onychoteuthis species.

Wakabayashi et al. (2007) conducted a similar study (using COI) on nine onychoteuthid species from three genera (including Gen. nov. 1 knipovitchi), and also found that three 'Moroteuthis'/Onykia species - Ok. (Ok.) robusta, Ok. (Ok.) robsoni and Ok. (Ok.) loennbergii - grouped together, with several paralarvae of classic Onykia morphology genetically identical to Ok. (Ok.) robusta; Ok. (M.) ingens was also related to this group, though more distantly. The Onychoteuthis species also grouped together, and Gen. nov. 1 knipovitchi grouped with Onychoteuthis rather than Onykia 
- in fact, Gen. nov. 1 knipovitchi was nearly as closely related to Onychoteuthis as was Ok. (M.) ingens to other Onykia taxa.

So little concurrence between studies in which half of the analysed taxa overlapped is remarkable. Both Bonnaud et al. (1998) and Wakabayashi et al. (2007) found that two or three Onychoteuthis species grouped together, as did two or three Onykia species, and that Gen. nov. 1 knipovitchi was far more distantly related to 'Moroteuthis'/Onykia than previously believed. However, the studies disagreed on the position of Ok. (M.) ingens, which grouped closely with Ok. (Ok.) robsoni in Bonnaud et al. (1998) and far from it in Wakabayashi et al. (2007), and on the position of Gen. nov. 1 knipovitchi, which grouped with Gen. nov. 2 sp. 1 and the Onykia species in Bonnaud et al. (1998) and with the Onychoteuthis species in Wakabayashi et al. (2007).

However complementary or contrary these findings are, it is important to note that these studies presented the first molecular data on onychoteuthids, and were constrained by available material. Between them only ten to 12 species (the identities of Wakabayashi et al.'s 'Os. banksii' and 'Onykia sp.' specimens are unconfirmed), from four of the seven now-recognised genera, were analysed; therefore genetic data remain unreported for half of the recognised onychoteuthid taxa, including Ancistroteuthis, Kondakovia and Notonykia. Although a genetic component to this study was prevented by the absence of fresh material of most species examined, samples were collected wherever possible in the hope of undertaking a molecular component in the future.

Nesis (2000) assessed relationships within the family based on morphology and zoogeography, discussing primitive versus advanced character states and likely phylogeny. He considered dense, muscular tissues, smooth skin on the mantle, and the absence of photophores and secondary occipital folds all to be primitive states, with ammoniacal tissues, sculptured skin, secondary occipital folds, and photophores representing more advanced states. Nesis also discussed the presence of both 'primitive' and 'advanced' genera in every zoogeographical zone, although he considered Onykia primitive and 'Moroteuthis' advanced, and concluded that the family evolved in the tropics.

From the combined observations of these earlier authors and the morphological and zoogeographical findings herein, several patterns emerge. Most onychoteuthid genera seem to follow one of two general body plans: small-bodied (ML 60-320mm at 
maturity), smooth-skinned, with muscular, negatively buoyant tissues, secondary occipital folds, and relatively low numbers (15-28) of relatively large tentacular hooks (length of largest 15-25\% CL) (Onychoteuthis, Ancistroteuthis and Notonykia); or large-bodied (ML 250mm-2m at maturity), with sculptured skin (where adults are known), ammoniacal tissues, no secondary occipital folds, and relatively high numbers (22-36) of relatively small tentacular hooks (largest 5-11\% CL) (Onykia and Kondakovia). Two genera are somewhat intermediate, being small- to medium-sized (Gen. nov. 2 to ML $150 \mathrm{~mm}$, Gen. nov. 1 knipovitchi to ML $>320 \mathrm{~mm}$ at maturity) and smooth-skinned, but lacking secondary occipital folds and possessing intermediate numbers (24-27 and 25-31, respectively) of intermediate-sized hooks (largest 10$20 \% \mathrm{CL}$ and $\sim 12 \% \mathrm{CL}$, respectively).

Each general body plan is represented by one relatively diverse genus (Onychoteuthis has nine currently recognised species, Onykia seven) and one or two genera with one or two species each. Most species of the diverse genus in each case are found within a particular latitudinal range (Onychoteuthis species are mostly tropical, Onykia species mostly sub-Antarctic to sub-tropical, and largely notalian), with one or several species found along the edge(s) of this range. The smaller genus or genera presently appear restricted to certain latitudes overlapping with or adjacent to those of the large genus (Ancistroteuthis is at present considered a boreal Atlantic species, while Notonykia appears exclusively notalian, in sub-tropical to sub-Antarctic waters; Kondakovia is exclusively Antarctic to sub-Antarctic). Of the two remaining genera, Gen. nov. 2 appears distributed throughout sub-tropical to tropical waters worldwide, while Gen. nov. 1 knipovitchi has been found only in the sub-Antarctic.

These distribution and morphological patterns reveal a latitudinal morphological gradient within the Onychoteuthidae. In the high latitudes of the Southern Hemisphere, species following the larger body plan are more prevalent; the southern-most smallbodied genus (Notonykia, which also possesses the lowest number of secondary occipital folds of any small-bodied genus) appears in the northern-most sub-Antarctic and southern sub-tropical waters. Gen. nov. 1 knipovitchi, with its intermediate body plan, also occurs in the sub-Antarctic latitudes. Continuing north, the southern-most Onychoteuthis species (Os. bergii and Os. aequimanus) first occur in the sub-tropical waters of the Southern Hemisphere, along with the southern-most Gen. nov. 2 species (Gen. nov 2 rancureli). Approaching the southern boundaries of the tropics, Onykia species are no longer in the majority; three to four species of Onychoteuthis are found in the equatorial Atlantic and Pacific Oceans. To the north, even fewer Onykia species 
are found, with only Ok. (Ok.) robusta inhabiting the northern North Pacific, while three to five Onychoteuthis species are present; similarly, in the north Atlantic, two Onychoteuthis species are present as well as A. lichtensteinii, while only Ok. (Ok.) robsoni and Ok. (OK.) carriboea remain, and only in the tropical to southern subtropical waters. In spite of the family's success in the high southern latitudes, few onychoteuthid species are found in northern sub-tropical to sub-Arctic waters, and none has been recorded from the Arctic itself.

\section{Biology \& ecology}

The life history of onychoteuthids is poorly known. Based on collection data for the material examined herein, most species appear to occur in surface waters in early ontogenetic stages. Adults seem to follow diverse strategies; maturing Onychoteuthis are most frequently caught near the surface at night and frequently 'fly' or are washed aboard vessels, while adult Onykia are commonly caught at depths of several hundred metres, some mostly by bottom trawl (e.g. Ok. (Ok.) robsoni). Literature reporting nonsystematic aspects of onychoteuthids has concentrated mainly on OK. (M.) ingens (Jackson 1993, 1995, 1997, 2001; Jackson \& Jackson 2004; Jackson \& Mladenov 1994; Jackson et al. 1997, 1998; Cherel \& Duhamel 2003) and Os. borealijaponica (Murata \& Ishii 1977, Murata et al. 1982, Okutani \& Murata 1983), with several other studies also reporting on 'Chaunoteuthis mollis' (most likely encompassing spent specimens from a range of taxa, given their diverse geographic origins). Some information on onychoteuthid ecology (life cycle, ontogenetic depth migration) has been gleaned from studies on their predators (Clarke 1980, Cherel \& Weimerskirsch 1999), and most specimens of large species like $K$. longimana have in fact been recovered from gut contents of cetaceans, seals and seabirds (pers. obs.). Dietary studies have been conducted on very few species; Ok. (M.) ingens is known to feed on crustaceans, myctophids and other squid (Jackson et al. 1998; Phillips et al. 2001, 2002, 2003a, 2003b; Cherel \& Duhamel 2003), and K. longimana relies at least partially on euphausids (Nemoto et al. 1998). Almost nothing is known of the diet of any other species. Accounts of reproductive behaviour are likewise sparse, with studies on $O k$. (M.) ingens once again comprising the majority of reports (Jackson \& Mladenov 1994, Jackson \& Jackson 2004, Jackson et al. 2004), interspersed with several accounts of spermatophore implantation in 'Chaunoteuthis mollis' and other spent Onychoteuthis specimens (Nesis 1970, Murata et al. 1982, Okutani \& Ida 1986). 


\section{CONCLUSIONS}

This revision is the most comprehensive treatment of the Onychoteuthidae to date, describing 25 onychoteuthid species, nearly double the number treated in most recent accounts (Nesis 1987, Kubodera et al. 1998, Vecchione et al. 2003). Most extant type material has been examined, and each taxon attributed to the family for the past 200 years has been critically appraised (see Appendix E). External characters and states are described herein for each taxon considered taxonomically valid, augmented by internal and ontogenetic characters where the condition and number of specimens permitted.

This study is necessarily restricted by the material available. Some geographic regions (New Zealand, the North Pacific) were well represented in collections, while specimens from others were sparse (e.g. South Atlantic and Indian Oceans), more likely indicating relative collection/fishing effort than the actual abundance or scarcity of onychoteuthids. Similarly, individuals appear more vulnerable to collection at certain ontogenetic stages (paralarvae to small juveniles and sub-adults), and are conspicuously absent at others (e.g. mid-size to large juveniles of several Onykia species); once again, most collection effort appears to have been made within the upper $100 \mathrm{~m}$ of the water column or between several hundred metres and the bottom. Some species are known from a single specimen and/or ontogenetic stage, or from small numbers of specimens largely taken from the gut contents of predators, and it is possible that these species occupy a position in the water column that is not regularly sampled or trawled. Specifically, Os. mollis is known from a sole spent female lacking most systematic characters; of the seven Onykia species, five are known solely from specimens of early or late ontogeny; and the status of Gen. nov. 2 spp. 1-3 is also uncertain.

Given these constraints, it is likely that the true number of onychoteuthid species is intermediate between the 26 species (including $K$. nigmatullini) reported herein and the 12-14 generally accepted by recent authors, but is likely to exceed 20 species.

Additional material and molecular analysis may resolve the identity of adult Ok. (Ok.) carriboea and result in synonymising one of the Onykia species found in the central Atlantic and known only from large juvenile to adult specimens; Ok. (Ok.) indica may likewise ultimately be attributed to a known older Onykia species, although few adult Onykia specimens are known from the Indian Ocean. 
Considerable systematic confusion has ensued from the past two centuries' worth of synonymies and partial descriptions. Each taxon treated herein has recognisable unique combinations of traits that are described consistently, permitting detailed comparison between taxa. Synonymies have been constructed conservatively and new taxa have been formally described only where sufficient material exists. Ongoing collection and study of onychoteuthid material from the more poorly represented geographic areas, and increasing availability of tissue samples, will undoubtedly reveal new information and assist in further understanding this systematically complex family.

One final noteworthy consideration is the historical span of onychoteuthid collections (Fig. 1), and the resulting temporal trend in onychoteuthid research. Only $13 \%$ of the material examined in this study was collected prior to 1950 , while $\sim 20 \%$ of the available material was collected in 1950-1969 and an additional $20 \%$ in each of the three decades thereafter. Although species belonging to the Onychoteuthidae have been known for over 200 years, only recently has sufficient material become available to begin understanding onychoteuthid diversity. Four of the seven genera recognised herein (including Gen. nov. 1 and Gen. nov. 2) have been recognised/described since 1970 and 12 of the $\sim 26$ species (excluding Gen. nov. 2 spp. 1-3 and a new species of Kondakovia) since 1960. Even if some of the taxa treated above do ultimately prove allied, additional unknown taxa are likely to exist and recognised onychoteuthid diversity is expected to increase, provided collection efforts continue. 


\section{ACKNOWLEDGEMENTS}

I am profoundly grateful to many people for their assistance and support. Without my mentor and friend Steve O'Shea, this project would never have started, continued or finished. My partner Gary Peebles, my parents Gary and Kristi Bolstad, and my sister J. Berit Bolstad have all endured and even encouraged esoteric squid talk far above and beyond the call of duty. Bruce Marshall (NMNZ) and Dick Young (University of Hawai'i) have been willing and inexhaustible founts of systematic knowledge. Drs T. Kubodera, G. Jackson, S. Bizikov and V. Laptikhovsky have also provided advice and answers; further thought-provoking discussions have come from fellow teuthological postgrads J. Horstkotte, F. Gómez-Villota, A. Lindgren and H.J. Hoving. My secondary supervisors B. Bollard-Breen and S. Cook have been kind enough to review and comment on sections of this thesis, as have R. Young, T. Kubodera, B. Marshall, N. Voss, E. Shea, N. Moltschaniwskyj, M. Norman, and two anonymous reviewers.

Thanks also to everyone who assisted with and facilitated my domestic and overseas research visits and loans, including: P. Callomon (ANSP); J. Ablett and K. Way (BMNH); B. Van Syoc and C. Piotrowski (CASIZ); G. Jackson (IASOS); M. Pernice, L. Bonnaud and R. Boucher (MNHN); K. Schnabel and A.-N. Lörz (NIWA); M. Seki (NMFS); B. Marshall (NMNZ); M. Norman and C. Rowley (NMV); T. Kubodera and T. Okutani (NSMT); L. Hoenson and M. Cochrane (SAM); E. Hochberg (SBMNH); N. Voss (UMML); M. Vecchione (NMFS/USNM), L. Cole, M. Schotte and L. Ong (USNM); F. Köhler and M. Glaubrecht (ZMB); E. Willassen and J. Kongsrud (ZMBN); B. Hausdorf and V. Miske $(\mathrm{ZMH})$; and P. Young in Hawai'i. SEM assistance was provided by C. Hobbis, A. Turner and B. James (University of Auckland), and administrative assistance by the saintly B. Lorigan (AUT).

My heartfelt gratitude goes also to the institutions who provided financial support for this research: Auckland University of Technology and its Earth \& Oceanic Sciences Research Institute, Discovery Channel, the American Museum of Natural History (and N. Landman in particular), the Malacological Society of London, and the Harvard Museum of Comparative Zoology.

Several colleagues assisted me in translating foreign references, including S. Gulyaev and S. Dewas. I. Anderson (University of Auckland), D. Ivanov and A. Sysoev (ZMMGU) allowed the use of several photographs, and K.H. Moore (NMFS) several illustrations.

Finally, I must thank the late Dr. Brian Kensley (USNM), my first systematic mentor, who introduced me to the world of invertebrate taxonomy and the joys of studying small dead things in jars. 


\section{REFERENCES}

Adam, W. 1942. Notes sur les Cephalopodes, XXI.- A propos d'une publication peu connue de A. Risso, 1854. Bulletin du Musee royal d'Histoire naturelle de Belgique 18 (25): 1-36.

Adam, W. 1952. Cephalopodes. Expedition Oceanographique Belge dans les Eaux Cotieres Africaines de l'Atlantique Sud (1948-1949), Resultats scientifiques, 3 (3): 1-142.

Adam, W. 1962. Céphalopodes de l'Archipel du Cap.-Vert, de l'Angola et du Mozambique. Memórias da Junta de Investicacões do Ultramar, second series, 33: 9-64, PI. 1.

Adam, W. 1972. Notes sur les Céphalopodes: 25. Contribution à la connaissance de Chaunoteuthis mollis Appellöf, 1891. Mededelingen van het Koninklijk Belgisch Instituut voor Natuurwetenschappen, 48 (12): 1-7.

Anderson, O.F.; Bagley, N.W.; Hurst, R.J.; Francis, M.P.; Clark, M.R.; McMillan, P.J. 1998. Atlas of New Zealand fish and squid distributions from research bottom trawls. NIWA Technical Report 42 (unpaginated).

Akimushkin, I.I. 1965. Cephalopods of the seas of the U.S.S.R. (English translation) Israel Program for Scientific Translations. Jerusalem. 223 pp.

Appellöf, A. 1891. Teuthologische Beiträge II: Chaunoteuthis n.g. Oegopsidarium. Bergens Museums Årsberetning, 1891 (1): 29 pp.

Arkhipkin, A.I.; Nigmatullin, C.M. 1997. Ecology of the oceanic squid Onychoteuthis banksii and the relationship between the genera Onychoteuthis and Chaunoteuthis (Cephalopoda: Onychoteuthidae). Journal of the Marine Biological Association of the United Kingdom 77: 839-869.

Bello, G. 1998. Chaunoteuthis mollis: Re-examination of a Mediterranean specimen and its identity with Onychoteuthis banksii (Cephalopoda: Onychoteuthidae). Journal of the Marine Biological Association of the UK 78: 1027-1030.

Belon, P. 1553. De aquatilibus. Libro duo cum eiconibus ad viuam iposorum effigiem, quoad eius fieri potuit, expressis. Ad amplissimum Cardinalem Castillionaeum. Paris. 448 pp.

Berry, S.S. 1912. A review of the Cephalopods of Western North America. Bulletin of the Bureau of Fisheries 30: 265-336.

Berry, S.S. 1913. Some New Hawaiian Cephalopods. Proceedings of the U.S. National Museum 45: 563-566.

Berry, S.S. 1914. The Cephalopoda of the Hawaiian Islands. Bulletin of the Bureau of Fisheries 32: 253-362.

Berry, S.S. 1916. Cephalopoda of the Kermadec Islands. Proceedings of the Academy of Natural Sciences of Philadelphia Feb. 1916: 45-67.

Bigelow, K.A. 1994. Age and growth of the oceanic squid Onychoteuthis borealijaponica in the North Pacific. Fisheries Bulletin (U.S.) 92: 13-25. 
Bizikov, V.A. 1996. Atlas of Morphology and Anatomy of the Gladius of Squids. VNIRO Publishing, Moscow. 247 pp.

Blainville, H.D. de 1823. Memoire sur les especes du genre Calmar (Loligo, Lamarck). Journal de Physique, de Chimie et d'Histoire Naturelle, 96: 116-135.

Bolstad, K.S. 2006. Sexual dimorphism in the beaks of Moroteuthis ingens Smith, 1881 (Cephalopoda: Oegopsida: Onychoteuthidae). New Zealand Journal of Zoology 33: 317-327.

Bolstad, K.S. 2007. Systematics and distribution of the New Zealand onychoteuthid fauna (Cephalopoda: Oegopsida), including a new species, Notonykia nesisi sp. nov. Reviews in Fish Biology and Fisheries 17: 305-335.

Bolstad, K.S. (in press) Two new species and a review of the squid genus Onychoteuthis Lichtenstein, 1818 (Oegopsida: Onychoteuthidae) from the Pacific Ocean. Bulletin of Marine Science.

Bonnaud, L.; Rodhouse, P.G.; Boucher-Rodoni, R. 1998. A Phylogenetic Study of the Squid Family Onychoteuthidae (Cephalopoda: Oegopsida). Proceedings of the Royal Society of London 265: 1761-1770.

Carlini, D. B.; Graves, J. E. 1999. Phylogenetic analysis of cytochrome c oxidase I sequences to determine higher-level relationships within the coleoid cephalopods. Bulletin of Marine Science 64: 57-76.

Cherel, Y.; Duhamel, G. 2003. Diet of the squid Moroteuthis ingens (Teuthoidea: Onychoteuthidae) in the upper slope waters of the Kerguelen Islands. Marine Ecology Progress Series 250: 197-203.

Cherel, Y.; Duhamel, G. 2004. Antarctic jaws: cephalopod prey of sharks in the Kerguelen waters. Deep-Sea Research I 51: 17-31.

Cherel, Y.; Weimerskirsch, H. 1999. Spawning cycle of onychoteuthid squids in the Indian Ocean: new information from seabird predators. Marine Ecology Progress Series 188: 93-104.

Clarke, M. 1962. The identification of cephalopod "beaks" and the relationship between beak size and total body weight. Bulletin of the British Museum (Natural History) Zoology 8 (10): 422-480, Pls 13-22.

Clarke, M. 1965. "Growth rings" in the beaks of the squid Moroteuthis ingens (Oegopsida: Onychoteuthidae). Malacologia 3: 287-307.

Clarke, M. 1966. A review of the systematics and ecology of oceanic squids. Advances in Marine Biology 4: 91-300.

Clarke, M.R. 1980. Cephalopoda in the diet of sperm whales of the southern hemisphere and their bearing on sperm whale biology. Discovery Reports 37 : $324 \mathrm{pp}$.

Clarke, M.R. 1986. A handbook for the identification of cephalopod beaks. Clarendon Press, Oxford. 273 pp. 
Clarke, M.R.; Goodall, N. 1994. Cephalopods in the diet of three odontocete cetacean species stranded at Tierra del Fuego, Globicephalia melaena (Traill, 1809), Hyperoodon planifrons Flower, 1882 [sic], and Cephalorhynchus commersonii (Lacepede, 1804). Antarctic Science 6 (2): 149-154.

Clarke, M.R.; MacLeod, N. 1980. Cephalopod remains from sperm whales caught off Western Canada. Marine Biology 59: 241-246.

Clarke, M.R.; MacLeod, N. 1982a. Cephalopod remains in the stomachs of eight Weddell seals. British Antarctic Survey Bulletin 57: 33-40.

Clarke, M.; MacLeod, N. 1982b. Cephalopod remains from the stomachs of sperm whales caught in the Tasman Sea. Memoirs of the National Museum of Victoria 43: $25-42$.

Clarke, M.R.; MacLeod, N. 1982c. Cephalopods in the diet of southern elephant seals Mirounga leonina at Signy Island, South Orkney Islands, South Atlantic Ocean. British Antarctic Survey Bulletin 57: 27-32.

Clarke, M.R.; MacLeod, N.; Paliza, O. 1976. Cephalopod remains from the stomachs of Sperm whales caught off Peru and Chile. Journal of Zoology London 180: 477-493.

Clarke, M.R.; Martins, H.R.; Pascoe, P. 1993. The diet of sperm whales (Physeter macrocephalus Linnaeus 1758) off the Azores. Philosophical Transactions of the Royal Society of London B 339: 67-82.

Clarke, M.R.; Roper, C.F.E. 1998. Cephalopods represented by beaks in the stomach of a sperm whale stranded at Paekakariki, North Island, New Zealand. South African Journal of Marine Science 20: 129-133.

Dall, W.H. 1871. Descriptions of sixty new forms of mollusks from the West Coast of North America and the North Pacific Ocean, with notes on others already described. American Journal of Conchology, 7 (2): 93-160, 4 Pls.

Dall, W.H. 1873. Aleutian Cephalopods. The American Naturalist 7: 484-485.

Dell, R.K. 1951. The New Zealand Cephalopoda. Tuatara 4: 91-102.

Dell, R.K. 1952. The Recent Cephalopoda of New Zealand. Dominion Museum Bulletin 16: $157 \mathrm{pp}$.

Engeser, T.S.; Clarke, M.R. 1988. Cephalopod hooks, both Recent and fossil. In: Clarke, M.R.; Trueman, E.R. (eds) The Mollusca. Vol. 12. Paleontology and Neontology of Cephalopods. Academic Press, New York. 355 pp.

Férussac, A.E. de; d'Orbigny, A. 1834-1848. Histoire naturelle générale et particulière des Céphalopodes Acétabulifères Vivants et Fossiles. Ballière, Paris. Ivi + 361 pp., Atlas with 144 Pls.

Filippova, J.A. 1972. New data on the squids (Cephalopoda: Oegopsida) from the Scotia Sea (Antarctic). Malacologia 11 (2): 391-406. 
Filippova, J.A.; Alekseev, D.O.; Bizikov, V.A.; Khromov, D.N. 1997. Commercial and mass cephalopods of the world ocean. A manual for identification. VNIRO Publishing, Moscow. 271 pp.

Fiscus, C. H.; Rice, D. W.; Wolman, A. A. 1989. Cephalopods from the stomachs of sperm whales taken off California. NOAA Technical Report NMFS 83: 1-12.

Gabb, W.M. 1862. Description of two new species of Cephalopods in the Museum of the California Academy of Natural Sciences. Proceedings of the California Academy of Natural Sciences, 2: 170-172.

Gabb, W.M. 1868. Description of a new species of cephalopod from the South Pacific. American Journal of Conchology 4 (1): 23-24, 1 PI.

Gaskin, D.E.; Cawthorn, M.W. 1967a. Diet and feeding habits of the sperm whale (Physeter catodon L.) in the Cook Strait region of New Zealand. New Zealand Journal of Marine and Freshwater Research 2: 156-179.

Gaskin, D.E.; Cawthorn, M.W. 1967b. Squid mandibles from the stomachs of sperm whales (Physeter catodon L.) captured in the Cook Strait region of New Zealand. New Zealand Journal of Marine and Freshwater Research 1: 59-70.

Gillespie, G.E. 1997. Review of Biology, Fisheries and Assessment of Oceanic Squids, particularly Ommastrephes bartrami, Onychoteuthis borealijaponica, Gonatopsis borealis and Berryteuthis magister. 45 pp.

Glaubrecht, M.; Salcedo-Vargas M.A. 2000. Annotated type catalogue of the Cephalopoda (Mollusca) in the Museum für Naturkunde, Humboldt University of Berlin. Mitteilungen aus dem Museum für Naturkunde Berlin, Zoologischen, 76 (2): 269-282.

Gómez-Villota, F. 2007. Sperm whale diet in New Zealand. Thesis (MSc), Auckland University of Technology, Auckland, New Zealand. 236 pp.

Gould, A.A. 1852. Mollusca and shells. United States Exploring Expedition during the years 1838, 1839, 1840, 1841, 1842. Under the command of C. Wilkes, U.S.N. Vol. 12,510 pp., atlas of 16 pp. and 52 Pls.

Gray, J.E. 1847. A list of the genera of Recent Mollusca, their synonyma and types. Proceedings of the Zoological Society of London 15: 129-219.

Gray, J.E. 1849. Catalogue of the Mollusca in the Collection of the British Museum, I: Cephalopoda Antepedia. London: British Museum (Natural History). 164 pp.

Grimpe, G. 1921. Das Leipziger Stück von Chaunoteuthis mollis Apellöf. Zoologischer Anzeiger 52 (12/13): 289-296.

Guerra Sierra, A. 1992. Mollusca, Cephalopoda. In: Ramos, M.A. et al. (eds.) Fauna Ibérica 1. 327 pp., 12 pl. Museo Nacional de Ciencias Naturales, CSIC. Madrid.

Guerrero-Kommritz, J.G. 2001. Catalogue of the cephalopod type specimens in the Zoological Museum Hamburg. Mitteilungen. Hamburgisches Zoologische Museum and Institut, 98: 171-174. 
Haimovici, M.; Costa, P.A.S.; Santos, R.A.; Martins, A.S.; Olavo, G. 2007. Composição de espécies, ditribuição e abundância de cefalópodes do talude de região central do Brasil. In: Costa, P.A.; Olavo, G.; Martins, A.S. (eds). Biodiversidade da fauna marinha profunda na costa central brasiliera. $24^{\text {th }}$ ed. Rio de Janeiro, Museo Nacional. 109-132.

Harman, R.F.; Young, R. E. 1985. The larvae of ommastrephid squids (Cephalopoda: Teuthoidea) from Hawaiian waters. Vie et Milieu 35: 211-222.

Hess, S.C. 1987. Comparative morphology, variability, and systematic applications of cephalopod spermatophores (Teuthoidea and Vampyromorpha). Dissertation (PhD). University of Miami. 581 pp.

Hoyle, W.E. 1904. A Diagnostic Key to the Genera of Recent Dibranchiate Cephalopoda. Manchester Memoirs 48: 1-21.

Hoyle, W.E. 1910. A list of the generic names of Dibranchiate Cephalopoda with their type species. Abhandlungen der Senckenbergischen Naturforschenden Gesellschaft 32: 407-413.

Hoyle, W.E. 1912. The Cephalopoda of the Scottish National Antarctic Expedition. Transactions of the Royal Society of Edinburgh 48: 273-283.

Imber, M.J. 1976. Comparison of prey of the black Procellaria petrels of New Zealand. New Zealand Journal of Marine and Freshwater Research 10 (1): 119-130.

Imber, M.J.; Russ, R.R. 1975. Some foods of the wandering albatross (Diomedea exulans). Notornis 22 (1): 27-36.

Ishikawa, C. \& Wakiya, Y. 1914. On a New Species of Moroteuthis from the Bay of Sagami, M. Iönnbergii. Journal of the College of Agriculture, Imperial University of Tokyo, 4 (7): 445-460, Pls 45, 46.

Iverson, I.L.K.; Pinkas, L. 1971. A pictorial guide to beaks of certain Eastern Pacific cephalopods. Fish Bulletin 152: 83-105.

Jackson, G.D. 1993. Growth Zones within the Statolith Microstructure of the Deepwater Squid Moroteuthis ingens (Cephalopoda: Onychoteuthidae): Evidence for a Habitat Shift? Canadian Journal of Fisheries and Aquatic Science 50: 1-9.

Jackson, G.D. 1995. The use of beaks as tools for biomass estimation in the deepwater squid Moroteuthis ingens (Cephalopoda: Onychoteuthidae) in New Zealand waters. Polar Biology 15: 9-14.

Jackson, G.D. 1997. Age, growth and maturation of the deepwater squid Moroteuthis ingens (Cephalopoda: Onychoteuthidae) in New Zealand waters. Polar Biology 17: $268-274$.

Jackson, G.D. 2001. Confirmation of winter spawning of Moroteuthis ingens (Cephalopoda: Onychoteuthidae) in the Chatham Rise of New Zealand. Polar Biology 24: 97-100.

Jackson, G.D.; Buxton, N.G.; George, M.J.A. 1997. Beak length analysis of Moroteuthis ingens (Cephalopoda: Onychoteuthidae) in the Falkland Islands 
region of the Patagonian Shelf. Journal of the Marine Biological Association of the UK 77: 1235-1238.

Jackson, G.D.; Jackson, C.H. 2004. Mating and spermatophore placement in the onychoteuthid squid Moroteuthis ingens. Journal of the Marine Biological Association of the UK 84: 783-784.

Jackson, G.D.; Lu, C.C. 1994. Statolith microstructure of seven species of Antarctic squid captured in Prydz Bay, Antarctica. Antarctic Science 6 (2): 195-200.

Jackson, G. D.; McKinnon, J. F.; Lalas, C.; Ardern, R.; Buxton, N. G. 1998. Food spectrum of the deepwater squid Moroteuthis ingens (Cephalopoda: Onychoteuthidae) in New Zealand Waters. Polar Biology 20: 56-65.

Jackson, G.D.; Mladenov, P.V. 1994. Terminal spawning in the deepwater squid Moroteuthis ingens (Cephalopoda: Onychoteuthidae). Journal of Zoology, London 234: 189-201.

Jackson, G.D.; Semmens, J.M.; Phillips, K.L.; Jackson, C.H. 2004. Reproduction in the deepwater squid Moroteuthis ingens, what does it cost? Marine Biology 145: 905-916.

Jarre, A.; Clarke, M.R.; Pauly, D. 1991. Re-examination of growth estimates in oceanic squids: the case of Kondakovia longimana (Onychoteuthidae). ICES J. Marine Science. 48: 195-200.

Johnson, R.I. 1964. The Recent Mollusca of Augustus Addison Gould. United States National Museum Bulletin 239: 182 pp., 45 Pls.

Joubin, L. 1900. Cephalopodes provenant des campagnes de la Princesse-Alice (1891-1897). Resultats des campagnes scientifiques accomplies sur son yacht par Albert I Prince souverain de Monaco 17: 1-135, 15 Pls.

Köhler, F.; Glaubrecht, M. 2004. Addendum to the catalogue of cephalopod types in the Museum für Naturkunde, with remarks on Onychoteuthis taxa described by Hinrich Lichtenstein. Mitteilungen aus dem Museum für Naturkunde in Berlin Zoologische Reihe 80 (2): 275-282.

Kubodera, T. 1990. Onychoteuthidae, Lepidoteuthidae, Histioteuthidae, Cranchiidae. Pp. 336-339. In: Amaoka, K.; Matsuura, K.; Inada, T.; Takeda, M.; Hatanaka, H.; Okada, K. (eds) Fishes collected by the R/V Shinkai Maru around New Zealand. Japan Marine Fishery Resource Research Centre, Tokyo: 410 pp.

Kubodera, T.; Piatkowski, U.; Okutani, T.; Clarke, M.R. 1998. Taxonomy and Zoogeography of the Family Onychoteuthidae (Cephalopoda: Oegopsida). Smithsonian Contributions to Zoology 586 (2): 277-291.

Laptikhovsky, V.; Arkhipkin, A.; Bolstad, K.S. 2008. A second species of the squid genus Kondakovia (Cephalopoda: Onychoteuthidae) from the sub-Antarctic. Polar Biology 40: [pagination unknown; online only at present].

Latreille, P.A. 1825. Familles naturelles du regne animal, exposees succinctement et dans un ordre analytique, avec l'indication de leurs genres. Paris, J.B. Bailliere, $570 \mathrm{pp}$. 
Leach, W.E. 1817. Synopsis of the orders, families, and genera of the class Cephalopoda. The Zoological Miscellany; being descriptions of new or interesting animals 3 (30): 137-141.

Lesson, R.P. 1830. Centurie Zoologique, ou choix d'animaux rares nouveaux ou imparfaitement conus. Paris, F.G. Lavrault. 244 pp.

Lesueur, C.A. 1821. Description of Several New Species of Cuttlefish. Journal of the Academy of Natural Sciences of Philadelphia 2 (1): 86-101.

Lesueur, C.A.; Petit, N.M. 1807. Atlas Historique, 41 plates. In: F. Peron (ed.) Voyage de decouvertes aux terres Australes sur les corvettes le Geographe, le Naturaliste, et la Geolette le Casuarina, pendant 1800-1804. 2 volumes, atlas and 14 maps, 1807-1816.

Lichtenstein, K.M.H. 1818. Onychoteuthis, Sepien mit Krallen. Isis, 1818 (9): $1591-$ 1592.

Lichtenstein, H.C. 1820. Von den Sepien mit Krallen. Abhandlungen der physikalischen Klasse der Königlich-Preußischen Akademie der Wissenschaften aus den Jahren 1818-1819. 211-224. Berlin (Realschul-Buchhandlung).

Lindgren, A.R.; Giribet, G.; Nishiguchi, M.K. 2004. A combined approach to the phylogeny of Cephalopoda (Mollusca). Cladistics 20: 454-486.

Lipinski, M.R.; Linkowski, T.B. 1986. Some aspects of the biology of the squid Moroteuthis ingens (Onychoteuthidae) from New Zealand waters. Reports of the Sea Fisheries Institute, Gdynia 21: 97-105.

Lipinski, M.R.; Naggs, F.A.; Roeleveld, M.A. 2000. Catalogue of types of Recent cephalopods in the collection of the Natural History Museum, London. Annales Zoologici (Warszawa), 50 (1): 101-120.

Lönnberg, E. 1898. On the Cephalopods collected by the Swedish Expedition to Tierra del Fuego 1895-96. Svenska Expedition en till Magellanslanderna 2 (4): 49-64.

Lu, C.C.; Boucher-Rodoni, R.; Tillier, A. 1995. Catalogue of types of recent Cephalopoda in the Museum national d'Histoire Naturelle (France). Bulletin du Museum national d'Histoire naturelle, Paris, series 4, 17 (3-4): 307-343.

Messenger J.B.; Young, J.Z. 1999. The radular apparatus of cephalopods. Philosophical Transactions of the Royal Society of London. 354: 161-182.

Middendorff, A.T. 1849. Beiträge zu einer Malacozoologica Rossica. II-III. Aufzahlung und Beschreibung der zur Meeresfauna Russlands gehörigen Einschaler. Memoires de l'Academie imperiale des Sciences de St. Petersbourg, (series 6) 6: 329-610, 21 Pls.

Molina, G.I. 1782. Saggio sulla storia naturale del Chili. Bologna, Stamperia di S. Tommaso d'Aquino, $367 \mathrm{pp}$.

Moore, G.J.; Robertson, G.; Wienecke, B. 1998. Food requirements of breeding king penguins at Heard Island and potential overlap with commercial fisheries. Polar Biology 20: 293-302. 
Murata, M.; Ishii, M. 1977. Some information on the oceanic squid, Ommastrephes bartrami (Lesueur) and Onychoteuthis borealijaponicus Okada, in the Pacific Ocean off Northeastern Japan. Bulletin of the Hokkaido Regional Fisheries Research Library 42: 1-23.

Murata, M.; Ishii, M.; Osako, M. 1982. Some information on copulation of the oceanic squid Onychoteuthis borealijaponicus Okada. Bulletin of the Japanese Society of Scientific Fisheries, 48 (3): 351-354.

Naef, A. 1921/1923. Die Cephalopoden. Fauna e Flora de Golfo di Napoli 35 [English translation] Parts 1, 2. Berlin. 863 pp.

Nemoto, T.; Okiyama, M.; Iwasaki, N.; Kikuchi, T. 1988. Squid as predators on krill (Euphausia superba) and prey for sperm whales in the Southern Ocean. In: Sahrhage, D. (ed.) 1988. Antarctic Ocean and resources variability. Berlin: Springer-Verlag 292-296.

Nemoto, T.; Okiyama, M.; Takahashi, M. 1985. Aspects of the roles of squid in food chains of marine Antarctic ecosystems. In: Siegfried, W.R.; Condy, P.R.; Laws, R.M. (eds) Antarctic nutrient cycles. Springer, Berlin Heidelberg New York Tokyo. 415-420.

Nesis, K.N. 1970. Occurrence of a rare squid Chaunoteuthis mollis Appellöf (family Onychoteuthidae) in the Indian Ocean. Veliger 12 (3): 290-292.

Nesis, K.N. 1995. Mating, spawning and death in oceanic cephalopods: a review. Ruthenica 6 (1): 23-64.

Nesis, K.N. 1987. Cephalopods of the world (English translation). Tropical Fish Hobbyist (T.F.H.) publications, Neptune City. 352 pp.

Nesis, K.N. 2000. Squids of the family Onychoteuthidae: phylogeny, biogeography, and way of life. Zoologichesky Zhurnal 79 (3): 272-281.

Nesis, K.N.; Nikitina, I.V. 1986. A New Family of Abyssal Squids (Cephalopoda, Oegopsida) from the South-eastern Atlantic. Zoologichesky Zhurnal, 65 (1): 4754.

Nesis, K. N.; Nikitina, I.V. 1992. New records of oceanic squids Walvisteuthis virilis Nesis et Nikitina, 1986 and Nototeuthis dimegacotyle Nesis et Nikitina, 1986 (Cephalopoda, Oegopsida) from the South Atlantic and the South Pacific. Ruthenica 2: 55-58.

Nesis, K.N.; Roeleveld, I.V.; Nikitina. I.V. 1998. A new genus and species of onychoteuthid squid (Cephalopoda, Oegopsida) from the Southern Ocean. Ruthenica, 8 (2): 153-168.

Nixon, M.; Dilly, P.N. 1977. Sucker surfaces and prey capture. In: Nixon, M.; Messenger, J.B. (eds). The Biology of Cephalopods. Symposium of the Zoological Society of London 38: 447-511.

Okada, Y.K. 1927. Contribution a l'étude des Céphalopodes lumineux (notes preliminaries), 1: Organes photogenes d'Onychoteuthis; Etablissement d'une nouvelle espèce, Onychoteuthis borealijaponicus. Bulletin de l'Institut Océanographique de Monaco 494: 1-7. 
Okutani, T. 1978. Ilustrated book of the useful and latent squids in the world. Towa Denkai Co., Ltd. 166 pp.

Okutani, T. 1981. Two New Species of the Squid Genus Onykia from the Tropical Indian Ocean (Cephalopoda, Onychoteuthidae). Bulletin of the National Science Museum, Tokyo, series A (Zoology) 9 (3): 105-112, PI.1.

Okutani, T. 1983. A new species of an oceanic squid, Moroteuthis pacifica from the North Pacific (Cephalopoda: Onychoteuthidae). Bulletin of the National Science Museum, (series A) 9 (3): 105-112, 10 figures, 1 PI.

Okutani, T. 2005. Cuttlefishes and squids of the world. Publication for the $40^{\text {th }}$ Anniversary of the Foundation of National Cooperative Association of Squid Processors. Tokyo. 253 pp.

Okutani, T.; Ida, H. 1986. Rare and Interesting Squid in Japan - IX. A Mass Occurrence of Chaunoteuthis mollis Appellöf, 1891 (Oegopsida: Onychoteuthidae) from off Japan. Venus (Japanese Journal of Malacology) 45 (1): 53-60.

Okutani T.; Murata, M. 1983. A review of the biology of the oceanic squid Onychoteuthis borealijaponica. Memoirs of the National Museum of Victoria 44: 189-195.

Okutani, T.; Clarke, M.R. 1985. Identification Key and Species Description for Antarctic Squids. Biomass Handbook 21. 57 pp.

Okutani, T.; Tagawa, M.; Horikawa, H. 1987. Cephalopods from the continental shelf and slope around Japan. Japan Fisheries Resource Conservation Association, Tokyo. $194 \mathrm{pp}$.

Okutani, T.; Tsukada, S. 1988. Squids eaten by lancetfish and tunas in the tropical Indo-Pacific Oceans. Journal of the Tokyo University of Fisheries. 75 (1): 1-44, Pls 1-7.

O'Shea, S. 1999. The Marine Fauna of New Zealand: Octopoda (Mollusca: Cephalopoda). National Institue of Water and Atmospheric Research Biodiversity Memoir 112. 280 pp.

Owen, R. 1836. Descriptions of some new or rare Cephalopoda, collected by Mr. George Bennett, Corr. Memb. Z.S. Proceedings of the Zoological Society of London 37: 19-24.

Owen, R. 1881. Descriptions of some new and rare Cephalopoda (Part II). Transactions of the Zoological Society of London 11(5): 131-170, 4 Figs, 13 Pls.

Pfeffer, G. 1884. Die Cephalopoden des Hamburger Naturhistorischen Museums. Abhandlungen aus dem Gebiete der Naturwissenschaften Hamburg 8 (1): 1-30, 37 Figs.

Pfeffer, G. 1900. Synopsis der oegopsiden Cephalopoden. Mitteilungen aus dem Naturhistorischen Museum Hamburg 17 (2): 147-198. 
Pfeffer, G. 1908a. Die Cephalopoden. Nordisches Plankton 2 (9): 9-116, 120 Figs.

Pfeffer, G. 1908b. Teuthologische Bemerkungen. Mitteilungen aus dem Naturhistorischen Museum Hamburg 25: 289-295.

Pfeffer, G. 1912. Results of the Plankton Expedition of the Humboldt Foundation Vol. 2 F.a., The Cephalopoda of the Plankton Expedition [English Translation]. Smithsonian Institution Libraries and the National Science Foundation, Washington, D.C. 618 pp.

Phillips, J. 1961. Two unusual cephalopods taken near Monterey. California Fish and Game 47 (4): 416-417.

Phillips, K.L.; Jackson, G.D.; Nichols, P.D. 2001. Predation on myctophids by the squid Moroteuthis ingens around Macquarie and Heard Islands: stomach contents and fatty acid analysis. Marine Ecology Progress Series 215: 179-189.

Phillips, K.L.; Nichols, P.D.; Jackson, G.D. 2002. Lipid and fatty acid composition of the mantle and digestive gland of four Southern Ocean squid species: implications for food-web studies. Antarctic Science 14 (3): 212-220.

Phillips, K.L.; Nichols, P.D.; Jackson, G.D. 2003a. Dietary variation of the squid Moroteuthis ingens at four sites in the Southern Ocean: stomach contents, lipid and fatty acid profiles. Journal of the Marine Biological Association of the UK 83: 532-534.

Phillips, K.L.; Nichols, P.D.; Jackson, G.D. 2003b. Size-related dietary changes in the squid Moroteuthis ingens at the Falkland Islands: stomach contents and fattyacid analyses. Polar Biology 26 (7): 474-485.

Piatkowski, U.; Heinemann, H.; Pütz, K. 2001. Cephalopod prey of king penguins (Aptenodytes patagonicus) breeding at Volunteer Beach, Falkland Islands, during austral winter 1996. Fisheries Research. 52: 79-90.

Quoy, J.R.; Gaimard, J.P. 1824-1826. Zoologie. In: Louis de Freycinet. Voyage autour du Monde...execute sur les corvettes de S.M. L'Uranie et la Physicienne, pendant les annees 1817, 1818, 1819, et 1820. Paris. 712 pp., atlas of 96 Pls.

Rancurel, P. 1970. Les contenus stomacaux d'Alepisaurus ferox dans le sud-ouest Pacifique (Céphalopodes). Cahiers O.R.S.T.O.M. Océanographie, 8 (4): 3-87.

Rancurel, P.; Okutani, T. 1990. A New Species of Squid Genus Onychoteuthis from the Southwest Pacific. Venus, the Japanese Journal of Malacology 49 (1): 2630 .

Reynaud, A. 1831. L'Onychotheute de Fleury. In: R.P. Lesson, Centurie Zoologique ou choix d'animaux rares, nouveaux ou imparfaitement connus. 244 pp, Paris: F.G. Levrault. pp. 61-62, PI. 17.

Risso, A. 1854. Mollusques Cephalopodes vivants observes dans les parage mediterraneen du Comte de Nice. Nice. 81 pp., 33 Pls.

Robson, G.C. 1924. On the Cephalopoda obtained in South African Waters by Dr. J.D.F. Gilchrist in 1920-21. Proceedings of the Zoological Society of London 1924: 589-686. 
Roper, C.F.E.; Sweeney, M.J. 1978. A catalog of the type-specimens of Recent Cephalopoda in the National Museum of Natural History. Smithsonian Contributions to Zoology, 278: 1-19.

Roper, C.F.E.; Sweeney, M.J.; Clarke, M.R. 1985. Cephalopoda. FAO Species Identification Sheets for Fishery Purposes 1: 117-205.

Roper, C.F.E.; Sweeney, M.J.; Nauen, C.E. 1984. Cephalopods of the world. An annotated and illustrated catalogue of species of interest to fisheries. FAO Species Catalogue. Vol. 3. FAO Fisheries Synopsis 125 (3): 277 pp.

Roper, C.F.E.; Vecchione, M. 1993. A geographic and taxonomic review of Taningia danae Joubin, 1931 (Cephalopoda: Octopoteuthidae), with new records and observations on bioluminescence. In: T. Okutani, R.K. O'Dor and T. Kubodera (eds). The Recent Advances in Cephalopod Fishery Biology. Tokai University Press, Tokyo. pp. 441-456.

Roper, C.F.E.; Voss, G.L. 1983. Guidelines for taxonomic descriptions of cephalopod species. Memoirs of the National Museum of Victoria 44: 49-63.

Rüppell, E. 1844. Intorno ad alcuni cefalopodi del mare di Messina. Lettera del Dr. Eduardo Rüppell di Frankfort sue Meno al Prof. Anastasio Cocco. Giornale del Gabinetto Letterario di Messina 5 (27-28): 129-135.

Salcedo-Vargas, M.A. 1995. Systematic value of the ultrastructure of the sucker surface in the squid family Mastigoteuthidae (Mollusca: Cephalopoda). Contributions to Zoology 65 (2): 65-77.

Sánchez, P.; Molí, B. 1984. Cefalópodos de las costas de Namibia (Atlántico sudoriental). Resultados Expediciones Cientificas 12: 3-22.

Sands, C.J.; Jarman, S.N.; Jackson, G.D. 2003. Genetic differentiation in the squid Moroteuthis ingens inferred from RAPD analysis. Polar Biology 26: 166-170.

Sasaki, M. 1929. A monograph of the dibranchiate cephalopods of the Japanese and adjacent waters. Journal of the Faculty of Agriculture, Hokkaido Imperial University 20 (Supplement 10): 357 pp.

Seki, M.P. 2001. Distribution patterns of pelagic cephalopods through the Subarctic and Subtropical Frontal Zones in the central North Pacific. Thesis (MSc), University of Hawai'i, Honolulu, Hawai'i, 102 pp.

Smith, E. 1881. Account of the Zoological collections made during the survey of H.M.S. "Alert" in the Strait of Magellan, and on the coast of Patagonia. IV. Mollusca and Molluscoidea. Proceedings of the Zoological Society of London 1881 (1): 22-44, PI. 3.

Souleyet, F.L. 1841-1852. Mollusques, volume 2, 664 pages (1852). In: Voyage autour du monde execute pendant les annees 1836 et 1837 sur la corvette La Bonite. Zoologie par MM. Eydoux et Souleyet. Paris. 2 volumes atlas of 101 Pls.

Spamer, E.E.; Bogan, A.E. 1992. Catalog of the types of neontological Mollusca of the Academy of Natural Sciences of Philadelphia. Part 7: Cephalopoda and Scaphopoda. Tryonia 24: 1-13. 
Steenstrup, J. 1882. 9. Notae Teuthologicae 1-4. Oversigt over det Kongelige Danske Videnskabernes Selskabs Forhandlinger 1882: 143-168.

Strugnell, J.; Norman, M.; Drummond, A.J.; Cooper, A. 2004. Neotenous origins for pelagic octopuses. Current Biology 14 (8): 300-301.

Sweeney, M.J.; Roper, C.F.E. 1998. Classification, type localities and type repositories of Recent Cephalopoda. Smithsonian Contributions to Zoology 586 (2): 561-599.

Sweeney, M.J.; Roper, C.F.E.; Mangold, K.M.; Clarke, M.R.; Boletzky, S.V. 1992. "Larval" and Juvenile Cephalopods: A Manual for Their Identification. Smithsonian Contributions to Zoology 513: 282 pp.

Sweeney, M.J.; Young, R.E. 2003. Taxa associated with the family Onychoteuthidae Gray, 1849. [Internet] Tree of Life Web Project 2003, accessed 1 September 2007. Available from: http://tolweb.org/accessory/Taxa associated with the family Onychoteuthidae?acc id=1358.

Taki, I. 1964. On Eleven New Species of the Cephalopoda from Japan, Including Two New Genera of Octopodinae. Journal of the Faculty of Fisheries and Animal Husbandry of Hiroshima University 5: 277-343.

Thiele, J. 1920. Die Cephalopoden der Deutschen Südpolar Expedition. Deutsche Südpolar Expedition, Zoologie, 16: 433-465, PI. 53, 54.

Thiele, J. 1935. Handbuch der Systematischen Weichtierkunde 2. Fischer, Berlin. pp. 780-1154.

Thompson, D.W. 1900. On a rare Cuttlefish, Ancistroteuthis robusta (Dall) Steenstrup. Proceedings of the Zoological Society of London. 1900: 992-998.

Toll, R.B. 1982. The comparative morphology of the gladius in the Order Teuthoidea (Mollusca: Cephalopoda) in relation to systematics and phylogeny. Dissertation (PhD), University of Miami, 390 pp.

Toll, R.B. 1998. The gladius in teuthoid systematics. Smithsonian Contributions to Zoology 586 (1): 55-67.

Torchio, M. 1967. Eccezionale reperto di Chaunoteuthis mollis (Appelöff [sic]) nello Stretto di Messina (Cephalopoda: Dibranchiata) Natura (Milano), 58 (3): 193207.

Tsuchiya, K.; Okutani, T. 1992. Growth stages of Moroteuthis robusta (Verrill, 1881) with the re-evaluation of the genus. Bulletin of Marine Science 49 (1/2): 137-147.

Vacchi, M.; Greco, S.; La Mesa, M. 1994. Kondakovia longimana Filippova, 1972 (Cephalopoda: Onychoteuthidae) from Terra Nova bay, Ross Sea. In: Rodhouse, P. G., Piatkowski, U. \& Lu, C.C. (eds.) 1994. Southern Ocean cephalopods: life cycles and populations. Antarctic Science 6: 283.

Vecchione, M., Young, R.E., Tsuchiya, K., and K.S. Bolstad; Onychoteuthidae Gray, 1849 [Internet]. Tree of Life Web Project, 2003, accessed 1 September 2007 (under construction). Available from:

http://tolweb.org/tree?group=Onychoteuthidae\&contgroup=Decapodiformes. 
Vérany, J.B. 1847. [On six new species from the Mediterranean.] Atti della Ottava Riunione degli Scienziati Italiani tenuta in Genova dal XIV al XXXIX Settembre MDCCXLVI, pp. 512-514. (Plates in Vérany, 1846 Catalogo degli animali ...)

Vérany, J.B. 1847. Cephalopodes de la Mediterranee. Mollusques Mediterraneans Observes, Decrits, Figures et Chromolithographies a après le vivant ouvrage dedii ASM le roi Charles Albert I: 132 pp., 41 Pls.

Verrill, A.E. 1876. Note on Gigantic Cephalopods, a Correction. American Journal of Science 12: 236.

Verrill, A.E. 1879. The Cephalopods of the North-Eastern coast of America, Part I. The gigantic squids (Architeuthis) and their allies; with observations on similar large species from foreign localities. Transactions of the Connecticut Academy of Arts and Sciences 5 (5): 177-257.

Verrill, A.E. 1881. The Cephalopods of the North-Eastern coast of America, Part II. The smaller cephalopods including the "squids" and the octopi, with other allied forms. Transactions of the Connecticut Academy of Arts and Sciences 5: 259446, Pls xxvi-Ivi, 3 Text-figs.

Verrill, A.E. 1882. Report on the cephalopods of the north-eastern coast of America. Annual Report of the Commissioner for Fish and Fisheries for 1879: 211-455, 46 Pls.

Verrill, A.E. 1885. Third Catalogue of Mollusca, Recently Added to the Fauna of the New England Coast and the Adjacent Parts of the Atlantic, Consisting Mostly of Deep Sea Species, with Notes on Others Previously Recorded. Transactions of the Connecticut Academy of Sciences, 6 (2): 385-452, 3 Pls.

Voss, G.L. 1956. A review of the cephalopods of the Gulf of Mexico. Bulletin of Marine Science of the Gulf and Caribbean 6 (2): 85-178.

Voss, G.L. 1962. List of the types and species of cephalopods in the collections of the Academy of Natural Sciences of Philadelphia. Notulae Naturae 356: 1-7.

Wadley, V. 1990. Squid from the West and North West Slope Deepwater Trawl Fisheries. CSIRO Division of Fisheries publications. 12 pp.

Wakabayashi, T.; Kubodera, T.; Sakai, M.; Ichii, T.; Chow, S. 2007. Molecular evidence for synonymy in the genera Moroteuthis and Onykia and identification of their paralarvae from northern Hawaiian waters. Journal of the Marine Biological Association of the UK 87: 959-965.

Young, R.E. 1972. The Systematics and Areal Distribution of Pelagic Cephalopods from the Seas of Southern California. Smithsonian Contributions to Zoology 97: 108 pp. 37 pls.

Young, R.E.; Harman, R.F. 1987. Descriptions of the larvae of three species of the Onychoteuthis banksii complex from Hawaiian waters. Veliger 29 (3): 313-321.

Young, R. E.; Harman, R. 1998. The phylogeny of the "enoploteuthid families." Smithsonian Contributions to Zoology 586 (2): 257-270. 
Young, R.E.; Vecchione, M.; Tsuchiya, K. Ancistroteuthis lichtensteinii: Description Continued [Internet] Tree of Life Web Project 2003[a], accessed 1 September 2007. Available from: http://tolweb.org/ accessory/Ancistroteuthis lichtensteinii Description?acc id=1362.

Young, R.E.; Vecchione, M.; Tsuchiya, K. Walvisteuthis Nesis and Nikitina 1986. [Internet] Tree of Life Web Project 2003[b], accessed 1 September 2007. Available from: http://tolweb.org/ Walvisteuthis/19957.

Zuev, G.V.; Nesis, K.N. 1971. Volume II. Squid (Biology and Fishing) (English translation). Moscow, Pishchevaya Promyshlennost. 360 pp. 


\title{
APPENDIX A
}

\author{
'Onychoteuthis, Sepia with claws'
}

H.C. Lichtenstein, 1818, pp. 1591-2

\section{Translated from German}

'Following a short note in Molina's Natural History of Chile, one Sepia unguiculata has appeared in the thirteenth edition of the Linnaean System; yet so little attention has been paid to it that even otherwise well-travelled zoologists barely know of such an animal's existence. Denys Montfort and our Schneider have also spoken of it, then all became quiet and the clawed Sepia drifted into the society of countless other forgotten animals. Resurrecting such cases has always appealed to me - bringing the neglected or awkwardly pushed-aside descriptions from honourable older authors out of the darkness of their inadequate verbal descriptions and into the light, by providing a clarified explanation, or simply by referring to them. I had just such an opportunity when I discovered, in July of this year (occupied as I was with clarifying descriptions by Maregrav and Piso), reference to two Sepia that I must believe closely related to Sepia unguiculata. I presented a detailed description of these, together with an appraisal of older and newer reports of similar occurrences (which are even more poorly known than that of Molina), to the Royal Academy of Sciences in their open meeting on the third of August. Until this presentation appears in print, may the following preliminary note serve.

'The hooked Sepia belong, without exception, to those forms designated by Aristotle as 'teuthis,' which were called Loligo or Lolliolus by Roman authors and the newer systematists. The hooks are situated only on the two long tentacles, and there take the place of suckers. They are in actuality merely a modified version of the latter, and in the various species of this new genus, gradual transitions in development from an Acetabuli to a hard, horny ring may be observed. The horny ring, which was first recognised by Swammerdam and Needham as the main functional/support structure of the sucker for the purposes of attaching and holding fast (and upon the outer circumference of which they discovered small teeth), departs in this case from its circular form, becomes first elliptical, then pointed distally, and soon protrudes from the surrounding skin and musculature as a well-formed hook. The aperture of the sucker ring may still be observed in the wide base of the hook, surrounded by the solid shank of the hook. These hooks are also in double, alternating rows, exactly like the sucker rings in the earlier named Loligo species. The longest of these on our example spans four lines, the smallest a minimum of one and a half. They move freely within a circular depression, spanning half of its diameter, and attached in its middle by a fleshy stalk. They do not appear able to move independently from the general contraction of the tentacle itself, since no muscles are apparent that could serve as flexors or extensors. However, one could interpret the fleshy sheath (modified from the fleshy outer ring of the sucker), which encloses the hook nearly to its tip, as capable of controlling the hook's movement to some extent. There is also a broad fleshy protective keel that folds over all the hooks in their relaxed state, from the outer margin of the manus.

'One cannot help but divide the end portion of the tentacle, on which the claws are situated, into its three obviously different regions, and to call these carpus (literally 'root of the hand'), manus ('middle hand') and dactylus ('finger') (see illustration). The first of these is indicated by an outward and backward bend, and on the thus created protrusion there is a disk of 16 small warts, of which eight are hemispherical and closed, and eight are open, smooth suckers. Their regular organisation into rows is particularly pleasing to the eye when viewed under the magnifying glass. 
'The second, doubly long section, possesses eleven claws (5 in the outer, 6 in the inner row); the final claw in the outer row is the longest of all.

'There follows then a considerable clawless interspace, so to speak a throat for the inward bend that marks the final region of the hand. This is noticeably flattened and furnished with 12 claws in two rows (6 and 6); the third and fourth of the outer rows are the largest of these.

'These arms cannot bring what they catch to the mouth, but must rather give it over to the eight shorter arms. I consider them more as organs for holding the body still on the less rocky ocean floor, like anchors, and prefer to call them 'latching arms' rather than 'catching arms.' Aristotle observed that Sepia may cling to boulders, being at the mercy of the waves over sandy and gravelly substrates; these animals may have an advantage in this case. Of course these arms may also assist in attacking while swimming in the open ocean, but they probably have little effect on crabs, mussels and snails, upon which these Sepia mostly subsist.

'Our two specimens came to us as part of the rich inheritance of specimens from our brave Bergius, who died in the Cape of Good Hope in January of this year of tuberculosis, the victim of his own tireless efforts at collecting and observing plants and animals. His journal from the voyage reveals that these specimens flew on board the ship one night. One was found the following morning on the foredeck, the other in the crow's nest, thirty feet above the sea surface, more evidence to support Aristotle's remarks on their power of flight. The very elastic lateral flaps or fins may be of particular use in this respect. This occurred in May, 1816, approximately 100 miles west of the Cape. I have heard from other seafarers that other similar animals were collected in this way at about the same time. (Having crept?)

'The genus Loligo deserves a careful review; it contains many more species than have been recognised so far. I myself know of three, of which nothing is written in the literature. These clawed squids should be described in their own genus, however, the characters of which are easy to differentiate, and for which I suggest the name Onychoteuthis. This genus is also rather diverse, when one researches carefully and considers the good work of older authors. I interpret the following:

1. O. bellonii (Bellon, de Aquatilibus, p. 430). Quite unmistakeably described, with three robust spikes on each tentacle.

2. O. fabricii (O. fabricii Fauna Groenlandica p. 359). On each tentacle there are two longitudinally compressed suckers, armed with a long, crooked spike, between many small unarmed suckers.

3. O. molinae; Sepia unguiculata Lin. Gmel. (Molina Saggio sulla storia naturale de Chili p. 199; German translation p. 174). The description is very meagre; from the second edition (in 4.1810, p. 175), one may see for the first time that Molina refers to a Loligo, and that the entire description is courtesy of Sir Joseph Banks.

4. O. bergii. The species described above. It is not possible to state with complete certainty that it differs from that of Molina, since his work leaves so much doubt.'

[Figure legend follows]

'Berlin, October 1818

Lichtenstein' 
'Teuthological Contributions II. Chaunoteuthis n.g. Oegopsidarum'

A. Appellöf, 1891, pp. 1-29

Relevant morphological sections translated from German; references and figure numbers given are from the original reference.

'Among the collection of locally occurring cephalopods, I discovered a form that deserves great attention. According to the label, the specimen had been brought from the Mediterranean by ship's captain Lampe. I immediately noticed the unusually weak, soft consistency of the mantle, and the rudimentary tentacles. At first I believed the flaccid consistency to be a result of poor preservation, but even the most cursory examination convinced me that the specimen had been preserved well and that the weak nature of the mantle was truly its natural state. The tentacles, which exist only as two equal stumps, show no evidence of damage through injury, in that the surfaces are evenly rounded and pigmented. In addition, upon finding the normal cartilaginous connection between head and mantle, it became clear to me that this was an as yet undescribed form, which I wish to describe presently in detail. I suggest the name Chaunoteuthis for the new genus.

\section{Chaunoteuthis mollis, n.g. \& sp.}

'Mantle of remarkably soft and limp consistency, fairly broad. Eyes naked, eye opening with small anterior sinus. Head connected to mantle with normal funnel- and mantlecartilage. Funnel cartilage composed of an elongate disc, deepened into a simple groove in the middle. Fins abnormally extensive, in that their width is nearly the length of the mantle; they span more than half the mantle in length. Funnel with large valve (Fig. 9 v.). Arms with two rows of suction cups (Fig. 4); sucker rings completely toothless. The inner sucker-bearing surface of the arms, like the upper portion of the suckers, is pigmented dark brown. Tentacles rudimentary, evident only in two $10 \mathrm{~mm}$ long stumps (Fig. $4 t$.). All arms except the dorsal pair bear fleshy aboral keels. The dorsal arms are the shortest, the third (ventro-lateral) the longest. On each side of the nuchal midline are 11 longitudinal folds of the same form as in Onychoteuthis.

'The shell (Fig. 2) is very similar to that of Onychoteuthis; broadest in the middle part, by contrast greatly narrowed posteriorly and excavated in a groove. (Footnote: the endcone of the shell (Fig. 2a) from the sole specimen was slightly damaged and I was thus unable to determine its exact form. To judge from the fragments, the endcone was essentially similar to that of Onychoteuthis.) The body has a dark reddish-brown colour, stemming from densely accumulated chromatophores. On the inner surfaces of the arms, by contrast, there are no distinctive chromatophores, but rather a uniform dark brown colour. The interior of the mantle and many of the internal organs are also dark brown in colour.

'Concerning the rudimentary tentacles, I wish to emphasise foremost that they were, in all probability, not lost by coincidence. As previously mentioned, both stumps are of exactly the same length, and this condition alone suggests that they cannot have been torn away by chance. This supposition is supported even more strongly by their appearance. Specifically, the surface bears not even the smallest trace of scarring, which should have necessarily been the case, had they been torn away at some point. Rather, they are very similarly rounded and on the proximal end partly covered with chromatophores. I must therefore consider the rudimentary condition of the tentacles to be their normal state. 
'Another abnormality of the present specimen, which is a female, lies in the method of spermatophore attachment. On the underside of the mantle, on both sides of the emargination, the spermatophores are implanted in longitudinal rows (Fig. $5 \mathrm{sp}$.). The manner in which this occurs deserves a more exact description.

'The mantle is so thickly covered with chromatophores externally that it appears entirely reddish-brown. However, two groove-like depressions (one anteriorly forked), which extend back from the anterior mantle margin, are an exception. The spermatophores are embedded in these grooves. They are not, however, held loosely to the mantle with a mass of mucous, but are rather implanted for most of their length directly into the mantle musculature, running parallel to the mantle's surface. The lower half is relatively broad, but toward the front the spermatophore suddenly becomes narrow and whip-shaped, ending finally in a small bell-shaped thickening, which is open at the tip (Fig. 6); this thickening and a small section of the narrow portion protrude freely from the muscle mass. The spermatophores are completely full of spermatozoa, of which only the tails are visible - proof that the animal is well preserved and that the loose consistency of the mantle cannot result from poor conservation.

'Steenstrup demonstrated that, in the Ommastrephidae and Enoploteuthidae [Footnote: Orient. i de Ommatostr.-agtige Blaekspr. Indb. Forhold. - Overs. Kgl. Danske Vid. Selsk. Forhandl. 1880, PI. 3 with explanation], the spermatophores are fastened to the inner mantle surface of the female, and that this occurs with the help of a mucous mass, at least in enoploteuthids. The manner in which the spermatophores are delivered, and whether they are even attached to the female's body, has to the best of my knowledge not been observed in the Onychoteuthidae, Taoniidae, or Cranchiidae. From the figure and text, it is clear that this form deviates from those previously known in similar condition.

'How do the spermatophores come to be embedded, for most of their length, in the female's mantle? I can only offer the following as the most probable explanation. The groove in which the spermatophores sit is without chromatophores; the musculature of the mantle is exposed and without epithelial covering; the groove appears to have been torn into the mantle. The holes in which the spermatophores are lodged also show signs of such a tearing, in that microscopic examination reveals loose muscle fibres protruding into the lumen - obviously torn.

'The only method I can think of, by which the spermatophores could be so introduced into the mantle musculature, is that the male somehow creates a tear in the female's mantle, most likely with the help of a of a special modification of the hectocotylised arm or arms, presently unknown. Despite careful examination, I could discover no preexisting openings through which spermatophores could perhaps be conducted. I assume therefore that, most probably, the male creates a rift in the mantle and deposits the spermatophores in it; later the rift grows back together and the spermatophores are stored each in its own sac, or together in a collective cavity.

'Since the present specimen possesses so many external abnormalities relative to the usual cephalopod morphology, I also undertook an anatomical examination, whose results I wish to present. I have also examined Ommastrephes and Onychoteuthis specimens for comparison.'

[Descriptions follow of the supra- and sub-oesophageal ganglia and the nerves.]

'Of the digestive apparatus, we will describe the radula first. It has seven rows of teeth, the middle being a single large cusp with or without rudimentary lateral cusps. The 
shapes of the various tooth [plates] is best described by the illustration (Fig. 18). A comparison with common radular forms is not easy to make, since examinations in this area have not been continuous enough to allow the description of general rules for radular construction by genus. For example, I may adduce that, according to Troschel's examinations [Footnote: Ueber die Mundtheile der Cephalop. - Arch. F. Naturg. Jahrg. 19. 1853.], the radula of Onychoteuthis bergii is characterised by having two lateral cusps on the middle plate, and that Troschel also records, as characteristic for the genus, that the large tooth of the middle plate distinguishes itself by its linear narrowness and length. In the meantime, I have examined an Onychoteuthis specimen from the heart of the Bengalese ocean, with especial attention to the tongue, and found the middle plate to be completely lacking lateral cusps; the middle tooth is also relatively broad. Thus, even before examining an entire series of specimens, I believe it invalid to determine relationships between forms based on the radular construction, when there are no striking peculiarities. It can only be said of this specimen that its radula completely conforms to the oegopsid type.'

[Descriptions of the oesophagus, stomach, and digestive gland follow.]

'The funnel contains a very clearly pronounced funnel organ, made up of a middle section and two lateral sections. The middle part (Fig. 9, b) is composed of two shanks which lie on either side of the dorsal midline and unite anteriorly, after which they end in a small, unattached point $(f)$. The lateral parts $(a, a)$ are made of two symmetrically placed oval pads. The middle and side parts stand out visually, being ringed with a small, brown-pigmented border. They are easy to remove from the funnel.'

[Descriptions of the heart and vessels follow.]

'The gill lamellae, which are in rows on either side of the gill vessels, are peculiar in that their free edges are pigmented brown, giving them a delicate appearance.'

\section{[Descriptions of the reproductive organs follow.]}

'The remaining musculature is weakly developed, corresponding to the condition of the mantle. I have not examined the span and insertion of individual muscles.

'From the description above, it is evident that the new genus agrees with the onychoteuthids and ommastrephids in many respects, but possesses several peculiarities that have not been observed in the examined forms, or only in forms from a completely different group. First there is the condition of the tentacles to consider. A similar condition is observed in the cranchiid Leachia - here, too, these organs are represented by small stumps and, in the specimens examined to date, show no evidence of having been forcefully torn off. It is also peculiar that, in spite of the rudimentary condition of the tentacles in Chaunoteuthis, the tentacular nerves are completely developed. Several years ago, in the national Museum in Stockholm, I examined a specimen of Leachia - only briefly, but as far as I can remember, the tentacular nerve was also externally obvious thought the transparent rudimentary tentacle.' [Comparisons between Chaunoteuthis and other 'truly tentacle-less' forms are made; Appellöf concludes that Chaunoteuthis is a form truly without tentacles.]

'The abnormally slack consistency of the mantle and organs is a state that is reminiscent of the Chiroteuthidae; it is well known that the ommastrephids and onychoteuthids are generally characterised by a strongly muscular mantle.

'Also noteworthy is a character of unknown significance, which however is not known in other forms as far as I know - namely, the strong pigmentation of the suckers, the 
oral face of the arms and the interior surface of the mantle, as well as some of the inner organs. Even if this condition is not isolated - it may be, I do not know - I believe that is it rare enough to warrant a particular mention.'

[A brief review of anatomical peculiarities is given - arm nerves, lack of brachial heart ganglion, etc.]

'In spite of the differences in mantle and anatomical features, our form possesses characters that indicate Onychoteuthis as most closely related. First the shell, which corresponds to that of a typical Onychoteuthis. Furthermore, the skin folds at the neck are entirely identical in form and position to those of Onychoteuthis, as is the funnellocking apparatus.

'Our form agrees with the onychoteuthids also in many anatomical aspects.' [A brief discussion of similarities in reproductive organs follows.]

'Regarding the systematic position of Chaunoteuthis, I consider this form as most closely related to the onychoteuthids. If more forms of this type become known, then they should be placed together into a separate family.'

[The final paragraphs discuss tentacular development as observed by Brock and Steenstrup.] 


\title{
APPENDIX C
}

\section{'The Cephalopods of the German South Polar Expedition'}

\author{
J. Thiele, 1920, pp. 442-444
}

Relevant morphological sections translated from German; references and figure numbers given are from the original reference.

\section{‘Family Onychoteuthidae}

'Moroteuthis aequatorialis, n. sp.

'Plate 53, Fig. 17; Plate 54, Figs 1-4

'A large animal that was found drifting dead at the surface, in the Atlantic Ocean near the equator $\left(0^{\circ} 16^{\prime} \mathrm{N}, 18^{\circ} 07^{\prime} \mathrm{W}\right)$; the tentacles were missing, but it doubtless belongs to the Onychoteuthidae. Regarding the genus, the structure of the gladius is particularly important, and allows the species to be identified as Moroteuthis, since it is the most similar to Moroteuthis ingens (Edg. Smith) (compare Pfeffer (1912) PI. 12, Figs 1 and 2). Apart from this species, which occurs in Patagonia, M. robusta Verrill (from Unalaska) belongs [to this genus]; both are very large. Based on these, Pfeffer (p. 104) developed a new diagnosis for the genus, which however includes several isolated points that do not apply to the present species and perhaps therefore should be more generalised, including the warty skin, which (as in the genus Polypus) is a diagnostic at the species level.

'It cannot be doubted that the present species differs from both the previously mentioned species occurring in cold seas: the endcone of the gladius is substantially different in form, as are the neck-folds, the posterior portion of the mantle, which is more slender; the head is also visibly smaller.

'The skin of the animal is brown-coloured and smooth, without warts. The tail is long and slender, pointed posteriorly. The dorsal mantle length is $40 \mathrm{~cm}$, of which the fins make up almost exactly half. They are broad and triangular; the posterior margin is approximately $20 \mathrm{~cm}$, the anterior $15.5 \mathrm{~cm}$ long, and the breadth of both together is 28 $\mathrm{cm}$.

'The anterior mantle circumference is approximately $30 \mathrm{~cm}$; the diameter is approximately $10 \mathrm{~cm}$. Posteriorly, on the ventral side of the mantle, is a fleshy, keel-like protrusion that terminates $3.5 \mathrm{~cm}$ before the posterior point of the tail.

The funnel has $5 \mathrm{~cm}$-long and $12 \mathrm{~cm}$-wide, posteriorly thin cartilage with a shallow groove; however I cannot discern the corresponding cartilage on the mantle. The nuchal cartilage is produced like a keel, with a central longitudinal groove; it is $57 \mathrm{~mm}$ long and $13 \mathrm{~mm}$ wide. The corresponding, thin mantle cartilage is approximately $60 \mathrm{~mm}$ long, $17 \mathrm{~mm}$ wide posteriorly, and narrows anteriorly into a rounded point.

'The posterior portion of the gladius is well preserved, the anterior less so. It is rounded anteriorly, corresponding to the cartilage, then widening and strengthening posteriorly, its greatest breadth $3 \mathrm{~cm}$. In the centre is a broad, flat protrusion, bisected by a groove. The initially broad-set lateral margins curve and gradually narrow so that the middle forms a strong keel with a longitudinal groove. In the region encompassed by the anterior end of the endcone (Fig. 1), the keel is acutely angled, has lost its groove, and thickens near the lateral margins. The posterior cavity is $11 \mathrm{~mm}$ deep. In this region, the solid, amber-coloured, cartilaginous endcone has a $4 \mathrm{~mm}$-wide dorsal groove, which gradually disappears toward the posterior end; next to the posterior end 
of the conus, the cartilaginous portion is separated from the broader, lamelliform, produced lateral margin by a deep groove.

'The endcone is $9 \mathrm{~mm}$ high and $5 \mathrm{~mm}$ wide at its anterior end, gradually and evenly shrinking posteriorly. In cross-section, it narrows ventrally and the posterior end is blunted; the entire length from the anterior end of the conus is $61 \mathrm{~mm}$.

'The neck is remarkably weak, and the head is only $5 \mathrm{~cm}$ wide. The eyes are barely discernible. Next to the funnel aperture, from the base of the ventral arm on each side, emerges a fairly broad skin-fold, diverging posteriorly into an arc; above its end, a small, $1 \mathrm{~cm}$-long transverse fold is visible, which forms a small, forward-directed round lappet in the middle; and above the transverse fold is a small lateral fold, the ventral portion of which forms an arc from beneath the eye, curving forward. These three folds demarcate the head posteriorly.

'In the funnel, there is a large, rounded valve. The strong brown buccal membrane has seven short lappets.

'The arms are strong and moderately long. The ventral pairs have keel-like skin-folds, particularly wide in the middle, on their aboral surfaces. The sucker-bearing oral face is bordered by a small membrane. The suckers are in two longitudinal rows, but are incompletely preserved. On the proximal portion of the arms, they are quite small and widely spaced, but increase evenly in size and density, then toward the arm-tips become smaller and more numerous. The largest are approximately $3.5 \mathrm{~mm}$ (their form is illustrated in Figs 2, 3); they are short-stalked, irregularly round, with a weak muscle ring. The opening is quite small, approximately $2 \mathrm{~mm}$ in diameter, with a smooth, nonprotruding chitin ring.

'The tentacles have been severed, leaving stumps.

'The beaks are illustrated in Fig. 4; their form is the usual.

'The radula (PI. 53, Fig. 17) has three teeth on each side of the rachidian, that are somewhat distanced from each other. The rachidian has an approximately half-circular base, with a moderately large, slender, pointed central cusp and two smaller lateral cusps. The first lateral tooth has a somewhat smaller base, with a larger and smaller cusp, whose length corresponds approximately to that of the rachidian. The second lateral tooth has only one strong, curved, pointed cusp. The marginal tooth has, as usual, a longer, curved cutting edge. Marginal plates are absent.

\section{'Teleoteuthis caribaea (Lesueur) \\ 'Plate 54, Fig. 5}

'I have attributed an animal that was caught on 7 October, $1903\left(15^{\circ} 06^{\prime} \mathrm{N}, 27^{\circ} 44^{\prime} \mathrm{W}\right)$ in the Atlantic Ocean, near the Cape Verde Islands, in spite of several deviations (as these may simply be abnormalities) to the above-named species, which is broadly distributed in the Atlantic. The dorsal mantle length is $28 \mathrm{~mm}$, the anterior diameter $12 \mathrm{~mm}$.

'The red-brown chromatophores are dense dorsally, growing more sparse and pale ventrally, allowing the anterior half of the ventral side to shine silver. Each fin is $11 \mathrm{~mm}$ long and wide, with an anterior lobe and rounded laterally. Next to the edge of the funnel groove, which is $6 \mathrm{~mm}$ long and wide, is a single thickening that diminishes laterally and posteriorly into a corner-shaped skin fold. No other lateral skin folds are apparent. 
'The gladius also differs somewhat from Pfeffer's description (PI. 1, Fig. 9-11). It is strong and sharply keeled, evenly broadened posteriorly, so that the greatest breadth is at the beginning of the posterior third (Fig. 5). The small endcone is not directed posteriorly, but rather anteriorly. It is triangular and its ventral side is merged with the keel (Fig. 5a).

'The funnel cartilages are long and thin, and the cartilaginous ridges in the mantle extend over nearly half the mantle cavity.

'A young animal that was caught on 11 August, $1903\left(28^{\circ} 46^{\prime} \mathrm{S}, 10^{\circ} 17^{\prime} \mathrm{E}\right)$ may also belong to this species. Its mantle is $10.5 \mathrm{~mm}$ long.' 


\section{APPENDIX D}

'Cephalopods of the Archipelago of Cape Vert, of Angola and of Mozambique' W. Adam, 1962, pp. 19-30

Relevant morphological sections translated from French; references and figure numbers given are from the original reference.

'Ancistroteuthis lichtensteinii (Férussac and d'Orbigny, 1839)'

[synonymy follows]

'Geographical distribution: Mediterranean: Nice, Corsa, Messina, Sicily, Catalogne; ?Western Atlantic: $27^{\circ} 18^{\prime} \mathrm{N}, 8^{\circ} 25^{\prime} \mathrm{W}$ (G.L. Voss 1956).

'Origin and material: Angola, MEPA II, M.c.10: Ponta do Egito, 18/09/1952, chalut, depth 439-456m, 1 specimen.

'Relative dimensions:

$\begin{array}{lll}\text { Mantle: } & \begin{array}{l}\text { dorsal length in } \mathrm{mm} \\ \text { ventral length }\end{array} & 67 \\ & \text { width } & 96 \\ \text { Head: } & \text { length } & 29 \\ & \text { width } & 22.5 \\ \text { Fins: } & \text { length } & 22.5 \\ & \text { total width } & 58 \\ & \text { point of greatest width } & 86 \\ \text { Arms: length } & \text { I } & 40 \\ & \text { II } & \text { L 58, R 54 } 66, \text { R 68 } \\ & \text { III } & \text { L 60, R 60 } \\ & \text { IV } & \text { L 63, R 63 } \\ \text { Tentacles: } & \text { total length } & \text { L 134, R 134 } \\ & \text { club length } & \text { L 36, R 36 } \\ \text { Suckers: } & \text { diameter } & 1.5\end{array}$

'Description:

'The body is subcylindrical in the anterior portion, elongate conical in the posterior portion. The head is approximately as long as wide. The length of the fins is greater than half the mantle length, and they are broadly rhombic in shape. The anterior points of fin attachment are separated by a space of $9 \mathrm{~mm}$; the free margin is anteriorly convex, then becomes nearly a straight line at the level of the greatest fin breadth; the posterior margin is slightly convex, becoming slightly concave near the tip of the body, where the fins join.

'The anterior margin is essentially straight dorsally, and strongly emarginated ventrally near the siphon.

'The head is flattened on its different surfaces. On each side of the midline there are six longitudinal nuchal folds. Behind the eye there are also three longitudinal folds, very strongly developed, joined by a transverse membrane at the posterior edge.

'The funnel groove lacks any folds or membranes. 
'The eyes do not project: the ocular aperture is slightly rectangular, with a deep sinus in the lower third of the anterior margin.

'The dorsal arms are flattened at the base of the exterior face and they do not have a swimming membrane.

'The dorso-lateral arms are equally flattened at the base of the exterior face and have a swimming membrane on the ventral edge.

'The ventro-lateral arms are flattened laterally and possess a swimming membrane that is very strongly developed near the midpoint of the arms.

'The ventral arms are flattened at the base of the exterior face and possess a welldeveloped lateral membrane.

'All of the arms have protective membranes. The suckers are arranged in two longitudinal rows, and the sucker rings are smooth. The tentacles are laterally compressed. The interior face of the tentacle stalk is narrow, compressed, transversely striated and furnished with a longitudinal groove. The exterior face of the stalk is rounded, with a weak membranous crest that terminates at the base of the club; it is not continuous with the swimming membrane of the tentacle club.

'The base of the club possesses a flattened carpal oval, measuring $2 \times 2.8 \mathrm{~mm}$, bearing nine small smooth-ringed suckers and nine rounded, flattened papillae. The main portion of the club has two longitudinal rows of hooks. In the ventral series of the right tentacle, the first hook is very small, smaller than its dorsal partner; the rest of the hooks increase rapidly in size and then diminish again toward the distal extremity of the club. The ventral series of hooks is composed of nine hooks that measure, respectively, $0.7,1.0,2.0,3.5,4.5,4.5,3.7,2.5$ and $1.4 \mathrm{~mm}$. In the dorsal series the hooks remain small and are subequal, measuring $1 \mathrm{~mm}$, apart from the two distal hooks, which measure respectively 0.8 and $0.5 \mathrm{~mm}$. The distal extremity of the right club bears a group of 19 miniscule suckers whose diameter varies from 0.1 to $0.2 \mathrm{~mm}$.

'On the left club the ventral hooks measure respectively $0.6,1.0,1.5,3.0,4.2,4.5,4.1$, 3.0 , and $2.0 \mathrm{~mm}$; the hooks in the dorsal row have approximately the same dimensions as those on the right club.

'The ventral membrane is well developed and extends from the carpus to the distal extremity of the club; the dorsal is barely visible and present only along the basal third of the club. The swimming membrane is well developed; it commences level with the space between the first two hooks and migrates toward the dorsal face of the club, and is not continuous with the membranous crest on the tentacle stalk.

'The seven points of the buccal membrane are attached to the dorsal face of the dorsal and dorso-lateral arms, and to the ventral face of the ventro-lateral and ventral arms.

'The funnel cartilage forms an elongate oval, with a longitudinal groove in the middle that receives the palleal component to make a connection; the palleal component comprises a narrow longitudinal bar whose length surpasses that of the funnel cartilage.

'The genital organs are not well developed.

'The radula is represented in Fig. 1. 
'Remarks: This animal corresponds generally to the detailed description by Pfeffer (1912, p. 92). The fins are relatively more broad than those of a specimen of the same size described by that author; the hooks are slightly lower in number, but the differences do not exceed normal variations for this species, although the number of known specimens that have been described is very small.

'At present this species is known only from the Mediterranean and has not been found off the Atlantic coast of Africa. C.L. Voss [sic] (1956, p.130) described a young specimen from the Gulf of Mexico that he attributed to Ancistroteuthis lichtensteini. In comparing that description with the young specimen described by Pfeffer and with the one I am presently describing, several differences are notable: the fins are relatively much broader and the arms much longer. It is difficult to interpret the description of the tentacle club because the author does not distinguish between the swimming membrane and the protective membrane. According to him (p. 131) "The tentacles are long, triangular, flattened on their oral surface and equipped with a strong swimming membrane on the outer surface." Now, in the specimen from Angola, the tentacle stalk bears a weak membranous crest that is not continuous with the well-developed swimming membrane on the club. On a large specimen from Monaco, the swimming membrane on the tentacle stalk is very strongly developed.

'Regarding the tentacle club, according to G.L. Voss (1956, p. 131), "The tentacular clubs are expanded, bordered dorsally by a swimming membrane which occupies about the distal $2 / 3$ of the club and ventrally bordered by a swimming membrane which borders the entire club, having its origin about midway of the carpal cluster of adhesive buttons and cups." On the specimens that I have examined, the swimming membrane extends $3 / 4$ of the way along the length of the club and is situated on the dorsal face at its extremity, but does not border on the oral face of the club. In reality, the oral face is bordered dorsally by a feeble protective membrane that extends along the basal half of the oral face, and ventrally by strong protective membrane along its entire length. On the specimen from Monaco, which is in a perfect state of conservation, the two protective membranes are prolonged over the entire length of the oral face of the tentacle stalk. The example from Angola, less well preserved, does not retain the protective membrane on the tentacle stalk. According to G.L. Voss (1956, p. 131), the club bears 14 pairs of hooks; G. Pfeffer (1912, p. 95) indicates 10 to 12 pairs, and the specimen from Angola possesses nine pairs. As for the small distal suckers, G.L. Voss (1956, p. 131) only indicates 11 , while G. Pfeffer $(1912$, p. 96) cites a number that varies from 15 to 18; the example from Angola possesses 19. According to G.L. Voss, the rings of the small suckers are armed with miniscule teeth, but they are smooth in the specimen I have.

'G.L. Voss (1956, p. 132) did not observe the nuchal membranes; these are quite apparent in the material I am studying. Finally, I do not understand how that author was able to discern that his specimen was a female; in the specimen from Angola, which is somewhat larger, I cannot discern the genital organs.

'In my opinion it is necessary to capture other specimens before deciding that Ancistroteuthis lichtensteini is present in the Gulf of Mexico. 
'Moroteuthis robsoni, sp. nov.

'(Figs 2-3, pl. I, Figs 1-4)

'Moroteuthis sp. A Robson, G.C., 1924, p.2; 1924a, p.595

'Geographical distribution: Cape Town (135 fathoms) (G.C. Robson)

'Origin and material: Angola: Holotype: MBM - 1957 NO, M 7: 16³5.6'S, 11ํ19.5'E, 26/02/1957, chalut, depth 485-550m, 1 specimen.

'Relative dimensions:

\begin{tabular}{|c|c|c|c|}
\hline & & Holotype & $\underline{\text { Robson's }}$ \\
\hline \multirow[t]{3}{*}{ Mantle: } & dorsal length in $\mathrm{mm}$ & 470 & - \\
\hline & ventral length & 440 & 558 \\
\hline & width & $17(18)$ & 17.5 \\
\hline \multirow[t]{2}{*}{ Head: } & length & $16(17)$ & - \\
\hline & width & $13(13.5)$ & - \\
\hline \multirow[t]{3}{*}{ Fins: } & length & $60(64)$ & 70 \\
\hline & total width & $48(51)$ & 54 \\
\hline & point of greatest width & 15 & - \\
\hline \multirow[t]{4}{*}{ Arms: length } & $\mathrm{i}$ & L 53 (57), R 49 (52) & L 61, R - \\
\hline & II & L 60 (64), R 51 (55) & L 72, R 66 \\
\hline & III & L 47 (50), R 64 (68) & L - , R 70 \\
\hline & IV & L 64 (68), R 66 (70) & L 75, R 77 \\
\hline \multirow[t]{2}{*}{ Tentacles: } & total length & L 160 (170), R 160 (170) & L 165 \\
\hline & club length & L 30 (32), R 28 (30) & - \\
\hline Gladius: & length of cone & 22.5 & $<25$ \\
\hline Suckers: & diameter & 0.85 & - \\
\hline \multirow[t]{4}{*}{ Tentacles: } & carpal papillae & L 8, R 9 & - \\
\hline & carpal suckers & L 9, R 8 & - \\
\hline & hook pairs & L 121/2, R 13 & 14 \\
\hline & distal suckers & L 15, R 14 & $13-15$ \\
\hline
\end{tabular}

'Description:

'It is difficult to describe the form of the body exactly (PI. I, Fig. 1); the specimen has been compressed and folded several times during the expedition. The body is perhaps more or less cylindrical in the anterior part, but perhaps was originally conical along its entire length, gradually narrowing from the very broad mantle opening to the posterior extremity. The pallial margin is slightly angular dorsally and forms two points ventrally, at the level of the pallial cartilages. These last have a length of $6 \mathrm{~cm}$ on the left margin and $9 \mathrm{~cm}$ on the right.

'The fins, whose length surpasses half the mantle length, attain their greatest breadth at a quarter of their length. Their anterior margins are convex, the posterior first convex, then concave. They are strongly attenuate at the distal extremity of the body, and on the dorsal surface, over a distance of $15 \mathrm{~mm}$, there are weak depressions reminiscent of alvaeolae.

'Large flattened papillae, more or less irregular in form and attaining a diameter of $5 \mathrm{~mm}$, cover the dorsal and ventral surfaces of the mantle (PI. I, Fig. 2). They are not present on the fins or on the surface of the mantle posteriorly beneath the fins. 
'The head is somewhat damaged, the left eye absent. The right eye opening has a sinus in the middle of the anterior margin, with the dorsal part covering the ventral part.

'The medio-dorsal cartilage is $5 \mathrm{~cm}$ long, subcircular in its anterior third, where the width reaches $1.5 \mathrm{~cm}$, and in the posterior two-thirds the margins are parallel, with a width of $1 \mathrm{~cm}$. Along the entire length is a flattened medial crest, with a width of 4 to $5 \mathrm{~mm}$, with a longitudinal groove.

'The funnel cartilages measure $4 \times 1.5 \mathrm{~cm}$, are fusiform and possess a medial groove.

'The funnel groove is without folds or pockets. On each side are three longitudinal nuchal folds, but the poor conservation does not permit a more detailed description; they appear completely separate from each other, not joined by a transverse membrane.

'The dorsal and dorso-lateral arms are flattened at the base of the exterior face, with a weak swimming membrane on the outer face. The ventro-lateral arms are laterally compressed, with a swimming membrane that very strongly developed, especially near the midpoint of the arms.

'The ventral arms are flattened at the ventral edge and possess a well-developed exterior swimming membrane.

'The suckers, globular with smooth rings, are arranged in two longitudinal rows, widely spaced at the bases of the arms. The protective membranes are slightly developed. The tentacle stalks are laterally compressed and furnished with a weak membranous crest along the entire length of the external surface. This crest continues onto the club and enlarges into a swimming membrane in the distal half of the club.

'The base of the club possesses a flattened carpal oval, measuring $2 \times 1 \mathrm{~cm}$, bearing on the left: eight papillae and nine suckers, on the right: nine papillae and eight suckers. The main part of the club (PI. I, Fig. 3) bears three pairs of obliquely set hooks at its base; the first pair begins with a very small hook near the ventral [club] margin, in the second pair the sizes are equal, and in the third pair the ventral hook is the larger. Thereafter there is a change in the directions of the hook pairs, as if one were missing from the ventral row, but this is not the case. In total there are 12 pairs of hooks and an additional hook distally on the dorsal side. In the dorsal series, the size of the hooks increases through the $3^{\text {rd }}$ or $4^{\text {th }}$ and then diminishes gradually to the $13^{\text {th }}$. The ventral series commences with a very small hook, then the size increases rapidly through the $6^{\text {th }}$ or $7^{\text {th }}$, then diminishes to the $12^{\text {th }}$. The largest hook is $14 \mathrm{~mm}$ long.

'On the right tentacle club (PI. I, Fig. 4) there are 13 pairs of hooks, obliquely set.

'The first pair commences with a small hook on the dorsal side; apart from this there is little difference from the left club. The distal extremity bears three rows of four and one row of three small suckers on the left club; on the right, three rows of four and one row of two small suckers. The protective membranes are slightly developed, the dorsal extending from most of the carpus to the level of the largest hooks, the larger ventral best developed at two-thirds of the club length.

'The buccal membrane has seven points, attached to the dorsal side of the dorsal and dorso-lateral arms, and to the ventral side of the ventro-lateral and ventral arms.

'The gladius is too poorly conserved to allow a detailed description. The cone has a dorso-ventral diameter of $12.5 \mathrm{~mm}$, with a thickness of $7.5 \mathrm{~mm}$. It is more or less 
triangular in cross-section, with the dorsal edge rounded and divided in two in the anterior third by a deep groove. It curves toward the dorsal surface in the posterior third.

'The beak and radula (Fig. 2) exactly correspond to those described by G.C. Robson (1924a, p.597. Figs 1-2). The cuticular sheath of the buccal wall bears strong teeth, directed toward the back (Fig. 3).

'Similarities and differences:

'The place of capture of this specimen is the closest to that of Moroteuthis aequatorialis Thiele, 1920 (p. 443). This last species was caught at $0^{\circ} 16^{\prime} \mathrm{N}, 18^{\circ} 07^{\prime} \mathrm{W}$, floating dead at the surface and without tentacles. It is distinguished by its smooth skin, the short fins $(+/-50 \%)$ but very broad $(70 \%)$, and the cone of the gladius being relatively short $(15 \%)$.

'The specimen Robson described as Moroteuthis sp. A, originating from Cape Town, appears to be the same species as this specimen from Angola; there are no notable differences as far as I can judge, given the poor state of conservation.

G.C. Robson (1924a, p. 598) discussed the similarities and differences between his Moroteuthis sp. A and the other related species. He concludes (p. 599): "Although our data for comparison with other forms is not very complete, I consider we know enough to assume this form is specifically different. I do not consider, however, that a single specimen is enough material upon which to base a specific definition, especially as the form in question exhibits some vague affinities with other species."

'The general form of the body and fins most closely resemble those of Moroteuthis lönnbergii Ishikawa \& Wakiya (1914, PI. 45). The form of the body does not differ much from that of Moroteuthis robusta (Verrill, 1876) (fide M. Sasaki, 1929, p. 231, Fig. 171), but the fins of the latter species attain their greatest width just a little before the middle of their length, which gives them a very different appearance, contrary to what G.C. Robson claims (1924a, p.595). The relative length of the fins is $+/-50 \%$ of the mantle in the large species of Moroteuthis robusta described by M. Sasaki (1929, p. 235). It is the same in the specimen figured by A.E. Verrill (1880, PI. 23, Fig. 2), although in the specimen described by d'Arcy W. Thompson (1900, p. 992), the fins are longer, +/$56 \%$. The total width of the fins varies from 42 to $45 \%$ the length of the mantle in these specimens of Moroteuthis robusta and is smaller in the African species. In Moroteuthis ingens (Smith, 1881), the total width of the fins surpasses their length (fide C. Ishikawa \& Y. Wakiya, 1914, pp. 457-458).

'The characteristic sculpture of the mantle skin is unique to the African species, although, as in Moroteuthis lönnbergii, it extends over the head and the base of the arms. The sculpture is otherwise quite different, however: on the African species, the large papillae are flattened and attain a diameter of $5 \mathrm{~mm}$; in Moroteuthis lönnbergii the papillae are found on the head in ten very small rows, whereas on the mantle they become elongate ridges with a diameter of 0.3 to $0.8 \mathrm{~mm}$, anastomosing and reuniting obliquely.

'The radula of Moroteuthis lönnbergii, according to its description by M. Sasaki (1929, p. 237, Fig. 119), differs from that of the African species in having smaller medial and admedial teeth and longer marginal teeth. 


\section{'Diagnosis:}

'Moroteuthis robsoni sp. nov. is characterised by its conical body, very attenuate at the rear, with sculpture on the mantle skin comprised of large flattened papillae, of more or less irregular form, by the long fins with their greatest width at a quarter of their length, by the form of the tentacle club, and by the gladius, whose cone attains nearly the quarter of its entire length.' 


\section{APPENDIX E}

\section{Systematic summary}

Possible additional synonyms

Each species treated herein can be identified by unique combinations of characters/states at known ontogenetic stages. However, since some taxa are known only from small numbers of specimens and/or single life stages (particularly early or late), some species presently considered distinct may ultimately prove synonymous with other taxa.

The species accepted herein with the least certainty, and their most likely senior names, are:

- Onychoteuthis bergii (?= Os. banksii)

- Onychoteuthis mollis (?= an older Onychoteuthis species)

- Onykia (Onykia) robsoni (?= Ok. (Ok.) carriboea)

- Onykia (Onykia) aequatorialis (?= Ok. (Ok.) carriboea)

- Onykia (Onykia) indica (?= an older Onykia species)

- Gen. nov 2 spp. 1-3 (?= Gen. nov. 2 rancureli)

Pending additional material, Gen. nov. 2 may also prove synonymous with Walvisteuthis Nesis \& Nikitina, 1986 (currently in its own family, the Walvisteuthidae).

\section{Unresolved names with possibly extant types}

Several unresolved species names have reportedly extant types that were not available for examination in this study. Should this material become available for examination in the future, the following names may also prove taxonomically valid:

- 'Loligo' cardioptera Lesueur \& Petit in Peron, 1807 - MNHN 3-2-647 (see table below); Lesueur (1821) suggested affinity with Sepiola; considered onychoteuthid by Lu et al. (1995)

- 'Onykia' angulatus Lesueur, 1821 - MNHN 3-1-628, 3-1-643 (see table below); $\mathrm{ML} \sim 125 \mathrm{~mm}$, Canary Islands, $36^{\circ} 40^{\prime} \mathrm{S}, 29^{\circ} \mathrm{E}$; may prove taxonomically valid Onychoteuthis species or junior synonym of Os. banksii or Os. bergii

- 'Teleonychoteuthis' krohnii Vérany, 1847 - ?MHNN type (see table below)

- 'Loligo' bianconii Vérany, 1847 — ?MHNN type (see table below)

- 'Teleoteuthis' appelloefi Pfeffer, 1900 - supposedly deposited in Leipzig Museum, ML 38mm, Atlantic origins; accepted by Voss (1956: 128)

- 'Teleoteuthis' caroli Joubin, 1900 - 3 MOM ?syntypes (see table below), Bay of Biscay, $\sim 45^{\circ} \mathrm{N}, 5^{\circ} \mathrm{W}$; appears to be Onychoteuthis from illustrations

Information on the type material and current systematic status of each species name historically attributed to the Onychoteuthidae is chronologically summarised below. 


\begin{tabular}{|c|c|c|c|c|}
\hline Species & $\begin{array}{c}\begin{array}{c}\text { Current systematic } \\
\text { status }\end{array} \\
\end{array}$ & Type specimen(s) & Condition & Remarks \\
\hline $\begin{array}{l}\text { Onykia cardioptera } \\
\text { Lesueur \& Petit in } \\
\text { Peron, } 1807\end{array}$ & Unresolved & $\begin{array}{l}\text { Syntype, MNHN 3-2-647, } \\
\text { fide Lu et al. (1995: 313) }\end{array}$ & Unknown & $\begin{array}{l}\text { Not relocated for examination in } 2007 \text {; } \\
\text { Steenstrup (1880: } 96 \text { ) synonymised } \\
\text { with Ok. (OK. ) carriboea }\end{array}$ \\
\hline $\begin{array}{l}\text { Loligo banksii } \\
\text { Leach, } 1817\end{array}$ & $\begin{array}{l}\text { Onychoteuthis } \\
\text { banksii }\end{array}$ & Holotype, BMNH 1986266 & $\begin{array}{c}\text { Fair } \\
\text { (pers. obs.) }\end{array}$ & $\begin{array}{l}\text { Redescribed and -diagnosed by Bolstad } \\
\text { (in press) }\end{array}$ \\
\hline $\begin{array}{l}\text { Onychoteuthis bellonii } \\
\text { Lichtenstein, } 1818\end{array}$ & Nomen nudum & None designated & - & fide Lu et al. 1995: 312 \\
\hline $\begin{array}{l}\text { Onychoteuthis bergii } \\
\text { Lichtenstein, } 1818\end{array}$ & Onychoteuthis bergii & $\begin{array}{l}\text { Lectotype, ZMB Moll.121; } \\
\text { paralectotype, ZMB } \\
\text { Moll.123 }\end{array}$ & $\begin{array}{c}\text { Good } \\
\text { (pers. obs.) }\end{array}$ & $\begin{array}{l}\text { Köhler \& Glaubrecht (2004: 280) } \\
\text { designated lectotype }\end{array}$ \\
\hline $\begin{array}{l}\text { Onychoteuthis molinae } \\
\text { Lichtenstein, } 1818\end{array}$ & Nomen dubium & $\begin{array}{l}\text { ? Royal College of } \\
\text { Surgeons RCSHC/308 } \\
\text { (Enoploteuthis cookii) }\end{array}$ & Unknown & $\begin{array}{l}\text { New name for Sepia unguiculata } \\
\text { Molina, 1782; fide Roper \& Vecchione } \\
\text { (1993: 443); holotype is buccal bulb }\end{array}$ \\
\hline $\begin{array}{l}\text { Loligo bartlingii } \\
\text { Lesueur, } 1821\end{array}$ & Unresolved & ANSP, fide Voss 1962: 1 & Not extant & $\begin{array}{l}\text { No type; original description non- } \\
\text { diagnostic }\end{array}$ \\
\hline $\begin{array}{l}\text { Onykia carriboea } \\
\text { Lesueur, } 1821\end{array}$ & $\begin{array}{l}\text { Onykia (Onykia) } \\
\text { carriboea }\end{array}$ & ANSP, fide Voss 1962: 1 & Not extant & \\
\hline $\begin{array}{l}\text { Onykia angulatus } \\
\text { Lesueur, } 1821\end{array}$ & Unresolved & $\begin{array}{l}\text { Syntype, MNHN 3-2-647, } \\
\text { fide Lu et al. (1995: 313) }\end{array}$ & Unknown & Not relocated for examination in 2007 \\
\hline $\begin{array}{l}\text { Loligo felina } \\
\text { Blainville, } 1823\end{array}$ & Unresolved & $\begin{array}{l}\text { MNHN, fide Lu et al. } \\
\quad(1995: 316)\end{array}$ & Not extant & $\begin{array}{l}\text { Holotype illustrated in d'Orbigny (1848, } \\
\mathrm{PI} .1 \text { ) as Onychoteuthis angulata }\end{array}$ \\
\hline $\begin{array}{l}\text { Loligo uncinata } \\
\text { Quoy \& Gaimard, } 1825\end{array}$ & Unresolved & $\begin{array}{l}\text { MNHN, fide Lu et al. } \\
\text { (1995: 316) }\end{array}$ & Not extant & $\begin{array}{l}\text { No type; original description non- } \\
\text { diagnostic }\end{array}$ \\
\hline $\begin{array}{l}\text { Onychoteuthis lessonnii } \\
\text { Férussac, } 1830\end{array}$ & Nomen nudum & $\begin{array}{l}\text { MNHN, fide Lu et al. } \\
\text { (1995: 316) }\end{array}$ & Not extant & fide Lu et al. 1995: 316 \\
\hline $\begin{array}{l}\text { Onychoteuthis fleuryi } \\
\text { Reynaud in Lesson, } 1831\end{array}$ & Unresolved & $\begin{array}{l}\text { MNHN, fide Lu et al. } \\
\quad(1995: 316)\end{array}$ & Not extant & $\begin{array}{l}\text { No type; original description non- } \\
\text { diagnostic }\end{array}$ \\
\hline $\begin{array}{l}\text { Onychoteuthis peratoptera } \\
\text { d'Orbigny in Férussac \& } \\
\text { d'Orbigny, } 1834\end{array}$ & Unresolved & $\begin{array}{l}\text { MNHN, fide Lu et al. } \\
\text { (1995: 316) }\end{array}$ & Not extant & $\begin{array}{l}\text { No type; original description non- } \\
\text { diagnostic }\end{array}$ \\
\hline $\begin{array}{l}\text { Onychoteuthis platyptera } \\
\text { d'Orbigny in Férussac \& } \\
\text { d'Orbigny, } 1834\end{array}$ & Unresolved & $\begin{array}{l}\text { MNHN, fide Lu et al. } \\
\text { (1995: 316) }\end{array}$ & Not extant & $\begin{array}{l}\text { No type; original description non- } \\
\text { diagnostic }\end{array}$ \\
\hline $\begin{array}{l}\text { Onychoteuthis leachii } \\
\text { d'Orbigny in Férussac \& } \\
\text { d'Orbigny, } 1835\end{array}$ & Unresolved & $\begin{array}{l}\text { MNHN, fide Lu et al. } \\
\text { (1995: 316) }\end{array}$ & Not extant & $\begin{array}{l}\text { No type; original description non- } \\
\text { diagnostic }\end{array}$ \\
\hline $\begin{array}{l}\text { Onychoteuthis lesueurii } \\
\text { d'Orbigny in Férussac \& } \\
\text { d'Orbigny, } 1835\end{array}$ & $\begin{array}{l}\text { Onykia angulatus } \\
\text { (unresolved, see } \\
\text { above) }\end{array}$ & $\begin{array}{l}\text { Syntype, MNHN 3-2-647, } \\
\text { fide Lu et al. (1995: 313) }\end{array}$ & Unknown & $\begin{array}{l}\text { Same type specimen as Onykia } \\
\text { angulatus; not relocated for } \\
\text { examination in } 2007\end{array}$ \\
\hline $\begin{array}{l}\text { Onychoteuthis lichtensteinii } \\
\text { Férussac, } 1835\end{array}$ & $\begin{array}{l}\text { Ancistroteuthis } \\
\text { lichtensteinii }\end{array}$ & $\begin{array}{c}\text { Holotype, MNHN } 3.1 .620 \\
\text { (and 3.1.621, gladius of } \\
\text { same) }\end{array}$ & $\begin{array}{c}\text { Good } \\
\text { (pers. obs.) }\end{array}$ & \\
\hline $\begin{array}{l}\text { Loligo laticeps } \\
\text { Owen, } 1836\end{array}$ & Unresolved & None designated & Unknown & $\begin{array}{l}\text { Pfeffer (1912: } 37) \text { synonymised with } \\
\text { Onykia carriboea }\end{array}$ \\
\hline $\begin{array}{l}\text { Loligo bianconii } \\
\text { Vérany, } 1847\end{array}$ & Unresolved & $\begin{array}{c}\text { ? MHNN, fide Sweeney \& } \\
\text { Young (2003) }\end{array}$ & Unknown & $\begin{array}{l}\text { Steenstrup (1880: 100) considered } \\
\text { Onychoteuthis sp. }\end{array}$ \\
\hline $\begin{array}{l}\text { Teleonychoteuthis krohnii } \\
\text { Vérany, } 1847\end{array}$ & Unresolved & $\begin{array}{l}\text { MHNN (M. Roeleveld, } \\
\text { unpubl. data*) }\end{array}$ & Unknown & $\begin{array}{l}\text { Type material not available for } \\
\text { examination in this study; Pfeffer (1912: } \\
\text { 55) and Adam (1952: } 77 \text { ) synonymised } \\
\text { with Os. banksii }\end{array}$ \\
\hline
\end{tabular}

* unpublished notes stored at SAM 
(continued)

\begin{tabular}{|c|c|c|c|c|}
\hline Species & $\begin{array}{c}\begin{array}{c}\text { Current systematic } \\
\text { status }\end{array} \\
\end{array}$ & Type specimen(s) & Condition & Remarks \\
\hline $\begin{array}{l}\text { Onychoteuthis dussumieri } \\
\text { d'Orbigny, } 1849\end{array}$ & Unresolved & $\begin{array}{l}\text { MNHN, fide Lu et al. } \\
\text { (1995: 315) }\end{array}$ & Not extant & Illustration clearly pholidoteuthid \\
\hline $\begin{array}{l}\text { Onychoteuthis } \\
\text { kamtschatica } \\
\text { Middendorff, } 1849\end{array}$ & $\begin{array}{c}\text { Gonatus } \\
\text { kamtschaticus }\end{array}$ & $\begin{array}{l}\text { Unknown (fide Sweeney \& } \\
\text { Roper 1998: 572) }\end{array}$ & Unknown & \\
\hline $\begin{array}{l}\text { Loligo plagioptera } \\
\text { Souleyet, } 1852\end{array}$ & Unresolved & $\begin{array}{l}\text { Syntypes, MNHN 3-2-648 } \\
\text { and 3-2-649 }\end{array}$ & $\begin{array}{c}\text { Poor } \\
\text { (pers. obs.) }\end{array}$ & $\begin{array}{l}\text { Pfeffer (1912: } 37 \text { ) synonymised with } \\
\text { Onykia carriboea }\end{array}$ \\
\hline $\begin{array}{l}\text { Onychotetuthis brevimanus } \\
\text { Gould, } 1852\end{array}$ & Unresolved & $\begin{array}{l}\text { Unknown; not found by } \\
\text { Johnson (1964: 32) }\end{array}$ & Not extant & $\begin{array}{l}\text { No type; original description non- } \\
\text { diagnostic }\end{array}$ \\
\hline $\begin{array}{l}\text { Onychoteuthis rutilus } \\
\text { Gould, } 1852\end{array}$ & Unresolved & Unknown & Not extant & $\begin{array}{l}\text { No type; not traced by Johnson (1964); } \\
\text { original description non-diagnostic }\end{array}$ \\
\hline $\begin{array}{l}\text { Onychoteuthis hamatus } \\
\text { Risso, } 1854\end{array}$ & $\begin{array}{l}\text { Ancistroteuthis } \\
\text { lichtensteinii }\end{array}$ & $\begin{array}{l}\text { ? MHNN, fide Sweeney \& } \\
\text { Young (2003) }\end{array}$ & Unknown & \\
\hline $\begin{array}{l}\text { Onychoteuthis perlatus } \\
\text { Risso, } 1854\end{array}$ & Chiroteuthis veranyi & None designated & Unknown & fide Adam (1952: 111) \\
\hline $\begin{array}{l}\text { Onychoteuthis fusiformis } \\
\text { Gabb, } 1862\end{array}$ & Unresolved & CASIZ & Not extant & $\begin{array}{l}\text { No type; original description non- } \\
\text { diagnostic }\end{array}$ \\
\hline $\begin{array}{l}\text { Onychoteuthis aequimanus } \\
\text { Gabb, } 1868\end{array}$ & $\begin{array}{l}\text { Onychoteuthis } \\
\text { aequimanus }\end{array}$ & Syntypes (2), ANSP 6444 & $\begin{array}{c}\text { Good } \\
\text { (pers. obs.) }\end{array}$ & \\
\hline $\begin{array}{l}\text { Onychoteuthis lobipennis } \\
\text { Dall, } 1871\end{array}$ & Unresolved & None designated & Unknown & Watercolor plate in USNM library \\
\hline $\begin{array}{l}\text { Ommastrephes robustus } \\
\text { Verrill, } 1876\end{array}$ & $\begin{array}{l}\text { Onykia (Onykia) } \\
\text { robusta }\end{array}$ & $\begin{array}{c}\text { Syntypes, USNM } 576952, \\
576974\end{array}$ & $\begin{array}{c}\text { Good } \\
\text { (pers. obs.) }\end{array}$ & $\begin{array}{l}\text { Syntypes comprise two beaks, one } \\
\text { buccal mass, one gladius rostrum and } \\
\text { two arm fragments }\end{array}$ \\
\hline $\begin{array}{l}\text { Onychoteuthis ingens } \\
\text { Smith, } 1881\end{array}$ & $\begin{array}{l}\text { Onykia } \\
\text { (Moroteuthopsis) } \\
\text { ingens }\end{array}$ & $\begin{array}{c}\text { Holotype, BMNH } \\
1880.10 .8 .1\end{array}$ & $\begin{array}{l}\text { Fair (pers. } \\
\text { obs.) }\end{array}$ & $\begin{array}{l}\text { Holotype consists of head \& brachial } \\
\text { crown }\end{array}$ \\
\hline $\begin{array}{l}\text { Onychoteuthis raptor } \\
\text { Owen, } 1881\end{array}$ & Unresolved & $\begin{array}{l}\text { ? Royal College of } \\
\text { Surgeons }\end{array}$ & Not extant & $\begin{array}{l}\text { No type; original description non- } \\
\text { diagnostic }\end{array}$ \\
\hline $\begin{array}{l}\text { Steenstrupiola chilensis } \\
\text { Pfeffer, } 1884\end{array}$ & Nomen dubium & $\mathrm{ZMH}$ & Not extant & fide Nesis et al. (1998: 154) \\
\hline $\begin{array}{l}\text { Onychoteuthis brachyptera } \\
\text { Pfeffer, } 1884\end{array}$ & Nomen dubium & $\mathrm{ZMH}$ & Not extant & fide Nesis et al. (1998: 154) \\
\hline $\begin{array}{l}\text { Onykia binotata } \\
\text { Pfeffer, } 1884\end{array}$ & Unresolved & ? ZMH & ? Not extant & $\begin{array}{l}\text { Not traced by Guererro-Kommritz } \\
\text { (2001); Pfeffer (1912: } 37 \text { ) synonymised } \\
\text { with Onykia carriboea }\end{array}$ \\
\hline $\begin{array}{l}\text { Onykia curta } \\
\text { Pfeffer, } 1884\end{array}$ & Unresolved & ? ZMH & ? Not extant & $\begin{array}{l}\text { Not traced by Guererro-Kommritz } \\
\text { (2001); Pfeffer (1912: 37) synonymised } \\
\text { with Onykia carriboea }\end{array}$ \\
\hline $\begin{array}{l}\text { Steenstrupiola atlantica } \\
\text { Pfeffer, } 1884\end{array}$ & Unresolved & ? ZMH & ? Not extant & $\begin{array}{l}\text { Not traced by Guererro-Kommritz } \\
\text { (2001); Pfeffer (1912: 37) synonymised } \\
\text { with Onykia carriboea }\end{array}$ \\
\hline $\begin{array}{l}\text { Teleoteuthis agilis } \\
\text { Verrill, } 1885\end{array}$ & $\begin{array}{l}\text { Onykia (Onykia) } \\
\text { carriboea }\end{array}$ & Holotype, USNM 40129 & $\begin{array}{c}\text { Good } \\
\text { (pers. obs.) }\end{array}$ & $\begin{array}{l}\text { Best available reference specimen for } \\
\text { Onykia (Onykia) carriboea, fide Voss } \\
(1956: 126)\end{array}$ \\
\hline $\begin{array}{l}\text { Chaunoteuthis mollis } \\
\text { Appellöf, } 1891\end{array}$ & $\begin{array}{l}\text { Onychoteuthis mollis } \\
\text { (new comb.) }\end{array}$ & Holotype, ZMBN 2428 & $\begin{array}{c}\text { Fair } \\
\text { (pers. obs.) }\end{array}$ & \\
\hline
\end{tabular}


(continued)

\begin{tabular}{|c|c|c|c|c|}
\hline Species & $\begin{array}{c}\begin{array}{c}\text { Current systematic } \\
\text { status }\end{array} \\
\end{array}$ & Type specimen(s) & Condition & Remarks \\
\hline $\begin{array}{l}\text { Teleoteuthis appelloefi } \\
\text { Pfeffer, } 1900\end{array}$ & Unresolved & ZMH or Leipzig Museum & ? Not extant & $\begin{array}{l}\text { Type material not available for } \\
\text { examination in this study }\end{array}$ \\
\hline $\begin{array}{l}\text { Teleoteuthis caroli } \\
\text { Joubin, } 1900\end{array}$ & Unresolved & $\begin{array}{l}\text { syntypes (3), MOM, fide } \\
\text { Sweeney \& Young (2003) }\end{array}$ & Unknown & $\begin{array}{l}\text { Type material not available for } \\
\text { examination in this study }\end{array}$ \\
\hline $\begin{array}{l}\text { Teleoteuthis jattai } \\
\text { Joubin, } 1900\end{array}$ & Unresolved & $\begin{array}{l}\text { MOM, fide M. Roeleveld } \\
\text { (unpubl. data*) }\end{array}$ & Not extant & $\begin{array}{l}\text { Pfeffer (1912: } 37 \text { ) synonymised with } \\
\text { Onykia carriboea }\end{array}$ \\
\hline $\begin{array}{l}\text { Teleoteuthis verrilli } \\
\text { Pfeffer, } 1900\end{array}$ & Nomen dubium & $\mathrm{ZMH}$ & Not extant & fide Nesis et al. (1998: 154) \\
\hline $\begin{array}{l}\text { Teleoteuthis intermedia } \\
\text { Pfeffer, } 1912\end{array}$ & Nomen dubium & $\mathrm{ZMH}$ & Not extant & fide Nesis et al. (1998: 154) \\
\hline $\begin{array}{l}\text { Teleoteuthis compacta } \\
\text { Berry, } 1913\end{array}$ & $\begin{array}{l}\text { Onychoteuthis } \\
\text { compacta }\end{array}$ & Holotype, USNM 214381 & $\begin{array}{c}\text { Poor } \\
\text { (pers. obs.) }\end{array}$ & $\begin{array}{l}\text { Juvenile specimen (ML } 21 \mathrm{~mm} \text { ), } \\
\text { extensively damaged, no remaining } \\
\text { systematic characters }\end{array}$ \\
\hline $\begin{array}{l}\text { Moroteuthis loennbergii } \\
\text { Ishikawa \& Wakiya, } 1914\end{array}$ & $\begin{array}{l}\text { Onykia (Onykia) } \\
\text { loennbergii } \\
\text { (new comb.) }\end{array}$ & None designated & - & \\
\hline $\begin{array}{l}\text { Moroteuthis aequatorialis } \\
\text { Thiele, } 1920\end{array}$ & $\begin{array}{l}\text { Onykia (Onykia) } \\
\text { aequatorialis } \\
\text { (new comb.) }\end{array}$ & $\begin{array}{l}\text { Holotype, ZMB Moll.- } \\
110000\end{array}$ & $\begin{array}{c}\text { Fair } \\
\text { (pers. obs.) }\end{array}$ & $\begin{array}{l}\text { Holotype is spent female, lacking } \\
\text { tentacle clubs; radula missing }\end{array}$ \\
\hline $\begin{array}{l}\text { Onychoteuthis } \\
\text { borealijaponicus } \\
\text { Okada, } 1927\end{array}$ & $\begin{array}{l}\text { Onychotethis } \\
\text { borealijaponica }\end{array}$ & None designated & & \\
\hline $\begin{array}{l}\text { Moroteuthis robsoni } \\
\text { Adam, } 1962\end{array}$ & $\begin{array}{l}\text { Onykia (Onykia) } \\
\text { robsoni } \\
\text { (new comb.) }\end{array}$ & $\begin{array}{l}\text { Deposition unknown } \\
\text { ('MBM - } 1957 \text { NO, M 7') }\end{array}$ & ? Not extant & \\
\hline $\begin{array}{l}\text { Onykia japonica } \\
\text { Taki, } 1964\end{array}$ & $\begin{array}{l}\text { Onykia (Onykia) } \\
\text { robusta } \\
\text { (new comb.) }\end{array}$ & $\begin{array}{l}\text { Holotype, private collection } \\
\text { of I. Taki, Kyoto, fide } \\
\text { Sweeney \& Young (2003) }\end{array}$ & Unknown & $\begin{array}{l}\text { Type material not available for } \\
\text { examination in this study; Tsuchiya \& } \\
\text { Okutani (1992: 141) syonymised with } \\
\text { Ok. (Ok. ) robusta }\end{array}$ \\
\hline $\begin{array}{l}\text { Moroteuthis knipovitchi } \\
\text { Filippova, } 1972\end{array}$ & $\begin{array}{l}\text { Gen. nov. } 1 \\
\text { knipovitchi } \\
\text { (new comb.) }\end{array}$ & ZMMGU & Unknown & $\begin{array}{l}\text { Type material not available for } \\
\text { examination in this study }\end{array}$ \\
\hline $\begin{array}{l}\text { Kondakovia longimana } \\
\text { Filippova, } 1972\end{array}$ & $\begin{array}{l}\text { Kondakovia } \\
\text { longimana }\end{array}$ & ZMMGU & Unknown & $\begin{array}{l}\text { Type material not available for } \\
\text { examination in this study }\end{array}$ \\
\hline $\begin{array}{l}\text { Onykia rancureli } \\
\text { Okutani, } 1981\end{array}$ & $\begin{array}{l}\text { Gen. nov. } 2 \text { rancureli } \\
\text { (new comb.) }\end{array}$ & $\begin{array}{l}\text { Holotype, NSMT } \\
\text { Mo61210, } 3 \text { paratypes, } \\
\text { Mo59490 to } 59492\end{array}$ & $\begin{array}{c}\text { Fair } \\
\text { (pers. obs.) }\end{array}$ & \\
\hline $\begin{array}{l}\text { Onykia indica } \\
\text { Okutani, } 1981\end{array}$ & $\begin{array}{l}\text { Onykia (Onykia) } \\
\text { indica }\end{array}$ & $\begin{array}{c}\text { Holotype, NSMT } \\
\text { Mo59493; } 2 \text { paratypes, } \\
\text { NSMT Mo59494, } \\
\text { Mo59495 }\end{array}$ & $\begin{array}{c}\text { Fair } \\
\text { (pers. obs.) }\end{array}$ & \\
\hline $\begin{array}{l}\text { Moroteuthis pacifica } \\
\text { Okutani, } 1983\end{array}$ & $\begin{array}{l}\text { Onykia (Onykia) } \\
\text { robusta }\end{array}$ & $\begin{array}{l}\text { Holotype, NSMT } \\
\text { Mo61210; } 3 \text { paratypes, } \\
\text { Mo61211 to } 61213\end{array}$ & $\begin{array}{l}\text { Excellent } \\
\text { (pers. obs.) }\end{array}$ & $\begin{array}{l}\text { Tsuchiya \& Okutani (1992: 141) } \\
\text { syonymised with Ok. (Ok. ) robusta; } \\
\text { present data concur }\end{array}$ \\
\hline $\begin{array}{l}\text { Onychoteuthis } \\
\text { meridiopacifica } \\
\text { Rancurel \& Okutani, } 1990\end{array}$ & $\begin{array}{l}\text { Onychoteuthis } \\
\text { meridiopacifica }\end{array}$ & $\begin{array}{l}\text { Holotype, NSMT } \\
\text { Mo67008; } 4 \text { paratypes, } \\
\text { Mo67009 to } 67112\end{array}$ & Unknown & $\begin{array}{l}\text { Type material not available for } \\
\text { examination in this study }\end{array}$ \\
\hline $\begin{array}{l}\text { Notonykia africanae } \\
\text { Nesis, Roeleveld } \\
\text { \& Nikitina, } 1998\end{array}$ & Notonykia africanae & $\begin{array}{l}\text { Holotype, SAM S3414; } 13 \\
\text { paratypes, SAM S3412 to } \\
\text { S3421, S3452 }\end{array}$ & $\begin{array}{c}\text { Good } \\
\text { (pers. obs.) }\end{array}$ & \\
\hline $\begin{array}{l}\text { Notonykia nesisi } \\
\text { Bolstad, } 2007\end{array}$ & Notonykia nesisi & $\begin{array}{c}\text { Holotype, NMNZ M.91597; } \\
3 \text { paratypes, NMNZ } \\
\text { M.183110 }\end{array}$ & $\begin{array}{l}\text { Excellent } \\
\text { (pers. obs.) }\end{array}$ & \\
\hline $\begin{array}{l}\text { Onychoteuthis lacrima } \\
\text { Bolstad \& Seki, in press }\end{array}$ & $\begin{array}{l}\text { Onychoteuthis } \\
\text { lacrima }\end{array}$ & $\begin{array}{c}\text { Holotype, SBMNH } \\
\text { 423106; } 4 \text { paratypes, } \\
\text { SBMNH 423107, 423108; } \\
\text { KSB-062 }\end{array}$ & $\begin{array}{l}\text { Excellent } \\
\text { (pers. obs.) }\end{array}$ & $\begin{array}{l}\text { KSB-062 ( } 2 \text { paratypes) to be } \\
\text { accessioned and registered at USNM }\end{array}$ \\
\hline $\begin{array}{l}\text { Onychoteuthis prolata } \\
\text { Bolstad, Vecchione \& } \\
\text { Young, in press }\end{array}$ & $\begin{array}{l}\text { Onychoteuthis } \\
\text { prolata }\end{array}$ & $\begin{array}{c}\text { Holotype, SBMNH } \\
\text { 423101, paratype, USNM } \\
1102755\end{array}$ & $\begin{array}{l}\text { Excellent } \\
\text { (pers. obs.) }\end{array}$ & \\
\hline
\end{tabular}

